\title{
Novel synthetic approaches for fabrication of polymer brushes on gold surfaces via RAFT polymerization: A new era for gold modification
}

\author{
Dissertation \\ for the award of the degree \\ "Doctor rerum naturalium" (Dr.rer.nat.) \\ of the Georg-August-Universität Göttingen
}

within the doctoral program

of the Georg-August University School of Science (GAUSS)

submitted by

Candan Catli

from Istanbul

Göttingen, 2016 
$\underline{\text { Thesis Committee }}$

Prof. Dr. Philipp Vana

Macromolecular Chemistry, Physical Chemistry Institute

Prof. Dr. Jörg Enderlein

Single Molecule Spectroscopy for Biophysics and Complex Systems, III. Institute of Physics

\section{Members of the Examination Board}

Reviewer: Prof. Dr. Philipp Vana,

Macromolecular Chemistry, Physical Chemistry Institute

Second Reviewer: Prof. Dr. Jörg Enderlein

Single Molecule Spectroscopy for Biophysics and Complex Systems, III. Institute of Physics

Further members of the Examination Board:

Prof. Dr. Michael Buback

Technical and Macromolecular Chemistry Group, Physical Chemistry Institute

Prof. Dr. Konrad Samwer

Disordered Systems and Magnetic Thin Films Group, I. Physics Institute

Prof. Dr. Annette Zippelius

Statistical Physics and Complex Systems Group, Institute for Theoretical Physics

Prof. Dr. Karl-Henning Rehren

Mathematical Physics Group, Institute for Theoretical Physics

Date of the oral examiniation: 15.12.2016 
To three wonderful women in my life;

my mother, Nuran Catli

my aunt, Nuray Altiok

and my grandmother, Fatma Altiok

for their unconditional love and encouragement 

$1 \quad$ Abstract..................................................................................................................................11

2 General Introduction .......................................................................................12

3 Theoretical Background................................................................................................16

3.1 Free Radical Polymerization........................................................................16

3.2 Controlled Radical Polymerization .........................................................................21

3.2.1 Atom Transfer Radical Polymerization ............................................................23

3.2.2 Reversible Addition Fragmentation Chain Transfer Polymerization ...................23

3.2.2.1 The Mechanism of RAFT Polymerization....................................................

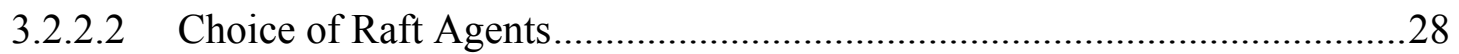

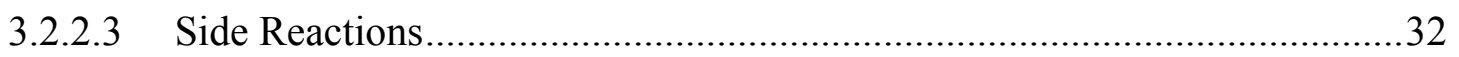

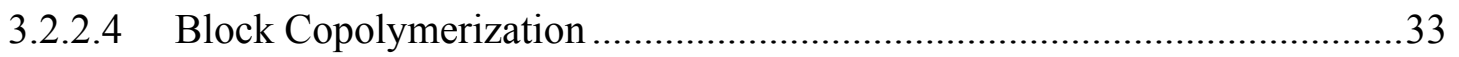

3.3 Molecular Self Assembly ...................................................................................34

3.3.1 Self Assembly of Thiol and Raft Agent Monolayer on Gold Surface..................37

3.3.1.1 Nature of Affinity of Thiols and Raft Agents toward Gold Surface.............37

3.3.1.1.1 An Overview of Main Bond Types.......................................................... 37

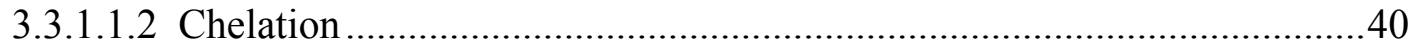

3.3.1.1.3 Metal-Thiolate Complex ........................................................................4

3.3.1.1.4 Organosulphur Compounds on Gold ....................................................42

3.3.1.2 Self Assembly of Thiols on Gold Surface ...................................................4

3.3.1.3 Self Assembly of Raft Agent Monolayer on Gold Surface ..........................47

3.4 Polymer Architectures........................................................................................49

3.5 Polymer Brushes .....................................................................................................52

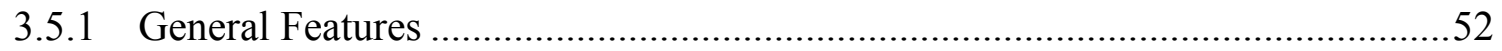

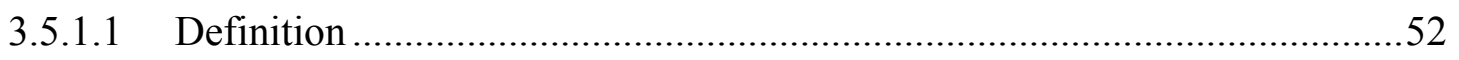

3.5.1.2 Theoritical Description of Polymer Brushes..............................................52

3.5.1.2.1 Conformation Types of Polymer Brushes...............................................54

3.5.1.2.2 Calculation of Grafting Density ..........................................................56

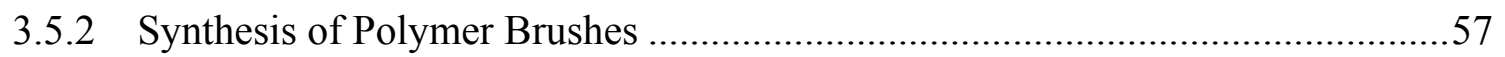

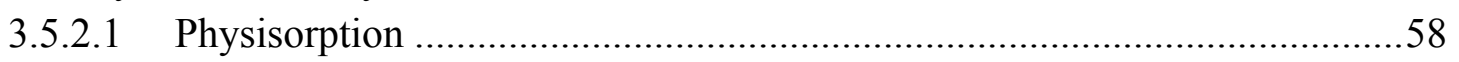

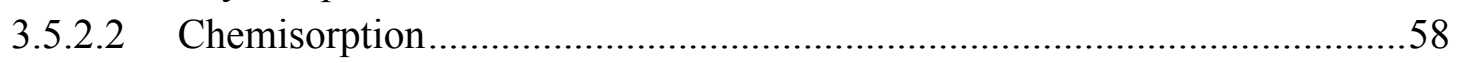

3.5.2.2.1 "Grafting To" Technique ..................................................................59

3.5.2.2.2 "Grafting From" Technique ....................................................................60

3.5.2.2.3 Synthesis of Polymer Brushes via SI-CRP ..............................................61

3.5.2.2.3.1 Synthesis of Polymer Brushes via SI-RAFT ........................................61

3.5.2.2.3.2 Control of Architecture via SI-CRP .................................................64

3.5.2.2.3.2.1 Block Copolymer Brushes via SI-CRP .......................................65

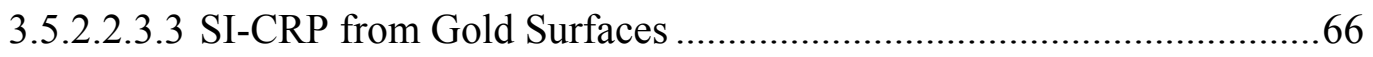

3.5.2.2.3.3.1 SI-RAFT from Gold Planar Surfaces............................................68

3.5.3 Properties and Applications of Polymer Brushes .................................................73

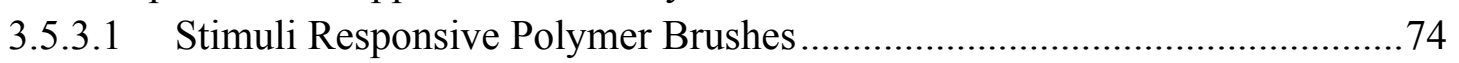

3.5.3.1.1 General Features .................................................................................. 74

3.5.3.1.2 Solvent Responsive Polymer Brushes ....................................................75

3.5.3.1.2.1 Solvent Responsive Homopolymer Brushes ......................................75 
3.5.3.1.2.2 Solvent Responsive Binary Mixed Homopolymer Brushes ..............75

3.5.3.1.2.3 Solvent Responsive Block Copolymer Brushes ................................ 77

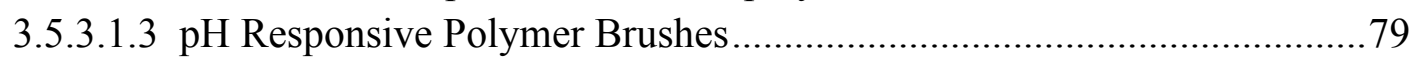

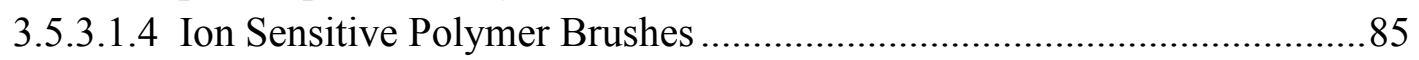

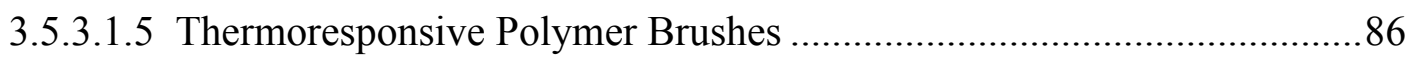

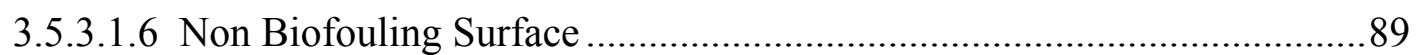

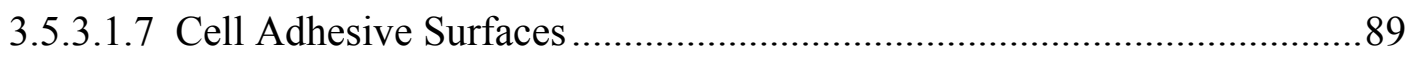

3.5.3.1.8 Protein Binding and Immobilization........................................................90

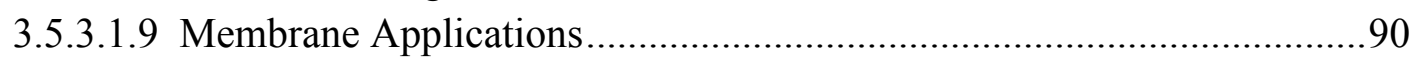

3.5.3.1.10 Antibacterial Coatings .................................................................. 91

3.6 Star Shaped Polymers.................................................................................92

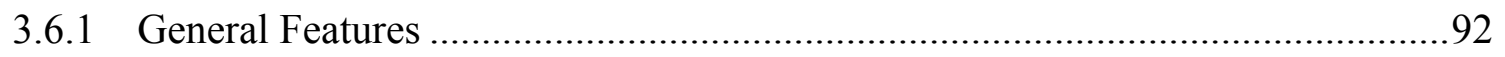

3.6.2 Synthetic Approaches of Star Shaped Polymers............................................... 93

3.7 Protein Fouling.................................................................................................94

4 Scientific Strategies for Polymer Brush Formation on Gold ........................................95

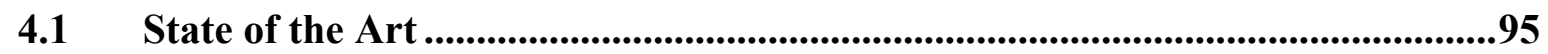

4.2 Scope of the Thesis ....................................................................................100

4.3 Motivation and Aim of the Work for Each Chapter ............................................103

4.3.1 Preparation of Polymer Brushes on Gold via Traditional SI-RAFT (R Group) Approach (Chapter 5) ....................................................................................... 103

4.3.2 Preparation of Polymer Brushes on Gold via Novel Chemisorption Based Approach (Chapter 6) .............................................................................................. 104

4.3.3 Synthesis of Polymer Brushes on Gold via Novel DTBA Mediated Polymerisation Approach (Chapter 7) .............................................................................................. 107

4.3.4 Synthesis of Star Polymer Brushes on Gold via Novel Z-RAFT Polymerisation

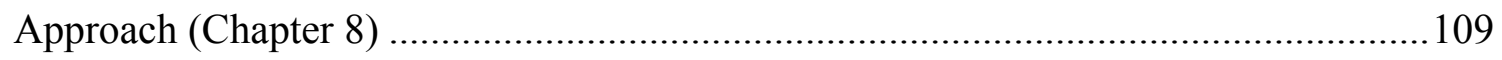

5 Characterization of Homopolymer and Diblock Copolymer Brushes Prepared on Gold via Traditional SI-RAFT (R Group Approach) .....................................................114

5.1 Characterization of Self Assembling Monolayer (MUD) and Raft Agent (CTA) Immobilized Gold Surfaces ................................................................................115

5.2 Kinetic Investigation of Poly (BuMA) Brush Growth on Gold-MUD-CTA Surface ...........................................................................................................116

5.3 Kinetic Investigation of Poly (DMAEMA) Brush Growth on Gold-MUD-CTA Surface ..........................................................................................................................120

5.4 Characterization of Poly (PEGMA) Synthesized on Gold-MUD-CTA

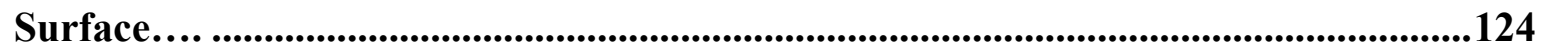

5.5 Characterization of Diblock Copolymer Brush (PPEGMA-b-PBuMA) Synthesized on Gold-PPEGMA Surface ..........................................................125

5.6 Characterization of Diblock Copolymer Brush (PDMAEMA-b-PPEGMA) Synthesized on Gold-PDMAEMA Surface............................................................................127

5.7 Investigation of Impact of Monomer / Raft Agent Ratio in Feed on Resulting Thickness and Grafting Density Values of Polymer Brushes ......................................130

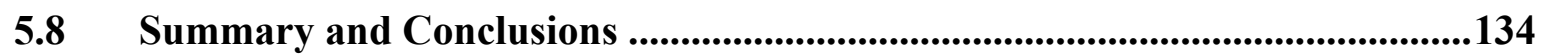


6 Characterization of Homopolymer and Diblock Copolymer Brushes Prepared on Gold via Novel Chemisorption Based Approach ...........................................................136

6.1 Characterization of Raft Agent Immobilized Gold Surfaces............................138

6.2 Kinetic Investigation of Polymer Brush (PBuMA) Growth on GoldEBPEDTTC Surface in the Presence of Bifunctional Free CTA (EBPEDTTC) .......141

6.3 Characterization of Homopolymer Brushes Prepared on Gold ........................145

6.3.1 Characterization of Poly (DMAEMA) Brushes Synthesized in the Presence of Free CTA (CDB) from Gold-CDB Surface .............................................................. 145

6.3.2 Characterization of Poly (DMAEMA) Brushes Synthesized in the Presence of Bifunctional Free CTA (EBPEDTTC) from Gold-EBPEDTTC Surface ....................... 147

6.3.3 Characterization of Poly (PEGMA) Brushes Synthesized in the Presence of Bifunctional Free CTA (EBPEDTTC) and in the Presence of Monofunctional Free CTA (DSTCSPA) from Gold-EBPEDTTC Surface..........................................................149

6.3.4 Characterization of Poly (PEGMA) Brushes Synthesized in the Presence of Tetra Functional Free CTA (PETSPETTCP) and in the Presence of Monofunctional Free CTA (DSTCSPA) from Gold-PETSPETTCP Surface

6.3.5 Characterization of Poly (PEGMA) Brushes Synthesized in the Presence of Tetra Functional Free CTA (PETSPETTCP) and in the Presence of Monofunctional Free CTA (DSTCSPA) from Gold-DPEHPETTC Surface 154

6.4 Characterization of Diblock Copolymer (PDMAEMA-b-PPEGMA) Brush Prepared in the Presence of Bifunctional Free CTA (EBPEDTTC) on GoldEBPEDTTC-PDMAEMA Surface ...........................................................................................157

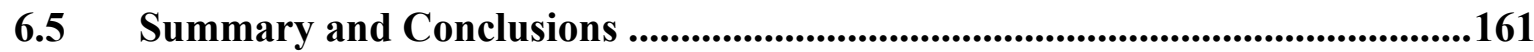

7 Characterization of Homopolymer and Diblock Copolymer Brushes Prepared on Gold via Novel DTBA Based Approach..............................................................164

7.1 Characterization of DTBA Immobilized Gold Surface ....................................165

7.2 Kinetic Investigation of Polymer Brush (PMMA) Growth in the Presence of Free DTBA on Gold-DTBA Surface 166

7.3 Characterization of Homopolymer Brushes Prepared on Gold ........................170

7.3.1 Characterization of Poly (BuMA) Brush Synthesized on Gold-DTBA Surface via DTBA Mediated Polymerization Technique. 170

7.3.2 Characterization of Poly (PEGMA) Brush Synthesized on Gold-DTBA Surface via RAFT Mediated Polymerization Technique ....................................................... 172

7.3.3 Investigation of Impact of Monomer / Raft Agent Ratio in Feed on Resulting Thickness and Grafting Density Values of Polymer Brushes Prepared via DTBA

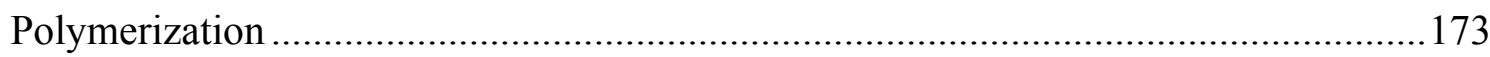

7.4 Characterization of Diblock Copolymer Brushes Prepared on Gold ..............178

7.4.1 Characterization of Diblock Copolymer Brush (PBuMA-b-PDMAEMA) Synthesized on Gold-DTBA-PBuMA Surface in the Presence of Free DTBA via DTBA

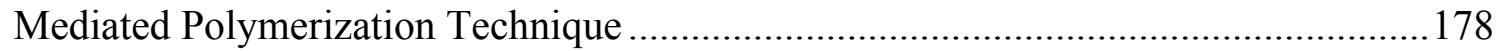

7.4.2 Characterization of Diblock Copolymer Brush (PPEGMA-b-PDMAEMA) Synthesized on Gold-DTBA-PPEGMA Surface in the Presence of Free CTA (CDB) via RAFT Mediated Polymerization Technique. 181 
8 Characterization of Star Polymer Brushes Prepared on Gold via Novel Z-Raft Star Polymerization Approach

8.1 Characterization of Self Assembling Monolayer and Raft Agents Immobilized on Gold Surface. .187

8.2 Kinetic Investigation of Synthesis of Tetrafunctional Star Poly (PEGMA) Brush from Gold-MUD-CTA Surface.

8.3 Characterization of Poly (PEGMA) Brushes Synthesized in the Presence of Linear and Multifunctional Raft Agents from Gold-MUD-CTA.

8.4 Investigation of Protein Resistivity of Star Poly (PEGMA) Brushes Prepared on Gold Surface ..............................................................................197

8.5 Summary and Conclusions ............................................................................199

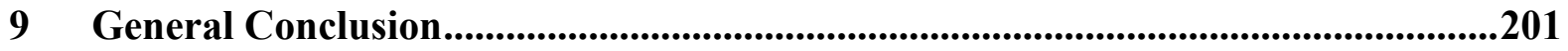

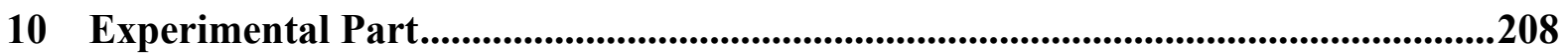

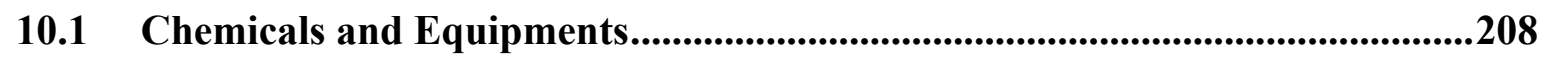

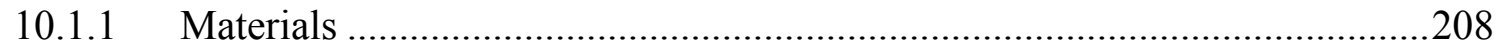

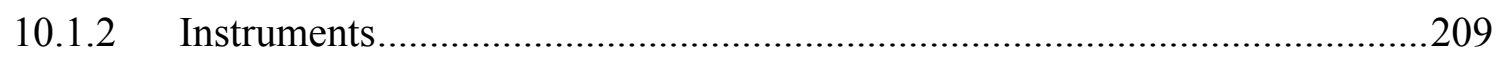

10.1.2.1 Gel Permeation Chromatography (GPC) ...............................................209

10.1.2.1.1 Working Principle and Data Analysis ...............................................209

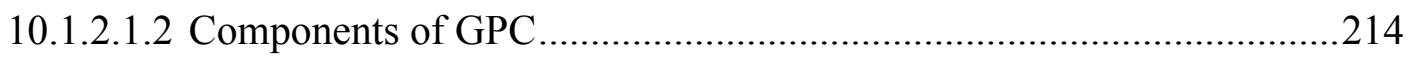

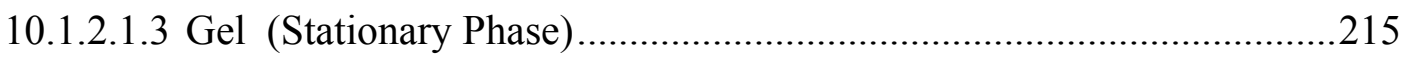

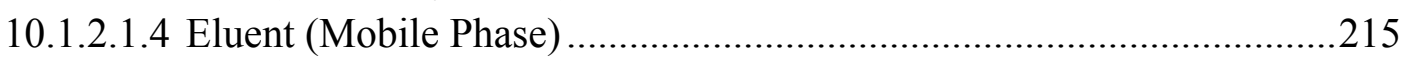

10.1.2.1.5 Column Selection ...............................................................................2. 215

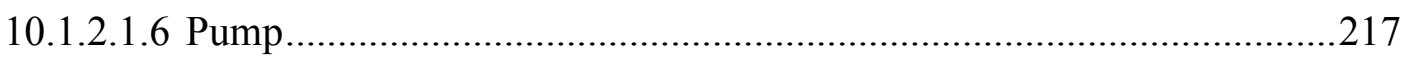

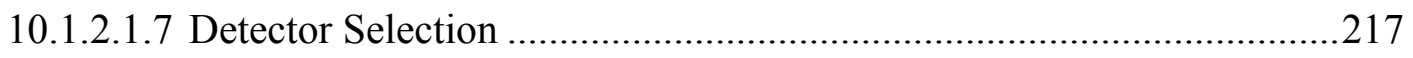

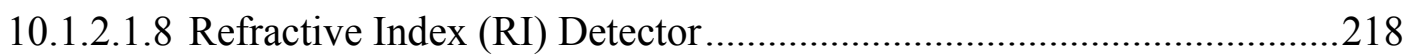

10.1.2.1.9 Ultraviolet Absorbance (UV) Detector .............................................219

10.1.2.1.10 Equipment Properties and Measurement Conditions ........................220

10.1.2.2 High Pressure Liquid Chromatography (HPLC) Mass Spectrometry .......220

10.1.2.2.1 Definitions $\backslash$ Basic Principle..............................................................22

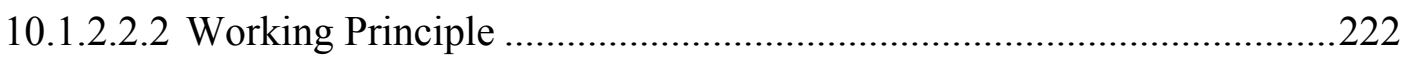

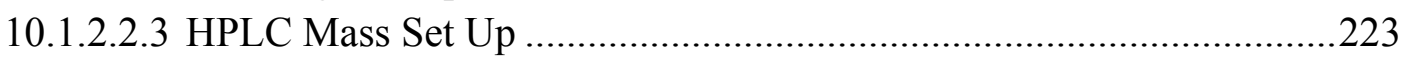

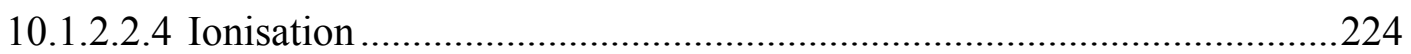

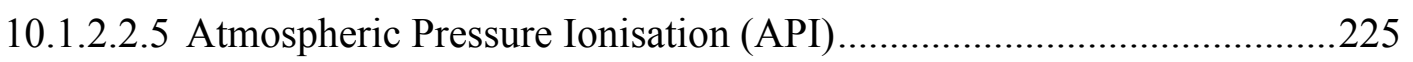

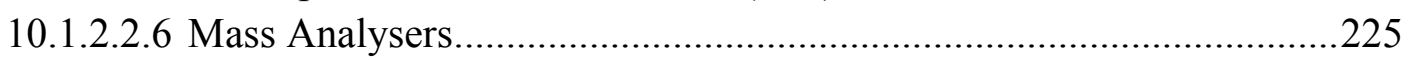

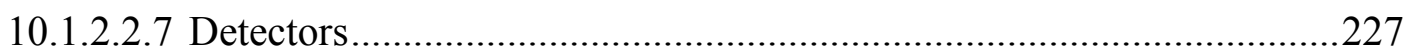

10.1.2.2.8 Equipment Properties $\backslash$ Measurement Conditions.................................228

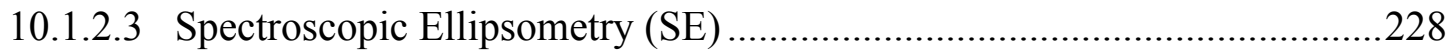

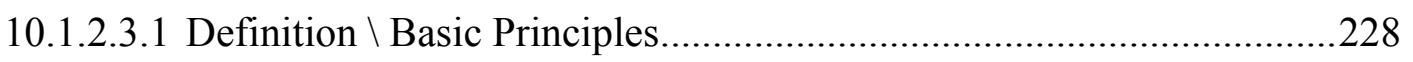

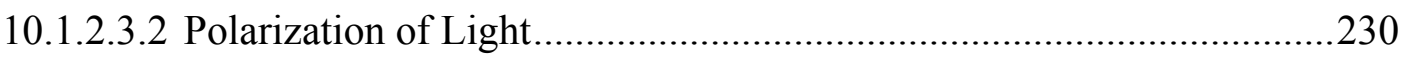

10.1.2.3.3 The Proper Coordinate System.........................................................230

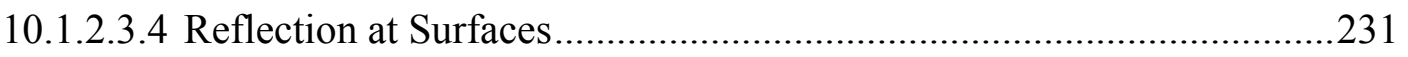


10.1.2.3.5 Ellipsometry Set up $\backslash$ Components ..................................................232

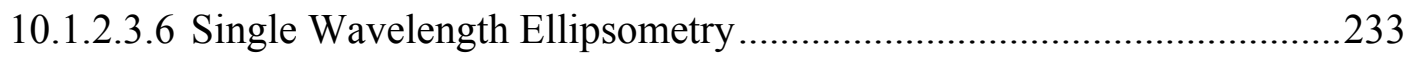

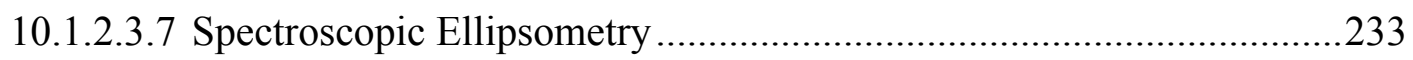

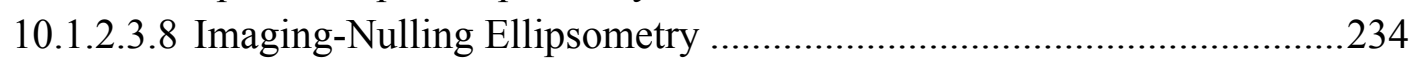

10.1.2.3.9 Data Analysis \Optical Modelling .....................................................2237

10.1.2.3.10 Equipment Properties $\backslash$ Measurement Conditions ...............................239

10.1.2.4 Attaneuted Total Reflectance Infrared (ATR-IR) Spectroscopy ...............239

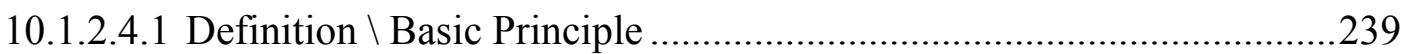

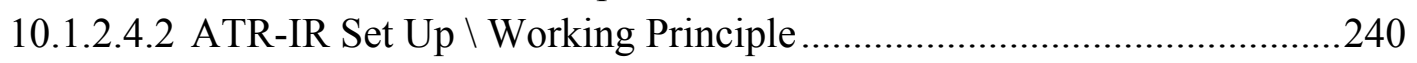

10.1.2.4.3 Equipment Properties $\backslash$ Measurement Conditions ..................................242

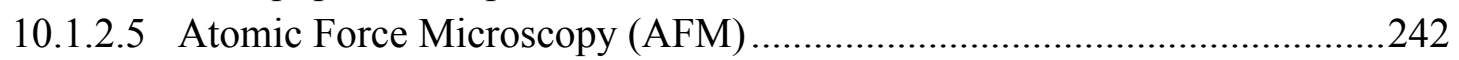

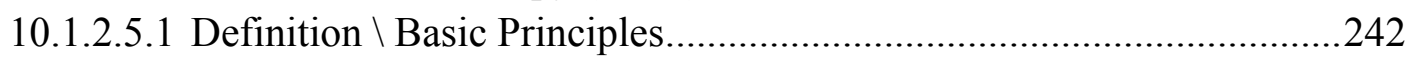

10.1.2.5.2 AFM Imaging Modes .......................................................................243

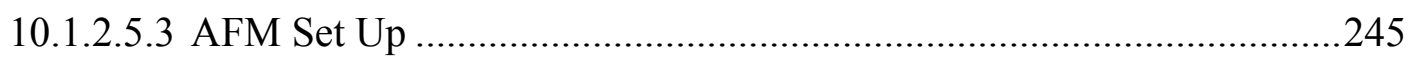

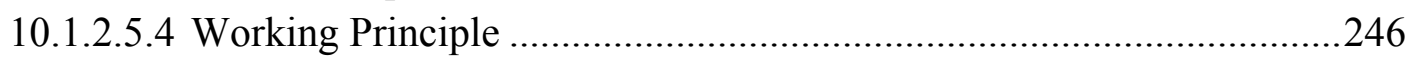

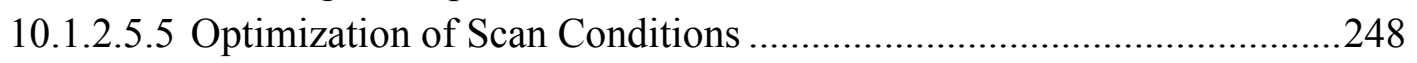

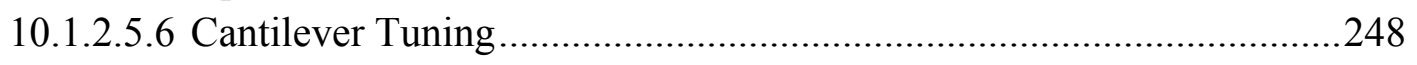

10.1.2.5.7 Scan Parameters and Imaging Conditions ............................................249

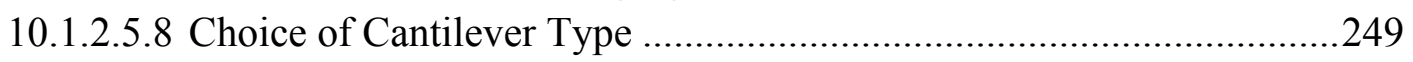

10.1.2.5.9 Equipment Properties $\backslash$ Measurement Conditions .................................250

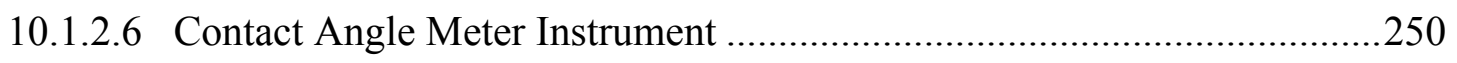

10.1.2.6.1 Definition and Basic Principle ...........................................................250

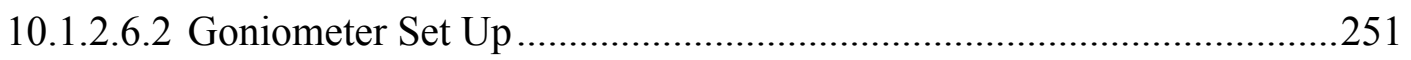

10.1.2.6.3 Dynamic or Static Measurement ........................................................252

10.1.2.6.4 Selecting Method and Drop Type ......................................................253

10.1.2.6.5 Shape Analysis and Baseline.........................................................253

10.1.2.6.6 Models for Contour Analysis (Young-Laplace Method) .....................254

10.1.2.6.7 Equipment Properties $\backslash$ Measurement Conditions...............................255

11 Self Assembly Processes and Polymerisations................................................................255

11.1 Self Assembly Process............................................................................................255

11.1.1 Self Assembly of MUD onto Gold Surface .................................................255

11.1.2 Self Assembly of Dithiobenzoicacid (DTBA) onto Gold Surface..................255

11.1.3 Self Assembly of Raft Agents onto Gold Surface .......................................255

11.1.4 Immobilization of Raft Agent onto Gold-MUD Surface .............................256

11.2 Polymerizations .....................................................................................................2256

11.2.1 Polymerization of BuMA in the Presence of Linear Free CTA from Gold-MUD-

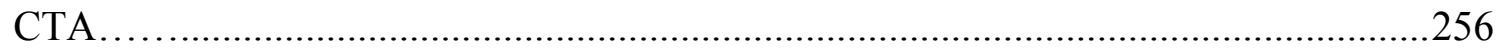

11.2.2 Polymerization of DMAEMA in the Presence of Linear Free CTA from Gold-

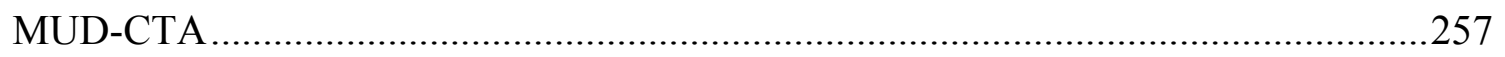

11.2.3 Copolymerization of BuMA from Gold-PPEGMA Surface...........................257

11.2.4 Copolymerization of PEGMA from Gold-PDMAEMA Surface....................258

11.2.5 Polymerization of DMAEMA in the Presence of Free CTA (CDB) from Gold-

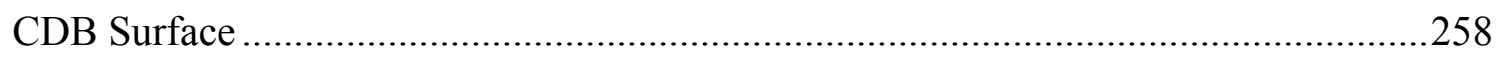


11.2.6 Polymerization of BuMA in the Presence of Bifunctional Free CTA (EBPEDTTC) from Gold-EBPEDTTC Surface ......................................................259

11.2.7 Polymerization of DMAEMA in the Presence of Bifunctional Free CTA (EBPEDTTC) from Gold-EBPEDTTC Surface ........................................................2.259

11.2.8 Polymerizations of PEGMA in the Presence of Monofunctional Free CTA (DSTCSPA) from Gold-EBPEDTTC, Gold-PETSPETTCP, Gold-DPEHPETTC

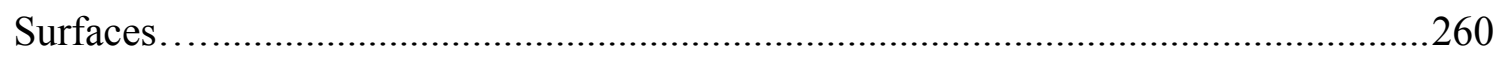

11.2.9 Polymerization of PEGMA in the Presence of Bifunctional Free CTA (EBPEDTTC) from Gold-EBPEDTTC Surface ..........................................................260

11.2.10 Polymerization of PEGMA in the Presence of Tetrafunctional Free CTA (PETSPETTCP) from Gold-PETSPETTCP and Gold-DPEHPETTC Surface ..............261

11.2.11 Copolymerization of PEGMA in the Presence of Bifunctional Free CTA (EBPEDTTC) from Gold-EBPEDTTC-PDMAEMA Surface .....................................261

11.2.12 Polymerization of MMA in the Presence of Free DTBA from Gold-DTBA

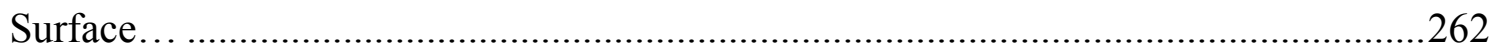

11.2.13 Polymerization of BuMA in the Presence of Free DTBA from Gold-DTBA

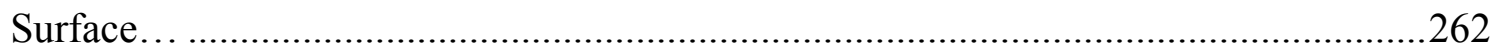

11.2.14 Polymerization of PEGMA in the Presence of Free CTA (DSTCSPA) from

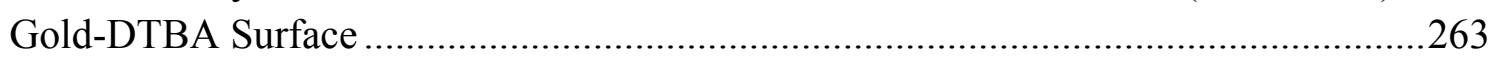

11.2.15 Copolymerization of DMAEMA in the Presence of Free DTBA from Gold-

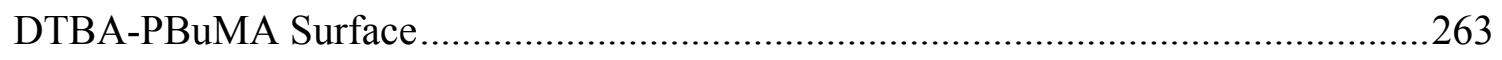

11.2.16 Copolymerization of DMAEMA in the Presence of Free CTA (CDB) from

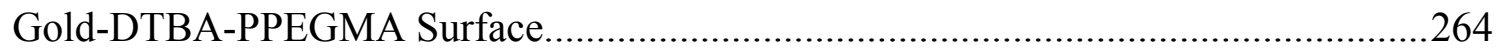

11.2.17 Polymerization of PEGMA in the Presence of Bifunctional Free CTA (EBPEDTTC) from Gold-MUD-CTA Surface..........................................................264

11.2.18 Polymerization of PEGMA in the Presence of Tetrafunctional Free CTA (PETSPETTCP) from Gold-MUD-CTA Surface …...................................................265

11.2.19 Polymerization of PEGMA in the Presence of Hexafunctional Free CTA (DPEHPETTC) from Gold-MUD-CTA Surface .......................................................265

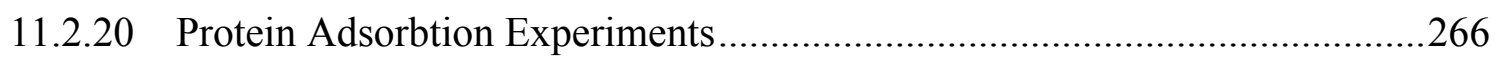

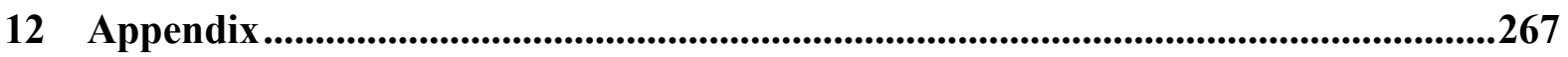

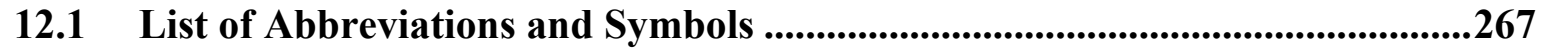

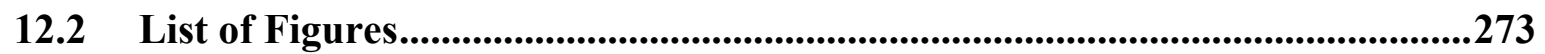

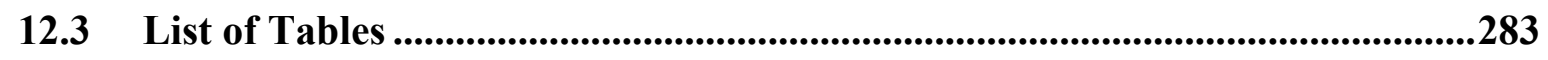

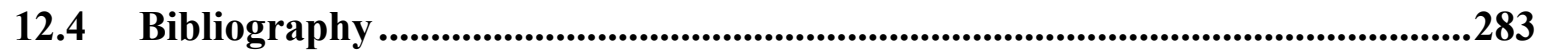

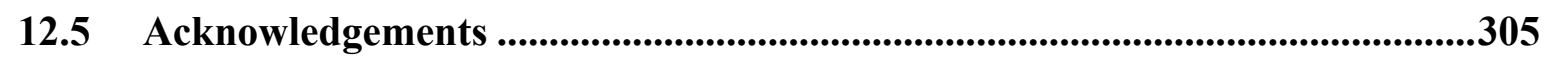

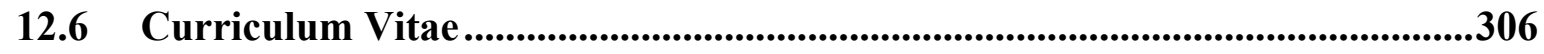




\section{PART I: INTRODUCTION}




\section{Abstract}

Nanobiotechnology is an important branch of nanotechnology, which has been dramatically developed for creating functional nanoscale materials for various biomedical applications. The past few decades have witnessed significant advances in the development of various functionalized surfaces for applications in a wide range of fields such as chemistry, biology, pharmacy and physics. There has recently been extensive research to modify gold surfaces, thereby opening up opportunities to enhance breadth of their applicability. Recently developed methods have allowed the modification of gold nanoparticles with suitable functionalizing agents, facilitate their applications in different areas such as chemical and biological sensing, imaging labelling, delivering, heating and biomedical applications such as cancer diagnostics and therapy, drug delivery, gene delivery, DNA and RNA analysis, antibacterial agent, vaccine preparation, brain implants, artificial skin and improving electrical signaling in the heart. Apart from gold nanoparticle surfaces, there are various blood contacting biomedical devices used for applications such as heart valves, vascular grafts, stents, in vivo biosensors etc. The lack of hemo compatibility is main problem of cardiovascular and other blood contacting medical devices. Congestion of small diameter vascular grafts and failure of blood contacting biosensors due to thrombus formation on device surface might be counted as some examples for this hemo compatibility problem. Non specific protein adsorption can decrease device performance, such as in the case of in vivo biosensor and stent surfaces. For this reason, improvement of anticoagulant devices or drugs is required for long term applications.

Surface modification is an essential process in biotechnological fields such as tissue engineering, biosensors, or implant manufacturing. Covalently bound polymer films offer an efficient and convenient way of modifying physicochemical characteristics of material surfaces used for various applications such as stabilization of colloidal particles, non-fouling coatings, and responsive films for sensors. Sofar, several strategies were developed for modification of device surfaces with an aim of reducing non-specific protein adsorbtion. Synthetic polymers serve as excellent candidates for surface modification because of their tunable mechanical properties, the variability of film thickness, degree of functionality and because of the potential multifunctional stimuli responsivity. Furthermore, synthetic polymers and their hybrids with biological molecules have been widely used in biotechnology, biomedical, and pharmaceutical technologies. In terms of the role of nanoscale properties in applications, it is important to tailor the properties of polymers at molecular level to fulfill the performance criteria better for any given application. The design requirements of polymers vary widely according to the application. The key properties for various applications can be counted as the molecular weight, molecular architecture, composition and chemical functionality. The uniformity in these key properties is mandatory for most biological applications of polymers (e.g. biomaterial surfaces), as it enables the performance to be correlated with structure. 
Among the variety of techniques that allow the formation of polymer thin films for surface modification, polymer brushes have gained special attention along the past decades due to their unique structures in combination with the possibility offered by controlled / "living" radical polymerization techniques (CLRP) to generate polymeric thin films with precisely controlled thickness, composition and architecture. Polymer brushes can be defined as an assembly of polymer chains which are tethered by one ends to a surface. Despite their interesting properties and the numerous reports describing synthetic pathways of polymer brushes via different CLRP techniques, polymer brush formation via Reversible Addition Fragmentation Chain Transfer (RAFT) polymerization technique has received little attention so far. RAFT polymerization is the most versatile platform for controlled synthesis of polymers for biological applications, with respect to monomer types and reaction conditions. When it comes to polymer brush formation on gold planar surfaces via RAFT technique, there has been a few studies in literature. This thesis describes how RAFT polymerization technique is successfully adapted onto gold surfaces with an aim of construction of dense polymer brush layers.

So far, the privileged way to tune the desired features, functionalities of a surface has been the growth of tethered polymer chains by the combination of a CRP technique with "grafting from" approach. However, this approach is experimentally rather complex as it involves multi-step synthetic procedures. With this background in mind, the main objective of this thesis work was to develop novel Raft based synthetic approaches for fabrication of polymer brushes with different architectures on gold surface. The interest to develop new synthetic strategies, which are relatively easier than multi-step procedures in literature used for synthesis of brushes, lies in the possibility of utilization of these novel straightforward techniques for the design and production of novel biomaterials used for advanced biomedical applications. The originality of this thesis work stems from the fact that the chemisorption tendency of raft agents toward gold and the specific mechanistic principles of RAFT, which allows for various synthetic strategies of performing surface-confined RAFT polymerizations, were successfully combined in order to develop novel straight forward pathways for synthesis of polymer brushes with complex topologies on gold.

\section{General Introduction}

Considering every single item used in everyday life, its properties are not only determined by the bulk material but also by its surface properties. The control of surface properties of materials and devices has a critical significance in many areas of science such as biomaterials used in tissue engineering (1), (2), controlled cell growth (3), controlled gene-drug delivery (4), stimuli responsive coatings for sensors (5), nonfouling coatings (6), photolithographic masks (7), adhesion promoters (8), lubricants (9), stabilization of colloidal particles (10). The smaller the item the higher the impact of surface properties as the surface-to-volume ratio increases. For this reason, 
adjustment of surface properties becomes more and more important in many fields such as device miniaturization (e.g. in electronic devices), nanobiotechnology (e.g. in drug delivery systems, biomedical devices).

Applications of coatings vary from large area coverage (e.g. in automotive industry or building exteriors) to defined functionalization of (sub-) micrometer surfaces (e.g. microelectronics or microfluidics). In order to produce more sophisticated materials and devices; it is very important to achieve well-defined or unique surface characteristics, which determine the materials characteristics to a large extend (11). Almost any kind of object can be coated with an aim to obtain desired specific surface characteristics. The requirements on the desired coating, e.g. specific wettability; chemical resistance; transparency; conductivity; color; defined permeability; corrosion protection; etc. are determined by the application area of material. (12) The specific function and stability of the coating on the underlying material as well as a cost effective and simple processability are important parameters of a high quality coating. Adhesion phenomena can vary depending on underlying material type due to specific chemical functions at the interface and different degrees of surface roughness. This variety of underlying materials and desired surface functions lead to various coating formation procedures such as physical or chemical vapor deposition; powder coatings; electrochemical deposition. (13), (14) However, the most common technique used for coating is deposition from a liquid state (e.g. solution or melt). This transition from the liquid state to a continuous solid film is usually followed by a shift of the glass transition temperature $\left(T_{g}\right)$ of polymers in the coating material. (15) The $T_{g}$ of the polymeric coating material is increased either by solvent evaporation or chemical reactions during the film formation. (16), (17) This type of film formation technique based on solvent evaporation (thermoplastic coating) is not that efficient due to problems in processability caused by the fact that effective polymer entanglement only occurs for high molecular weight polymers results in highly viscous polymer solutions. In general, all these techniques used for film formation suffer from the delamination of the polymer from substrate due to lack of covalent attachment of film to the underlying material. In addition to these procedures metioned above for coating substrates with polymers without covalent attachment, different specialized approaches are used to create well defined coatings on various substrates for various applications. Self-assembled monolayers (SAM) became a useful tool to create desired surface properties. (18), (19) (Figure 1) This self assembly method is based on the controlled adsorption of specific functionalities which match with that of the desired substrate. 


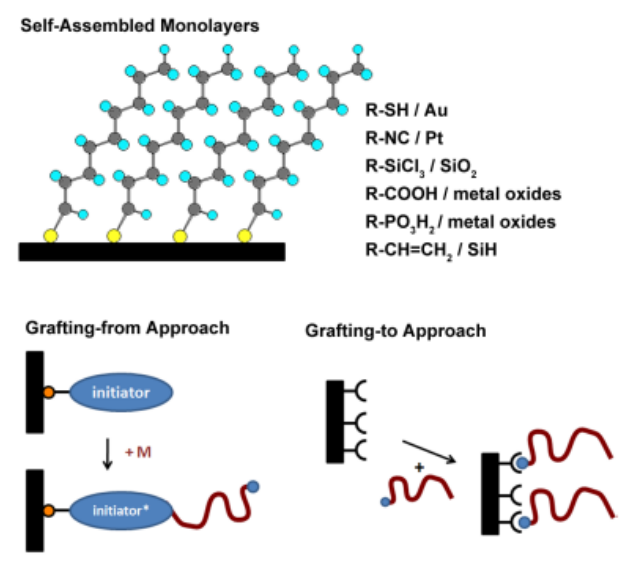

Figure 1 Different approaches to functionalize specific surfaces with small molecules or polymers.

Most popular SAMs used for gold surfaces are thiols (or disulfides) (20) whereas they are chloro- or alkoxy-silanes for glass or silicon surface (generally on hydroxylated surfaces) (21), (22) In case of organosulfur on metal surfaces (e.g. thiols on gold), SAMs form via the chemisorption which is most likely due to Au (I) thiolate (RS-) species. (23), (24) In the case of organosilicon derivatives on metal oxide surfaces, in situ covalent attachment ( $\mathrm{Si}-\mathrm{O}-\mathrm{Si}$ bonds) formes between surface silanol groups ( $\mathrm{Si}$ $\mathrm{OH}$ ) and organosilicon derivatives. The most promising way of tuning physical and chemical properties of surfaces is deposition with grafted polymer layers. Synthetic polymers serve as excellent candidates for surface modification because of their tunable mechanical properties, the variability of film thickness, degree of functionality and because of the potential multifunctional stimuli-responsivity. Polymer brushes are long polymer chains tethered by one end to a surface with high enough density of attachment points so that the chains are crowded and forced to stretch away from the surface (from a collapsed state so-called "mushroom" to an extended configuration socalled "brushlike"). (25)-(27) This type of polymer brushes provides unique characteristics and exhibits different properties in comparison with flexible polymer chains in solution. (5), (25) As a result of these unique properties, polymer brush films have been used in advanced applications such as smart surfaces, ion sensors (28), external stimuli responsive coatings (5), controlled cell growth (3) and controlled gene-drug delivery. (4) Generally, polymer brushes can be prepared either by physisorption or covalent attachment. Covalently tethered polymer brushes are more stable than physically adsorbed polymer brushes where the interaction (e.g. van der Waals force and hydrogen bonding) between the substrate and the polymers is weak. As a result of this stability, covalently bound polymer films offer an efficient and convenient way of modifying physicochemical characteristics of material surfaces and thus play an important role in many areas of science. (29) This stability deals with entropy loss caused by chain extension in densely grafted polymer brushes which experience significant steric repulsion. Since this entropic tension is on the order of pico-Newtons (30), it is too small to break a covalent bond. However, osmotic stress, 
electrostatic repulsion, and unfavorable interaction between the polymers and the substrate may cause additional tension which might lead to the detachment of polymer chains off the substrate, if it exceeds the strength of a covalent bond. "Grafting from" or "grafting to" are the basic methods used to form polymer brushes from functional thiol molecules anchored on gold surfaces. (31) The "grafting to" technique is based on the chemical adsorption of preformed polymer chains onto a substrate. The "grafting from" so called surface initiated polymerization (SIP) relies on the growth of polymer chains from initiators anchored at surface of the substrate. The conformation of the polymeric chains in dry state depend on the grafting density. The grafting of polymer brushes to terminal functionalities (anchoring sites) of self assembled monolayer (SAM) provides better control over the surface grafting density and consequently the resulting conformation of the grafted polymer as well as the thickness. (32), (33) The aim of a coating application is to fulfill a certain function on the surface (e.g. protein resistivity, corrosion protection) by attachment of a defined chemical moiety onto the substrate. This defined surface chemistry is directly responsible for the coating's function. Depending on desired function, coating formulation requires different types of polymers, polymer architectures or functionalities within the polymer (e.g. thermosetting resins require cross-linkable moieties within the polymer, polymers which will be grafted to a surface need one certain end-group functionality). Various polymeric materials with different architectures or functionalities usually require different polymerization techniques. Recent developments in the field of controlled polymerization techniques enable to tune the desired features and functionalities of these polymer brushes and thus, the properties of a surface. Up to now, many techniques including Ring-Opening Polymerization (34), (35) (ROP), Nitroxide Mediated Polymerization (36) (NMP), Reversible Addition-Fragmentation Chain Transfer (37) (RAFT) and Atom Transfer Radical Polymerization (38) (ATRP) have been used to prepare well-defined polymer brushes initiated from a surface. Various monomer types polymerized with these different techniques enable to form polymer brushes with a variety of functionalities and properties which lead to the development of new functional materials in many fields of research such as anti-fouling coatings (39), protection against corrosion or other chemical degradation (40), release systems (41) etc. Based on these CRP methods, it is possible to produce mechanically and chemically robust coatings with desired functional groups. Up to date, a wide variety of polymer brushes namely homopolymer, block copolymer, mixed homopolymer and random copolymer has been prepared in the literature. However, studies relying on Raft polymerization from surfaces have not been extensively carried out. The present thesis investigates new concepts for surface functionalization basing on Raft mechanism and describes new synthetic approaches for fabrication of functional polymer brushes on gold. 


\section{Theoretical Background}

A polymer is a large molecule or macromolecule composed of many repeated subunits that are joined by the same type of covalent linkage. Polymers have found numerous applications in the construction, automobile, and packaging industries due to their mechanical and thermal properties, their easy processability, and low cost. New polymeric materials must fulfill certain requirements in terms of specific performance aspects such as mechanical, optical, surface, electrical, and thermal properties depending on the particular needs for a desired application. Therefore, new polymerization tools are required in order to prepare advanced polymeric structures and architectures for various desired applications. At that point, it is very important to be able to control the macromolecular architecture by controlling the molar masses, polydispersities and terminal functional groups of the polymer. Recent developments in the field of controlled polymerization techniques enable to tune the desired features and functionalities of polymers whereas it is not possible in the case of Conventional Free Radical Polymerization (FRP) which is a chain-growth polymerization technique.

\subsection{Free Radical Polymerization}

A chain growth reaction is assembled of different reactions taking place in parallel, which are in competition to each other. Due to its wide ranging applicability and versatility, FRP is generally preffered for the commercial production of high molecular weight polymers. (42) Main advantage of FRP in comparision with other chain growth polymerization techniques such as anionic or cationic polymerization is the fact that it can polymerize a very wide range of monomers under a wide variety of experimental conditions at relatively mild conditions, such as near ambient temperatures. In addition to these, it is generally less sensitive to impurities and contaminants due to the lack of highly active catalysts in the system. Moreover, it can either be performed in a homogeneous system, such as bulk or solution polymerization, or in a heterogeneous system such as emulsion or suspension polymerization. (43) The FRP system consists of monomer, initiator and, if necessary, solvent. Monomers are molecules bearing a reactive double bond. Common initiators preffered in FRP are based on azo- or peroxycompounds, e.g. azoisobutyronitrile (AIBN) or benzoyl peroxide (BPO) due to their unstability. (44) The mechanism can be roughly divided into 4 steps: (Figure 2)

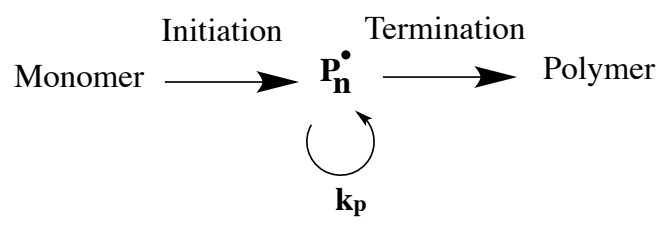

Figure 2 Conventional Free Radical Polymerization. (45) 
- Initiation: Radicals are formed by the decomposition of the initiator at reasonable rates which generally occurs between $40{ }^{\circ} \mathrm{C}$ and $90{ }^{\circ} \mathrm{C}$. Besides thermal decomposition, electrochemical or photo activation might be used aswell. In the case of thermal initiation, it is a first order reaction related to the initiator concentration. High reactivity of the formed radicals lead not only to the addition of monomer but also the backwards reactions and the termination reactions with other radicals. The addition of monomer is a very fast reaction compared to the decomposition reaction, which is negligible. The rate of the initiation reaction can be described as the following (Equation 1):

$$
r_{\mathrm{d}}=-\frac{1}{2} \cdot \frac{\mathrm{d}[\ln ]}{\mathrm{d} t}=\frac{\mathrm{d}[\mathrm{I} \cdot]}{\mathrm{d} t}=2 \cdot k_{\mathrm{d}} \cdot f \cdot[\ln ]
$$

where $t$ is the reaction time, $[\mathrm{In}]$ is the initiator concentration, $[\mathrm{I} \cdot]$ the primary radical concentration and $k_{\mathrm{d}}$ the rate coefficient of the decomposition. The term of 'initiator effectiveness $f$ ' is used to describe the probability with which half of the initiator molecule start chains. This effectiveness depends on the system parameters including the initiator, monomer and solvent. (46)

- Propagation: The radicals formed in initiation step react with the double bonds in the vinyl monomers and leads to formation of growing polymer chains. The generated free radical $(R \cdot)$ initiates the polymerization by addition of the first monomer $(R-M \cdot)$, this active chain end propagates further polymerization of monomers $(\mathrm{Pn} \cdot)$. The radical of the growing chain may also be transferred to another molecule (e.g. to a solvent molecule, to a chain transfer agent or another polymer, monomer or initiator). By radical transfer, the chain growth of one chain is stopped, but the concentration of radicals remained equal. The polymerization proceeds until termination of the radical chain end occurs. The propagation rate depends on the radical $([\mathrm{R} \cdot])$ and monomer concentration ([M]). (Equation 2) In an ideal case the rate coefficient $k_{\mathrm{p}}$ is independent on the length of the chain radical, monomer concentration and conversion.

$$
r_{\mathrm{p}}=-\frac{\mathrm{d}[\mathrm{M}]}{\mathrm{d} t}=k_{\mathrm{p}} \cdot[\mathrm{M}] \cdot[\mathrm{R} \cdot]
$$

- Termination: The radical species are destroyed through various means leading to dead polymer. Two radical chain ends may undergo recombination or disproportionation. In case of recombination, two chain radicals react directly via their radical functions to form one polymer strand with a saturated bond. On the other hand in the disproportionation, two chain radicals react under $\mathrm{H}$-abstraction to form two polymer strands, one with a double bond and one saturated. In case of recombination the molecular weight doubles whereas disproportionation has no influence on the 
molecular weight. Termination is a diffusion-driven process as the radicals have to be next to each other for the reaction. The rate constant of the termination reaction $\left(\mathrm{k}_{\mathrm{T}}\right)$ is usually five orders of magnitude higher than the rate constant of the propagation reaction $\left(\mathrm{k}_{\mathrm{P}}\right)$. (47) As a summary the rate law for the termination can be stated as (Equation 3):

$$
r_{\mathrm{t}}=-\frac{\mathrm{d}[\mathrm{R} \cdot]}{\mathrm{d} t}=-2 \cdot k_{\mathrm{t}} \cdot[\mathrm{R} \cdot]^{2}
$$

The propagation rate is directly proportional to the concentration of active growing chain ends whereas the termination rate is proportional to the concentration of active growing chain ends squared.

- Chain Transfer: The radical species might move from one molecule to another without termination of the radicals. In that case one growing chain is stopped but the radical functionality is transferred to another monomer, solvent or polymer molecule. The new radical generated via chain transfer adds monomers in order to form a polymer chain. In the case of transfer to polymer chains, monomer addition might occur not only on the chain ends but also on side chains of polymers. These transfer reactions can proceed via intramolecular (so called back biting) and intermolecular mechanisms. (48) The rate of the transfer reaction is described as (Equation 4):

$$
r_{\mathrm{tr}}=-\frac{\mathrm{d}[\mathrm{X}]}{\mathrm{d} t}=2 \cdot \mathrm{k}_{\mathrm{tr}} \cdot[\mathrm{R} \cdot] \cdot[\mathrm{X}]
$$

where $[\mathrm{X}]$ is the concentration of the transferring agent. The transfer constant $C_{\text {tr }}$ describes the effectiveness of the agent in the case of special CTAs are used (Equation $5)$ :

$$
C_{\mathrm{tr}}=\frac{k_{\mathrm{tr}}}{k_{\mathrm{p}}}
$$

The transfer reaction doesn't affect the rate of polymerization, $R p$ since the active radical concentration remains constant. The main difference in polymerization including transfer reactions is the length of the resulting polymer chains. Raft polymerization technique takes advantage of this chain transfer mechanism in which chain transfer agents are used to control the molar weight and with that control over material properties. (49) To receive the rate of polymerisation as a whole the rate of initiation and the rate of termination are equated with the assumption that the radical concentration is quasi-stationary (Equation 6): 


$$
2 \cdot k_{\mathrm{d}} \cdot f \cdot[\ln ]=2 \cdot k_{\mathrm{t}} \cdot[\mathrm{R} \cdot]^{2}
$$

Solving for $[\mathrm{R} \cdot]$ and inserting into the rate equation of polymerisation reveals the brutto-polymerisation rate (Equation 7):

$$
r_{\mathrm{b}}=-\frac{\mathrm{d}[\mathrm{M}]}{\mathrm{d} t}=k_{\mathrm{p}} \cdot\left(f \cdot \frac{k_{\mathrm{d}}}{k_{\mathrm{t}}} \cdot[\ln ]\right)^{0.5} \cdot[\mathrm{M}]
$$

In FRP, the molecular weights of chains formed in the early stages of polymerization are relatively high, which subsequently decreases with increasing monomer conversion. Main drawbacks of FRP are:

* Distribution of molecular weights (Polydispersity index=PDI) is relatively broad. $\mathrm{PDI}=\mathrm{M}_{\mathrm{w}} / \mathrm{M}_{\mathrm{n}}>2$, where $\mathrm{Mw}$ is the weight average molecular weight and $\mathrm{Mn}$ is the number average molecular weight. Due to the different reactions which take place simultaneously, there is a variety of chain lengths and that of the molecular mass of the chains which lead to relatively broader PDIs. These broad distributions are adversarial for some applications which require identical chain lengths.

* In addition, FRP techniques are limited in their ability to synthesize complex architecture polymers such as block copolymers, star copolymers or dendrimers. (42)

In the case of Raft polymerization, controlling agents which enable planning of molecular weight and the resulting material properties are added into reaction in order to gain control over FRP. These agents react with the radical species in a reversible manner and form the dormant species which are stable enough to keep the radical species dormant, but also reactive enough for re-initiation. The resulting reaction equilibrium is shown in Figure 3.

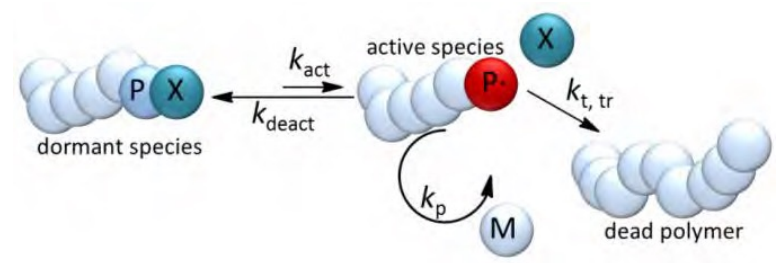

Figure 3 Reaction equilibrium in a $C R P(50)$ 
It is assumed that

$$
k_{\text {act }} \cdot[\mathrm{P}-\mathrm{X}]=k_{\text {deact }} \cdot[\mathrm{P} \cdot]
$$

As a result of the fact that the radical concentration is approximately 105 times smaller than the concentration of the dormant species, the equilibrium is mainly on the side of the dormant species. Thus, termination and transfer reactions are strongly minimized due to the low radical concentration. As a consequence, the life span of the growing polymer chains is prolonged. The fact that all the chains are started simultaneously and exhibit synchronized growth under the controlled equilibrium leads to yielding of equal sized chains and therefore narrower molecular weight distributions (MWD) in comparision to the FRP case (Figure 4).

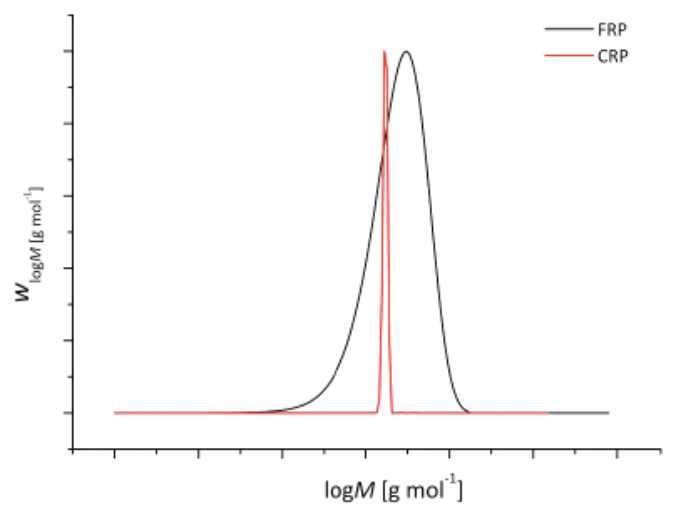

Figure 4 Molecular weight distributions resulting from a FRP (black) and a CRP (red) (50)

Therefore, living polymerizations have recently got tremendous academic and industrial interest. The bimolecular termination step is the major limiting factor for the control of all chain polymerization techniques. The concept of controlled / "living" radical polymerization is based on lowering the number of active growing chain ends, which results in dramatic decrease in termination as well as depression of propagation. In most types of living radical polymerization techniques, this is carried out by a reversible reaction between the active growing chain end and a corresponding dormant chain end which is not capable to propagate or terminate. The most popular methods for living radical polymerization are Atom Transfer Radical Polymerization (ATRP), Niroxide-Mediated Polymerization (NMP) and Reversible Addition Fragmentation Chain Transfer (RAFT) polymerization. Living radical polymerizations enable a defined built-up of specific polymer architectures, in addition to the advantages of controlling the molecular weight and obtaining narrow molecular weight distributions. Polymer chains synthesized via CRP usually have defined end groups which can also be activated in a second polymerization step for block-copolymer synthesis. (51) 


\subsection{Controlled Radical Polymerization}

Living polymerization behavior has been defined as: (52)

* Since the polymerization proceeds to complete conversion, further monomer addition leads to continued polymerization which enables to produce homopolymers with longer chains or block copolymers in the case of using a different monomer.

* The number average molecular weight $\left(\mathrm{M}_{\mathrm{n}}\right)$ directly increases with conversion/time.

* The polydispersity of the molecular weight distribution is low (PDI 1.2).

* Polymers with chain end functionality can be obtained quantitatively.

Ionic polymerization is one of living polymerization techniques, which allows to synthesize complex macromolecular architectures in a controlled way. However, there are some drawbacks of the technique such as the high sensitivity to impurities, the relatively limited range of monomers that can be used and the requirement of the extreme polymerization conditions (often around $-30^{\circ} \mathrm{C}$ or even lower). (53) In 1982, Otsu combined the advantages of both free radical and ionic polymerization techniques with an attempt to apply the idea of living polymerization to the free radical system. (54) For a living polymerization, the termination reactions must be negligible in comparision to the propagation and activation/deactivation steps. (55), (56) In an ideal living polymerization, all chains are initiated syncronised at the beginning of the polymerization, grow simultaneously at the same rate, and have no termination step. All processes that terminate chains irreversibly should be eliminated in order to assure living character of a radical polymerization. Thus, living radical polymerization takes place in the presence of reagents which are capable of reacting with the propagating radicals $\left(\mathrm{Pn}^{*}\right)$ by reversible deactivation (Figure 5) or reversible chain transfer (Figure 6) and thus keeping the majority of chains in a dormant form $(\mathrm{P}-\mathrm{X})$.

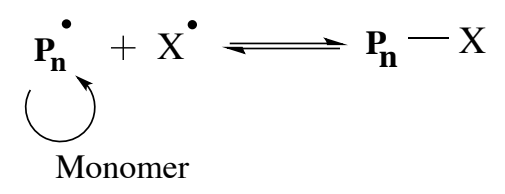

Figure 5 Reversible deactivation. (57) 


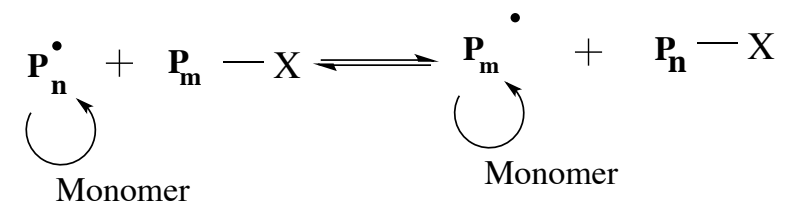

Figure 6 Reversible chain transfer. (57)

A rapid equilibrium between the active and dormant forms of the polymer chain ensures that all chains will grow until all monomer is consumed. Under these conditions, the molecular weight increases linearly with time (conversion) and the molecular weight distribution can be narrow (Figure 7).

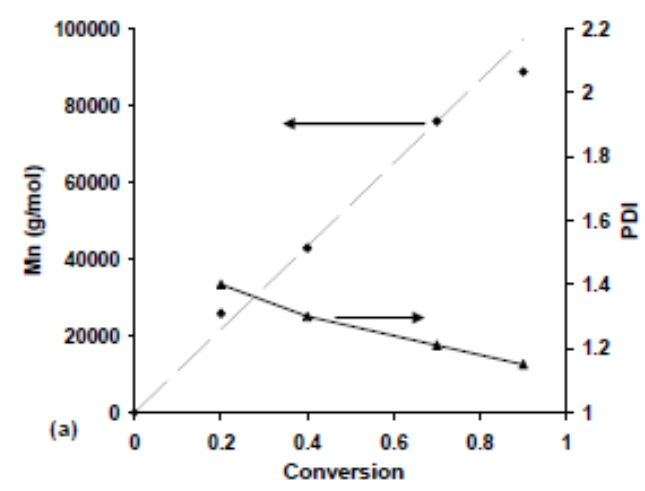

Figure 7 Typical molecular weight and PDI vs. conversion for a living radical polymerization. (58)

As described above, living behavior is achieved by minimizing the normal bimolecular termination, and thus prolonging the lifetime of propagating radicals into hours or longer. This kind of polymerization has excellent commercial potential for synthesis of very well-defined polymers with complex architectures and a variety of novel materials as a result of that many more monomer types might undergo radical polymerization compared to ionic polymerization.

As mentioned above, controlled / living radical polymerization (CLRP) relies on two principles: reversible deactivation (Figure 5), and reversible chain transfer (Figure 6). (45) The basis of this mechanism is rapid deactivation of any radical species which leads to low radical concentration which limits the bimolecular termination step and thus prolonges the lifetime of propagating radicals. Here only two types of Controlled Radical Polymerisations (CRP) that currently receive the greatest attention will be presented: Atom Transfer Radical Polymerization (ATRP) and Reversible AdditionFragmentation Chain Transfer (RAFT). 


\subsubsection{Atom Transfer Radical Polymerization}

ATRP has been used to polymerize styrenic (59), acrylamides (59), acrylates, acrylonitrile, and diene monomers with controlled molecular weights and low polydispersities. (60) Radicals are generated via an organic halide undergoing a reversible redox process catalyzed by a transition metal compound. The catalysts commonly used in ATRP are $\mathrm{Cu}, \mathrm{Fe}$, and $\mathrm{Ni}$. The initiator is activated by the $\mathrm{CuBr}$ metal center undergoing an electron transfer with simultaneous halogen atom abstraction and expansion of its coordination sphere (Figure 8). $\mathrm{R}^{\circ}$ is the reactive radical that initiates polymerization whereas $\mathrm{CuBr} 2(\mathrm{~L})$ is the deactivator which reduces the steady-state concentration of propagating radicals in order to minimize termination of living polymers. (61) In ATRP, the redox reaction of alkyl halides (R$\mathrm{Br}$ ) with transition-metal complexes generate radicals which then add monomers and form growing polymeric radicals which are then rapidly deactivated by the oxidized form of the transition-metal catalyst (CuBr2 (L)). (62)

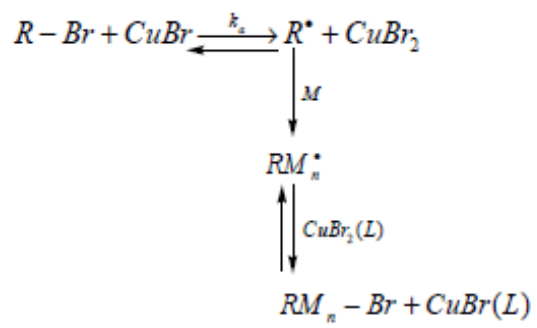

Figure 8 ATRP polymerization mechanism. (57)

ATRP is based on a reversible mechanism which lies heavily on the dormant side $(\mathrm{RMn}-\mathrm{Br})$, thus reducing the concentration of radicals and consequently minimizing bimolecular termination. The advantage of ATRP is its wide applicability for the production of advanced and novel polymeric structures whereas the main limitation is the employment of a relatively high amount of transition metal complex $(0.1-1 \%$ in the reaction mixture) that is toxic and has to be removed from the final polymer.

\subsubsection{Reversible Addition Fragmentation Chain Transfer Polymerization}

Another promising method to perform controlled / "living" radical polymerization is the Reversible Addition Fragmentation Chain Transfer (RAFT) polymerization which has been developed in Australia by Ezio Rizzardo's team at CSIRO in 1998. (63)- (67) On the contrary of ATRP and SFRP, it is possible to polymerize a wide range of monomers including styrenic, acrylamides, (59) acrylates, acrylonitrile, vinyl acetates, vinyl formamide, vinyl chlorides as well as a range of other vinyl monomers, (68), (69) via RAFT polymerization technique. RAFT has been shown to control molecular weight and molecular weight distributions, providing PDI's in the range of 1.03-1.25. 
(66) In terms of Controlled Radical Polymerization (CRP) techniques, there are two ways to control the polymerization: lowering the radical concentration with capping agents or with a degenerative chain transfer. In contrast to ATRP and NMP techniques, the RAFT process does not control the amount of active growing chain ends by reversible termination (no radical is present) but by reversible chain transfer (the persistent radical in the dormant species is a real radical species). In an ideal living polymerization, all chains are initiated at the beginning of the reaction and grow at a similar rate. Moreover, there is no irreversible chain transfer or termination. If initiation is rapid enough with respect to propagation, the molecular weight distribution will be very narrow. In RAFT polymerization, the majority of living chains are maintained in a dormant form by utilization of the reagents, which are so called raft agents and capable of reversibly deactivating propagating radicals. Thus, during reaction, a rapid equilibrium between the active and dormant chains is assured. (Figure 9)

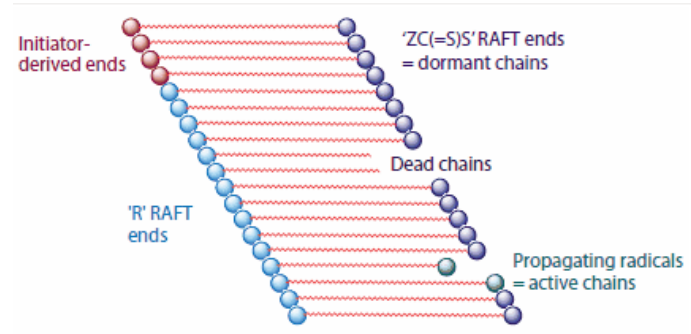

Figure 9 Raft polymerization schematic. The number of chains of each type shown here is not in proportion to that expected for a well-designed experiment. On average, all living chains grow simultaneously and have equal chain length because equilibration of the dormant and active chain ends is rapid with respect to propagation. A raft agent is represented as ' $\mathrm{ZC}(=\mathrm{S}) \mathrm{S}$ '. (45)

In Raft polymerization, molecular weight increases linearly with conversion and molecular weight distributions can be very narrow. (70) (Figure 10)

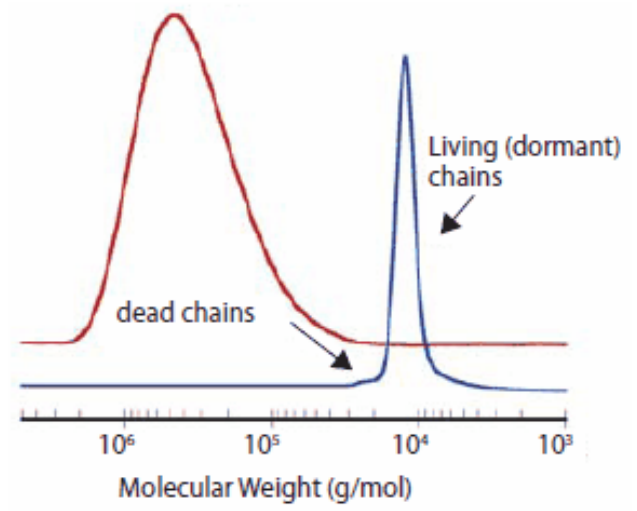

Figure 10 Typical molecular weight distrubions for a conventional and a RAFT polymerization of styrene under identical experimental conditions. (45) 
Another advantage of the RAFT process is that it can be carried out in the same conditions as a classical free radical polymerization, except for the addition of a RAFT agent, which is also referred to as the chain transfer agent (CTA). (Figure 11) Similar to a free radical polymerization; RAFT needs common radical initiators such as AIBN or BPO; additionally a chain transfer agent (CTA), which consists of a stabilising group $(\mathrm{Z})$ and a leaving group $(\mathrm{R})$.

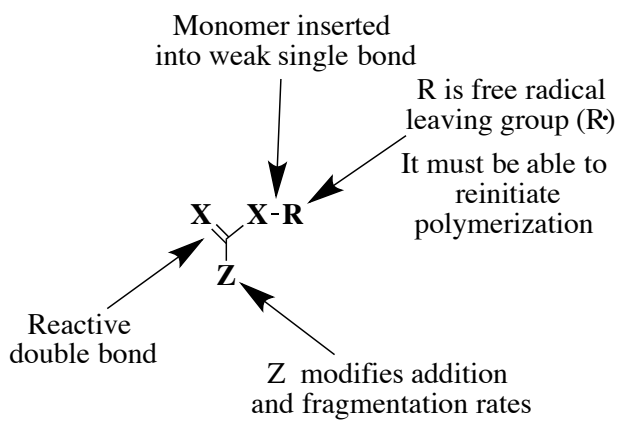

Figure 11 General structure of a chain transfer agent.

These RAFT agents include macromonomers (71), allyl sulfides (72), allyl bromides (73), allyl peroxides (74), and thionoesters. (75) (Figure 12) As a result, RAFT polymerizations have been carried out in bulk, aqueous solutions (76), organic solutions, suspensions, emulsions, mini and micro emulsions, and ionic liquids. (68) Complex macromolecular architectures can be generated ranging from block to star copolymers by using the RAFT technique. (64), (77)- (79)

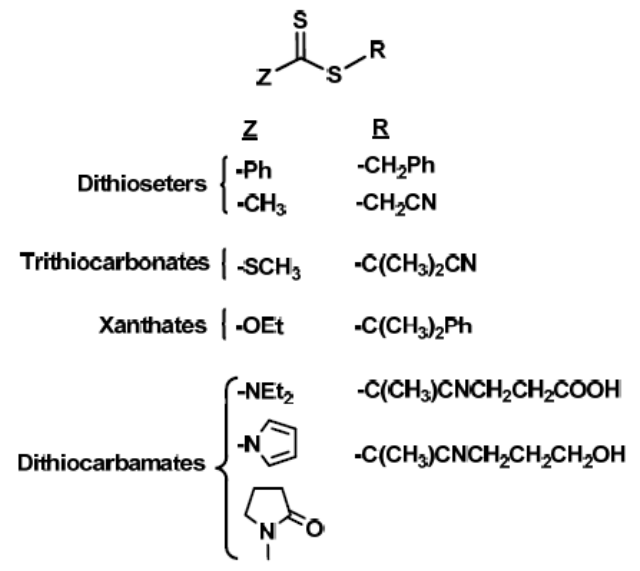

Figure 12 Examples of the different classes of thiocarbonylthio RAFT agents. (50)

In RAFT living polymerizations, the control over chain growth is achieved through reversible chain transfer. (Figure 13) The RAFT polymerization accomplishes "living" growth starting from the initial chain transfer agents which are activated by radicals generated from a traditional initiator (e.g. AIBN). (66) A chain-transfer agent reversibly transfers a labile end group to a propagating chain. The $\mathrm{R}$ group initiates the 
growth of polymeric chains, while the $\mathrm{Z}$ group activates the thiocarbonyl bond toward radical addition and then stabilizes the resultant adduct radical. (68)

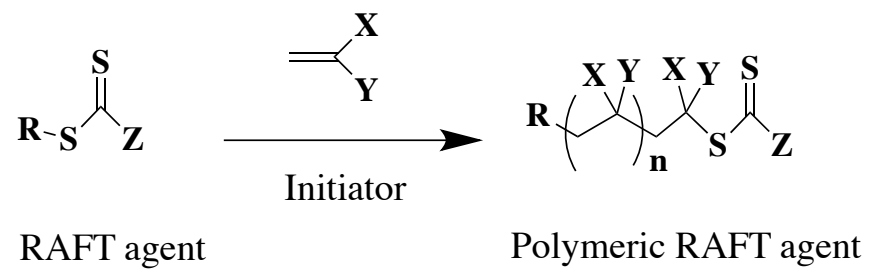

Figure 13 Overall reaction in RAFT polymerization. (34)

\subsubsection{The Mechanism of RAFT Polymerization}

As mentioned above, the control mechanism in RAFT polymerization differs significantly from that in SFRP and ATRP, which are based on reversible deactivation of propagating radical by radical — radical reaction (SFRP) or atom transfer (ATRP). In RAFT polymerization, the deactivation-activation equilibria is chain transfer reaction. The mechanism of the RAFT polymerization is based on a sequence of addition and fragmentation reactions, which enables the transfer of the active growing chain end to a dormant radical species and further to a new active growing chain end. (80) Radicals are neither formed nor destroyed in these steps, with an external source of free radicals required to initiate and maintain polymerization. (45) The mechanism of the RAFT polymerization was suggested by Vana (80) and this kinetic scheme consists of the: the initiation, the propagation and the termination steps just like in the case of FRP. Additionally, there is a transfer equilibrium taking place between the transfer agent and the growing radicals. (Figure 14)
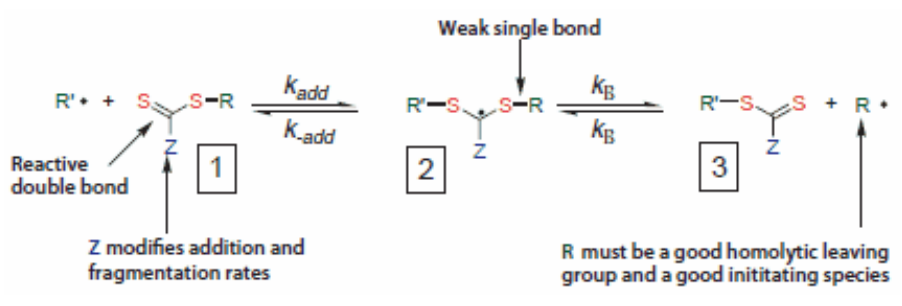

Figure 14 Mechanism for reversible addition-fragmentation chain transfer (RAFT). (45)

The reaction scheme including all these reactions are shown in Figure 15. In the initiation step, just like in the case of the free radical polymerization, an initiator (I) decomposes into two free radicals $\left(I^{*}\right)$ which might add first monomer molecules and form macroradicals (active propagating radicals) $\left(\mathrm{P}_{\mathrm{m}}{ }^{*}\right)$. After the initiation step, in the first reversible addition reaction, the growing active radicals $\left(\mathrm{P}_{\mathrm{m}}{ }^{*}\right)$ react with the CTAs (1) to form intermediate (persistent) radicals (2) which are not able to propagate or to terminate. Further fragmentation of this intermediate radical (2) might result in two 
possibilities: The radical intermediate (2) can fragment back to the original RAFT agent (1) and a polymeric radical $\mathrm{P}_{\mathrm{m}}$ *, or fragment to a polymeric thiocarbonylthio compound $\left(\mathrm{P}_{\mathrm{m}} \mathrm{SC}-(\mathrm{Z})=\mathrm{S}\right)(3)$ and a reinitiating $\mathrm{R}^{*}$ radical. The structure of $\mathrm{R}$ should be such that it is a good reinitiating group, which can subsequently initiate the monomer. (73) In re-initiation step, a new propagating radical $\left(\mathrm{P}_{\mathrm{n}}{ }^{*}\right)$ is generated sothat it can be exchanged by $\mathrm{Pm}$ in the compound $\mathrm{P}_{\mathrm{m}} \mathrm{SC}-(\mathrm{Z})=\mathrm{S}$ in order to produce a new polymeric thiocarbonylthio compound $\left(\mathrm{P}_{\mathrm{n}} \mathrm{SC}(\mathrm{Z})=\mathrm{S}\right)$. In the second reversible addition reaction, the chain equilibrium between active propagating radicals $\left(\mathrm{P}_{\mathrm{n}}{ }^{*}\right),\left(\mathrm{P}_{\mathrm{m}}{ }^{*}\right)$ and the persistent radical (4) allows all chains to grow at approximately the same rate, leading to a linear evolution of the molecular weight with conversion, and a low PDI. In termination step, some dead polymer chains are formed via either a combination or disproportionation event depending on the monomer.

(I) Initiation

Initiator $\stackrel{\mathrm{hVor} \triangle}{\longrightarrow} 2 \mathrm{I}^{\bullet} \stackrel{\mathrm{M}}{\longrightarrow} \mathrm{P}_{\mathrm{m}}^{\bullet}$

(II) Pre-equilibrium

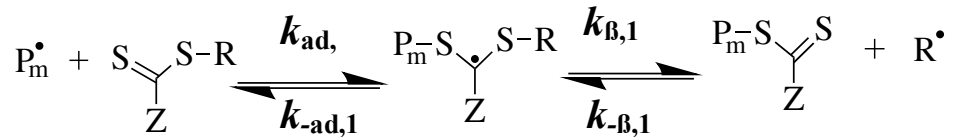

(1)

(2)

(III) Propagation

$$
\mathrm{R}^{\cdot} \underset{\boldsymbol{k}_{\mathrm{i}}}{\mathrm{M}}=\mathrm{P}_{\mathrm{n}}^{\bullet}
$$

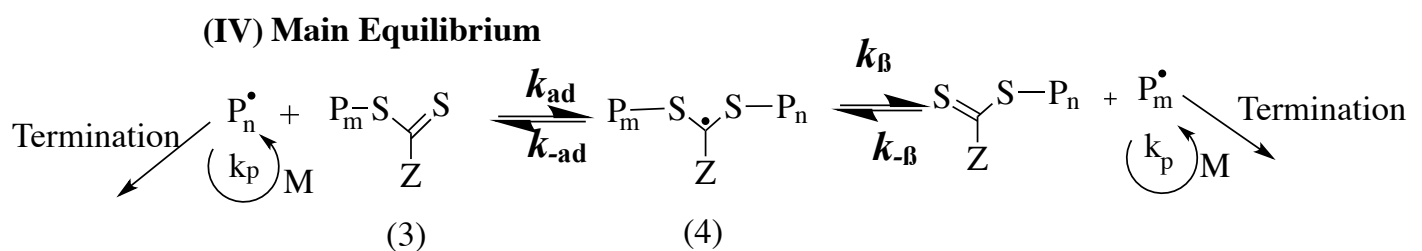

(V) Termination

$$
\mathrm{P}_{\mathrm{n}}^{\cdot}+\mathrm{P}_{\mathrm{m}}^{\cdot} \longrightarrow \mathrm{P}_{\mathrm{n}+\mathrm{m}}
$$

Figure 15 Mechanism of RAFT polymerization. (80)

The control of RAFT polymerisations relies on the fast equilibrium between controlling agent and growing radical chains $\left(\mathrm{P}_{\mathrm{m}}{ }^{*}\right.$ and $\left.\mathrm{P}_{\mathrm{n}}{ }^{*}\right)$. The kinetics of the FRP reaction doesn't differ from the kinetics of RAFT reaction which additionally has the new introduced equilibrium. The only difference is the molecular mass due to control over the high equilibrium velocity, which provides equal probability for growth of all chains. Thus, narrow molecular weight distributions can be obtained. (81) As 2a 
consequence, the chain length will be proportional to the monomer conversion as shown in Figure 16.

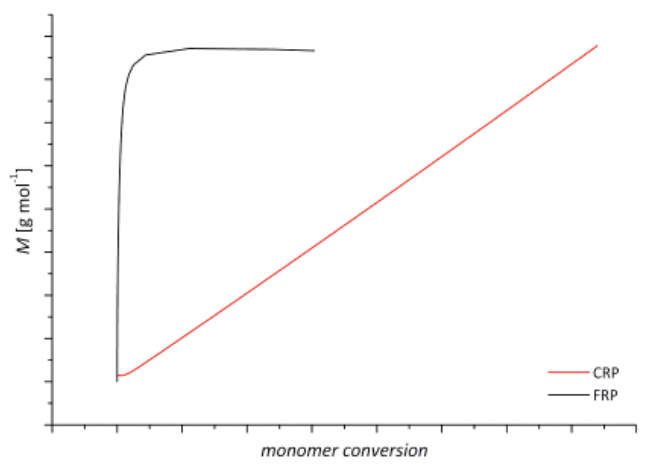

Figure 16 Comparison of the developments of the molecular mass of the polymers with consumed monomer in the FRP-case (black) and the CRP-case (red). (57)

The RAFT agent concentration remains constant during polymerization. When the polymerisation is stopped, there will be two types of polymer chains in reaction mixture: the living chains bearing RAFT endgroups and the dead chains resulting from termination. The ratio of living chains to dead ones can be determined by the concentrations of RAFT-agent and initiatior. (82) The living chains can be employed as marcoRAFT-agents which can be reactivated for further polymerization which allows preparation of blockcopolymers. (81)

\subsubsection{Choice of Raft Agents}

The choice of RAFT agent (CTA) is the key point which assures an efficient RAFT polymerization. (Figure 15) The effectiveness of the RAFT agent depends on the monomer being polymerized and the properties of the free-radical leaving group $\mathrm{R}$ and the group $\mathrm{Z}$. These groups should be chosen in such a way that the reactivity of the $\mathrm{C}=\mathrm{S}$ bond might be activated or deactivated towards addition and the stability of the intermediate radicals might be modified. (83) (Figure 17)

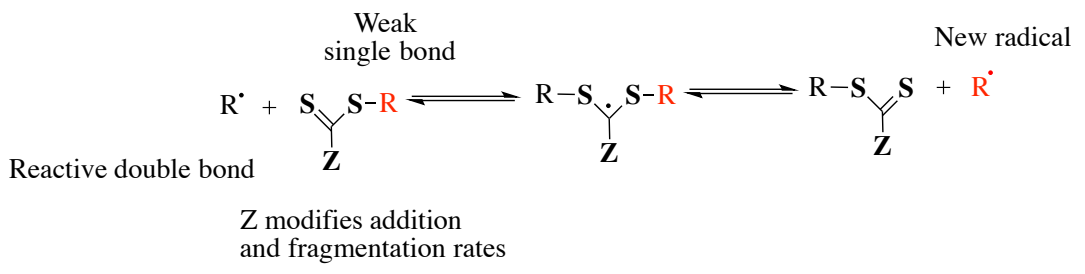

Figure 17 Schematic of general RAFT polymerization. (50)

A wide variety of RAFT agents with different $\mathrm{Z}$ and $\mathrm{R}$ groups have been synthesized and evaluated for their effectiveness in controlling the polymerization of vinyl monomers. (Figure 18) (63), (79) 


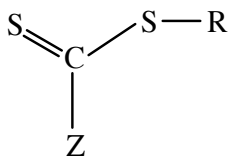

Dithioester

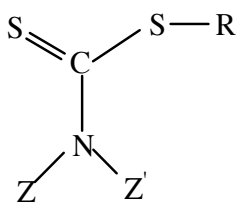

Dithiocarbamate
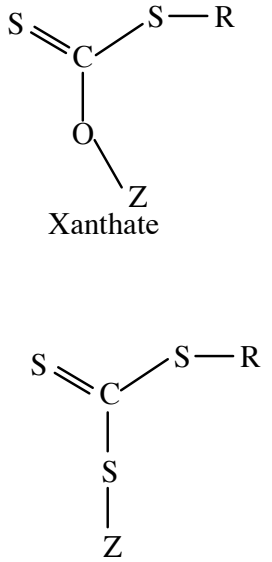

Trithiocarbonate

Figure 18 Structure of different classes of reagents currently used as RAFT agents. (57)

The following requirements have to be fulfilled for an efficient RAFT polymerisation:

* $\mathrm{C}=\mathrm{S}$ double bond in the initial RAFT and the polymeric RAFT agents should be reactive. (high $\mathrm{k}_{\mathrm{add}}$ )

* The intermediate radicals should fragment quickly (high $\mathrm{k}_{\beta}$, weak $\mathrm{S}-\mathrm{R}$ bond in intermediate) and should not undergo side reactions.

The intermediate of the pre-equilibrium (2) should decompose in favour of the products side of the reaction. (84) $\left(\mathrm{k}_{\beta} \geq \mathrm{k}_{\text {-add }}\right)$

* The expelled radical $\mathrm{R}^{*}$ should be able to re-initiate polymerization effectively.

The values of the equilibrium constants which are associated with radical addition to the thiocarbonylthio compound, is the basic parameter that determines the ability of RAFT agents to control polymerization. For this reason, the transfer constant is highly dependent on monomer and groups used in R- and Z-positions of Raft agents and the backbone of Raft agent. The leaving group $R$ of Raft agent is released when a radical attacks $\mathrm{C}=\mathrm{S}$ double bond of the dithio unit. $\mathrm{R}$ has to fragmentate easily and form a relatively stable radical that might initiate a new chain. (Figure 17) The leaving/reinitiating ability of the $\mathrm{R}$ group is determined by steric factors, radical stability, and polar effects. (85) The leaving ability of $\mathrm{R}$ group increases with the increased radical stability; on the other hand, it may not effectively add monomer and reinitiate polymerization if the radical is too stabilized. Increased steric bulk enables $\mathrm{R}$ group to leave easily, but it might also result in a decrease on the reinitiating capability due to steric hindrance. In the ideal case the radical $\mathrm{R}$ should initiate as fast as the polymer chain grows. (85) Electron withdrawing substituents within the R group 
affect the electrophilicity of the resulting derived radical. For example, the RAFT agent where $\mathrm{R}$ is $\mathrm{CH} 2 \mathrm{Ph}$ (e.g. benzyl dithiobenzoate) might be a reasonable leaving group and suitable for polymerization including the styryl and acrylyl propagating radicals whereas it is a poor leaving group for polymerization including the methacrylyl propagating radical. (74) The ability of the homolytic leaving group $\mathrm{R}$ is tertiary $>>$ secondary $>$ primary. (Figure 19) (86)
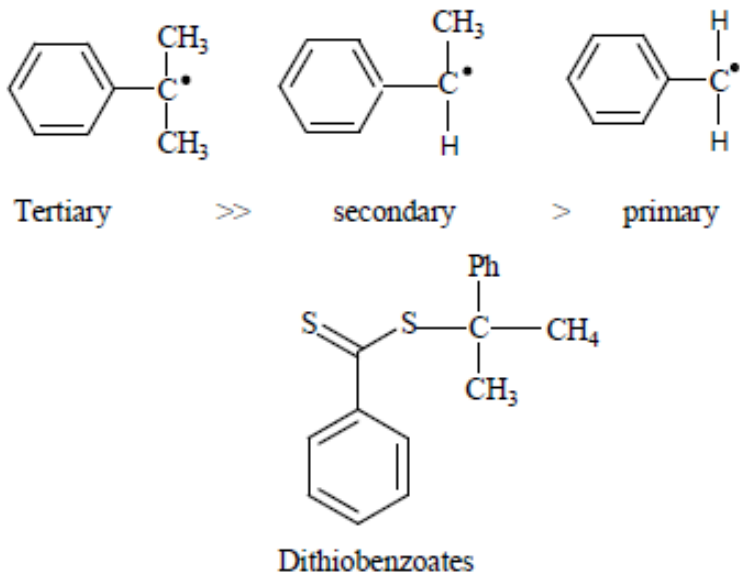

Figure 19 Structure of some R groups and dithiobenzoates. (57)

The Z-group is the functional group that remains attached to the RAFT agent throughout the polymerization, and which is responsible for stabilization of the radical intermediate formed in the equilibrium. (Figure 17) This group should be chosen so that it is able to activate the $\mathrm{C}=\mathrm{S}$ bond toward radical addition and then provide stabilization of the adduct radical formed. The degree of stabilization has great importance for the entire polymerization process. If the stabilisation of the intermediate is too weak, it is not possible to form the intermediate at all. As a result of that there is no chain transfer, polymerization won't be in a controlled manner. In the case of the stabilization is too strong, the intermediate will be unreactive and won't fragmentate at all. As a consequence, the reaction will stop at that point. (63), (87)(90) The rate of activation ( of $\mathrm{C}=\mathrm{S}$ bond toward radical addition) is higher when the $\mathrm{Z}$ group is aryl, alkyl (dithioesters), or S-alkyl (trithiocarbonates), whereas it is lower when the $\mathrm{Z}$ group is $O$-alkyl (xanthates) or $N, N$-dialkyl (dithiocarbamates). This is due to the interaction between $\mathrm{O}$ or $\mathrm{N}$ lone pairs and the $\mathrm{C}=\mathrm{S}$ double bond, leading to delocalization of this bond; that is, the phenomenon in which electrons in some molecules are not fixed to specific atoms or bonds but are spread out over several atoms or bonds. (Figure 20) 
<smiles>[R]SC(=O)OCCPSC1(C)CS1</smiles>

(a)

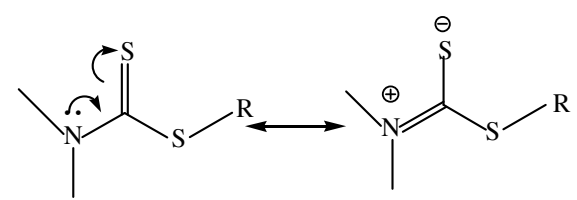

(b)

Figure 20 Canonical form of (a) xanthates and (b) dithiocarbamates. (57)

Rizzardo et al. prepared guidelines based on the monomer structure, leaving group R, and group $Z$ in RSC-(Z) =S. (Figure 21) (45) For $Z$ group, the addition rate decreases and the fragmentation rates increase from left to right. In general, the stability of $Z$ group of RAFT agent decreases in the order dithiobenzoates $>$ trithiocarbonates $\sim$ dithioalkanoates $>$ dithiocarbonates (xanthates) $>$ dithiocarbamates. That can be attributed to the electron-withdrawing $\mathrm{Z}$ substituents with lone pairs directly conjugated to the $\mathrm{C}=\mathrm{S}$ double bond (O-, N-) and which have low stability. (63) For R, the fragmentation rates decrease from left to right. Dashed lines in the below figure indicates partial control of molecular weight but poor polydispersity.

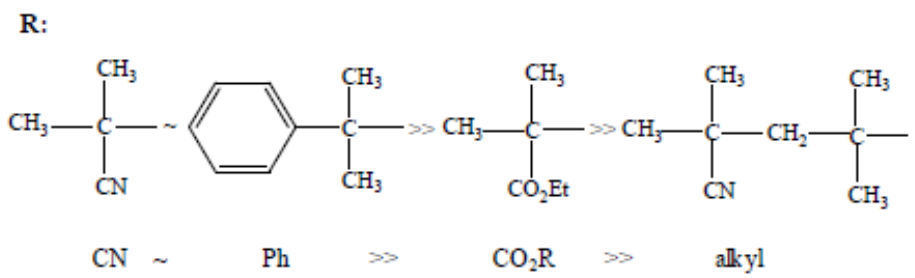

Chain length $n>2>1$ (strong penultimate unit effect)

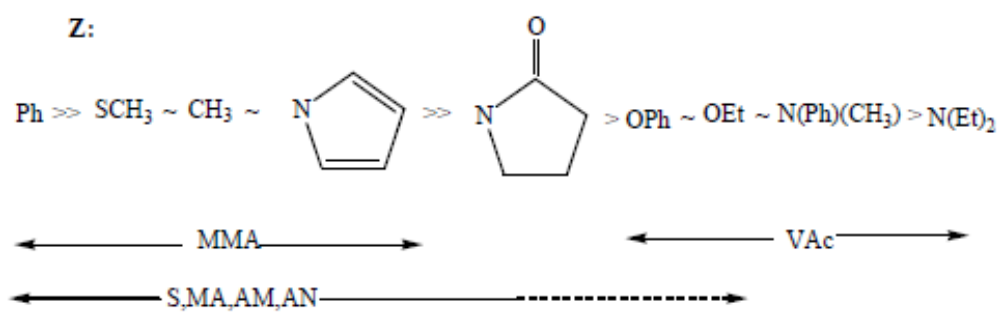

Figure 21 Guideline for selection of RAFT agents. (45)

Rates of radical addition to the thiocarbonylthio compound are typically high $\left(10^{6}\right.$ $10^{8} \mathrm{M}^{-1} \mathrm{~s}^{-1}$ ). Thus a high equilibrium constant generally implies a low fragmentation rate for the radical adduct and increases the tendency to retardation and/or side reaction involving this species. (45) The transfer constants of the starting RAFT agent and the macroRAFTs should be taken into account in order to optimize the conditions for a RAFT polymerization. 


\subsubsection{Side Reactions}

A variety of side reactions can potentially complicate the RAFT mechanism causing retardation, by products and anomalies in the molecular weight distributions depending on the particular RAFT agentlmomomer combination and the reaction conditions. In the initialisation step, the amount of RAFT-agents with the original R-group is decreased by the attack of propagating radicals to the $\mathrm{C}=\mathrm{S}$ double bond of Raft agents. (91) In the period of initialisation all original R-groups are exchanged with propagating radicals. During this exchange process, different type of scenarios can be observed:

* The fact that no polymerization activity is observed in the initial phase of many Raft polymerizations is called inhibition. (92) The stability and thus the average lifetime of the intermediate RAFT radicals (2) and (4) (Figure 15) have significant importance for both inhibition and retardation effects in RAFT polymerization. It was found that an inhibition period did not occur, when using polymeric RAFT agents (3) in RAFT polymerization. Based on this finding, inhibition effects can be attributed to the individual reactions of the pre-equilibrium. (93)

* In the case of that the leaving group $\mathrm{R}$ is less stabilised than the propagating radical, the formed intermediate (2) (Figure 15) will decompose back to the original RAFT agent, and the propagating radical. As a result, the chains will grow unequally. Thus, polymer chains with above average high molecular mass will form at low monomer conversion.

* If the rate coefficient for the propagation of the R-group is high, a fast transition towards the main equilibrium takes place. (91)

* If the release of $\mathrm{R}$ is favoured or the pre-equilibrium has no preference in direction, there is an accumulation of small radicals which terminate much faster than larger radicals. This results in a radical loss during the initial period of polymerization leading to uneffective start of polymerization. This effect is called induction and is intensified by slowly propagating leaving groups. (39)

* Another effect observed in RAFT polymerisations is called retardation. Rate retardation may occur where $R p$ decreases slightly with increasing concentration of the RAFT agent. (70) On the other hand, rate retardation may either be explained by slow fragmentation of the intermediate radicals (2), (4) (Figure 15) or by additional side reactions, such as cross-termination and self-termination. (70), (94) 
There are three possibilities which might occur in the system (Figure 22):

-An irreversible termination reactions of the intermediate RAFT radical (2), (4) with growing macroradicals, Pm* (Figure 22 (a), (b)) leading to formation of a three armed star. (89), (90), (95)

-The reversible termination reaction of the intermediate RAFT radical (4) with itself (Figure 22 (c)). (95)- (98)

(a)

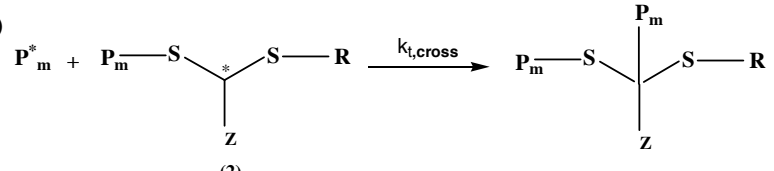

(b)

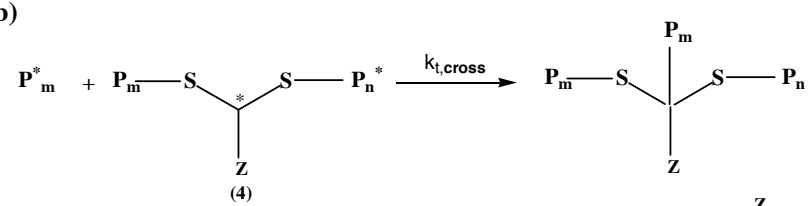

(c)

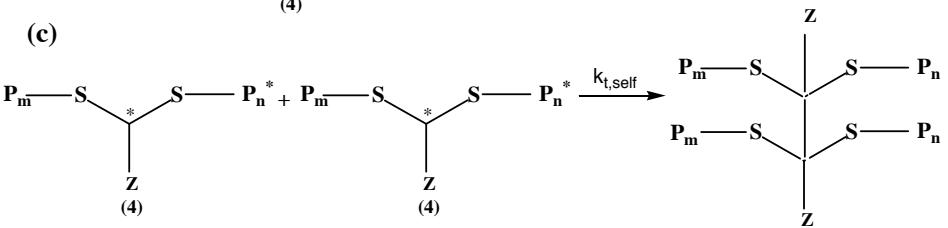

Figure 22 Schematic of (a) cross-termination between propagating and intermediate radicals of the pre-equilibrium, (b) the core-equilibrium, and (c) self-termination reaction between intermediate radicals of the core-equilibrium. (45)

\subsubsection{Block Copolymerization}

A copolymer is a type of polymer formed when two or more different monomers combine together to polymerize. Copolymers can be classified according to the arrangement styles of these different types of monomer units along the chain. (Figure 23)

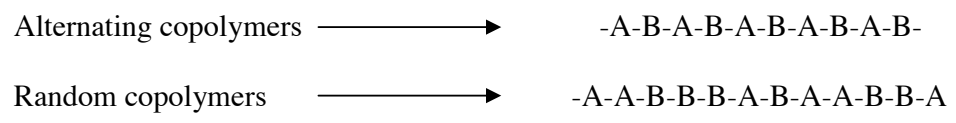

Figure 23 Schematic illustration of copolymers types. (57)

Block copolymers are a class of polymers which consist of sequences or blocks of identical repeating units. Due to their unique properties, block copolymers gain tremendous academic importance as well as commercial interest. There are many different applications areas of block copolymers such as surfactants, dispersants, 
coatings, adhesives and materials for pharmaceuticals and microelectronics. (99) It is called linear block copolymer when two blocks of different chemical structures are linked together through a common junction point. In order to obtain a linear block copolymer, first monomer is polymerized. After complete consumption of first monomer, the second monomer is added into reaction medium and polymerized. It is not possible to prepare block copolymers by free radical polymerization. Therefore, living radical polymerization is utilized for the synthesis of blockcopolymers with a narrow PDI as well as the preparation of functional polymers with pre-determined molecular weight and complex structures, such as block, graft, and star polymers. (100) Living radical/controlled polymerization (LCP) techniques including ATRP, SFRP and RAFT have been sucsessfully used for the synthesis of block copolymers. (45), (101)- (104)

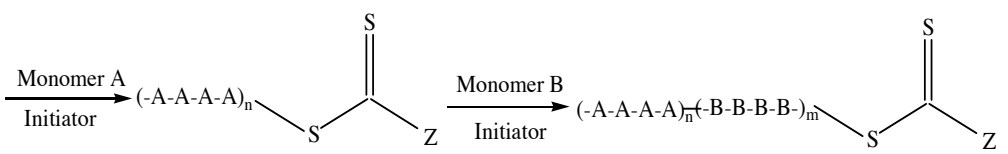

Figure 24 Schematic illustration of linear diblock copolymerization via RAFT. (57)

RAFT-functional polymer obtained via polymerization of first monomer can further act as a polymeric RAFT agent (macro-CTA) in the presence of a desired second monomer. (105) Here, second monomer is inserted between the thiocarbonyl thio $(\mathrm{S}(\mathrm{S}) \mathrm{C})$ moiety, and the last inserted monomer of the first block. (Figure 24) The RAFT-functionality, which might be used for immobilization of the block copolymer to surfaces, remains at the chain end of block copolymer during polymerization. The order of monomer addition is quite significant in block copolymerization. (45) The first block should act as a better leaving group than the block formed in the second step. (79) For example, in order to prepare the poly(methacrylate)-b-poly(styrene) copolymer, the methacrylate block should be prepared first due to the fact that styrene propagating radicals are very poor leaving groups compared to methacrylate propagating radicals. (106) During the past decade, amphiphilic block copolymers gained great interest in academic and industrial research due to their unique properties. (107)- (109) Amphiphilic block copolymers consist of at least one hydrophilic and one hydrophobic block which are covalently linked. (110) Basic industrial applications of Amphiphilic copolymers might be counted as detergents, personal care, paints, pharmaceuticals, oil recovery, environmental protection, agrochemicals, food industry, etc.

\subsection{Molecular Self Assembly}

During the last decade, the molecular assembly has opened new doors for the fabrication of functional materials through non-covalent interactions such as stacking, hydrogen and halogen bonding. (111) In the 1980's, Nuzzo \& Allara found that 
organo-sulphuric compounds spontaneously react with noble metals such as gold and achieved the formation of a well ordered thiol monolayer on gold surface via the selfassembly of di-n-alkyl disulfides from dilute solutions, which further would become the most popular SAM system. (20) Interest in these monolayer films has tremendously increased since that time. The film structure and growth process of monolayers were understood better with the development and application of surface-sensitive experimental techniques (e.g. scanning probe microscopy, infrared spectroscopy and X-ray photoelectron spectroscopy). (112) This finding led to development of convenient, and simple tools for a variety of applications in the field of surface science such as surface wetting (i.e. hydrophilic/hydrophobic), non-fouling property, electrochemistry, molecular electronics, etc. (113) When it comes to define self assembly process, there are different definitions in literature:

* In chemical solutions, self assembly refers to the joining of complementary surfaces via nano-molecular interactions, which stems from the affinity of binding sites of molecules for one another. (114)

* A method of integration in which the components spontaneously assemble onto a substrate from a solution or gas phase, until a stable structure of minimum energy is reached. (115)

* Self-assembly is the process by which a system of non-living chemical components has become a living, biological system. (116)

Basing on definitions above, a self-assembled monolayer (SAM) can be defined as a well ordered and densely packed layer of molecules which exhibit a high degree of orientation, and which are formed by the spontaneous adsorption of an active surfactant on a solid surface from solution or a gas phase. (Figure 25) The assembly is produced by a spontaneous chemical reaction at the interface, as the system approaches equilibrium. The compounds capable of interacting with the substrate consist of three specific functionalities: head group, end-group, and backbone. Each one has an important contribution on the formation of SAMS. Head-group must have an affinity toward the surface (e.g. metal, metal oxide, mica, etc.) such as thiol toward gold surface. In general, the backbone is typically made up of alkyl chain $\left(\mathrm{C}_{n} \mathrm{H}_{2 n}\right)$ whose length determines the degree of order of resulting monolayer. SAMs prepared by using molecules with short alkyl chains (fewer than 10 carbons) lack sufficent attractive interactions (4), whereas longer alkane chains with methyl or trifloromethyl end groups have greater dispersion interactions than shorter chains, thus leading to a lower free energy and a more ordered monolayer. (117) Moreover, the chemical nature of the end-group (e.g. $\mathrm{CH} 3, \mathrm{COOH}, \mathrm{OH}, \mathrm{NH}_{2}$ etc.), determines and influences the surface properties such as the wettability, the protein-repelling, etc. This terminal group can also be used to introduce initiator or raft agent to the surface with the purpose of polymerization via the "grafting from" method. Until the early 1990's, SAMs 
research has been mainly focused on the determination of new types of molecules and surfaces. The interactions between subunits might be either covalent or non-covalent such as hydrogen bonds, electrostatic and van der Waals forces. Deposition of SAMs might be performed by either liquid-phase or vapor-phase deposition. In the case of vapor-phase deposition, it requires a special designed reactor and gas flow lines. Another drawback of vapor-phase technique is the fact that it is limited to volatile, lowmolecular-weight adsorbates. Despite these disadvantages, it can overcome the limitations of liquid-phase deposition, such as non-uniform distribution of reactants, and incomplete coverage of surfaces. On the other hand, Liquid-phase deposition is a very fast, easy and convenient process which makes SAMs inherently manufacturable and thus technologically attractive for surface engineering.

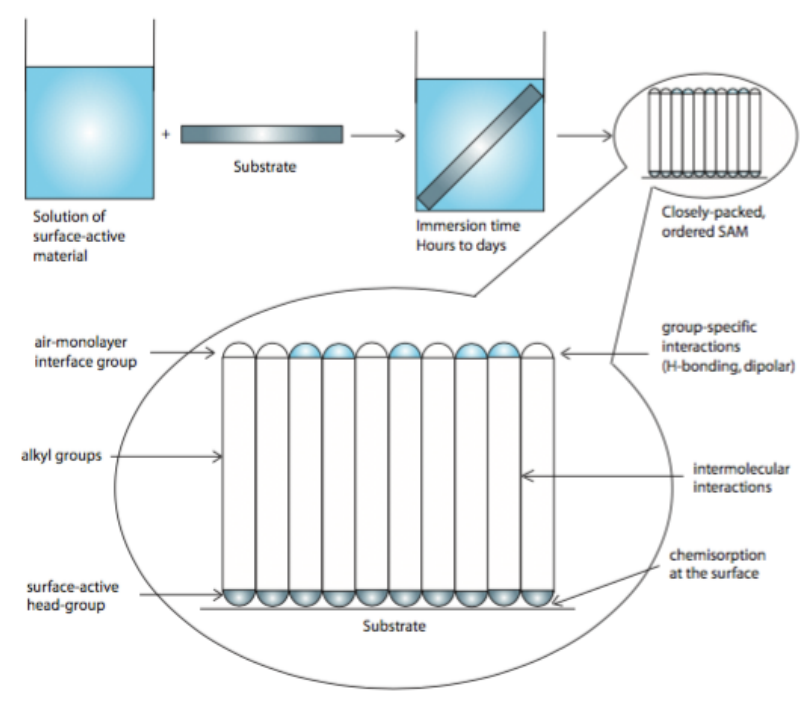

Figure 25 Self-assembled monolayers are formed by simply immersing a substrate into a solution of the surface-active compound. The driving force for the spontaneous formation of the $2 \mathrm{D}$ assembly includes chemical bond formation of molecules with the surface and intermolecular interactions. (19)

Sofar, self-assembled monolayers (SAMs) have been extensively investigated due to their well-defined structures (19) using various surface probes such as X-ray photoelectron spectroscopy (118), grazing incidence infrared reflection absorption spectroscopy (IR-RAS) (119), ellipsometry (120), surface plasmon resonance spectroscopy (SPR) (121), or atomic force microscopy (AFM). (117) The main purpose of the preparation of organic thin layers on solid surfaces is to alter the physicochemical properties of the surfaces (e.g the stability and wettability of solid surfaces), which leads to variation of application areas. Today, selfassembly chemistries allow formation of different type of monolayers on various surfaces such as thiols (122), disulfides and sulfides (123) on gold; fatty acids (124) and phosph(on)ates on metal oxides (125); silanes on silicon dioxide (126) and hydroxy functionalized surfaces (127); and isonitriles on platinum (128), catechol derivatives on $\mathrm{TiO} 2$ surfaces (129). The sucsess of self assembly process highly depends on the 
structure and composition of the metal surface. It is a well known fact that oxide-free metal surfaces and metals covered with stable oxides provide favorable conditions for self-assembly. (124) Metals covered with unstable, porous oxides might cause the inhomogenity and instability of the metal substrate which further result in disordering of the adsorption layers during self assembly process. Alkanethiols have been succesfuly assembled onto copper and reduced iron surfaces with an attempt to improve resistance of the underlying surfaces aganist corrosion. (125)- (128), (130), (131) Self-assembling monolayers, one end attached to the metal surface and the other chain end connected to an organic coating (e.g polymer coating), can inhibit electrochemical reactions at the interface and result in very stable bonds between the organic coating and the metal surface.

\subsubsection{Self Assembly of Thiol and Raft Agent Monolayer on Gold Surface}

\subsubsection{Nature of Affinity of Thiols and Raft Agents toward Gold Surface}

\subsection{An Overview of Main Bond Types}

Chemical bond refers to the forces holding atoms together to form molecules and solids. This force is of electric nature, and the attraction between electrons of one atom to the nucleus of another atom contribute to what is known as chemical bonds. Although electrons of one atom repell electrons of another, but the repulsion is relatively small. So is the repulsion between atomic nuclei. This attraction may be seen as the result of different behaviors of the outermost or valence electrons of atoms. Covalent, ionic and metallic bonding are stronger than other type of bonds shown in Table 1. (132)

Table 1 Classification of Chemical Bonds

\section{Chemical Bonds}

\begin{tabular}{|c|c|c|}
\hline 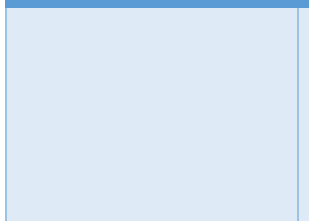 & By symmetry & $\begin{array}{ll}\text { - } & \text { Sigma }(\sigma) \\
\text { - } & \operatorname{Pi}(\pi) \\
\text { - } & \text { Delta }(\delta) \\
\text { - } & \operatorname{Phi}(\varphi)\end{array}$ \\
\hline & & $\begin{array}{ll}\text { - } & 1 \text { (single) } \\
\text { - } & 2 \text { (double) } \\
\text { - } & 3 \text { (triple) } \\
\text { - } & 4 \text { (quadruple) } \\
\text { - } & \text { 5(quintuple) }\end{array}$ \\
\hline
\end{tabular}




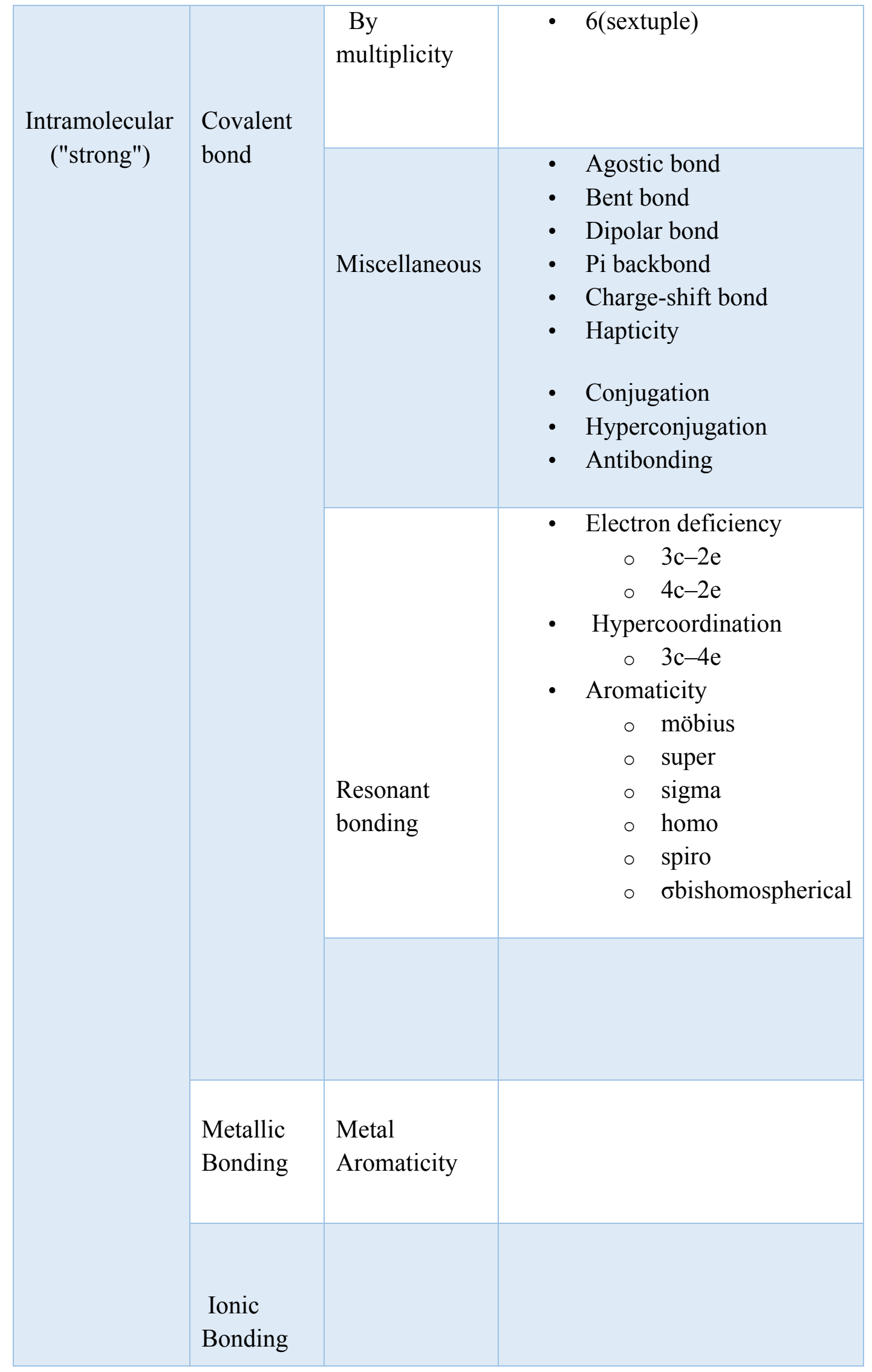




\begin{tabular}{|c|c|c|}
\hline \multirow{3}{*}{$\begin{array}{l}\text { Intermolecular } \\
\text { ("weak") }\end{array}$} & $\begin{array}{l}\text { Van der } \\
\text { Waals } \\
\text { forces }\end{array}$ & London dispersion force \\
\hline & $\begin{array}{l}\text { Hydrogen } \\
\text { bond }\end{array}$ & $\begin{array}{l}\text { - Low-barrier } \\
\text { - Resonance-assisted } \\
\text { - Symmetric } \\
\text { - Dihydrogen bonds } \\
\\
\text { C-H } \cdots \text { O interaction }\end{array}$ \\
\hline & $\begin{array}{l}\text { Other non- } \\
\text { covalent }\end{array}$ & $\begin{array}{ll}\text { - } & \text { Mechanical bond } \\
\text { - } & \text { Halogen bond } \\
\text { - } & \text { Aurophilicity } \\
\text { - } & \text { Stacking } \\
\text { - } & \text { Cation-pi bond } \\
\text { - } & \text { Anion-pi bond } \\
\text { - } & \text { Salt bridge }\end{array}$ \\
\hline
\end{tabular}

Nature of an ionic bond electrostatic is attraction between atoms which become positive or negatively charged ions as a result of that the bonding electron is transferred between these atoms instead of being shared. This transfer causes one atom to assume a net positive charge, and the other to assume a net negative charge. Ionic bonds may be seen as extreme examples of polarization in covalent bonds. In metallic bonding, each atom in a metal donates one or more electrons to a "sea" of electrons which are situated between many metal atoms. In this sea, each electron is free (by virtue of its wave nature) to be connected with many atoms at once without being part of any given atom. The metal atoms become positively charged due to loss of their electrons while the electrons still remain in contact with many atoms. Metallic bonding may be viewed as delocalization of electrons over a large system of covalent bonds, in which every atom participates. In the case of a covalent bond one or more electrons (often a pair of electrons) are shared by two atoms via being pulled into the space between the two atomic nuclei. The formation of a bond is generally exothermic process which results in energy loss. This reduction in overall energy (and hence stability of the bond) stems from the reduction in kinetic energy due to the electrons being in a more spatially distributed (i.e., longer deBroglie wavelength) orbital compared with each electron being confined closer to its respective nucleus. (133) Covalent bonds often lead to the formation of molecules which are composed of better-connected atoms. Molecules in solids and liquids are bound to other molecules by much weaker forces than the covalent bonds that hold the molecules internally together. Such weak intermolecular 
bonds result in organic molecular substances, such as waxes and oils, their soft bulk character, and their low melting points. When covalent bonds link long chains of atoms in large molecules (as in polymers such as nylon), the result may be both strong and tough and the melting points of such covalent polymers increase.

A special class of covalent bond is called as dipolar bond (134) (also known as a dative covalent bond (135), "dative bond" or coordinate bond (136)) As explained above, a covalent bond is formed by two atoms sharing a pair of electrons. The atoms are held together because the electron pair is attracted by both of the nuclei. In the formation of a simple covalent bond, each atom supplies one electron to the bond but that doesn't have to be the case. A coordinate bond is a covalent bond (a shared pair of electrons) in which both electrons come from the same atom. In another words, it is a kind of 2-center, 2-electron covalent bond in which the two electrons are donated from the same atom.

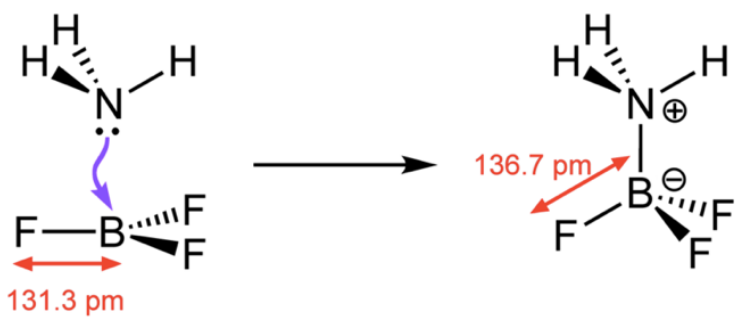

Figure 26 Adduct of ammonia and boron trifluoride. The arrow $\rightarrow$ indicates that both electrons in the bond originate from the amine moiety. (137)

The name dipolar bond arises from the electronic structure which has an electric dipole. The atoms carry fractional charges and the more electronegative atom of the two involved in the bond usually carries a fractional negative charge. An example of a dative covalent bond is the adduct of ammonia and boron trifluoride. (Figure 26) The interaction between a molecule of ammonia, a Lewis base with a lone pair of electrons on the nitrogen atom, and boron trifluoride, a Lewis acid stems from the fact that the boron atom has an incomplete octet of electrons. The boron atom obtains an octet configuration via forming the adduct. The electronic structure of a coordination complex is determined by the set of ligands each donating a pair of electrons to a metal centre. For example, in Hexaamminecobalt (III) chloride, each ammonia ligand donates its lone pair of electrons to the cobalt (III) ion. In this case, the bonds formed are called as coordinate bonds. In all cases the bond is a covalent bond.

\subsection{Chelation}

Chelation is a type of covalent bonding between ions or molecules with metal ions. It involves the formation of two or more separate coordinate covalent bond between a polydentate ligand and a metal atom. (138) These ligands are usually organic 
compounds, and are called chelants, chelators, chelating agents whereas the complex produced by this process is called a chelate. Chelating agents are ligands that have two or more atoms with donating electron pairs that are able to attach a metal ion at the same time. A ligand is an ion (anion or rarely cation) or neutral molecule with electron pair available that binds to a metal to form a complex. The ligand provides both of the electrons for the bond that forms between itself and the metal atom or ion. Ligands (which are neutral or negatively charged) are viewed as Lewis bases (electron pair donors) attracted to the metal atoms which act as Lewis acids (electron pair acceptor). They are classified according to number of donor atoms which are used to bond to metal atom or ion. Monodentate ligands bind through only one donor atom. Monodentate means "one-toothed". $\mathrm{H}_{2} \mathrm{O}$ and $\mathrm{NH}_{3}$ are examples of neutral monodentate ligands. When $\mathrm{H}_{2} \mathrm{O}$ is a ligand, oxygen is the donor atom binding to the metal. When $\mathrm{NH}_{3}$ is a ligand, nitrogen is the donor atom binding to the metal. Examples of monodentate ligands which begin with charges are halide ions, such as: $\mathrm{F}^{-}, \mathrm{Cl}^{-}, \mathrm{Br}^{-}, \mathrm{I}^{-}$, and cyano, $\mathrm{CN}^{-}$. A ligand molecule with more than one donor atom is a called a polydentate ligand. These are given specific names, depending on how many donor atoms they contain. Bidentate ligands bind through two donor sites. Bidentate means "two-toothed". An example of a bidentate ligand is ethylenediamine (Figure 27)

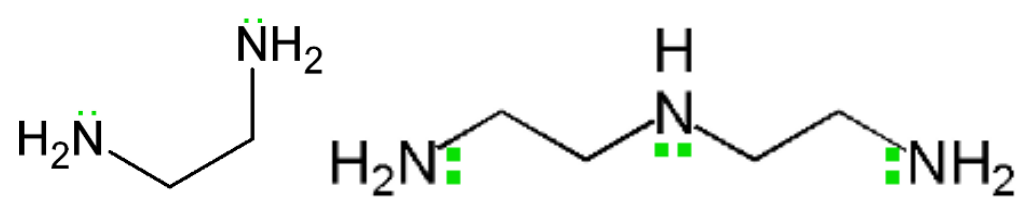

Figure 27 Examples of a) a bidentate ligand - Ethane-1,2-diamine has two lone pairs of electrons shown in green b) a tridentate ligand. 1,4,7-triazaheptane (also known as diethylenetriamine) has three lone pairs of electrons shown in green. (139)

Both of the nitrogens in this molecule can act as electron donors, binding with a central metal atom or ion. It can bind to a metal via two donor atoms at once. Bidentate binding allows a ligand to bind more tightly. Tridentate ligands, which bind through three donors, can bind even more tightly, and so on. This phenomenon is generally called the "chelate effect". The chemical reasons for this "chelate effect" stems from relative enthalpy and entropy changes upon binding a multidentate ligand. In terms of enthalpy, two coordinate bonds must be broken in order to completely remove a bidentate ligand. Breaking two bonds of the bidentate ligand requires more energy than breaking one coordinate bond for a monodentate ligand. Entropy deals with the distribution of energy within a system. From the entropic point of view, bringing two molecules together (a bidentate ligand and a metal complex) costs less than bringing three molecules together (two monodentate ligands and a metal complex). That's because individual molecules are free to move around, tumble and vibrate independently. Once they come together, they have to do all these things together. Since these different types of motion represent different ways of distributing energy, if the system becomes more restricted, energy can't be distributed in as many states. 
Energy is lowered even more by two bonding interactions. Compared to two separate donors, bidentate donation is entropically favoured. (140)

\subsection{Metal-Thiolate Complex}

The ability of the metal to exist in multiple oxidation states and the redox obstinacy of the organic elements work wonderfully together. Transition metals can take on additional covalent bonds (oxidation), switch out ligands (substitution), then release new covalent bonds (reduction). Although a complex indicates a reversible association of molecules, atoms, or ions through such weak chemical bonds, some metal complexes are formed irreversibly and many are bound together by bonds that are quite strong. (141)-(143) Transition metal thiolate complexes are metal complexes including thiolate ligands which can be classified as soft Lewis bases. Therefore, thiolate ligands coordinate most strongly to metals that behave as soft Lewis acids as opposed to those that behave as hard Lewis acids. Metal thiolate complexes are commonly prepared by redox reactions of metal complexes with appropriate organosulfur compounds, thiols (RSH), thiolates $\left(\mathrm{RS}^{-}\right)$, and disulfides $\left(\mathrm{R}_{2} \mathrm{~S}_{2}\right)$ etc. These reactions probably proceed via oxidative addition of the thiol. (144), (145) In organic chemistry, good reducing agents are reagents that deliver $\mathrm{H}_{2}$. Thiols and especially thiolate salts are reducing agents. Consequently, they induce redox reactions with certain transition metals. Thiolates are relatively basic ligands, being derived from conjugate acids with $\mathrm{pK}_{\mathrm{a}}$ 's of 6.5 (thiophenol) to 10.5 (butanethiol). Consequently, thiolate ligands often bridge pairs of metals. When nonbridging, they are susceptible to attack by electrophiles including acids, alkylating agents, and oxidants.

\subsection{Organosulphur Compounds on Gold}

It is a well known fact that sulfur and selenium compounds have a strong affinity to transition metal surfaces (146)- (150) because of the possibility to form multiple bonds with surface metal clusters. (151) Different types of organosulfur compounds that form monolayers on gold which include di- $n$-alkyl sulfide, (152), (153) di- $n$-alkyl disulfides, (20) thiophenols, (154), (155) mercaptopyridines, (155) mercaptoanilines, (156) thiophenes, (157) cysteines, (158), (159) xanthates (160) thiocarbaminates, (161) thiocarbamates, (162) thioureas, (163) mercaptoimidazoles, (164)-(166) and alkaneselenols (167) have been reported in recent years. (19) (Figure 28) 


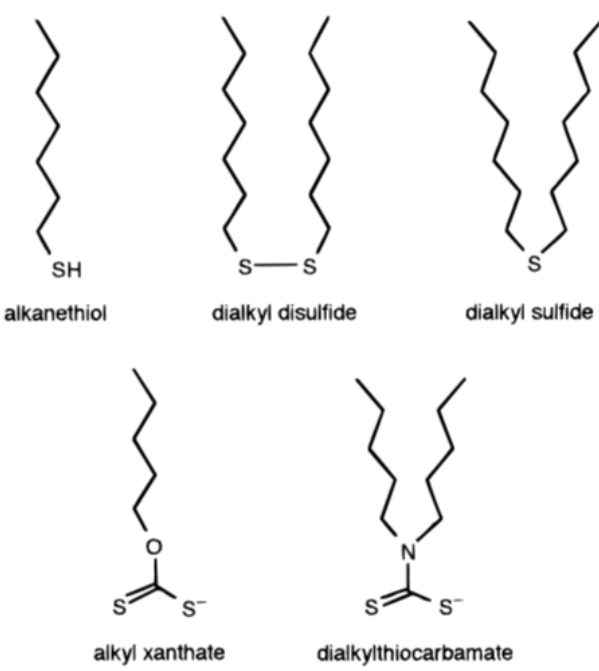

Figure 28: Surface active organosulfur compounds that form monolayers on gold. (19)

Apart from gold surfaces, organosulfur compounds coordinate very strongly also to silver, (168)-(172) copper, (171)-(175) platinum, (176) mercury, (177), (178) iron, (179), (180) nanosize $\gamma$-Fe2O3 particles, (181) colloidal gold particles, (182) GaAs, (183) and InP surfaces. (184) However, most studied SAMs have been carried out on that of thiolates on $\mathrm{Au}(111)$ surfaces. Chemisorption of alkanethiols as well as of di$n$-alkyl disulfides on clean gold gives indistinguishable monolayers. (185) It was reported that 4-aminobenzenethiol is spontaneously oxidized to 4,4'-diaminodiphenyl disulfide in the presence of gold powder. (186) This result indicates that the stability of thiolate SAMs on gold may be related to the electron density on the thiolate sulfur. (19)

\subsubsection{Self Assembly of Thiols on Gold Surface}

In the self-assembly method, alkanethiols, $\mathrm{SH}\left(\mathrm{CH}_{2}\right)_{\mathrm{n}} \mathrm{X}$ (e.g. thiols, disulfides, or sulfides) are spontaneously assembled onto gold surface. SAMs which are highly stable, densely packed and ordered, can be simply prepared via solution adsorption of sulfides onto zerovalent gold substrates which are inert toward corrosion or oxidation. (Figure 29) (187), (188), (19) 


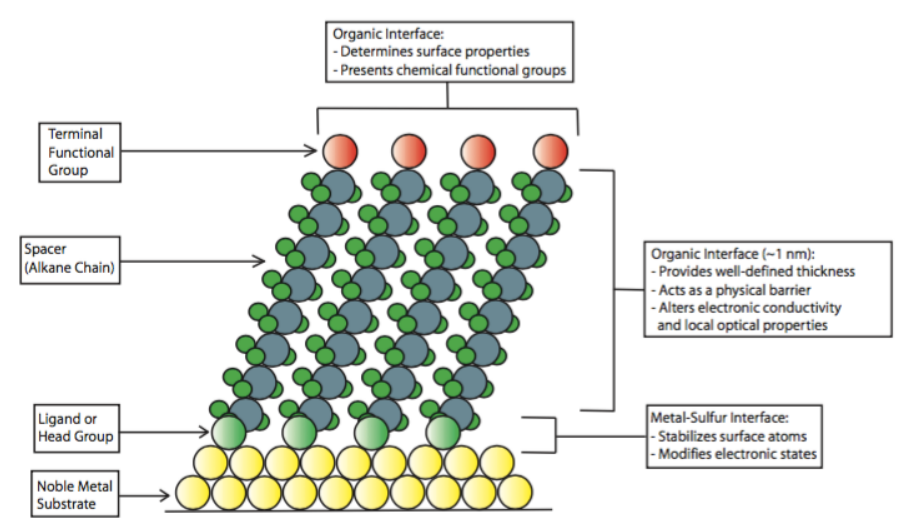

Figure 29 Schematic diagram of an ideal, single-crystalline SAM of alkanethiolates supported on a gold surface with a (111) texture. (19)

Main advantages of thiols for gold surfaces are summarized below (189):

* Well-ordered SAMs can be obtained via utilization of various sulphur containing species (i.e., thiols, dialkylsulfides, and disulfides)

* The self-assembly process of thiols is reproducible and can be easily performed at ambient temperature

* Large variety of thiol end-groups are commercially available, providing different chemical natures of the surface after self-assembly

Main advantages of gold as a substrate are summarized below (190):

Thin films of gold are easily obtained by physical vapor deposition, sputtering, or electrodeposition.

* Due to its own chemical inert nature, gold does not have an oxidized surface which allows easier cleaning process of the physically and/ or chemicallyadsorbed contaminants on gold surface. (191)

The formation of a covalent bond between sulphur and gold atom has been extensively investigated. (19) In the case of self assembly of alkanethiole, the reaction is considered formally as an oxidative addition of the $\mathrm{S}-\mathrm{H}$ bond to the gold surface, followed by a reductive elimination of the hydrogen. (19) (Figure 30) By means of various diffraction, microscopy and spectroscopy techniques, formation and structure of SAMs on gold were characterized in detailed with an attempt to obtain more informations about Au-S bond, loss of surface hydrogen and delocalization of surface charge. (192) (193) 


$$
\left.\mathrm{Au}_{\mathrm{n}}+\mathrm{HS}-\left(\mathrm{CH}_{2}\right)_{11} \mathrm{OH} \rightleftharpoons \mathrm{Au}_{\mathrm{n}-1} \mathrm{Au}-\mathrm{S}-\mathrm{CH}_{2}\right)_{11} \mathrm{OH}+1 / 2 \mathrm{H}_{2}
$$

Figure 30 Chemical reaction of thiolate bond formation. (19)

The fact that the thiolate $\left(\mathrm{RS}^{-}\right)$is the adsorbing species has been proven by XPS, (194)-(196) Fourier transform infrared (FTIR) spectroscopy, (197) Fourier transform mass spectrometry, (119) Electrochemistry, (198) and Raman spectroscopy. (199)(201) The resulting bond between gold and the thiolate group is very strong, stable, semi-covalent bond and the homolytic bond strength is on the order of $40 \mathrm{kcal} \mathrm{mol}^{-1}$. (202) Although the sulphur-gold interaction is quite strong, there is a limitation of stability of the Au-S bond (up to $60^{\circ} \mathrm{C}$ ). Regarding the structure of the assembly of thiols on gold (111) substrate with a full coverage, they tend to organize in a hexagonal close packed crystal lattice with a (_3 x _3) R30 arrangement. (Figure 31) (202)(204) The distance between the gold atoms is $2.88 \AA$ and the sulphur atoms arrange with a separation of $5.01 \AA$. Thiols form monolayers with a "tail-up" orientation and a tilt angle of the hydrocarbon chains of about $30^{\circ}$ with respect to the surface normal in order to maximize the van der Waals forces between the alkyl chains. (19)

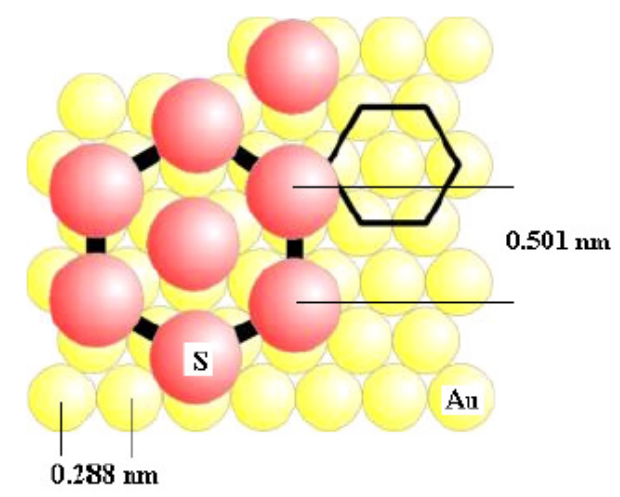

Figure 31 Schematic representation of the organisation of gold and sulphur atoms.

In addition to the affinity of sulfur for the gold surface, there are several driving forces for the assembly process of alkanethiols onto gold, which is ruled by inter- and intramolecular forces that drive the molecules into a stable, low energy state. The other driving force for assembly is the hydrophobic, van der Waals interactions between the methylene carbons on the alkane chains. This interaction results in tilting of the thiol chains in order to maximize the interaction between the chains and lower the overall surface energy. An alkane chain of at least 10 carbons might form a well-ordered monolayer where hydrophobic interactions between the chains can overcome the molecules' rotational degrees of freedom. (194), (205) 
Alkanethiols form closely packed monolayers on not only gold but also other metal surfaces such as silver, (206) copper, (207) and nickel. (208) On the contrary of gold surface, these metals oxidize under ambient temperature. The fresh solutions of thiol solution should be prepared and bubbled with Ar or $\mathrm{N}_{2}$ prior to use in order to prevent any oxidization of sulphur-metal bonds and to assure the formation of good quality, stable and reproducible monolayers. In the case of the chemisorption of disulfide species on gold surface, the formation of a packed monolayer just like thiol monolayer is observed. (209), (210) The mechanism of formation of the disulfide monolayer is explained by the cleavage of their S-S bond to form thiolate bond subsequent to the simple oxidative addition of the sulphur-sulphur bond to the gold surface. (211) The immersion of a clean gold substrate into a dilute solution $(\sim 1-10 \mathrm{mM})$ of the desired alkanethiol for 24 hours at room temperature is the most common procedure for preparation of a thiol monolayer. The kinetics of formation of dense monolayers from dilute thiol solution is divided into two steps including rapid coverage of the surface by thiol molecules takes milliseconds to minutes and then slow reorganization process of the adsorbed molecules to form a dense monolayer. (Figure 32)

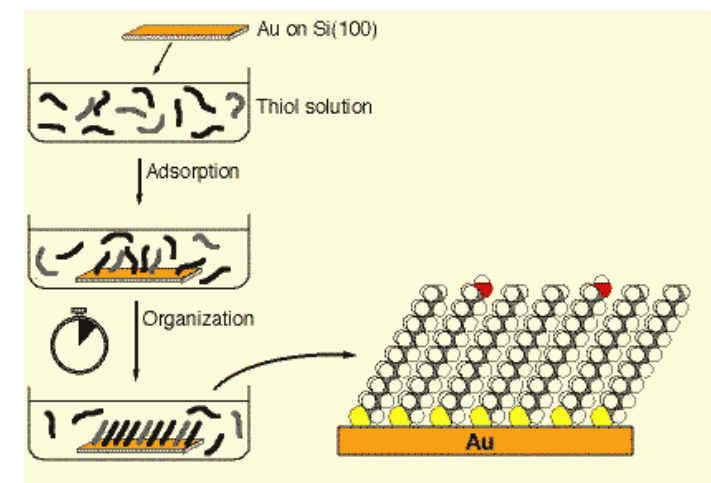

Figure 32 Illustration of the organization of the thiol self-assembly in solution. (212)

There are various experimental parameters which affect the growth rate of the monolayer (190):

* Solvent: Ethanol is the most common solvent preferred for the preparation of thiol monolayer due to its various advantages such as non-toxicity, low cost, the opportunity to be able to dissolve various alkanethiols with different chain length and polarity. Moreover, it provides well organized layers in comparison to apolar solvent based solutions. (120), (213)

* Temperature: The formation of monolayers is commonly performed at ambient temperature. (214) However, Kawasaki demonstrated that temperatures above $25{ }^{\circ} \mathrm{C}$ improve the kinetics, and reduce the number of defects in the monolayer. (215) 
Concentration vs immersion time: As a result of that these two parameters are inversely related; a low concentration of thiol requires a longer immersion time.

\subsubsection{Self Assembly of Raft Agent Monolayer on Gold Surface}

Preparation of monolayers based on a sulfur - Au bond has been extensively investigated (19), (190) including the use of thiols, (216) disulfides, (217) sulfides, (218) thiophene, (219) thiocarboxylic acids, (220) dithiocarboxylic acids, (221) xanthates, (222) thiocarbamates, (223) and dithiocarbamates. (224) For the first time, Duwez et al. demonstrated that dithioesters and trithiocarbonates bind to flat gold surfaces. (225) They investigated the specific binding modes of two different CTAs (benzyldithiobenzoate (BDTB) and dibenzyl trithiocarbonate (DBTTC)) using XPS and concluded that binding was bidentate in which both sulfur groups of the dithioester are involved. The S $2 p$ spectras recorded on monolayers of BDTB and DBTTC is shown in Figure 33. On the spectrum of BDTB, there is only one peak, which is monomodal, indicating that both sulfur atoms experience the same chemical environment. Moreover, the location of this peak, around $162 \mathrm{eV}$, is in very good agreement with the value reported in the literature for chemisorbed dithiocarboxylic acid on gold. (221) These findings demonstrate that BDTB is chemisorbed with both sulfurs attached to the gold substrate.

In the case of DBTTC, the peak is much broader than the resolution of $1.5 \mathrm{eV}$, which is associated with a bimodal structure. The reconstruction of the spectrum clarifies the presence of two doublets in a 2:1 ratio located around 162 and $163.5 \mathrm{eV}$. The binding energy and the width of the most intense doublet are identical to those observed for BDTB and is therefore associated with chemisorbed sulfurs whereas the minor doublet, located at $163.5 \mathrm{eV}$, is associated with the sulfur of the C-S bond not interacting with the gold substrate. Another additional evidence for lack of interaction with gold is that its binding energy $(163.5 \mathrm{eV})$ is in very good agreement with the values reported for a C-S bond in bulk compounds. Based on these observations, it is concluded that the chemisorption of DBTTC happens to be in a configuration where two of three sulfur atoms interact with the gold substrate. (Figure 33) This bridged configuration is reminiscent of the one found for xanthates and dithiocarbamates where only the two sulfur atoms are chelating gold. (222) - (224) This is likely due to geometrical constraints.
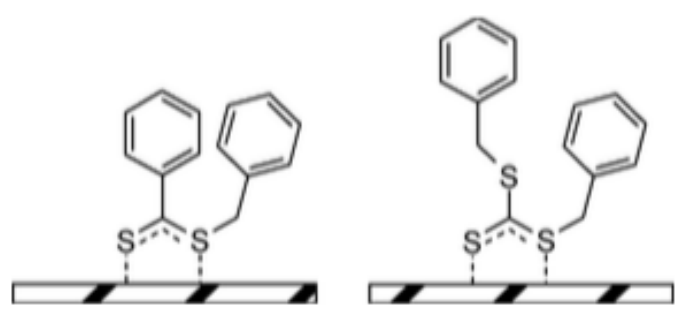
Figure 33 Chemisorption Configuration of the BDTB (Left) and of the DBTTC (Right).

There have also been reports conflicting with the results of Duwez metioned above. The specific mode of binding of dithiocarbamates onto gold nanoparticle surfaces was investigated by using surface enhanced Raman spectroscopy (SERS). (226) These studies concluded that the mode of binding could be either monodentate or bidentate and the particular binding mode was dependent on the density of ligands. (227), (228) Similar studies have also been reported for bis- and tris (thiol) compounds. (229) It is found out from these SERS experiments that the $\mathrm{C}=\mathrm{S}$ stretching band exhibits a significant shift compared to the normal Raman, whereas the C-S band and bands associated with the aromatic ring exhibit no significant shift. The shift of $\mathrm{C}=\mathrm{S}$ band ranged from 6 to $25 \mathrm{~cm}^{-1}$ depending on the structure of the phenyl dithioester. Based on these findings, it is concluded that the mode of binding for the small molecule phenyldithioesters is monodentate through the $\mathrm{C}=\mathrm{S}$ sulfur and there is no significant interaction of the phenyl ring, or the $\mathrm{C}$-S sulfur, with the gold nanoparticle surface. In each case the shift observed for the $\mathrm{C}=\mathrm{S}$ stretch as a result of binding of raft agent onto gold was to approximately $1210-1212 \mathrm{~cm}^{-1}$, regardless of the frequency of the band in the normal Raman spectrum. This is a powerful indicator of strong influence of the gold-sulfur interactions on the nature of the $\mathrm{C}=\mathrm{S}$ vibrational modes of the $\mathrm{C}=\mathrm{S}$ sulfur bound to gold. Another interesting finding coming out of these experiments is the fact the dithioester doesn't disassociate upon binding as a result of that the $\mathrm{C}=\mathrm{S}$ and $\mathrm{C}-\mathrm{S}$ stretches are still clearly observible. This data indicating that the binding of phenyldithioesters to AuNps is monodentate through the $\mathrm{C}=\mathrm{S}$ sulfur, is in contrast to the results reported by Duwez explained above (225) who used X-Ray photoelectron spectroscopy (XPS) and concluded that the particular phenyldithioester they used binds in a bidentate fashion.

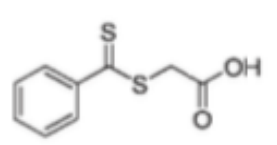

PDE-1

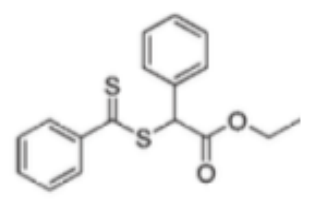

PDE-2

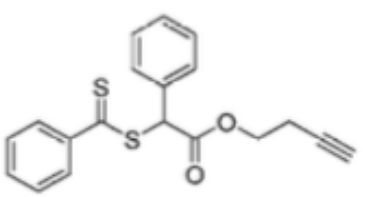

PDE-3

Figure 34 Structures of phenyldithioester derivatives used in SERS based study.

The differences in the results obtained from XPS and SERS might stem from a number of reasons: 
* The first reason is impact of surface coverage of the phenyldithioester on binding type. The degree of coverage has been shown to affect the mode of binding of thiols (192) and dithiocarbamates; (227) hence, it is likely to have an effect for the binding of phenyldithioesters to AuNPs aswell. Duwez and co workers experiments were carried out on flat gold surfaces. Although the surface coverage of the phenyl-dithioester small molecules was not specifically investigated, the experimental conditions and choice of a non charged phenyldithioester for self-assembled monolayer formation would result in a high degree of surface coverage. In the case of SERS studies, the maximum coverage of PDE-1 (Figure 34), a negatively charged molecule, was $71 \%$. It was not possible to measure the coverage of the PDE-2 and -3 (Figure 34), but the mode of preparation is not likely to result in a high coverage of molecules in the nanogap between two AuNPs. As a consequence of low coverage, it might result in monodentate binding of raft agents onto gold in the case of SERS experiments.

Other factors that may explain the conflicting results, is that the XPS has been carried out at high vacuum whereas the SERS experiments have been carried out in solution. In the case of XPS measurements, the incident X-rays or photoelectrons formed during analysis may result in X-Ray induced degradation of the phenyldithioester molecules on gold surfaces, which could lead to a different binding mode. This makes interpretation of the spectra difficult, in addition to the fact that the XPS spectra have been taken close to the limit of detection for sulfur.

\subsection{Polymer Architectures}

A polymer is defined as a macromolecule made up of many segments connected eachother. These segments contain a carbon backbone, which is the 'spine' of the polymer chain formed by carbon atoms, from which other elements/molecules dangle.

(Figure 35)

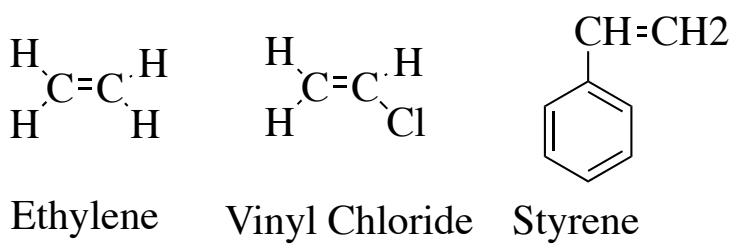

Figure 35 Some monomers illustrating the concept of carbon backbone.

Polymerization is name of the process which all the segments are connected eachother to form a polymer chain. The architecture of resulting polymer chains depend on the structure of the monomer and on the polymerization method employed. A polymer's architecture strongly affects many of its physical properties such as 
solution viscosity, melt viscosity, solubility in various solvents, glass transition temperature and the size of individual polymer coils in solution. If the segments are connected through the Carbon atoms, then a linear polymer chain results. The high density polyethylene is a good example of a linear polymer chain. (Figure 36)

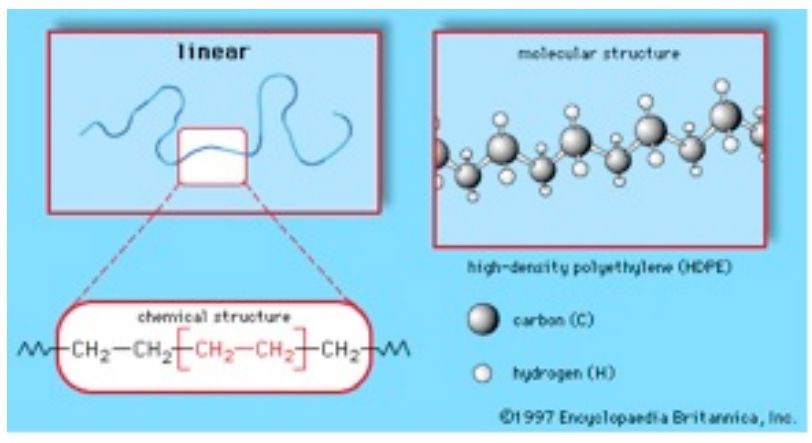

Figure 36 The structure of high density polyethylene. (230)

If a polymer chain has segments branching off of the main carbon backbone, it is called as a branched polymer. Due to the fact that forces holding branched polymer molecules together are much weaker than that of linear molecules, they cannot pack together closely. This makes branched polymers more flexible in comparison to linear ones. The low density polyethylene is a good example of a branched polymer chain. (Figure 37) Polymer chain gets more compact with increasing degree of branching. Branching also affects the chain entanglement, which strongly influences the bulk physical properties. In case of long chain branches, there is an increase in the number of entanglements per chain, which directly increase polymer strength, toughness, and the glass transition temperature $\left(\mathrm{T}_{\mathrm{g}}\right)$. On the other hand, a random and short chain length between branches reduce polymer strength as a result of disruption of the chains' ability to interact with each other or crystallize.

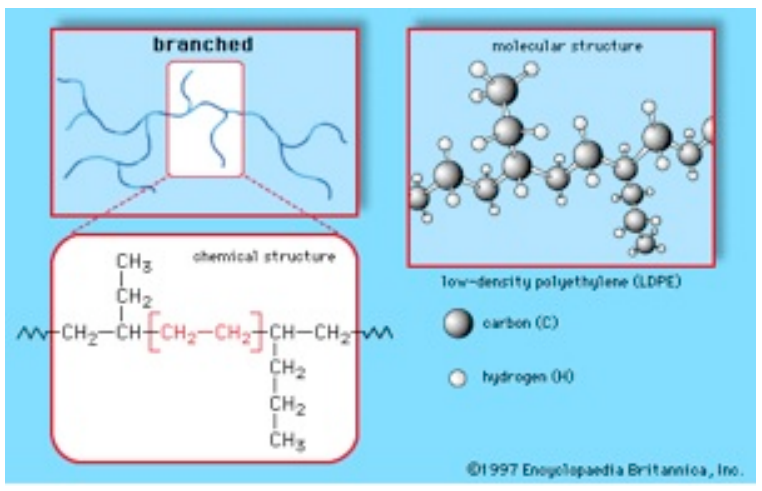

Figure 37 The structure of low density polyethylene. (230)

Polyethylene can be a good example of the effect of branching on physical properties. High-density polyethylene has a very low degree of branching, is relatively though, and is used in applications such as bottles or toys. On the other hand, since Low-density 
polyethylene is highly branched, it is relatively flexible, and is used as packaging film and plastic grocery bags. As seen in polyethylene case, by utilization of one single monomer type, it is possible to synthesise polymers which exhibit different macroscopic properties. With an attempt to vary applications, it is possible to combine different type of monomers, which can alter the properties of resulting material. (Figure 38-left hand column)

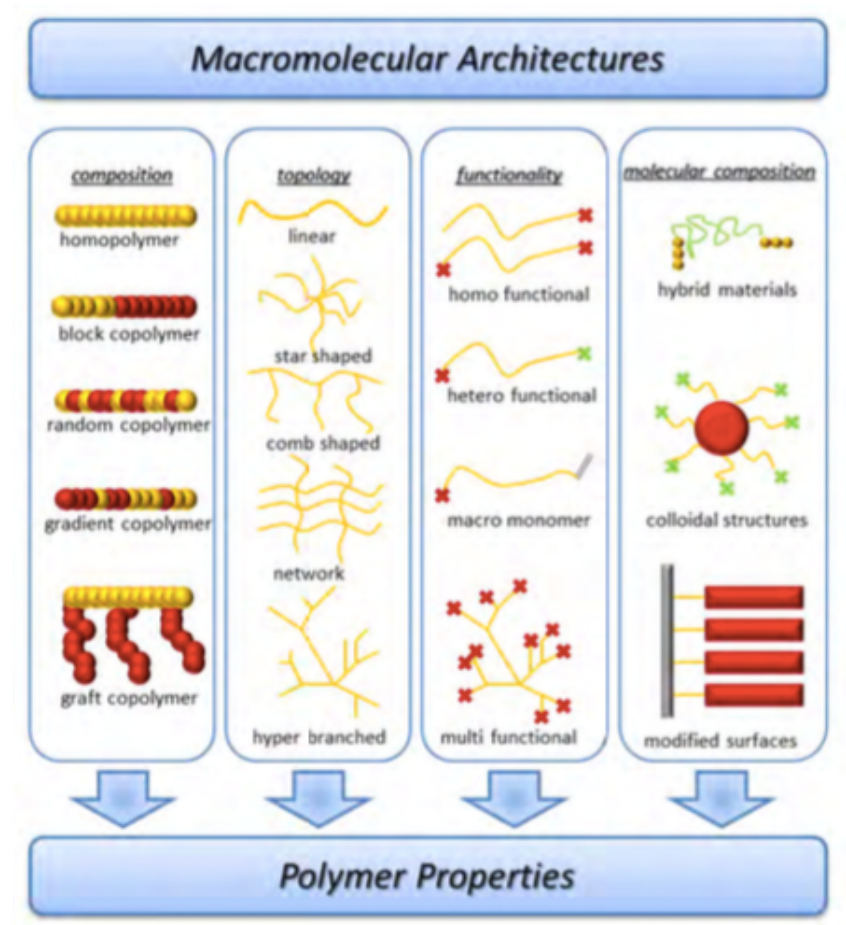

Figure 38 Various architectures of polymers. (230)

The properties of the resulting polymeric materials depend on many parameters such as the monomers used (left-hand column), the procedure with which the polymer is synthesized, additives (right-hand column), the topology of the single polymer molecules and the functionalities resulting from the monomers, starters (columns in the centre). (231) The chemical nature and behaviour of the polymer can be altered by utilization of different functional groups (e.g. hydrophilic end groups support a hydrophilic behaviour of the polymer). Moreover, it is possible to achieve complex hybrid structures (Figure 38, right-hand column) by growth of polymers from the surface of, e.g. silica, (232) or gold particles. (233) 


\subsection{Polymer Brushes}

\subsubsection{General Features}

\subsubsection{Definition}

Polymer brushes refer to an architecture in which polymer chains are terminally tethered to a surface at a very high density. The chains can be grafted to different substrates that may result in planar brushes (Figure 39) for planar or "reasonably flat" substrates whose root mean square roughness should be much smaller than end-to-end distance of grafted polymer chains, "molecular brushes" in the case of cylindrical brushes for fiber- or rod-like and spherical brushes for substrates of spherical shape. The grafting can be realized through covalent bonds, electrostatic interactions, hydrogen bonds, or strong van der Waals interactions.

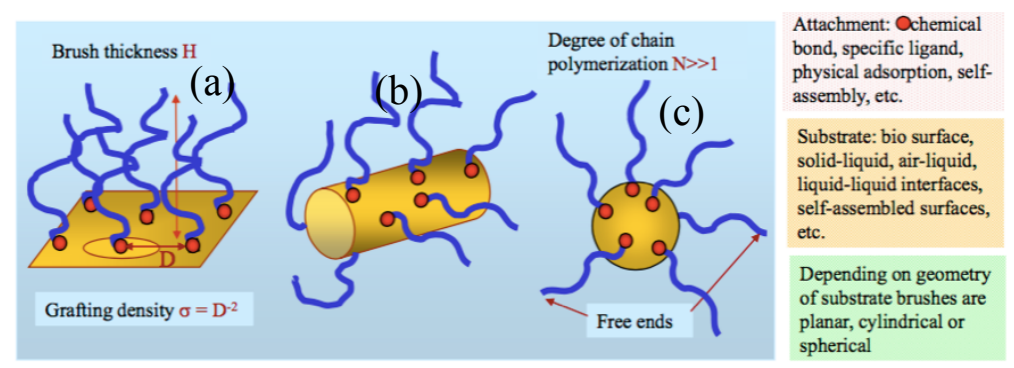

Figure 39 Schematic illustration of brush types with respect to geometry of substrate (a) Planar brushes (b) Cylindrical brushes (c) Spherical brushes. (234)

\subsubsection{Theoritical Description of Polymer Brushes}

The parameters used for description of brush systems, are the segment density profile $(\Phi(\mathrm{z}))$ of the surface-attached chains andlor the brush height $\mathrm{H}$ as a function of the graft density $\sigma$, the molecular weight (Idegree of polymerization) of the surfaceattached chains, and the solvent quality of the contacting medium. (Figure 40)

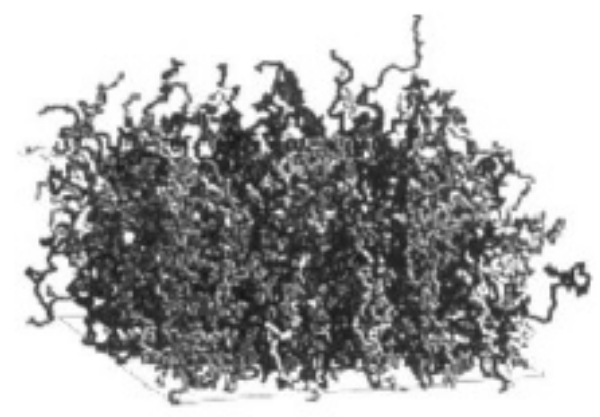

Figure 40 Two hundred chains of a polymer brush (chain length $\mathrm{N}=100$ ) under good solvent conditions. (234) 
Alexander de Gennes described a brush system for monodisperse chains consisting of $\mathrm{N}$ segments, which are attached to non adsorbing surface with an average distance of the anchor points ( $d$ or D) much smaller than the radius of gyration of the same unperturbed chains not in contact with the surface. (235), (236) (Figure 41)

(a)

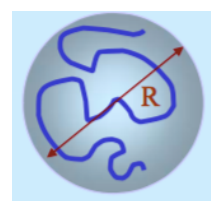

(a)

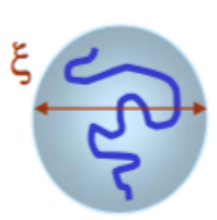

(b)

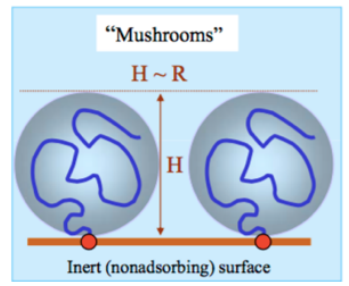

(c)

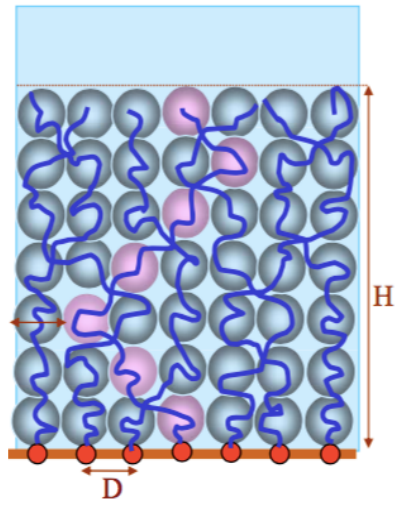

Figure 41 Schematic illustration of the Alexander de Gennes model for the theoretic description of polymer brushes. The chain segments with the "blobs" (indicated by the circles) behave as random ("Gaussian") coils. (D represents the average distance between anchor points) (234) (a) Average end to end distance of a non-grafted polymer chain in a given solvent (b) If the brush height $(\mathrm{H})$ of grafted chain is not higher than the end-to-end distance $(\mathrm{R})$ of the identical non-grafted polymer chain in the same solvent, resulting conformation is a mushroom (c) If tethering points are high enough, the macromolecular chains stretch away from the surface so that in the given solvent the brush height $(\mathrm{H})$ is larger than the end-to-end distance of the identical non-grafted chains dissolved in the same solvent. If the distance between grafting points (D) is smaller than the chain end-to-end distance, it leads to formation of a real brush conformation. (25), (237)

Basing on Flory theory, the radius of gyration is dependent on the number of monomer $(\mathrm{N})$ with following equation 9:

$$
\mathrm{R}_{\mathrm{g}} \sim \mathrm{N}^{\mathrm{v}} \mathrm{a}
$$


Where a is the size of the monomer and $v$ reflects the quality of solvent. The value of $v$ for good, theta and bad solvent of the tethered polymer chains is respectively $3 / 5$, $1 / 2$ and $1 / 3$.

Basing on Alexander de Gennes model, the size of a blob $\xi$ is defined with equation 10:

$$
\xi \sim 1 / \sqrt{\sigma}
$$

The number of monomers $(\mathrm{g})$ in a blob is determined with equation 11:

$$
\mathrm{g} \sim(\xi / \mathrm{a})^{1 / v} \sim \sigma^{-1 /(2 v)} \mathrm{a}^{-1 / v}
$$

The number of correlation blobs per chain is $\mathrm{N} / \mathrm{g}$ is defined with equation 12 :

$$
\mathrm{N} / \mathrm{g} \sim \mathrm{N} \mathrm{\sigma}{ }^{1 /(2 v)} \mathrm{a}^{1 / v}
$$

The height of the brush is the size of a correlation blob times the number of these blobs per chain (equation 13):

$$
\mathrm{H} \sim \xi \mathrm{N} / \mathrm{g} \sim \mathrm{No}(1-v) /(2 v) \mathrm{a}^{1 / v}
$$

The height $\mathrm{H}$ increases linearly with the number of monomers $\mathrm{N}$ per chain at a constant grafting density.

\subsection{Conformation Types of Polymer Brushes}

The characteristic parameter for brush systems is the anchoring or grafting density $\sigma$, which is the inverse of the average area available for each polymer at the surface. The conformational behaviour of the polymer chains is a function of the grafting density and the radius of gyration (the average size of the chains) (Figure 41), (Figure 42). Depending on the grafting density, three different type conformational behavior might be observed (25), (237):

* For small grafting densities, $\sigma<\sigma^{*}$, in another words, If the distance between two anchoring sites is larger than the size of the surface-tethered polymers, the segments of the individual chains will be far apart from each other and hardly interact. Thus, each chain will be isolated from the surrounding chains and 
behave just like single chains pinned onto the surface by one end. Depending on the strength of interactions between polymer segments and the surface, resulting conformation types might be divided into two categories: If the interaction between chains and surface is weak, the chains form a typical random coil that is linked to the surface through a "stem" of varying size and conformation will be called "mushroom". (Figure 42 (a)) In case that the segments of surface tethered chains strongly adsorb onto the the underlying surface, a "pancake" like conformation is obtained. (Figure 42 (b)) In this case, conformation of tethering chains is similar to the one in solution.

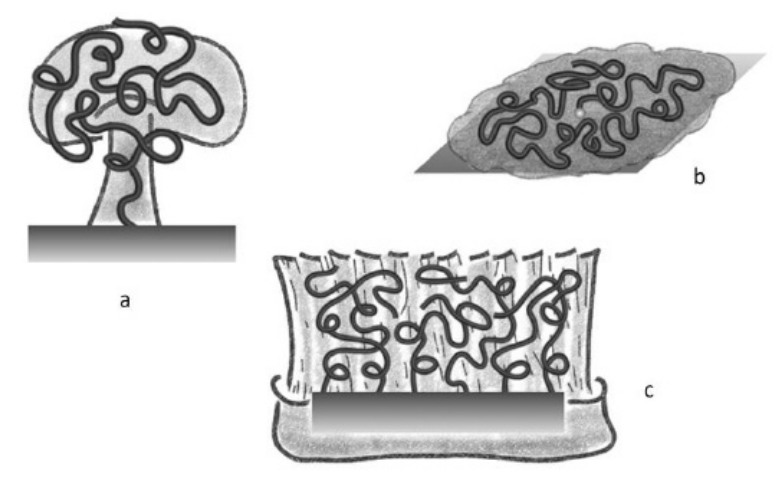

Figure 42 (a) "mushroom", (b) "pancake" and (c) "brush" used for the different possible conformations of surface-attached polymers. (234)

As the size of grafted polymer chains approaches the distance between grafting points, the grafted chains overlap. The grafting density at which chains just start to overlap is determined with equation 14 :

$$
\sigma^{*} \sim \mathrm{R}_{\mathrm{g}}^{-2}
$$

where $\mathrm{Rg}$ is the typical radius or size of a tethered chain at specific experimental conditions of solvent and temperature. In good solvent conditions (that is for swollen chains), the chain radius follows the Flory prediction $R_{g} \sim$ $\mathrm{aN}^{3 / 5}$ where $\mathrm{N}$ is the polymerization index or monomer number of the chain, and $\mathrm{a}$ is the size of monomer. The crossover grafting density for a polymer under good-solvent conditions is determined with equation 15 :

$$
\sigma^{*} \sim \mathrm{a}^{-2} \mathrm{~N}^{-6 / 5}
$$

This point so called as cross-over regime is a transition point between mushroom regime and brush regime. A commonly used literature parameter for quantitative characterization of this transition is the reduced tethered density defined by equation 16 : 
(1) $\sum<1$ refers to weakly interacting regime which is so called the "mushroom" regime, (2) $\sum \sim 1$ is equivalent to the crossover regime, whereas (3) $\sum>1$ corresponds to the highly stretched regime. However, in real systems, the transition between single grafted chains and a polymer brush is less sharp because of the statistical characteristic of grafting and polydispersity of the tethered chains. (238)

For large grafting densities, $\sigma>\sigma^{*}$, in another words, If the distance between two anchoring sites is such short that the chain segments are very close to each other, the chains will be strongly overlapping. Due to this overcrowding, assuming the solvent to be good, monomers repel each other. Since the lateral separation between the polymer coils is fixed by the grafting density, the polymer chains try to minimize the segment-segment interactions in order to avoid each other by extending away from the grafting surface. The chains stretch perpendicular to the surface until the loss of possible conformations (loss in entropy) balances the enthalpic gain of segment dilution. (239) This loss of entropy leads to a retracting force trying to keep the chains coiled just like in the case of response of a stretched rubber. Thus, a new equilibrium at a higher energy level is obtained in which the chains are stretched perpendicular to the surface. The resulting structure is called a real polymer brush, with a vertical height $\mathrm{h}$ which greatly exceeds the unperturbed coil radius $\mathrm{R}$. (69) (Figure 42 (c)) In this case, conformation of tethering chains is significantly different to the one in solution. (38) The main parameters which determine and influence the conformation of the polymer brushes in solution are the quality of the solvent, the chain stiffness and the degree of polymerization $(\mathrm{N})$.

\subsection{Calculation of Grafting Density}

The structure of a surface-immobilized polymer can be determined by calculation of grafting density $(\sigma)$. (Figure 43) The grafting density is determined by equation 17 :

$$
\sigma=\left(h \rho N_{A}\right) \backslash M_{n}
$$

where $\mathrm{h}$ is the brush thickness; $\rho$, bulk density of the brush composition; and $\mathrm{N}_{\mathrm{A}}$, Avogadro's number and $\mathrm{M}_{\mathrm{n}}$ is number average molecular weight of polymer. 


$$
\begin{aligned}
& A\left(\mathrm{mg} / \mathrm{m}^{2}\right)=h(\mathrm{~nm}) \times \rho\left(\mathrm{g} / \mathrm{cm}^{2}\right) \\
& \sigma\left(\text { chains } / \mathrm{nm}^{2}\right)=\frac{(6.023 \mathrm{~A} \times 100)}{\bar{M}_{n}} \\
& \mathrm{D}=2(\pi \sigma)^{-0.5}
\end{aligned}
$$

The coverage (A, $\mathrm{mg} \backslash \mathrm{m}^{2}$ ), grafting density $\left(\sigma\right.$, chains $\left./ \mathrm{nm}^{2}\right)$ and anticipated average distance between grafting points $(\mathrm{D}, \mathrm{nm})$ of the polymer brushes are calculated from ellipsometric thickness $(\mathrm{h}, \mathrm{nm})$ and number-average molar weight $\left(\mathrm{M}_{\mathrm{n}}, \mathrm{g} / \mathrm{mol}\right)$ and the bulk density of polymer $(\rho)$ values using equations 18,19 and 20. (240) - (243)

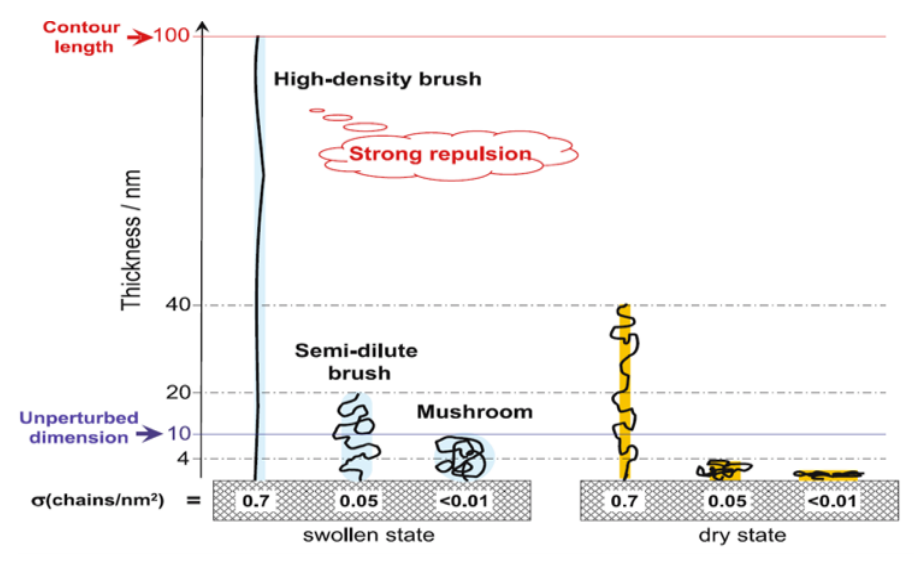

Figure 43 Classification of polymer brushes with respect to grafting density.

\subsubsection{Synthesis of Polymer Brushes}

The formation of these brushes is generally obtained by two types of techniques, namely physisorption and chemisorption (covalent attachment). (26) (Figure 44)

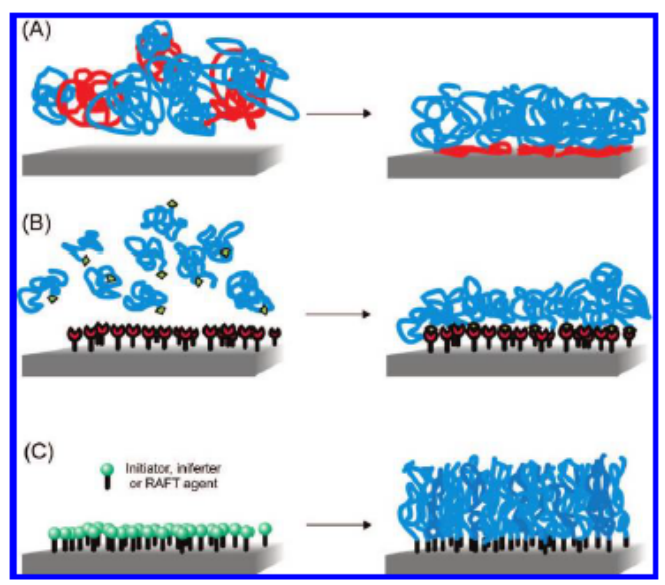


Figure 44 Synthetic strategies for the preparation of polymer brushes: (a) physisorption of diblock copolymers via preferential adsorption of the red blocks to the surface (grafting to approach); (b) chemisorption via reaction of appropriately endfunctionalized polymers with complementary functional groups at the substrate surface (grafting to approach); (c) polymer brushes grown via surface-initiated polymerization techniques (grafting from approach). (33)

\subsubsection{Physisorption}

Physisorption method relies on a physical, selective adsorption of a polymer chain where one part strongly coheres onto the surface, whereas the second part interacts weakly or has no interaction at all. This selective adsorption was generally achieved with block copolymers. (Figure 45)

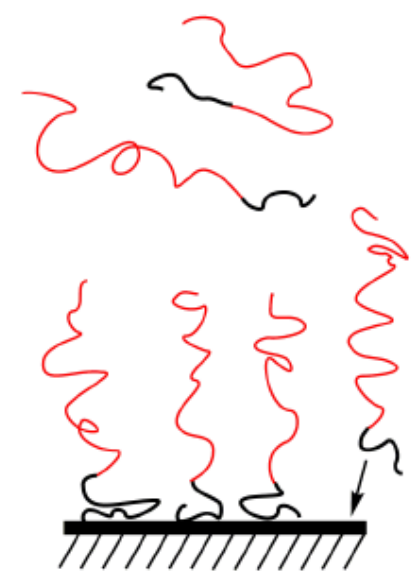

Figure 45 Schematic description of the physisorption of a block copolymer. (25)

The polymer brush formation through physisorption method has some drawbacks such as requirement for design of diblock copolymers which consist of special polymeric segments, one of which has high and selective affinity towards the surface and the other one which might interact only with the solvent. Secondly, polymer brushes prepared via physisorption suffer from thermal and solvent instabilities due to the weak interactions (van der Waals and hydrogen bond) between the substrate and polymer chains. Furthermore, the physisorption is a reversible process implying a poor grafting density of chains leading to mushroom type conformation (not a real brush).

\subsubsection{Chemisorption}

Chemisorption strategy relies on the creation of covalent bonds between the surface and polymer molecules. Main advantage of this method over physisorption is the fact that resulting macromolecular brushes are robust and resistant against most chemical 
environments due to covalent bonding. In this approach, covalent grafting can be achieved either grafting "to" or "from" techniques. (Figure 46)

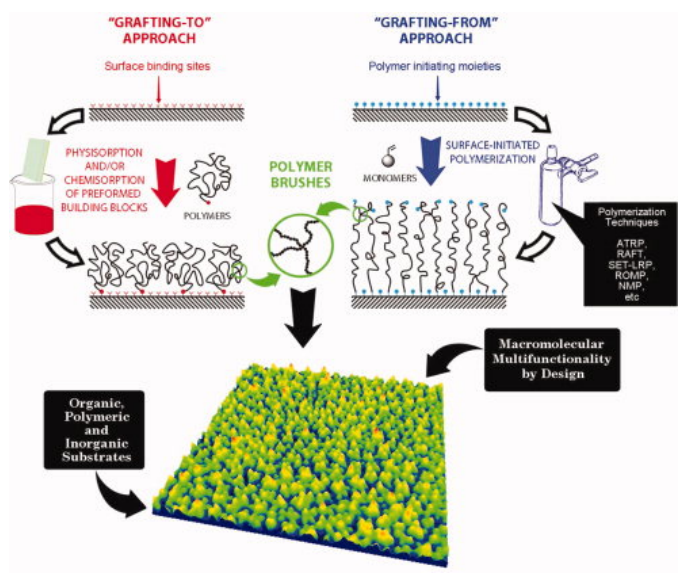

Figure 46 Preparation of polymer brushes on surfaces via SI-CRP through (a) grafting to (b) grafting from approaches. (244)

\subsubsection{1 "Grafting To" Technique}

The "grafting to" technique is based on the reaction of a preformed chain bearing a complemental end-functionality suitable for functionality of the substrate. (25) (Figure 47)

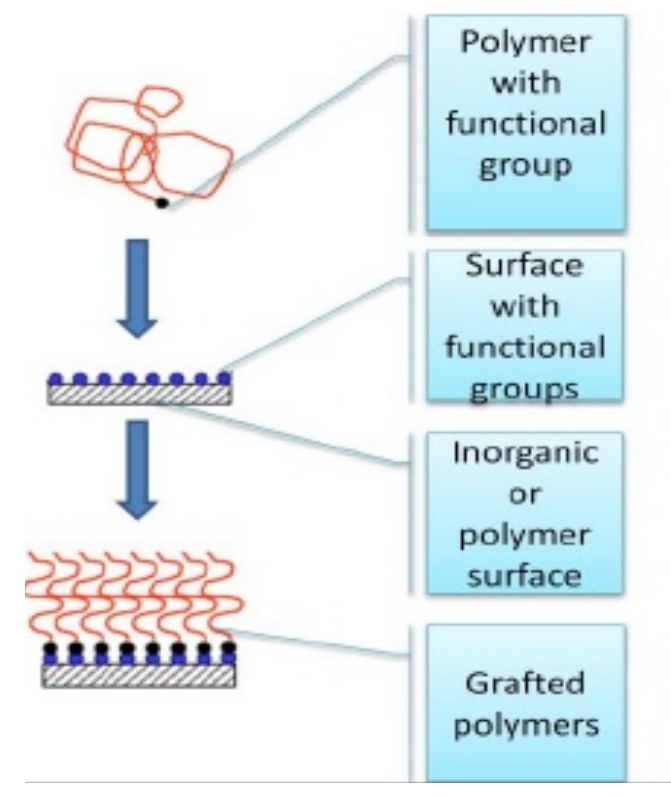

Figure 47 Schematic description of the "grafting to" process of a polymer chain. (244)

Several studies were performed to form polymer brushes using the "grafting to" technique. (245)- (247) Main drawback of the "grafting to" method is low grafting density resulting from the steric hindrances between broad polymer chains diffusing 
onto surface. This low surface density might lead to brush degrafting as a result of penetration of small molecules inside the polymeric films toward the surface. Because of these disadvantages, "grafting from" method is mostly preffered.

\subsubsection{2 "Grafting From" Technique}

The "grafting from" technique so called surface initiated polymerization relies on a chain-growth polymerization from an initiator anchored on the surface. (26), (248) (Figure 48) In this technique, monomer easily diffuses to the initiator fixed on the surface in order to generate tethered polymers with high grafting density, on the contrary of the "grafting to" method in which broad polymer chains try to approach surface. Many different pathways were developed for preparation of polymer brushes on various type of substrates such as gold, glass, particles, etc. by utilization of various initiator introducement methods and a variety of polymerization techniques. Main advantages of this approach is the possibility to obtain high grafting density brushes and control the chemical composition (homopolymers, copolymers, etc.) as well as the macromolecular architectures (linear, branched, etc.).

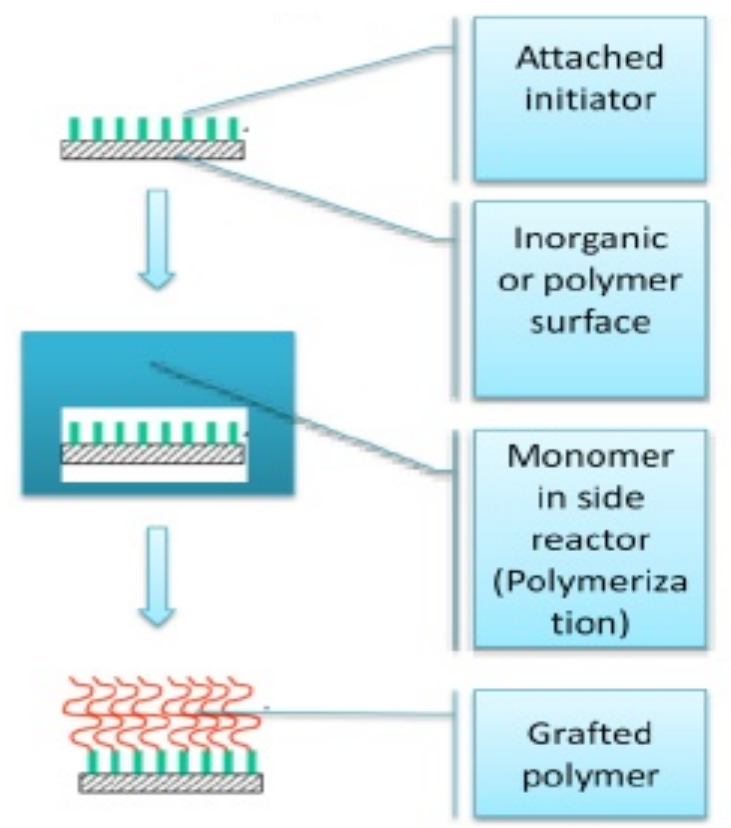

Figure 48 Schematic representation of the "grafting from" process of a polymer chain. (249)

A variety of synthetic strategies were improved for preparation of polymer brushes via the "grafting from" method. Some examples for controlled polymerizations are cationic and anionic polymerization, (250), (251) ring-opening polymerization (ROP), (252), (253) ring-opening metathesis polymerization (ROMP), (254), (255) atom transfer radical polymerization (ATRP), (256), (257) nitroxide mediated polymerization (NMP) (258), (259) and reversible addition-fragmentation chain transfer (RAFT). (66), (260) 


\subsection{Synthesis of Polymer Brushes via SI-CRP}

\subsection{Synthesis of Polymer Brushes via SI-RAFT}

Several living radical/controlled polymerization techniques have been employed in order to have surface-initiating graft polymerization on surfaces. On the contrary of ATRP, where the equilibrium between the dormant and active propagating chains is based on reversible termination, RAFT (reversible-addition fragmentation chain transfer) polymerization relies on reversible chain transfer. (45), (66), (115) Main advantage of RAFT polymerization is its relative simplicity and versatility leading to the opportunity to polymerize various monomer types. Conventional free radical polymerizations can be easily converted into a RAFT process by adding an appropriate RAFT agent (e.g. a dithioester, dithiocarbamate, or trithiocarbonate compound) while other reaction parameters, such as monomer, initiator, solvent, and temperature, can be kept constant. RAFT polymerization has currently gained significant interest to prepare polymer brushes from surfaces due to the control ability of the final polymer product with respect to molecular weight, polydispersity, and macromolecular architecture. (66) Moreover, raft polymerization technique enables the opportunity to utilize not only surface anchored initiator but also surface anchored Raft agent. Anchoring of a RAFT agent to a surface can be accomplished via either the $\mathrm{Z}$ or $\mathrm{R}$ group. (70), (261), (262) (Figure 49) However, the R group approach is mostly preffered due to the fact that its mechanism is very similar to "grafting from" polymerization whereas the polymerization mechanism of surface immobilized $\mathrm{Z}$ group imitates that of the "grafting to" approach which has a limited ability to obtain high density grafted polymer. (70) In the case of the "grafting from" approach, low molar mass monomer molecules can easily diffuse onto the functionalized surface to start polymerization and grow the polymer chains.

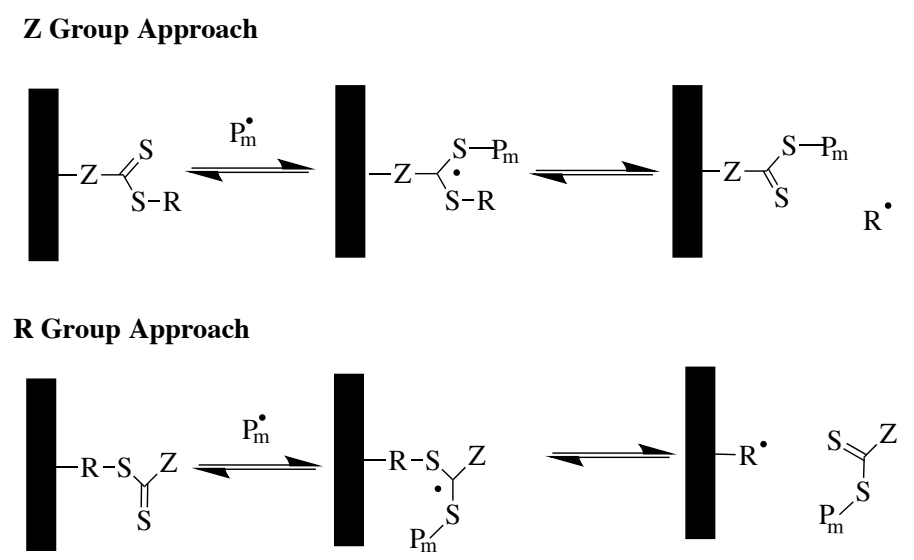

Figure 49 Anchoring types of a Raft agent to a surface via $\mathrm{Z}$ and $\mathrm{R}$ group approach.

As a consequence, in comparison to other CRP methods in which only initiator might be introduced onto surface, immobilization opportunity of the RAFT agent through 
either $\mathrm{Z}$ group or $\mathrm{R}$ group on a solid support (e. g. gold, silica or titania etc.) may therefore be an attractive alternative way for synthesis of brushes from surfaces. In this study, two kinds of commercial RAFT agents (1) and (2) were used whereas three multifunctional RAFT agents were synthesized. (3), (4), (5) (Figure 50) There are many examples of SI-RAFT polymerization from initiator anchored surfaces. Forexample, Baum and Brittain prepared 30-nm-thick PMMA brushes as from azofunctionalized silicon wafers in the presence of the chain transfer agent (CTA) 2phenylprop-2-yl dithiobenzoate and free initiator (2,2'-azoisobutyronitrile (AIBN)) via SI-RAFT polymerization (Figure 51). (263) It was shown that addition of free initiator (e.g., AIBN) is essential to support polymer brush growth on surfaces in terms of two aspects: The fact that the concentration of the surface initiators is not high enough, might result in early termination by CTA capping. In order to avoid this termination, the amount of radicals should be increased in the system by utilization of free initiators.

Secondly, free initiator acts as a scavenger for impurities in the polymerization mixture. The surface anchored initiator approach is sucsessfullly employed to prepare PMMA, (266) poly (poly (ethylene glycol) methyl ether methacrylate) (PPEGMEMA), (120) and poly (2-(dimethylamino) ethyl methacrylate) (PDMAEMA) brushes. (266) Besides the use of free radical initiator-modified substrates, SI-RAFT can also be carried out from surface-immobilized RAFT agents.

The RAFT agent can be immobilized in two different ways socalled the R-group and Z-group approaches. In the R-group approach, the RAFT agent is tethered to the surface via the leaving and reinitiating $R$ group. A variety of polymer brushes were sucsessfully prepared via $\mathrm{R}$ group approach from dithiobenzoate- or trithiocarbonatebased Raft agent immobilized substrates such as silicon wafers, (267)- (270), titania (271), gold nanoparticles (260), cellulose. (272) In the case of the Z-group approach, RAFT agent is immobilized via the stabilizing $Z$ group. This approach has been successfully employed for fabrication of a variety of methacrylic, acrylic, styrenic based brushes. (265), (273)- (278) The use of surface-immobilized CTAs might have specific limitations. In the case of R-group approach, since it is comparable to a grafting from process, it is possible that RAFT agent might be detached from surface during the polymerization. Therefore, the molecular weight distribution might be broadened via bimolecular termination at high rate. On the other hand, Z-group approach is similar to a grafting to approach. It might suffer from a decrease of brush grafting densities with increasing brush length, as a result of the surface tethered RAFT agent will be less accessible for longer growing polymeric radicals which are sterically hindered both by eachother and by pre-approached (already grafted) chains on surface, while trying to approach back to surface. (265), (274) 


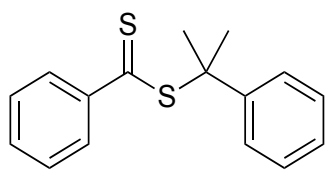

Cumyl dithiobenzoat<smiles>CCCCCCCCCCCCSC(=S)SC(C)(C#N)CCC(=O)O</smiles>

4-cyano-4-(((dodecylthio)carbonothioyl)thio) pentanoic acid<smiles>CC(SC(=S)SCCSC(=S)SC(C)c1ccccc1)c1ccccc1</smiles>

Ethane-1,2-diyl bis(1-phenylethyl) ditrithiocarbonate<smiles>CC(SC(=S)SCC(=O)OCC(COC(=O)CSC(=S)SC(C)c1ccccc1)(COC(=O)CSC(=S)SC(C)c1ccccc1)COC(=O)CSC(=S)SC(C)c1ccccc1)c1ccccc1</smiles>

Penta-erythritol-tetrakis-(3-(S-benzyl trithiocarbonate))-propanoate<smiles>COC(=O)C(C)SC(=S)SCCC(=O)OCC(COCC(COC(=O)CCSC(=S)SC(C)C(=O)OC)(COC(=O)CCSC(=S)SC(C)C(=O)OC)COC(=O)SC(=S)SC(C)C(=O)OC)(COC(=O)CCSC(=S)SC(C)C(=O)OC)COC(=O)CCSC(=S)SC(C)C(=O)OC</smiles>

Dipentaerythriol-hexakis(3-(S-methoxopropionate-trithiocarbonate)) -propanoate(hexa functional) (DPEHSMPETTC)

Figure 50 Structures of Raft agents used in this study. 


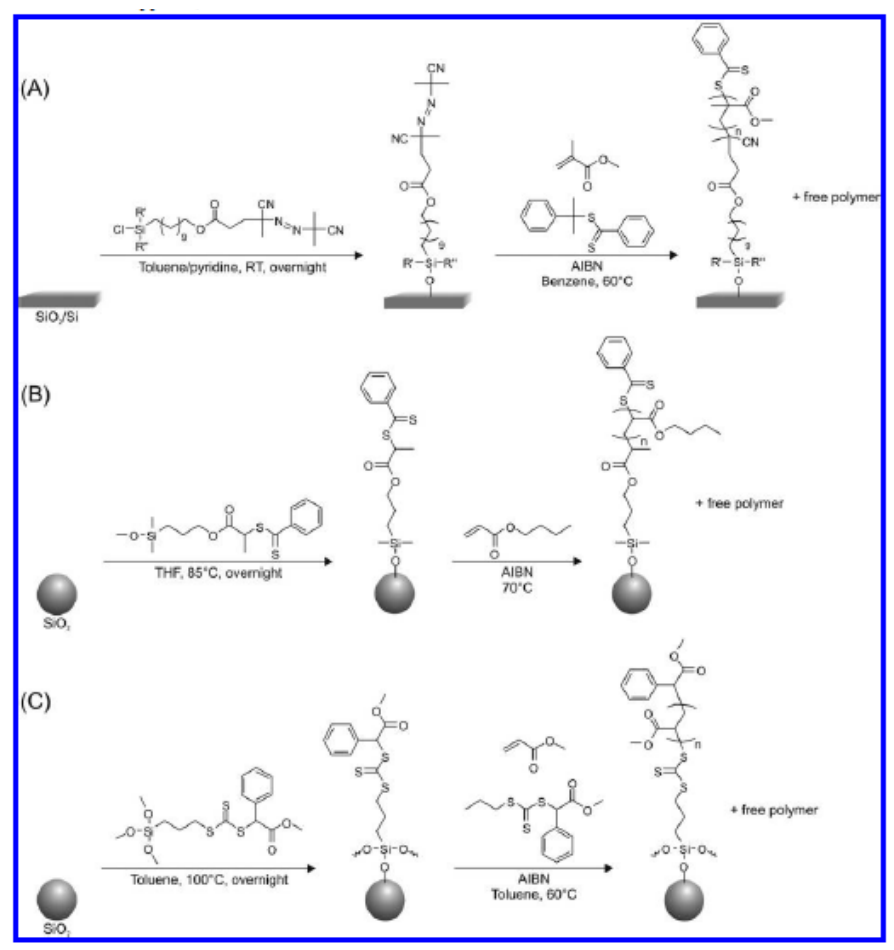

Figure 51 SI-RAFT Polymerization: (A) Bimolecular Process as reported by Baum and Brittain (263) for the Preparation of PMMA Brushes from Azo-Functionalized Silicon Wafers; (B) R-Group Approach To Grow PBA Brushes from Dithiobenzoate Modified Silica Nanoparticles as Described by Li and Benicewicz; (264) (C) Z-Group Approach for the Grafting of PMA Brushes from Silica Particles Supported Trithiocarbonate Derivative. (265)

\subsection{Control of Architecture via SI-CRP}

The utilization of surface-initiated controlled radical polymerization (SI-CRP) such as ATRP, RAFT techniques provides control over not only synthesis parameters (molecular weight distribution, brush thickness) but also variation of the architecture of polymer brushes. The density of surface anchored initiator or raft agent (in the case of RAFT polym) directly affects the configuration and the thickness of the resulting brushes. It was reported that the density of initiating sites strongly influences the growth rate of the chains and the morphology of the resulting polymer film. (279) In this study, gold surface was coated with a mixed monolayer of two thiols where the quantity of each thiol was known and subsequently polymerized with methyl methacrylate and glycidyl methacrylate via SI-ATRP. The key parameter here to vary grafting density of resulting brushes is the fact that only one of these two thiols which has a bromoisobutyrate end-group, is able to initiate polymerization on the surface. Thus, a linear relationship between the initiator density and the thickness of the polymer brush has been established. In another study, the dependence of protein repelling property of poly (oligo (ethylene glycol) methyl methacrylate) brushes to 
film thickness and polymer surface density was demonstrated. (280) Preparation of several architectures such as block, random copolymer brushes, gradient brushes, binary brushes as well as various branched polymer brush is possible via SI-RAFT.

(33) (Figure 52)

(A)
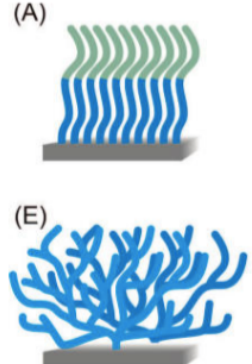

(1)

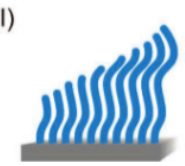

(B)

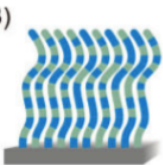

(F)

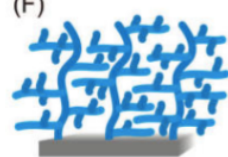

$(J)$

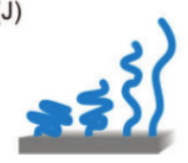

(C)

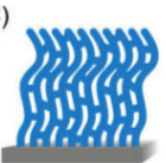

(G)

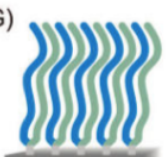

(K)

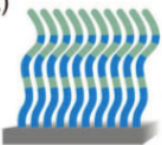

(D)

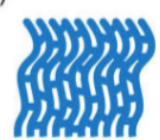

(H)

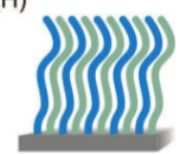

(L)

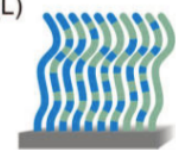

Figure 52 Overview of different polymer brush architectures that can be prepared via surface-initiated controlled radical polymerization. (A) block copolymer brushes; (B) random copolymer brushes; (C) cross-linked polymer brushes; (D) free-standing polymer brushes; (E) hyperbranched polymer brushes; (F) highly branched polymer brushes; (G) Y-shaped binary mixed polymer brushes; $(\mathrm{H})$ standard binary mixed brushes; (I) molecular weight gradient polymer brushes; (J) grafting density gradient polymer brushes; $(\mathrm{K}, \mathrm{L})$ chemical composition gradient polymer brushes. (33)

\subsection{1 Block Copolymer Brushes via SI-CRP}

In order to confirm the livingness of the brushes obtained via SI-CRP, blockcopolymer brushes have been prepared aswell. The first diblock copolymer brush synthesized via SI-CRP was reported by Otsu et al. in 1986. (281) In this work, PS- $b$ PMMA diblock copolymer was prepared on a cleavable photoiniferter immobilized on a PS gel. A variety of block copolymers were synthesized via different SI-CRP techniques. First diblock copolymer brush examples of each SI-CRP techniques have been synthesized by Nakayama and Matsuda from dithiocarbamated PS films via SIPIMP, (282) by Husseman from TEMPO-functionalized silicon substrates via SINMP, (283) by Matyjaszewski from 2-bromoisobutyrate-derivatized silicon wafers via SI-ATRP, (284) by Baum and Brittain from azo-functionalized silicon wafers via SIRAFT. (263) Moreover, the ability of a macroinitiator to reinitiate a homopolymer brush was also investigated. It has been found that the nature of the surface attached macroinitiator and the nature of the monomer used for the subsequent block strongly affect the success of block copolymerization process. It turned out that the preparation of PMMA- $b$-PDMAEMA brushes is difficult as a result of that chains terminated with 
PDMAEMA did not reinitiate polymerization of MMA, the chain ends remained intact and would allow further polymerization of DMAEMA. (285)

Another approach for formation of block copolymers were developed by Baker and coworkers. In this work, two controlled polymerization techniques were combined in order to accomplish desired "bottle-brush" morphology. Poly (hydroxyethyl methacrylate) (PHEMA) was synthesized via ATRP from the bromide functionalized SAMs on gold substrate.(25) (Figure 53) Subsequently, ROP was carried out to grow poly(lactide) brushes using the hydroxyl groups of PHEMA layer as initiators.

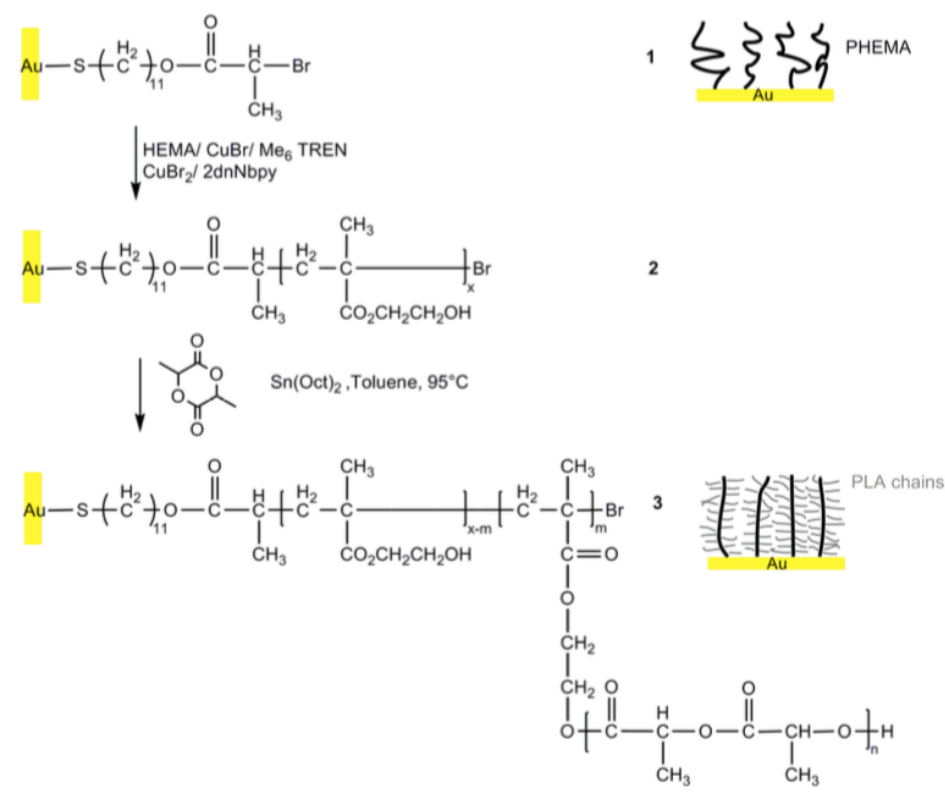

Figure 53 Schematic represantation of bottle brush formation by polymerization of HEMA and LA. (25)

\subsection{SI-CRP from Gold Surfaces}

Gold has been extensively utilized as a substrate to graft polymer brushes via SI-CRP. The gold substrates that have been used for polymer brush growth might be divided into two categories: (i) gold films deposited onto flat substrates such as silicon wafers, glass, and quartz slides etc. (ii) gold nanoparticles. SI-ATRP is the most widely used technique for fabrication of brushes on gold surfaces, whereas only a few reports describe the modification of gold surfaces via SI-PIMP or SI-RAFT. Table 2 gives a summary of the initiators, iniferters, and RAFT agents that have been employed for the modification of both planar gold substrates and gold nanoparticles via SI-CRP.(33)

In the case of ATRP, different type two-step protocols have been used to tether ATRP initiators onto planar gold substrates. In the first step, ATRP initiator-functionalized disulfides or thiols are synthesized and then directly grafted onto gold surfaces. The most common ATRP initiator-functionalized disulfide and thiol are 
$\left(\mathrm{BrC}\left(\mathrm{CH}_{3}\right)_{2} \mathrm{COO}\left(\mathrm{CH}_{2}\right)_{11} \mathrm{~S}\right)_{2}(286)$ and $\mathrm{BrC}\left(\mathrm{CH}_{3}\right)_{2} \mathrm{COO}-\left(\mathrm{CH}_{2}\right)_{11} \mathrm{SH}$ (279) respectively. The second multistep approach consists of the immobilization step of a hydroxylfunctionalized disulfide or thiol SAM on gold surface and subsequent esterification reaction with the ATRP initiator. (287)

A variety of strategies have been developed to modify the surface of gold nanoparticles with functional groups which further allow SI-CRP. Ligand-exchange reaction is the most common way used to modify the surface of gold nanoparticles with functional groups that can initiate or mediate SI-CRP. These exchange reactions take place between stabilizing ligands and ligands functionalized with initiators, iniferters, or RAFT agents and might be divided into two categories: the direct ligandexchange strategy and two step postmodification strategies. For example, in the case of use of dodecanethiol-stabilized gold nanoparticles, this alkanethiol has been replaced with the ATRP initiator-functionalized thiol $\left(\mathrm{BrC}\left(\mathrm{CH}_{3}\right)_{2} \mathrm{COO}\left(\mathrm{CH}_{2}\right)_{11} \mathrm{SH}\right)$ via direct ligand-exchange strategy. (288) Citrate-stabilized gold nanoparticles have also been sucsessfully modified via ligand exchange reaction which relies on the fact that the bond strength between $\mathrm{Au}$ and $\mathrm{S}$ is stronger than that between Au and citrate. In this case, citrates can be exchanged with disulfide-functionalized ATRP initiators such as $\left.\left(\mathrm{BrC}\left(\mathrm{CH}_{3}\right)_{2} \mathrm{COO}\left(\mathrm{CH}_{2}\right)_{x}\right)_{11} \mathrm{~S}\right)_{2}$. (289)- (296)

Gold nanoparticles can also be functionalized with ATRP initiating or RAFT groups via two step postmodification strategies. As an example, gold nanoparticles have been modified with 11-mercapto-1-undecanol and subsequently esterified with 2bromoisobutyryl bromide as initiator for ATRP (297). A third strategy for the modification of gold nanoparticles with functional groups which can mediate SI-CRP is based on the utilization of the ATRP initiators functionalized with thiols or disulfides as ligands. The limitation of this strategy is requirement of mild conditions to avoid cleavage of ester bonds. As an example, coating of gold nanoparticles with an ATRP initiator group was accomplished by the simple one-pot reduction of $\mathrm{HAuCl}_{4} \cdot 4 \mathrm{H}_{2} \mathrm{O}$ with slow addition of sodium borohydrate in the presence of an ATRP initiatorfunctionalized disulfide $\left(\mathrm{BrC}\left(\mathrm{CH}_{3}\right)_{2} \mathrm{COO}-\left(\mathrm{CH}_{2}\right)_{11} \mathrm{~S}\right)_{2}$. (298), (299) Another approach includes the rapid addition of sodium borohydride to an ethyl acetate solution of $\mathrm{HAuCl}_{4} \cdot 4 \mathrm{H}_{2} \mathrm{O}$ and the ATRP initiator-functionalized disulfide $\left(\mathrm{BrC}\left(\mathrm{CH}_{3}\right)_{2} \mathrm{COO}\left(\mathrm{CH}_{2}\right)_{6} \mathrm{~S}\right)_{2} \cdot(300)$

Table 2 Overview of initiators, iniferters, and Raft agents that have been used to grow polymer brushes from gold surfaces 


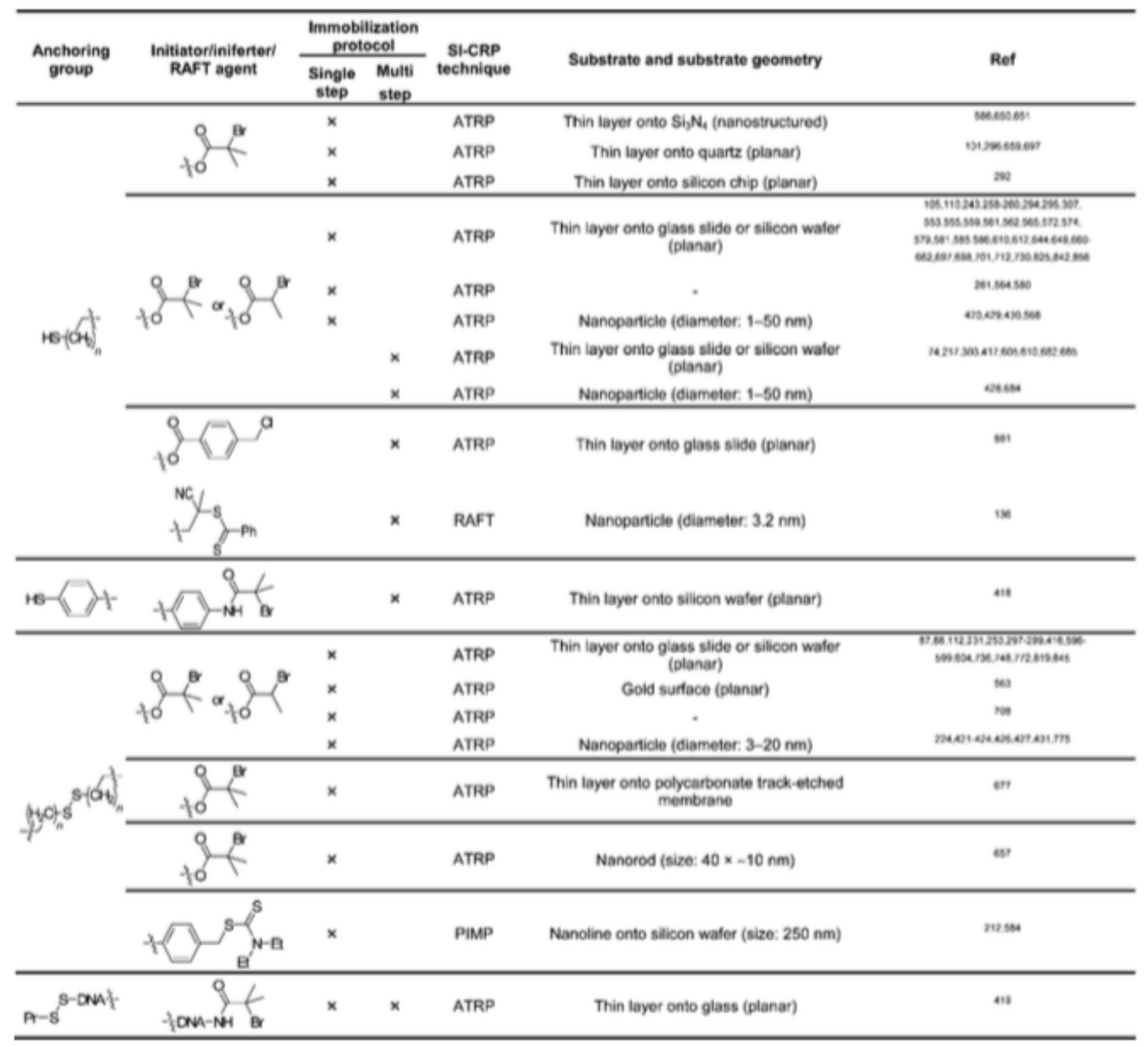

\subsection{1 SI-RAFT from Gold Planar Surfaces}

In 2003, Summerlin and coworkers reported the immobilization of poly (sodium 4styrenesulfonate), poly((ar-vinylbenzyl) trimethylammonium chloride), $\operatorname{poly}(N, N$ dimethyl- acrylamide), and poly(3-[2-( $N$-methylacrylamido)-ethyldimethyl ammonio]propane sulfonate- $b-N, N$ - dimethylacrylamide) onto gold planar surfaces via in situ reduction of RAFT-prepared (co)polymers. (301) (Figure 54) By virtue of the RAFT mechanism, (co)polymers prepared by this technique bear dithioester end groups. The reduction of these dithioesters to thiols in the presence of a suitable transition metal complex leads to the formation of (co)polymer-stabilized metal surfaces. The presence of the immobilized (co)polymers was confirmed by atomic force microscopy, attenuated total reflectance fourier transform infrared spectroscopy, and surface contact angle measurements.

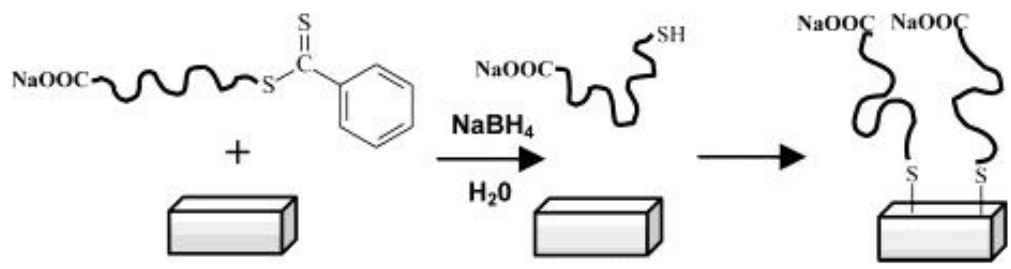


Figure 54 Basic mechanism describing the in situ reduction and immobilization of a RAFT-prepared (co)polymer on a gold surface. (301)

In 2008, Peng and coworkers used a complex multistep approach to perform RAFT polymerization on gold surfaces via grafting from approach. (302) (Figure 55) In this work, the disulfide bonds at the 3 '-ends of the oligonucleotide probes were reduced to generate free thiol groups for surface immobilization. (303), (304)

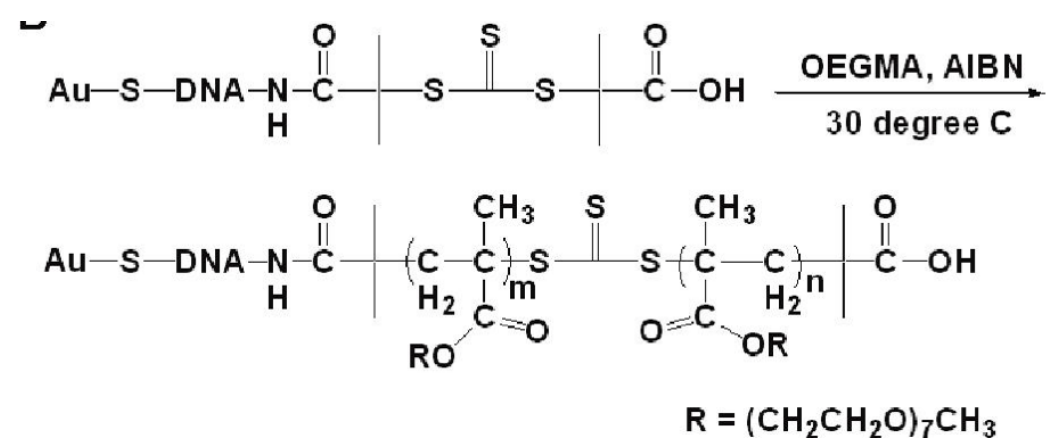

Figure 55 (a) Schematic Illustration of RAFT-Based DNA Detection and (b) the Chemical Reaction of RAFT Polymerization Grafting on DNA-Immobilized Surfaces. (302)

Another similar multi-step study involving modification of gold surfaces with RAFT prepared-DNA-polymer bioconjugates via grafting from technique was reported in 2009 by He Peng and He Lin. (305) (Figure 56) In this work, in order to examine the formation of polymer bioconjugates on a solid support, single-stranded DNA molecules (ssDNAs) were used as the model molecule to study surface-anchored polymer growth. The DNA molecules contained dual functional groups were allowed CTA coupling to the 5'-end amino group and gold surface immobilization with the 3 'end thiol group. First of all, CTA was coupled with NHS. This NHS-coupled CTA was mixed with the amino-terminated DNA molecules at a 1:1 stoichmetric ratio in the presence of EDC / NHS. Formation of CTA-coupled DNA molecules was confirmed by MALDI-MS. Freshly reduced CTA-coupled ssDNAs were immobilized onto gold substrate and polymerized via Raft polymerization technique. The thickness of resulting POEGMA film was $212 \mathrm{~nm}$. 


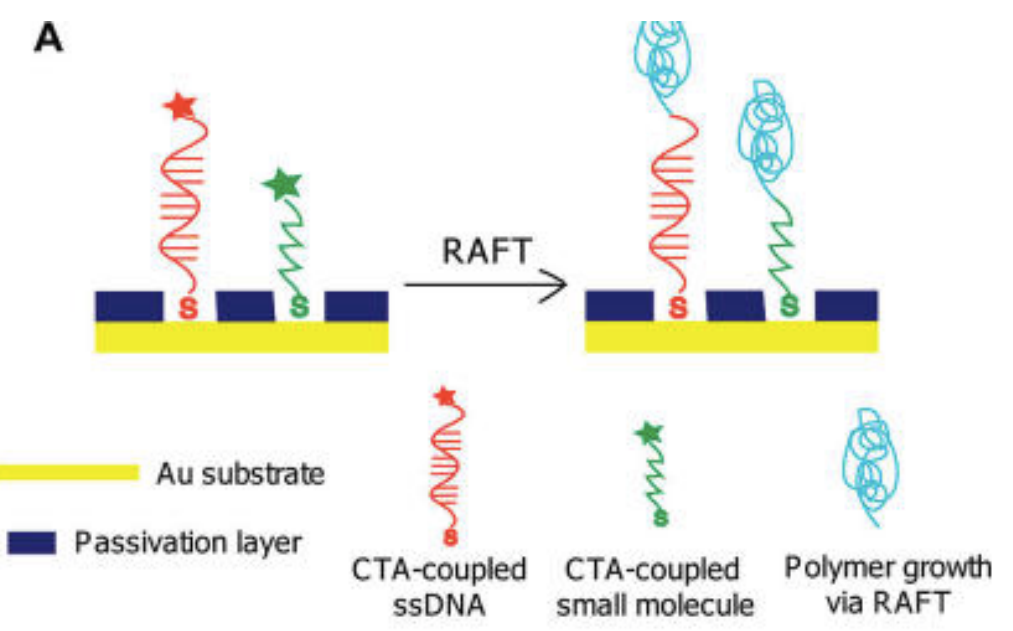

B

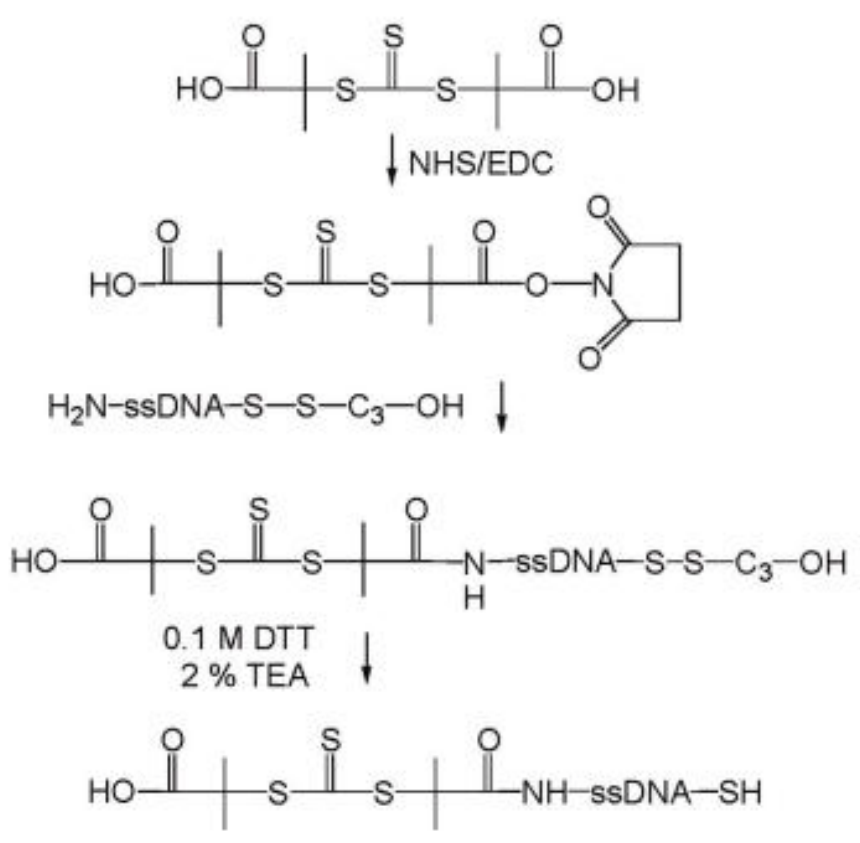

Figure 56 (A) DNA-polymer conjugates grafted on the surface using RAFT polymerization; (B) Chemical steps in preparation of CTA-coupled ssDNA molecules. (305)

In 2010, Advincula and coworkers reported a novel and versatile method of grafting protein and cell-resistant poly (poly ethylene glycol methyl ether methacrylate) (PPEGMEMA) brushes on gold surface. (306) (Figure 57) In this work, gold surface was covered with an electro-active chain transfer agent (CTA) via the electrochemical deposition technique. The electrochemical behavior of the CTA was investigated by cyclic voltammetry (CV) while the deposition and stability of the CTA on the surface were confirmed by ellipsometry, contact angle, and X-ray photoelectron spectroscopy 
(XPS). The capability of the electrodeposited CTA to mediate surface-initiated reversible addition-fragmentation chain transfer (SI-RAFT) polymerization on both the polymethyl methacrylate (PMMA; model polymer) and PPEGMEMA brushes was demonstrated by the increase in thicknesses of the films after polymerization ( 99 and $26 \mathrm{~nm}$ respectively). The formation of polymer layers were investigated via Contact angle, XPS and atomic force microscopy (AFM) analyses aswell. Furthermore, the ability of the PPEGMEMA-modified Au surface to resist nonspecific adhesion of proteins and cells was monitored and confirmed by XPS, ellipsometry, contact angle, AFM, and fluorescence imaging.

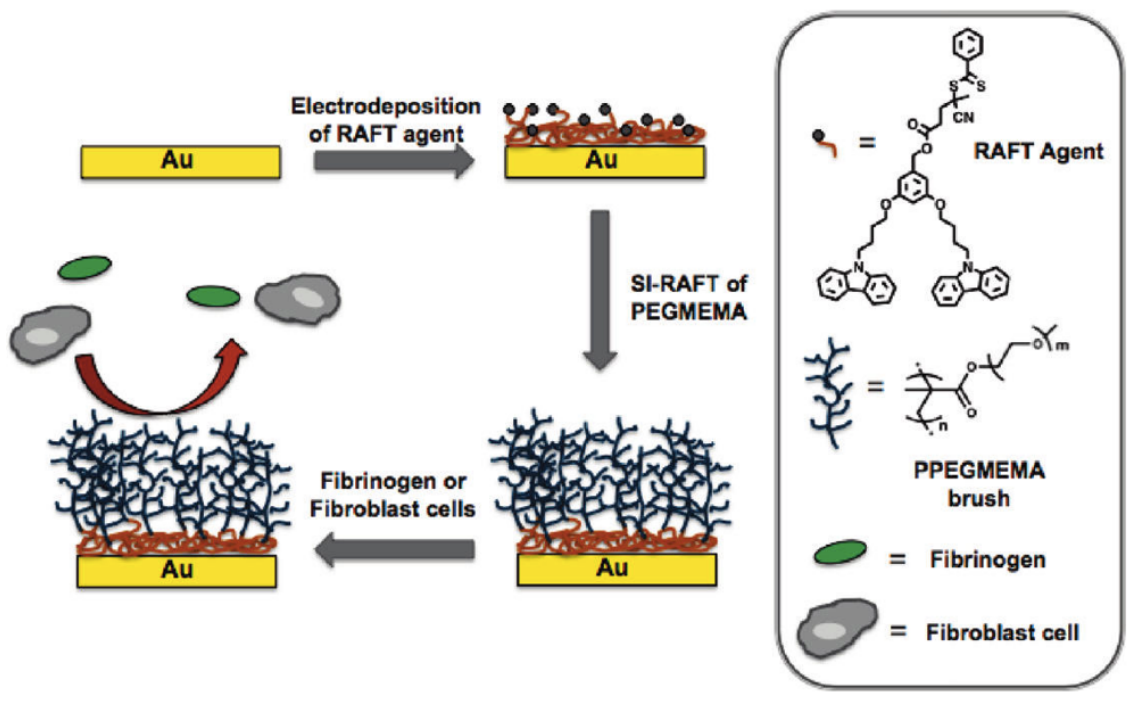

Figure 57 General scheme for the preparation of protein and cell-resistant PPEGMEMA brushes from the electrodeposited RAFT agent. (306)

In 2012, Caykara and coworkers demonstrated the successful synthesis of poly [(oligoethylene glycol) methyl ether acrylate] [poly(OEGA)] brushes on gold planar surfaces via surface initiated reversible addition-fragmentation chain transfer (RAFT) polymerization and the selective immobilization of streptavidin proteins. (307) (Figure 58) In this method, gold surfaces were modified with 11-mercapto-1undecanol as first layer and subsequent coupling with a trithiocarbonate-based RAFT chain transfer agent (CTA) by using an ester reaction. Poly(OEGA) brushes were then prepared via RAFT-mediated polymerization from the surface-immobilized CTA. The immobilization of CTA on the gold surface and the subsequent polymer formation were investigated by ellipsometry, X-ray photoelectron spectroscopy, grazing angleFourier transform infrared spectroscopy, atomic force microscopy, and water contactangle measurements. Main advantage of utilization of RAFT-mediated polymerization method is the convertion possibility of terminal CTA groups of polymer brushes to various biofunctional groups. The terminal carboxylic acid groups of poly(OEGA) chains were functionalized with amine-functionalized biotin units to provide selective attachment points for streptavidin proteins. Fluorescence microscopy measurements confirmed the successful immobilization of streptavidin molecules on the polymer 
brushes. It is demonstrated that this fabrication method may be successfully applied for specific protein recognition and immobilization.

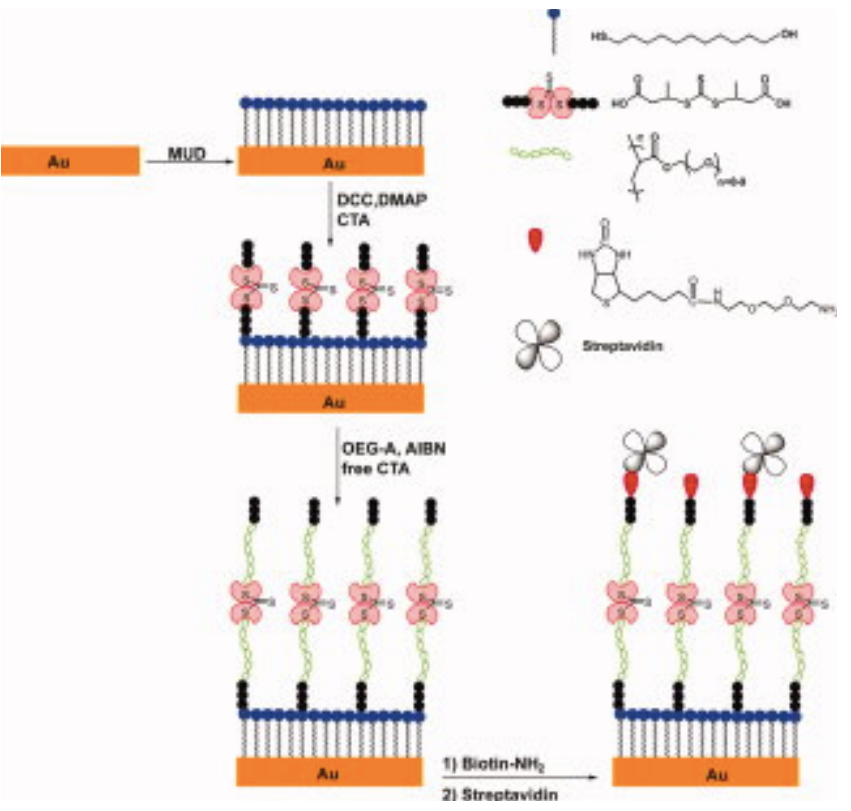

Figure 58: Stepwise fabrication process for creating streptavidin-immobilized poly(OEGA) brushes. (307)

In 2016, Wiarachai and co-workers reported a work including modification of gold surface via "grafting to" approach with a functional copolymer platform, namely, poly [(propargyl methacrylate)-ran-(2-methacryloyloxyethyl phosphoryl- choline)] (PPgMAMPC) prepared by reversible addition- fragmentation chain-transfer polymerization. (308) (Figure 59) Propargyl methacrylate (PgMA) was preffered for its alkyne moiety which serves as an active site for binding azide-containing molecules via a click reaction (i.e., Cu-catalyzed azide/alkyne cycloaddition (CuAAC)) whereas 2-methacry-loyloxyethyl phosphorylcholine (MPC) was employed for its hydrophilic nature which enables the resulting copolymer to suppress nonspecific adsorption. The copolymers were characterized using Fourier transform infrared (FTIR) and ${ }^{1} \mathrm{H}$ NMR spectroscopies. Thiol-terminated Raft polymers (PPgMAMPC-SH) were obtained by aminolysis and were immobilized on a goldcoated substrate using a "grafting to" approach via self-assembly. Azide-containing species, namely, biotin and peptide nucleic acid (PNA), were then immobilized on the alkyne-containing copolymeric platform via $\mathrm{CuAAC}$. The potential use of surfaceattached PPgMAMPC in biosensing applications was shown by detection of specific target molecules (i.e., streptavidin (SA) and DNA) by the developed sensing platform using a surface plasmon resonance technique. 

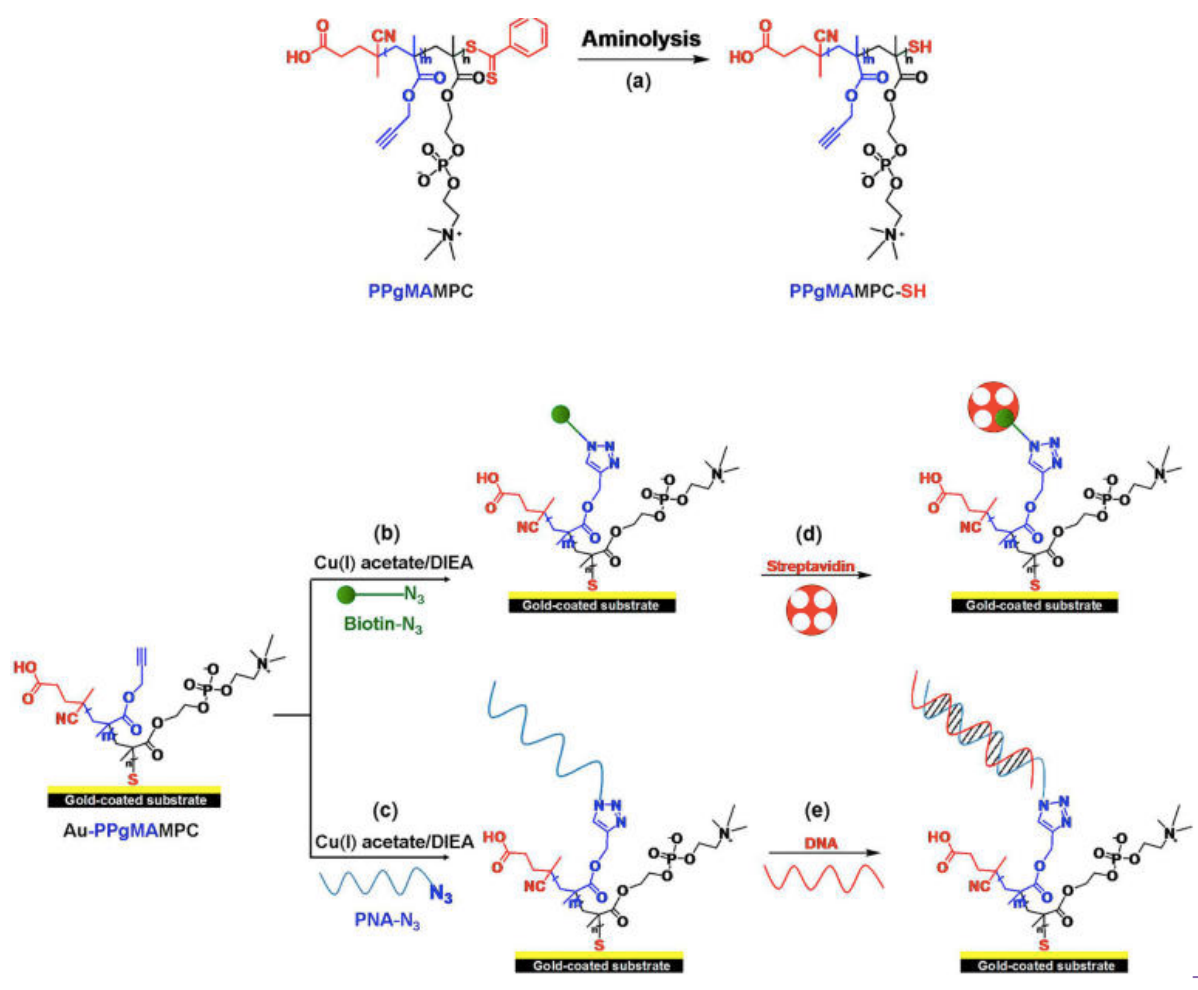

Figure 59: Schematic diagram of (a) preparation of thiol terminated polymer (PPgMAMPC-SH), (b,c) immobilization of Biotin- $\mathrm{N}_{3}$ and PNA-N 3 on PPgMAMPCmodified gold surface, and $(\mathrm{d}, \mathrm{e})$ specific binding between conjugated probe and target molecule. (308)

\subsubsection{Properties and Applications of Polymer Brushes}

In order to develop new applications, surface properties can be altered by tuning the synthesis parameters including grafting density, the chemical composition, the chemical nature of the monomer or the substrate. Some examples of polymer brushes produced for spesific applications are summarized below. (Figure 60)
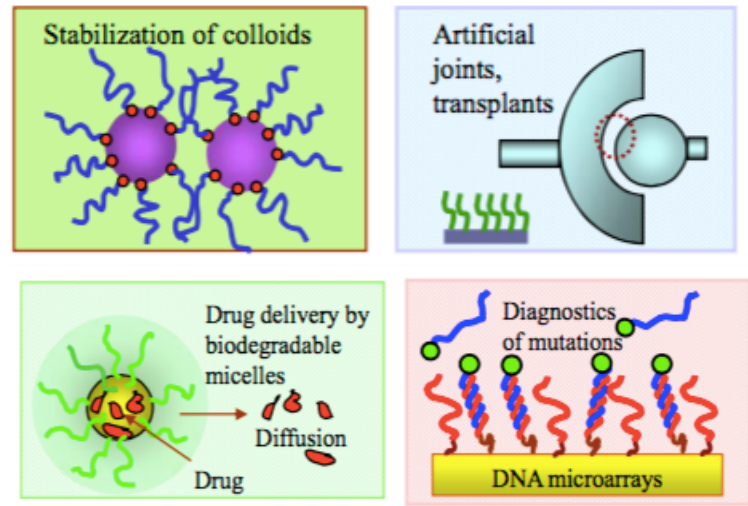

Figure 60 Some examples for applications of polymer brushes. (309) 


\subsubsection{Stimuli Responsive Polymer Brushes}

\subsection{General Features}

Different applications in the field of biomaterials and engineering materials require the design and development of "smart systems" which exhibit environmentally responsive behavior. The increasing demand has generated a large interest for synthetic polymers that can respond with conformational and property changes when being exposured to external stimuli such as temperature-, $\mathrm{pH}$-, ionic strength-, light-, electric- and magnetic field (Figure 61). Stimuli-responsive polymers can be prepared for various applications against a variety of external stimuli by tuning the chemical composition and the architecture such as homo-polymers, statistical / block copolymers, graft copolymers and molecular brushes. In the case of stimuli responsive brushes, they can also be grafted on/from surfaces, or be used as chemically or physically cross-linked gels. (310)

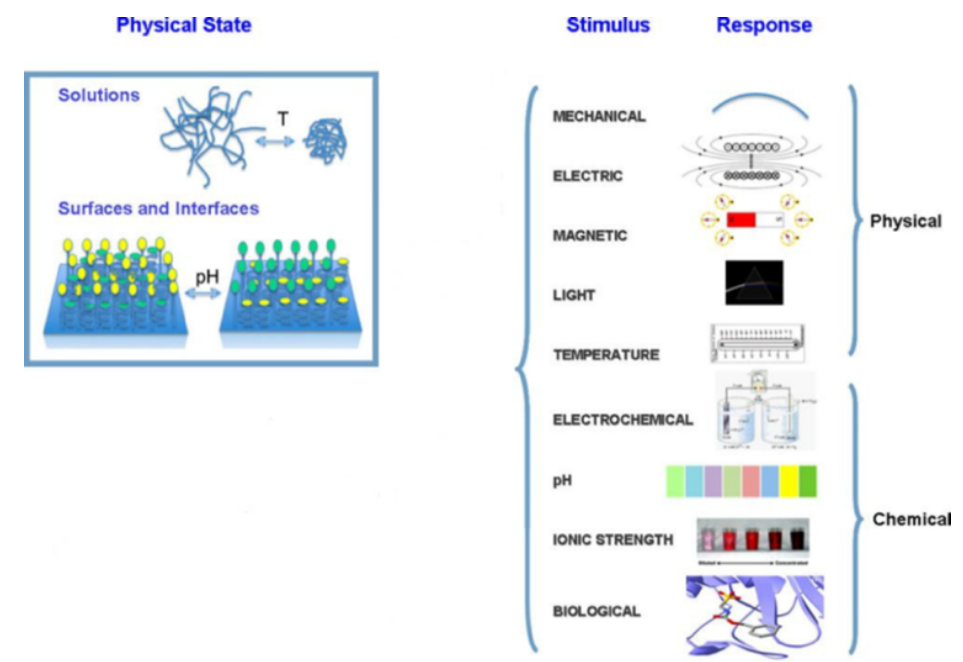

Figure 61 Schematic representation of physical and chemical stimuli in solution, and surfaces \& interfaces state. (311)

In solutions, the conformation of brush molecules is controlled by the excluded volume repulsion. On the other side, the conformation of adsorbed molecules depends on the interaction of the side chains with the substrate and changes in response to variation of the substrate surface energy. (41) In terms of the function of the stimuli that polymer brushes can develop, they can be utilized for a range of applications such as surface wettability (312), chemical sensing (313), drug release (314), cell growth (315) etc. 


\subsection{Solvent Responsive Polymer Brushes}

The interaction between polymer brush chains and the solvent strongly affects the conformation of brushes. Thus, resulting conformation is highly dependent on the solvent quality. In the presence of a good solvent, the polymer chains maximize the polymer/solvent interactions, leads to an extent configuration and swell. In case of that polymer brushes are exposured to a poor solvent, they tend to minimize polymer/solvent interaction and therefore they will collapse.

\subsection{Solvent Responsive Homopolymer Brushes}

Chen et al. prepared patterned PMMA brush on silicon substrate, and investigated the behavior of the homopolymer brushes in two different type solvents via AFM and ellipsometry. One poor (water) and one good (THF) solvent for the PMMA brushes, were chosen for measurements. (316) In water, a decrease in the thickness and the roughness of the layer was observed, which was an indication of the collapse of the brush whereas it adopts an extended conformation in case of being exposured to THF. The behavior of polymer brushes in the presence of different types of solvents were also investigated. These solvents are isopropanol/ethyl acetate mixture (317), (318) benzene/acetonitrile, (319) etc. and characterized by micromechanical cantilever (317), (318) or fluorescence depolarization. (319)

Aoki et al. investigated the dynamic swelling properties of PMMA brushes in benzene (a good solvent) and acetonitrile (a bad solvent). (319) It was found that the thickness of the polymer layer was around two times lower in acetonitrile (poor solvent) than in benzene (good solvent) where the motion of the polymer chains was faster. The influence of brush density on the swelling properties were also investigated. A fast response to solvent-exchange was observed for chains of low density polymer brushes which are able to easily change their conformation. In the case of high density brushes, it was observed that the chains were almost nonresponsive to solventexchange due to the fact that the polymer chains interact strongly with each other and adopt a stretched conformation, even in a poor solvent.

\subsection{Solvent Responsive Binary Mixed Homopolymer Brushes}

The solvent responsiveness of binary mixed homopolymer brushes is a more complex system in comparison to that of the simple homopolymer brush. Exposure to a specific solvent triggers a selective swelling behavior of one of the components of mixed brush system whereas leading to a simultaneous collapse of the other polymer chains, which results in a phase separation and the formation of nanoscale surface patterns. (Figure 62) 


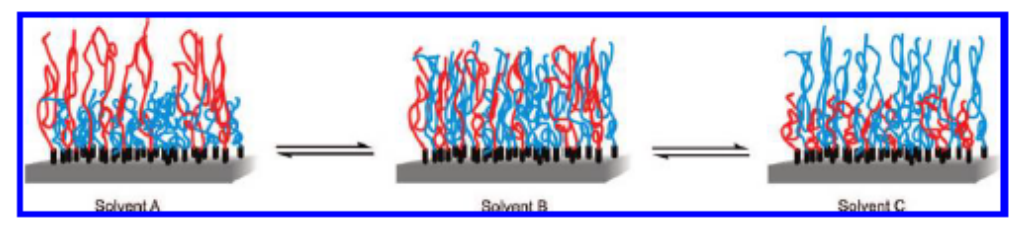

Figure 62 Solvent responsiveness of a binary mixed homopolymer brush: solvent $\mathrm{B}$ is a nonselective solvent, whereas solvents $\mathrm{A}$ and $\mathrm{C}$ are selective for the red and blue segments, respectively. (33)

It was found out that the brushes tend to minimize the interactions with the solvent and adopt the collapsed state in the presence of non-selective solvent. (Figure 63 (a)) (246) In the case of selective solvents, one of the polymers preferentially segregates from the top of the brush, whereas the other polymer forms clusters segregated onto the surface. (Figure 63 (b), (c))

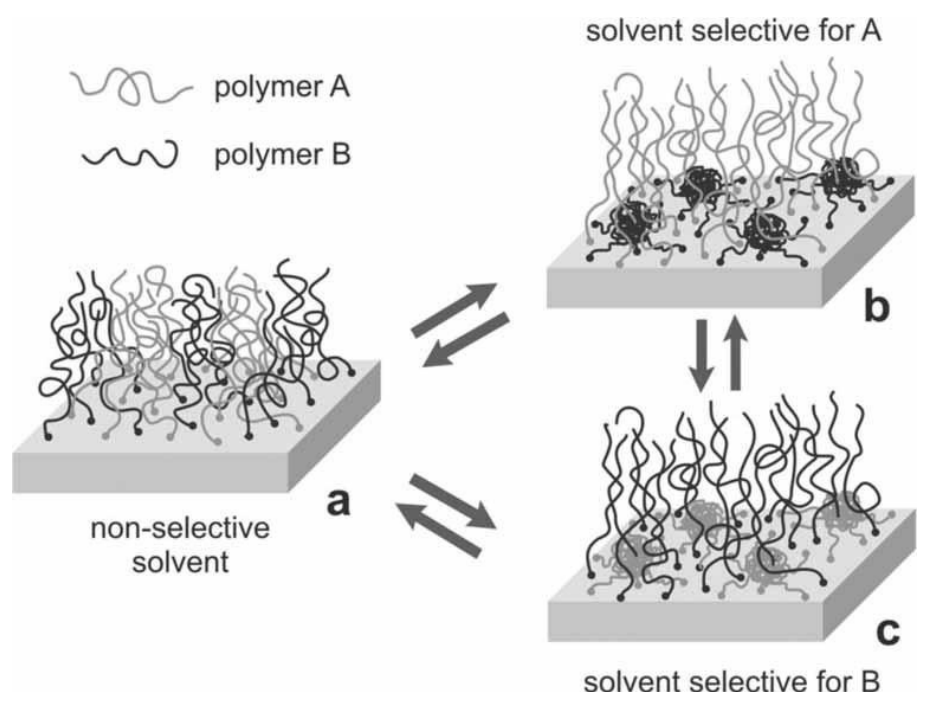

Figure 63 Solvent-responsive behavior of binary mixed homopolymer brushes. (41)

An example for solvent-responsive behavior of brushes is the study of mixed PMMA/PS brushes grown from initiator anchored flat silicon wafer via SIP. (320) A series of mixed brushes which are combinations of a constant PMMA number-average molecular weight and a variety of different PS number-average molecular weights, were exposured to chloroform (good solvent for both brushes which is a non-selective solvent) and cyclohexane (good solvent for PS above $35^{\circ}$ whereas bad for PMMA, which represents a selective solvent). AFM analysis revealed smooth surface with root mean square of $0.5 \mathrm{~nm}$ for all samples. Water contact angle measurements of mixed polymer brushes being exposed to chloroform, exhibited a gradual transition from the value of pure PMMA to the value for pure PS, with increasing PS molecular weight. When the PS chain was much shorter than that of PMMA, water contact angle value of the sample after treatment in cyclohexane at $45^{\circ} \mathrm{C}$ is the same with that of pure PMMA. When the molecular weight of the PS is slightly smaller than that of PMMA, 
the water contact angle exhibits the same value with that of pure PS due to the fact that PS brushes were swollen and disposed on the outermost layer. Another result of this brush configuration is a slight increase in the roughness. Besides Zhao, Santer et al. have also studied solvent-induced topographical changes in PS/PMMA binary brushes. (321), (322) Another study of Zhao and co-workers on solvent responsiveness of binary mixed polymers involves growth of mixed PAA/PS brushes from a "Y-shaped" initiator anchored silica nanoparticles. (323) In this study, the ability of the particle surface-tethered mixed binary polymer chains to undergo structural changes in response to changes in solvent quality has been revealed via Tyndall scattering and ${ }^{1} \mathrm{H}$ NMR spectroscopy measurements. It was observed that the mixed binary brush-coated particles could be dispersed both in chloroform (a PS selective solvent) and in methanol (a PAA selective solvent).

\subsection{Solvent Responsive Block Copolymer Brushes}

The solvent responsiveness of block copolymer brushes is different from that of homopolymer and mixed homopolymer brushes. (Figure 64) In the presence of nonselective solvent (B) which is a good solvent for both blocks, both segments of brush swell and lead to full extension of the system. In case of being exposured to a selective solvent (A), which is a good solvent for the blue part of the brush but a poor solvent for the red one, the blue segment swells whereas the red block collapses. Since these collapsed chains have a tendency to minimize as much as possible its contact with the solvent, they might penetrate into the other blue segment (depending on the nature of blue part and the interaction parameter between the two blocks).

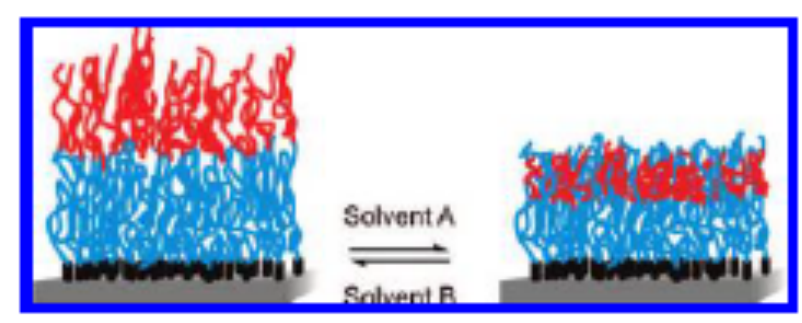

Figure 64 Structural changes in a diblock copolymer brush upon variations in solvent quality; solvent B is a good solvent for both blocks, while solvent A is a good solvent for the blue block but a nonsolvent for the red block. (33)

The solvent responsive behavior of various diblock copolymer brushes such as (PS$b$-PPFS, PS- $b$ - PHDFDA, PS- $b$-PPFEA, PS- $b$-PPFPA, PMA- $b$-PPFS, PMA- $b$ PDHFDA, PMA- $b$-PPFEA, and PMA- $b$-PPFPA) (324) and PS- $b$-PAA, PS- $b$ PNIPAM, and PMA- $b$-PDMAEA was investigated. (325) In these studies, the brushes were first exposed to a non selective solvent (good solvent for both blocks) and then to a selective solvent which is a poor solvent for the outer segment whereas a good solvent for the inner block. The contact angle of the brush after this second step was close to the value expected for the inner block, which is an indication of both the 
swelling behavior of the inner block and a strong collapse of the outer one. These observations were confirmed by XPS measurements, which demonstrated a change in the surface atomic composition upon the solvent treatment. Another study investigated the wetting properties of three groups of PBMA- $b$-PDMAEMA brushes which are combinations of a fixed molecular weight PBMA inner block and a variety of different molecular weights PDMAEMA outer blocks. (326) The block copolymer brushes were exposed to hexane and water, respectively. Water contact angle measurements revealed three different response regimes. When the PDMAEMA block was shorter than PBMA, the PBMA segment dominated the surface after hexane treatment. In the partial response regime, the PDMAEMA and PBMA blocks coexisted at the air interface. It was found that further gradual increase in the PDMAEMA block length reduced the rearrangement of the PBMA blocks after hexane treatment.

Gao et al. investigated nanoscale patterns formed via solvent responsive behavior of PPEGMA- $b$-PMMA diblock copolymer brushes. (327) These brushes which consisted of an inner PPEGMA block with a thickness of $23.4 \mathrm{~nm}$ and an outer PMMA blocks with various thicknesses (ranging from 1.6 to $31.0 \mathrm{~nm}$ ) were prepared on a silicon wafer via SI-ATRP. The formation of nanoscale patterns in these brushes was characterized via AFM, ellipsometry, and water contact angle measurements. Resulting phase segregation regimes differ from eachother since these patterns are highly dependent on the PMMA block length. Spherical PMMA domains were observed when the PMMA layer thicknesses are around $4 \mathrm{~nm}$. The size of these spherical features increased with increasing PMMA block length until the point which they come into contact and merge into "wormlike" structures (at PMMA layer thicknesses of $10.5 \mathrm{~nm}$ ). Further increase in the PMMA layer thickness led to the formation of striped patterns as a result of the fact that the PMMA chains minimized the contact with the solvent but could not go inside the PPEGMA layer because of relatively long ethylene glycol side chains in this block. Other block copolymer types whose topographical switching properties were investigated, are PMMA- $b$-PGMA (328), (329) and PMMA/PHEMA gradient copolymer brushes. (330)

In addition to diblock copolymer brushes, the swelling behavior of triblock copolymer brushes such as PS $b$-PMMA- $b$-PS, PMMA- $b$-PS- $b$-PMMA (331) and PMMA- $b$-PDMAEMA- $b$-PMMA, PMA- $b$-PMMA- $b$-PHEMA (332) were examined aswell. In these studies, brushes were exposed to a solvent that was a good solvent for the middle block but a poor solvent for the bottom and outer blocks. For both systems, a conformational rearrangement including migration of the nonsoluble blocks inside the brush and the extension of the soluble block to the outer surface was observed via the contact angle measurements. AFM demonstrated an increase in roughness upon the solvent treatment, indicating the formation of micellar structures which stem from the migration of the outer blocks inside the layer. Moreover, changes in the surface atomic concentration was examined via XPS measurements. Gas sensors which were made of carbon nanotubes coated with poly (butyl acrylate) or poly (acrylic acid) brushes is a good example of an application of solvent responsive homopolymer 
brushes. (313) The electrical resistance of the polymer brush-coated carbon nanotubes increased when being exposed to organic vapors such as acetone, chloroform, methanol, or toluene. A correlation between the maximum response values of the polymer brush-modified nanotubes toward organic vapors and the solubility of the pure polymers in the respective solvents were found out.

\subsubsection{3 pH Responsive Polymer Brushes}

Polyelectrolytes (PELs) are macromolecules with ionizable subunits which dissociate to give charged macroions and counterions when dissolved in a polar solvent. Proteins and DNA are some examples of biopolymer PELs. PELs have attracted increasing interest in recent years because of their importance in materials science, and molecular biology steming from their relevance to many biological systems. (333) In the case of charged macromolecules, interactions are strongly modified by Coulomb forces. The charge density on a polymer chain in a polar solvent depends on the chain constitution and degree of dissociation ( $f$ ) of ionizable groups. With respect to their different dissociation behaviors, PELs can be classified as strong or weak. (334) If ionizable groups are strong acids or bases (strong PEL), $\mathrm{f}$ is equal to 1 and is not affected by the environment. In another words, variation of $\mathrm{pH}$ or ionic strength will not influence the number of charges in the case of strong polyelectrolyte brushes, where the number and position of charges along the chain is fixed. If ionizable groups are weak acids or bases (weak PEL), f depends on local $\mathrm{pH}$. For the latter case charges are mobile within the polymer chain. The charge density can be tuned by changing the $\mathrm{pH}$ or the ion concentration of the medium. (33)

For the dense strong PEL brush (high $\mathrm{f}$ and grafting density) all counterions are trapped inside the brush (Figure 65 (b)). The brush height is determined by the balance between osmotic pressure of the trapped counter-ions and the stretching entropy of the chains (so called osmotic brush regime). The contribution of the excluded volume effect depends on the grafting density. At very high densities the excluded volume effect may dominate while at moderate densities the electrostatic nature will have a major contribution. The latter will be reflected in the prefactor in the scaling relationship $\mathrm{h}, \mathrm{N}$. These brushes are insensitive to local $\mathrm{pH}$. Added salt does not affect the brush unless the ionic strength of the solution approaches the level of the ionic strength inside the brush (Figure 65 (d)). In that case the prefactor is an inverse cubic root function of the external salt concentration and the grafting density (so called salted brush regime). (334), (335) Thus, in terms of responsive applications strong PEL are interesting for design of responsiveness to humid and aqueous environments when the high swelling of the brush in water or a humid atmosphere is resulted from strong osmotic pressure of trapped counterions. (Figure 65 (a), (b)) 


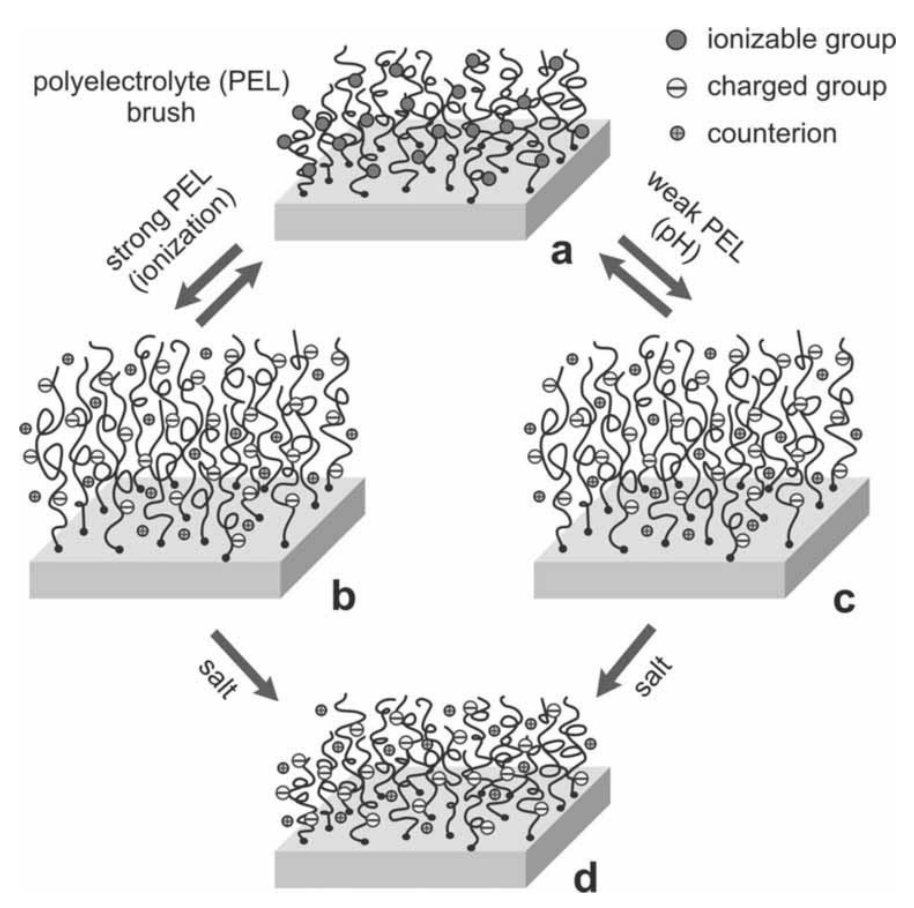

Figure 65 Planar polyelectrolyte (PEL) brush with ionizable groups (a) swells when the groups dissociate a polar solvent (b); the dissociation is $\mathrm{pH}$ dependent for weak polyelectrolytes (c); addition of salt leads to the shrinkage of the PEL brush. (41)

Weak PEL brushes represent one of the most interesting responsive behaviors. They demonstrate responsiveness to changes in external $\mathrm{pH}$ and ionic strength. Weak PEL brushes carrying basic functionalities expand upon a decrease of $\mathrm{pH}$, while acidic PEL brushes expand upon an increase of $\mathrm{pH}$ (Figure 65 (c), (d)). At a high salt concentration weak PEL brushes shrink due to the same mechanism as strong PEL brushes. However, it is noteworthy, that in some range of $\mathrm{pH}$ values they shrink also at no salt added or at very small salt concentrations, thus, expressing non-monotonous dependence of the brush height vs. salt concentration. This behavior originates from the sensitivity of $\mathrm{f}$ for weak PEL(s) to the local electric field. (336) At a low ionic strength electrostatic interactions result in the condensation of counterions in the brush (the neutral polymer brush regime). If ionic strength is increased the screening of the electrostatic interactions affects an increase of $f$ and brings the system in the osmotic regime. The graph of the brush thickness vs. salt concentration has a maximum. (337) In mixed brushes (Figure 66) two or more different polymers grafted to the same substrate constitutes the brush. The mechanism of responsiveness of the mixed brushes is very different from homopolymer brushes. Unlike polymers in the mixed brush segregate into nanoscopic phases. The size of the nanophases scales with $\mathrm{kr}^{2}{ }^{1 / 2}$ value. 


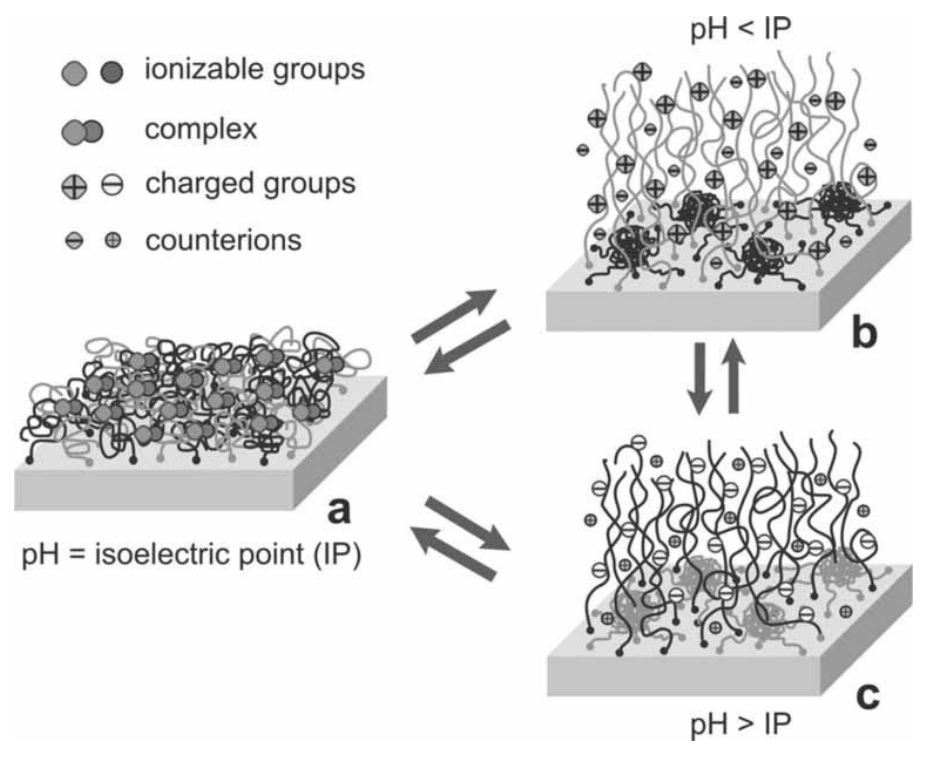

Figure 66 Mixed two-component brush: lateral phase segregation of chains in nonselective solvent (a), and combined lateral and layered segregation in a selective solvent (b, c). (41)

The phase segregation is a lateral segregation in a nonselective solvent when unlike polymers form spherical or elongated clusters. Both the polymers are exposed on the top of the brush. (Figure 66 (a)) In selective solvents the mixed brush structure may be seen as a combination of lateral and layered segregation mechanisms. (338) In the latter case one polymer preferen- tially segregates to the top of the brush, while another polymer forms clusters segregated to the grafting surface. (Figure 66 (b), (c)) The most important difference of the mixed brush as compared to the homopolymer brush is that not only the height and density profile but also the composition profile depends on solvent quality. In other words, the surface composition of the brush is switched by a change in its environment. If two unlike polymers are of the same chemical constitution but they differ by molecular weight the brush stratifies. (339), (341) The polymer of the largest molecular weight will occupy the top of the brush. If two unlike polymers are of different chemical compositions the lateral and layered segregation takes place. A combination of both kinds of heterogeneity (by chemical composition and molecular weight) results in the more complex phase diagram when the whole range of parameters of the system can be used to design the brush responsiveness. (342) The further modification of the responsiveness can be obtained for mixed PEL brushes. (Figure 67) In this case electrostatic interactions are used to regulate the mechanism of the phase segregation. Weak mixed PEL brushes are of special interest. At isoelectric points they form a neutral PEL complex. (Figure 67 (a)), while outside the isolectric point one PEL dominates in the topmost layer and affects the elec- trical charge of the brush (Figure 67 (b), (c)). Thus, the surface composition of the mixed PEL brush can be switched just by a change of external pH. (343), (345) 


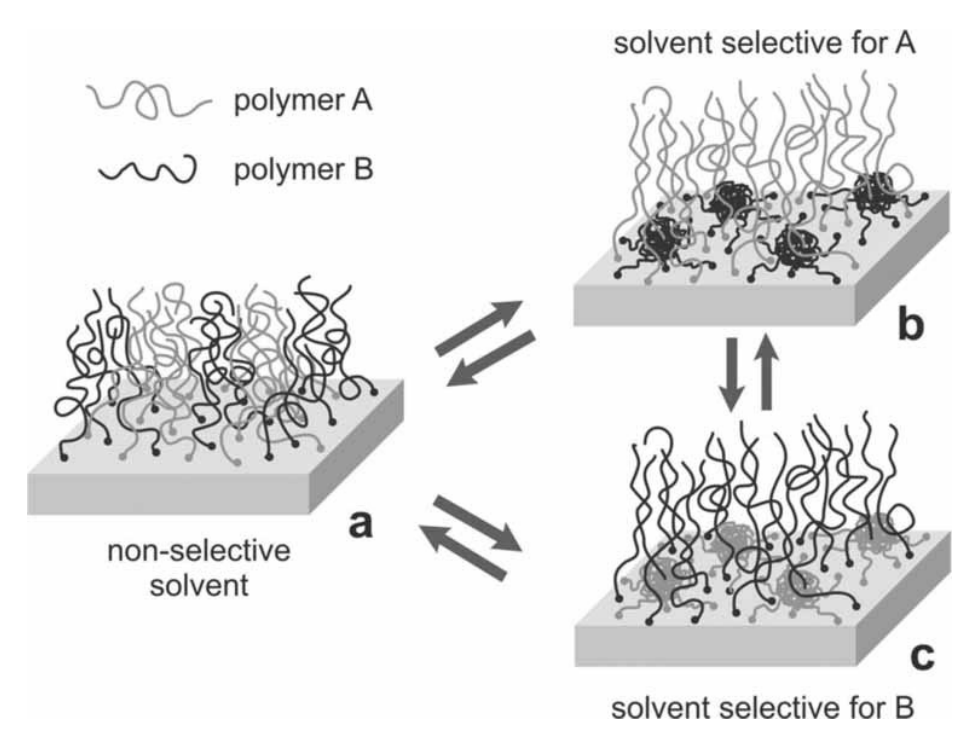

Figure 67 Mixed weak polyelectrolyte brush forms a neutral complex in the isoelectric point (a); in acidic or basic solutions positively or negatively charged polymers, respectively, are stretched while a counterpart chains are collapsed and segregated to the grafting surface $(b, c)$. (41)

In the case of blockcopolymer brushes, two or more chemically different polymers constitute a polymer brush with block-copolymer architecture. The responsiveness of these brushes is determined by phase segregation of unlike polymers, however, the mechanism depends on whether the AB block copolymer is tethered by the more (A) or the less (B) soluble block. (346), (347) In poor solvents the brushes grafted by the B blocks form dumbbell-like micelles with the B-polymer segregated to the grafting surface and the A-polymer micelles on the top (Figure $\mathbf{6 8}(\mathrm{b})$ ). The brushes grafted by the A blocks form B-polymer micelles shielded by A-polymers (Figure 68 (a)). If the solvent quality is improved (increased selectivity of solvent) for the A-polymers the dumbbell-like micelles of the B-grafted brushes are transformed to the structures which are similar to the mixed brushes in selective solvents. The B-polymers form pinned micelles segregated to the grafted surfaces and shielded by the brush of the Apolymers. In general, the block-copolymer brushes have a complex phase diagram affected by the ratio between the molecular weights of the A and B blocks and the grafting density. The major difference between mixed and block-copolymer brushes can be found for the case of the A-grafted brushes in a selective solvent for the Ablocks. The mixed brushes of the $\mathrm{A}$ and $\mathrm{B}$ polymers form pinned B-micelles segregated to the grafting surface. The AB block- copolymer brushes form B-micelles located at the top of the brush (Figure 68 (a)). Consequently, block-copolymer brushes introduce unique mechanisms of the phase segregation. 


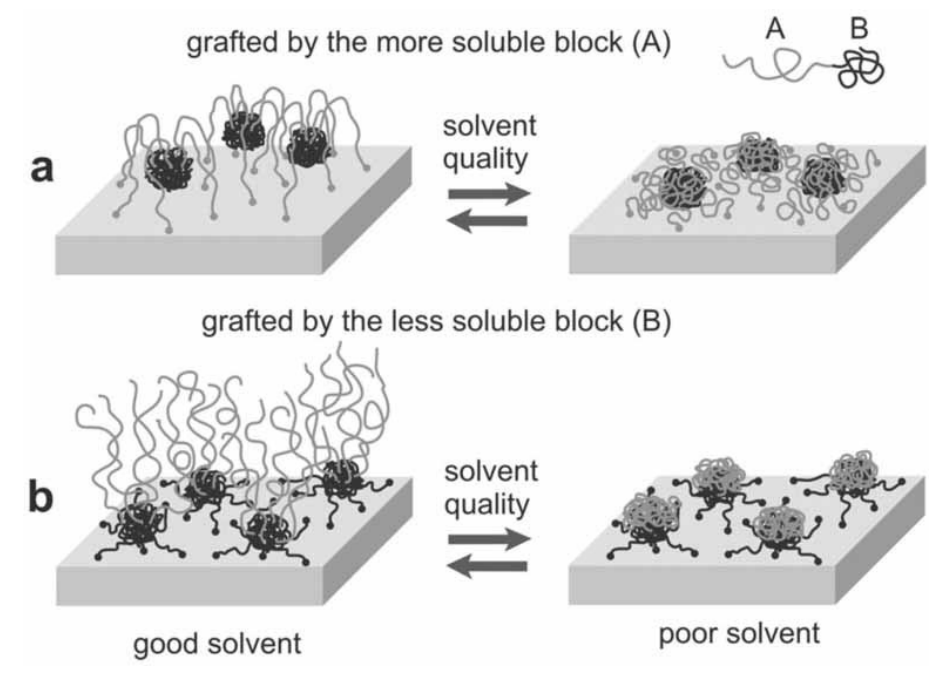

Figure 68 The block-copolymer brush constituted of more soluble A blocks and less soluble B blocks. The brushes grafted by the A-blocks (a) form B-polymer micelles shielded by the A-polymers (a, right). In good solvent for the A-polymers B-micelles are located at the top of the brush (a, left). The brushes grafted by the B-polymers (b) form dumbbell-like micelles (b, right). In good solvent for the A-polymers the Ablocks are stretched and the B-blocks form micelles segregated to the grafting surface (b, left). (41)

The experimental studies (331), (348), (350) have demonstrated reach responsive behavior of block- copolymer brushes based on the rearrangement of segregated phases in good agreement with theoretical predictions. PEL block-copolymer brushes are less investigated. The A-B PEL block-copolymer brushes grafted by the neutral blocks A demonstrated no rearrangement upon treatment by different solvents. (351) However, it seems to be a specific case of the brush structure. Generally, electrostatic interaction can modify inter- actions in the block copolymer brushes resulting in additional responsive mechanisms that could be a subject of future investigations. The $\mathrm{pH}$ effect on the conformation of $\mathrm{pH}$ responsive poly (acrylic acide) (PAA) brushes were investigated by Brittain and co-workers. (Figure 69) In this study, the poly (tertbutyl acrylate) chains were converted to poly (acrylic acid) via pyrolysis and then immersed in alkaline solution. The pending acidic groups along the polymer brush backbone were deprotonated by the addition of base. As a result of being introduced charges within layer, the polymer brush swells due to Coulombic repulsions between the charged polymer chains. A linear increase in PAA brush thickness from $\sim 16$ to $\sim 26$ $\mathrm{nm}$ with increasing $\mathrm{pH}$ (from 2 to 8 ) was observed. (352) The nature and concentration effect of electrolyte on the behavior of the brushes were investigated aswell. Moreover, the effect of grafting density on the $\mathrm{pH}$-induced conformational changes of PAA brushes was studied. (353) It was found that the degree of swelling of the PAA brushes was dependent on brush density at $\mathrm{pH} 4$ and 5.8, whereas it was independent of grafting density at $\mathrm{pH} 10$, indicating that the PAA brush behaves as a weak polyelectrolyte at pH 4 and 5.8, whereas it tends to act as a strong polyelectrolyte at pH 10. 


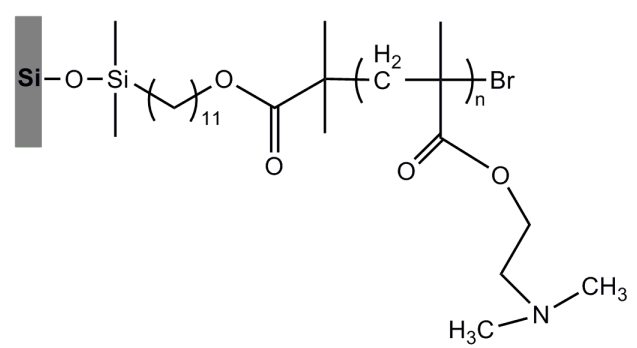

Figure 69 Poly (acrylic acid) brushes from a silica substrate. (353)

When it comes to pH-response of polybase brushes such as PDMAEMA, conformational change of the brush in solution is opposite to that of polyacid brushes due to the fact that wet thickness of polyalkaline brush decreases with increasing $\mathrm{pH}$ due to deprotonation of the side groups. The $\mathrm{pH}$-induced conformational changes of PDMAEMA brushes have been studied using various techniques. The swelling behavior of PDMAEMA brushes prepared on silica substrate via SI-ATRP was investigated using ellipsometry and neutron reflectivity by Sanjuan. (354) (Figure 70) It was revealed that the wet thickness of the brush increases with decreasing $\mathrm{pH}$ (from 10 , to 7 and to 2) due to the protonation of the amine pendant groups. PDMAEMA brushes adopt an extended conformation in acidic solution whereas they resemble a less extended conformation as the $\mathrm{pH}$ of the solution became more basic.

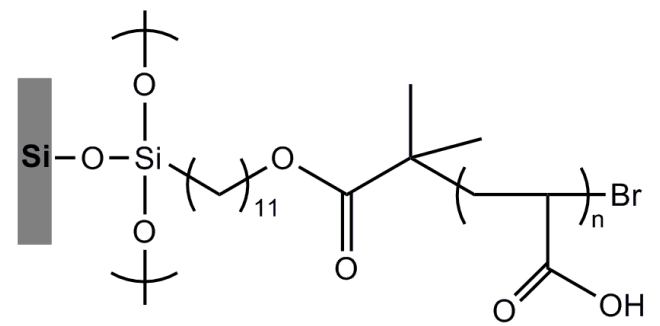

Figure 70 Poly-(N,N'-(dimethylamino)ethyl methacrylate) from silica substrate. (354)

Moreover, the more densely grafted brush swells at a lower $\mathrm{pH}$ than the other brush, reflecting a shift in the $\mathrm{pK}_{\mathrm{a}}$ as a function of grafting density. (355) When it comes to applications of $\mathrm{pH}$-induced conformational changes of polyelectrolyte homopolymer brushes, they can be used for various applications such as quartz crystal microbalance (QCM)-based $\mathrm{pH}$-sensors, which were produced by modification of the resonator with a PAA brush coating. (356), (357) Furthermore, the pH-induced swelling/collapse of polyelectrolyte brushes can be used to control the flocculation behavior of the corresponding polymer brush-coated particles (292), (358) or actuation of AFM cantilevers by modification with a poly (2-methacryloyloxyethyl phosphate) (PMEP) brush coating. (359) 
The protonation/deprotonation of the surface-tethered polyelectrolyte chains can be employed in order to tune the wettability of the polymer brushes just like in the case of PMEP brushes which exhibit a three stage switching of wettability. (360) The brushes exhibit relatively hydrophobic behavior when being exposured to $\mathrm{pH} 1$ solutions (advancing contact angle $65^{\circ}$ ) whereas they become more hydrophilic (contact angle $\sim 49^{\circ}$ ) after treatment with a $\mathrm{pH} 4$ solution. Immersion into basic aqueous solution ( $\mathrm{pH}$ 13) led to almost completely wetting surfaces. In addition to all these studies based on $\mathrm{pH}$ responsive homopolymer brushes, the $\mathrm{pH}$ effect on the conformation of $\mathrm{pH}$-responsive diblockcopolymers such as PNIPAMco-PNVI (361), PMAA-co-PDMAEMA (362), poly (acrylic acid)- $b$-poly(vinylpyridine) block copolymer brushes (363) were extensively examined.

\subsection{Ion Sensitive Polymer Brushes}

In addition to $\mathrm{pH}$, polyelectrolyte brushes are also sensitive to variation of ionic strength. (353), (364) According to theoretical studies, strong and weak polyelectrolyte brushes exhibit different behaviors. For strong polyelectrolytes, the brush behaves as a neutral, i.e. collapsed, brush at high salt concentrations whereas an unbalance between the ion concentration inside and outside the brush is observed at low salt concentrations. As a consequence of this unbalance, electrostatic interactions lead to swelling of the brush. At this specific concentration, the brush enters the osmotic brush regime, where co-ions are expelled from the brush and the layer thickness reaches a limiting value. In the case of weak polyelectrolyte brushes, for the neutral and salted brush regimes, the salt concentrations inside and outside the brush are approximately equal and the internal degree of dissociation is the same as in bulk solution. On the other hand, in the osmotic brush regime, there is a significant electric potential difference between the brush and the bulk solution, and in addition, the salt concentration inside the brush is considerably higher. These unfavorable electrostatic conditions result in a discharge of the electrolyte groups and a collapse of the layer thickness. Experimental investigations of the wet thickness of PAA brushes at different $\mathrm{pH}$ values and a range of salt concentrations were in good agreement with the predicted behavior of weak polyelectrolyte brushes. In the salted brush regime, Szleifer, Genzer, and co-workers found that above the mushroom-to-brush transition, which was observed at a brush density $(\sigma)$ of 0.08 chains $/ \mathrm{nm}^{2}$, the wet PAA layer thickness $(H)$ increased with increasing brush density. (353) The increase in wet PAA thickness followed a scaling law $H \sim \sigma n$ with $n \approx 0.29-0.31$, which was in good agreement with the theoretically predicted $1 / 3$. The behavior of the PAA brushes in the osmotic brush regime was more complex. In contrast to theory, which predicted a decrease in wet thickness with increasing grafting density and an increase in wet thickness with increasing ionic strength, the experimental results revealed an increase in brush swelling with increasing brush density. Furthermore, the increase in wet layer thickness at high brush densities was found to increase with increasing ionic strength. Ayres et al. reported the effects of mono- and divalent salts on the behavior of PMAA 
brushes. (352), (365) Upon decreasing the salt concentration, it was found that the threshold concentration that marks the onset of brush expansion was higher for the monovalent salt. Huck and co-workers have extensively studied the influence of the counterion on the structure and properties of PMETAC brushes. (366)- (368) In contrast to many other studies that use highly hydrated and mobile counterions, these authors investigated scarcely hydrated anions, which can undergo ion-pairing interactions with the quaternary ammonium groups in the brush. (369), (370) The characteristics of the brush (e.g., wettability) were found to be very sensitive to the nature of the counterion. Upon exchanging the original chloride counterion with a variety of other counterions, it was found that the wettability of the counterionmodified brushes increased from $\mathrm{ClO} 4$-, SCN- , I- , Br- , Cl- , PO4 3- which correlates with the Hofmeister classification of the hydrophobicity of these anions. (371)

\subsection{Thermoresponsive Polymer Brushes}

The thermoresponsive properties of various surface-attached polymer brushes such as homopolymer, random copolymer, and block copolymer brushes have been extensively investigated. (372) Thermoresponsive polymer brushes prepared by SICRP have been employed in a variety of fields including biomedicine, (373), (374) chromatography, (375)- (378); controlled cell adhesion, (379), (380), catalysis, (381) and membrane transport. (382) PNIPAM is one of the most studied thermoresponsive polymers, and surface-tethered PNIPAM brushes have attracted much attention in the past two decades due to its LCST behavior. (383) At temperatures below the LCST, these brushes are hydrophilic, whereas they exhibit hydrophobic character at the temperatures above the LCST due to the collapse of the brushes when being exposed to water. Free PNIPAM in solution shows a sharp LCST at $32{ }^{\circ} \mathrm{C}(384)$, whereas the LCST transitions of PNIPAM brushes are broader and occur over a wider temperature range (from $\sim 29$ to $\sim 40{ }^{\circ} \mathrm{C}$ ). (260), (311), (385), (386)

The phase transition temperature of PNIPAM brushes depends on the salt concentration just like in the case of the free polymer in solution. (387) Phase transition temperature of free polymers in solution linearly decreases the with increasing salt concentration whereas surface-attached PNIPAM brushes exhibit a nonlinear behavior. Also, it was found that varying $\mathrm{pH}$ between 3 and 8 has almost negligible impact on the swelling properties. (388) This difference between the solution properties of free PNIPAM and the properties of surface-attached PNIPAM brushes stems from the high chain density of brushes. The LCST transition of a PNIPAM brush leads to an increase in the water contact angle of $\sim 10-30^{\circ}(385),(389)$ as well as a decrease of the polymer brush thickness (385), (386), (390), (391) and stiffness. (392)

The collapse of PNIPAM brushes upon temperature increase was investigated via neutron reflectivity. (393), (394) It was found that the brush shrinkage was not a 
monoton process. A phase separation upon heating or cooling was observed in the temperature range of $\sim 30-33{ }^{\circ} \mathrm{C}$. (393), (394) In addition to these, the impact of the polymer molecular weight and brush density on the temperature-induced conformational changes of PNIPAM brushes were investigated via neutron reflectivity aswell. (395), (396) It was found out that high density PNIPAM brushes with lower molecular weight undergo larger conformational changes upon varying the temperature across the LCST in comparision to higher molecular weight PNIPAM brushes. (395) Also, low molecular weight brushes present a more complex behavior and exhibit phase separation. (394) On the other hand, low density PNIPAM brushes with different molecular weights represented a contradictory behavior. (396) The high molecular weight (152 $000 \mathrm{~g} / \mathrm{mol})$ brush underwent conformational changes whereas there was no conformational change observed for the low molecular weight (33000 $\mathrm{g} / \mathrm{mol}$ ) brush. Conformational changes were most prominent for brushes with intermediate grafting densities and high molecular weights.

Besides neutron reflectivity based studies, PNIPAM chain collapse as a function of brush molecular weight and grafting density was investigated via using water contact angle and surface force measurements, aswell. (377), (397) According to surface force measurements, the chain collapse above the LCST decreased with decreasing grafting density and molecular weight. Above the LCST, the increase in the advancing water contact angle of low molecular weight brushes at lower densities is less pronounced than that of high molecular weight and dense PNIPAM brushes, whose advancing water contact angles increase sharply. The wettability of PNIPAM brushes is strongly affected by the roughness of the substrate from which they are grafted. (312) Water contact angles of PNIPAM brushes grown from flat surfaces are $63.5^{\circ}$ at $25{ }^{\circ} \mathrm{C}$ and $93.2^{\circ}$ at $40^{\circ} \mathrm{C}$. When these brushes were grown from structured (patterned) surfaces, the water contact angles demonstrated a dramatic change from $63.5^{\circ}$ to $0^{\circ}$ (for $25^{\circ} \mathrm{C}$ ) and from $93.2^{\circ}$ to $149.3^{\circ}$ (for $40^{\circ} \mathrm{C}$ ). As seen from results, it is possible to reversibly switch brushes from a superhydrophilic to a superhydrophobic state. The LCST of PNIPAM brushes can also be affected by cross-linking. The influence of the amount of cross-linker on the LCST of the PNIPAM-co-PMBAM random copolymer brushes was studied. (293) It was found that $0.5 \mathrm{~mol} \%$ (molar ratio in polymerization mixture) of MBAM did not affect the LCST value of the polymer brush, whereas the LCST increased with increasing amount of MBAM. In addition to NIPAM, another monomer that has been widely used to prepare thermosensitive polymer brushes is poly (poly (ethylene glycol) methyl ether methacrylate) (PPEGMEMA).

The thermosensitivity of poly (di (ethylene glycol) methyl ether methacrylate) (PPEGMEMA2) and poly (tri (ethylene glycol) methyl ether methacrylate) (PPEGMEMA3) brush-coated silica particles were investigated by means of comparement of the phase transitions of the polymer brushes with those of the corresponding free polymers in water. (398) Both PPEGMEMA brushes didn't exhibit sharp transitions on the contrary of the free polymer in solution. The transition 
began at lower temperature than the case of the free polymer and occurred over a broader temperature range. These differences stem from the close packing of the chains in the brush compared to the case of the free chains in solution.

The effect of the nanoconfinement on the thermoresponsive behavior of PPEGMEMA2 brushes was examined by Jonas et al. (399) Compared to a nonstructured polymer brush, patterned brushes demonstrated an increased temperature-induced vertical swelling. This phenomenon is attributed to the different packing of the chains. Since the chains in patterned brushes are initially less stretched than that in nonstructured brush, they are able to swell more. Poly (sulfobetaine methacrylate) (PSBMA) brushes which displays upper critical solution temperature (UCST) behavior has been grafted from gold surfaces via SI-ATRP and thermo induced behavior was examined via contact angle measurements. (400) PSBMA brushes are hydrophobic at room temperature (water contact angle $\sim 79^{\circ}$ ) due to its UCST behavior whereas it is more hydrophilic at high temperature (water contact angle $\sim 58^{\circ} \mathrm{C}$ ). As for the LCST transition, it was observed that the UCST of PSBMA brushes is different from the free PSBMA in solution (i.e., $\left.33{ }^{\circ} \mathrm{C}\right)(401)$ and occurs over a wider temperature range (from 40 to $50{ }^{\circ} \mathrm{C}$ ).

In addition to these thermoresponsive homopolymer brushes, it is also possible to utilize surface-initiated random copolymerization to tune the thermosensitive properties of polymer brushes. For example, polymer brushes with LCSTs between 32 and $40{ }^{\circ} \mathrm{C}$ were sucsessfully prepared by surface-initiated atom transfer radical copolymerization of di (ethylene glycol) methyl ether methacrylate and poly (ethylene glycol) methacrylate. (402) One of important findings of this study is that LCST values of the copolymer brushes depend linearly on the comonomer composition. It is possible to produce dual responsive surfaces if the second monomer used for the preparation of the copolymer brushes is sensitive to another stimulus than temperature.

This dual responsive brush was obtained by Xia et al., who grafted PNIPAM-based brushes containing $3 \mathrm{~mol} \%$ acrylic acid from silicon substrates. (403) The copolymerization of acrylic acid introduced a $\mathrm{pH}$-sensitivity to the brush in addition to temperature sensitivity, which leads to a variation in the LCST of the brushes (from 21 to $45{ }^{\circ} \mathrm{C}$ ) depending on the $\mathrm{pH}$. Besides homopolymer and random copolymer brushes, also thermosensitive block copolymer brushes have been prepared and investigated. One example for that is preparation of PDMAM- $b$-PNIPAM-modified PS latex particles via SI-ATRP. (404) The hydrodynamic thickness of the brush layer was evaluated as a function of temperature. A gradual decrease in layer thickness was observed over a broad temperature range $\left(20-38^{\circ} \mathrm{C}\right)$, in contrast to free PNIPAM in solution whose LCST is sharp.

Dual thermosensitive block copolymer brushes of NIPAM and PEGMEMA have been prepared from initiator-modified gold nanoparticles via SI-ATRP. (291) Two 
thermal transitions which correspond to the LCSTs of the different blocks were observed in course of temperature-dependent dynamic light scattering experiments. Other double responsive diblock copolymer brushes consist of a $\mathrm{pH}$-sensitive block and a thermosensitive block. In another study, the dual thermoresponsiveness of symmetric poly (2-succinyloxyethyl methacrylate)- $b$-poly( $N$-isopropylacrylamide) brushes was examined by means of AFM. (405) A decrease in film thickness with increasing temperature (from 25 to $50{ }^{\circ} \mathrm{C}$ ) was observed at $\mathrm{pH} 9$, whereas there isn't any change in the thickness of brush observed at $\mathrm{pH} 4$. Brush seemed to be temperature-insensitive. This loss of thermal responsiveness was attributed to hydrogen bonding between the constituent blocks. Dual ( $\mathrm{pH} /$ temperature) responsive block copolymer brushes were also studied by Rahane et al. (388) In contrast to previous the example, the PMAA- $b$-PNIPAM brushes exhibited temperaturedependent swelling properties between $\mathrm{pH} 3$ and 8. It was reported that, hydrogen bonding interactions did not influence the LCST of the PNIPAM blocks (even if the transition was broad), despite the fact that the $\mathrm{pH}$-dependent actuation was strongly affected by hydrogen bonding.

\subsection{Non Biofouling Surface}

Non-biofouling surface can be defined as a surface that is resistive to the non-specific adsorption of proteins, cells, or other biological species. The materials with nonbiofouling surfaces are extremely important in biomedical area for a wide variety of applications such as medical implants, contact lenses, drug delivery, biosensors. Polymer brushes are very attractive candidates for the development of nonbiofouling coatings. Improvement of SI-CRP techniques provides possibility for preparation of polymer brushes with well-defined thickness, composition, and architecture.

Hydrophilic polymer brushes have special significance due to their highly hydrated nature that provides an effective enthalpic and entropic barrier to nonspecific protein adsorption. As a consequence, polymer brushes prepared via SI-CRP might be very promising to provide better nonbiofouling properties in comparision to oligo (ethylene glycol) self-assembled monolayers (SAMs). Sofar, many studies supporting this hypothesis have been reported. (406)- (408) The potential of 'smart polymers' as biofouling-release agents was also examined. When it comes to design of protein resistive "smart" systems, PNIPAM has a special importance due to its LCST behavior, which allows, as a function of the work temperature, to switch the surface properties from a hydrophilic (protein and cell resistant state) to a hydrophobic state that readily adsorbs proteins and cells. (409), (410)

\subsection{Cell Adhesive Surfaces}

The surface of artificial biomaterials is of critical importance in the field of regenerative medicine and tissue engineering. This importance stems from its 
influence in guiding and directing cellular behavior and function. (411), (412) When it comes to improvement of surfaces of artificial materials in order to provide better control and direction of cell adhesion, polymer brush based coatings obtained via SICRP are attractive tools. Cell adhesive polymer brushes are generally classified into three categories: the first group includes nonbiofouling polymer brushes functionalized with an extracellular matrix (ECM) protein or a cell adhesion peptide derived thereof whereas the second group consists of various nonbiofouling polymer brushes that have been used to pattern cell adhesive substrates and geometrically control cell adhesion. The third category includes thermoresponsive polymer brushes which exhibit a lower critical solution temperature (LCST) behavior, which allows thermal triggering action to change surface property from a hydrophobic cell adhesive to a hydrophilic nonbiofouling state.

\subsection{Protein Binding and Immobilization}

Polymer brushes have special importance in terms of biomedical applications, which require protein binding, such as protein microarrays or tools used for protein purification. There are several advantages of utilization of brushes for these applications in comparison to the use of self assembling monolayers: One of them is the possibility of preparation of polymer brushes with excellent nonbiofouling properties, which is essential for microarray applications. Otherwise, nonspecific protein adsorption could lead to an enhanced background noise and to prevent denaturation of surface-immobilized proteins. Furthermore, polymer brushes possess a very high surface concentration of functional groups that can be used to bind or immobilize proteins.

\subsection{Membrane Applications}

SI-CRP has been widely used to prepare or vary the properties of membranes as well as the derivatization of stationary phases used for chromatography applications. There are many studies that involve modification of different porous inorganic and polymerbased support membranes with polymer brushes by using SI-CRP techniques. Forexample, SI-ATRP was used to modify the surface of porous alumina membranes with a 50-100-nm-thick cross-linked PPEGDMA or linear PHEMA brush. (413) According to gas permeation studies, the PPEGDMA-based membranes have $\mathrm{CO}_{2} / \mathrm{CH}_{4}$ selectivities of $\sim 15-20$ and $\mathrm{O}_{2} / \mathrm{N}_{2}$ selectivities of $\sim 2$ whereas the selectivity of PHEMA-modified membranes is very little. The performance of the PHEMA-based membranes was significantly improved by postmodification of the hydroxyl side-chain functional groups with pentadecafluorooctanoyl chloride. The $\mathrm{CO}_{2} / \mathrm{CH}_{4}$ selectivity of post-modified PHEMA reached value of $\sim 6-8$.

Post-modification of PHEMA brushes from alumina membranes with octanoyl chloride, palmitoyl chloride were also reported. (414) These postmodified polymer 
brush-coated alumina supports were utilized as pervaporation membranes for the separation of volatile organic compounds (VOCs) from water. (415) Modification of regenerated cellulose ultrafiltration membranes with a PPEGMA brush coating was achieved via SI-ATRP. (416) It was demonstrated that the water flux across these modified membranes decreased with increasing polymerization time (i.e., brush thickness). Thermosensitive PNIPAM brushes was sucsessfully grafted from the exterior surface of gold-coated polycarbonate track-etched membranes with the purpose of taking advantage of the LCST behavior of the PNIPAM coating in order to control the flow properties across the membrane.

Chloromethylated poly (phthalazinone ether sulfone ketone) membranes have been modified with a thin PPEGMEMA coating by using SIATRP. (417) The hydrophilic polymer brush coating led to a decrease in pore size, increased solute rejection, and an improved fouling resistance. In addition to all these membrane types used for different applications, proton conducting membranes have also been prepared by sulfonation of polystyrene brushes grown from poly (vinylidene fluoride)-g-poly- (vinylbenzyl chloride) supports. (418) PVDF microfiltration membranes was modified with PMMA and PPEGMEMA brush coatings by using surface-initiated reverse ATRP. (419) It was reported that PPEGMEMA-modified membranes exhibited more resistive character aganist protein fouling compared to the PMMA-modified supports.

\subsection{Antibacterial Coatings}

One of the important topics in in modern healthcare is prevention and treatment of bacterial infections. There is an increasing interest in strategies to modify material surfaces with antibacterial coatings which prevent biofilm formation. The antibacterial brushes prepared via SI-CRP sofar can be subdivided into three categories: The first group consists of biocidal polymer brushes which kill bacteria. The second class of antibacterial polymer brushes are nonbiofouling brushes that are able to prevent bacterial adhesion. The third class of antibacterial polymer brushes is a combination of biocidal and nonbiofouling properties.

The most common polymer used as antibacterial coating is PDMAEMA brushes which were grafted on various substrates, including filter paper, glass slides, and silicon wafers via SI-ATRP. (420), (421) Quartenary ammonium-modified surfaces with substantial biocidal activity was obtained via quaternization of these brushes with ethyl bromide. A combinatorial screening was developed with an attempt to clarify the influence of polymer brush chain length and grafting density on the bacterial killing properties. It was found that one of the important parameters which determine biocidal activity is surface charge density. The most effective brush coatings had charge densities greater than $1-5 \times 1015$ accessible quaternary ammonium groups per square centimeter. (421) An alternative approach was developed for preparation of biocidal polymer brush coatings against Gram positive and Gram negative bacterias. (422) In 
this study, PSPMA $(\mathrm{K})$ brushes were prepared via SI-ATRP in order to be used as reservoirs that could be loaded with silver ions. It was reported that these silver-loaded polymer brush coatings effectively inhibited the growth of both Gram positive and Gram negative bacteria. In addition to utilization of biocidal functional groups or releasing biocidal agents, a second strategy to prevent biofilm formation is to take advantage of the nonbiofouling properties of certain polymer brushes.

Bacterial adhesion and biofilm formation was investigated on various substrates such as bare glass slides, substrates coated with SAMs of methyl-terminated, mixed sulfate/trimethylammonium-functionalized, and oligo (ethylene glycol)-containing alkanethiols and zwitterionic poly (sulfobetaine methacrylate) (PSBMA) and PPEGMEMA brush-coated substrates. (423) It was found that bacterial adhesion on the polymer brush coatings was significantly reduced compared to the bare glass reference and the SAM-modified slides. Van der Mei and co-workers investigated the microbial deposition, adhesion, and detachment of two bacterial strains ( $S$. aureus and S. saliVarius) and one yeast strain (C. albicans) on the poly (acrylamide) (PAM) brush-coated substrates. It was found that PAM coating reduced the microbial adhesion by $70-92 \%$ compared to unmodified silicon surfaces. (424)

Examples of other antibacterial polymer brushes, which have been reported sofar are PMAA, (425) PAM, (424), (426) and poly (2-(tert-butylamino) ethyl methacrylate) brushes. (427) Besides the examples mentioned above, also several antibacterial polymer coatings which combine both biocidal and nonbiofouling features have been reported aswell. Antibacterial coatings that consisted of a PHEMA brush, which was functionalized with the antibiotics gentamicin or penicillin was prepared by Zhang et al. (428) Another important example is preparation of a bifunctional antibacterial polymer brush coating which consists of both biocidal quaternary ammonium groups as well as nonbiofouling properties, which was obtained by block copolymerization and subsequent quaternization of PPEGMA and PDMAEMA from polypropylene substrates. (429)

\subsection{Star Shaped Polymers}

\subsubsection{General Features}

Star polymers are a special class of branched polymers, which gains an increasing interest in material science since their behaviour is quite different compared to their linear relatives. (430)- (432) For instance, star polymers exhibit a lower hydrodynamic volume and a smaller size in solution due to higher segment density than their linear polymer relatives of the same molecular mass. (433) This effect is more pronounced with an increasing number of arms. (434) They can be determined as linear polymer chains which are covalently connected to a central point. (434)- (436) 


\subsubsection{Synthetic Approaches of Star Shaped Polymers}

There are two basic routes for preparation of star-shaped polymers; the arm-first and the core-first approaches. (Figure 71) (437) In the arm-first approach linear polymer chains are synthesised first and than connected to a multifunctional core. The main drawback of this method is high probability that not all functionalities of the core's surface are occupied due to steric hindrance caused by the coiled polymer chains. In the case of the core first approach, since the core carries the controlling functionalities, the polymerisation takes place right on the core's surface. As a result of that all chains start at the same time, the core is covered with polymer chains of similar lengths and the final structure is predetermined. (438)- (440) This strategy provides a predefined number of arms and an opportunity to modify the end groups of the arms. (231)

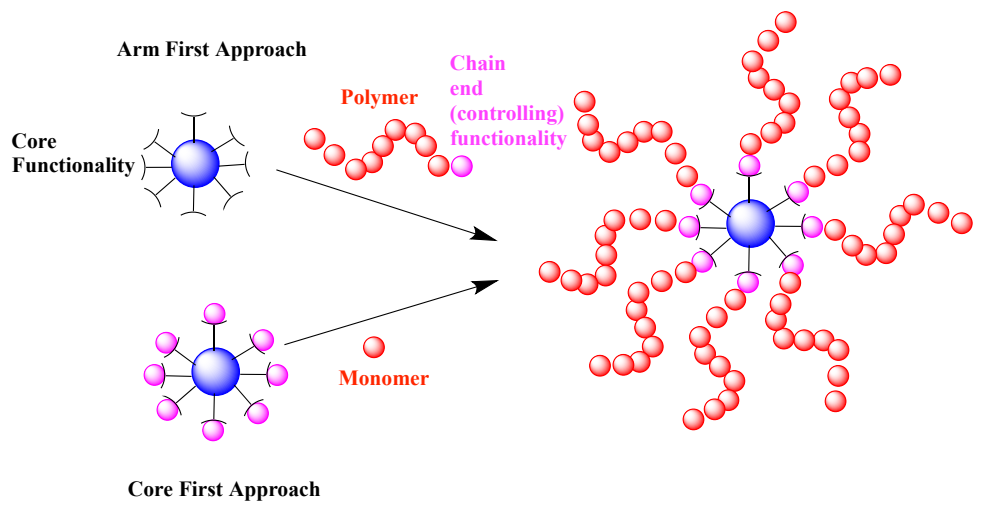

Figure 71 Approaches used to prepare star-shaped polymers. (50)

In case of RAFT polymerisation the core-first approach is further divided into two categories socalled as the R-group- and the Z-group approaches. (440) Figure 72 and Figure 73 illustrates these two concepts respectively where the red represents free radical functionalities, blue illustrating the Z-groups and green symbolising the Rgroups. In the R-group approach (Figure 72), the core is the leaving group R (441) whereas $\mathrm{Z}$ group represents the core in the case of $\mathrm{Z}$ group approach. (Figure 73)

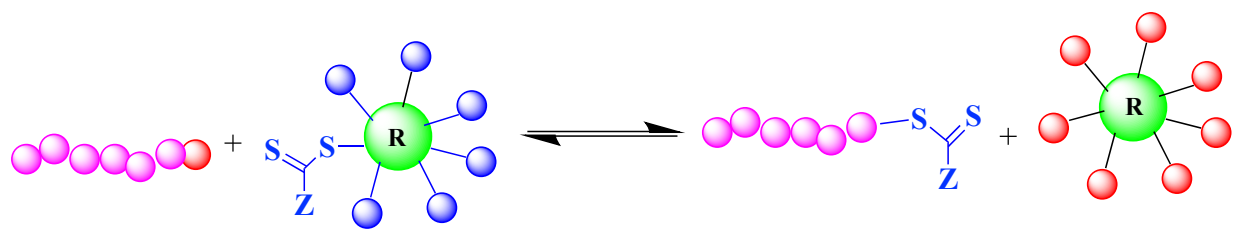

Figure 72 R-group approach. (50)

During this R (core) - RAFT star polymerization, the RAFT-unit with the Z-group departs from the core and transferred to the chain whereas radicals are generated on the core. At that point, it is highly likely that the radical functionality on the core can lead to star-star-coupling via termination by combination. This results in a variety of 
products including dead chains, living chains, single- and double-star polymers as well as stars with a mixture of dead and living arms.

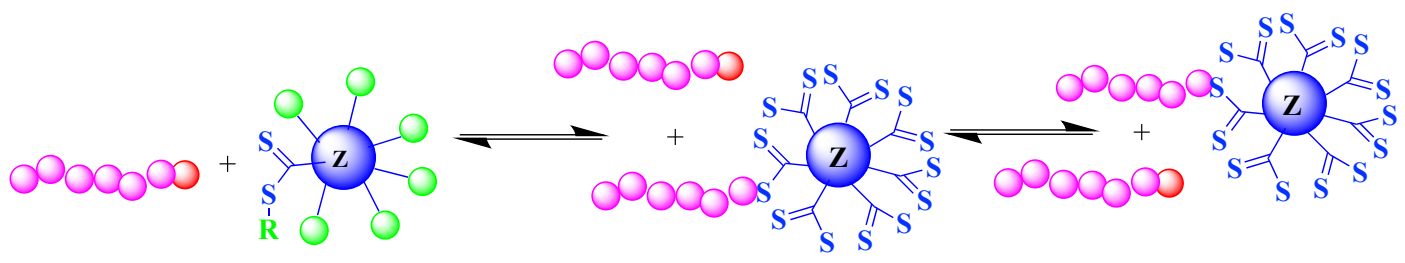

Figure 73 Z-group approach. (50)

The most important adventageous of Z-group approach is the fact that star-starcoupling is avoided. (437), (442) The radical functionality is never at the core since the core is the stabilising $(Z)$ group. As a result, a high homogeneity of the stars is achieved. (442) The resulting products of this process are single-star polymers and dead chains. Due to the fact that the RAFT-units remain on the core throughout the reaction, the whole star keeps its livingness, which further allows block extention of star polymer.

\subsection{Protein Fouling}

Non-biofouling surface can be defined as a surface that is resistive to the non-specific adsorption of proteins, cells, or other biological species. The materials with nonbiofouling surfaces are extremely important in biomedical area for a wide variety of applications such as medical implants, contact lenses, drug delivery, biosensors. Poly (ethylene glycol) (PEG) and poly (2-hydroxyethyl methacrylate) (PHEMA) are the most widely used polymer brush systems in the field of non-fouling materials (443), (444) due to their hydrophilic character which has low interfacial energy and provides an effective barrier to non-specific protein adsorption.

This fouling resistance characteristics of PEG chains is attributed to a "steric" repulsion effect. Water molecules confined in hydrophilic polymer chains are released while biological organisms approach the hydrophilic brush coating. With this release of water molecules, polymer chains are compressed. (445) Both the dehydration (resulting in an increase in enthalpy) and the chain compression (resulting in a decrease in entropy), which provides the thermodynamic basis for the non-biofouling properties of the coating, are unfavorable for biomolecules adsorption. (445) Non-biofouling effect was strongly influenced by grafting density and polymer chain length. (446) It was reported that the growth of high grafting densities and high poly (2methacryloyloxyethyl phosphorylcholine) chains length resulted in a dramatic reduction in the fibrinogen adsorption. (447) Another report demonstrated that a star PEG polymer covalently grafted to silicon surface exhibited, in some cases, a better protein resistivity than the linear form does. (448) Basing on these two reports, it is 
concluded that the control over the synthesis parameters of the polymeric brush has a direct impact on the properties and applications of the substrates.

In surface engineering, it is important to understand and control the mechanism of protein adsorbtion and protein $\backslash$ cell-surface interactions with the aim of developing methods for the design of surfaces for different biomedical applications. However, it is not easy task to control the protein surface interactions due to complex and dynamic interactions between proteins and a surface. The main force driving protein adsorbtion to a solid surface is hydrophobic dehydration which stems from the interaction between hydrophobic part of a protein and a hydrophobic surface. It is a general trend that protein adsorbtion increases with increasing hydrophobicity of the surface. Moreover, electrostatic interactions between charged groups of a protein and the surface might be counted as another force. (449), (450) The efficiency of protein adsorbtion via electrostatic interactions depends on the factors such as the solution $\mathrm{pH}$, ionic strength and the electrostatic properties of the protein and the surface. In addition to these, protein adsorption also depends on protein concentration. (451), (452) At low concentrations, protein-surface interactions are maximized due to protein's orientation and unfolding that leads to denaturation and irreversible adsorption of the protein at the surface. At high protein concentrations, proteins undergo fewer interactions with the surface, thereby, they keep their stable conformations and are desorbed easily.

\section{Scientific Strategies for Polymer Brush Formation on Gold}

\subsection{State of the Art}

The control of surface properties of materials and devices has a critical significance in many areas of science such as stimuli responsive coatings for sensors, (5) colloid stabilization, (10) adhesion, (8) lubrication (9). The most promising way of tuning physical and chemical properties of surfaces is deposition with grafted polymer layers. Covalently bound polymer films offer an efficient and convenient way of modifying physicochemical characteristics of material surfaces and thus play an important role in many areas of science. (453), (454) Synthetic polymers serve as excellent candidates for surface modification because of their tunable mechanical properties, the variability of film thickness, degree of functionality and because of the potential multifunctional stimuli responsivity. Polymer brushes are polymeric chains that are chemically anchored or physisorbed onto a surface at one or more tethering points. (25)- (27) The grafting of chains closer to each other results in steric repulsion which leads to the free chain ends stretching away from a collapsed state so-called "mushroom" to an extended configuration so-called "brushlike". If these grafting points are not high enough, mushroom type brushes form instead of a "real brush". It is possible to tune properties of brushes by changing grafting parameters. (26) "Brushlike" polymer brush provides unique characteristics that differs from similar bulk systems. (25), (26) As a result of these unique properties, polymer brush films have been used in advanced 
applications in many fields such as colloidal particles, (455) non-fouling coatings, (456) and responsive films for sensors, (5), (100) controlled cell growth, (3) controlled gene-drug delivery, (4) biomaterials used in tissue engineering. (457), (458)

There are two basic strategies to prepare polymer brushes: covalent attachment by chemical reaction at the surface and physisorption of the polymer to the surface. (3)(5), (25)- (28), (459)- (465) It is a well known fact that physically absorbed polymer brush systems have major drawbacks such as thermal, solvent based instabilities and low grafting densities leading to "mushroom" type brushes. On the other hand, covalently bound polymers overcome these drawbacks due to irreversible-stronger bond formed between surface and polymeric chains. The synthetic approaches of covalently grafting polymer chains onto surfaces are divided into two categories: "grafting to" and "grafting from". The grafting to technique involves a chemical coupling reaction of the functionalized surface with polymer chains bearing suitable terminal functionalities. It is difficult to achieve high grafting densities by using "grafting to" approach, because of steric congestion resulting from already attached polymer chains on the surface. (Figure 74 (a)) On the other hand, the "grafting from" technique is based on the diffusion of a small monomer molecule into already growing polymer chains covalently attached to the surface, which constitutes a powerful alternative for control of functionality, density and thickness of polymer brushes with high precision. (Figure 74 (b)) For this reason, "grafting from" is more common and powerful method compared to "grafting to" approach.

The combination of "grafting from" approach with a controlled radical polymerization (CRP) technique is highly advantageous in order to achieve maximum control over brush density, polydispersity, composition and form surface-tethered block copolymers. (239), (283), (284), (466) The advent of controlled radical polymerization techniques (CRP) had a significant impact on the fast growing field of surface-confined polymerizations leading to terminally grafted polymers on solid surfaces. CRP techniques provide control over the molecular weight, composition and architecture of these tethered polymers. The basic advantage of CRP over traditional free radical polymerization is a reversible activation and deactivation process which provides controlled growth of polymer chains with less termination reactions, leading to presence of "living" end groups. The most widely used polymerization CRP methods to prepare polymer brushes via the "grafting from" technique are atom transfer radical polymerization (ATRP), nitroxide-mediated polymerization (NMP) and reversible addition-fragmentation chain transfer (RAFT) polymerization. Although ATRP is arguably the most widely used CRP technique for preparation of surface initiated polymer brushes, RAFT has more advantegouses over ATRP in terms of diversity in polymerization of functional monomers. The main advantages of RAFT polymerization over other CLRP techniques are (i) the ability to polymerize a wide variety of monomer types, including styrenics, acrylamides, acrylates, methacrylates and vinyl esters; and the facts that (ii) polymerizations may be conducted under bulk, solution, emulsion, and miniemulsion conditions; (iii) it may be conducted over a wide 
range of temperatures; (iv) it has exceptional functional group tolerance which allows for the preparation of highly functional materials; (v) it allows for the preparation of materials with complex and advanced architectures. (7), (81), (263), (467) Despite these advantages, utilization of SI-RAFT polymerization for the preparation of polymer brushes has not been very common. (26), (260), (264), (268), (273), (276)(278), (468)- (476) In addition to all benefits of Raft polymerization technique over other CRP methods, it multiplies all these advances when it comes to preparation of polymer brushes on surfaces especially on gold surfaces. The specific mechanistic principles of RAFT allow for various synthetic strategies of performing surfaceconfined RAFT polymerizations: It might be classified according to type of species immobilized onto substrate: (i) initiator (ii) CTA through the $\mathrm{Z}$ group and (iii) CTA through the R group.

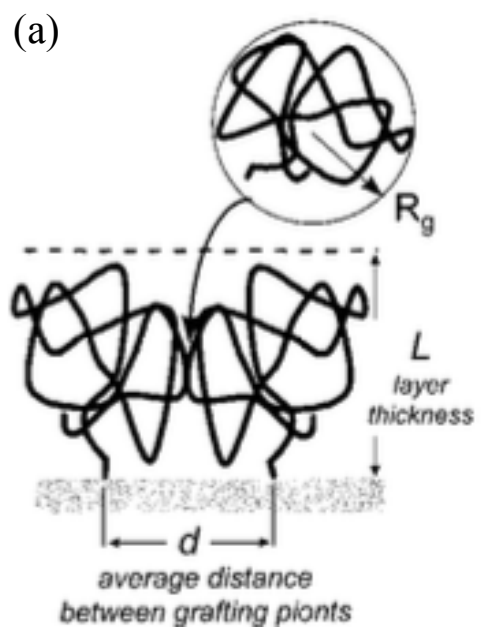

(b)

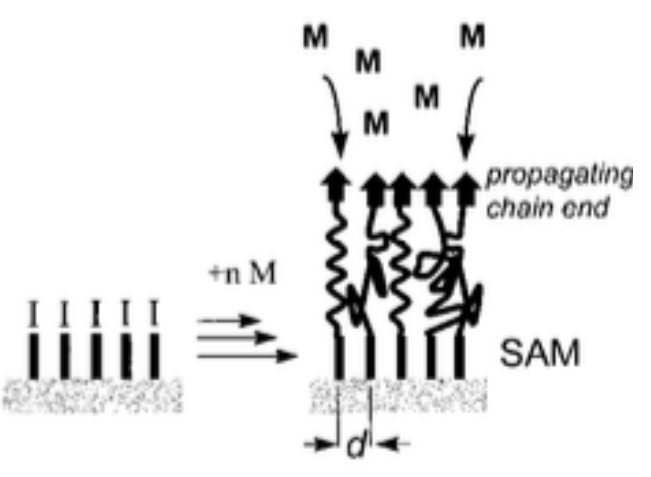

(c)

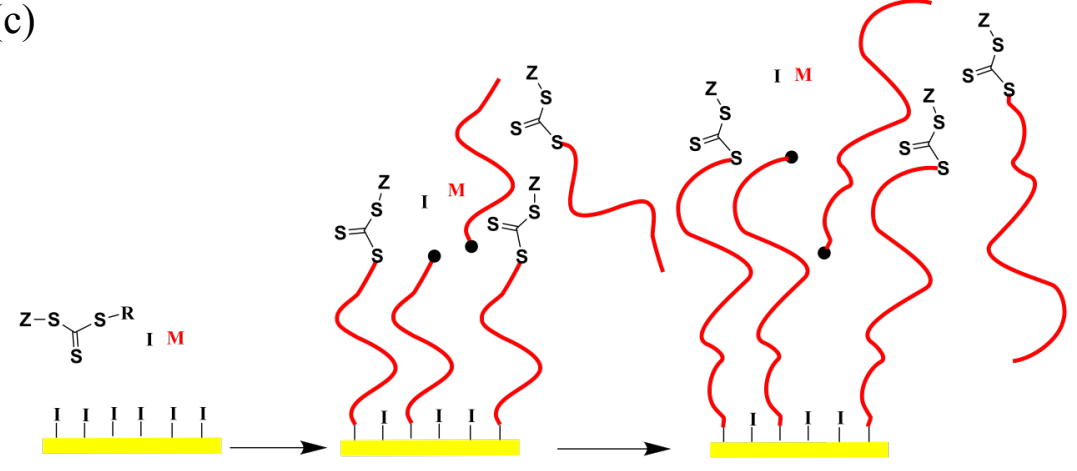

Figure 74 Schematic depiction of the formation of polymer brushes via (a) "grafting to" (b) "grafting from" technique with $\mathrm{R}_{\mathrm{g}}$ as the radius of gyration of a random coiled polymer in a good solvent, $L$ as the layer thickness, $d$ the average lateral distance between grafting points, $\mathbf{M}$ an arbitrary monomer, and $\mathbf{I}$ an initiation site able to induce a surface initiated polymerization (c) Initiator group approach on gold via RAFT. 
The first synthetic route includes a combination of a surface tethered free radical initiator with free CTA in solution and subsequent polymerization (Figure 74 (c)) whereas the others are combinations of a surface tethered CTAs (through the R or Z groups) with free CTA and free radical initiator in solution. The main drawback of the first approach is that thermal free radical initiators dissociate and initiate chains over a wide temperature range, which results in the fact that all chains can't be initiated at the same time, which is essential to form a well defined brush. Another problem might be radical-radical couplings on surface leading to low initiation efficiency and low grafting density. Consequently, this late initiation problem and steric hindrance effect of early initiated longer chains over the propagation of new shorter chains which inititiates relatively later, causes nonuniformity. (463), (468)- (472), (477) The attachment style of surface-tethered raft agents ( $\mathrm{R}$ or $\mathrm{Z}$ group) influence whether the polymerization from surfaces has a real grafting from nature or not, in another words whether the growing polymeric chains are growing on the surface or in solution phase. Regarding $Z$ group approach, the raft agent is irreversibly bounded to surface through its $\mathrm{Z}$ group and it is more similar to "grafting to" approach than a "grafting from". (468) - (470) (Figure 75) In this case, growing polymeric radical chains are the mobile species which break off the surface, add monomer, approach back to surface and interact with the Raft unit immobilized at the surface. In the latter stages of polymerization, as the time goes by, this mobility of growing polymeric radicals become more difficult due to increase in molecular weight, length and size, which leads to a decrease in the accessibility toward raft units immobilized at the surface, so called as the autophobic effect. (478) Consequently, Z group approach leads to low grafting density and poor film uniformity. The most promising approach to form high density and uniform polymer brushes using RAFT polymerization is attachment of CTA through the $\mathrm{R}$ group. In the case of introducement of raft agent through its $\mathrm{R}$ group to the surface, monomers and the cleavable dithioester/trithiocarbonate moieties of raft agents are mobile species whereas the $\mathrm{R}$ group of Raft agent is irreversibly bounded on to surface, which assures growth of polymeric radicals on the surface. (Figure 76) The initiation step takes place in solution phase with or without a free cta and than these growing polymeric radicals attack the raft agents immobilized at the surface. This way, growing polymeric radicals remain on the surface whereas the dithioester or trithiocarbonate moiety cleaves off and departs from surface. Since the both the raft moieties and monomers are such small molecules which can easily be diffused into the growing polymeric radicals on surface and come into contact with the chain ends of these growing polymer chains, there isn't a pronounced steric hindrance problem leading to a decrease in accessibility. In comparision to others, $\mathrm{R}$ group approach is the best way to obtain thicker polymer brushes via Raft polymerization technique. (479), (480)

Compared with the large number of reports in which ATRP was used for preparation of polymer brushes, there are surprisingly few reports on the application of RAFT technique to modify surfaces, although RAFT offers the greatest versatility with respect to monomer and solvent. Up to now, some procedures including multistep 
approaches for the introducement of CTAs onto substrate were improved in order to obtain brushes via RAFT technique. The first approach relies on the reaction between an end functionalized raft agent and native functional group, which originally presents on substrate surface. In this case, there is no need for a prior-extra treatment of surface in order to functionalize it. Another approach relies on the formation of a monolayer consisting of functional groups active toward the terminally functionalized raft agents (e.g. amine, hydroxyl, carboxyl functionality). Self assembled silane and thiol based monolayers are highly preffered for functionalization of surfaces.

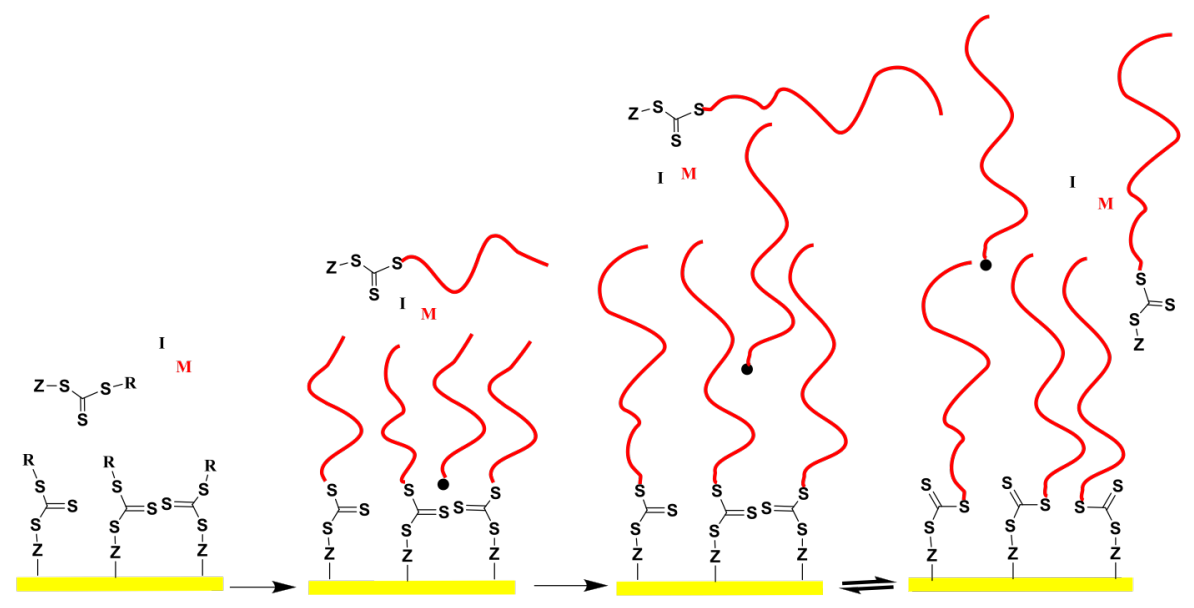

Figure $75 \mathrm{Z}$ group approach on gold.

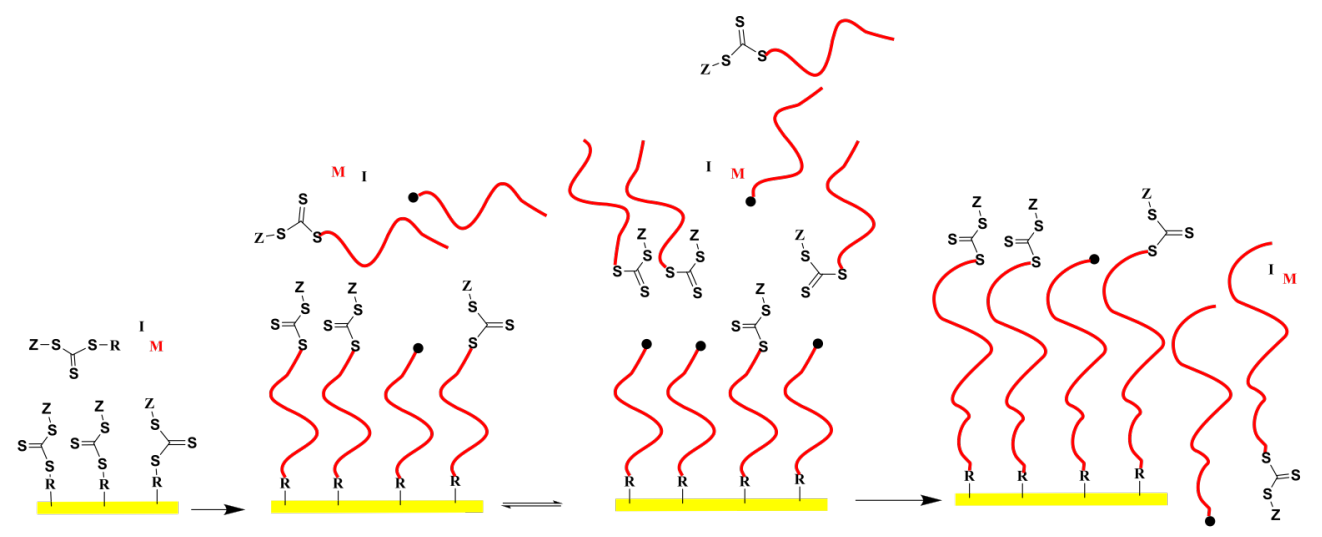

Figure 76 R group approach on gold.

The coupling methods of raft agents through their $\mathrm{R}$ or $\mathrm{Z}$ groups onto functionalized surfaces are relatively complex and specific for each substrate-raft agent combinations. Forexample, R-group attachment of the CTA was performed via condensation reactions between surface hydroxyl $(-\mathrm{OH})$ and amino $\left(-\mathrm{NH}_{2}\right)$ functionalities and 4cyanopentanoic acid dithiobenzoate. (260), (268), (466) Another approach improved was conversion of surface immobilized ATRP end groups into RAFT CTAs. (481), (482) Recently, Caykara and coworkers reported modification of gold substrate with a 
CTA through its R group by using an ester reaction. (482) All these works reported so far for synthesis of polymer brushes on gold rely on multistep approaches including self assembly of thiols and subsequent introducement of raft agents onto these functional surfaces through their $\mathrm{R}$ or $\mathrm{Z}$ groups, which are complex and specific for each substrate functionality-raft agent combinations.

\subsection{Scope of The Thesis}

In the past ten years, thiols and derivatives have attracted growing interest due to their high affinity toward gold surfaces whose importance stems from the applications in the many fields (e.g. medical devices). The self-assembly of thiols/thiol derivatives onto gold and subsequent polymerization of these monolayers leading to longer polymeric thin films have potential for design of specific interface architectures in sensor technology and in general for composite gold-polymer interfaces. With this background in mind, the main objective of this thesis work was to develop novel synthetic approaches for fabrication of polymer brushes with various architectures on gold surfaces. As previously discussed in the state of the art, the privileged way to tune the desired features and functionality of a surface has been the growth of polymer chains by a combination of a CRP and "grafting from" technique. The interest to develop new synthetic strategies which are relatively easier than ones in literature lies in the possibility of utilization of these novel straightforward techniques for design and production of advanced biomedical devices. The novelty in this work is successful combination of the chemisorption mechanism of raft agents toward gold and the specific mechanistic principles of RAFT, which is the basis for novel straight forward synthetic strategies developed for fabrication of polymer brushes with complex topologies on gold.

The specific tasks comprising this research are as follows:

(1) Self-assembly of thiols, raft agents and raft agent precursors on gold.

(2) Polymerisation of SAMs on the substrate via RAFT polymerization technique.

(3) Copolymerization of homopolymers formed on gold via RAFT polymerization technique.

In this work, synthesis of methacrylate based brushes were investigated extensively. Poly (buthyl) and (methyl) based brushes were investigated both for their hydrophobicity whereas poly (N, N' dimethylamino ethyl methacrylate) brush was examined for its hydrophilicity and external stimuli-responsive characteristics. Furthermore, poly (PEGMA) brush was utilized for its hydrophilicity and proteinresistive characteristics. Formation of homopolymer, blockcopolymer and star brushes on gold planar surfaces via Raft polymerization is an interesting and little studied class 
of polymeric thin films. Few studies have been performed sofar (explained in detailed above in the part of "SI-RAFT from gold surfaces"). In the frame of this work, novel straightforward strategies were elaborated for the preparation of various brush architectures on gold planar surfaces. (Figure 77) Chapters 5, 6, 7 and 8 describe characterizations of all polymeric coatings prepared via novel strategies, whereas chapter 9 gives a compherensive summary of all approaches;

* In chapter 5, a two-step approach was presented and applied onto gold surfaces with an aim to develop homopolymer and diblockcopolymer brushes (Figure 77 (Traditional multistep approach)). As first step, the preparation of homogeneous monolayers derived from thiols and subsequent introducement of raft agents on gold surfaces were investigated. In the second step, the "grafting from" synthesis of homopolymer and diblockcopolymer brushes from raft agents tethered on surfaces were carried out.

* In chapter 6, a straight forward approach based on direct attachment of raft agents was presented and applied onto gold surfaces in an effort to develop homopolymer and diblockcopolymer brushes. (Figure 77 (1), (2)) As first, the preparation of homogeneous monolayers derived from raft agents on gold surface was investigated. In the second step, synthesis of homopolymer and diblockcopolymer brushes from surface confined raft agents was carried out.

* In chapter 7, a second straight forward approach based on different methodology than previous one, which relies on utilization of raft agent precursor was presented and applied onto gold surfaces with an aim to develop homopolymer and diblockcopolymer brushes. (Figure 77 (3)) As first, the preparation of homogeneous monolayers derived from raft agent precursor on gold surface was investigated. In the second step, synthesis of homopolymer and diblockcopolymer brushes from surface confined raft agent precursors was carried out.

* In chapter 8, a novel synthetic strategy for formation of star polymer layer was presented and applied on gold surface in an effort to reduce nonspecific protein adsorbtion. (Figure 77 (4)) As first, the preparation of homogeneous monolayers derived from thiols and subsequent introducement of raft agents on gold surfaces was investigated. In the second step, synthesis of star polymer brushes from linear raft agents tethered on surface was carried out. Finally, as the last step, protein resistive properties of star brushes were investigated.

* Chapter 9 describes the experimental procedures used for formation of the selfassembling monolayers and synthesis of all polymer brush types on gold surfaces whereas chapter 10 describes the various analytical methods and tools to characterize polymers and the modified surfaces. These techniques include Spectroscopic Ellipsometry (SE), Atomic Force Microscopy (AFM), 
Attenauted Total Reflectance Infrared spectroscopy (ATR-IR), Contact Angle measurement (CA) for characterization of surfaces whereas Gel Permation Chromatography (GPC) and High Pressure Liquid Chromatography-Mass (HPLC-Mass) spectrometry for characterization of polymers and proteins.

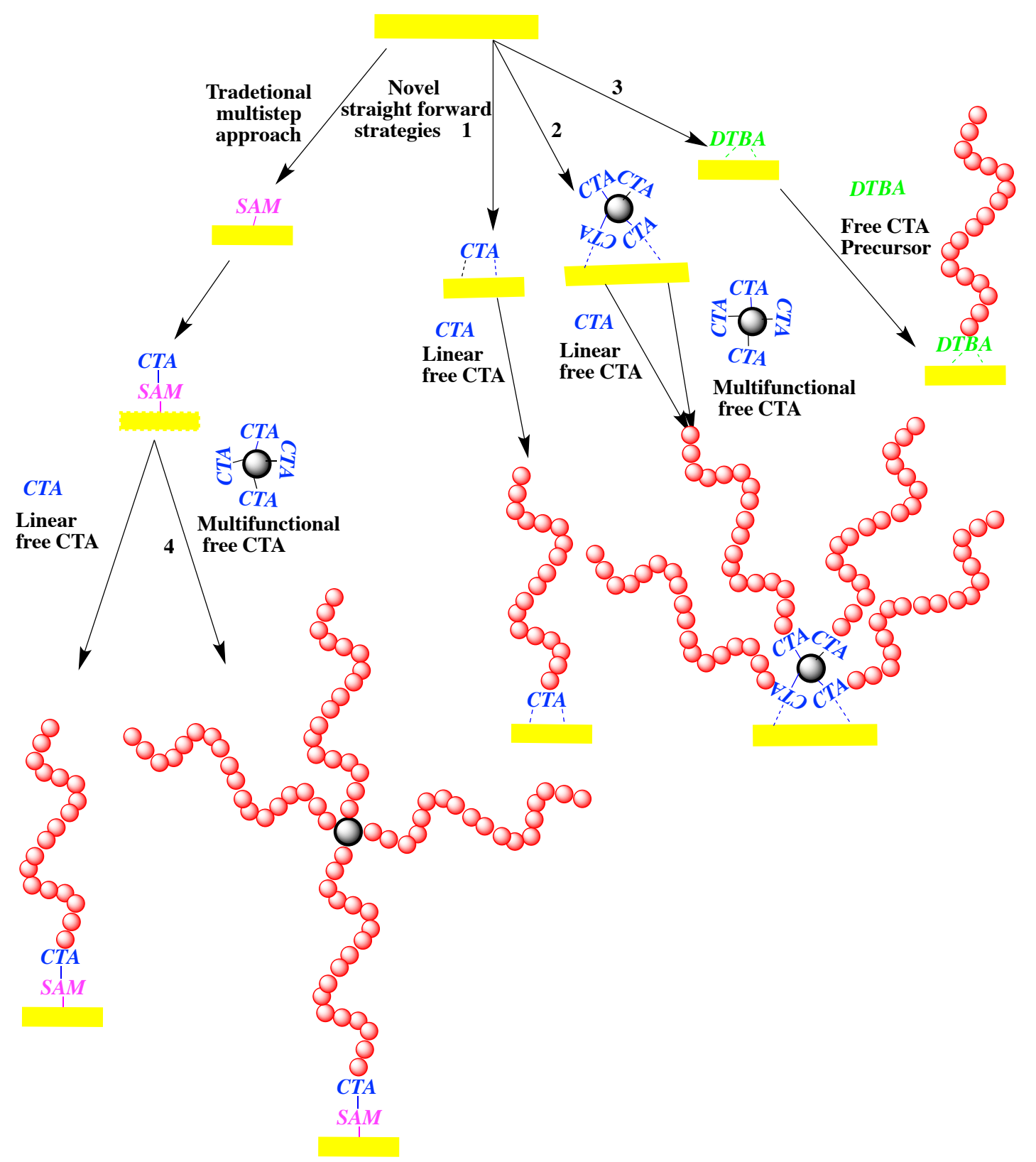

Figure 77 Novel straightforward strategies developed for preparation of various brush architectures on gold planar surfaces. 


\subsection{Motivation and Aim of the Work for Each Chapter}

\subsubsection{Preparation of Polymer Brushes on Gold via Traditional SI-RAFT ( $R$ Group) Approach}

Up to now, although a remarkable amount of studies was performed on SI-RAFT polymerization, there was not much work on investigation of "livingness" of brushes prepared via RAFT on gold surfaces, which has a basic importance for design of materials of high complexity such as smart block copolymer brushes as well as for post-functionalization of the brushes through their end groups with the purpose of tuning surface properties for any desired application. To best of our knowledge, for the first time, well-defined diblock copolymer brushes on gold planar surfaces have been prepared via SI-RAFT polymerization in order to prove the ability of RAFT technique which can effectively reinitiate the polymerization in an effort to tune physicochemical properties of homopolymer brushes and therefore underlying surfaces. It is known that dithioester and trithiocarbonate moieties are highly susceptible to be chemisorbed on gold surface. (225) This impedes the direct coupling of raft agent through $\mathrm{R}$ group toward gold surface, which results in low grafting density of resulting brushes. In order to overcome chemisorption of raft agents on gold, a two step approach was used. The complete coverage of gold surface with a self assembling monolayer (SAM) of 11Mercapto-1-undecanol (MUD) was applied as first step while the second step includes a subsequent coupling of a commercial trithiocarbonate-type Raft agent (4-Cyano-4[dodecylsulfanylthiocarbonyl) sulfanyl] pentanoicacid) (DSTCSPA). Butyl methacrylate (BUMA), 2-(dimethylamino)ethyl methacrylate (DMAEMA) and poly (ethyleneglycol) methyl ether methacrylate (PEGMA) were chosen as model monomers for the synthesis of homopolymer and diblock copolymer brushes from flat gold substrates. In every case, either DSTCSPA or 2-phenyl-2-propylbenzodithioate (CDB) was added into reaction medium as sacrificial CTA in order to control the polymerization and form polymer in solution to be used for molecular weight estimation. (Figure 78) Scheme 1 (page 114) illustrates the general route taken for the synthesis of diblock copolymer brushes from flat gold surfaces utilizing SI-RAFT polymerization technique.

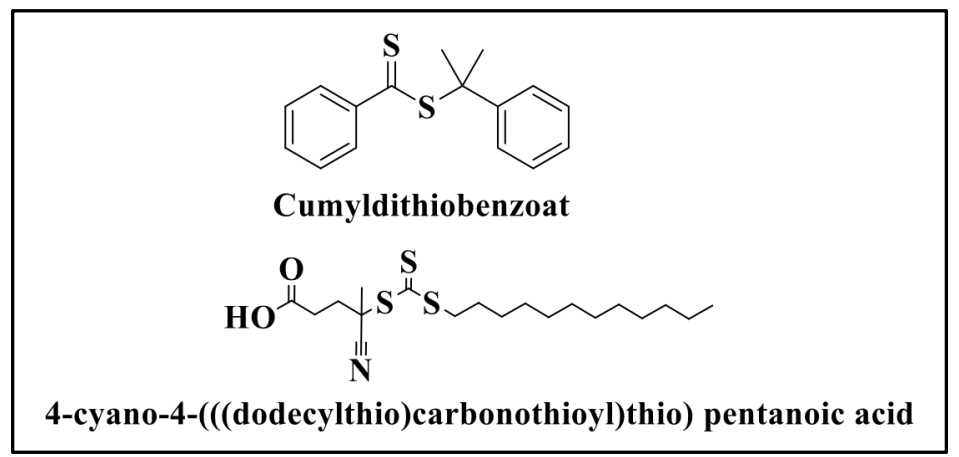


Figure 78 Chemical structure of the "free" chain transfer agents used in this work.

\subsubsection{Preparation of Polymer Brushes on Gold via Novel Chemisorption Based Approach}

Reversible addition fragmentation chain transfer (RAFT) polymerization is an extremely versatile, controlled radical polymerization technique due to its tolerance toward functional monomers which allows the synthesis of a vast range of (co) polymers. RAFT technique is based on a degenerative transfer mechanism in which a thiocarbonylthio compound acts as a chain transfer agent (CTA). (66) As a consequence of this mechanism, polymers prepared by this technique contain a dithioester end group or a trithiocarbonate group in the middle of the chain depending on the type of CTA used. Polymers produced by RAFT are thus highly interesting for the functionalization of metal, and in particular gold surfaces via the "grafting to" approach. Preparation of monolayers based on a sulfur -Au bond has been extensively investigated (19), (190) including the use of thiols, (216), (217) sulfides, (218) disulfides, (219) thiocarboxylicacids, (220) dithiocarboxylic acids, (221) xanthates, (222) thiocarbamates, (223) dithiocarbamates (224) and Raft polymers. (483)- (486) Up to now, the reported procedures to modify gold surfaces with RAFT polymers involve the prior transformation of the sulfur containing group coming from the CTA into thiols by reaction with nucleophiles such as primary amines. (483)- (486) However, this step is incompatible with some polymers such as poly (acrylic acid) or polymers bearing activated esters due to the reactivity of the main chain and restricts thus the range of application. Moreover, it has been shown that it is rather difficult to selectively obtain thiols by this procedure and that disulfides are often formed, resulting from the coupling between two thiol-functionalized polymer chains. (487)(489)

Duwez and coworkers showed that the prior transformation of dithioesters and trithiocarbonates into thiols is not mandatory for the functionalization of metal surfaces, since these two species are able to chemisorb onto gold. (225) In the work of Duwez, firstly, polymers were synthesized via RAFT and then these raft polymers were self-assembled onto gold via 'grafting to' approach without any transformation process of sulfur containing group in CTA into thiols. However, it is a well known fact that 'grafting to'approach results in low grafting densities compared to 'grafting from' approach due to steric hindrance caused by already grafted polymer chains on surface. (245)- (247) The most common synthetic strategy used to form polymer brushes on gold is 'grafting from' based RAFT technique, which consists of multi step procedures including the complete coverage of gold surface with a self assembling monolayer (SAM) as first step and a subsequent coupling of initiator or a Raft agent through its $\mathrm{R}$ group. 
In this work, a novel straight forward method is introduced for preparation of well defined homopolymer and diblock copolymer brushes on gold planar surfaces via SIRAFT polymerization in an effort to add a second route to literature. In addition to all the benefits of Raft mediated polymerizations in terms of preparation of polymer brushes previously mentioned in the part of "state of art", it multiplies these advantageouses when it comes to gold surfaces due to the sulphur based nature of Raft agents, which has a natural affinity toward gold. Duwez and coworkers have shown that dithioester and trithiocarbonate moieties are highly susceptible to be chemisorbed on gold surface. (225) However, introducement of raft polymers onto gold via 'grafting to' technique is not the most efficient way for preparation of dense layers. The chemisorption tendency of raft agents toward gold is taken advantage and combined with 'grafting from' approach in order to reduce multi-step procedures into an easier, direct approach. Chemisorption of raft agents on gold through their dithioester and trithiocarbonate moieties eliminate any extra special pre-treatments for gold surfaces, use of any first self assembling monolayer and any special catalysts or chemical reactions to graft raft agent through its $\mathrm{R}$ or $\mathrm{Z}$ group onto gold. This chemisorption based SI-RAFT approach automatically eliminates the requirement for design, synthesis and utilization of any specific raft agent bearing a specific functionality in the $\mathrm{R}$ or $\mathrm{Z}$ groups so that they might be reacted with the end functionality of self assembling monolayers pre-formed on gold surface. This approach opens up the possibility to introduce any desired raft agent onto gold without the limitation of "appropriate functionality" requirement, which leads to great versatility of the monomers which might be polymerized from gold surfaces.

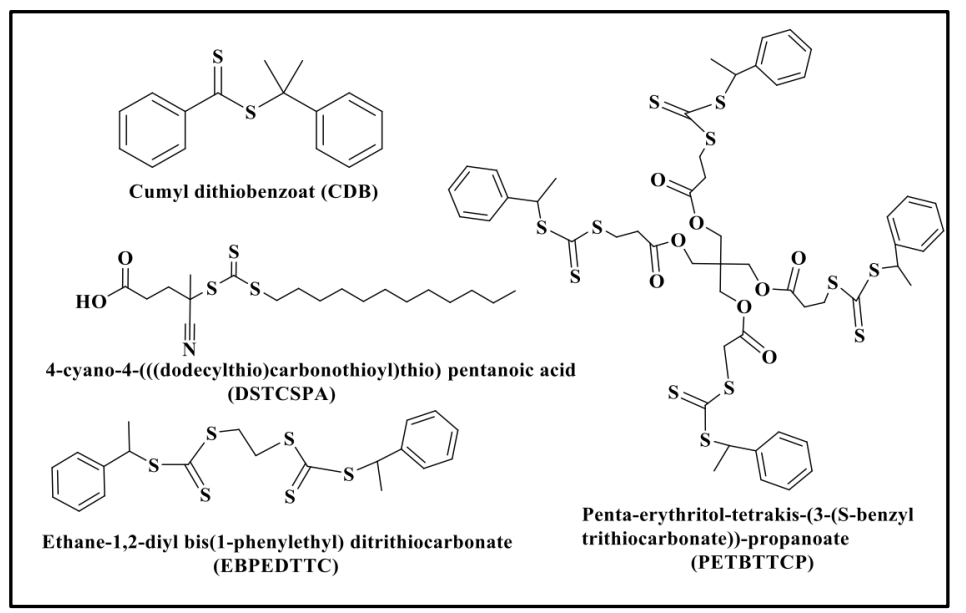

Figure 79 Chemical structure of the "free" chain transfer agents used in this work.

Butyl methacrylate (BuMA), 2-(dimethylamino) ethyl methacrylate (DMAEMA) and poly (ethylene glycol) methyl ether methacrylate (PEGMA) were chosen as model monomers for the synthesis of homopolymer and diblock copolymer brushes from flat gold substrates via direct attachment of raft agents through their dithioester and trithiocarbonate moieties. In every case, either 4-Cyano-4[dodecylsulfanylthiocarbonyl) sulfanyl] pentanoicacid (DSTCSPA) or 2-phenyl-2- 
propylbenzodithioate $(\mathrm{CDB})$ or star shaped raft agents were utilized both as surface anchored CTAs on gold and as sacrificial CTAs in reaction medium in order to control both polymerisation at the the surface and in solution. (Figure 79)

(a)

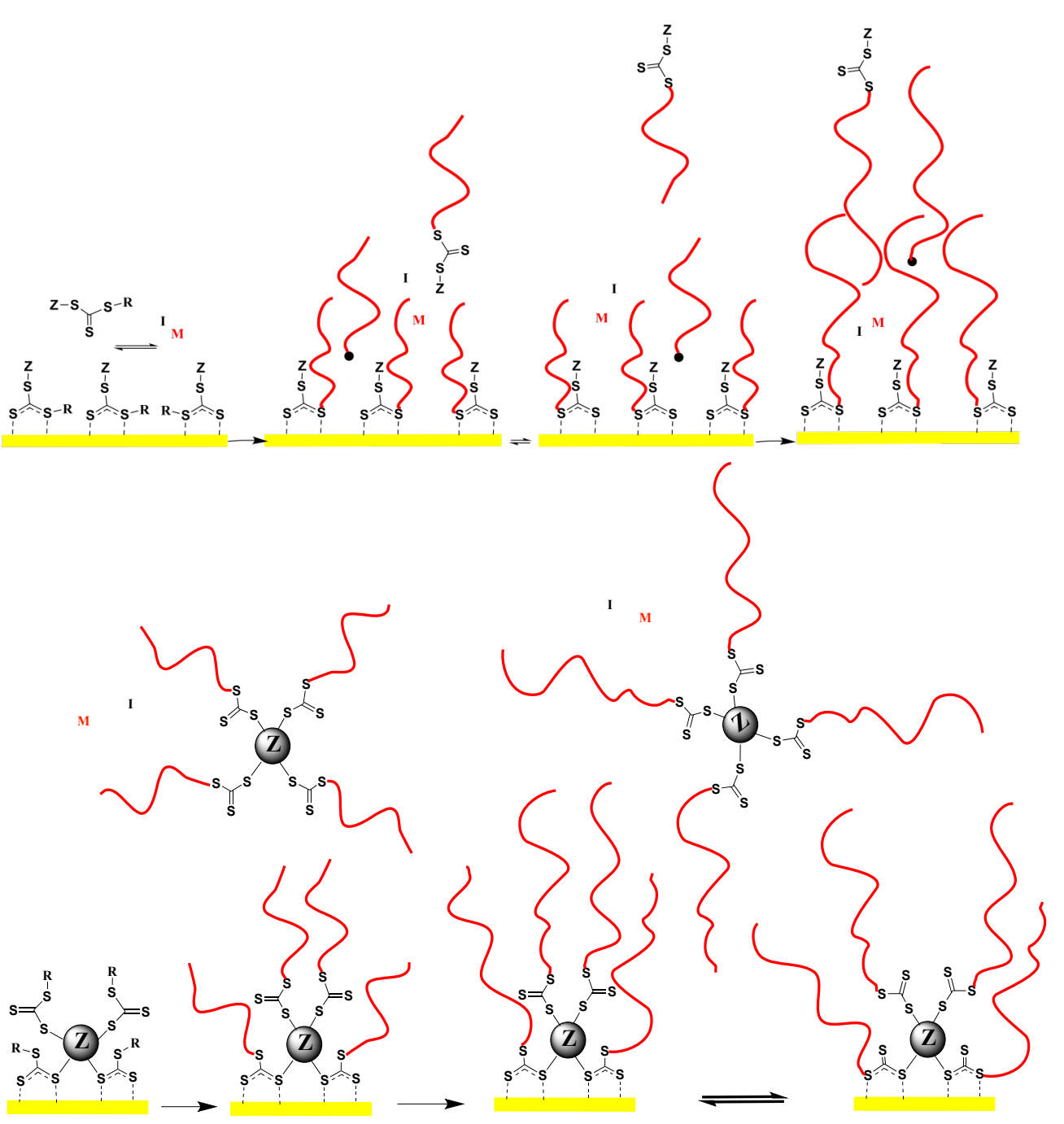

Figure 80 Polymer brush formation on gold via novel approach based on chemisorption of (a) linear and (b) multifunctional raft agent.

The main reason of chemisorption of both monofunctional, bifunctional and star shaped raft agents onto gold is to investigate the impact of increasing arm numbers of surface confined CTAs over resulting thickness and grafting densities of polymer brushes formed. (Figure 80) The cores of star shaped CTAs used in this work as both surface anchored CTA and free CTA in reaction mixture are Z groups and therefore, the approach is called as $\mathrm{Z}$ group approach. The most important benefit of the Z-group approach is the fact that star-star-coupling is avoided. (437), (442) The radical functionality is never at the core since the core is the stabilising $(Z)$ group. As a result, a high homogeneity of the stars is achieved. (442) The resulting products of this process are single-star polymers and dead chains. Due to the fact that Raft units remain on the core throughout the reaction, the whole star keeps its livingness, which allows 
block extention of star polymer. In addition to variation of CTAs chemisorbed on surfaces, free CTAs were also tuned for polymerization of two identical surfaces with an aim to investigate impact of free CTA type used in solution phase on resulting thicknesses, PDIs and grafting densities of resulting polymer brushes. (Scheme 2) For this reason, one of two identical gold flats covered with same CTA was polymerized in the presence of free CTA which is identical with the one chemisorbed on gold whereas second flat was polymerized in the presence of a monofunctional free CTA which is different from the one chemisorbed on gold.

\subsubsection{Synthesis of Polymer Brushes on Gold via Novel DTBA Mediated Polymerisation Approach}

In this work, a straight forward method is introduced to prepare well defined homopolymer and diblock copolymer brushes on gold planar surfaces without utilization of any chain transfer agent neither on surface as gold confined CTA nor in solution phase as free CTA. Despite all the benefits of Raft mediated polymerizations in terms of preparation of polymer brushes mentioned in the part of "state of art", there is no need to use Raft mediated polymerization for preparation of well defined brushes on gold. Therefore, there is no need for design and utilization of a specific raft agent bearing suitable $\mathrm{R}$ or $\mathrm{Z}$ group functionality. Vana had reported a novel protocol which allows for the generation of well controlled poly (MMA) using a raft agent precursor dithiobenzoic acid (DTBA) as the mediating agent, which is another alternative to classical RAFT polymerizations of MMA, as DTBA is accessible with less effort and at lower costs than dithioesters with tertiary leaving groups. (490) Also, it is a well known fact that dithioester and trithiocarbonate moieties are highly susceptible to be chemisorbed on gold surface. (19), (225)

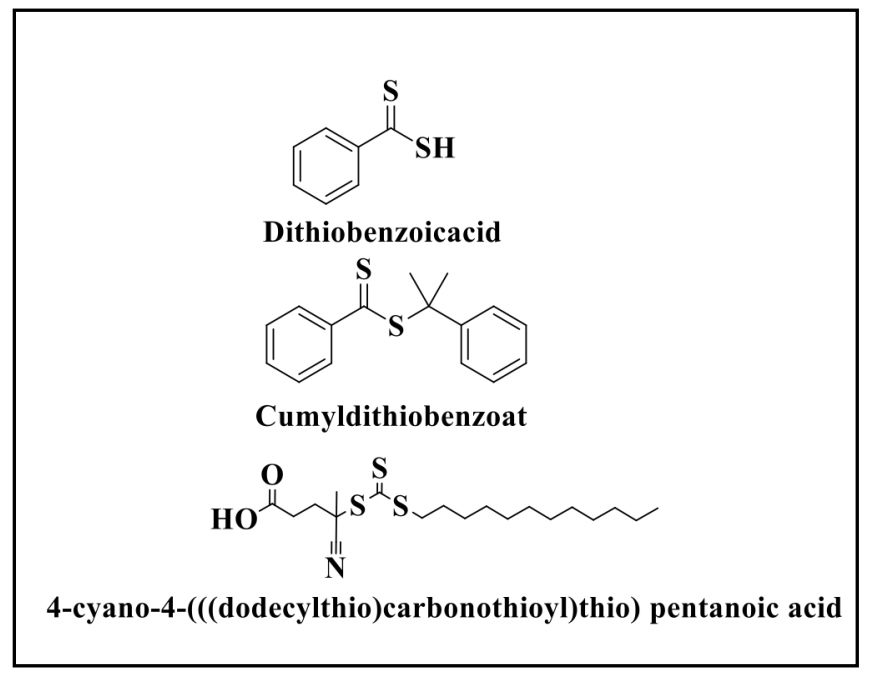

Figure 81 Chemical structure of the "free" chain transfer agent precursor and chain transfer agents used in this work. 
Both this chemisorption property of dithiobenzoicacid toward gold and its control ability in polymerization of methacrylates were successfully combined in order to reduce multi-step procedures required for preparation of polymer brushes on gold into a straight forward approach. (Scheme 3) Instead of utilization of raft agents either anchored on surface and $\backslash$ or used in solution phase, DTBA has been utilized both as surface anchored agents and free agents in solution to conduct polymerization in a controlled manner. (Figure 81) Chemisorption of dithiobenzoicacid on gold through its dithioester moiety eliminates any extra special pre-treatments for gold surfaces, use of any self-assembling monolayer as first layer and any special catalysts or any subsequent chemical reactions to couple raft agents through their $\mathrm{R}$ or $\mathrm{Z}$ groups onto first layer. Moreover, it eliminates the obligation for design or use of any specific raft agent containing a specific functionality in the $\mathrm{R}$ or $\mathrm{Z}$ groups so that it might react with self assembling monolayers pre-attached onto gold surface.

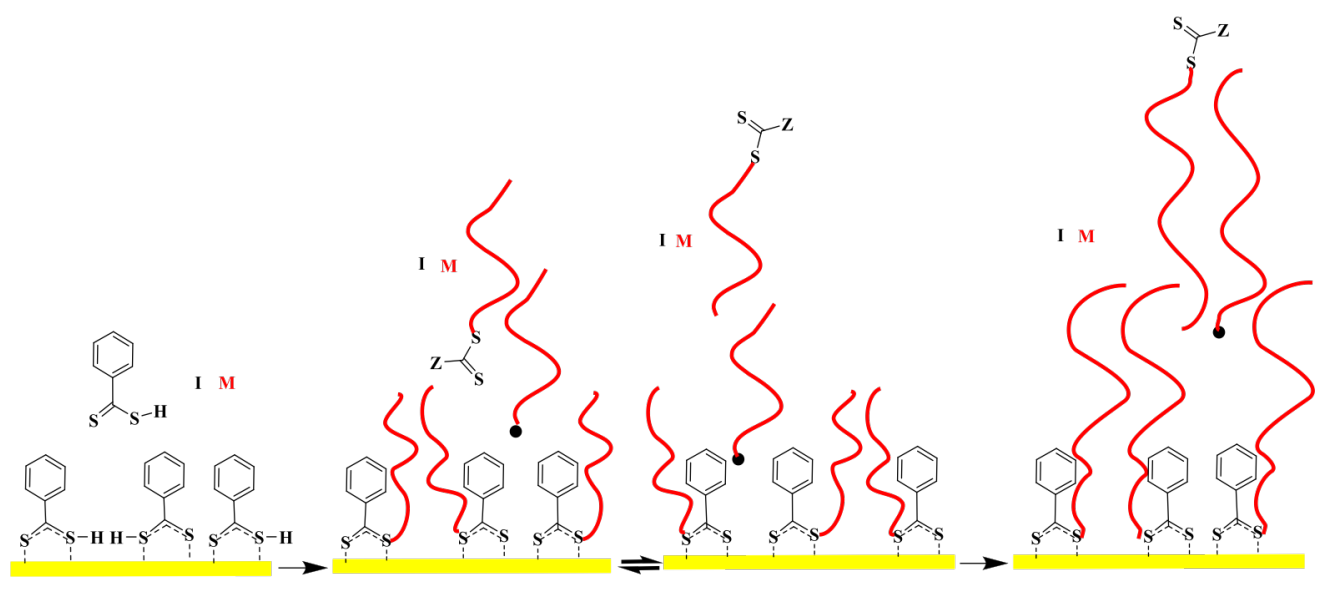

Figure 82 Polymer brush formation on gold via novel approach based on chemisorption of DTBA.

Methyl methacrylate (MMA), butyl methacrylate (BUMA), 2-(dimethylamino)ethyl methacrylate (DMAEMA) and poly (ethyleneglycol) methyl ether methacrylate (PEGMA) were chosen as model monomers for the synthesis of homopolymer and diblock copolymer brushes from gold surface confined DTBA. In every case, DTBA was immobilized onto gold surfaces. Either DTBA or 4-cyano-4[dodecylsulfanylthiocarbonyl)sulfanyl] pentanoicacid (DSTCSPA) or 2-phenyl-2propylbenzodithioate (CDB) were added into reaction mediums as sacrificial controlling agents in order to investigate the impact of utilization of free DTBA and free CTAs on resulting thicknesses and PDIs. (Figure 82) The thicknesses of resulting polymer brushes and polydispersity indexes of resulting free polymers synthesized either in the presence of free DTBA or in the presence of free CTAs were compared eachother in order to prove success of DTBA mediated polymerization over Raft mediated polymerizations. 


\subsubsection{Synthesis of Star Polymer Brushes on Gold via Novel Z-RAFT Polymerisation Approach}

During past century, blood contacting biomedical devices have been widely used for applications such as heart valves, vascular grafts, stents, in vivo biosensors. (491), (492) The lack of hemocompatibility is main problem of cardiovascular and other blood contacting medical devices. (493) Congestion of small diameter vascular grafts and failure of blood contacting biosensors due to thrombus formation on device surface might be counted as some examples for this hemocompatibility problem. (494), (495) Nonspecific protein/cell adsorption can decrease device performance, such as in the case of in vivo biosensor and stent surfaces. (496) For this reason, improvement of anticoagulant devices or drugs is required for long term applications. (492) Several strategies for modification of device surfaces were developed in order to reduce nonspecific protein adsorbtion. (497)- (499)

Covalently bound polymer films offer an efficient and convenient way of modifying physicochemical characteristics of material surfaces and thus play an important role in many areas of science. (25), (29) Polymer modified surfaces are used in a variety of applications such as stabilization of colloidal particles, (10) non-fouling coatings, (6) and responsive films for sensors. (32) Synthetic polymers serve as excellent candidates for surface modification because of their tunable mechanical properties, the variability of film thickness, degree of functionality and because of the potential multifunctional stimuli-responsivity. Poly (ethylene glycol) (PEG) based monomers are the most versatile monomer types used against nonspecific protein adsorbtion due to nonfouling character which is attributed to its conformation resulting in a very high dipole moment of the ethylene oxide repeats. (500) This leads to extensive hydration of the grafted polymer brush chains and thereby sterically repels the proteins that approach the surface. The non-fouling character of PEG depends on several factors, such as the polymer molecular weight, its graft density, the solution temperature and concentration of other solutes present in solution. (501)- (504)

Surface-grafted star polymers of poly (ethylene glycol) were found to prevent adsorption of proteins, due to a pronounced size-exclusion effect that is enhanced by the hard-sphere character of the stars. (505), (448) It was also found that relatively few star polymer molecules, which do not need to strongly overlap, are sufficient for preventing proteins from adsorbing. This finding is attracting increasing interest in surface polymer science and stimulated specialized surface modifications for biomedical applications. (506), (507) These approaches are experimentally rather complex, involving multi-step approaches of forming star polymer, functionalizing star polymers and subsequently binding the star polymer to the surface. The results were, however, very promising in that protein adsorption could almost quantitatively be prevented. (507) Star polymers are a special class of branched polymers, which gains an increasing interest in material science since their behaviour is quite different compared to their linear relatives. (430)- (432) They can be determined as linear 
polymer chains which are covalently connected to a central point. (434)- (436) There are two basic routes for preparation of star-shaped polymers; the arm-first and the corefirst approaches. (437) The core first approach is more advantageous since the polymerisation takes place right on the core's surface due to the fact that the core carries the controlling functionalities. As a result of that all chains start at the same time, the core is covered with polymer chains of similar lengths and the final structure is predetermined. (438), (439)

In case of RAFT polymerisation the core-first approach is further divided into two categories socalled as the R-group- and the Z-group approaches. (440) The most important adventage of $\mathrm{Z}$ group approach is the fact that the radical functionality is never at the core since the core is the stabilising $(Z)$ group. As a result, star-starcoupling is avoided (437), (442) and a high homogeneity of the stars is achieved. (442) The resulting products of this process are single star polymers and dead chains. Due to the fact that the RAFT units remain on the core throughout the reaction, the whole star keeps its livingness, which allows block extention of star polymer. In this work, basing on the Z-RAFT process, a novel and more viable pathway was designed in order to control the polymer topology and obtain star polymer on gold planar surface which is of special biomedical interest. In this approach, star-shaped trithiocarbonate type RAFT agents (Figure 83) in which the central core constitutes the stabilizing Z-group of the mediating dithio-compound were developed (231), (442) and used as free chain transfer agents in solution phase whereas a monofunctional raft agent was used as a surface-anchored chain transfer agent, which was immobilized onto gold through its R group. (Scheme 4)

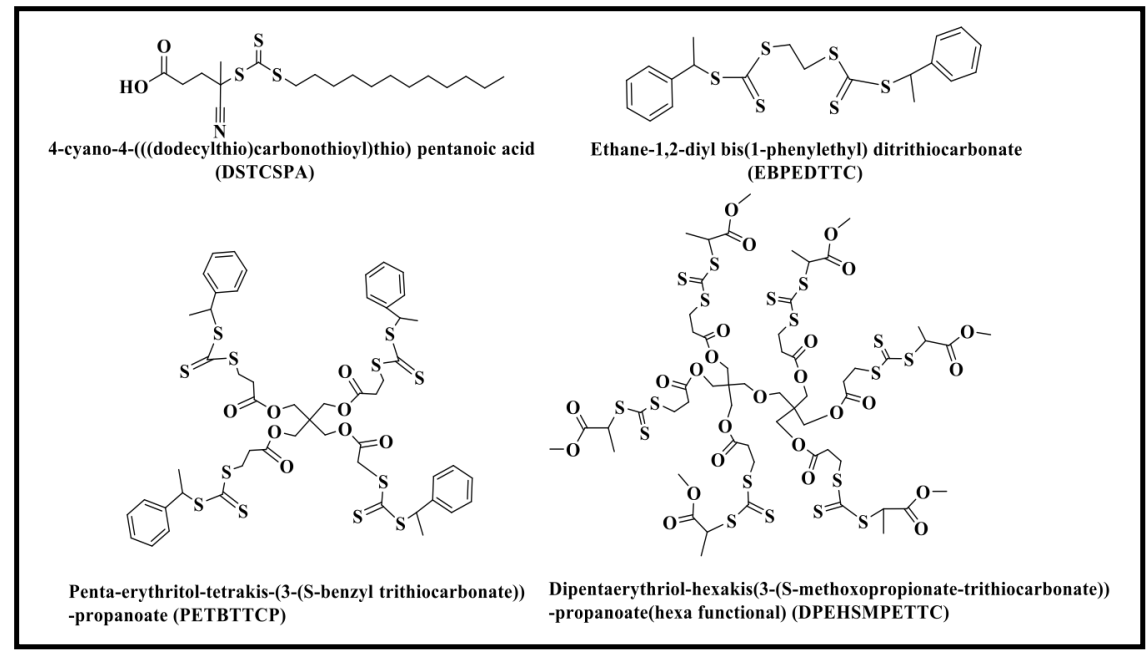

Figure 83 Chemical structures of the "free" chain transfer agents used in this work.

Employing such star shaped compounds induces a RAFT equilibrium, in which the propagating radicals, the star polymer arms, separate from the central core. Thus, no radical functionality is located on the actual star polymer at any time, whereby starstar coupling reactions are avoided. In addition to that, the raft moiety remains at the 
central core, preventing the controlled formation of additional linear polymer. Very uniform star polymeric material with high microstructural homogeneity became thus accessible. In this work, surface-confined Z-RAFT star polymerizations $(2,4$ and 6 arms) of poly (ethylene glycol) methyl ether methacrylate macromonomers $\left(M_{\mathrm{n}} \sim 500\right.$ $\left.\mathrm{g} \cdot \mathrm{mol}^{-1}\right)$ were performed following the novel approach mentioned above, which is related to the polymer loop formation. (Figure 84) When performing a RAFT polymerization using surface-tethered controlling agents (R-group approach) in the presence of a star-shaped RAFT agent ( $Z$ group approach) (231), (442) in the monomer solution phase, the RAFT process inherently led to surface-linked star polymers. In the case that one RAFT star polymer reacts with more than one surface-tethered polymer chains, looped structures formed, however, still having further dangling arm polymers. Throughout the process, more and more surface tethered polymer chains were incorporated into the star polymer, resulting in strongly bound star polymer.

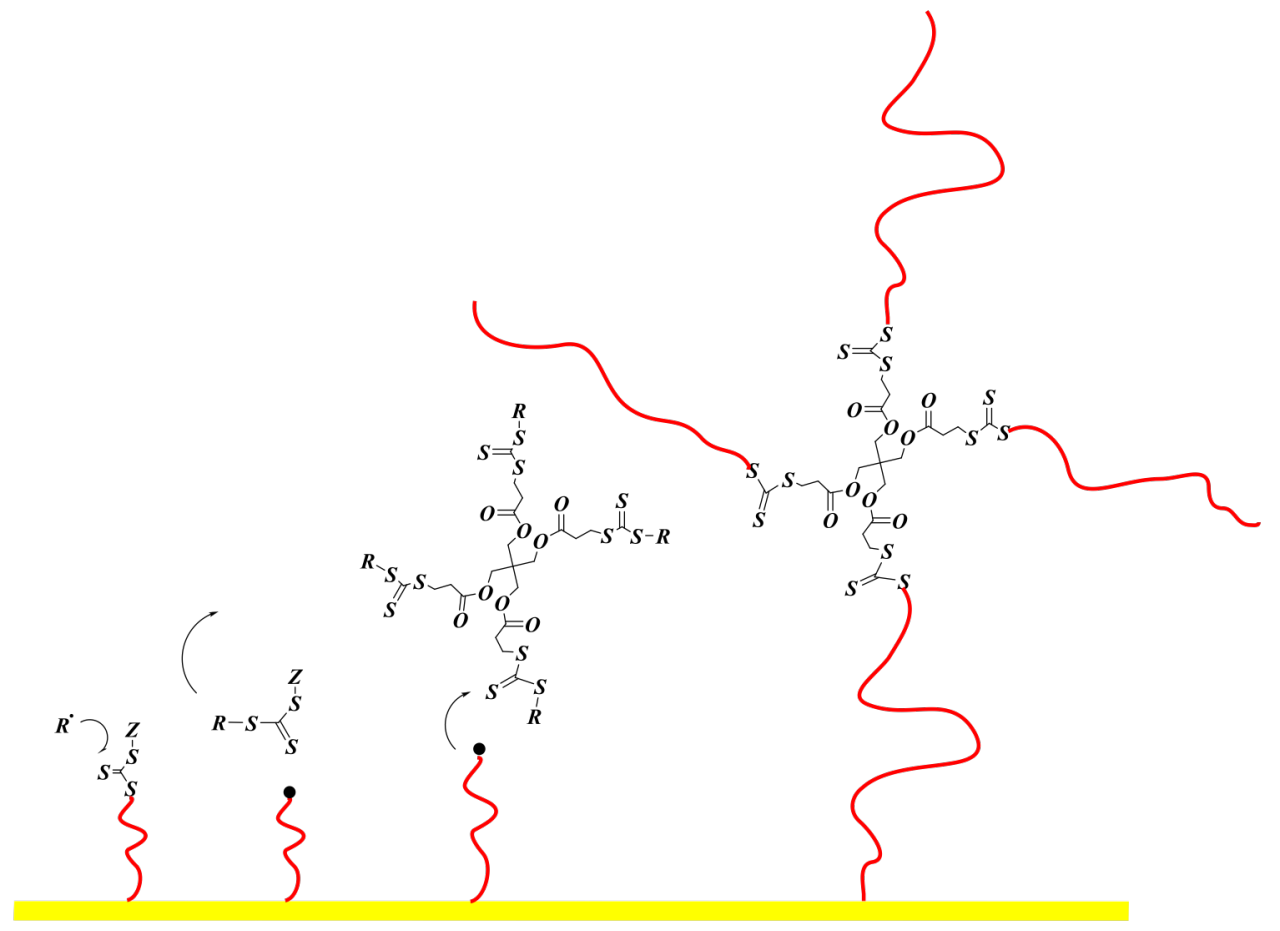

Figure 84 The mechanism of novel Z-Raft approach leading to surface-confined star polymers.

As a consequence, this work focused on a novel synthetic strategy for the development of star polymeric coatings which can prevent or reduce non-specific protein adsorption which is considered to be one of the major causes for the failure of various blood-contacting implantable devices. Moreover, the beneficial features of PEGMA star polymer surfaces, whose generation requires complex procedures, could effectively be retained by using this much easier approach, which is a new avenue to prepare anti-fouling surfaces having extreme significance for biomedical applications. 
Anything will work if you fiddle with it long enough

Murphy's Law 
PART II: RESULTS AND DISCUSSIONS 
5 Characterization of Homopolymer and Diblock Copolymer Brushes Prepared on Gold via Traditional SI-RAFT (R Group Approach)

(c)
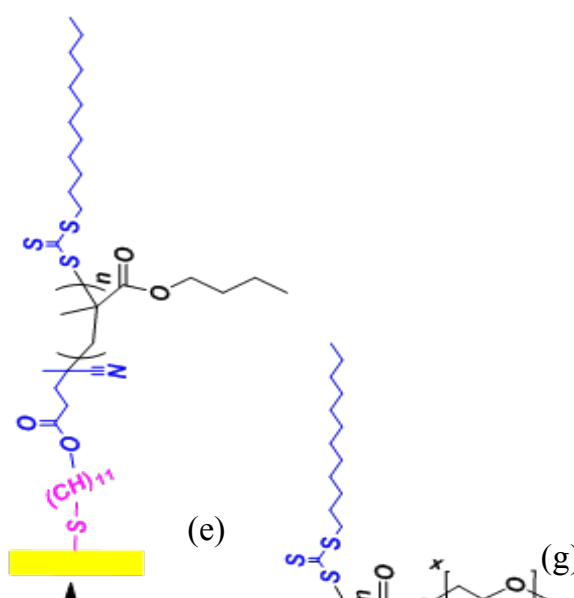

(b)

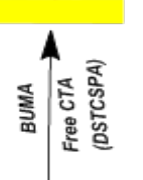

(a)

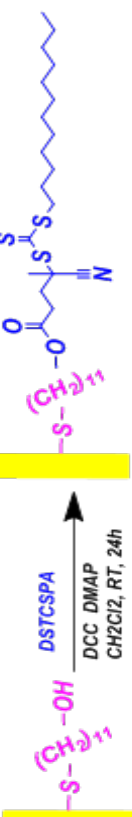

(e)
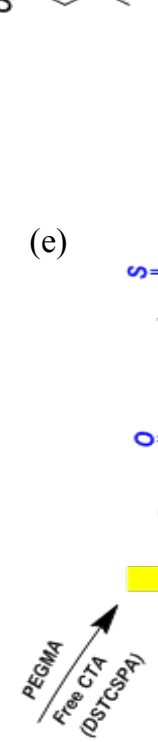

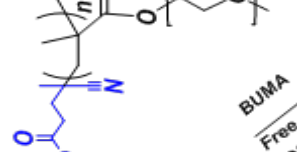

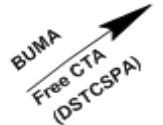

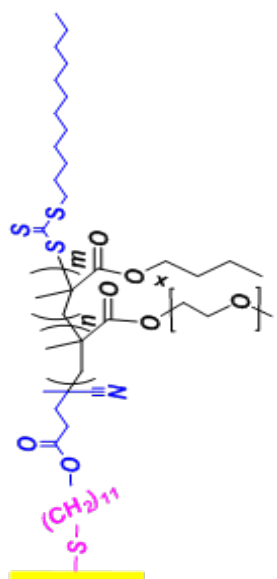

(f)

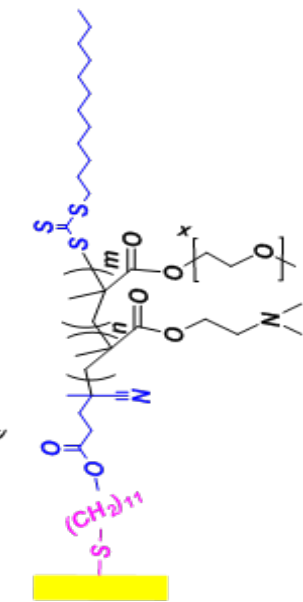

Scheme 1 Schematic diagram illustrating the processes of immobilization of (a) MUD (b) CTA 4-Cyano[dodecylsulfanylthiocarbonyl)sulfanyl] pentanoicacid (DSTCSPA) and the RAFT-mediated polymerization of (c) BuMA in the presence of free CTA (DSTCSPA), (d) DMAEMA in the presence of free CTA (CDB), (e) PEGMA in the presence of free CTA (DSTCSPA) from the gold-MUD-CTA surface blockcopolymerization of (f) PEGMA from the gold-MUD-CTA-PDMAEMA surface and (g) BuMA from the gold-MUD-CTA-PPEGMA surface. 


\subsection{Characterization of Self Assembling Monolayer (MUD) and Raft Agent (CTA) Immobilized Gold Surfaces}

It is known that thiols are highly susceptible to be chemisorped onto gold surface. (19), (225) Mercaptoundecanol (MUD) molecules were immobilized on gold surface by Self Assembly process mentioned in the Experimental section. The resulting MUD thickness was measured by ellipsometry as $1.6 \pm 0.3 \mathrm{~nm}$. The formation of MUD layer on gold was also confirmed by the presence of the peaks at about $2600-2540 \mathrm{~cm}^{-1}$, which are assigned to the aliphatic $\mathrm{CH} 2$ stretching vibrations [Figure 86 (a)]. In the second step, Chain Transfer Agent (CTA) was immobilized to hydroxyl functional gold surface via Steiglich esterification reaction. The CTA immobilization was confirmed by the appearance of ester bands at 1734 associated with $\mathrm{C}=\mathrm{O}$ stretching vibration and $1259 \mathrm{~cm}^{-1}$ attributed to $\mathrm{C}-\mathrm{O}$ stretching vibrations. [Figure 86 (b)] The $\mathrm{C}=\mathrm{S}$ stretching band at around $1045 \mathrm{~cm}^{-1}$ further confirmed successful covalent coupling of the CTA on the reactive SAM. The thickness of CTA layer was measured by ellipsometry as $1.8 \pm 0.4 \mathrm{~nm}$. Surface morphology and cross section of bare gold, MUD coated gold and CTA modified gold substrates are shown in Figure 85 (a), (b), (c). Root-mean-square roughness (RMS) and roughness average of the bare gold is 0.6936 and 0.548 respectively whereas that of MUD layer on gold is 0.9021 and 0.6545. After CTA coupling, these values decreased to 0.3512 and 0.2612 . The sucsessful immobilization of CTA resulted in a change in surface wettability observed by a dramatic increase of static contact angle from $30.53^{\circ}$ (MUD) to $73.84^{\circ}$ (CTA layer) [(Figure 85 (b), (c)].

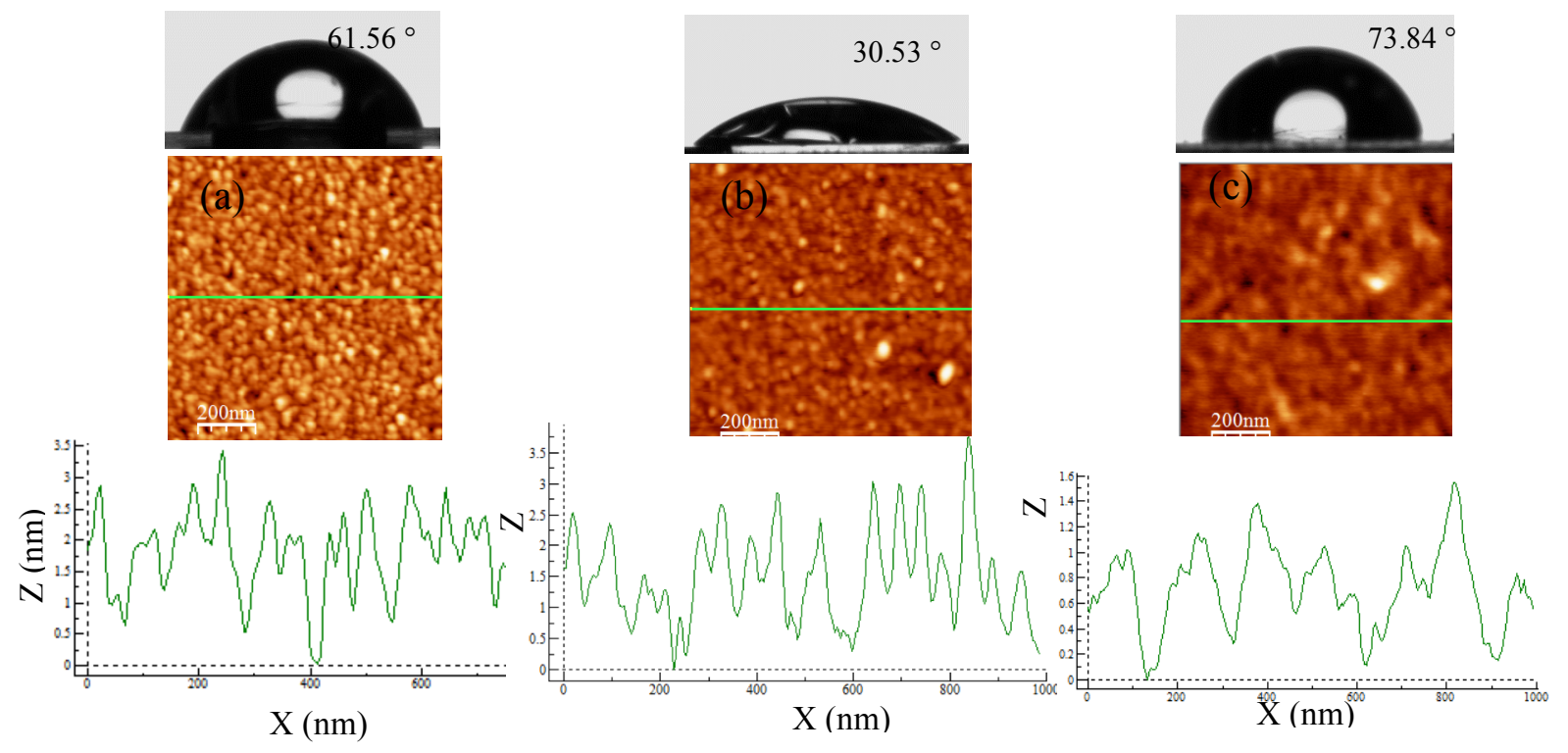

Figure 85 2D AFM topography images $(1 \mu \mathrm{m} \times 1 \mu \mathrm{m})$ in ambient conditions, surface profile analysis and photographs of $1 \mu \mathrm{L}$ water droplets for (a) Bare gold, (b) MUD layer, (c) CTA layer. 


\subsection{Kinetic Investigation of Poly (BuMA) Brush Growth on Gold-MUD-CTA Surface}

It is important to tether high amount of CTAs onto gold surface in order to obtain polymer brush with high grafting density. For this reason, it is important to use an appropriate reaction type for immobilization of CTA onto substrate in order to increase the coupling efficiency. A commercial trithiocarbonate based raft agent (DTSCSPA) was introduced onto MUD coated gold surfaces via Steiglich Esterification reaction to achieve better efficiency. RAFT-mediated polymerizations of BuMA, DMAEMA and PEGMA on gold surfaces were performed in the presence of "sacrificial" CTAs (DTSCSPA or CDB) to obtain better control over the polymerization. The presence of the sacrificial CTA provides the control of overall concentration of monomer and chain transfer agents which is directly connected to the control of molar mass and film thickness of tethered polymer brushes. The correlation of molecular weight of tethered chains with "free," unbounded polymers grown in solution allows indirect analysis of polymer brushes growing on the surface. It is a well known fact that Mn and PDI values of both tethered polymers on surface and bulk polymers in solution are comparable.

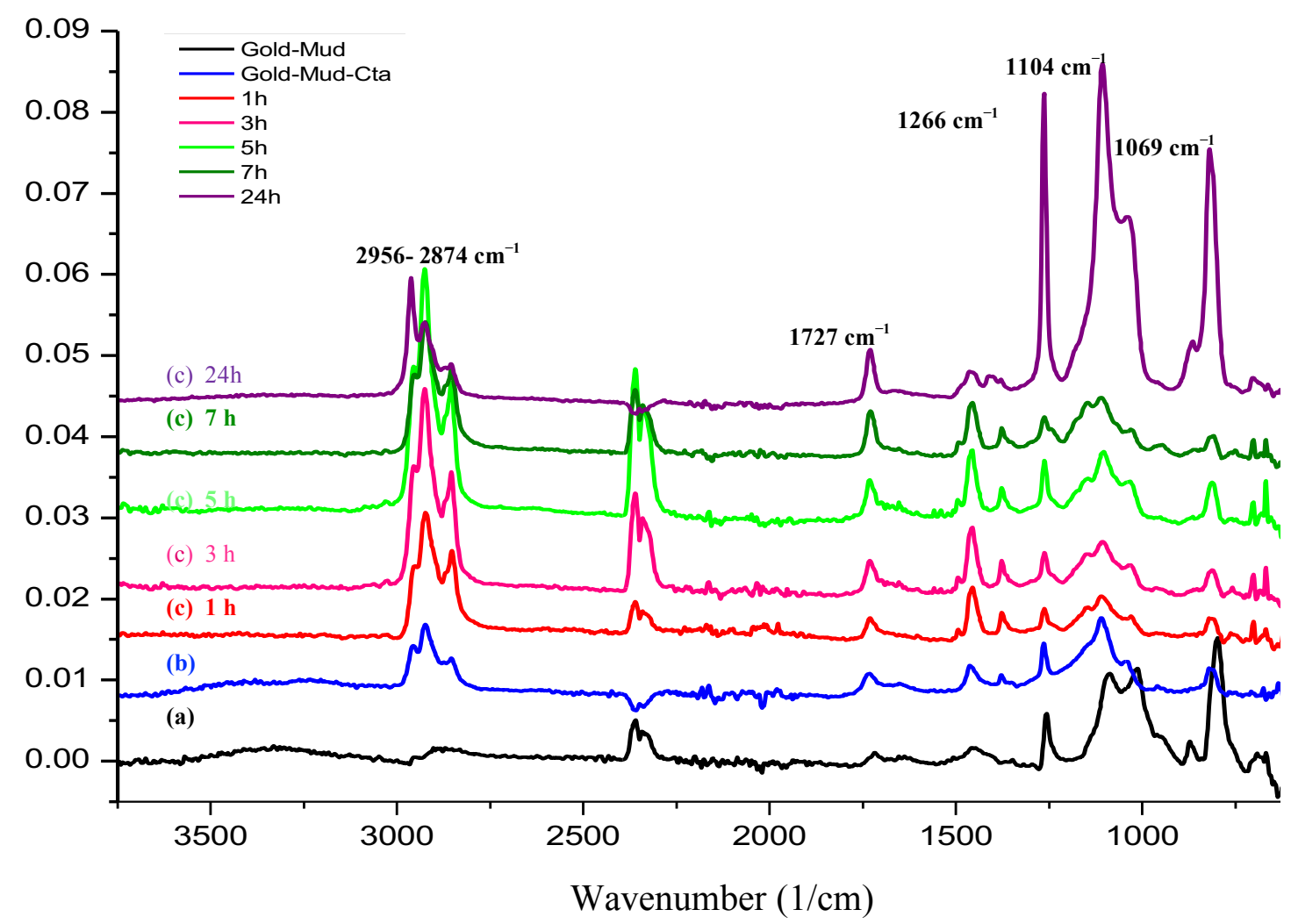

Figure 86 ATR-FTIR spectra of (a) MUD layer, (b) CTA layer, (c) poly (BuMA) brush kinetics synthesized for 1, 3, 5, 7, $24 \mathrm{~h}$. 
Polymerization kinetics were tracked with an attempt to achieve the characterization of SI-RAFT process from gold surfaces. The formation of poly (BUMA) brushes was confirmed by ATR-IR (Figure 86), AFM and water contact-angle measurements (Figure 87). The ATR-FTIR spectra of the poly (BUMA) brush demonstrated characteristic bands at $\sim 1727 \mathrm{~cm}^{-1}$, associated with the carbonyl stretch and the strong absorption band at $1069 \mathrm{~cm}^{-1}$ resulted from $\mathrm{C}=\mathrm{S}$ stretching vibrations suppressed by absorption bands at $1104 \mathrm{~cm}^{-1}$ and $1266 \mathrm{~cm}^{-1}$ associated with $\mathrm{C}-\mathrm{O}$ stretching of $\mathrm{C}(=\mathrm{O})$ $\mathrm{O}-\mathrm{C}$ bonds in monomer units. The bands at around 2956, 2927, $2874 \mathrm{~cm}^{-1}$ are considered to be due to aliphatic $\mathrm{CH}$ groups. The $2956,2874 \mathrm{~cm}^{-1}$ peaks are due to asymmetric and symmetric stretching vibrations of $\mathrm{CH}_{3}$, respectively, while the 2927 $\mathrm{cm}^{-1}$ peak is assigned to $\mathrm{CH}_{2}$ vibration. In addition to these bands, there are bands at around 701-609 $\mathrm{cm}^{-1}$ associated with C-S stretching and peaks at $1464 \mathrm{~cm}^{-1}, 1380$ $\mathrm{cm}^{-1}$ attributed to the $\mathrm{CH}$ bending and rocking respectively. An increase in water contact angle from $73.84^{\circ}$ (CTA layer) to $89.70^{\circ}$ (PBuMA layer) also confirmed that a hydrophobic polymer layer was obtained. The area shown in Figure 87 for 24 hours polymerization time and most of the surface areas for same sample (data not shown) display gaussian distribution which is an indicator of surface homogenity although small aggregates heterogeneously distributed over some areas of sample were observed in related AFM height image. Similar morphologies were also observed for $1 \mathrm{~h}$ polymerization time whereas these aggregations are not observable anymore for $3 \mathrm{~h}$, $5 \mathrm{~h}, 7 \mathrm{~h}$ polymerization time (data not shown). The change in film morphology is associated with a slight increase in the RMS roughness from $1.3837 \mathrm{~nm}$ (1h polymerization time) to $1.5497 \mathrm{~nm}$ ( $24 \mathrm{~h}$ polymerization time).

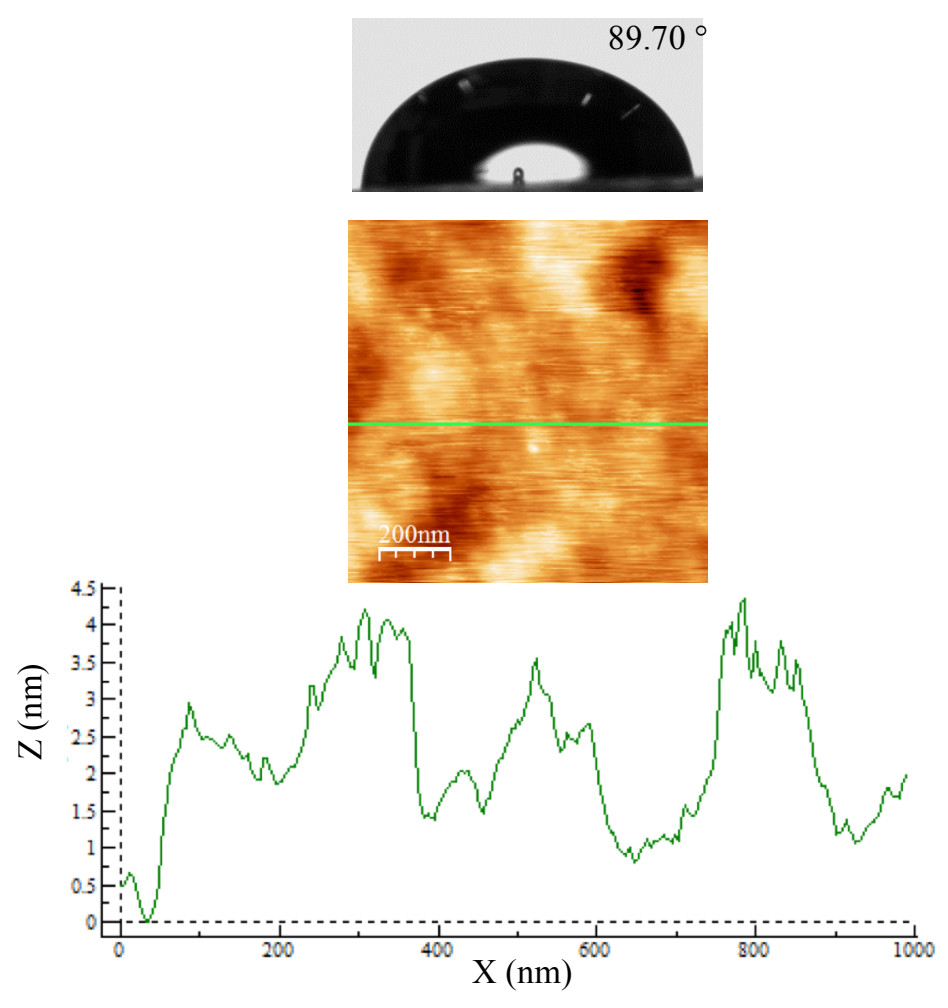


Figure 87 2D AFM topography image $(1 \mu \mathrm{m} \times 1 \mu \mathrm{m})$ in ambient conditions, surface profile analysis and photograph of $1 \mu \mathrm{L}$ water droplet for poly (BuMA) brushes synthesized for $24 \mathrm{~h}$ with the ratio of $[\mathrm{M}] /[\mathrm{CTA}] /[\mathrm{I}]=2000 / 4 / 1$ in feed.

The time evolution of number-average molecular weight, $M_{n}$ and polydispersity, PDI of the grafted polymer chains on gold, which is assumed to be the same as that of the unbound polymer chains in solution (263) was obtained by GPC analysis (shown in Table 3). Typical GPC traces of free polymers with the increase of polymerization were monomodal and had narrow molecular weight distributions, which are characteristics of well-defined polymers prepared via RAFT-mediated polymerization. The elution peaks shift to higher molecular weight with increasing reaction time (data not shown). After 24 hours polymerization, the monomer conversion is almost $88.5 \%$; GPC analysis of the free polymers reveals a $M_{n}$ of $41,925 \mathrm{~g} / \mathrm{mol}$ and a PDI of 1.32 . Figure 88 (d) presents the evolution of the $M_{n}$ of the free poly (BuMA) brushes as a function of monomer conversion. The fact that the experimental molecular weights of the free polymer determined by GPC increased linearly with monomer conversion and the PDI values are all below 1.32 indicate that the polymerization was conducted in a controlled manner.
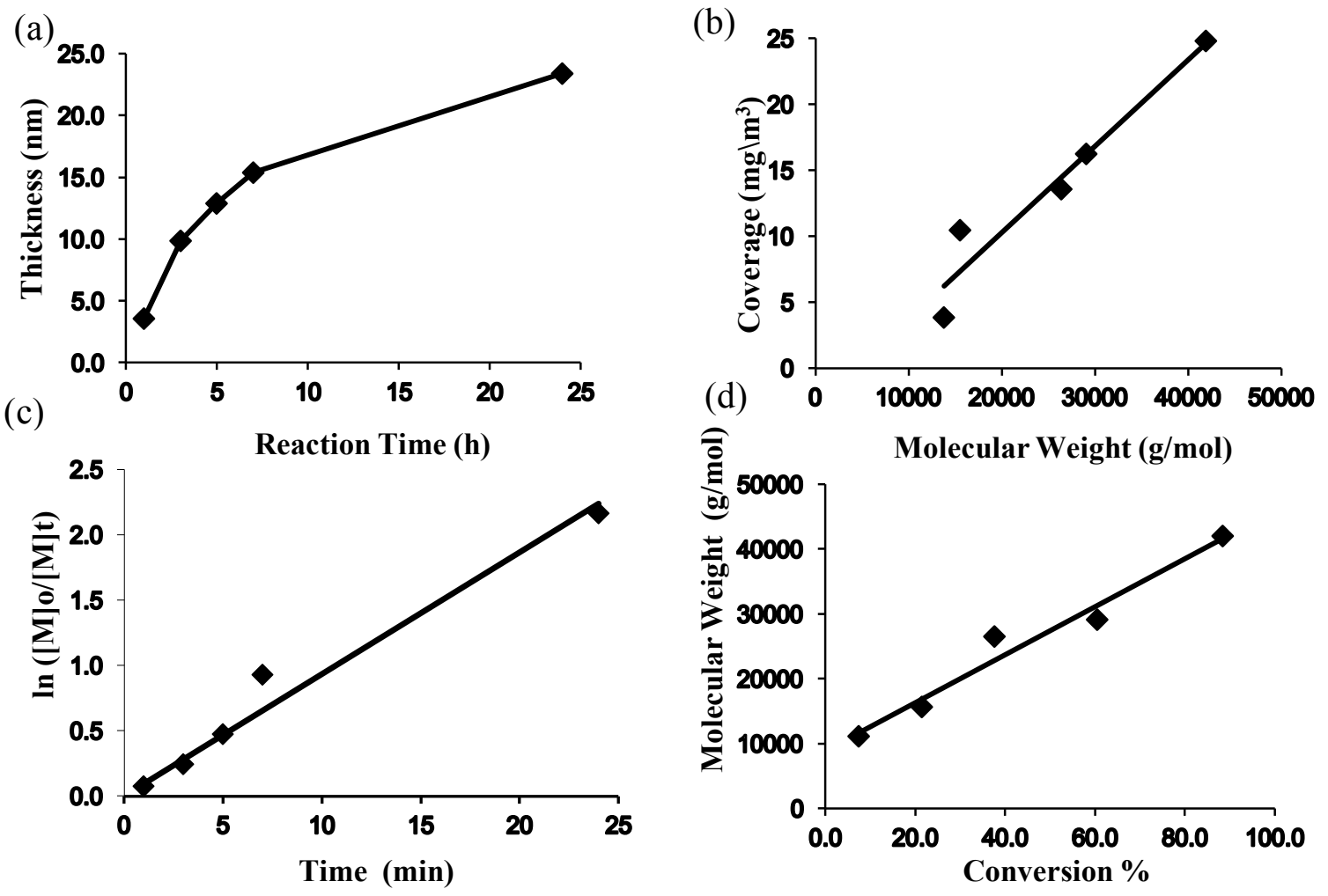

Figure 88 Results of Raft mediated polymerizations of BuMA in Toluen at $60{ }^{\circ} \mathrm{C}$ with the ratio of $[\text { Monomer }]_{\mathrm{o}} \backslash[\text { freeCTA }]_{\mathrm{o}} \backslash[\mathrm{AIBN}]_{\mathrm{o}}=2000 \backslash 4 \backslash 1$ in feed a) thickness profiles of poly (BuMA) brushes, $b$ ) the surface coverage plotted against molecular weight of 
poly (BuMA) brushes, c) plot of pseudo-first-order kinetics of poly (BuMA) brushes, d) molecular weights of poly (BuMA) brushes with inreasing monomer conversion.

Additional evidence of the controlled polymerization is also provided by the free poly (BuMA) brushes formed from the free raft agent. Figure 88 (c) shows the linear relationship between $\ln \left[\mathrm{M}_{\mathrm{o}}\right] \backslash\left[\mathrm{M}_{\mathrm{t}}\right]$ and time, where $\left[\mathrm{M}_{\mathrm{o}}\right]$ is the initial monomer concentration and $\left[\mathrm{M}_{\mathrm{t}}\right]$ is the monomer concentration remained in reaction mixture after $t$ hour polymerization time. The result indicates that the concentration of the growing species remains constant and first-order kinetic is obtained. The dependence of the Ellipsometric thickness of poly (BuMA) brush grown from the chain transfer agent immobilized surface via RAFT mediated polymerization on polymerization time is shown in Figure 88 (a).

The brush thickness initially increases linearly with polymerization time up to $7 \mathrm{~h}$ and deviates afterwards, which is consistent to with the literature data. (241), (508) The brush growth began to slow down and reached a plateau of $23.4 \mathrm{~nm}$ in film thickness. The reason for this deviation from linear behavior might be increase in steric hindrance to chain growth caused by increase in size, length and molecular weight of grafted polymer chains growing on surface, leading to limitation of monomer delivery in the latter stage of polymerization. Other reason might be enhanced recombination of polymeric radicals growing on surface. (508) It is also clearly observable that the surface coverage of polymer brushes displays linear increase with the increase in molecular weight. [Figure 88 (b)]

Table 3 Summary of polymerization kinetics of poly (BuMA) brushes

\begin{tabular}{|c|c|c|c|c|c|c|c|}
\hline $\begin{array}{l}\text { Reaction } \\
\text { Time } \\
\text { (h) }\end{array}$ & $\mathbf{M n} / \mathbf{M w}$ & PDI & $\begin{array}{l}\text { Thickness }{ }^{a} \\
\text { (nm) }\end{array}$ & $\begin{array}{c}\text { Coverage } \\
\left(\mathbf{m g} / \mathrm{m}^{2}\right)\end{array}$ & $\begin{array}{c}\text { Grafting } \\
\text { Density } \\
\text { (chains/ } \\
\mathbf{n m}^{2} \text { ) }\end{array}$ & $\begin{array}{c}\text { Distance } \\
\text { (nm) }\end{array}$ & $\begin{array}{l}\text { Static } \\
\text { Contact } \\
\text { Angle }^{b}\end{array}$ \\
\hline 1 & $13821 / 17983$ & 1.30 & 3.6 & 3.79 & 0.16 & 2.8 & $83.65^{\circ}$ \\
\hline 3 & $15572 / 19654$ & 1.26 & 9.9 & 10.42 & 0.40 & 1.8 & $84.17^{\circ}$ \\
\hline 5 & $26419 / 31603$ & 1.19 & 12.9 & 13.58 & 0.30 & 2 & $86.80^{\circ}$ \\
\hline 7 & $29056 / 33836$ & 1.16 & 15.4 & 16.21 & 0.33 & 1.96 & $88.74^{\circ}$ \\
\hline 24 & $41925 / 55695$ & 1.32 & 23.4 & 24.78 & 0.35 & 1.90 & $89.70^{\circ}$ \\
\hline
\end{tabular}

Polymerization of (buthyl methacrylate) BuMA with 4-Cyano-4[dodecylsulfanylthiocarbonyl)sulfanyl] pentanoicacid (DSTCSPA) and 2,2 ${ }^{\circ}$ 
azobis(isobutyronitrile) (AIBN) in toluene at $60{ }^{\circ} \mathrm{C}$ for $24 \mathrm{~h} .{ }^{a}$ Thicknesses were determined by ellipsometry and are an average of five samplings across the sample. Error of thicknesses measured was within $\pm 0.1 \mathrm{~nm}$. ${ }^{b}$ Static contact angles were taken using goniometry and are an average of five samplings across the sample. The standard deviation of contact angles was less than $1^{\circ}$.

The surface coverages, A $\left(\mathrm{mg} / \mathrm{m}^{2}\right)$, grafting densities, $\sigma$ (chains $\left./ \mathrm{nm}^{2}\right)$, and average distances between grafting sites, $D(\mathrm{~nm})$, of grafted poly(BUMA) brushes were calculated from the $M_{n}$ of the free polymer and the dry film thickness, $h(\mathrm{~nm})$, according to equations 18, 19 and 20 and summarized in Table 3. As a result of that the grafting densities range between 0.16 and 0.35 chains per $\mathrm{nm}^{2}$, poly(BUMA) brushes might be considered as a brush type between semi-dilute and a high density brush, which is closer to a high density conformation than semi-dilute. (509)

\subsection{Kinetic Investigation of Poly (DMAEMA) Brush Growth on Gold-MUD- CTA Surface}

Polymerization kinetics were tracked with an attempt to achieve the characterization of SI-RAFT process from gold surfaces. The formation of poly (DMAEMA) brushes was confirmed by ATR-FTIR (Figure 89), AFM and water contact angle measurements (Figure 90). The ATR-FTIR spectra for the poly (DMAEMA) brush demonstrated characteristic absorption bands at $\sim 1730 \mathrm{~cm}^{-1}$ associated with the carbonyl stretch, at $1046 \mathrm{~cm}^{-1}$ resulted from strong $\mathrm{C}=\mathrm{S}$ stretching vibrations suppressed by absorption bands at $1109 \mathrm{~cm}^{-1}, 1264 \mathrm{~cm}^{-1}$ associated with C-O stretching of $\mathrm{C}(=\mathrm{O})-\mathrm{O}-\mathrm{C}$ bonds in monomer units and bands at $1243-1023 \mathrm{~cm}^{-1}$ resulted from vibrations of C-N stretching. The bands at around 2953, 2922, $2852 \mathrm{~cm}^{-1}$ are considered to be due to aliphatic $\mathrm{CH}$ groups. The 2953, $2852 \mathrm{~cm}^{-1}$ peaks are due to asymmetric and symmetric stretching vibrations of $\mathrm{CH} 3$, respectively, while the $2922 \mathrm{~cm}^{-1}$ peak is assigned to $\mathrm{CH} 2$ vibration. A slight shift is observed in stretching vibrations of $\mathrm{CH} 3$ compared to PBuMA as a result of being overlapped by the characteristic peaks for the $-\mathrm{N}(\mathrm{CH} 3)_{2}$, which generally appears at $\sim 2830 \mathrm{~cm}^{-1}$. In addition to these bands, there are bands at $703-614 \mathrm{~cm}^{-1}$ associated with $\mathrm{C}-\mathrm{S}$ stretching while the peaks at $1457 \mathrm{~cm}^{-1}$ and $1374 \mathrm{~cm}^{-1}$ are attributed to the $\mathrm{CH}$ bending and rocking, respectively. The broad band at $3693-3113 \mathrm{~cm}^{-1}$ which is assigned to O-H stretching, results from water vapor which PDMAEMA brush coating absorbs due to its hydrophilic nature. The thicker the brush the more pronounced the band is.

A slight decrease in water contact angle from $73.84^{\circ}$ (gold-MUD-CTA layer) to $58.25^{\circ}$ (PDMAEMA layer) also confirmed that a relatively more hydrophilic polymer layer than CTA immobilized gold was obtained. The area shown in Figure 90 for 24 hours polymerization time and the other areas measured on same sample (data not shown) display gaussian distribution which is an indicator of surface homogenitiy. The 
change in film morphology is associated with a slight decrease in the RMS roughness from $2.3747 \mathrm{~nm}$ (1h polymerization time) to $1.4317 \mathrm{~nm}$ ( $24 \mathrm{~h}$ polymerization time).

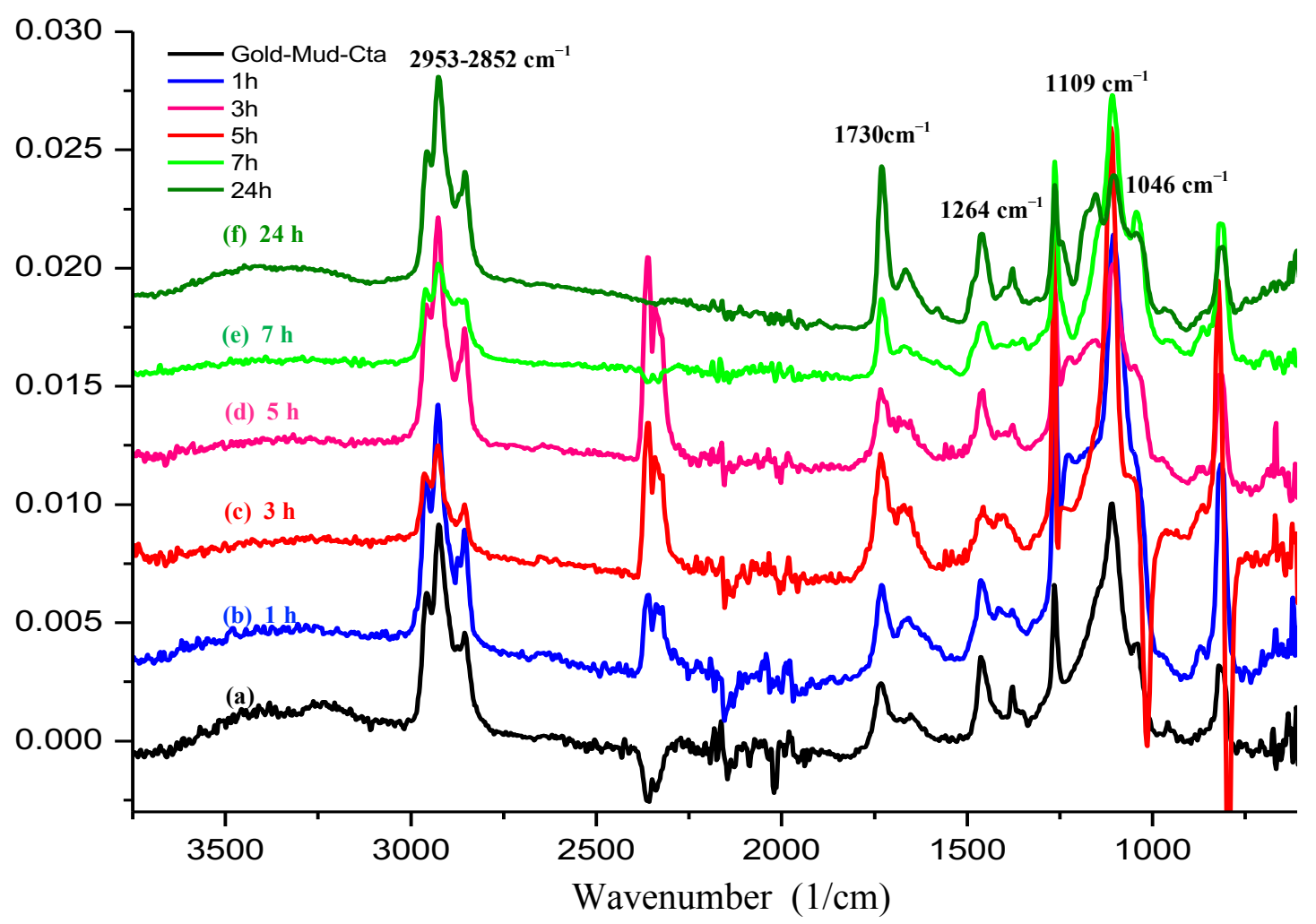

Figure 89 ATR-FTIR spectra of (a) CTA layer, (b),(c),(d),(e),(f) poly (DMAEMA) brush kinetics synthesized for 1, 3, 5, 7, 24 h respectively.

GPC traces of free polymers with the increase of polymerization were monomodal and had narrow molecular weight distributions, which are characteristics of welldefined polymers prepared via RAFT-mediated polymerization. The elution peaks shift to higher molecular weight with increasing reaction time (data not shown). After 24 hours polymerization, the monomer conversion is $89.3 \%$; GPC analysis of the free polymers reveals a $\mathrm{Mn}$ of 40,817 g/mol and a PDI of 1.19. Figure 91 (d) represents the evolution of the Mn of the free poly (DMAEMA) brushes as a function of monomer conversion. The fact that the experimental molecular weights of the free polymer determined by GPC increased linearly with monomer conversion and the PDI values are all below 1.33 indicate that the polymerization was conducted in a controlled manner. Additional evidence of the controlled polymerization is also provided by the free poly (DMAEMA) brushes formed from the free raft agent. Figure 91 (c) shows the linear relationship between $\ln [\mathrm{Mo}] \backslash[\mathrm{Mt}]$ and time, where $[\mathrm{Mo}]$ is the initial monomer concentration and $[\mathrm{Mt}]$ is the monomer concentration remained in reaction mixture after $t$ hour polymerization time. The result indicates that the concentration of the growing species remains constant and first-order kinetic is obtained. The dependence of ellipsometric thickness of poly (DMAEMA) brush grown from the 
chain transfer agent immobilized surface via RAFT mediated polymerization on polymerization time is shown in Figure 91 (a).

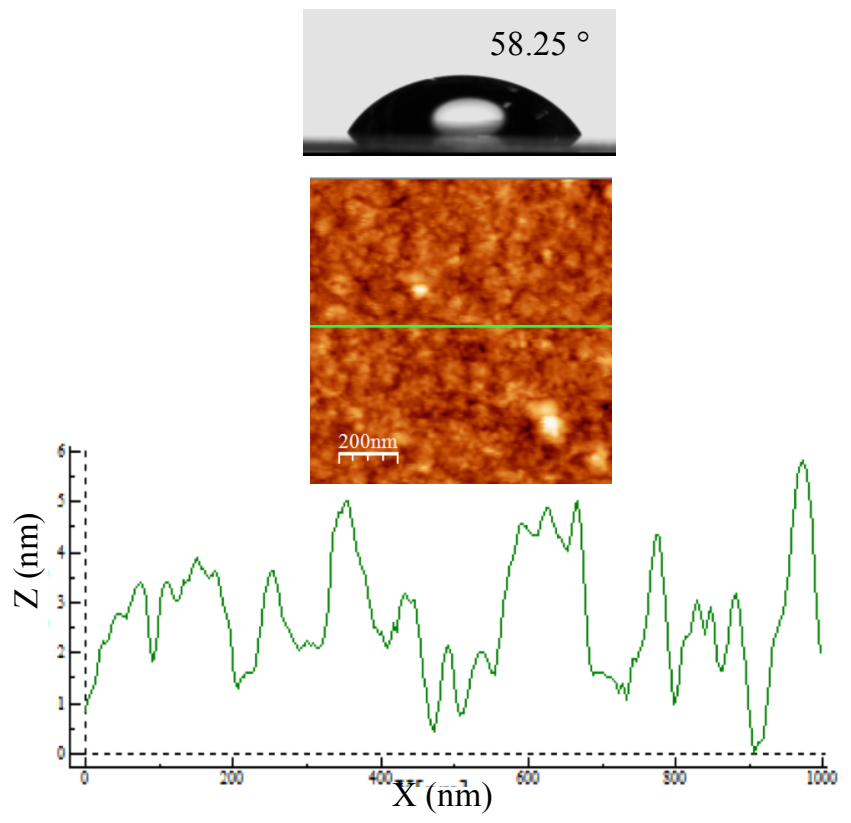

Figure 90 2D AFM topography image $(1 \mu \mathrm{m} \times 1 \mu \mathrm{m})$ in ambient conditions, surface profile analysis and photograph of $1 \mu \mathrm{L}$ water droplet for poly (DMAEMA) brushes synthesized for $24 \mathrm{~h}$ with the ratio of $[\mathrm{M}] /[\mathrm{CTA}] /[\mathrm{I}]=2000 / 4 / 1$ in feed.
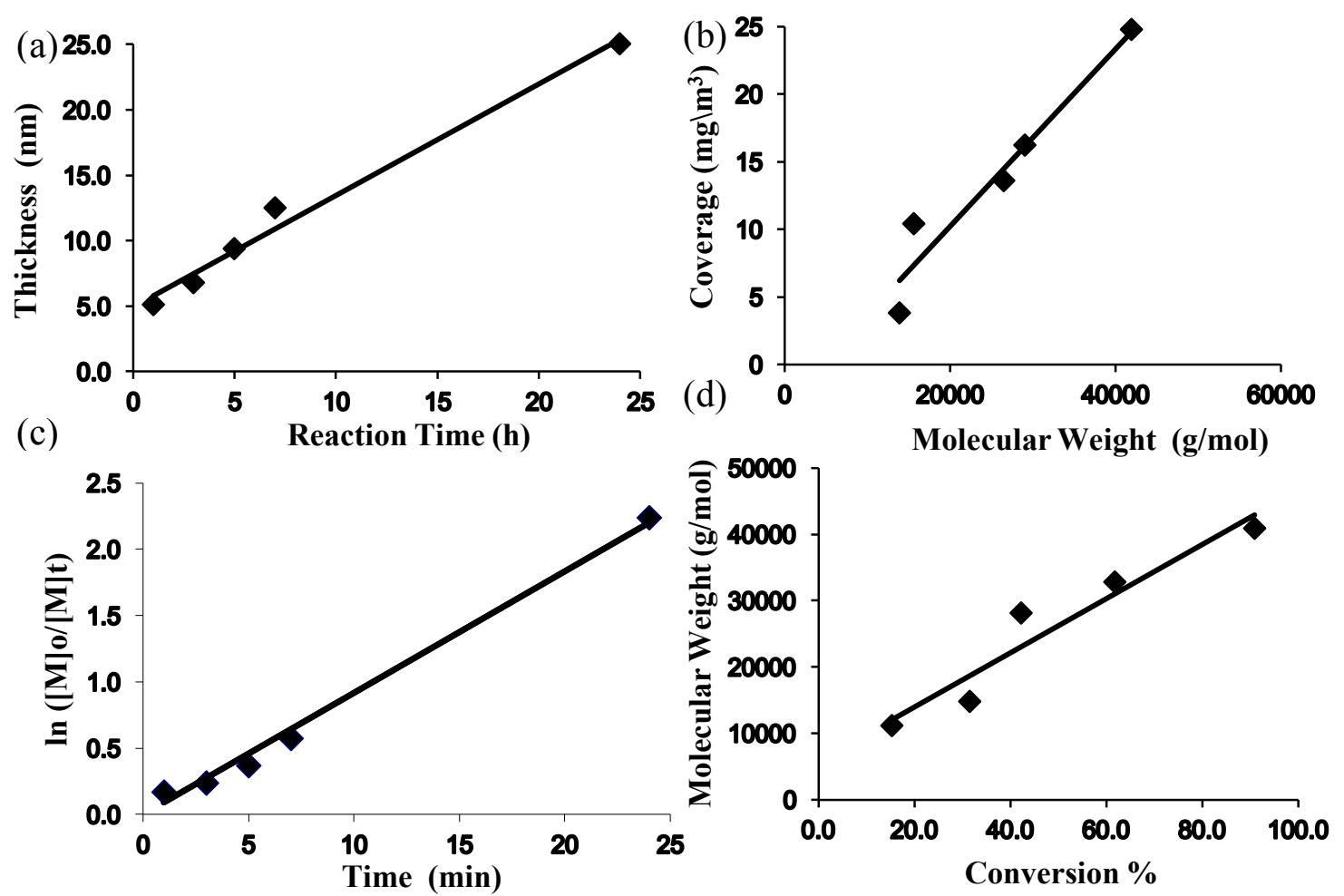

Figure 91 Results of Raft mediated polymerizations of DMAEMA in Toluen at $60{ }^{\circ} \mathrm{C}$ with the ratio of $[\text { Monomer }]_{0} \backslash[\text { freeCTA }]_{0} \backslash[\mathrm{AIBN}]_{\mathrm{o}}=2000 \backslash 4 \backslash 1$ in feed a) thickness 
profile of poly (DMAEMA) brushes, $b$ ) the surface coverage plotted against molecular weight of poly (DMAEMA) brushes, c) plot of pseudo-first-order kinetics of poly (DMAEMA) brushes, d) molecular weights of poly (DMAEMA) brushes with inreasing monomer conversion.

The brush thickness increases linearly with polymerization time and reaches $25 \mathrm{~nm}$, which is another proof of that polymerization proceeds in a controlled manner. It is also clearly observable that the surface coverage of polymer brushes displays linear increase with the increase in molecular weight [Figure 91 (b)]. The surface coverages, A $\left(\mathrm{mg} / \mathrm{m}^{2}\right)$, grafting densities, $\sigma$ (chains $\left./ \mathrm{nm}^{2}\right)$, and average distances between grafting sites, D (nm), of grafted polymer brushes were calculated from the Mn of the free polymer and the dry film thickness, $\mathrm{h}(\mathrm{nm})$, according to equations 18, 19 and 20 and summarized in Table 4. As a result of that the grafting density is 0.50 chains per $\mathrm{nm}^{2}$, poly (DMAEMA) brushes might be considered as a brush type between semi-dilute and a high density brush, which is closer to a high density conformation than semidilute. (509)

Table 4 Summary of polymerization kinetics of poly (DMAEMA) Brushes

\begin{tabular}{|c|c|c|c|c|c|c|c|}
\hline $\begin{array}{l}\text { Reaction } \\
\text { Time } \\
\text { (h) }\end{array}$ & $\mathbf{M n} / \mathbf{M w}$ & PDI & $\begin{array}{c}\text { Thickness }^{\text {a }} \\
\text { (nm) }\end{array}$ & $\begin{array}{c}\text { Coverage } \\
\left(\mathrm{mg} / \mathrm{m}^{2}\right)\end{array}$ & $\begin{array}{l}\text { Grafting } \\
\text { Density } \\
\text { (chains / } \\
\mathbf{n m}^{2} \text { ) }\end{array}$ & $\begin{array}{c}\text { Distance } \\
(\mathbf{n m})\end{array}$ & $\begin{array}{l}\text { Static } \\
\text { Contact } \\
\text { Angle }^{b}\end{array}$ \\
\hline 1 & $11126 / 13792$ & 1.23 & 5.1 & 6.88 & 0.37 & 1.8 & $66.63^{\circ}$ \\
\hline 3 & $14753 / 18932$ & 1.28 & 6.8 & 9.18 & 0.37 & 1.8 & $64.01^{\circ}$ \\
\hline 5 & $28073 / 37474$ & 1.33 & 9.4 & 12.7 & 0.27 & 2.2 & $60.65^{\circ}$ \\
\hline 7 & $32818 / 42216$ & 1.28 & 12.5 & 16.53 & 0.30 & 2 & $58.61^{\circ}$ \\
\hline 24 & $40817 / 48975$ & 1.19 & 25 & 33.75 & 0.50 & 1.6 & $58.25^{\circ}$ \\
\hline
\end{tabular}

Polymerization of (2-(dimethylamino) ethyl methacrylate) DMAEMA with cumyl dithiobenzoate (CDB) and 2,2 $2^{\circ}$-azobis(isobutyronitrile) (AIBN) in toluene at $60^{\circ} \mathrm{C}$ for $24 \mathrm{~h}$. a Thicknesses were determined by ellipsometry and are an average of five samplings across the sample. Error of thicknesses measured was within $\pm 0.1 \mathrm{~nm}$. b Static contact angles were taken using goniometry and are an average of five samplings across the sample. The standard deviation of contact angles was less than $1^{\circ}$. 


\subsection{Characterization of Poly (PEGMA) Synthesized on Gold-MUD-CTA Surface}

The formation of poly (PEGMA) brush was confirmed by ATR-IR [Figure 93 (a)], AFM and water contact angle measurements (Figure 92). The ATR-FTIR spectra for the poly (PEGMA) brush demonstrated characteristic absorption bands at $\sim 1730 \mathrm{~cm}^{-1}$ associated with the carbonyl stretch, at $1044 \mathrm{~cm}^{-1}$ resulted from strong $\mathrm{C}=\mathrm{S}$ stretching vibrations suppressed by absorption bands at $1262 \mathrm{~cm}^{-1}$ associated with $\mathrm{C}-\mathrm{O}$ stretching and $1104 \mathrm{~cm}^{-1}$ resulted from vibrations of C-O-C ether bonds in the ethylene glycol units. The bands at around 2960,2924, $2852 \mathrm{~cm}^{-1}$ are considered to be due to aliphatic $\mathrm{CH}$ groups. The $2960,2852 \mathrm{~cm}^{-1}$ peaks are due to asymmetric and symmetric stretching vibrations of $\mathrm{CH} 3$, respectively, while the $2924 \mathrm{~cm}^{-1}$ peak is assigned to $\mathrm{CH} 2$ vibration. The peaks at $1457 \mathrm{~cm}^{-1}$ and $1376 \mathrm{~cm}^{-1}$ are attributed to the $\mathrm{CH}$ bending and rocking respectively. In addition to these bands, there is absorption band at 703$620 \mathrm{~cm}^{-1}$ attributed to C-S stretching. The broad band at $3666-3108 \mathrm{~cm}^{-1}$ which is assigned to $\mathrm{O}-\mathrm{H}$ stretching, results from water vapor which PPEGMA brush coating absorbs due to its hydrophilic nature.

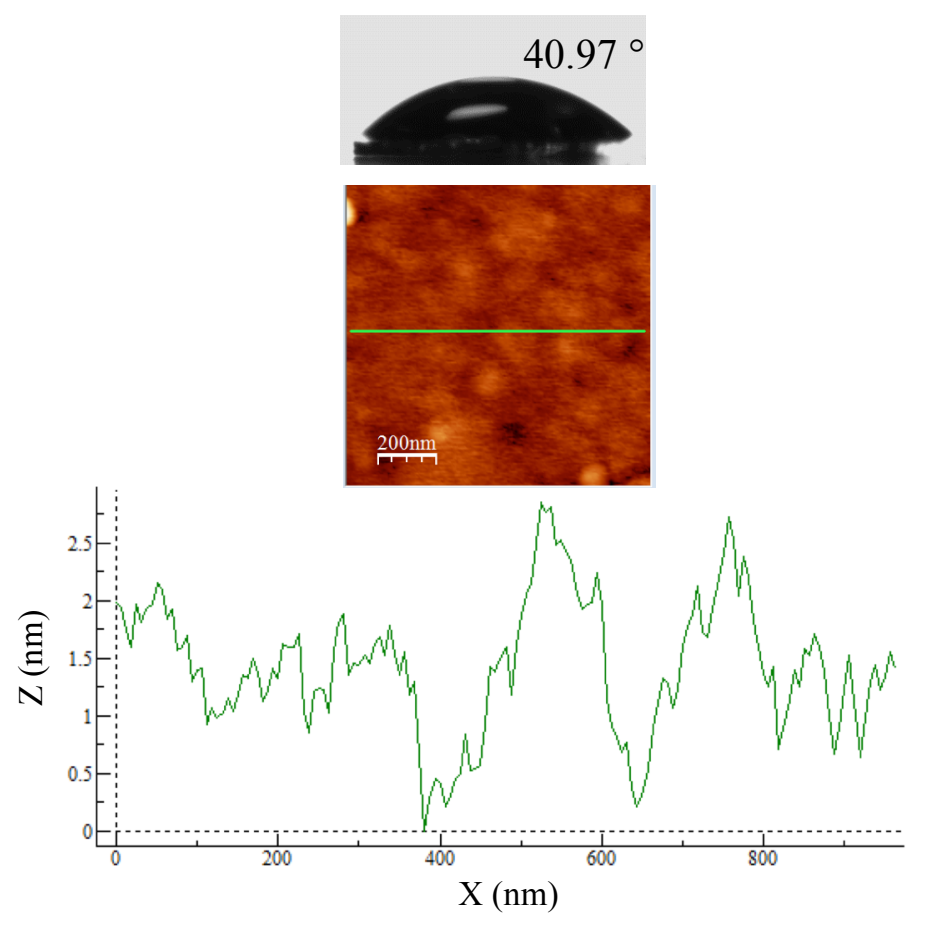

Figure 92 2D AFM topography image $(1 \mu \mathrm{m} \times 1 \mu \mathrm{m})$ in ambient conditions, surface profile analysis and photograph of $1 \mu \mathrm{L}$ water droplet for poly(PEGMA) brushes synthesized for $5 \mathrm{~h}$ with the ratio of $[\mathrm{M}] /[\mathrm{CTA}] /[\mathrm{I}]=2000 / 4 / 1$.

A drastic decrease in water contact angle from $73.84^{\circ}$ (CTA layer) to $40.97^{\circ}$ (PPEGMA layer) also confirmed that a hydrophilic polymer layer was obtained. The area shown in Figure 92 for 5 hours polymerization time and the other areas measured on same sample (data not shown) display gaussian distribution which is an indicator 
of surface homogenitiy and closely packed chains. The RMS roughness value is 0.7917 $\mathrm{nm}$. GPC trace of free polymers was monomodal and had narrow molecular weight distribution, which are characteristics of well-defined polymers prepared via RAFTmediated polymerization. After $5 \mathrm{~h}$ polymerization time, the monomer conversion is $20 \%$ while the thickness is $9.9 \mathrm{~nm}$. GPC analysis of the free polymers reveals a $\mathrm{M}_{\mathrm{n}}$ of $32850 \mathrm{~g} / \mathrm{mol}$ and a PDI of 1.35 . (Table 5) The low PDI value indicates that the polymerization was conducted in a controlled manner. The surface coverage, A $\left(\mathrm{mg} / \mathrm{m}^{2}\right)$, grafting density, $\sigma\left(\right.$ chains $\left./ \mathrm{nm}^{2}\right)$, and average distance between grafting sites, $\mathrm{D}(\mathrm{nm})$, of grafted poly (PEGMA) brushes were calculated from the $\mathrm{M}_{\mathrm{n}}$ of the free polymer and the dry film thickness, h (nm), according to equations 18, 19 and 20. (Table 5) As a result of that the grafting density is 0.20 chains per $\mathrm{nm}^{2}$, poly(PEGMA) brushes might be considered as a semi dilute brush. (241) The gold substrate covered with $9.9 \pm 0.1 \mathrm{~nm}$ poly(PEGMA) brushes, which was obtained from 5 hours homopolymerization step, was used as model substrate for RAFT mediated block copolymerization of BUMA.

\subsection{Characterization of Diblock Copolymer Brush (PPEGMA-b-PBuMA) Synthesized on Gold-PPEGMA Surface}

One of the advantages in using RAFT technique to prepare polymer brushes via the grafting from technique is the opportunity to produce block copolymer brushes by reactivation of the dormant chains. The preparation of a block copolymer brush is also another confirmation of whether the previous sample has maintained the living characteristics of a well defined RAFT. Thus, the gold substrate coated with $9.9 \mathrm{~nm}$ poly (PEGMA) brushes was used for subsequent formation of diblock copolymer brushes in order to check living nature of the surface bound homopolymer brushes prepared via surface immobilized RAFT polymerization.

The formation of blockcopolymer brush Poly (BuMA) as a second layer on the gold substrate covered with poly(PEGMA) brushes was confirmed by ATR-IR [Figure 93 (b)], AFM and water contact angle measurements. (Figure 94) The ATR-FTIR spectra for the PPEGMA-block-PBuMA brush demonstrated characteristic bands at $1728 \mathrm{~cm}^{-1}$ associated with the carbonyl stretch, at $1042 \mathrm{~cm}^{-1}$ resulted from strong $\mathrm{C}=\mathrm{S}$ stretching vibrations suppressed by absorption bands at $1113 \mathrm{~cm}^{-1}$ resulted from vibrations of C$\mathrm{O}-\mathrm{C}$ ether bonds in the ethylene glycol units which belongs to first PPEGMA layer and at $1262 \mathrm{~cm}^{-1}$ associated with C-O stretching. The bands at around 2958, 2929, $2850 \mathrm{~cm}^{-1}$ are considered to be due to aliphatic $\mathrm{CH}$ groups. The $2958,2850 \mathrm{~cm}^{-1}$ peaks are due to asymmetric and symmetric stretching vibrations of $\mathrm{CH}_{3}$, respectively, while the $2929 \mathrm{~cm}^{-1}$ peak is assigned to $\mathrm{CH}_{2}$ vibration. The peaks at $1464 \mathrm{~cm}^{-1}$ and 1379 $\mathrm{cm}^{-1}$ are attributed to the $\mathrm{CH}$ bending and rocking, respectively. In addition to these bands, there are bands at $718-620 \mathrm{~cm}^{-1}$ attributed to $\mathrm{C}-\mathrm{S}$ stretching. Moreover, dissappearence of the broad band at $3666-3108 \mathrm{~cm}^{-1}$ which is assigned to O-H stretching, results from water vapor which hydrophilic PPEGMA brush layer absorbs, 
is another confirmation of formation of a relatively hydrophobic second layer which doesnt absorb water vapor.

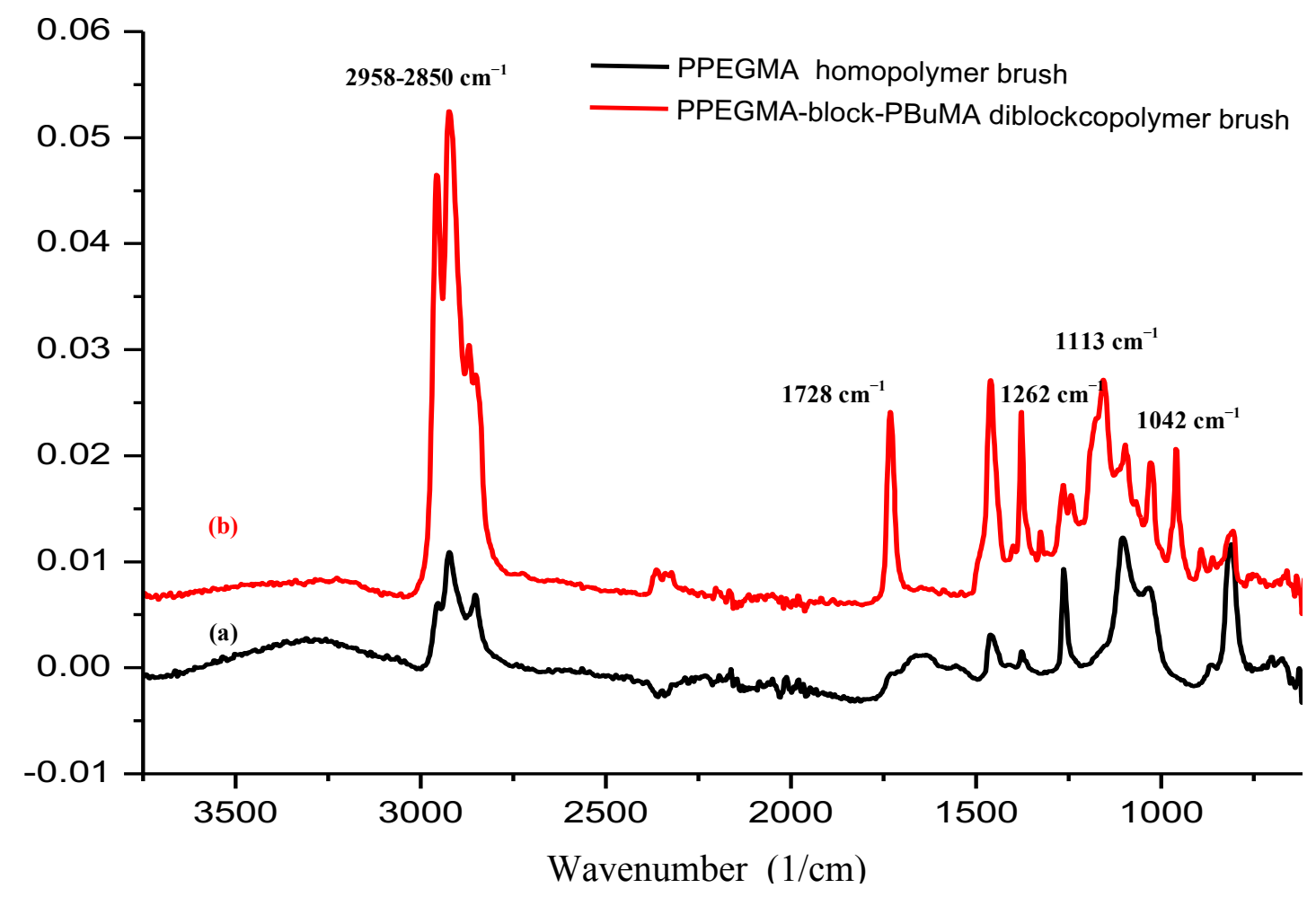

Figure 93 ATR-FTIR spectra of (a) PPEGMA brush synthesized for $5 \mathrm{~h}$ and (b) PPEGMA-block-PBuMA brush synthesized for $24 \mathrm{~h}$.

The goniometry demonstrated a huge increase in static water contact angle from $40^{\circ}$ (PEGMA) to $79.39^{\circ}$ (PPEGMA-block-PBuMA) as expected due to the more hydrophobic nature of second block (PBuMA). Ellipsometry indicated a thickness increase of $10.1 \mathrm{~nm}$ from that of the first (PPEGMA) homopolymer layer whereas the total thickness of blockcopolymer brush is $20 \mathrm{~nm}$ (Table 5). The area shown in Figure 94 for 24 hour polymerization displays small aggregates heterogeneously distributed over some areas of sample were observed in related AFM height image. The RMS roughness and roughness average values increased from 0.7917 to $2.3863 \mathrm{~nm}$ and from 0.5864 to 1.8666 respectively. GPC trace of free polymer was monomodal and had narrow molecular weight distribution, which are characteristics of well-defined polymers prepared via RAFT mediated polymerization. After 24 hours polymerization, the monomer conversion is $89 \%$. GPC analysis of the free polymers reveals a $M_{n}$ of $55798 \mathrm{~g} / \mathrm{mol}$ and a PDI of 1.11 (Table 5). 

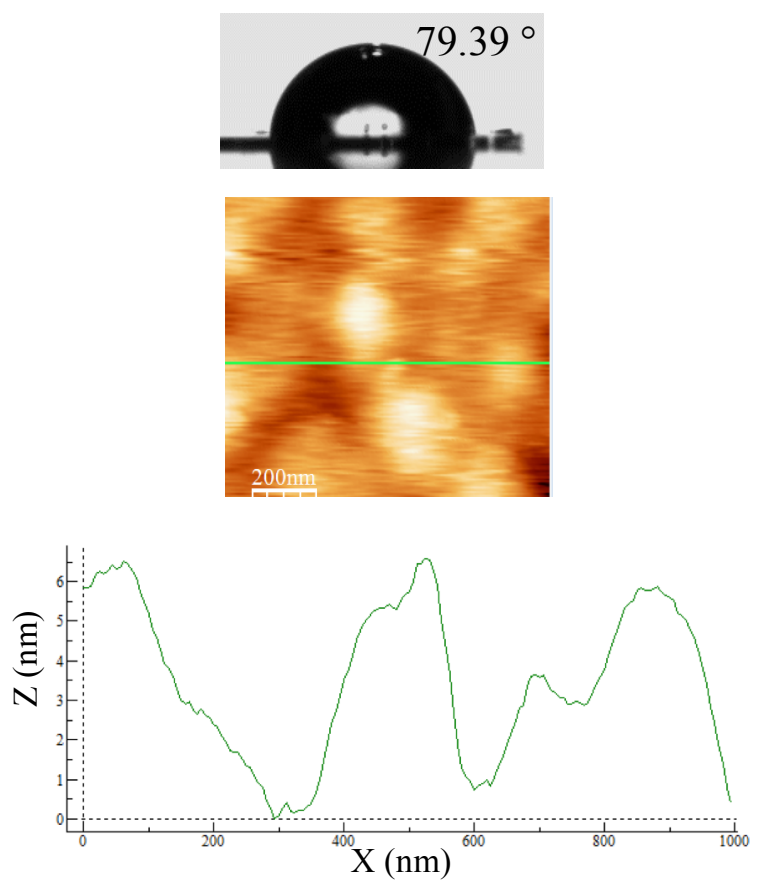

Figure 94 2D AFM topography image $(1 \mu \mathrm{m} \times 1 \mu \mathrm{m})$ in ambient conditions, surface profile analysis and photograph of $1 \mu \mathrm{L}$ water droplet for PPEGMA-block-PBuMA brushes synthesized for $24 \mathrm{~h}$ with the ratio of $[\mathrm{M}] /[\mathrm{CTA}] /[\mathrm{I}]=2000 / 4 / 1$ in feed.

The low PDI value indicates that the polymerization was conducted in a controlled manner. The surface coverage, A $\left(\mathrm{mg} / \mathrm{m}^{2}\right)$, grafting density, $\sigma$ (chains $\left./ \mathrm{nm}^{2}\right)$, and average distance between grafting sites, $D(\mathrm{~nm})$, of grafted poly(BUMA) brushes were calculated from the $M_{n}$ of the free polymer and the dry film thickness, $h(\mathrm{~nm})$, according to equations 18, 19 and 20 (Table 5). The grafting density of second block (PBuMA) is 0.1 chains per $\mathrm{nm}^{2}$ whereas it was 0.2 chains per $\mathrm{nm}^{2}$ for the first homopolymer layer (PPEGMA).

\subsection{Characterization of Diblock Copolymer Brush (PDMAEMA-b-PPEGMA) Synthesized on Gold-PDMAEMA Surface}

The livingness of homopolymer poly (DMAEMA) brushes synthesized for $24 \mathrm{~h}$ in previous step was checked by block extension with PEGMA. The formation of blockcopolymer brush poly (PEGMA) as a second layer on top of the gold substrate covered with $25 \mathrm{~nm}$ poly (DMAEMA) brushes was confirmed by ATR-IR [Figure 95 (b)], AFM and water contact-angle measurements. (Figure 96) The ATR-FTIR spectra for the PDMAEMA-block-PPEGMA brush demonstrated characteristic bands at 1729 $\mathrm{cm}^{-1}$ associated with the carbonyl stretch, at $1046 \mathrm{~cm}^{-1}$ resulted from strong $\mathrm{C}=\mathrm{S}$ stretching vibrations, suppressed by absorption bands at $1111 \mathrm{~cm}^{-1}$ and $1263 \mathrm{~cm}^{-1}$ resulted from vibrations of C-O-C ether bonds in the ethylene glycol units. The bands at around 2962, 2920, $2866 \mathrm{~cm}^{-1}$ are considered to be due to aliphatic $\mathrm{CH}$ groups. The $2962,2866 \mathrm{~cm}^{-1}$ peaks are due to asymmetric and symmetric stretching vibrations of $\mathrm{CH}_{3}$, respectively, while the $2920 \mathrm{~cm}^{-1}$ peak is assigned to $\mathrm{CH}_{2}$ vibration. The peaks at 
$1466 \mathrm{~cm}^{-1}$ and $1399 \mathrm{~cm}^{-1}$ are attributed to the $\mathrm{CH}$ bending and rocking respectively. In addition to these bands, there is an absorption band at 703-624 $\mathrm{cm}^{-1}$ attributed to C-S stretching. Moreover, a drastic increase in the broad band at $3666-3108 \mathrm{~cm}^{-1}$ which is assigned to $\mathrm{O}-\mathrm{H}$ stretching, resulting from water vapor which hydrophilic first brush layer (PDMAEMA) absorbs is another confirmation of formation of relatively more hydrophilic second blockcopolymer brush layer (PPEGMA) which absorbs more water vapor.

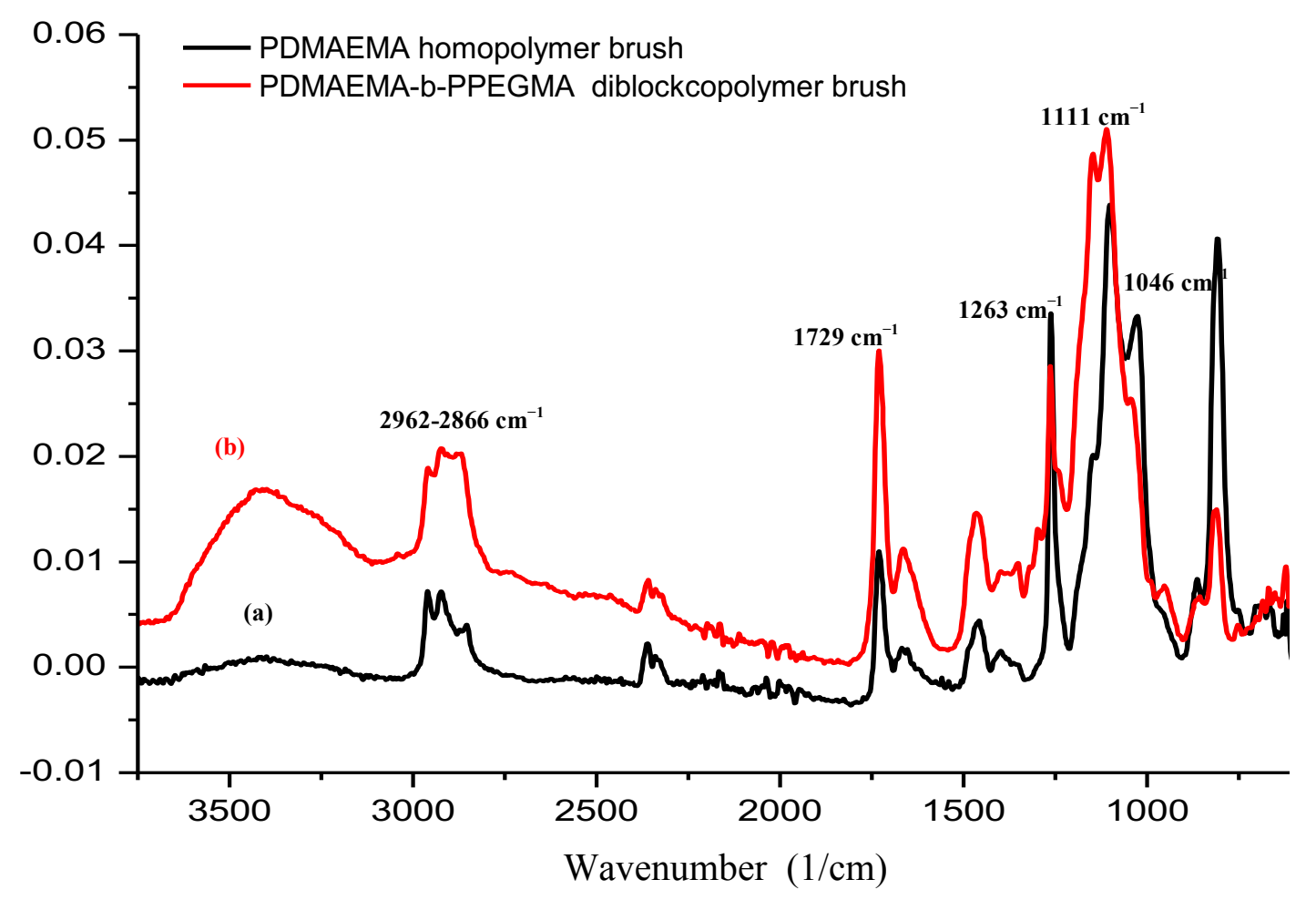

Figure 95 ATR-FTIR spectra of (a) PDMAEMA brush synthesized for $24 \mathrm{~h}$ and (b) PDMAEMA-block-PPEGMA brush synthesized for $24 \mathrm{~h}$.

Water contact angle decreased from 58.25 ${ }^{\circ}$ (PDMAEMA) to $36^{\circ}$ (PDMAEMAblock-PPEGMA), which is another proof for formation of a relatively more hydrophilic second polymer layer (PPEGMA) on top. Ellipsometry indicated a thickness increase of $15 \mathrm{~nm}$ from that of the first (PDMAEMA) homopolymer layer whereas the total thickness of blockcopolymer brush is $40 \mathrm{~nm}$. (Table 5) The area shown in Figure 96 for 24 hours polymerization display gaussian distribution which is an indication of surface homogenitiy although small aggregates heterogeneously distributed over some areas of sample were observed in related AFM height image. The RMS roughness and roughness average values increased from 1.4317 to 2.1225 and from 1.0792 to $1.5589 \mathrm{~nm}$ respectively. GPC trace of free polymer was monomodal and had narrow molecular weight distribution, which are characteristics of well-defined polymers prepared via RAFT mediated polymerization. 


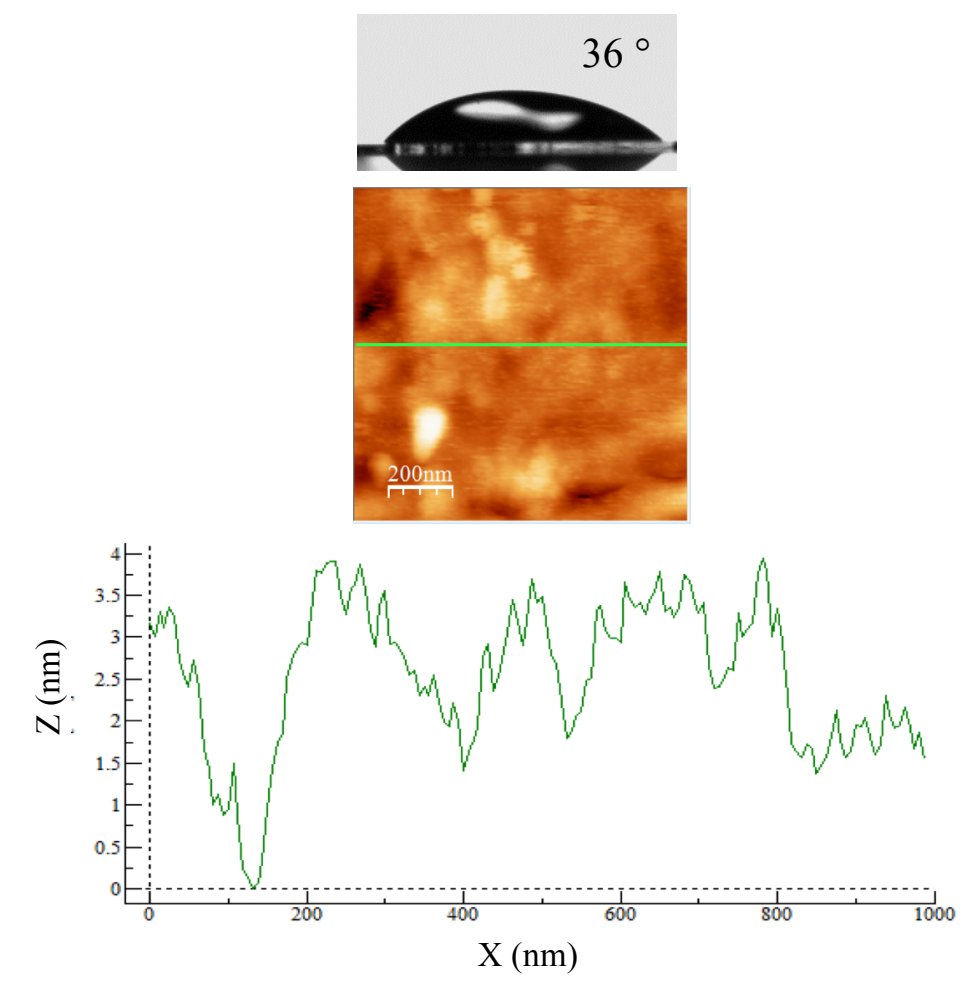

Figure 96 2D AFM topography image $(1 \mu \mathrm{m} \times 1 \mu \mathrm{m})$ in ambient conditions, surface profile analysis and photograph of $1 \mu \mathrm{L}$ water droplet for PDMAEMA-blockPPEGMA brush synthesized for $24 \mathrm{~h}$ with the ratio of $[\mathrm{M}] /[\mathrm{CTA}] /[\mathrm{I}]=2000 / 4 / 1$ in feed.

After $24 \mathrm{~h}$ polymerization, the monomer conversion is $96 \%$. GPC analysis of the free polymers reveals a $M_{n}$ of $83350 \mathrm{~g} / \mathrm{mol}$ and a PDI of 1.66. (Table 5) The low PDI value indicates that the polymerization was conducted in a controlled manner. The surface coverage, A $\left(\mathrm{mg} / \mathrm{m}^{2}\right)$, grafting density, $\sigma$ (chains $\left./ \mathrm{nm}^{2}\right)$, and average distance between grafting sites, $D(\mathrm{~nm})$, of grafted poly (PEGMA) brushes were calculated from the $M_{n}$ of the free polymer and the dry film thickness, $h(\mathrm{~nm})$, according to equations 18,19 and 20. (Table 5) The grafting density of second block (PPEGMA) is 0.1 chains per $\mathrm{nm}^{2}$ whereas it was 0.5 chains per $\mathrm{nm}^{2}$ for the first homopolymer layer (PDMAEMA).

Table 5 Summary of diblockcopolymer brushes

\begin{tabular}{|c|c|c|c|c|c|c|c|}
\hline $\begin{array}{l}\text { Polymer } \\
\text { type }\end{array}$ & $\mathbf{M n} / \mathbf{M w}$ & PDI & $\begin{array}{c}\text { Thickness }^{\mathrm{e}} \\
\text { (nm) }\end{array}$ & $\begin{array}{l}\text { Coverage } \\
\left(\mathrm{mg} / \mathrm{m}^{2}\right)\end{array}$ & $\begin{array}{l}\text { Grafting } \\
\text { Density } \\
\text { (chains/ } \\
\mathbf{n m}^{\mathbf{2}} \text { ) }\end{array}$ & $\begin{array}{l}\text { Dista } \\
\text { nce } \\
(\mathrm{nm})\end{array}$ & $\begin{array}{l}\text { Static } \\
\text { Contact } \\
\text { Angle }^{\mathrm{f}}\end{array}$ \\
\hline PDMAEMA $^{\text {a }}$ & $40817 / 48975$ & 1.19 & 25 & 33.75 & 0.5 & 1.6 & $58.25^{\circ}$ \\
\hline $\begin{array}{l}\text { PDMAEMA- } \\
\text { b-PPEGMA }\end{array}$ & $83350 / 138690$ & 1.66 & $\begin{array}{l}+15 \\
=40\end{array}$ & 16.2 & 0.1 & 3.57 & $36^{\circ}$ \\
\hline
\end{tabular}




\begin{tabular}{|l|l|l|l|l|l|l|l|}
\hline PPEGMA $^{\mathrm{c}}$ & $32850 / 44474$ & 1.35 & 9.9 & 10.42 & 0.2 & 2.5 & $40.97^{\circ}$ \\
\hline $\begin{array}{l}\text { PPEGMA-b- } \\
\text { PBuMA }^{\mathrm{d}}\end{array}$ & $55798 / 62455$ & 1.11 & $\begin{array}{l}+11 \\
=20\end{array}$ & 11.6 & 0.12 & 3.18 & $79.39^{\circ}$ \\
\hline
\end{tabular}

${ }^{a}$ Polymerization of (2-(dimethylamino) ethyl methacrylate) DMAEMA with cumyl dithiobenzoate (CDB) and 2,2 $2^{\circ}$-azobis (isobutyronitrile) (AIBN) in toluene at $60{ }^{\circ} \mathrm{C}$ for $24 \mathrm{~h} .{ }^{b}$ Copolymerization of (poly (ethylene glycol) methyl ether methacrylate) PEGMA with DSTCSPA and AIBN in toluene at $60{ }^{\circ} \mathrm{C}$ for $24 \mathrm{~h}$ (PDMAEMA-b-PPEGMA). ${ }^{c}$ Polymerization of (polyethyleneglycolmethyl ether methacrylate) PEGMA with 4Cyano-4-[dodecylsulfanylthiocarbonyl)sulfanyl] pentanoicacid (DSTCSPA) and 2,2‘azobis(isobutyronitrile) (AIBN) in toluene at $60{ }^{\circ} \mathrm{C}$ for $5 \mathrm{~h} .{ }^{d}$ Copolymerization of BuMA with DSTCSPA and AIBN in toluene at $60{ }^{\circ} \mathrm{C}$ for $24 \mathrm{~h}$ (PPEGMA-b-PBuMA). ${ }^{\mathrm{e}}$ Thicknesses were determined by ellipsometry and are an average of five samplings across the sample. Error of thicknesses measured was within $\pm 0.3 \mathrm{~nm} .{ }^{f}$ Static contact angles were taken using goniometry and are an average of five samplings across the sample. The standard deviation of contact angles was less than $1^{\circ}$.

\subsection{Investigation of Impact of Monomer / Raft Agent Ratio in Feed on Resulting Thickness and Grafting Density Values of Polymer Brushes}

In order to investigate and confirm controllability of thickness with increasing ratio of $[\mathrm{M}]$ / [CTA] in feed by utilizing SI-RAFT on gold surfaces, monomer amount per chain transfer agent of reaction mixtures were varied. (Table 6) Increase in water contact angles of the gold surfaces polymerized with BuMA with the ratio of [M]:2000 / [CTA]: 4 in feed $\left(89.70^{\circ}\right.$ ) (Figure 87), the ratio of [M] :6000 / [CTA]: 4 in feed $\left(87.61^{\circ}\right)$ [Figure $97(a)$, the ratio of [M] :10000 / [CTA]: 4 in feed $\left(88.87^{\circ}\right)$ [Figure 97 (b)] is confirmation of sucsessful growth of hydrophobic polymer layers whereas a drastic decrease in water contact angles of gold surfaces polymerized with DMAEMA with the ratio of [M] :2000 / [CTA]: 4 in feed $\left(58.25^{\circ}\right.$ ) (Figure 90) and with the ratio of $[\mathrm{M}]: 10000 /$ [CTA]: 4 in feed $\left(55.69^{\circ}\right)$ [Figure 97 (c)] is another evident of formation of relatively hydrophilic polymer layers. The areas shown in [Figure 97 (a)], [Figure 97 (b)] and [Figure 97 (c)] for 24 hour polymerization time display gaussian distribution which confirms surface uniformity although small aggregates heterogeneously distributed over some areas of sample were observed in related AFM height images. The changes in film morphology are associated with increases in the RMS roughnesses from $0.3512 \mathrm{~nm}$ (CTA immobilized gold surfaces) to 1.5497, $1.6268,1.0548,0.4254 \mathrm{~nm}$ respectively ( $24 \mathrm{~h}$ polymerization time). 

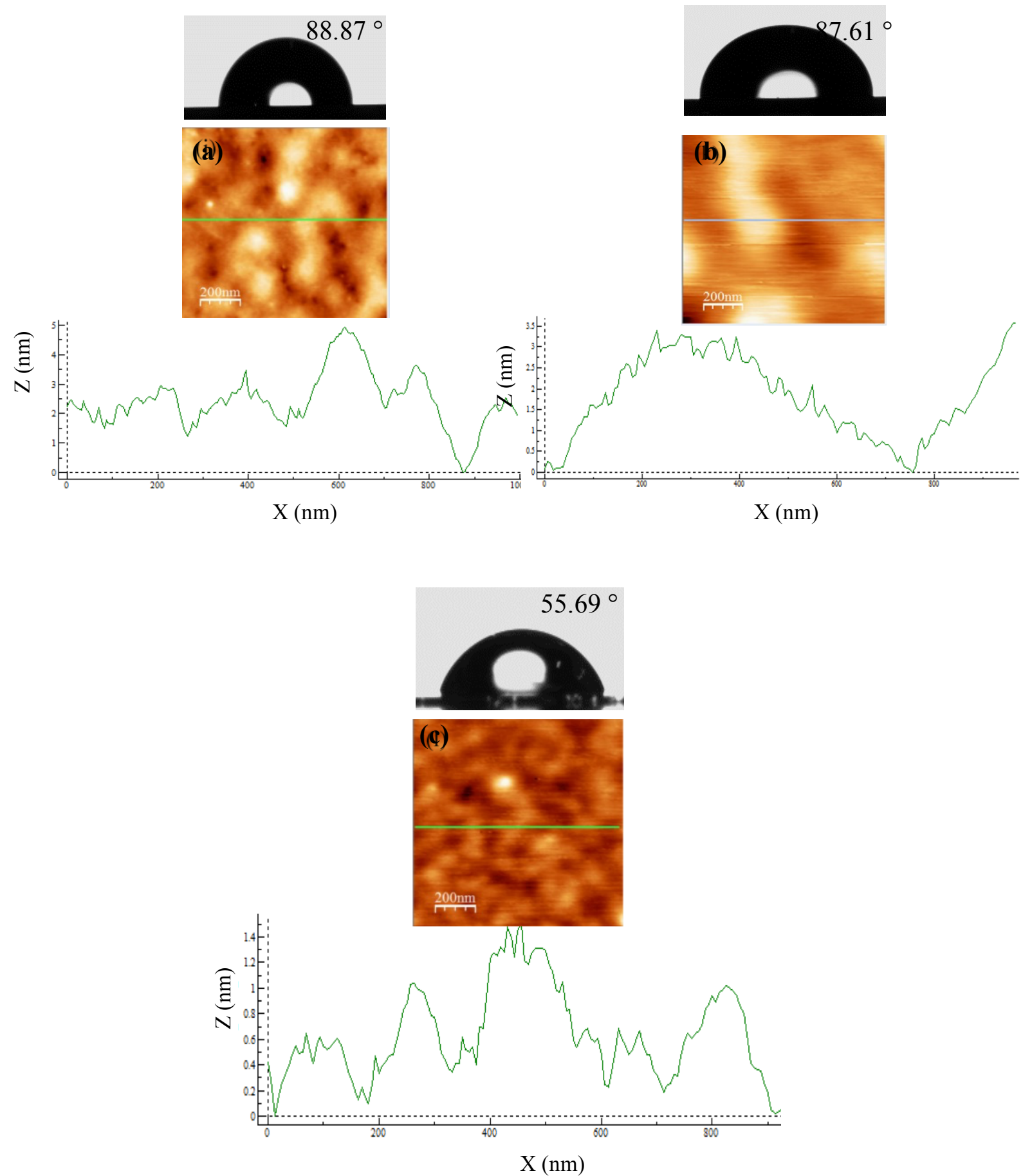

Figure 97 2D AFM topography images $(1 \mu \mathrm{m} \times 1 \mu \mathrm{m})$ in ambient conditions, surface profile analysis and photographs of $1 \mu \mathrm{L}$ water droplets for (a) poly (BuMA) brushes synthesized for $24 \mathrm{~h}$ with the ratio of $[\mathrm{M}] /[\mathrm{CTA}] /[\mathrm{I}]=6000 / 4 / 1$ in feed, (b) poly (BuMA) brushes synthesized for $24 \mathrm{~h}$ with the ratio of $[\mathrm{M}] /[\mathrm{CTA}] /[\mathrm{I}]=10000 / 4 / 1$ in feed, (c) poly (DMAEMA) brushes synthesized for $24 \mathrm{~h}$ with the ratio of $[\mathrm{M}] /[\mathrm{CTA}] /[\mathrm{I}]=10000 / 4 / 1$ in feed. 
The ATR-FTIR spectras of poly (BuMA) brushes [Figure 98 (e)] demonstrated characteristic bands at $\sim 1732 \mathrm{~cm}^{-1}$, associated with the carbonyl stretch and the strong absorption band at $1069 \mathrm{~cm}^{-1}$ resulted from $\mathrm{C}=\mathrm{S}$ stretching vibrations overlapped by absorption bands at $1108 \mathrm{~cm}^{-1}$ and $1266 \mathrm{~cm}^{-1}$ associated with $\mathrm{C}-\mathrm{O}$ stretching of $\mathrm{C}(=\mathrm{O})$ O-C bonds in monomer units. The bands at around 2960, 2926, $2872 \mathrm{~cm}^{-1}$ are considered to be due to aliphatic $\mathrm{CH}$ groups. The $2960,2872 \mathrm{~cm}^{-1}$ peaks are due to asymmetric and symmetric stretching vibrations of $\mathrm{CH}_{3}$, respectively, while the 2926 $\mathrm{cm}^{-1}$ peak is assigned to $\mathrm{CH}_{2}$ vibration. In addition to these bands, there are bands at $751-643 \mathrm{~cm}^{-1}$ associated with C-S stretching and peaks at $1460 \mathrm{~cm}^{-1}, 1379 \mathrm{~cm}^{-1}$ attributed to the $\mathrm{CH}$ bending and rocking respectively.

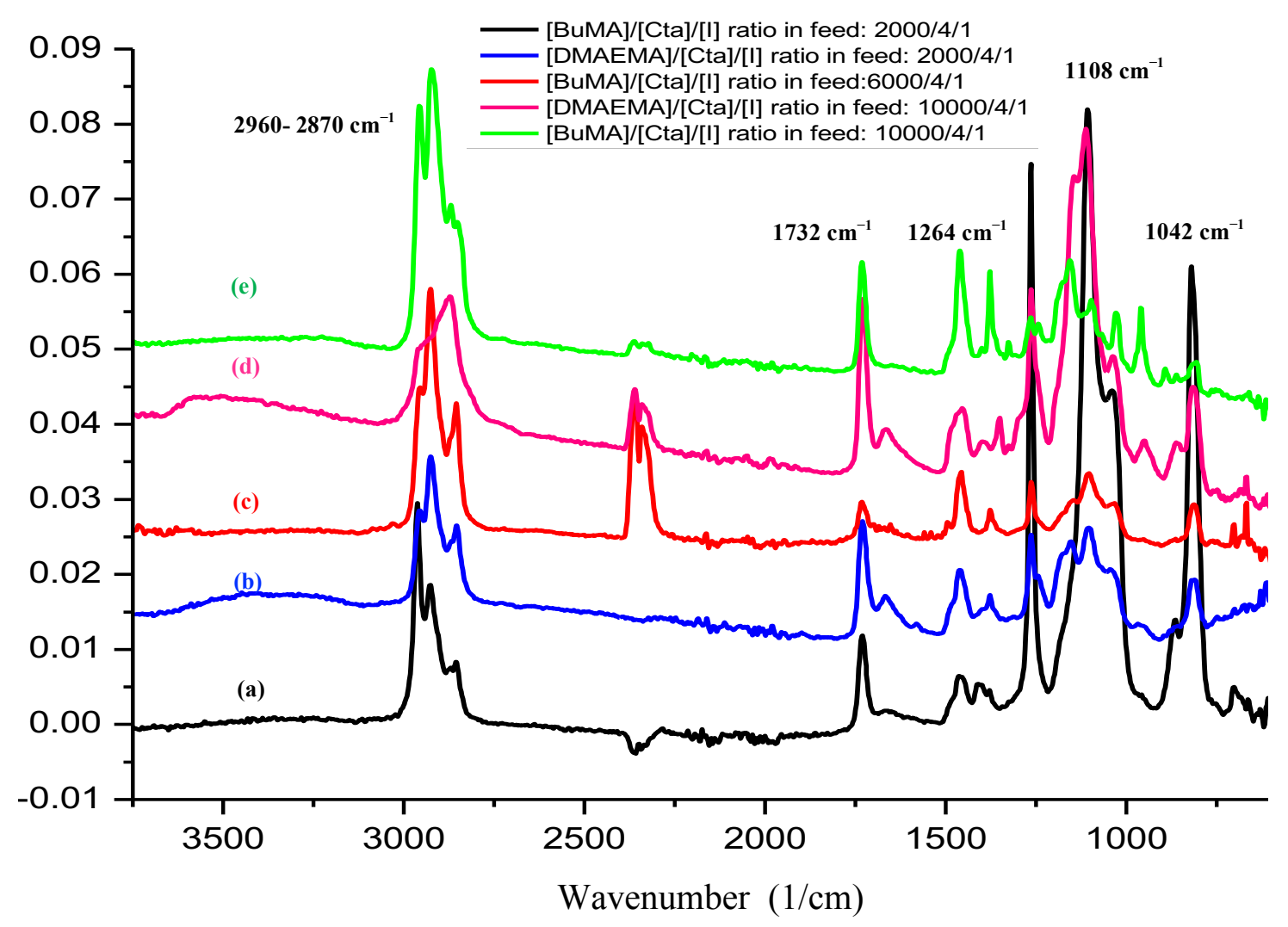

Figure 98 ATR-FTIR spectra of (a) poly (BuMA) brush synthesized for $24 \mathrm{~h}$ with the ratio of $[\mathrm{M}] /[\mathrm{CTA}] /[\mathrm{I}]=2000 / 4 / 1$ in feed, (b) poly (DMAEMA) brush synthesized for $24 \mathrm{~h}$ with the ratio of $[\mathrm{M}] /[\mathrm{CTA}] /[\mathrm{I}]=2000 / 4 / 1$ in feed (c) poly (BuMA) brush synthesized for $24 \mathrm{~h}$ with the ratio of $[\mathrm{M}] /[\mathrm{CTA}] /[\mathrm{I}]=6000 / 4 / 1$ in feed, (d) poly (DMAEMA) brush synthesized for $24 \mathrm{~h}$ with the ratio of $[\mathrm{M}] /[\mathrm{CTA}] /[\mathrm{I}]=10000 / 4 / 1$ in feed and (e) poly (BuMA) brush synthesized for $24 \mathrm{~h}$ with the ratio of [M]/[CTA] $/[\mathrm{I}]=10000 / 4 / 1$ in feed.

The ATR-FTIR spectras for the poly (DMAEMA) brushes [Figure 98 (d)] demonstrated characteristic absorption bands at $\sim 1732 \mathrm{~cm}^{-1}$, associated with the carbonyl stretch, and at $1063 \mathrm{~cm}^{-1}$ resulted from $\mathrm{C}=\mathrm{S}$ strong stretching vibrations overlapped by absorption bands at $1106 \mathrm{~cm}^{-1}, 1266 \mathrm{~cm}^{-1}$ associated with C-O stretching 
of $\mathrm{C}(=\mathrm{O})-\mathrm{O}-\mathrm{C}$ bonds in monomer units and bands at $1237-1036 \mathrm{~cm}^{-1}$ resulted from vibrations of C-N stretching. The bands at around 2951, 2924, $2854 \mathrm{~cm}^{-1}$ are considered to be due to aliphatic $\mathrm{CH}$ groups. The $2951,2854 \mathrm{~cm}^{-1}$ peaks are due to asymmetric and symmetric stretching vibrations of $\mathrm{CH}_{3}$, respectively, while the $2924 \mathrm{~cm}^{-1}$ peak is assigned to $\mathrm{CH}_{2}$ vibration. A slight shift is observed in stretching vibrations of $\mathrm{CH}_{3}$ $\left(2854 \mathrm{~cm}^{-1}\right)$ compared to PBuMA $\left(2872 \mathrm{~cm}^{-1}\right)$ as a result of being overlapped by the characteristic peaks for the $-\mathrm{N}\left(\mathrm{CH}_{3}\right)_{2}$, which generally appears at $\sim 2830 \mathrm{~cm}^{-1}$. In addition to these bands, there are bands at $722-624 \mathrm{~cm}^{-1}$ associated with C-S stretching while the peaks at $1462 \mathrm{~cm}^{-1}, 1379 \mathrm{~cm}^{-1}$ are attributed to the $\mathrm{CH}$ bending and rocking, respectively. The broad band at $3683-3014 \mathrm{~cm}^{-1}$ which is assigned to $\mathrm{O}-\mathrm{H}$ stretching, results from water vapor which PDMAEMA brush coating absorbs due to its hydrophilic nature.

Table 6 Summary of impact of monomer [M] / raft agent [CTA] ratio in feed on resulting thickness of polymer brushes

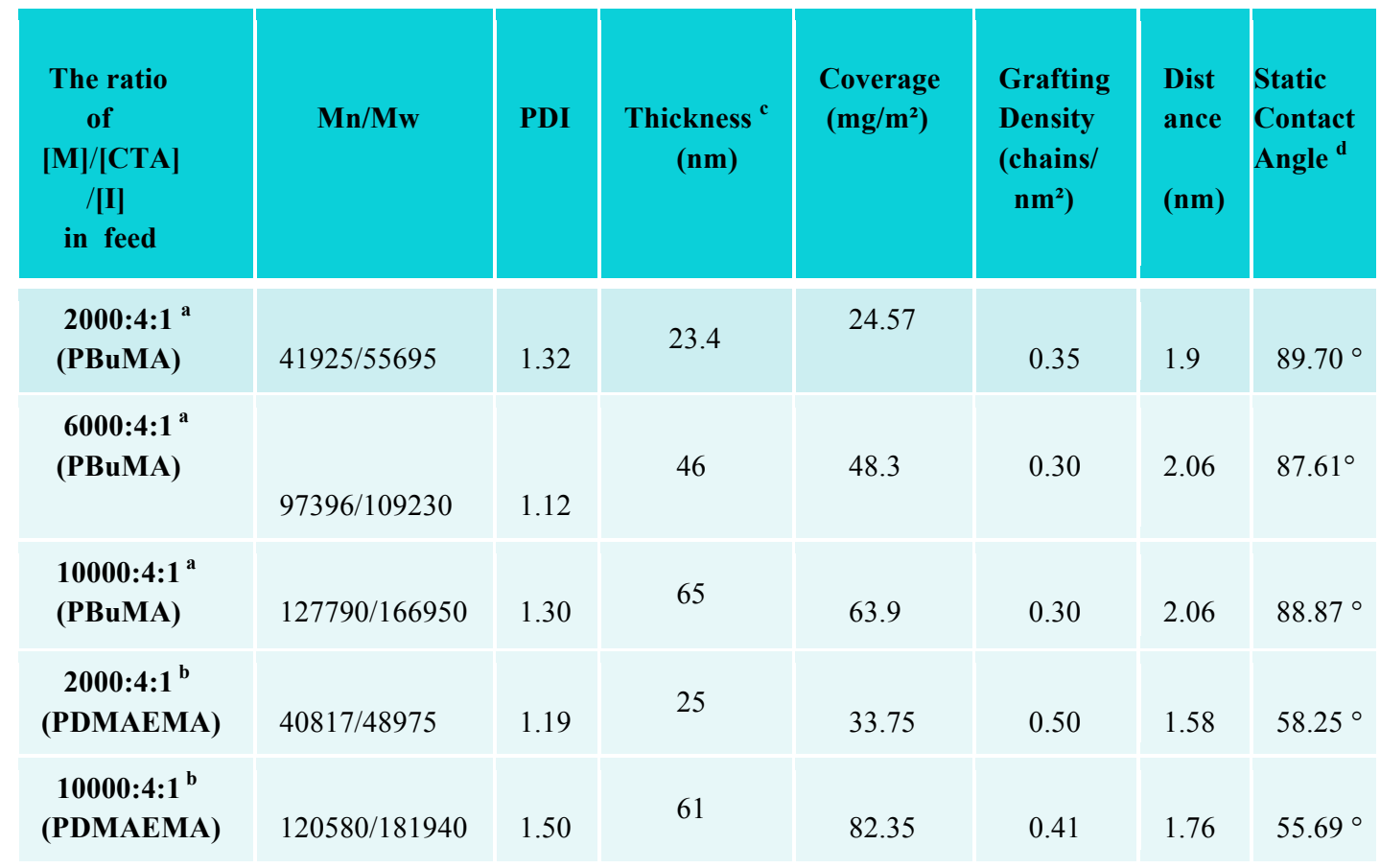

${ }^{a}$ Polymerization of (buthyl methacrylate) BuMA with 4-Cyano-4[dodecylsulfanylthiocarbonyl)sulfanyl] pentanoicacid (DSTCSPA) and 2,2 ${ }^{\circ}$ azobis(isobutyronitrile) (AIBN) in toluene at $60{ }^{\circ} \mathrm{C}$ for $24 \mathrm{~h} .{ }^{b}$ Polymerization of (2(dimethylamino) ethyl methacrylate) DMAEMA with cumyl dithiobenzoate (CDB) and 2,2 -azobis(isobutyronitrile) (AIBN) in toluene at $60{ }^{\circ} \mathrm{C}$ for $24 \mathrm{~h} .{ }^{c}$ Thicknesses were determined by ellipsometry and are an average of five samplings across the sample. Error of thicknesses measured was within $\pm 0.6 \mathrm{~nm} .{ }^{d}$ Static contact angles were taken using goniometry and are an average of five samplings across the sample. The standard deviation of contact angles was less than $1^{\circ}$. 
The time evolution of number average molecular weight, $M_{n}$ and polydispersity, PDI of the grafted polymer chains on gold, which is assumed to be the same as that of the unbound polymer chains in solution, was obtained by GPC analysis (shown in Table 6). GPC traces of free polymers for each sample were monomodal and had narrow molecular weight distributions, which is an indicator of well-defined polymers prepared via RAFT-mediated polymerization. The monomer conversions are ([BuMA]:2000/[CTA]:4) $88.5 \quad \%, \quad([\mathrm{BuMA}]: 6000 /[\mathrm{CTA}]: 4) \quad 70 \quad \%$, ([BuMA]:10000/[CTA]:4) $64.6 \%, \quad$ ([DMAEMA]:2000/[CTA]:4) $89.3 \%$, ([DMAEMA]:10000/[CTA]:4) $56.7 \%$ respectively; GPC analysises of the free polymers reveal molecular weights of $41925,97396,127790,40817,120580 \mathrm{~g} / \mathrm{mol}$ and PDIs of 1.32, 1.12, $1.30,1.19,1.50$ respectively. The fact that PDI values are all below 1.50 indicate that the polymerization was conducted in a controlled manner.

The gold surfaces polymerized with the ratio of [M] :2000 / [CTA]: 4 in feed resulted in a poly (BuMA) brush layer with a thickness of $23.4 \mathrm{~nm}$, the ratio of [M] :6000 / [CTA]: 4 in feed produced a layer with a thickness of $46 \mathrm{~nm}$, whereas the ratio of [M] $: 10000$ / [CTA]: 4 in feed resulted in $65 \mathrm{~nm}$ thick poly (BuMA) layer. The gold surfaces polymerized with the ratio of $[\mathrm{M}]: 2000 /$ [CTA]: 4 in feed resulted in a poly(DMAEMA) brush layer with a thickness of $25 \mathrm{~nm}$ whereas it is $61 \mathrm{~nm}$ thick for the one synthesized with the ratio of [M]:10000 / [CTA]: 4 in feed. (Table 6) This clear increase in thicknesses of brushes with increasing monomer amount per free CTA in solution is another proof of controllability of resulting thicknesses of polymer brushes synthesized from gold surfaces via utilization of SI-RAFT polymerization technique. The surface coverages, A $\left(\mathrm{mg} / \mathrm{m}^{2}\right)$, grafting densities, $\sigma$ (chains $/ \mathrm{nm}^{2}$ ), and average distances between grafting sites, $D(\mathrm{~nm})$, of grafted polymer brushes were calculated from the $M_{n}$ of the free polymer and the dry film thickness, $h(\mathrm{~nm})$, according to equations 18, 19 and 20 and summarized in Table 6. As a result of that the grafting densities vary in the range of $0.3-0.50$ chains per $\mathrm{nm}^{2}$, brushes might be considered as a brush type between semi-dilute and a high density brush, which is closer to a high density conformation than semi-dilute. (509)

\subsection{Summary and Conclusions}

In this part, a variety of homopolymer and diblockcopolymer brushes with different thicknesses and wettability properties were sucsessfully prepared via SI-RAFT polymerization technique. The homopolymer and diblockcopolymer brushes demonstrated linear growth of film thickness at the early stage of polymerization and the surface coverage controlled by the increase in molecular weight of polymer brushes. Block extension of homopolymer brushes obtained at first step further confirmed "living character" of brushes obtained via RAFT and the fact that postpolymerization is possible based on this "livingness" being maintained. 
In addition to that, impact of monomer [M] $\backslash$ Raft agent [CTA] ratio in feed on resulting thickness of polymer brushes was investigated. The presence of free CTA in reaction mixture, which results in free polymer chains in solution, is essential both in order to estimate molecular weights and to be able to control polymerization. The ellipsometric thicknesses and molecular weights were used to estimate grafting parameters of chains attached to the gold surface. As a consequence, RAFT mediated polymerization technique via $\mathrm{R}$ group approach can be utilized to obtain well-defined polymer and diblockcopolymer brushes polymer brushes of up to $60 \mathrm{~nm}$ thick. To best of our knowledge, for the first time, the thickest polymer layers on gold surfaces based on R-group approach were obtained in a SI-RAFT process. 
6 Characterization of Homopolymer and Diblock Copolymer Brushes Prepared on Gold via Novel Chemisorption Based Approach

(a)
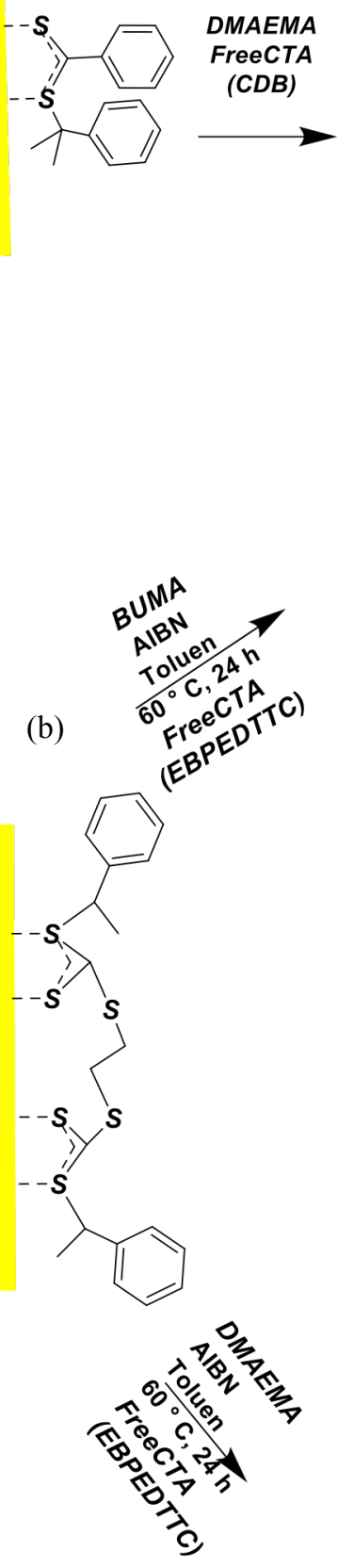

(e)

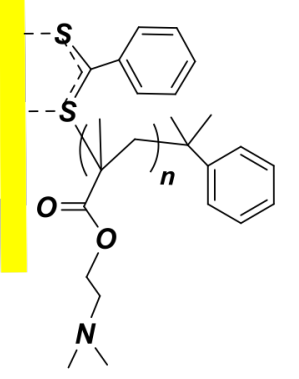

(f)

(g)

(h)
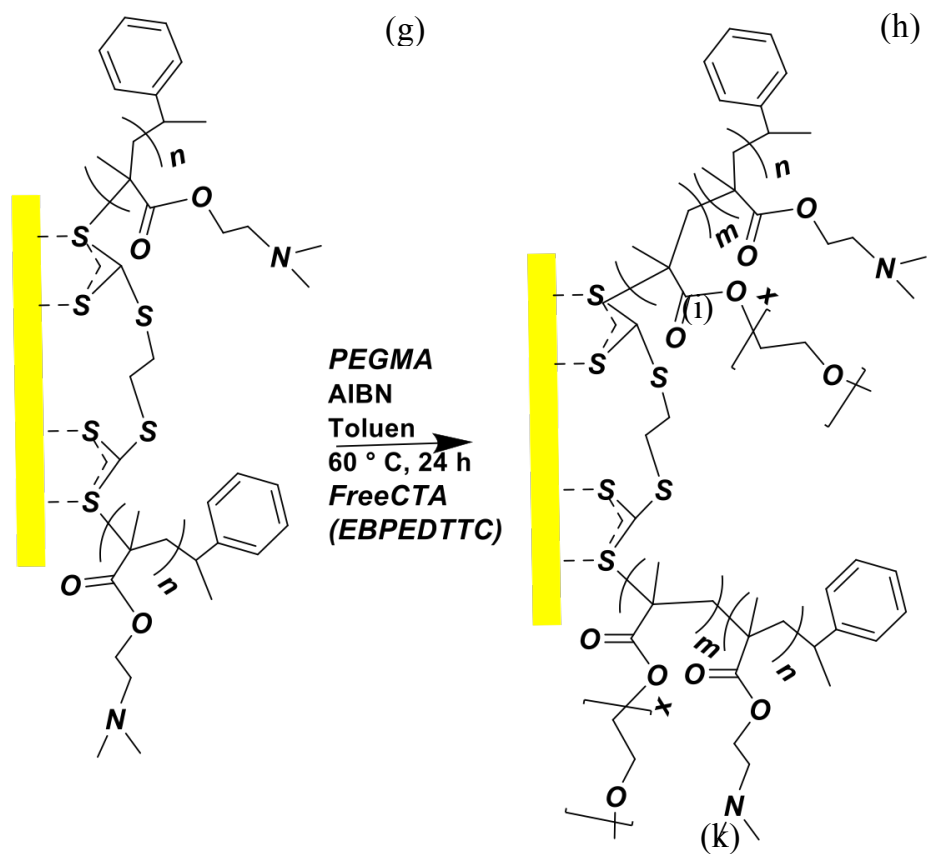
(c)
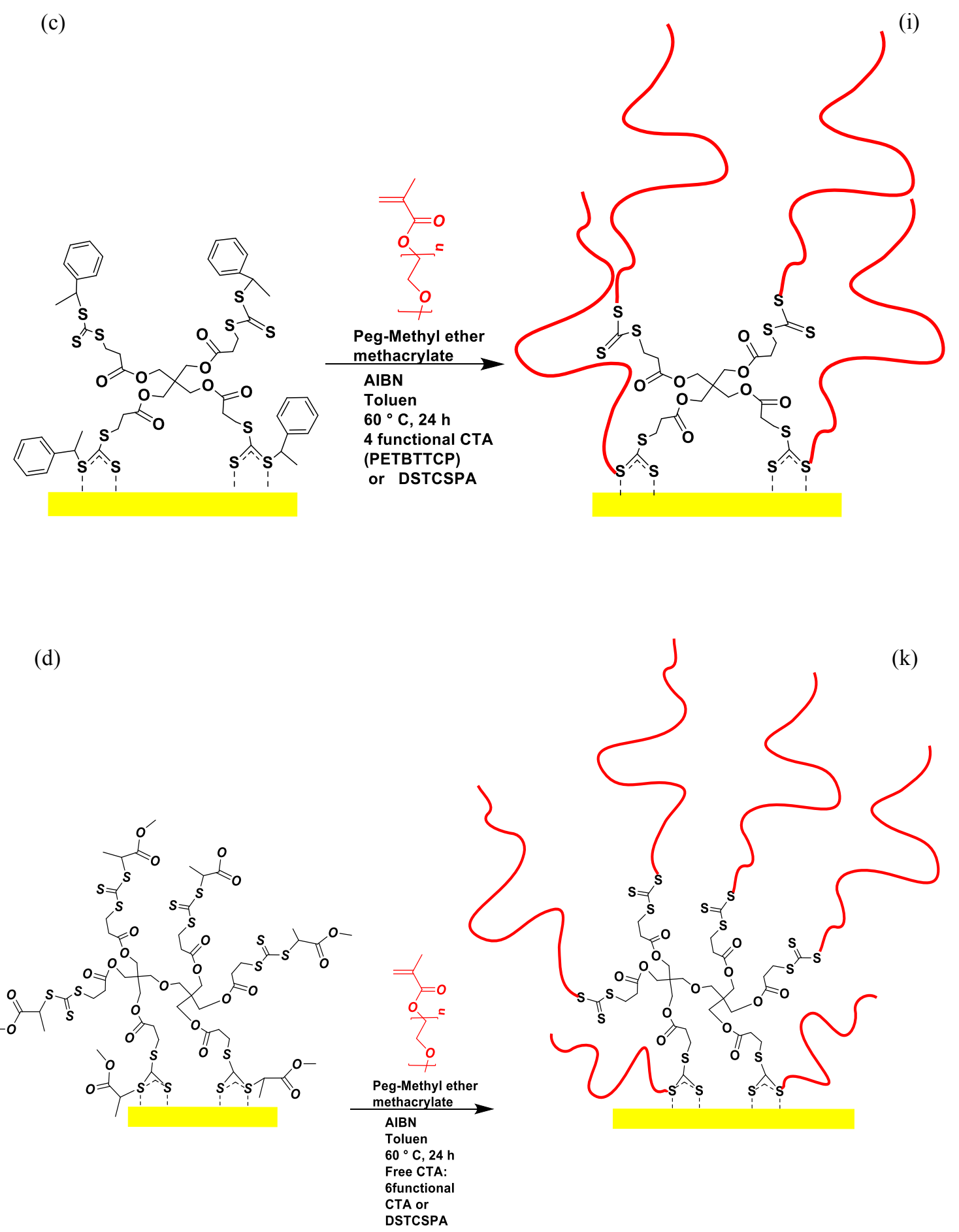

Scheme 2 Schematic diagram illustrating the processes of immobilization of CTAs (a) 2_Phenyl_2_Propylbenzodithioate (CDB), (b) Ethane-1,2-diyl bis(1-phenylethyl) ditrithiocarbonate (EBPEDTTC) (c) Pentaerythritol-tetrakis-(3-(S-phenylethyl trithiocarbonate))-propanoate (tetra functional)

(PETSPETTCP)

(d) Dipentaerythriol-hexakis(3-(S-methoxopropionate-trithiocarbonate))-propanoate (hexa functional) (DPEHSMPETTC) onto gold and the RAFT-mediated polymerization of (e) DMAEMA in the presence of free CTA (CDB) from gold-CDB surface, (f) BuMA in the presence of bifunctional free CTA (EBPEDTTC) from gold- 
EBPEDTTC surface (g) DMAEMA in the presence of bifunctional free CTA (EBPEDTTC) from the gold-EBPEDTTC surface, Raft-mediated blockcopolymerization of (h) PEGMA in the presence of bifunctional free CTA (EBPEDTTC) from the gold-EBPEDTTC-PDMAEMA surface and Raft-mediated polymerization of PEGMA in the presence of tetra functional free cta (PETSPETTCP) or in the presence of monofunctional free CTA (DSTCSPA) (i) from two identical goldPETSPETTCP surfaces and (k) from two identical gold-DPEHSMPETTC surfaces.

\subsection{Characterization of Raft Agent Immobilized Gold Surfaces}

It is known that dithioesters and trithiocarbonates are susceptible to be chemisorped onto gold surface. (225) CTAs were immobilized on gold surface through their dithioester and trithiocarbonate moieties by self assembly process mentioned in the Experimental section. The thicknesses of resulting CTA layers were measured by Ellipsometry as $1.2 \pm 0.1 \mathrm{~nm}$ for CDB [Scheme 2 (a)], $1.8 \pm 0.1 \mathrm{~nm}$ for EBPEDTTC [Scheme 2 (b)], $4.6 \pm 0.2 \mathrm{~nm}$ for PETSPETTCP [Scheme 2 (c)], $5.5 \pm 0.4 \mathrm{~nm}$ for DPEHSMPETTC. [Scheme 2 (d)] (page 137)

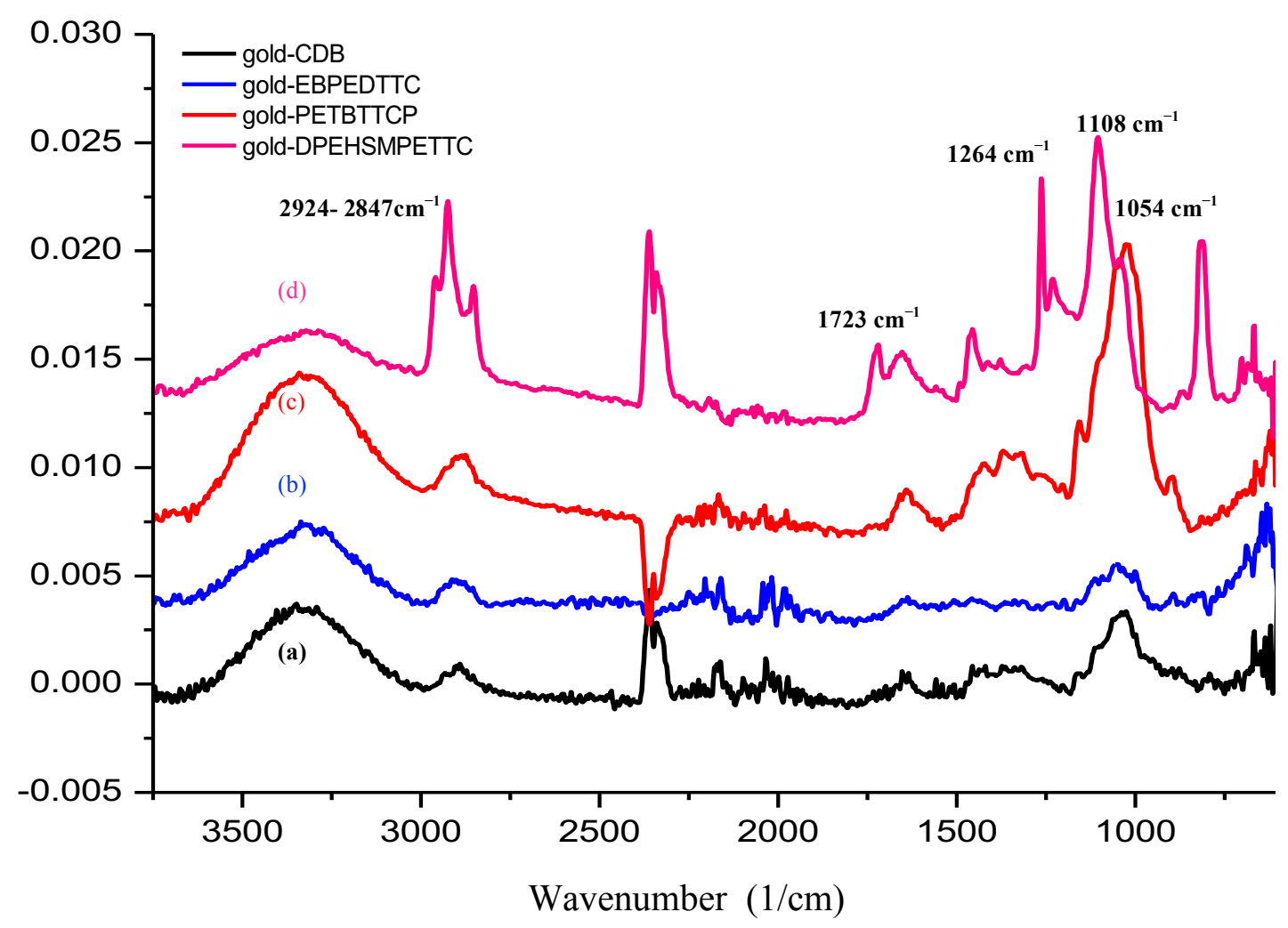

Figure 99 ATR-FTIR spectra of (a) gold-CDB (2_Phenyl_2_Propylbenzodithioate), (b) gold-EBPEDTTC (ethane-1,2-diyl bis(1-phenylethyl) ditrithiocarbonate) (c) goldPETSPETTCP (Penta-erythritol-tetrakis-(3-(S-phenylethyl trithiocarbonate))propanoate (tetra functional)) (d) gold-DPEHSMPETTC Dipentaerythriol-hexakis(3(S-methoxopropionate-trithiocarbonate))-propanoate(hexa functional). 
The formation of CTA layers (CDB and EBPEDTTC) were confirmed by the presence of the peaks at $1054 \mathrm{~cm}^{-1}$ resulted from strong $\mathrm{C}=\mathrm{S}$ stretching vibrations. The bands at around $2929,2912,2851 \mathrm{~cm}^{-1}$ are considered to be due to aliphatic $\mathrm{CH}$ groups. The $2929,2851 \mathrm{~cm}^{-1}$ peaks are due to asymmetric and symmetric stretching vibrations of $\mathrm{CH}_{3}$, respectively, while the $2912 \mathrm{~cm}^{-1}$ peak is assigned to $\mathrm{CH}_{2}$ vibration. In addition to these bands, there are bands at 714-624 $\mathrm{cm}^{-1}$ associated with C-S stretching while the peaks at $1455 \mathrm{~cm}^{-1}$ and $1380 \mathrm{~cm}^{-1}$ are attributed to the $\mathrm{CH}$ bending and rocking, respectively. The broad band at $3627-3034 \mathrm{~cm}^{-1}$ which is assigned to O$\mathrm{H}$ stretching, might result from water vapor being absorbed. The aromatic $\mathrm{C}-\mathrm{H}$ stretching peak expected at around $3100-3000 \mathrm{~cm}^{-1}$ is not clearly observable as a result of being suppressed by both aliphatic $\mathrm{C}-\mathrm{H}$ stretching and broad band of $\mathrm{O}-\mathrm{H}$ stretching whereas $\mathrm{C}=\mathrm{C}$ aromatic doublets, which are expected between $1480-1420 \mathrm{~cm}^{-1}$, are observed at $\sim 1455 \mathrm{~cm}^{-1}$, as being overlapped with $\mathrm{CH}$ bending vibration. [Figure 99 (a), (b)] The formation of CTA layers (PETSPETTCP and DPEHSMPETTC) were confirmed by the presence of the peaks at $1723 \mathrm{~cm}^{-1}$ associated with the carbonyl stretch, at $1054 \mathrm{~cm}^{-1}$ associated with strong $\mathrm{C}=\mathrm{S}$ stretching vibrations suppressed by absorption bands at $\sim 1108 \mathrm{~cm}^{-1}, 1264 \mathrm{~cm}^{-1}$ associated with $\mathrm{C}-\mathrm{O}$ stretching resulted from vibrations of C-O-C bonds. The bands at around 2924, 2906, $2847 \mathrm{~cm}^{-1}$ are considered to be due to aliphatic $\mathrm{CH}$ groups. The $2924,2847 \mathrm{~cm}^{-1}$ peaks are due to asymmetric and symmetric stretching vibrations of $\mathrm{CH}_{3}$, respectively, while the 2906 $\mathrm{cm}^{-1}$ peak is assigned to $\mathrm{CH}_{2}$ vibration.
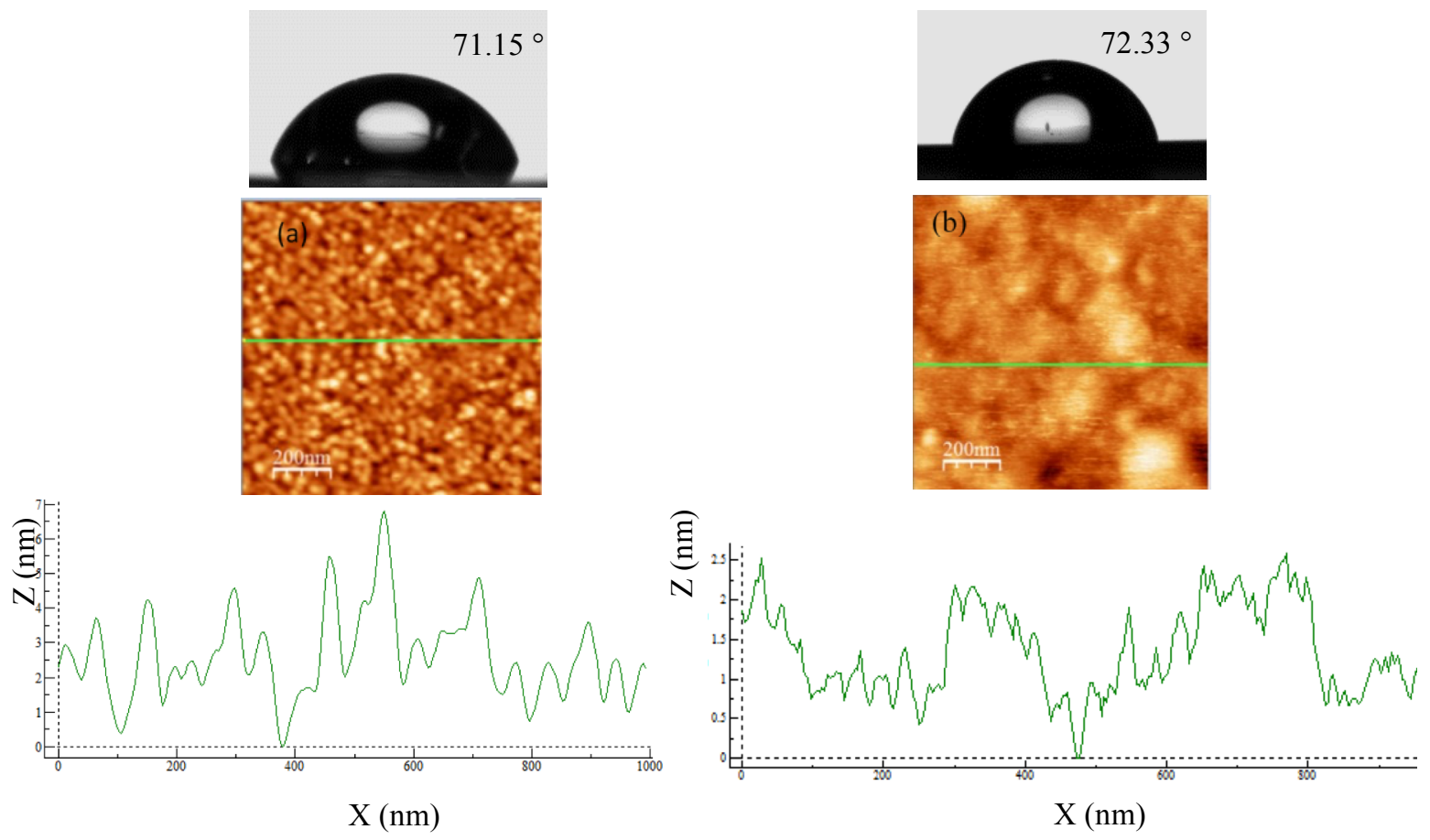

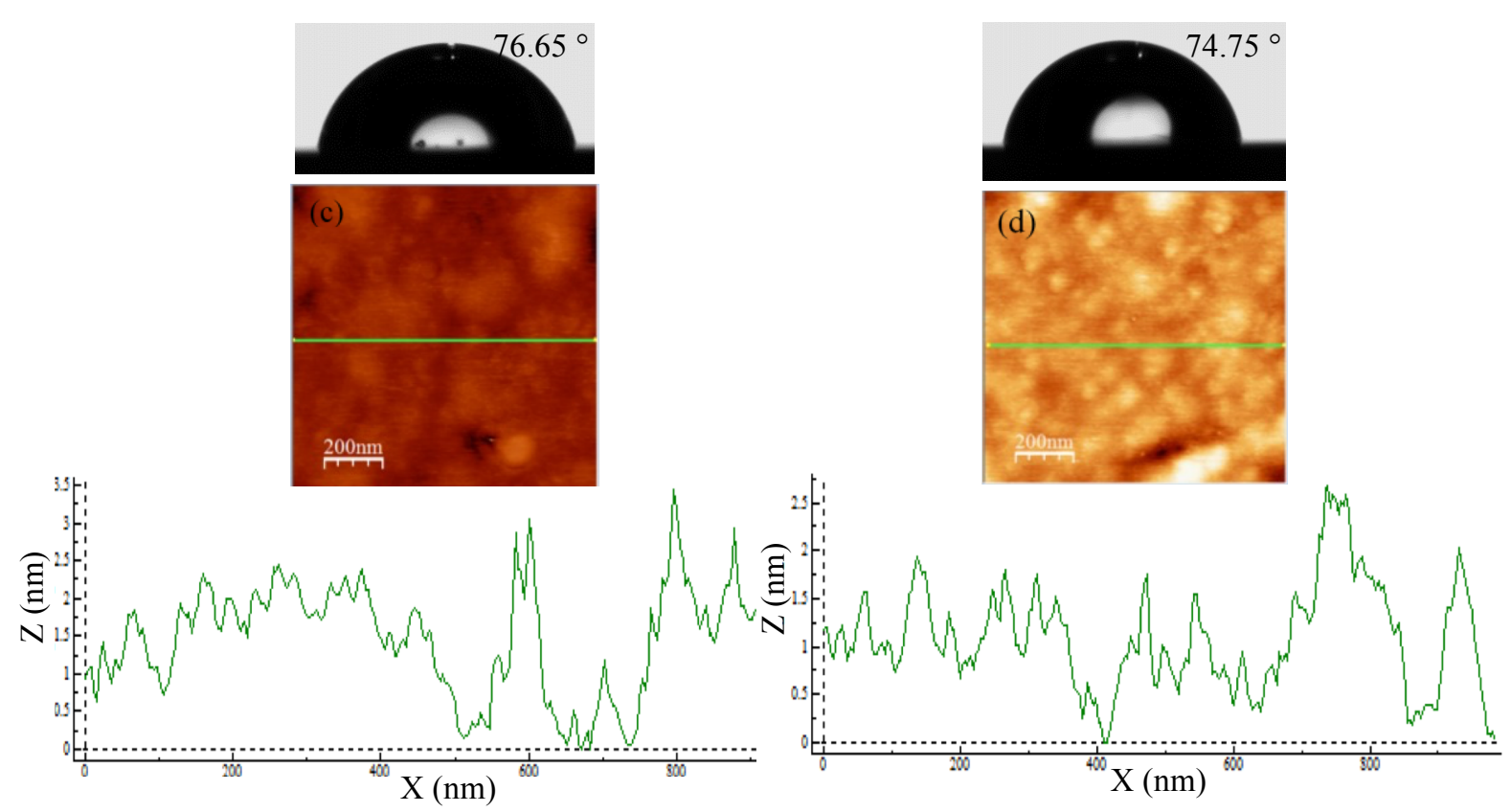

Figure $1002 \mathrm{D}$ AFM topography images $(1 \mu \mathrm{m} \times 1 \mu \mathrm{m})$ in ambient conditions, surface profile analysis, and photographs of $1 \mu \mathrm{L}$ water droplets for (a) gold-CDB (2_Phenyl_2_Propylbenzodithioate), (b) gold-EBPEDTTC (ethane-1,2-diyl bis(1phenylethyl) ditrithiocarbonate) (c) gold-PETSPETTCP (Penta-erythritol-tetrakis-(3(S-phenylethyl trithiocarbonate))-propanoate (tetra functional)), (d) goldDPEHSMPETTC Dipentaerythriol-hexakis(3-(S-methoxopropionatetrithiocarbonate))-propanoat (hexa functional).

In addition to these bands, there are absorption bands at $703-633 \mathrm{~cm}^{-1}$ attributed to $\mathrm{C}-\mathrm{S}$ stretching. The broad band at $3562-3100 \mathrm{~cm}^{-1}$ which is assigned to $\mathrm{O}-\mathrm{H}$ stretching, results from water vapor being absorbed. The aromatic $\mathrm{C}-\mathrm{H}$ stretching peak expected at around $3100-3000 \mathrm{~cm}^{-1}$ is slightly observable as a little neck at around 3116-3000 $\mathrm{cm}^{-1}$ as a result of being overlapped by both aliphatic $\mathrm{C}-\mathrm{H}$ stretching and broad band of $\mathrm{O}-\mathrm{H}$ stretching whereas $\mathrm{C}=\mathrm{C}$ aromatic doublets, which are expected at 1480-1420 $\mathrm{cm}^{-1}$, are observable at $1453 \mathrm{~cm}^{-1}$, overlapped with $\mathrm{CH}$ bending vibration. [Figure 99 (c), (d)] Surface morphology of CTA layers on gold substrates are shown in Figure 100 (a), (b), (c), (d). Analysises of CDB, EBPEDTTC, PETSPETTCP and DPEHSMPETTC layers showed a root-mean-square (RMS) roughnesses of 1.1518, $0.8494,1.0795,0.9731$ respectively whereas it is 0.6936 for pure gold (data not shown). An additional evidence of sucsessful chemisorption of CTAs through their dithioesters and trithiocarbonates onto bare gold is a change in surface wettability characterized by a slight increase of static contact angle from $61.56^{\circ}$ (bare gold) (data not shown) to $71.15^{\circ}$ (CDB), $72.33^{\circ}$ (EBPEDTTC), $76.65^{\circ}$ (PETSPETTCP) and $74.75^{\circ}$ (DPEHSMPETTC). 


\subsection{Kinetic Investigation of Polymer Brush (PBuMA) Growth on Gold- EBPEDTTC Surface in the Presence of Bifunctional Free CTA (EBPEDTTC)}

Polymerization kinetics were tracked with an attempt to investigate sucsess of controllability of SI-RAFT polymerization from CTA immobilized gold surfaces and characterize SI-RAFT process from gold surfaces. The formation of poly (BuMA) brushes was confirmed by ATR-IR (Figure 101), AFM and water contact-angle measurements. (Figure 102) The ATR-FTIR spectra of the poly (BuMA) brush demonstrated characteristic bands at $\sim 1733 \mathrm{~cm}^{-1}$, associated with the carbonyl stretch and the absorption band at $1036 \mathrm{~cm}^{-1}$ resulted from strong $\mathrm{C}=\mathrm{S}$ stretching vibrations suppressed by absorption bands at $1107 \mathrm{~cm}^{-1}$ and $1261 \mathrm{~cm}^{-1}$ associated with C-O stretching of $\mathrm{C}(=\mathrm{O})-\mathrm{O}-\mathrm{C}$ bonds in monomer units. The bands at around 2962, 2926, $2874 \mathrm{~cm}^{-1}$ are considered to be due to aliphatic $\mathrm{CH}$ groups. The $2962,2874 \mathrm{~cm}^{-1}$ peaks are due to asymmetric and symmetric stretching vibrations of $\mathrm{CH}_{3}$, respectively, while the $2926 \mathrm{~cm}^{-1}$ peak is assigned to $\mathrm{CH}_{2}$ vibration. In addition to these bands, there are bands at $703-608 \mathrm{~cm}^{-1}$ associated with $\mathrm{C}-\mathrm{S}$ stretching. The aromatic $\mathrm{C}-\mathrm{H}$ stretching peak expected at around $3100-3000 \mathrm{~cm}^{-1}$ is not clearly observable as a result of being suppressed by aliphatic $\mathrm{C}-\mathrm{H}$ stretching whereas $\mathrm{C}=\mathrm{C}$ aromatic doublets, which are expected at a value between $1480-1420 \mathrm{~cm}^{-1}$, which belong to chain transfer agent, were observed at $1463 \mathrm{~cm}^{-1}$.

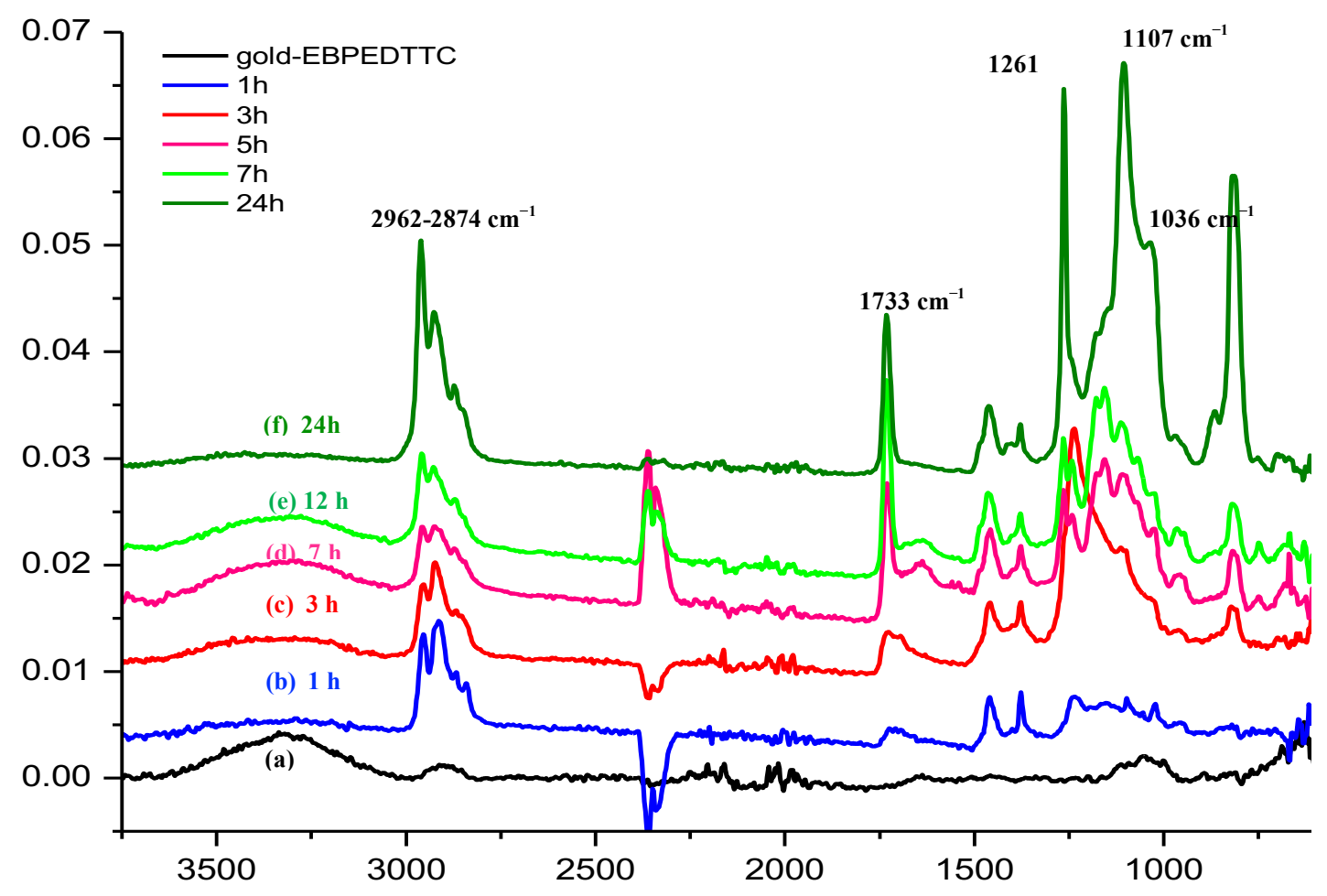

Figure 101 ATR-FTIR spectra of (a) gold-EBPEDTTC and (b),(c),(d),(e),(f) poly (BuMA) brush kinetics synthesized in the presence of free CTA (EBPEDTTC) from gold-EBPEDTTC surface for 1,3,7,12, $24 \mathrm{~h}$ respectively. 
A slight increase in water contact angle from $72.33^{\circ}$ (gold-CTA layer) to $89.22^{\circ}$ (PBuMA layer after $24 \mathrm{~h}$ polymerization) also confirmed that a relatively more hydrophobic polymer layer was obtained. The area shown in Figure 102 for 24 hours polymerization time displays gaussian distribution which is an indicator of surface uniformity although small aggregates heterogeneously distributed over some areas of sample were observed in related AFM height image. Similar morphologies were also observed for $1 \mathrm{~h}$ and $3 \mathrm{~h}$ polymerization time whereas these aggregations are not observable anymore for $5 \mathrm{~h}, 7 \mathrm{~h}$ polymerization time (data not shown). The change in film morphology is associated with a drastic increase in the RMS roughness from 0.8494 (gold-EBPEDTTC layer) to $2.1166 \mathrm{~nm}$ (after $1 \mathrm{~h}$ polymerization) and a subsequent slight decrease to $1.7822 \mathrm{~nm}$ (after $24 \mathrm{~h}$ polymerization).

The time evolution of number-average molecular weights, $M_{n}$ and polydispersities, PDIs of the grafted polymer chains on gold, which is assumed to be the same as that of the unbound polymer chains in solution (263) was obtained by GPC analysis (shown in Table 7) Typical GPC traces of free polymers with the increase of polymerization were monomodal and had narrow molecular weight distributions, which are characteristics of well-defined polymers prepared via RAFT-mediated polymerization. The elution peaks shift to higher molecular weight with increasing reaction time (data not shown). After 24 hours polymerization, the monomer conversion is $86.3 \%$ and the thickness value reached is $28 \mathrm{~nm}$; GPC analysis of the free polymers reveals a $M_{n}$ of $65899 \mathrm{~g} / \mathrm{mol}$ and a PDI of 1 .

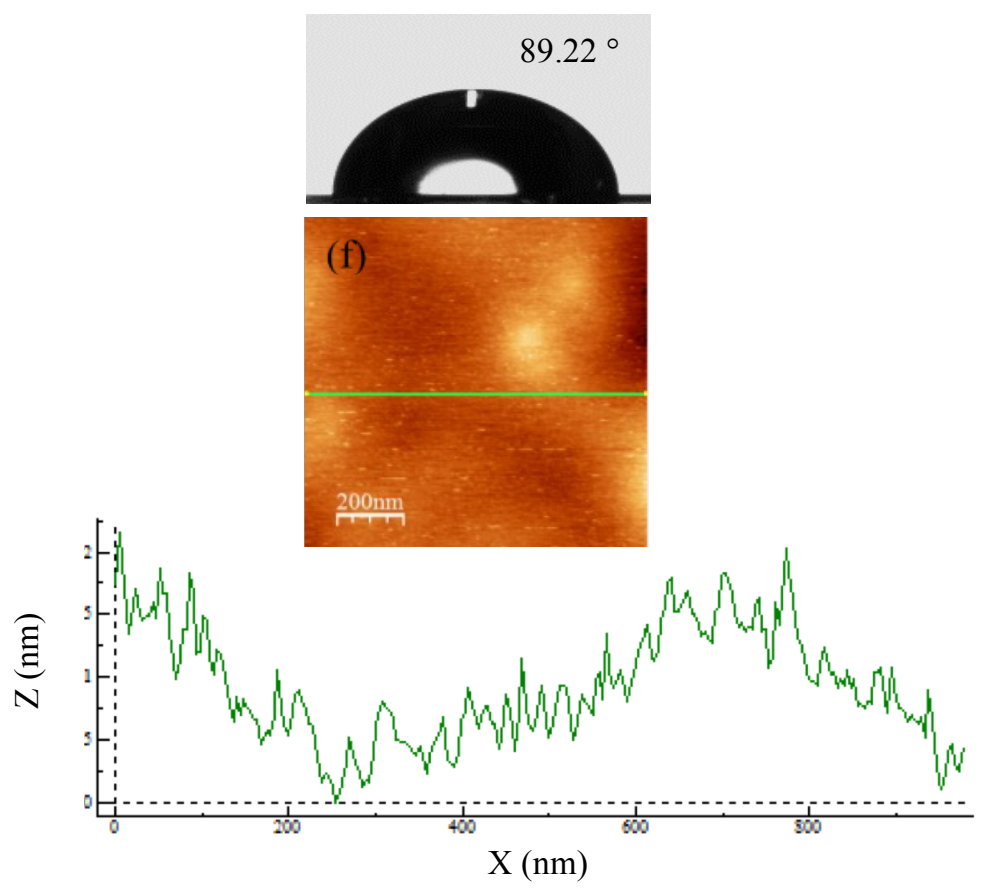

Figure $1022 \mathrm{D}$ AFM topography image $(1 \mu \mathrm{m} \times 1 \mu \mathrm{m})$ in ambient conditions, surface profile analysis, and photograph of $1 \mu \mathrm{L}$ water droplet for poly (BuMA) synthesized 
in the presence of bifunctional free CTA (EBPEDTTC) and AIBN in toluene at $60{ }^{\circ} \mathrm{C}$ for $24 \mathrm{~h}$ from gold-EBPEDTTC surface.

Figure 103 (d) presents the evolution of the $M_{n}$ of the free poly (BUMA) brushes as a function of monomer conversion. The fact that the experimental molecular weights of the free polymer determined by GPC increased linearly with monomer conversion and the PDI values are all below 1.16 indicate that the polymerization was conducted in a controlled manner. Figure 103 (c) shows the linear relationship between $\ln \left[M_{0}\right] \backslash\left[M_{t}\right]$ and time, where $\left[\mathrm{M}_{\mathrm{o}}\right]$ is the initial monomer concentration and $\left[\mathrm{M}_{\mathrm{t}}\right]$ is the monomer concentration remained in reaction mixture after $t$ hour polymerization time. The result indicates that the concentration of the growing species remains constant and first-order kinetic is obtained. The dependence of ellipsometric thickness of poly(BUMA) brush grown from the chain transfer agent immobilized surface on polymerization time is shown in Figure 103 (a). The brush thickness initially increases linearly with polymerization time up to $7 \mathrm{~h}$ and deviates afterwards, which is consistent to with the literature data. (263) The brush growth began to slow down and reached a plateau of $28 \mathrm{~nm}$ in film thickness.

Table 7 Summary of polymerization kinetics of poly (BuMA) brushes from goldEBPEDTTC surfaces

\begin{tabular}{|c|c|c|c|c|c|c|c|}
\hline $\begin{array}{l}\text { Reaction } \\
\text { Time } \\
\text { (h) }\end{array}$ & $\mathbf{M n} / \mathbf{M w}$ & PDI & $\begin{array}{c}\text { Thickness }^{\mathrm{a}} \\
\text { (nm) }\end{array}$ & $\begin{array}{c}\text { Coverage } \\
\left(\mathrm{mg} / \mathrm{m}^{2}\right)\end{array}$ & $\begin{array}{l}\text { Grafting } \\
\text { Density } \\
\text { (chains/ } \\
\mathbf{n m}^{\mathbf{2}} \text { ) }\end{array}$ & $\begin{array}{l}\text { Distance } \\
\text { (nm) }\end{array}$ & $\begin{array}{l}\text { Static } \\
\text { Contact } \\
\text { Angle }^{b}\end{array}$ \\
\hline 1 & $26006 / 29897$ & 1.14 & 5 & 5.26 & 0.12 & 3.2 & $78.53^{\circ}$ \\
\hline 3 & $29996 / 34398$ & 1.14 & 8 & 8.42 & 0.16 & 2.8 & $79.22^{\circ}$ \\
\hline 7 & $36540 / 41309$ & 1.13 & 11 & 11.6 & 0.19 & 2.6 & $83.59^{\circ}$ \\
\hline 12 & $49573 / 55892$ & 1.12 & 17.4 & 18.3 & 0.22 & 2.4 & $88.20^{\circ}$ \\
\hline 24 & $65899 / 76959$ & 1.16 & 28 & 29.4 & 0.26 & 2.2 & $89.22^{\circ}$ \\
\hline
\end{tabular}

Polymerization of (buthyl methacrylate) BuMA with Ethane-1,2-diyl bis(1phenylethyl) ditrithiocarbonate (EBPEDTTC) and 2,2'-azobis(isobutyronitrile) (AIBN) in toluene at $60{ }^{\circ} \mathrm{C}$ for $24 \mathrm{~h} .{ }^{a}$ Thicknesses were determined by ellipsometry and are an average of five samplings across the sample. Error of thicknesses measured was within $\pm 0.1 \mathrm{~nm} .{ }^{b}$ Static contact angles were taken using goniometry and are an 
average of five samplings across the sample. The standard deviation of contact angles was less than $1^{\circ}$.

The reason for this deviation from linear behavior might be increase in steric hindrance to chain growth for longer polymer brushes in the latter stage of polymerization as time goes on. Nature of the approach used in this work limits the accessibility of growing polymeric radicals onto the surface in the latter stage of polymerization since the mechanism becomes very similar to Z-group approach as polymerization proceeds. As a consequence of the fact that raft agents are attached to surface through their trithiocarbonate moieties, not through their R groups, polymeric radicals are not growing on the surface. They split up the surface, add monomers in solution phase and re-approach to the surface back. This "re-approach" process is relatively easier at the early stage of the polymerization. As time goes by and polymerization proceeds, both the length and size of polymeric radicals increase whereas the mobility decreases. This steric hindrance leads to the delivery of the limited amount of growing polymeric radicals back onto the surface in a slow motion for progressive stage of polymerization, which automatically results in an observable decrease in thickness increase, in another words, deviation from the linear behavior. It is also clearly observable that the surface coverage of polymer brushes displays linear increase with the increase in molecular weight. [Figure 103 (b)]

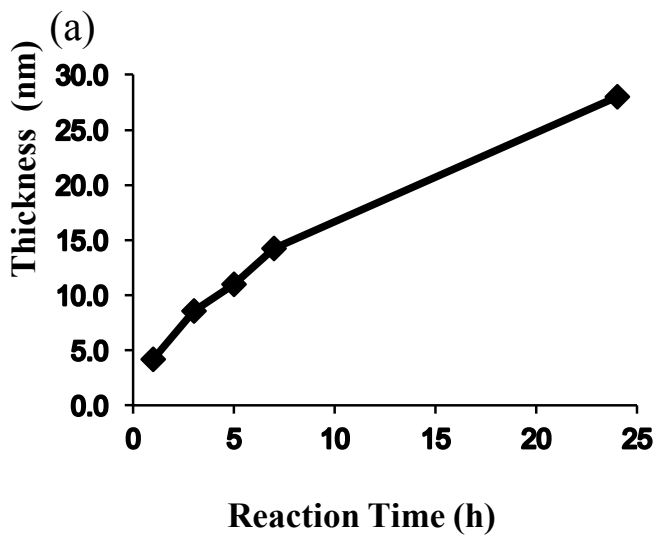

(c)

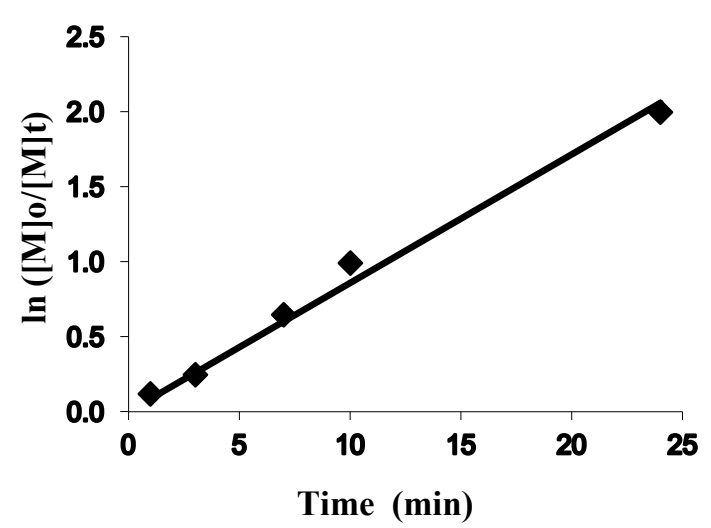

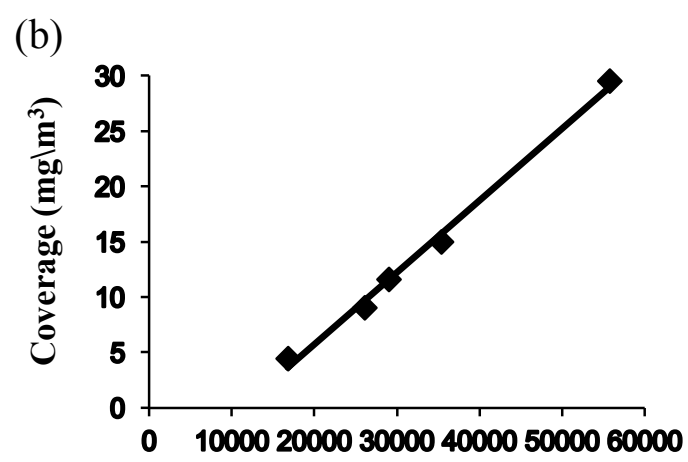

Molecular Weight (g/mol)

(d)

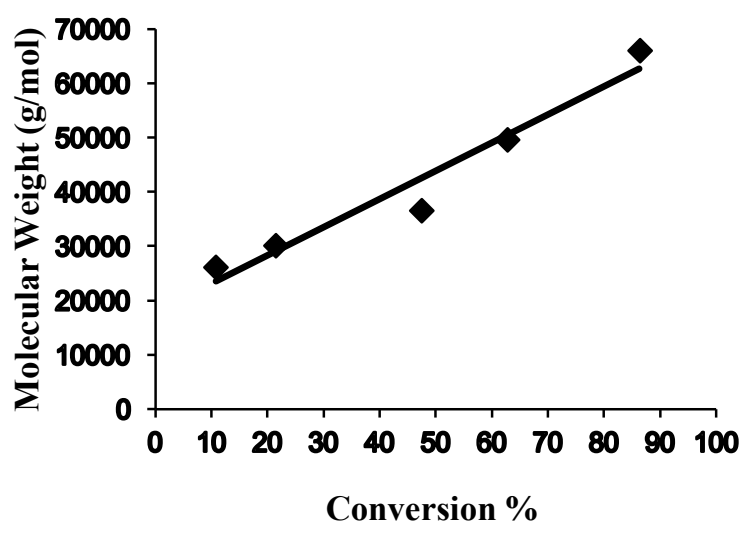


Figure 103 Results of RAFT mediated polymerizations of BuMA from goldEBPEDTTC surface in the presence of bifunctional free CTA ethane-1,2-diyl bis(1phenylethyl) ditrithiocarbonate (EBPEDTTC) in Toluen at $60{ }^{\circ} \mathrm{C}$ a) thickness profile of poly (BuMA) brushes, b) the surface coverage plotted against molecular weight of poly (BuMA) brushes, c) plot of pseudo-first-order kinetics of poly (BuMA) brushes, d) molecular weights of poly (BuMA) brushes with inreasing monomer conversion.

\subsection{Characterization of Homopolymer Brushes Prepared on Gold}

\subsubsection{Characterization of Poly (DMAEMA) Brushes Synthesized in The Presence of Free CTA (CDB) from Gold-CDB Surface}

It is important to tether high amount of CTA onto gold surface in order to obtain a polymer brush with high grafting density. For this reason, in traditional multistep approaches, it is important to use an appropriate reaction type for surface immobilization to increase the coupling efficiency of CTAs with self assembling monolayers (SAM) pre-tethered on gold surface. Here, in all these studies, there was no SAM layer used as first layer and no coupling reaction was required in order to couple CTAs onto gold surface. A commercial dithio-based raft agent (CDB) was chemisorbed onto gold surface via a simple self assembly process explained in Experimental section. RAFT-mediated polymerization of DMAEMA on gold surface was performed in the presence of "sacrificial"' CTA (CDB) to obtain better control over the polymerization. The presence of the sacrificial CTA provides the control of overall concentration of monomer and chain transfer agents which is directly connected to the control of molar mass and film thickness of tethered polymer brushes. The correlation of molecular weight of tethered chains with "free" unbounded polymers grown in solution allows indirect analysis of polymer brushes growing on the surface. It is a well known fact that Mn and PDI values of both tethered polymers on surface and bulk polymers in solution are comparable. (196)

The formation of poly (DMAEMA) brush was confirmed by ATR-FTIR [Figure 104 (b)], AFM and water contact angle measurements (Figure 105). The ATR-FTIR spectra for poly (DMAEMA) brush demonstrated characteristic absorption bands at $\sim 1732 \mathrm{~cm}^{-1}$, associated with the carbonyl stretch, at $1046 \mathrm{~cm}^{-1}$ resulted from strong $\mathrm{C}=\mathrm{S}$ stretching vibrations suppressed by absorption bands at $1109 \mathrm{~cm}^{-1}, 1264 \mathrm{~cm}^{-1}$ associated with $\mathrm{C}-\mathrm{O}$ stretching of $\mathrm{C}(=\mathrm{O})-\mathrm{O}-\mathrm{C}$ bonds in monomer units and peaks at $1235-1023 \mathrm{~cm}^{-1}$ resulted from vibrations of C-N stretching. The bands at around 2958, $2924,2853 \mathrm{~cm}^{-1}$ are considered to be due to aliphatic $\mathrm{CH}$ groups. The $2958,2853 \mathrm{~cm}^{-1}$ peaks are due to asymmetric and symmetric stretching vibrations of $\mathrm{CH}_{3}$, respectively, while the $2924 \mathrm{~cm}^{-1}$ peak is assigned to $\mathrm{CH}_{2}$ vibration. 


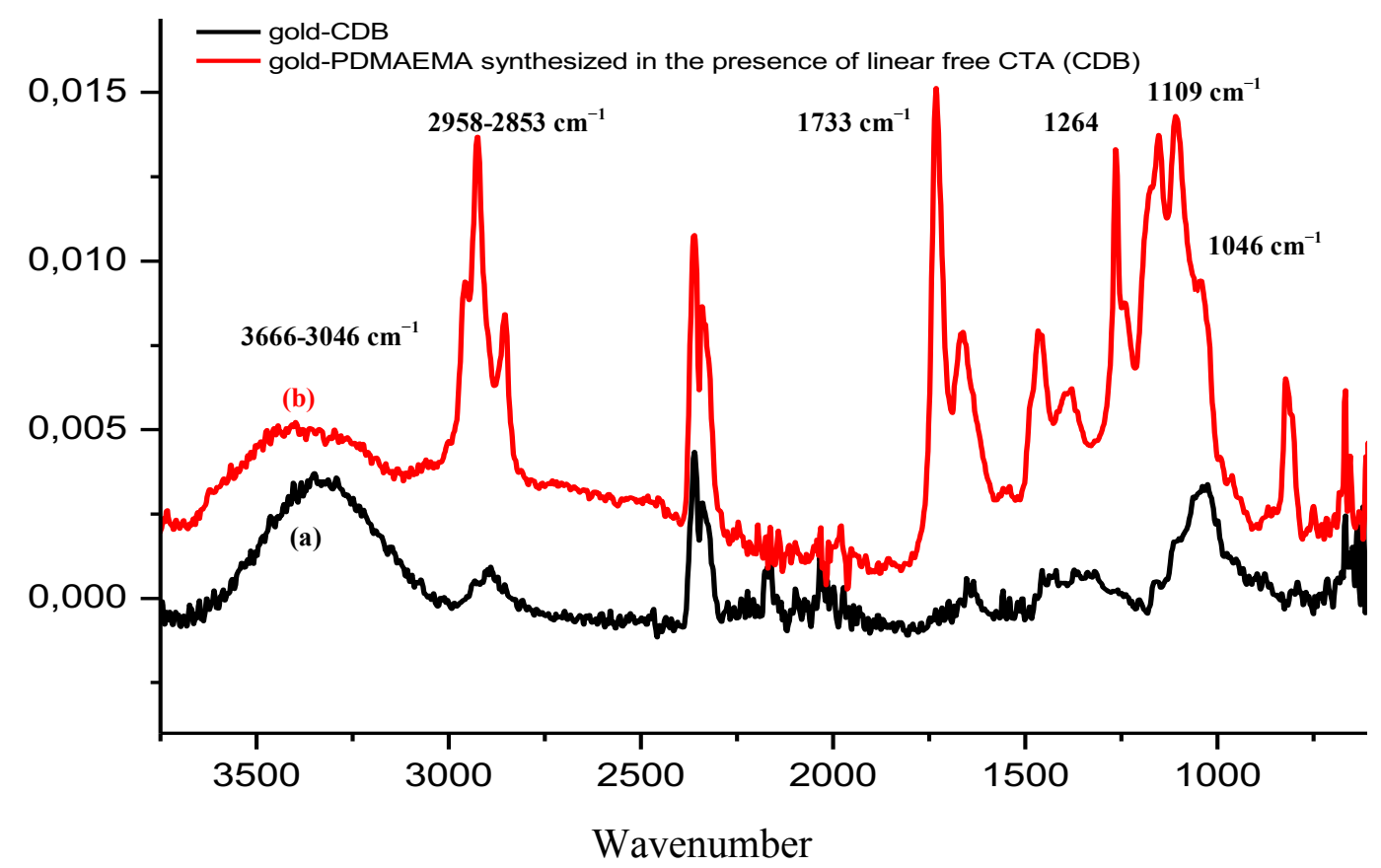

Figure 104 ATR-FTIR spectra of (a) gold-CDB and (b) poly (DMAEMA) brush synthesized in the presence of mono functional free CTA (CDB) from gold-CDB surface for $24 \mathrm{~h}$ with 2000:4:1 ratio of [DMAEMA] / [CTA] / [I] in feed.

The characteristic peaks for the $-\mathrm{N}\left(\mathrm{CH}_{3}\right)_{2}$ stretching vibrations, which generally appears at $\sim 2830 \mathrm{~cm}^{-1}$ is not clearly observable as a result of being overlapped. In addition to these bands, there are bands at $714-624 \mathrm{~cm}^{-1}$ associated with C-S stretching while the peaks at $1466 \mathrm{~cm}^{-1}$ and $1380 \mathrm{~cm}^{-1}$ are attributed to the $\mathrm{CH}$ bending and rocking, respectively. The broad band at $3666-3046 \mathrm{~cm}^{-1}$ which is assigned to $\mathrm{O}-\mathrm{H}$ stretching, results from water vapor which PDMAEMA brush coating absorbs due to its hydrophilic nature. The aromatic C-H stretching peak expected at around 3100$3000 \mathrm{~cm}^{-1}$ is not clearly observable as a result of being suppressed by both aliphatic $\mathrm{C}-\mathrm{H}$ stretching and broad band of $\mathrm{O}-\mathrm{H}$ stretching whereas $\mathrm{C}=\mathrm{C}$ aromatic doublets, which are expected at $1480-1420 \mathrm{~cm}^{-1}$, were observed at $1466 \mathrm{~cm}^{-1}$, being overlapped with $\mathrm{CH}$ bending vibration.

A slight decrease in water contact angle from $71.15^{\circ}$ (gold-CTA layer) to $63.57^{\circ}$ (PDMAEMA layer after $24 \mathrm{~h}$ polymerization) also confirmed that a relatively more hydrophilic polymer layer than CTA immobilized gold was obtained. The area shown in Figure 105 for 24 hours polymerization time and the other areas measured on same sample (data not shown) display gaussian distribution which is an indicator of surface homogenitiy. The change in film morphology is associated with a drastic decrease in the RMS roughness from $1.1518 \mathrm{~nm}$ (gold-CDB layer) to $0.4254 \mathrm{~nm}$ (PDMAEMA brush layer). 


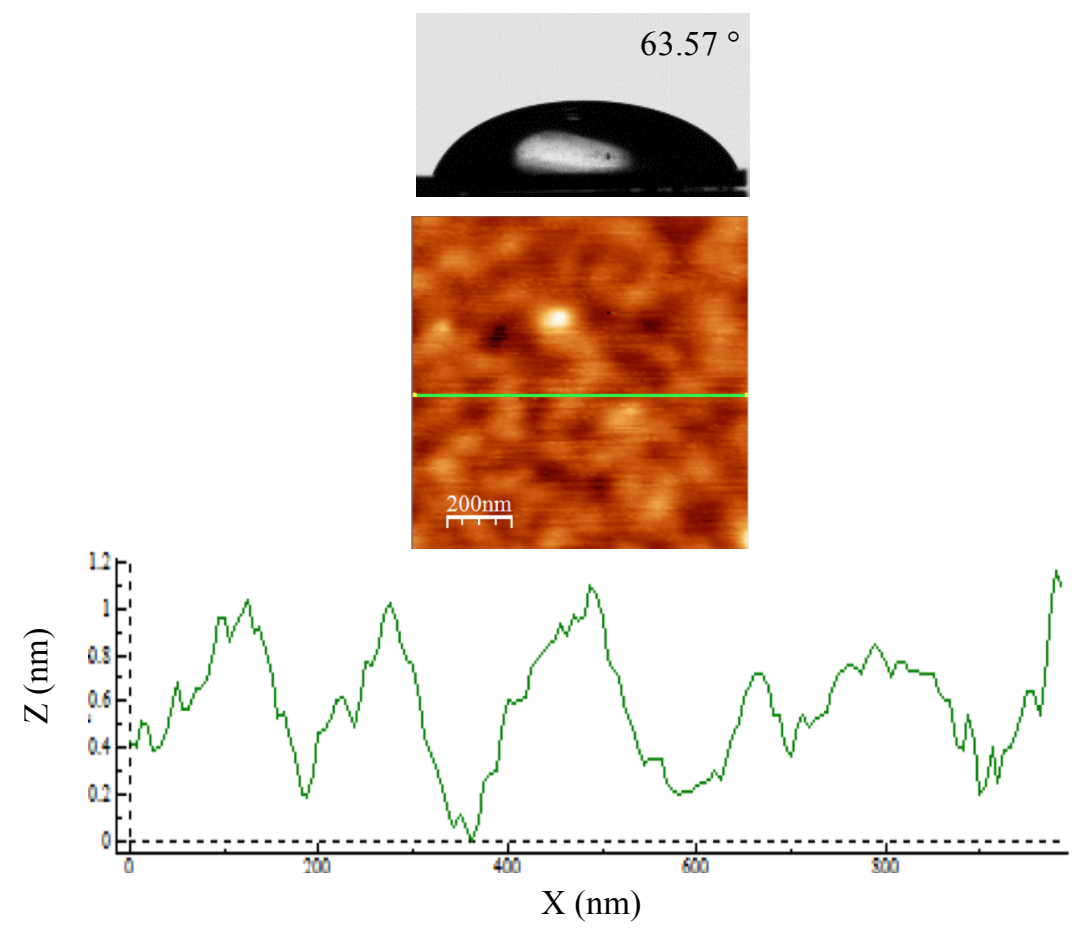

Figure 105 2D AFM topography image $(1 \mu \mathrm{m} \times 1 \mu \mathrm{m})$ in ambient conditions, surface profile analysis and photograph of $1 \mu \mathrm{L}$ water droplet for poly (DMAEMA) synthesized in the presence of linear free CTA (CDB) and AIBN in toluene at $60{ }^{\circ} \mathrm{C}$ for $24 \mathrm{~h}$ from gold-CDB surface.

GPC trace of free polymer was monomodal and had narrow molecular weight distribution, which are characteristics of well-defined polymers prepared via RAFTmediated polymerization. After $24 \mathrm{~h}$ polymerization time, the monomer conversion is $89.8 \%$ and the thickness value reached is $21 \mathrm{~nm}$; GPC analysis of the free polymers reveals a $M_{n}$ of $37714 \mathrm{~g} / \mathrm{mol}$ and a PDI of 1.31 (Table 8). The low PDI value indicates that the polymerization was conducted in a controlled manner. The surface coverage, A $\left(m g / m^{2}\right)$, grafting density, $\sigma$ (chains $\left./ \mathrm{nm}^{2}\right)$, and average distance between grafting sites, $D(\mathrm{~nm})$, of grafted polymer brushes were calculated from the $M_{n}$ of the free polymer and the dry film thickness, $h(\mathrm{~nm})$, according to equations 18,19 and 20 and summarized in Table 8. As a result of that the grafting density is 0.45 chains per $\mathrm{nm}^{2}$, poly(DMAEMA) brushes might be considered as a brush type between semi-dilute and a high density brush, which is closer to a high density conformation than semidilute. (509)

\subsubsection{Characterization of Poly (DMAEMA) Brushes Synthesized in The Presence of Bifunctional Free CTA (EBPEDTTC) from Gold-EBPEDTTC Surface}

The formation of poly (DMAEMA) brushes was confirmed by ATR-FTIR [Figure 113 (b)], AFM and water contact-angle measurements. (Figure 106) The ATR-FTIR 
spectra of the poly (DMAEMA) brush demonstrated characteristic absorption bands at $\sim 1729 \mathrm{~cm}^{-1}$, associated with the carbonyl stretch and at $1071 \mathrm{~cm}^{-1}$ resulted from strong $\mathrm{C}=\mathrm{S}$ stretching vibrations suppressed by absorption bands at $1111 \mathrm{~cm}^{-1}$ and $1264 \mathrm{~cm}^{-1}$ associated with $\mathrm{C}-\mathrm{O}$ stretching of $\mathrm{C}(=\mathrm{O})-\mathrm{O}-\mathrm{C}$ bonds in monomer units and bands at $1237-1023 \mathrm{~cm}^{-1}$ resulted from vibrations of $\mathrm{C}-\mathrm{N}$ stretching. The bands at around 2922, 2891, $2849 \mathrm{~cm}^{-1}$ are considered to be due to aliphatic $\mathrm{CH}$ groups. The $2922,2849 \mathrm{~cm}^{-1}$ peaks are due to asymmetric and symmetric stretching vibrations of $\mathrm{CH}_{3}$, respectively, while the $2891 \mathrm{~cm}^{-1}$ peak is assigned to $\mathrm{CH}_{2}$ vibration. A slight shift is observed in stretching vibrations of $\mathrm{CH}_{3}\left(2849 \mathrm{~cm}^{-1}\right)$ compared to PPEGMA (2870 $\mathrm{cm}^{-1}$ ) as a result of being overlapped by the characteristic peaks for the $-\mathrm{N}\left(\mathrm{CH}_{3}\right)_{2}$, which generally appears at $\sim 2830 \mathrm{~cm}^{-1}$. In addition to these bands, there are peaks at $710-620 \mathrm{~cm}^{-1}$ are associated with C-S stretching and at $1462 \mathrm{~cm}^{-1}, 1389 \mathrm{~cm}^{-1}$ attributed to the $\mathrm{CH}$ bending and rocking, respectively. The broad band at 3558-3021 $\mathrm{cm}^{-1}$ which is assigned to $\mathrm{O}-\mathrm{H}$ stretching, results from water vapor which PDMAEMA brush coating absorbs due to its hydrophilic nature. The aromatic $\mathrm{C}-\mathrm{H}$ stretching peak expected at around 3100-3000 is not clearly observable as a result of being suppressed by broad band of $\mathrm{O}-\mathrm{H}$ stretching whereas $\mathrm{C}=\mathrm{C}$ aromatic doublets which are expected between 1480-1420 $\mathrm{cm}^{-1}$, are observable at $1462 \mathrm{~cm}^{-1}$, being overlapped with $\mathrm{CH}$ bending vibration.

A slight decrease in water contact angle from $72.33^{\circ}$ (gold-CTA layer) to $65.11^{\circ}$ (PDMAEMA layer) also confirmed that a relatively more hydrophilic polymer layer than CTA immobilized gold surface was obtained. The area shown in for 24 hour polymerization time displays gaussian distribution which is an indicator of surface uniformity. The change in film morphology is associated with a drastic increase in the RMS roughness from $0.8494 \mathrm{~nm}$ (gold-EBPEDTTC layer) to $1.6883 \mathrm{~nm}$ (PDMAEMA layer).
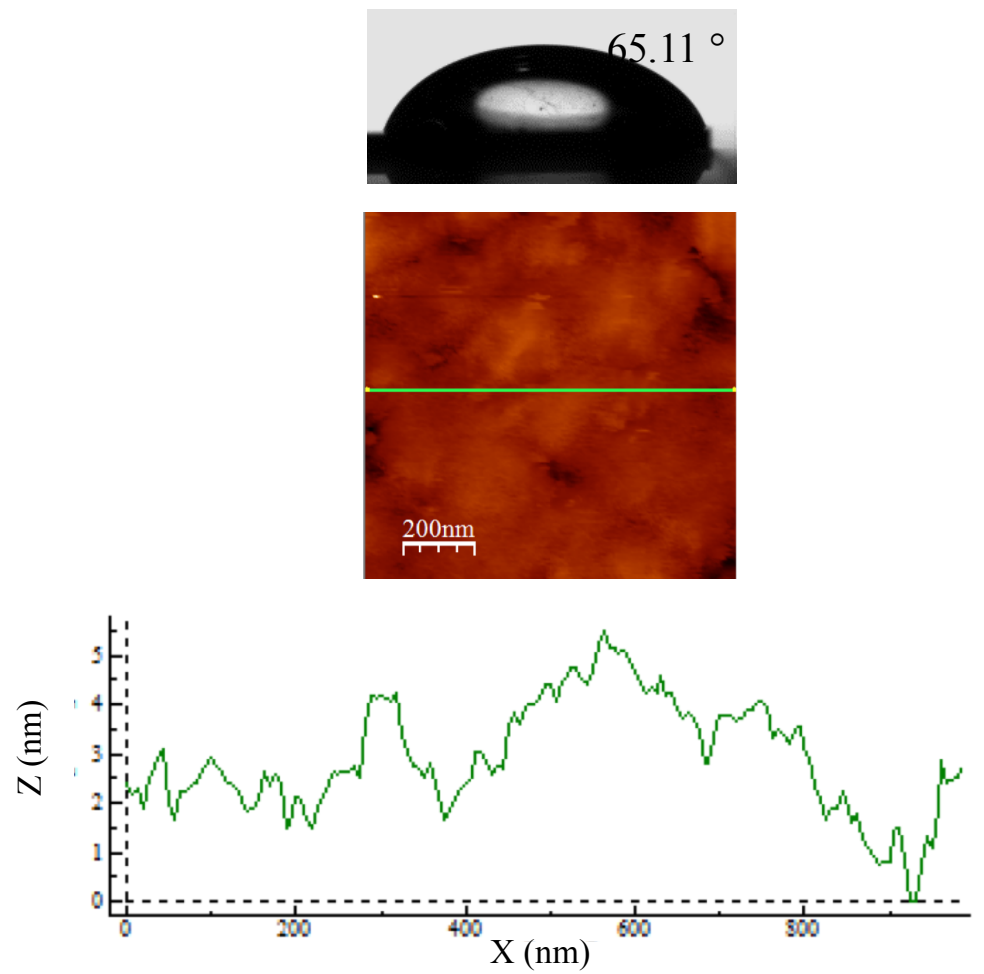
Figure $1062 \mathrm{D}$ AFM topography images $(1 \mu \mathrm{m} \times 1 \mu \mathrm{m})$ in ambient conditions, surface profile analysis and photograph of $1 \mu \mathrm{L}$ water droplet for poly (DMAEMA) brushes synthesized in the presence of bifunctional free CTA (EBPEDTTC) and AIBN in toluene at $60{ }^{\circ} \mathrm{C}$ for $24 \mathrm{~h}$ from gold-EBPEDTTC surface.

GPC trace of free polymers was monomodal and had narrow molecular weight distribution, which are characteristics of well-defined polymers prepared via RAFTmediated polymerization. After $24 \mathrm{~h}$ polymerization, the monomer conversion is 48.7 $\%$ whereas the ellipsometric thickness of the brush is $27 \mathrm{~nm}$. GPC analysis of the free polymers reveals a $M_{n}$ of $38409 \mathrm{~g} / \mathrm{mol}$ and a PDI of 1.40 . (Table 8) The low PDI value indicates that the polymerization was conducted in a controlled manner. The surface coverage, A $\left(\mathrm{mg} / \mathrm{m}^{2}\right)$, grafting density, $\sigma$ (chains $\left./ \mathrm{nm}^{2}\right)$ and average distance between grafting sites, $D(\mathrm{~nm})$ of grafted polymer brushes were calculated from the $M_{n}$ of the free polymer and the dry film thickness, $h(\mathrm{~nm})$, according to equations $18,19,20$ and summarized in Table 8. As a result of that the grafting density is 0.57 chains per $\mathrm{nm}^{2}$, poly (DMAEMA) brushes might be considered as a brush type between semi-dilute and a high density brush, which is closer to a high density conformation than semidilute. (509)

\subsubsection{Characterization of Poly (PEGMA) Brushes Synthesized in The Presence of Bifunctional Free CTA (EBPEDTTC) and in the Presence of Monofunctional Free CTA (DSTCSPA) from Gold-EBPEDTTC Surface}

The growth of poly (PEGMA) brushes from two identical gold-EBPEDTTC surfaces was conducted in two different reaction mixtures which include different type raft agents in order to check impact of free CTA type on resulting thickness and PDI. For this reason, PPEGMA brushes were prepared in the presence of bifunctional free CTA (EBPEDTTC) and monofunctional free CTA (DSTCSPA) in solution and characterized by Ellipsometry (Table 8), ATR-FTIR [Figure 107 (b), (c)], AFM and water contact-angle measurements. [Figure 108 (a), (b)] The ATR-FTIR spectra of poly (PEGMA) brush demonstrated characteristic absorption bands at $\sim 1728 \mathrm{~cm}^{-1}$, associated with the carbonyl stretch, at $1032 \mathrm{~cm}^{-1}$ resulted from strong $\mathrm{C}=\mathrm{S}$ stretching vibrations suppressed by absorption band at $\sim 1102 \mathrm{~cm}^{-1}, 1264 \mathrm{~cm}^{-1}$ resulted from C$\mathrm{O}$ stretching vibrations of C-O-C ether bonds in the ethylene glycol units. The bands at around 2922, 2897, $2868 \mathrm{~cm}^{-1}$ are considered to be due to aliphatic $\mathrm{CH}$ groups. The $2922,2868 \mathrm{~cm}^{-1}$ peaks are due to asymmetric and symmetric stretching vibrations of $\mathrm{CH}_{3}$, respectively, while the $2897 \mathrm{~cm}^{-1}$ peak is assigned to $\mathrm{CH}_{2}$ vibration. The peaks at $1457 \mathrm{~cm}^{-1}$ and $1379 \mathrm{~cm}^{-1}$ are attributed to the $\mathrm{CH}$ bending and rocking respectively. In addition to these bands, there are bands at $703-631 \mathrm{~cm}^{-1}$ attributed to C-S stretching. The broad band at $3685-3026 \mathrm{~cm}^{-1}$ which is assigned to $\mathrm{O}-\mathrm{H}$ stretching, results from water vapor which PPEGMA brush coating absorbs due to its hydrophilic nature. The aromatic C-H stretching peak expected at around $3100-3000 \mathrm{~cm}^{-1}$ is not clearly observable as a result of being suppressed by both aliphatic $\mathrm{C}-\mathrm{H}$ stretching and broad 
band of $\mathrm{O}-\mathrm{H}$ stretching whereas $\mathrm{C}=\mathrm{C}$ aromatic doublets which are expected at a value between 1480-1420 $\mathrm{cm}^{-1}$, are observable at $1457 \mathrm{~cm}^{-1}$, being overlapped with $\mathrm{CH}$ bending vibration. A drastic decrease in water contact angle from $72.33^{\circ}$ (gold-CTA layer) to $36.67^{\circ}$ (PPEGMA layer synthesized in the presence of bifunctional free CTA EBPEDTTC) and to $27.21^{\circ}$ (PPEGMA layer synthesized in the presence of monofunctional free CTA DSTCSPA) confirmed that a relatively more hydrophilic polymer layer than CTA immobilized gold. The areas shown in Figure 108 (a) and (b) for 24 hour polymerization time display gaussian distribution which is an indicator of surface uniformity. The change in film morphology is associated with a drastic decrease in the RMS roughness from $0.8494 \mathrm{~nm}$ (gold-EBPEDTTC layer) to $0.373 \mathrm{~nm}$ (PPEGMA brush layer synthesized in the presence of bifunctional free CTA EBPEDTTC) and to $1.7822 \mathrm{~nm}$ (PPEGMA layer synthesized in the presence of monofunctional free CTA DSTCSPA).

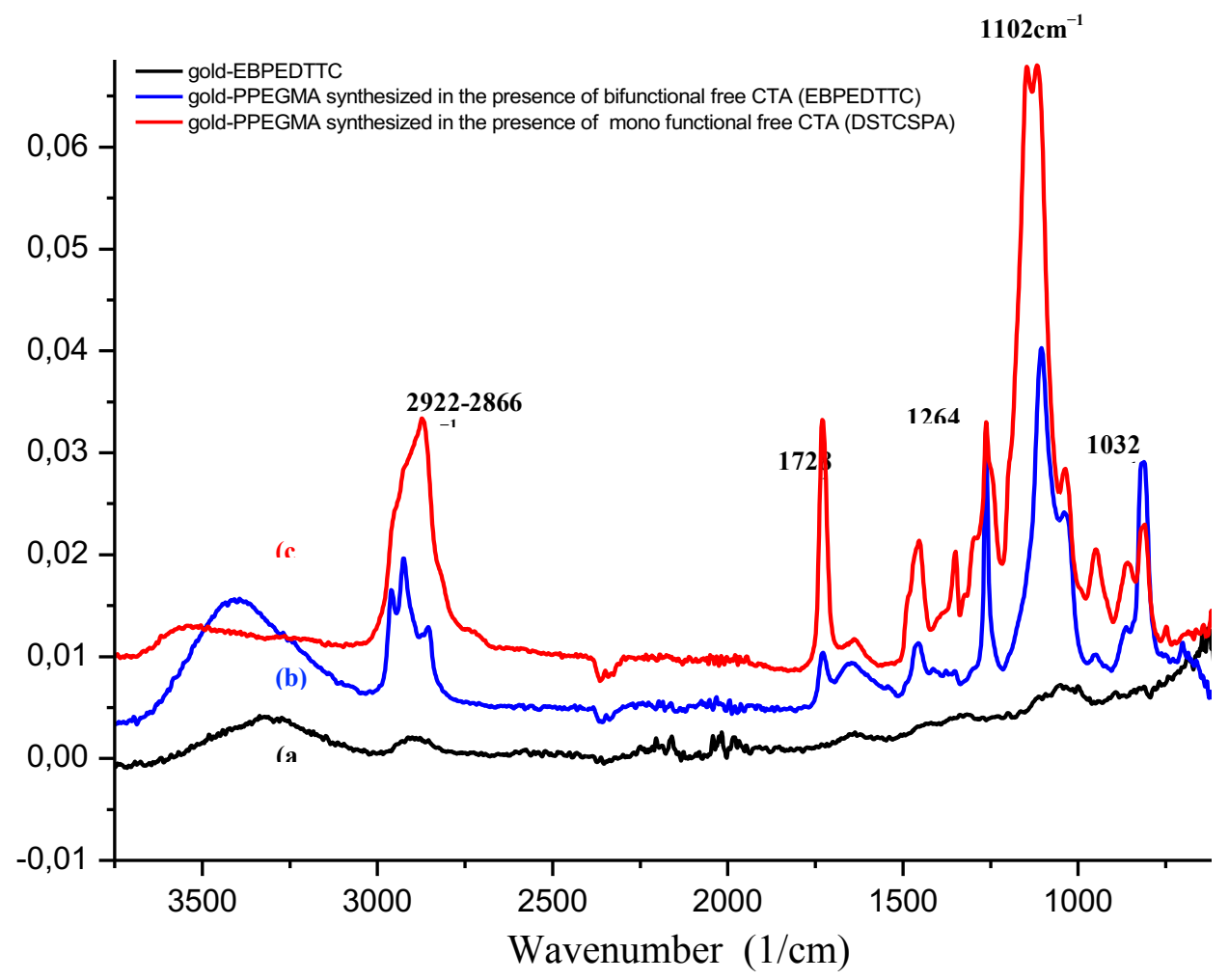

Figure 107 ATR-FTIR spectra of (a) gold-EBPEDTTC, (b) poly (PEGMA) brush synthesized in the presence of bifunctional free CTA (EBPEDTTC) from goldEBPEDTTC for $24 \mathrm{~h}$ and (c) poly (PEGMA) brush synthesized in the presence of mono functional free CTA (DSTCSPA) from gold-EBPEDTTC for $24 \mathrm{~h}$. 


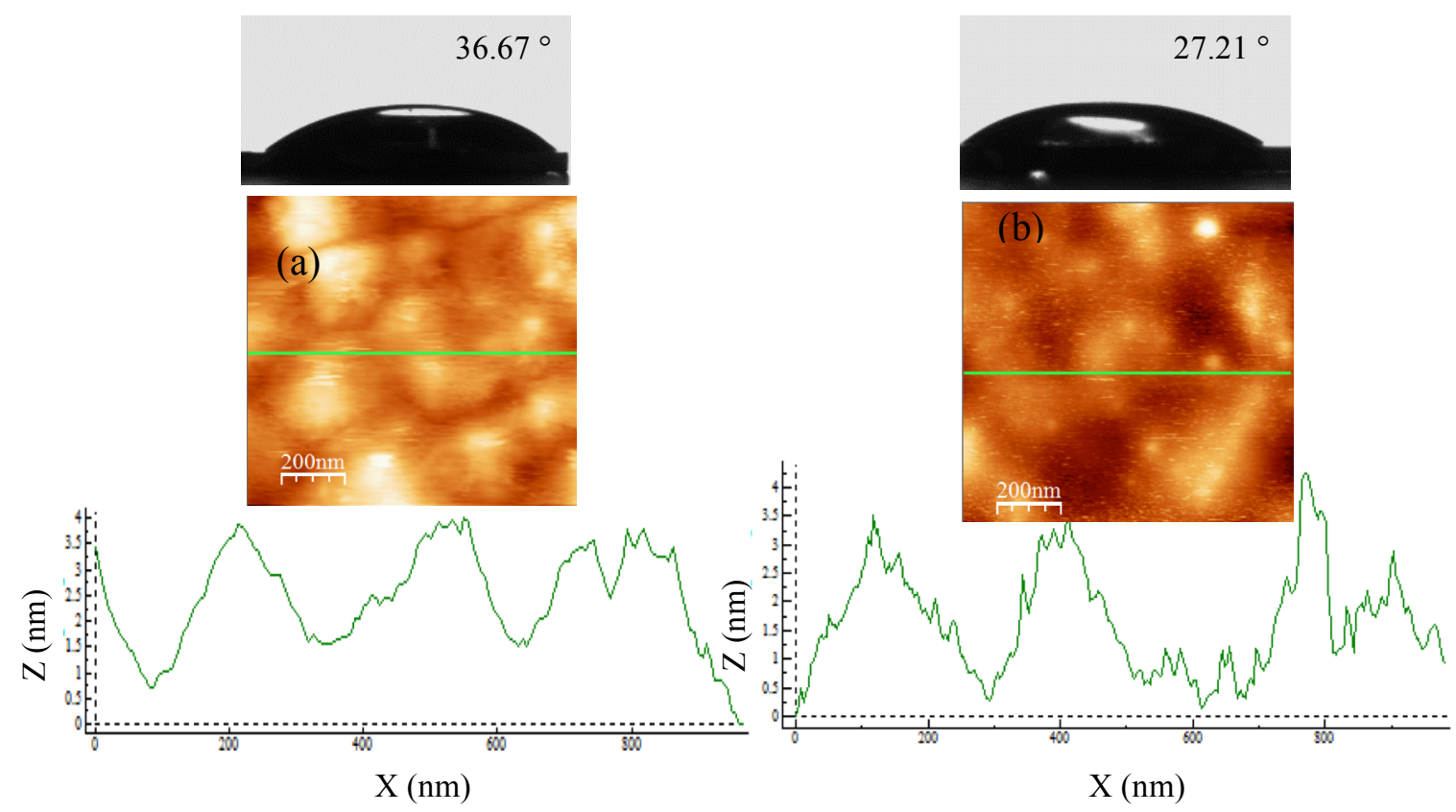

Figure $1082 \mathrm{D}$ AFM topography images $(1 \mu \mathrm{m} \times 1 \mu \mathrm{m})$ in ambient conditions, surface profile analysis and photographs of $1 \mu \mathrm{L}$ water droplets for (a) poly (PEGMA) synthesized in the presence of bifunctional free CTA (EBPEDTTC) and AIBN in toluene at $60{ }^{\circ} \mathrm{C}$ for $24 \mathrm{~h}$ from gold-EBPEDTTC surface (b) poly (PEGMA) synthesized in the presence of linear free CTA (DSTCSPA) and AIBN in toluene at 60 ${ }^{\circ} \mathrm{C}$ for $24 \mathrm{~h}$ from gold-EBPEDTTC surface.

GPC trace of free polymers was monomodal and had narrow molecular weight distribution, which are characteristics of well-defined polymers prepared via RAFTmediated polymerization. The monomer conversion for polymerization conducted in the presence of bifunctional free CTA (EBPEDTTC) is $31 \%$ and the resulting thickness $43.1 \mathrm{~nm}$, whereas they are $52.4 \%$ and $59.3 \mathrm{~nm}$ respectively for polymerization conducted in the presence of monofunctional free CTA (DSTCSPA). GPC analysis of the free polymers reveals a $M_{n}$ of $73162 \mathrm{~g} / \mathrm{mol}$ and a PDI of 1.20 for free polymers synthesized from bifunctional free CTA (EBPEDTTC) and a $M_{n}$ of $66395 \mathrm{~g} / \mathrm{mol}$ and a PDI of 1.40 for free polymers synthesized from monofunctional free CTA (DSTCSPA). (Table 8) The low PDI values indicate that both the polymerizations were conducted in a controlled manner.

The surface coverages, A $\left(m g / m^{2}\right)$, grafting densities, $\sigma$ (chains $\left./ \mathrm{nm}^{2}\right)$, and average distances between grafting sites, $D(\mathrm{~nm})$, of grafted polymer brushes were calculated from the $M_{n}$ of the free polymer and the dry film thickness, $h(\mathrm{~nm})$, according to equations 18, 19 and 20 and summarized in Table 8. As a result of that the grafting densities of both polymer brushes synthesized in the presence of bifunctional free CTA (EBPEDTTC) and in the presence of monofunctional free CTA (DSTCSPA) are 0.38 and 0.58 chains per $\mathrm{nm}^{2}$ respectively, poly (PEGMA) brushes might be classified as a brush type between semi-dilute and a high density brush, which is closer to a high 
density conformation than semi-dilute. (509) It is apperant that the resulting thickness $(59.3 \mathrm{~nm})$ and grafting density $\left(0.58\right.$ chains $\left./ \mathrm{nm}^{2}\right)$ of polymer brush synthesized in the presence of monofunctional free CTA (DSTCSPA) in solution is higher than that of the case of utilization of bifunctional free CTA (EBPEDTTC) in the solution due to higher conversion reached with monofunctional free CTA.

\subsubsection{Characterization of Poly (PEGMA) Brushes Synthesized in the Presence of Tetra Functional Free CTA (PETSPETTCP) and in the Presence of Monofunctional Free CTA (DSTCSPA) from Gold-PETSPETTCP Surface}

The growth of poly (PEGMA) brushes from two identical gold-PETSPETTCP surfaces was conducted in two different reaction mixtures which include different type raft agents in order to check impact of free CTA type on resulting thickness and PDI. For this reason, PPEGMA brushes were prepared in the presence of tetra functional free CTA (PETSPETTCP) and monofunctional free CTA (DSTCSPA) in solution and characterized by Ellipsometry (Table 8), ATR-FTIR [Figure 109 (c), (b)], AFM and water contact angle measurements. [Figure 110 Figure 110 (a), (b)] The ATR-FTIR spectra of poly (PEGMA) brushes demonstrated characteristic absorption bands at $\sim 1728 \mathrm{~cm}^{-1}$, associated with the carbonyl stretch, at $1038 \mathrm{~cm}^{-1}$ resulted from strong $\mathrm{C}=\mathrm{S}$ stretching vibrations suppressed by absorption bands at $\sim 1110 \mathrm{~cm}^{-1}, 1260 \mathrm{~cm}^{-1}$ associated with $\mathrm{C}-\mathrm{O}$ stretching resulted from vibrations of $\mathrm{C}-\mathrm{O}-\mathrm{C}$ ether bonds in the ethylene glycol units. The bands at around 2956,2924, $2870 \mathrm{~cm}^{-1}$ are considered to be due to aliphatic $\mathrm{CH}$ groups. The $2956,2870 \mathrm{~cm}^{-1}$ peaks are due to asymmetric and symmetric stretching vibrations of $\mathrm{CH}_{3}$, respectively, while the $2924 \mathrm{~cm}^{-1}$ peak is assigned to $\mathrm{CH}_{2}$ vibration. The peaks at $1457 \mathrm{~cm}^{-1}$ and $1379 \mathrm{~cm}^{-1}$ are attributed to the $\mathrm{CH}$ bending and rocking respectively. In addition to these bands, there are absorption bands at $749-631 \mathrm{~cm}^{-1}$ attributed to C-S stretching. The broad band at $3670-3172 \mathrm{~cm}^{-1}$ which is assigned to $\mathrm{O}-\mathrm{H}$ stretching, results from water vapor which PPEGMA brush coating absorbs due to its hydrophilic nature. The aromatic $\mathrm{C}-\mathrm{H}$ stretching peak expected at around 3100-3000 is not clearly observable as a result of being suppressed by both aliphatic $\mathrm{C}-\mathrm{H}$ stretching and broad band of $\mathrm{O}-\mathrm{H}$ stretching whereas $\mathrm{C}=\mathrm{C}$ aromatic doublets which are expected at a value between $1480-1420 \mathrm{~cm}^{-1}$, are observable at $1457 \mathrm{~cm}^{-1}$, being overlapped with $\mathrm{CH}$ bending vibration. 


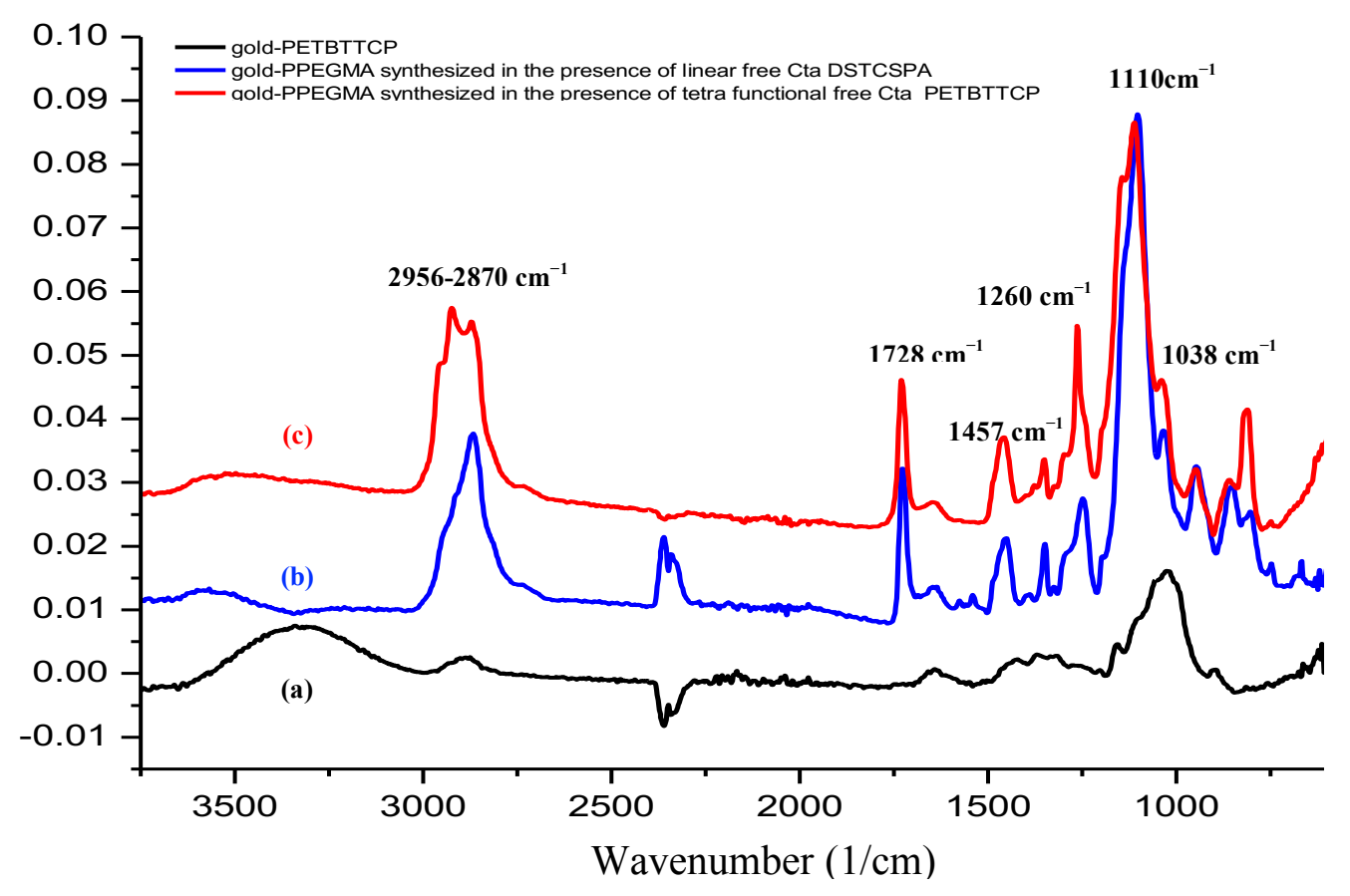

Figure 109 ATR-FTIR spectra of (a) gold-PETSPETTCP, (b) poly (PEGMA) brush synthesized in the presence of monofunctional free CTA (DSTCSPA) from goldPETSPETTCP surface for $24 \mathrm{~h}$, (c) poly (PEGMA) brush synthesized in the presence of tetra functional free CTA (PETSPETTCP) from gold-PETSPETTCP surface for 24 $\mathrm{h}$

A drastic decrease in water contact angle from $76.65^{\circ}$ (gold-CTA layer) to $36.64^{\circ}$ (PPEGMA layer synthesized in the presence of tetrafunctional free CTA (PETSPETTCP) and to $21.77^{\circ}$ (PPEGMA layer synthesized in the presence of monofunctional free CTA DSTCSPA) confirmed that a relatively more hydrophilic polymer layer than CTA immobilized gold was obtained. The area shown in Figure 110 (a) and (b) for 24 hours polymerization time display gaussian distributions which is an indicator of surface uniformity. The change in film morphology is associated with a slight increase in the RMS roughness from $1.0795 \mathrm{~nm}$ (gold-PETSPETTCP layer) to $1.1898 \mathrm{~nm}$ (PPEGMA brush layer synthesized in the presence of tetrafunctional free CTA (PETSPETTCP)) and to $1.4083 \mathrm{~nm}$ (PPEGMA brush layer synthesized in the presence of monofunctional free CTA DSTCSPA). GPC trace of free polymers was monomodal and had narrow molecular weight distribution, which are characteristics of well-defined polymers prepared via RAFT-mediated polymerization. The monomer conversion for polymerization conducted in the presence of tetrafunctional free CTA (PETSPETTCP) is $30 \%$ and the thickness value achieved is $46.5 \mathrm{~nm}$, whereas they are $70 \%$ and $81 \mathrm{~nm}$ for polymerization conducted in the presence of monofunctional free CTA (DSTCSPA). GPC analysis of the free polymers reveals a $M_{n}$ of $78161 \mathrm{~g} / \mathrm{mol}$ and a PDI of 1.74 for free polymers synthesized from tetrafunctional free CTA (PETSPETTCP); a $M_{n}$ of $83350 \mathrm{~g} / \mathrm{mol}$ and a PDI of 1.66 for free polymers synthesized from monofunctional free CTA (DSTCSPA). (Table 8) The low PDI values indicate that both the polymerizations were conducted in a controlled manner. 

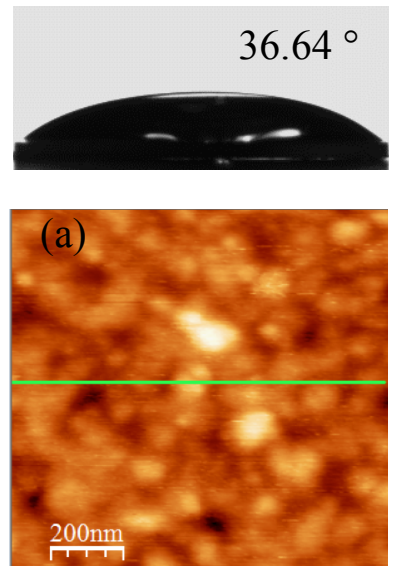

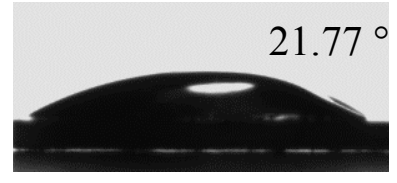

(b)

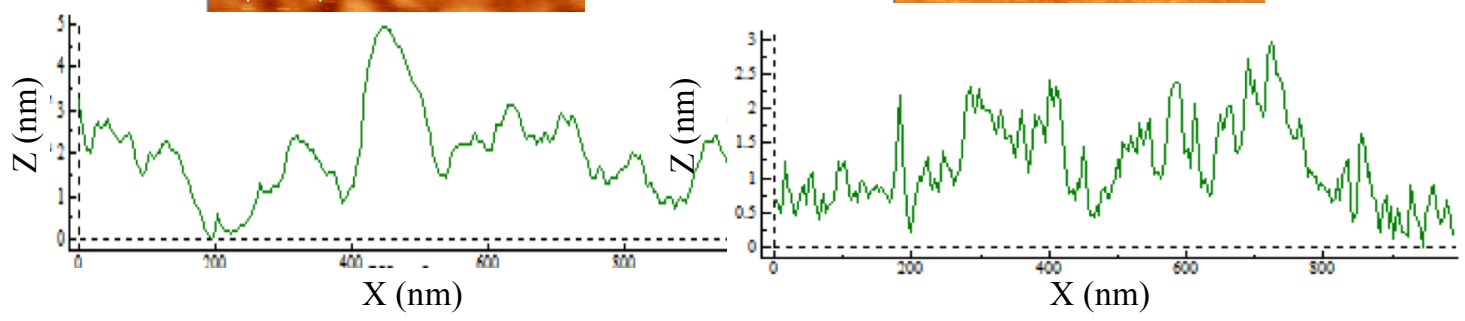

Figure $1102 D$ AFM topography images $(1 \mu \mathrm{m} \times 1 \mu \mathrm{m})$ in ambient conditions, surface profile analysis and photographs of $1 \mu \mathrm{L}$ water droplets for (a) poly (PEGMA) brushes synthesized in the presence of tetra functional free CTA (PETSPETTCP) and AIBN in toluene at $60{ }^{\circ} \mathrm{C}$ for $24 \mathrm{~h}$ from gold-PETSPETTCP surface, (b) poly (PEGMA) brushes synthesized in the presence of linear free CTA (DSTCSPA) and AIBN in toluene at 60 ${ }^{\circ} \mathrm{C}$ for $24 \mathrm{~h}$ from gold-PETSPETTCP surface.

The surface coverages, A $\left(m g / m^{2}\right)$, grafting densities, $\sigma$ (chains $\left./ \mathrm{nm}^{2}\right)$, and average distances between grafting sites, $D(\mathrm{~nm})$, of grafted polymer brushes were calculated from the $M_{n}$ of the free polymer and the dry film thickness, $h(\mathrm{~nm})$, according to equations 18, 19, 20 and summarized in Table 8. As a result of that the grafting densities of both polymer brushes synthesized in the presence of tetrafunctional free CTA (PETSPETTCP) and in the presence of monofunctional free CTA (DSTCSPA) are 0.38 and 0.63 chains per $\mathrm{nm}^{2}$ respectively, poly (PEGMA) brushes might be classified as a brush type between semi-dilute and a high density brush, which is closer to a high density conformation than semi-dilute. (509) It is apperant that the resulting thickness $(81 \mathrm{~nm})$ and grafting density $\left(0.63\right.$ chains $\left./ \mathrm{nm}^{2}\right)$ of polymer brush synthesized in the presence of monofunctional free CTA (DSTCSPA) in solution is higher than that of the case of utilization of tetrafunctional free CTA (PETSPETTCP) in the solution due to higher conversion reached with monofunctional free CTA.

\subsubsection{Characterization of Poly (PEGMA) Brushes Synthesized in the Presence of Tetra Functional Free CTA (PETSPETTCP) and in the Presence of Monofunctional Free CTA (DSTCSPA) from Gold-DPEHPETTC Surface}


The growth of poly (PEGMA) brushes from two identical gold-DPEHPETTC surfaces was conducted in two different reaction mixtures which include different type raft agents in order to check impact of free CTA type on resulting thickness and PDI. For this reason, PEGMA brushes were prepared in the presence of tetra functional free CTA (PETSPETTCP) and monofunctional free CTA (DSTCSPA) in solution and characterized by Ellipsometry (Table 8), ATR-FTIR [Figure 111 (c), (b)], AFM and water contact angle measurements [Figure 112 (a), (b)]. The ATR-FTIR spectra for the poly (PEGMA) brush demonstrated characteristic absorption bands at $\sim 1717 \mathrm{~cm}^{-1}$, associated with the carbonyl stretch, strong band at $1042 \mathrm{~cm}^{-1}$ resulted from $\mathrm{C}=\mathrm{S}$ stretching vibration suppressed by absorption bands at $\sim 1113 \mathrm{~cm}^{-1}, 1258 \mathrm{~cm}^{-1}$ resulted from $\mathrm{C}-\mathrm{O}$ stretching vibrations of C-O-C ether bonds in the ethylene glycol units. The bands at around 2951,2920,2856 $\mathrm{cm}^{-1}$ are considered to be due to aliphatic $\mathrm{CH}$ groups. The $2951,2856 \mathrm{~cm}^{-1}$ peaks are due to asymmetric and symmetric stretching vibrations of $\mathrm{CH}_{3}$, respectively, while the $2920 \mathrm{~cm}^{-1}$ peak is assigned to $\mathrm{CH}_{2}$ vibration. The peaks at $1460 \mathrm{~cm}^{-1}$ and $1376 \mathrm{~cm}^{-1}$ are attributed to the $\mathrm{CH}$ bending and rocking respectively. In addition to these bands, there is band at $720-620 \mathrm{~cm}^{-1}$ attributed to C$\mathrm{S}$ stretching. The broad band at $3679-3113 \mathrm{~cm}^{-1}$ which is assigned to $\mathrm{O}-\mathrm{H}$ stretching, results from water vapor which PPEGMA brush coating absorbs due to its hydrophilic nature. The aromatic C-H stretching peak expected at around $3100-3000 \mathrm{~cm}^{-1}$ is slightly observable as a little neck at around $3113-3024 \mathrm{~cm}^{-1}$ as a result of being overlapped by both aliphatic $\mathrm{C}-\mathrm{H}$ stretching and broad band of $\mathrm{O}-\mathrm{H}$ stretching whereas $\mathrm{C}=\mathrm{C}$ aromatic doublets which is expected as a value between $1480-1420 \mathrm{~cm}^{-1}$, are observable at $1462 \mathrm{~cm}^{-1}$, being overlapped with $\mathrm{CH}$ bending vibration.

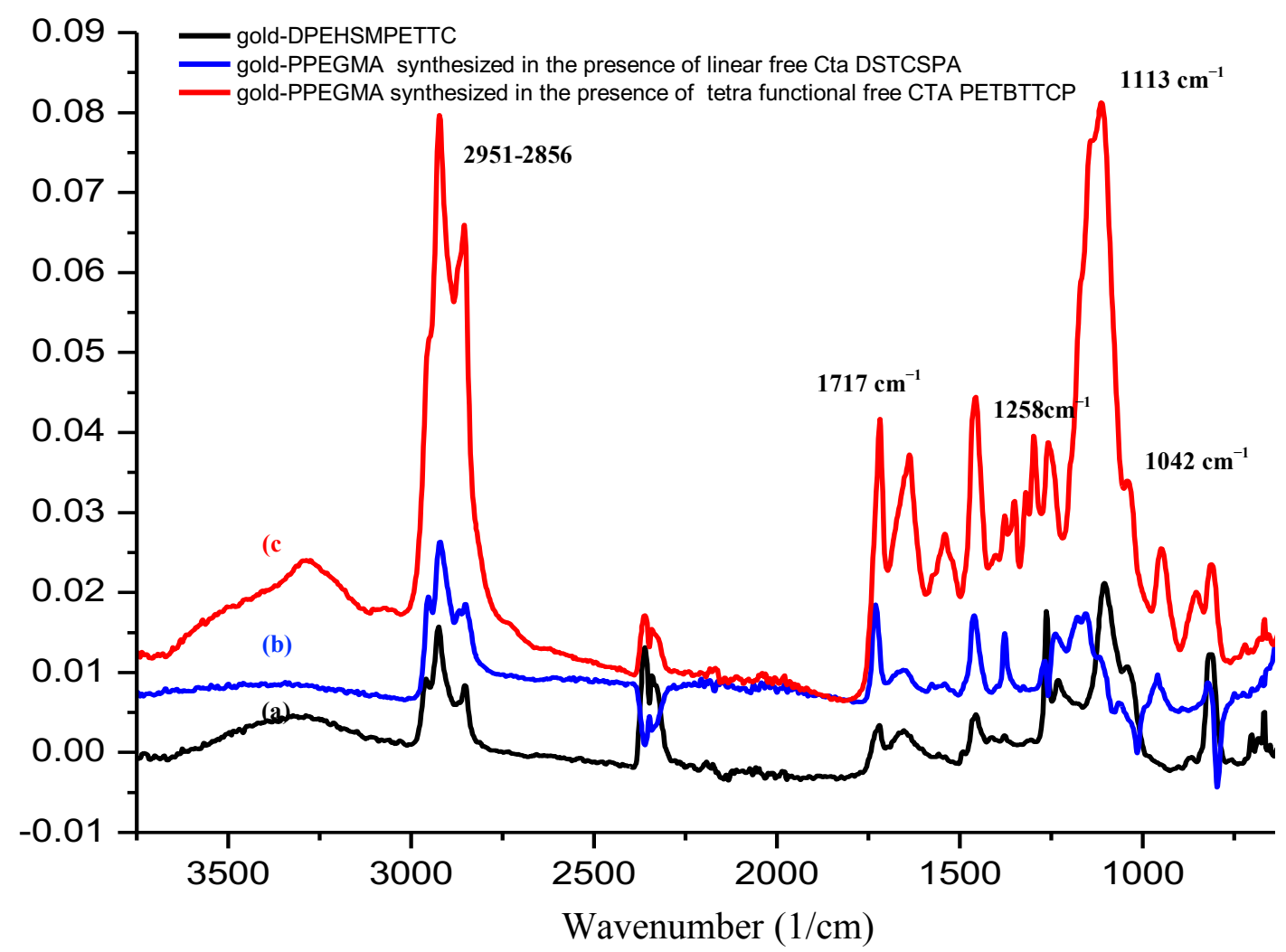


Figure 111 ATR-FTIR spectra of (a) gold-DPEHPETTC (b) poly (PEGMA) brush synthesized in the presence of monofunctional free CTA (DSTCSPA) from goldDPEHPETTC surface for $24 \mathrm{~h}$ and (c) poly (PEGMA) brush synthesized in the presence of tetra functional free CTA (PETSPETTCP) from gold-DPEHPETTC surface for $24 \mathrm{~h}$.

A drastic decrease in water contact angle from $74.75^{\circ}$ (gold-CTA layer) to $18.86^{\circ}$ (PPEGMA layer synthesized in the presence of tetrafunctional free CTA (PETSPETTCP) and to $20.90^{\circ}$ (PPEGMA layer synthesized in the presence of monofunctional free CTA DSTCSPA) confirmed that a relatively more hydrophilic polymer layer than CTA immobilized gold was obtained. The area shown in Figure 112 (a) and (b) for 24 hour polymerizations display gaussian distributions which is an indicator of surface uniformity. The change in film morphology is associated with a drastic increase in the RMS roughness from $0.9731 \mathrm{~nm}$ (gold-DPEHPETTC layer) to $4.0635 \mathrm{~nm}$ (PPEGMA brush layer synthesized in the presence of tetrafunctional free CTA (PETSPETTCP)) and a slight increase to $1.025 \mathrm{~nm}$ (PPEGMA brush layer synthesized in the presence of monofunctional free CTA DSTCSPA).
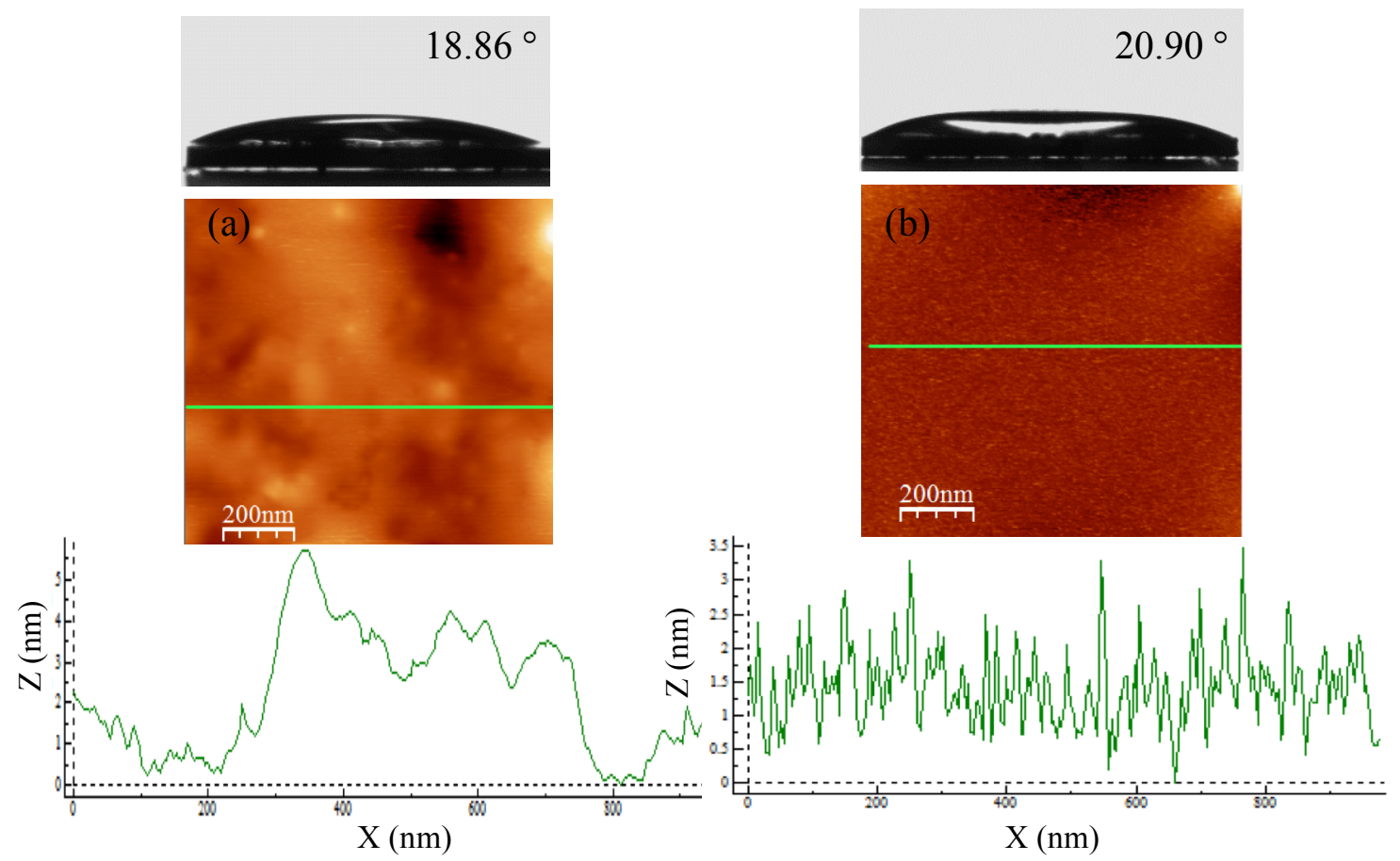

Figure 112 2D AFM topography images $(1 \mu \mathrm{m} \times 1 \mu \mathrm{m})$ in ambient conditions, surface profile analysis and photographs of $1 \mu \mathrm{L}$ water droplets for (a) poly (PEGMA) brushes synthesized in the presence of tetra functional free CTA (PETSPETTCP) and AIBN in toluene at $60{ }^{\circ} \mathrm{C}$ for $24 \mathrm{~h}$ from gold-DPEHPETTC surface, (b) poly (PEGMA) brushes synthesized in the presence of linear free CTA (DSTCSPA) and AIBN in toluene at 60 ${ }^{\circ} \mathrm{C}$ for $24 \mathrm{~h}$ from gold-DPEHPETTC surface. 
GPC traces of free polymers were monomodal which is characteristics of welldefined polymers prepared via RAFT mediated polymerization. The monomer conversion for polymerization conducted in the presence of tetrafunctional free CTA (PETSPETTCP) is $43.5 \%$ and the thickness value achieved is $166 \mathrm{~nm}$, whereas they are $63 \%$ and $83.6 \mathrm{~nm}$ for polymerization conducted in the presence of monofunctional free CTA (DSTCSPA). GPC analysis of free polymers reveals a $M_{n}$ of $127330 \mathrm{~g} / \mathrm{mol}$ and a PDI of 2.2 for free polymers synthesized from tetrafunctional free CTA (PETSPETTCP) and a $M_{n}$ of $75184 \mathrm{~g} / \mathrm{mol}$ and a PDI of 1.72 for free polymers synthesized from monofunctional free CTA (DSTCSPA). (Table 8) The low PDI value indicates that the polymerization in the presence of monofunctional free CTA (DSTCSPA) was conducted in a controlled manner whereas other one is deviated a bit from control due to utilization of star shape raft agent, which results in formation of a free polymer mixture of star shaped polymers bearing arms with different lengths and dead chains. As a result of utilization of $Z$ group based star polymerization approach, the radicals are not generated on the core of star shaped raft agent and thereby, polymeric radicals are not growing on the core. This might lead to formation of arms with different lengths for every free star shaped polymer molecule in solution phase or lack of some arms of star shaped polymers, due to steric hindrance problem which stems from increasing length and size of growing polymeric radicals while approaching back onto the core. This steric hindrance leads to limitation of the delivery of growing polymeric radicals(arms) back onto the cores of free star shaped raft agents in the latter stage of polymerizations. Another consequence of this problem might be low conversion.

The surface coverages, A $\left(\mathrm{mg} / \mathrm{m}^{2}\right)$, grafting densities, $\sigma$ (chains $\left./ \mathrm{nm}^{2}\right)$, and average distances between grafting sites, $D(\mathrm{~nm})$, of grafted polymer brushes were calculated from the $M_{n}$ of the free polymer and the dry film thickness, $h(\mathrm{~nm})$, according to equations 18, 19, 20 and summarized in Table 8. Poly (PEGMA) brushes might be classified as a high density brush (509) as a result of that the grafting densities of both polymer brushes synthesized in the presence of tetrafunctional free CTA (PETSPETTCP) and in the presence of monofunctional free CTA (DSTCSPA) are 0.85 and 0.72 chains per $\mathrm{nm}^{2}$ respectively. To best of our knowledge, for the first time, this is the thickest polymer brush with highest grafting density synthesized via RAFT polymerization technique both on gold and in general on surfaces.

\subsection{Characterization of Diblock Copolymer (PDMAEMA-b-PPEGMA) Brush Prepared in the Presence of Bifunctional Free CTA (EBPEDTTC) on Gold- EBPEDTTC-PDMAEMA Surface}

One of the advantages in using RAFT technique to prepare polymer brushes via the grafting from technique is the opportunity to produce block copolymer brushes by reactivation of the dormant chains. The preparation of a block copolymer brush is also another confirmation of whether the previous sample has maintained the living 
characteristics of a well defined RAFT. Thus, the gold substrate coated with $27 \mathrm{~nm}$ poly (DMAEMA) brushes was used for preparation of a diblock copolymer brush in order to check living nature of the surface bound homopolymer brushes synthesized via SI-RAFT polymerization. The formation of second block layer poly (PEGMA) on the gold substrate covered with poly (DMAEMA) brushes was confirmed by ATR-IR [Figure 113 (c)], AFM and water contact angle measurements. (Figure 114) The ATR-FTIR spectra for the PDMAEMA-block-PPEGMA brush demonstrated characteristic bands at $\sim 1732 \mathrm{~cm}^{-1}$, associated with the carbonyl stretch, and at 1034 $\mathrm{cm}^{-1}$ resulted from strong $\mathrm{C}=\mathrm{S}$ stretching vibrations suppressed by absorption bands at $\sim 1121 \mathrm{~cm}^{-1}$ and $1263 \mathrm{~cm}^{-1}$ associated with $\mathrm{C}-\mathrm{O}$ stretching resulted from vibrations of C-O-C ether bonds in the ethylene glycol units. The bands at around 2962, 2925, $2864 \mathrm{~cm}^{-1}$ are considered to be due to aliphatic $\mathrm{CH}$ groups. The $2962,2864 \mathrm{~cm}^{-1}$ peaks are due to asymmetric and symmetric stretching vibrations of $\mathrm{CH}_{3}$, respectively, while the $2925 \mathrm{~cm}^{-1}$ peak is assigned to $\mathrm{CH}_{2}$ vibration. The peaks at $1472 \mathrm{~cm}^{-1}$ and 1399 $\mathrm{cm}^{-1}$ are attributed to the $\mathrm{CH}$ bending and rocking respectively. In addition to these bands, there are bands at 704-620 $\mathrm{cm}^{-1}$ attributed to C-S stretching. The broad band at $3670-3190 \mathrm{~cm}^{-1}$ which is assigned to $\mathrm{O}-\mathrm{H}$ stretching, resulting from water vapor which blockcopolymer brush layer absorbs is another confirmation of formation of a hydrophilic blockcopolymer brush layer (PDMAEMA-block-PPEGMA). The aromatic C-H stretching peak expected at around $3100-3000 \mathrm{~cm}^{-1}$ is slightly observable as a little shoulder at the left side of $\mathrm{C}-\mathrm{H}$ stretching peak as a result of being overlapped by aliphatic $\mathrm{C}-\mathrm{H}$ stretching whereas $\mathrm{C}=\mathrm{C}$ aromatic doublets which are expected at a value between $1480-1420 \mathrm{~cm}^{-1}$, are observable at $1472 \mathrm{~cm}^{-1}$, being overlapped with $\mathrm{CH}$ bending vibration.

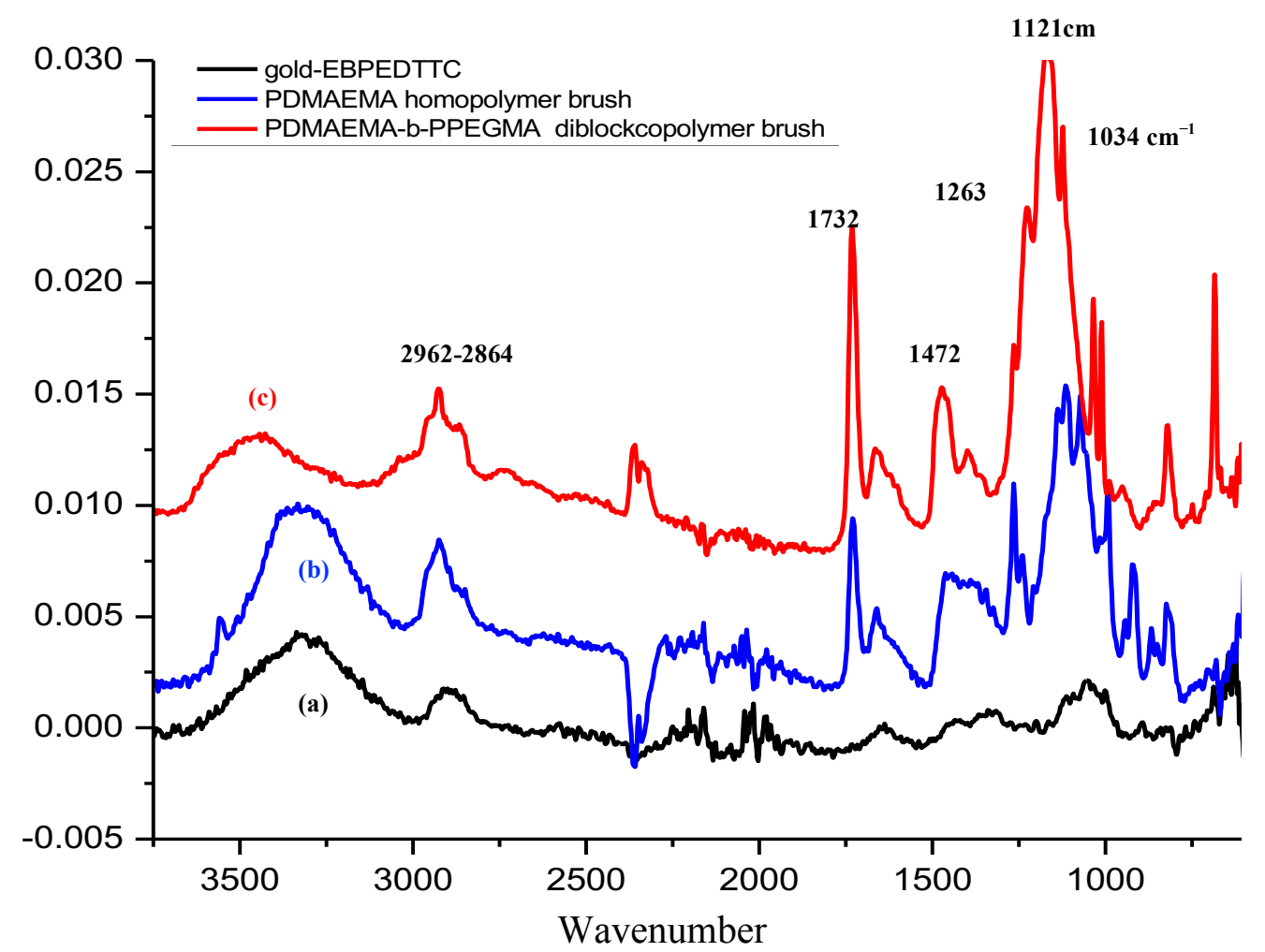


Figure 113 ATR-FTIR spectra of (a) gold-EBPEDTTC, (b) poly (DMAEMA) brush synthesized in the presence of bifunctional free CTA (EBPEDTTC) from goldEBPEDTTC surface for $24 \mathrm{~h}$ and (c) poly (DMAEMA)-block-poly (PEGMA) brush synthesized in the presence of bifunctional free CTA (EBPEDTTC) from goldEBPEDTTC-poly (DMAEMA) surface.

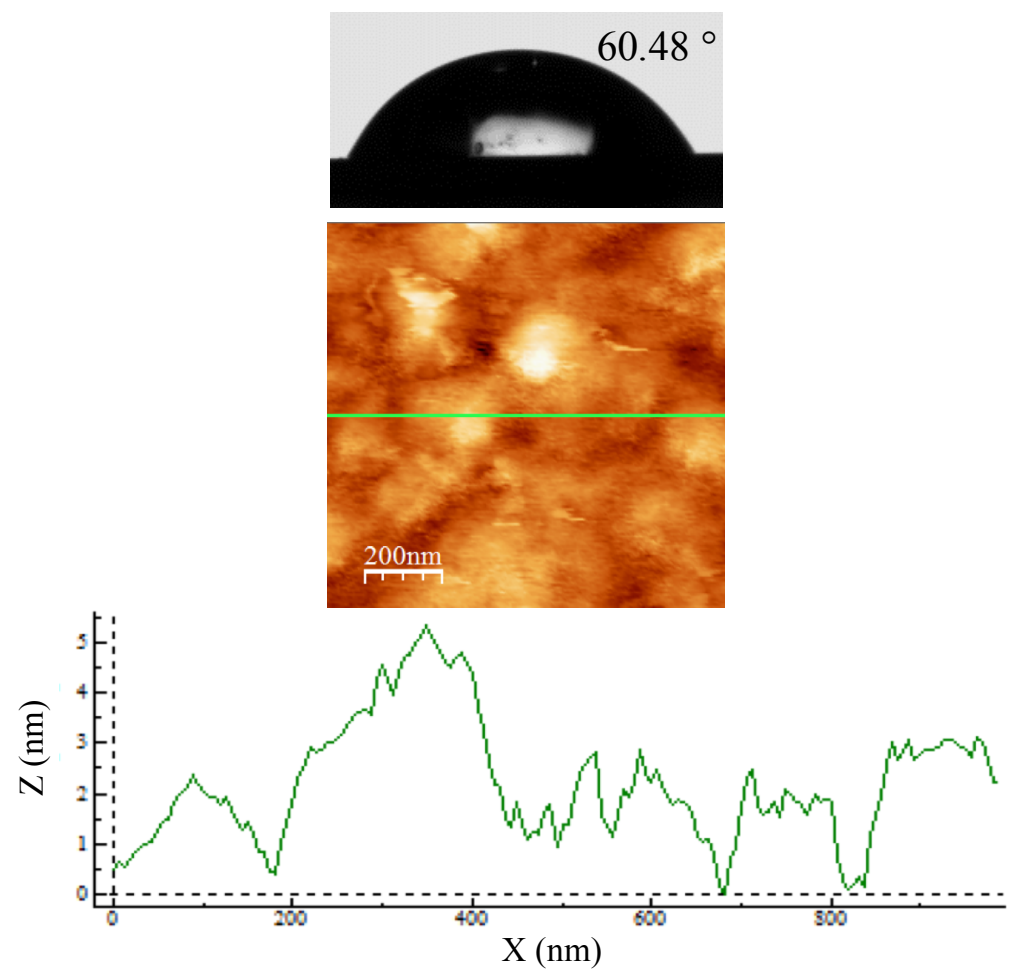

Figure $1142 \mathrm{D}$ AFM topography image $(1 \mu \mathrm{m} \times 1 \mu \mathrm{m})$ in ambient conditions, surface profile analysis and photograph of $1 \mu \mathrm{L}$ water droplet for poly (PEGMA) brush synthesized in the presence of bifunctional free CTA (EBPEDTTC) and AIBN in toluene at $60{ }^{\circ} \mathrm{C}$ for $24 \mathrm{~h}$ from gold-EBPEDTTC-PDMAEMA surface (PDMAEMAb-poly (poly (ethylene glycol) methyl ether methacrylate) (PPEGMA)).

The goniometry didnt demonstrate any significant change in static water contact angle measurements. Static contact angle slightly decreased from $65.11^{\circ}$ (PDMAEMA) to $60.48^{\circ}$ (PDMAEMA-block-PPEGMA), which is a supportive proof and an expected result of straight forward binding approach of raft agents via chemisorption onto gold surface leading to the fact that second layer (PPEGMA) is formed at the bottom instead of the top. Ellipsometry indicated a thickness increase of $28 \mathrm{~nm}$ from that of the first (PDMAEMA) homopolymer layer whereas total thickness of resulting blockcopolymer brush is $55 \mathrm{~nm}$. (Table 8) The area shown in Figure 114 for 24 hours polymerization displays gaussian distribution which is an indicator of surface homogenitiy. The RMS roughness and roughness average values increased from 1.6883 to $2.3956 \mathrm{~nm}$ and from 1.3109 to 1.7716 respectively. 
GPC trace of free polymer was monomodal and had narrow molecular weight distribution, which are characteristics of well-defined polymers prepared via RAFTmediated polymerization. After $24 \mathrm{~h}$ polymerization, the monomer conversion is 30 $\%$. GPC analysis of the free polymers reveals a $M_{n}$ of $50908 \mathrm{~g} / \mathrm{mol}$ and a PDI of 1.77 . (Table 8) The low PDI value indicates that the polymerization was conducted in a controlled manner. The surface coverage, A $\left(\mathrm{mg} / \mathrm{m}^{2}\right)$, grafting density, $\sigma$ (chains $/ \mathrm{nm}^{2}$ ), and average distance between grafting sites, $D(\mathrm{~nm})$, of grafted poly(BuMA) brushes were calculated from the $M_{n}$ of the free polymer and the dry film thickness, $h(\mathrm{~nm})$, according to equations 18,19 and 20. (Table 8) As a result of that the grafting density is 0.36 chains per $\mathrm{nm}^{2}$, second block layer poly (PEGMA) brushes might be considered as a brush type between semi-dilute and a high density brush, which is closer to a high density conformation than semi-dilute. (509)

Table 8 Summary of polymerizations and diblockcopolymerization from gold-CTA surfaces

\begin{tabular}{|c|c|c|c|c|c|c|c|c|}
\hline $\begin{array}{c}\text { CTA } \\
\text { immobilized } \\
\text { on gold }\end{array}$ & Monomer & $\mathbf{M n} / \mathbf{M w}$ & PDI & $\begin{array}{l}\text { Thick } \\
\text { ness }^{p} \\
(n m)\end{array}$ & $\begin{array}{l}\text { Cove } \\
\text { rage } \\
(\mathbf{m g} / \\
\left.\mathbf{m}^{2}\right)\end{array}$ & $\begin{array}{l}\text { Graftin } \\
\text { g } \\
\text { Density } \\
(\text { chains/ } \\
\left.\text { nm }^{2}\right)\end{array}$ & $\begin{array}{l}\text { Dist } \\
\text { anc } \\
\text { e }\end{array}$ & $\begin{array}{l}\text { Static } \\
\text { Contact } \\
\text { Angle }^{r}\end{array}$ \\
\hline $\mathrm{CDB}^{\mathrm{a}}$ & $\begin{array}{l}\text { DMAEMA } \\
\mathrm{e}\end{array}$ & $37714 / 49521$ & 1.31 & 21 & 28.4 & 0.45 & 1.67 & $63.57^{\circ}$ \\
\hline EBPEDTTC $^{b}$ & BUMA $^{f}$ & $65899 / 76959$ & 1.16 & 28 & 29.4 & 0.26 & 2.2 & $89.22^{\circ}$ \\
\hline EBPEDTTC $^{b}$ & PEGMA $^{\mathrm{g}}$ & $73162 / 88486$ & 1.20 & 43.1 & 46.5 & 0.38 & 1.83 & $36.67^{\circ}$ \\
\hline EBPEDTTC $^{b}$ & PEGMA $^{\mathrm{h}}$ & $66395-93023$ & 1.40 & 59.3 & 64 & 0.58 & 1.48 & $27.21^{\circ}$ \\
\hline PETSPETTCP $^{c}$ & PEGMA $^{\mathrm{i}}$ & $78161-136250$ & 1.74 & 46.5 & 50.2 & 0.38 & 1.81 & $36.64^{\circ}$ \\
\hline PETSPETTCP $^{c}$ & PEGMA $^{\mathrm{k}}$ & $83350 / 138690$ & 1.66 & 81 & 87.5 & 0.63 & 1.41 & $21.77^{\circ}$ \\
\hline DPEHPETTC $^{d}$ & PEGMA $^{1}$ & $127330 / 279960$ & 2.2 & 166 & 179.3 & 0.85 & 1.22 & $18.86^{\circ}$ \\
\hline DPEHPETTC $^{d}$ & PEGMA $^{\mathrm{m}}$ & $75184-129760$ & 1.72 & 83.6 & 90.28 & 0.72 & 1.32 & $20.90^{\circ}$ \\
\hline EBPEDTTC $^{b}$ & $\begin{array}{l}\text { PDMAEM } \\
\mathrm{A}^{\mathrm{n}}\end{array}$ & $38409 / 53851$ & 1.40 & 27 & 36.45 & 0.57 & 1.49 & $65.11^{\circ}$ \\
\hline EBPEDTTC $^{b}$ & $\begin{array}{l}\text { PDMAEM } \\
\text { A-b- } \\
\text { PPEGMA }^{\circ}\end{array}$ & $50908 / 90162$ & 1.77 & $\begin{array}{l}(27+28 \\
)=55\end{array}$ & 30.24 & 0.36 & 1.88 & $60.48^{\circ}$ \\
\hline
\end{tabular}


a 2_Phenyl_2_Propylbenzodithioate, b Ethane-1,2-diyl bis(1-phenylethyl) ditrithiocarbonate, ${ }^{c} \quad$ Penta-erythritol-tetrakis-(3-(S-phenylethyl trithiocarbonate))propanoate (tetra functional), ${ }^{\mathrm{d}}$ Dipentaerythriol-hexakis(3-(S-methoxopropionatetrithiocarbonate))-propanoate (hexa functional) and the RAFT-mediated polymerization of ${ }^{\mathrm{e}}$ (2-dimethylamino)ethyl methacrylate) DMAEMA with CDB and AIBN in toluene at $60{ }^{\circ} \mathrm{C}$ for $24 \mathrm{~h}$ from gold-CDB surface, ${ }^{\mathrm{f}}$ (buthyl methacrylate) BUMA with EBPEDTTC and AIBN in toluene at $60{ }^{\circ} \mathrm{C}$ for $24 \mathrm{~h}$ from goldEBPEDTTC surface, ${ }^{\mathrm{g}}$ (poly(ethylene glycol) methyl ether methacrylate) PEGMA with bifunctional CTA (EBPEDTTC) and AIBN in toluene at $60{ }^{\circ} \mathrm{C}$ for $24 \mathrm{~h}$ from gold-EBPEDTTC surface, ${ }^{\mathrm{h}}$ (poly(ethylene glycol) methyl ether methacrylate) PEGMA with DSTCSPA and AIBN in toluene at $60{ }^{\circ} \mathrm{C}$ for $24 \mathrm{~h}$ from goldEBPEDTTC surface, ${ }^{i}$ (poly(ethylene glycol) methyl ether methacrylate) PEGMA with tetra functional cta (PETSPETTCP) and AIBN in toluene at $60^{\circ} \mathrm{C}$ for $24 \mathrm{~h}$ from goldPETSPETTCP surface, ${ }^{\mathrm{k}}$ (poly(ethylene glycol) methyl ether methacrylate) PEGMA with DSTCSPA and AIBN in toluene at $60^{\circ} \mathrm{C}$ for $24 \mathrm{~h}$ from gold- PETSPETTCP surface, ${ }^{1}$ (poly(ethylene glycol) methyl ether methacrylate) PEGMA with tetra functional cta (PETSPETTCP) and AIBN in toluene at $60{ }^{\circ} \mathrm{C}$ for $24 \mathrm{~h}$ from goldDPEHPETTC surface ${ }^{\mathrm{m}}$ (poly(ethylene glycol) methyl ether methacrylate) PEGMA with DSTCSPA and AIBN in toluene at $60{ }^{\circ} \mathrm{C}$ for $24 \mathrm{~h}$ from gold- DPEHPETTC surface, ${ }^{n}$ (2 (dimethylamino)ethyl methacrylate) DMAEMA with EBPEDTTC and AIBN in toluene at $60{ }^{\circ} \mathrm{C}$ for $24 \mathrm{~h}$ from gold-EBPEDTTC surface and Blockcopolymerization of ${ }^{\circ}$ (poly(ethylene glycol) methyl ether methacrylate) PEGMA with DSTCSPA and AIBN in toluene at $60{ }^{\circ} \mathrm{C}$ for $24 \mathrm{~h}$ from goldEBPEDTTC-PDMAEMA surface (PDMAEMA-b-PPEGMA), ${ }^{\mathrm{p}}$ Thicknesses were determined by Ellipsometry and are an average of five samplings across the sample. Error of thicknesses measured was within $\pm 2 \mathrm{~nm}$, ${ }^{\mathrm{r}}$ Static contact angles were taken using goniometry and are an average of five samplings across the sample. The standard deviation of contact angles was less than $1^{\circ}$.

\subsection{Summary and Conclusions}

In this part, for the first time to our knowledge, a novel straight-forward method for immobilization of raft agents onto gold surface, which takes the advantage of chemisorption property of raft agents through their dithioester and trithiocarbonate moieties toward gold, was introduced as a better and easier alternative over multistep approaches commonly used for preparation of brushes on gold. The basic difference of the novel approach compared to the ones in literature is the fact that it doesn't require any special reaction or extra pre-treatments for immobilization of raft agents onto gold surface. In terms of the attachment style of tethered raft agents on gold (through R or Z group) which influence whether the polymerization from surfaces has a true grafting from nature or not, our route falls into a category between $\mathrm{R}$ and $\mathrm{Z}$ group 
approaches due to the fact that raft agents are introduced onto gold through neither $\mathrm{R}$ group nor $\mathrm{Z}$ group.

Regarding mechanism, this route might be classified as an approach similar to traditional $\mathrm{Z}$ group approach since the growing polymeric radicals depart from the surface instead of cleavage of trithiocarbonate moiety just like in the case of $\mathrm{R}$ group approach. However, the resulting grafting densities and thicknesses are by far better than that of traditional $\mathrm{Z}$ group. On the contrary, by utilizing this novel approach, it is possible to achieve grafting densities and thicknesses as high as ones obtained from traditional $\mathrm{R}$ group approach. Moreover, it is possible to reach higher thickness and grafting density values by utilization of star shaped raft agents both on the surface and in solution phase. RAFT mediated polymerization technique has been sucsessfully used on CTA-chemisorbed gold substrates to prepare well defined homopolymer and diblockcopolymer brushes with different thicknesses and wettability properties.

The homopolymer and diblockcopolymer brushes demonstrated the linear growth of film thickness at the early stage of polymerization and the surface coverage controlled by the increase in molecular weight of polymer brushes. Block extension of homopolymer brushes obtained at first step by further confirmed "living character" of brushes obtained via RAFT and the fact that postpolymerization is possible based on this "livingness" being maintained. The presence of free CTA in reaction mixture, which results in free polymer chains in solution, is essential both in order to estimate molecular weights and to be able to control polymerization. Free CTAs used in solution phase were varied with an attempt to investigate impact of free CTA type used in solution phase on resulting thicknesses, PDIs and grafting densities of polymer brushes. One of two identical gold flats coated with same raft agent was polymerized in the presence of free CTA which is same with one chemisorbed on gold whereas second flat was polymerized in the presence of a monofunctional free CTA which is different from the one chemisorbed on gold. Polymer brushes obtained via utilization of different free CTA types were compared to eachother in terms of the resulting thicknesses, PDIs and grafting densitities. It was found that utilization of monofunctional free CTA in solution instead of bifunctional or star shaped CTAs results in similar thickness values as high as ones obtained in the presence of star shaped CTAs and relatively lower PDIs as expected compared to that of starshaped free polymers. According to these results, Utilization of linear free CTAs is more advantageous than use of star shape raft agents in solution. There is only one exceptional result which is conflicting with these findings. Combination of the four functional free CTA in solution phase and hexa functional CTA chemisorbed on gold surface leads to the thickest polymer brush obtained sofar as high as $166 \mathrm{~nm}$. As conclusion, the combination of a monofunctional free CTA in solution and a bifunctional or star shaped CTA immobilized on gold leads to the formation of relatively thicker brushes than ones in literature obtained via SI-RAFT (R group or Z group) based on a classical multistep immobilization approaches. The ellipsometric thicknesses and molecular weights were used to estimate the grafting parameters of 
chains attached to the gold surface. SI-RAFT mediated polymerization technique can be utilized to obtain well-defined polymer and diblockcopolymer brushes of up to 166 $\mathrm{nm}$ and with a grafting density as high as 0.85 , which is classified as a high density brush. To best of our knowledge, for the first time, the advantages of raft process such as the living character and chemisorption property of raft agents onto gold surfaces were sucsessfully combined as a direct, relatively easier approach in order to construct the thickest polymer brushes including both homopolymer and diblockcopolymers on gold surfaces. This work is a new avenue for preparation of thick polymer brushes with high grafting densities for any desired application. 
7 Characterization of Homopolymer and Diblock Copolymer Brushes Prepared on Gold via Novel DTBA Based Approach

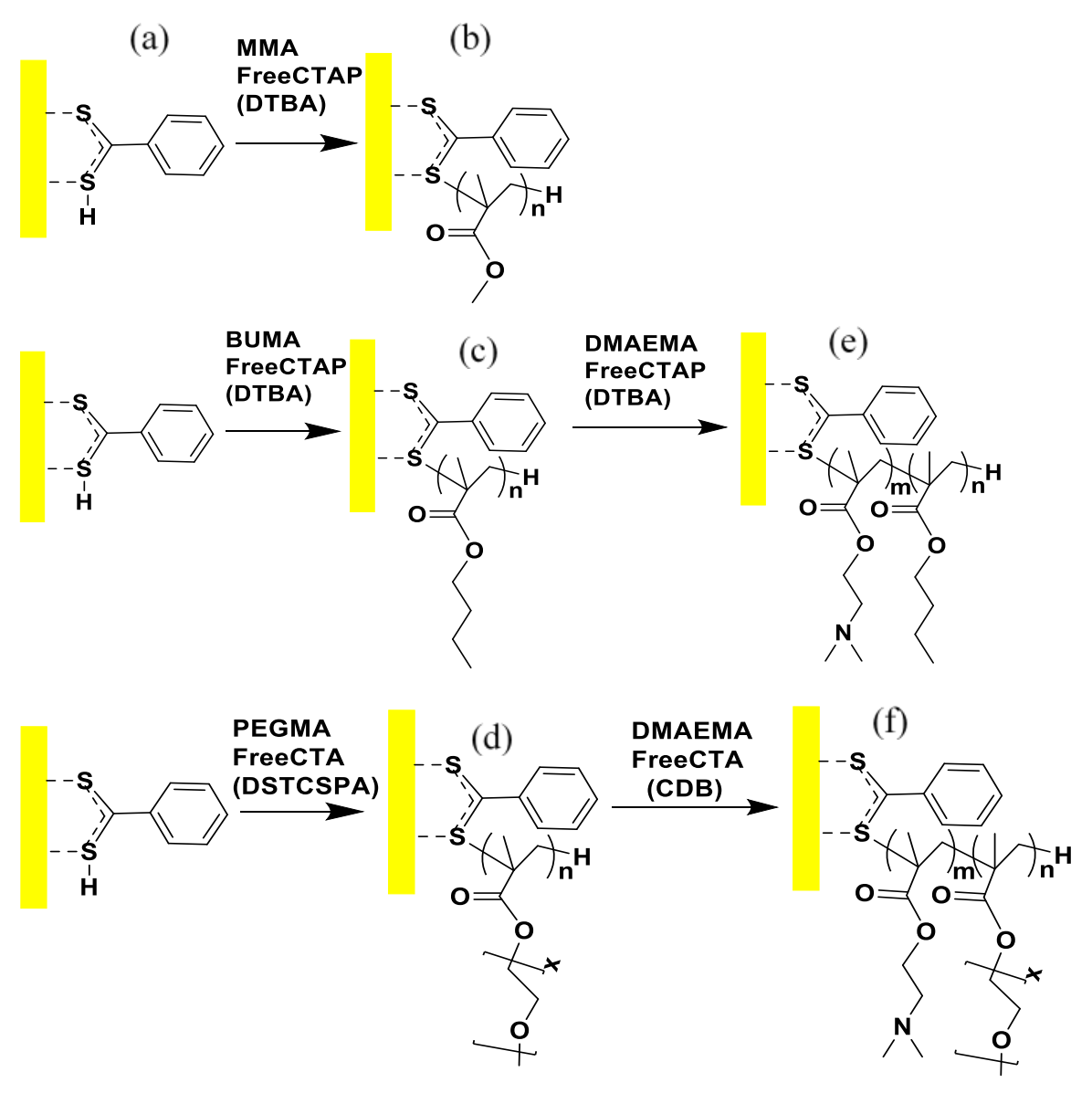

Scheme 3 Schematic diagram illustrating the processes of immobilization of (a) Chain Transfer Agent Precursor (CTAP) DTBA and DTBA-mediated polymerizations of (b) MMA in the presence of free DTBA from gold-DTBA surface, (c) BuMA in the presence of free DTBA gold-DTBA surface; Raft mediated polymerization of (d) PEGMA in the presence of free CTA (DSTCSPA) gold-DTBA surface, DTBA mediated blockcopolymerization of (e) DMAEMA in the presence of free DTBA from gold-DTBA-PBuMA surface and Raft mediated blockcopolymerization of (f) DMAEMA in the presence of free CTA (CDB) from gold-DTBA- PPEGMA surface. 


\subsection{Characterization of DTBA Immobilized Gold Surface}

It is known that dithioesters and trithiocarbonates are susceptible to be chemisorped onto gold surface. (225) Dithiobenzoicacid molecules were immobilized onto gold surface through their dithioester moieties by self assembly process mentioned in the Experimental section. (Scheme 3) The resulting DTBA thickness was measured by Ellipsometry as $1.2 \pm 0.1 \mathrm{~nm}$. The formation of DTBA layer was also confirmed by the presence of the peaks at around $1470-1433 \mathrm{~cm}^{-1}$ associated with $\mathrm{C}=\mathrm{C}$ aromatic doublets, which are expected at a value between $1480-1420 \mathrm{~cm}^{-1}$. The broad band at $3602-3001 \mathrm{~cm}^{-1}$ which is assigned to $\mathrm{O}-\mathrm{H}$ stretching, results from water vapor which DTBA coating absorbs due to its hydrophilic nature. The aromatic $\mathrm{C}-\mathrm{H}$ stretching which is supposed to be at around $3100-3000 \mathrm{~cm}^{-1}$, was not observable as a result of being suppressed by broad band of O-H stretching. [Figure 116 (a)] Surface morphology of pure gold is shown in Figure 115 (a) whereas that of DTBA layer on gold is presented in Figure 115 (b). Analysises of pure gold and DTBA immobilized gold showed a root-mean-square (RMS) roughness of 0.6936 and 1.3106 respectively. An additional evidence of sucsessful chemisorption of DTBAs through their dithioester moieties onto bare gold is a change in surface wettability characterized by a slight increase of static contact angle from $44.35^{\circ}$ (bare gold) to $61^{\circ}$ (DTBA layer on gold).
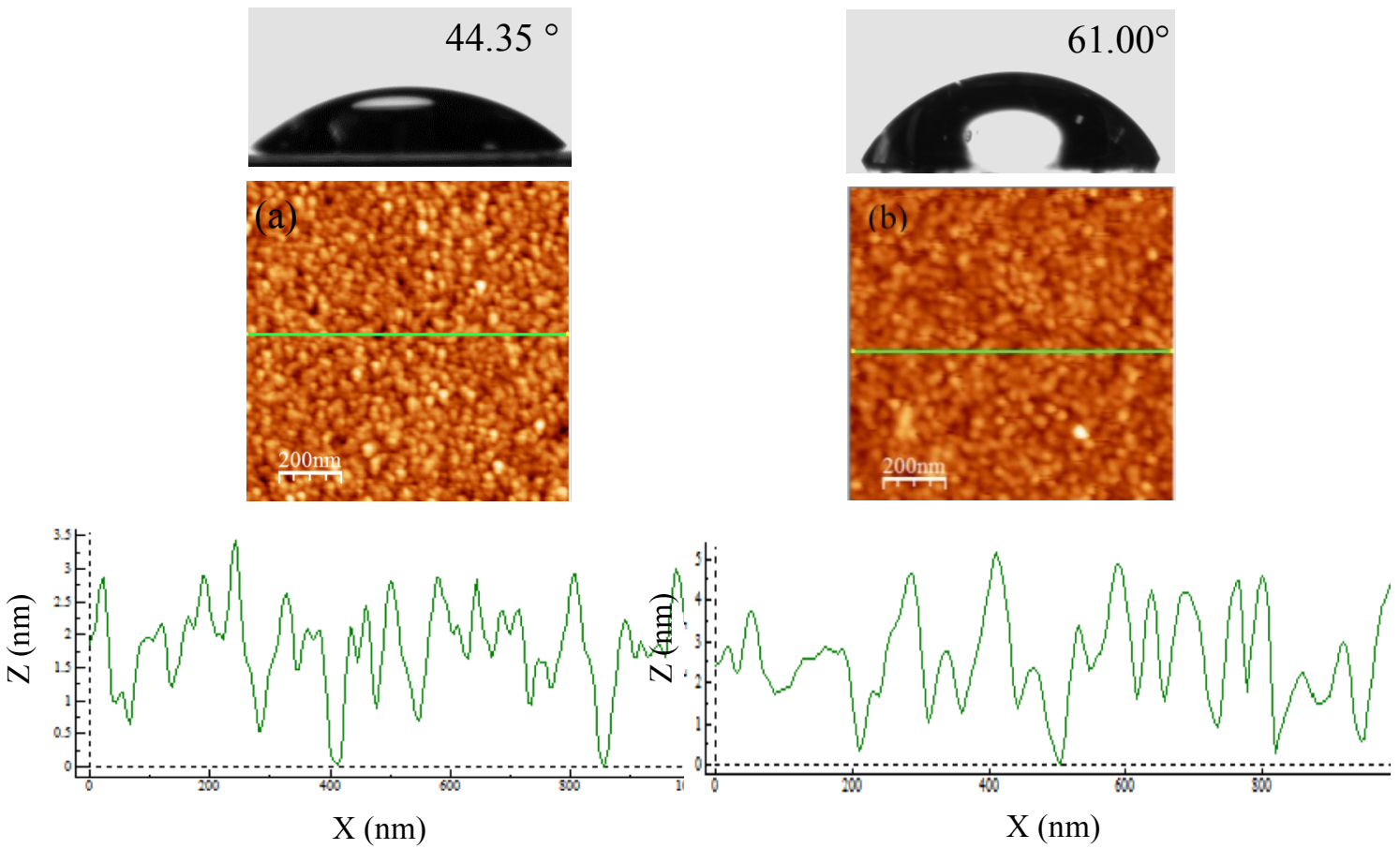

Figure 115 2D AFM topography images $(1 \mu \mathrm{m} \times 1 \mu \mathrm{m})$ in ambient conditions, surface profile analysises and photographs of $1 \mu \mathrm{L}$ water droplets for (a) bare gold, (b) DTBA on gold. 


\subsection{Kinetic Investigation of Polymer Brush (PMMA) Growth in the Presence of Free DTBA on Gold-DTBA Surface}

For Raft mediated polymerization on solid surface, it is important to tether high amount of CTAs onto gold surface in order to obtain a polymer brush with high grafting density. For this reason, in traditional multistep approaches, it is important to use an appropriate reaction type for surface immobilization to increase the coupling efficiency of CTAs with self assembling monolayers (SAM) pre-tethered on gold surface. Here, there is no SAM layer used as first layer and no coupling reaction is required in order to couple CTAs onto gold surface. Moreover, no CTA is attached onto gold surface as anchored CTAs. Instead of immobilization of any CTA onto gold surface, raft agent precursor (DTBA) is tethered via self assembly and than DTBA mediated polymerization of MMA on gold surface was performed in the presence of "sacrificial" DTBA to obtain better control over the polymerization. The presence of the sacrificial DTBA provides the control of overall concentration of monomer and DTBA which is directly connected to the control of molar mass and film thickness of tethered polymer brushes. The correlation of molecular weight of tethered chains with "free" unbounded polymers grown in solution allows indirect analysis of polymer brushes growing on the surface. It is a well known fact that $\mathrm{M}_{\mathrm{n}}$ and PDI values of both tethered polymers on surface and bulk polymers in solution are comparable. (263) The formation of poly (MMA) brushes for $24 \mathrm{~h}$ polymerization time with the ratio of $[\mathrm{M}] /[\mathrm{DTBA}] /[\mathrm{I}]=2000 / 4 / 1$ in feed was confirmed by ATR-FTIR [Figure 116 (b)], $\mathrm{AFM}$ and water contact angle measurements. (Figure 117)

The ATR-FTIR spectra of the poly (MMA) brush demonstrated characteristic bands at $\sim 1736 \mathrm{~cm}^{-1}$ associated with the carbonyl stretch and strong absorption band at 1065 $\mathrm{cm}^{-1}$ resulted from $\mathrm{C}=\mathrm{S}$ stretching vibrations suppressed by absorption bands at 1115 $\mathrm{cm}^{-1}$ and $1266 \mathrm{~cm}^{-1}$ associated with $\mathrm{C}-\mathrm{O}$ stretching of $\mathrm{C}(=\mathrm{O})-\mathrm{O}-\mathrm{C}$ bonds in monomer units. The bands at around 2997, 2924, $2858 \mathrm{~cm}^{-1}$ are considered to be due to aliphatic $\mathrm{CH}$ groups. The $2997,2858 \mathrm{~cm}^{-1}$ peaks are due to asymmetric and symmetric stretching vibrations of $\mathrm{CH}_{3}$, respectively, while the $2924 \mathrm{~cm}^{-1}$ peak is assigned to $\mathrm{CH}_{2}$ vibration. The peaks at $1451 \mathrm{~cm}^{-1}$ and $1387 \mathrm{~cm}^{-1}$ are attributed to the $\mathrm{CH}$ bending and rocking respectively. In addition to these bands, there are absorption bands at 705-618 $\mathrm{cm}^{-1}$ attributed to C-S stretching. The broad band at $3693-3055 \mathrm{~cm}^{-1}$ which is assigned to O-H stretching, results from water vapor which PMMA brush coating absorbs due to its hydrophilic nature. The little shoulder visible at around 3055-2997 is a part of the band associated with aromatic $\mathrm{C}-\mathrm{H}$ stretching which is supposed to be at around $3100-3000 \mathrm{~cm}^{-1}$. The rest of the peak is not observable as a result of being suppressed by broad band of $\mathrm{O}-\mathrm{H}$ stretching. Moreover, $\mathrm{C}=\mathrm{C}$ aromatic doublets, which belong to raft agent precursor DTBA, which are expected at a value between $1480-1420 \mathrm{~cm}^{-1}$, are observed at around $1487-1435 \mathrm{~cm}^{-1}$, being overlapped with $\mathrm{CH}$ bending vibration. 


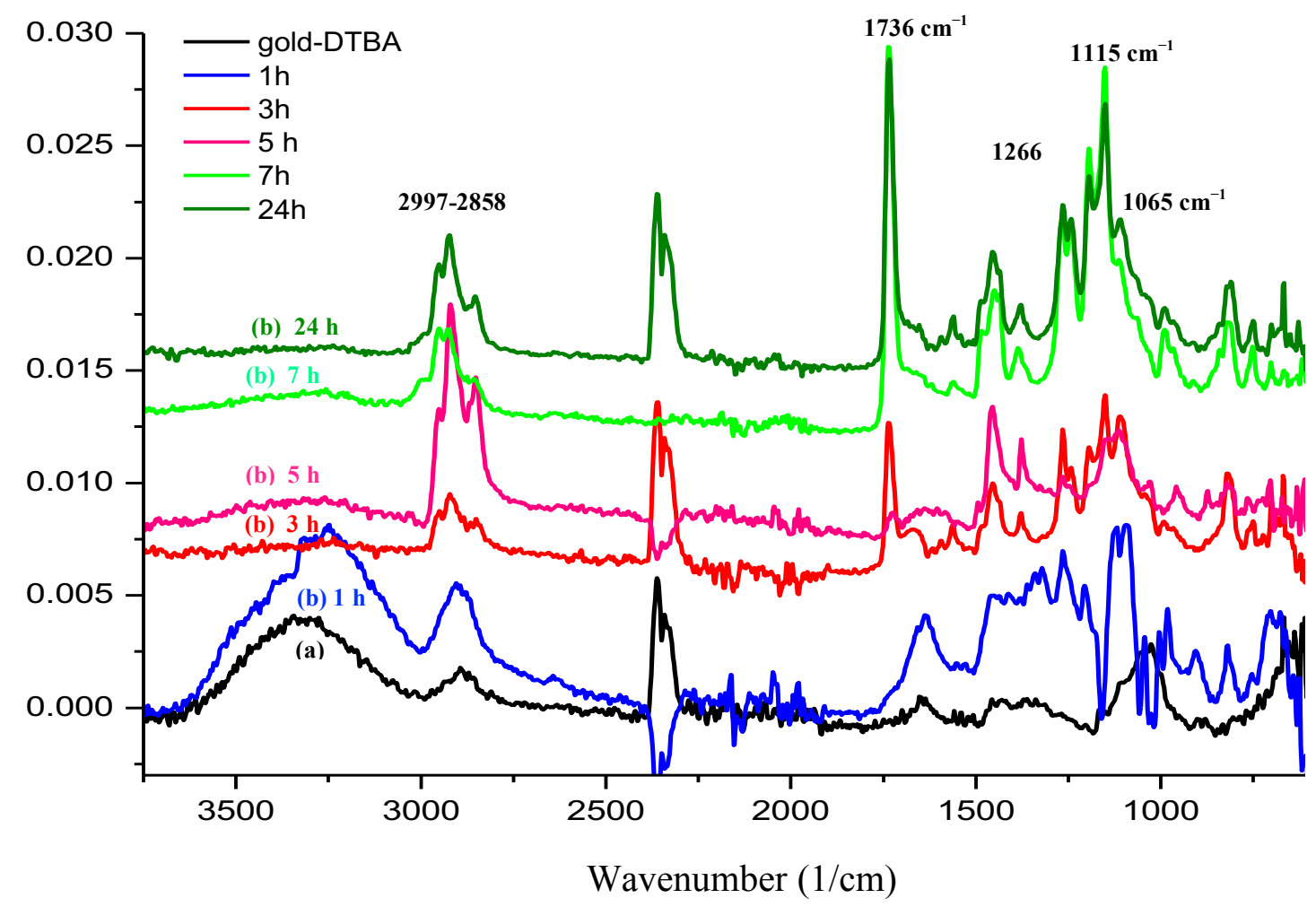

Figure 116 ATR-FTIR spectra of (a) DTBA on gold and (b) poly (MMA) brush kinetics conducted in the presence of free raft agent precursor (DTBA) for 1,3,5,7, 24 h.

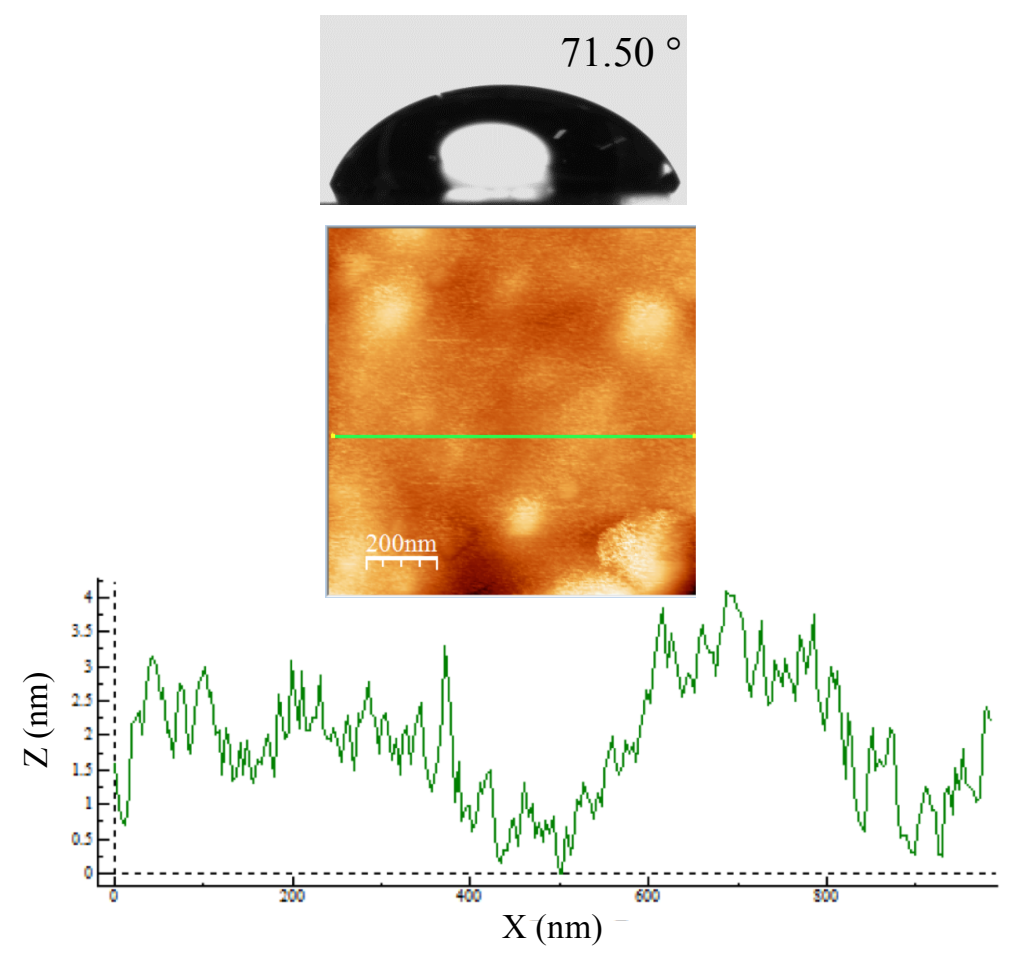

Figure 117 2D AFM topography image $(1 \mu \mathrm{m} \times 1 \mu \mathrm{m})$ in ambient conditions, surface profile analysis and photograph of $1 \mu \mathrm{L}$ water droplets for poly (MMA) brush 
synthesized in the presence of DTBA for $24 \mathrm{~h}$ with the ratio of $[\mathrm{M}] /[\mathrm{DTBA}] /[\mathrm{I}]=2000 / 4 / 1$ in feed.

A slight increase in water contact angle from $61^{\circ}$ (gold-DTBA layer) to $71.50^{\circ}$ (polymer brush layer) also confirmed that a relatively less hydrophilic polymer layer than DTBA coated gold was obtained. The area shown in Figure 117 for 24 hours polymerization time and the other areas measured on same sample (data not shown) display gaussian distribution which is an indicator of surface homogenitiy although small aggregates heterogeneously distributed over some areas of sample were observed in related AFM height image. The change in film morphology is associated with a drastic increase in the RMS roughness from $1.3106 \mathrm{~nm}$ (gold-DTBA layer) to 2.1821 nm (PMMA brush layer).

Table 9 Summary of polymerization kinetics of poly (MMA) brushes from goldDTBA surfaces

\begin{tabular}{|c|c|c|c|c|c|c|c|}
\hline $\begin{array}{l}\text { Reaction } \\
\text { Time } \\
\text { (h) }\end{array}$ & $\mathbf{M n} / \mathbf{M w}$ & PDI & $\begin{array}{c}\text { Thickness }^{\mathrm{a}} \\
\text { (nm) }\end{array}$ & $\begin{array}{c}\text { Coverage } \\
\left(\mathbf{m g} / \mathrm{m}^{2}\right)\end{array}$ & $\begin{array}{l}\text { Grafting } \\
\text { Density } \\
\text { (chains/ } \\
\mathbf{n m}^{2} \text { ) }\end{array}$ & $\begin{array}{c}\text { Distance } \\
(\mathbf{n m})\end{array}$ & $\begin{array}{l}\text { Static } \\
\text { Contact } \\
\text { Angle }^{\mathbf{b}}\end{array}$ \\
\hline 1 & - & - & 2 & - & - & - & $61.00^{\circ}$ \\
\hline 3 & $13597 / 14733$ & 1.08 & 4.4 & 4.64 & 0.20 & 2.5 & $62.55^{\circ}$ \\
\hline 5 & $20597 / 27410$ & 1.33 & 7.9 & 8.33 & 0.24 & 2.3 & $64.44^{\circ}$ \\
\hline 7 & $34859 / 49316$ & 1.41 & 9.9 & 10.44 & 0.18 & 2.65 & $69.51^{\circ}$ \\
\hline 24 & $49806 / 68447$ & 1.37 & 24.6 & 26 & 0.31 & 2 & $71.50^{\circ}$ \\
\hline
\end{tabular}

Polymerization of (methyl methacrylate) MMA with dithiobenzoicacid (DTBA) and $2,2^{\text {'}}$-azobis(isobutyronitrile) (AIBN) in toluene at $60{ }^{\circ} \mathrm{C}$ for $24 \mathrm{~h} .{ }^{a}$ Thicknesses were determined by ellipsometry and are an average of five samplings across the sample. Error of thicknesses measured was within $\pm 0.2 \mathrm{~nm} .{ }^{b}$ Static contact angles were taken using goniometry and are an average of five samplings across the sample. The standard deviation of contact angles was less than $1^{\circ}$.

GPC traces of free polymers were monomodal and had narrow molecular weight distributions, which is an indicator of well defined polymers prepared via DTBAmediated polymerization without utilization of a free CTA in solution. After $24 \mathrm{~h}$ polymerization time, the monomer conversion is $60 \%$. GPC analysis of the free polymers reveals a $M_{n}$ of $49806 \mathrm{~g} / \mathrm{mol}$ and a PDI of 1.37 (Table 9). The low PDI value indicates that the polymerization was conducted in a controlled manner in the presence of DTBA instead of a free CTA in solution. Figure 118 (d) presents the evolution of 
the $M_{n}$ of the free poly (MMA) brushes as a function of monomer conversion. The fact that the experimental molecular weights of the free polymer determined by GPC increased linearly with monomer conversion and the PDI values are all below 1.37 indicate that the polymerization was conducted in a controlled manner without a free CTA in solution. Figure 118 (c) shows the linear relationship between $\ln \left[\mathrm{M}_{\mathrm{o}}\right]\left[\mathrm{M}_{t}\right]$ and time, where $\left[\mathrm{M}_{\mathrm{o}}\right]$ is the initial monomer concentration and $\left[\mathrm{M}_{\mathrm{t}}\right]$ is the monomer concentration remained in reaction mixture after $t$ hour polymerization time. The result indicates that the concentration of the growing species remains constant and first-order kinetic is obtained.

(a)

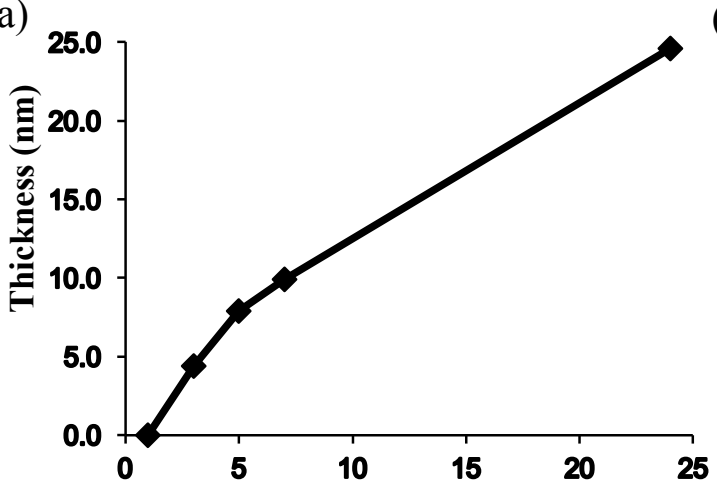

(c)

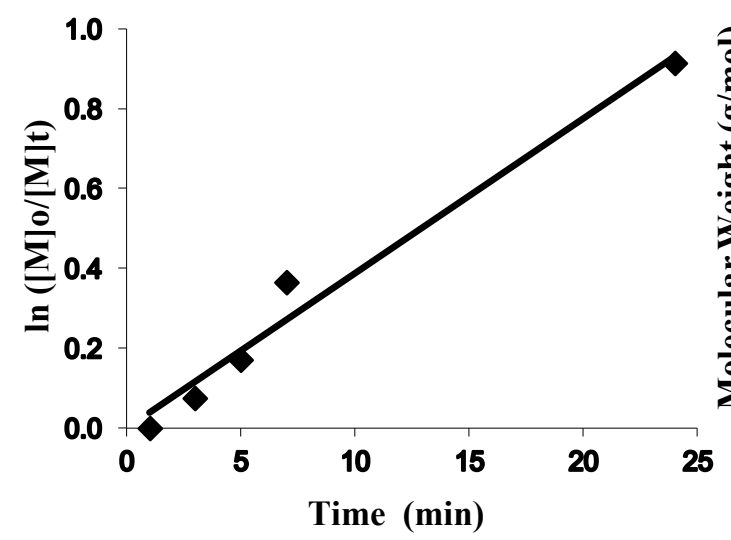

(b)

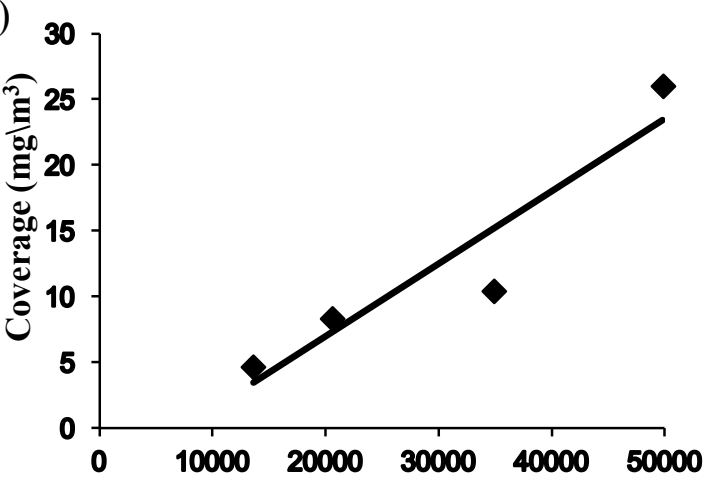

(d)

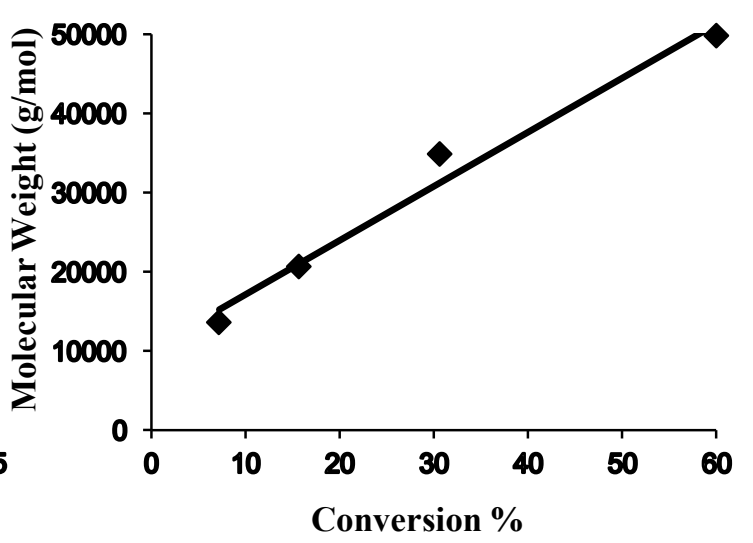

Figure 118 Results of DTBA mediated polymerizations of MMA in Toluen at $60{ }^{\circ} \mathrm{C}$, $[\text { Monomer }]_{\mathrm{o}} \backslash[\text { free } \mathrm{DTBA}]_{\mathrm{o}} \backslash[\mathrm{AIBN}]_{\mathrm{o}}=2000 \backslash 4 \backslash 1$ in the presence of free raft agent precursor dithiobenzoicacid (DTBA) a) thickness profiles and kinetics of poly (MMA) brushes, b) the surface coverage plotted against molecular weight of poly (MMA) brushes, c) plot of pseudo-first-order kinetics of poly (MMA) brushes, d) molecular weights of poly (MMA) brushes with increasing monomer conversion.

The dependence of ellipsometric thickness of poly (MMA) brush grown from DTBA immobilized surface on polymerization time is shown in Figure 118 (a). The brush thickness initially increases linearly with polymerization time up to $7 \mathrm{~h}$ and deviates afterwards, which is consistent to with the literature data. (508) The brush growth began 
to slow down and reached a plateau of $24.6 \mathrm{~nm}$ in film thickness. The reason for this deviation from linear behavior might be increase in steric hindrance to chain growth for longer polymer brushes as time goes on. Nature of the novel approach developed in this work limits the accessibility of growing polymeric radicals onto the surface since the mechanism is very similar to Z-group approach. As a consequence of the fact that raft agent precursors are attached to surface through their dithioester moieties, not through their R groups, polymeric radicals are not growing on the surface. They break off the surface, add monomers in solution phase and re-approach back to the surface. This process is relatively easier at the beginning of the polymerization. However, it gets more difficult in the latter stage of polymerization since both the length and size of polymeric radicals increase whereas the mobility decreases. This limited mobility automatically leads to slowing down of the increase in thickness, in another words, deviation from the linear behavior. It is also clearly observable that the surface coverage of polymer brushes displays linear increase with the increase in molecular weight. [Figure 118 (b)]

The surface coverage, A $\left(\mathrm{mg} / \mathrm{m}^{2}\right)$, grafting density, $\sigma$ (chains $\left./ \mathrm{nm}^{2}\right)$, and average distance between grafting sites, $D(\mathrm{~nm})$, of grafted polymer brushes were calculated from the $M_{n}$ of the free polymer and the dry film thickness, $h(\mathrm{~nm})$, according to equations 18, 19, 20 and summarized in Table 9. As a result of that the grafting density values are varied between $0.18-0.31$ chains per $\mathrm{nm}^{2}$, poly (MMA) brushes might be considered as a brush type between semi-dilute and a high density brush, which is closer to a high density conformation than semi-dilute. (509)

\subsection{Characterization of Homopolymer Brushes Prepared on Gold}

\subsubsection{Characterization of Poly (BuMA) Brush Synthesized on Gold-DTBA Surface via DTBA Mediated Polymerization Technique}

The growth of poly (BuMA) brush from the gold-DTBA surface in the presence of free DTBA was confirmed by ATR-FTIR [Figure 123 (b)], AFM and water contactangle measurements. (Figure 119) The ATR-FTIR spectra of the poly (BuMA) brush demonstrated characteristic bands at $\sim 1730 \mathrm{~cm}^{-1}$, associated with the carbonyl stretch, at $1034 \mathrm{~cm}^{-1}$ resulted from strong $\mathrm{C}=\mathrm{S}$ stretching vibrations suppressed by absorption bands at $1108 \mathrm{~cm}^{-1}$ and $1264 \mathrm{~cm}^{-1}$ associated with $\mathrm{C}-\mathrm{O}$ stretching of $\mathrm{C}(=\mathrm{O})-\mathrm{O}-\mathrm{C}$ bonds in monomer units. The bands at around 2960, 2926, $2872 \mathrm{~cm}^{-1}$ are considered to be due to aliphatic $\mathrm{CH}$ groups. The 2960, $2872 \mathrm{~cm}^{-1}$ peaks are due to asymmetric and symmetric stretching vibrations of $\mathrm{CH}_{3}$, respectively, while the $2926 \mathrm{~cm}^{-1}$ peak is assigned to $\mathrm{CH}_{2}$ vibration. The peaks at $1455 \mathrm{~cm}^{-1}$ and $1380 \mathrm{~cm}^{-1}$ are attributed to the $\mathrm{CH}$ bending and rocking respectively. In addition to these bands, there are absorption bands at 701-618 $\mathrm{cm}^{-1}$ attributed to $\mathrm{C}-\mathrm{S}$ stretching. Moreover, $\mathrm{C}=\mathrm{C}$ aromatic doublets which belong to raft agent precursor DTBA, which are expected at around 1480-1420 $\mathrm{cm}^{-1}$, were observed at around 1466-1455 $\mathrm{cm}^{-1}$, being overlapped with $\mathrm{CH}$ bending 
vibration. A drastic increase in water contact angle from $61^{\circ}$ (gold-DTBA layer) to $83.37^{\circ}$ (polymer brush layer) also confirmed that a relatively more hydrophobic polymer layer than DTBA immobilized gold was obtained. The area shown in Figure 119 (24 hours polymerization) displays uniformity. The change in film morphology is associated with a drastic decrease in the RMS roughness from $1.3106 \mathrm{~nm}$ (gold-DTBA layer) to $0.8059 \mathrm{~nm}$ (PBuMA brush layer).

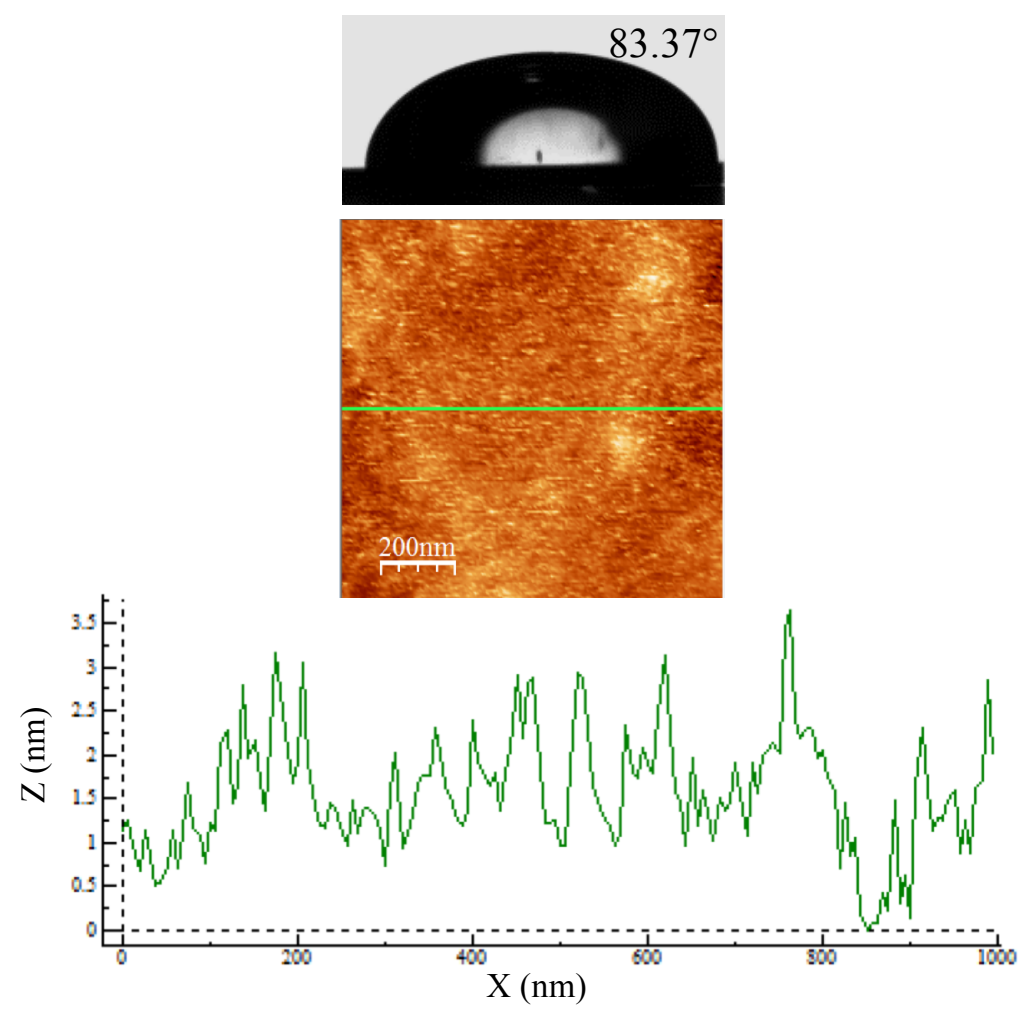

Figure 119 2D AFM topography image $(1 \mu \mathrm{m} \times 1 \mu \mathrm{m})$ in ambient conditions, surface profile analysis and photograph of $1 \mu \mathrm{L}$ water droplet for poly (BuMA) brush synthesized in the presence of DTBA for $24 \mathrm{~h}$ with the ratio of $[\mathrm{M}] /[\mathrm{DTBA}] /[\mathrm{I}]=2000 / 4 / 1$ in feed.

GPC trace of free polymer was monomodal and had narrow molecular weight distribution, which is a proof of well-defined polymers prepared via DTBA mediated polymerization without utilization of a free CTA in solution. After 24 hours polymerization, the monomer conversion is $42.2 \%$ whereas the thickness value reached is $20 \mathrm{~nm}$. GPC analysis of the free polymers reveals a $M_{n}$ of $44326 \mathrm{~g} / \mathrm{mol}$ and a PDI of 1.61. The low PDI value indicates that the polymerization was conducted in a controlled manner without any free CTA in reaction mixture.

The surface coverage, A $\left(\mathrm{mg} / \mathrm{m}^{2}\right)$, grafting density, $\sigma$ (chains $\left./ \mathrm{nm}^{2}\right)$, and average distance between grafting sites, $D(\mathrm{~nm})$, of grafted polymer brushes were calculated from the $M_{n}$ of the free polymer and the dry film thickness, $h(\mathrm{~nm})$, according to equations 18, 19, 20 and summarized in Table 11. As a result of that the grafting density is 0.28 chains per $\mathrm{nm}^{2}$, poly (BuMA) brushes might be considered as a brush type 
between semi-dilute and a high density brush. (509) This gold substrate covered with $20 \pm 0.1 \mathrm{~nm}$ poly (BUMA) brushes was used as model substrate for DTBA mediated block copolymerization of DMAEMA.

\subsubsection{Characterization of Poly (PEGMA) Brush Synthesized on Gold-DTBA Surface via RAFT Mediated Polymerization Technique}

4-Cyano-4-[dodecylsulfanylthiocarbonyl)sulfanyl] pentanoicacid (DTSCPA) is used as free CTA in solution instead of DTBA in order to investigate impact of used free CTA on controllability of polymerization, resulting PDI and thickness. The formation of poly (PEGMA) brush from gold-DTBA surface was confirmed by ATR-IR [Figure 125 (b)], AFM and water contact-angle measurements. (Figure 120) The ATR-FTIR spectra for poly (PEGMA) brush demonstrated characteristic bands at $\sim 1730 \mathrm{~cm}^{-1}$ associated with the carbonyl stretch, at $1032 \mathrm{~cm}^{-1}$ resulted from strong $\mathrm{C}=\mathrm{S}$ stretching vibrations suppressed by absorption bands at $\sim 1110 \mathrm{~cm}^{-1}, 1264 \mathrm{~cm}^{-1}$ associated with $\mathrm{C}-\mathrm{O}$ stretching resulted from vibrations of C-O-C ether bonds in the ethylene glycol units. The bands at around 2922, 2870, $2854 \mathrm{~cm}^{-1}$ are considered to be due to aliphatic $\mathrm{CH}$ groups. The $2922,2854 \mathrm{~cm}^{-1}$ peaks are due to asymmetric and symmetric stretching vibrations of $\mathrm{CH}_{3}$, respectively, while the $2870 \mathrm{~cm}^{-1}$ peak is assigned to $\mathrm{CH}_{2}$ vibration. The peaks at $1451 \mathrm{~cm}^{-1}$ and $1379 \mathrm{~cm}^{-1}$ are attributed to the $\mathrm{CH}$ bending and rocking respectively. In addition to these bands, there are bands at around $701-624 \mathrm{~cm}^{-1}$ attributed to C-S stretching. The broad band at $3660-3140 \mathrm{~cm}^{-1}$ which is assigned to O$\mathrm{H}$ stretching, results from water vapor which PPEGMA brush coating absorbs due to its hydrophilic nature.

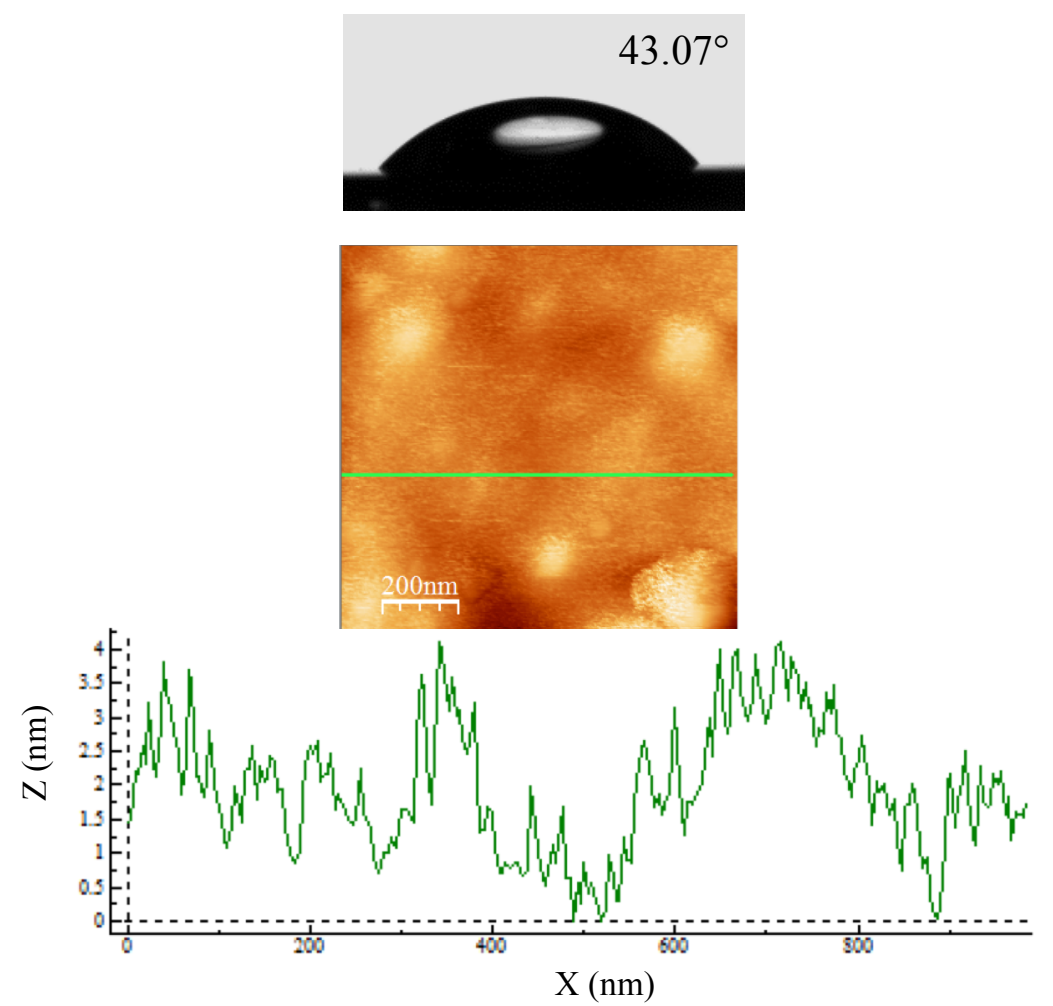


Figure 120 2D AFM topography images $(1 \mu \mathrm{m} \times 1 \mu \mathrm{m})$ in ambient conditions, surface profile analysis and photograph of $1 \mu \mathrm{L}$ water droplet for poly (PEGMA) brush synthesized in the presence of free CTA (DSTCSPA) for $24 \mathrm{~h}$ with the ratio of $[\mathrm{M}] /[\mathrm{CTA}] /[\mathrm{I}]=2000 / 4 / 1$ in feed.

The aromatic C-H stretching peak which belongs to DTBA, expected at around 3100$3000 \mathrm{~cm}^{-1}$ is not clearly observable as a result of being suppressed by broad band of O$\mathrm{H}$ stretching whereas $\mathrm{C}=\mathrm{C}$ aromatic doublets which is expected at a value between $1480-1420 \mathrm{~cm}^{-1}$, were observed at $1451 \mathrm{~cm}^{-1}$, being overlapped with $\mathrm{CH}$ bending vibration. A decrease in water contact angle from $61^{\circ}$ (gold-DTBA layer) to $43^{\circ}$ (poly(PEGMA) layer) also confirmed that relatively more hydrophilic polymer layer was obtained. The area shown in Figure $\mathbf{1 2 0}$ for 24 hours polymerization time display gaussian distribution which is an indicator of surface homogenitiy although small aggregates heterogeneously distributed over some areas of sample were observed in related AFM height image. The RMS roughness value is $2.1821 \mathrm{~nm}$. GPC trace of free polymers was monomodal and had narrow molecular weight distribution, which are characteristics of well-defined polymers prepared via RAFT-mediated polymerization. After $24 \mathrm{~h}$ polymerization, the monomer conversion is $74.3 \%$ and the thickness value reached is $24 \mathrm{~nm}$. GPC analysis of the free polymers reveals a $M_{n}$ of $53765 \mathrm{~g} / \mathrm{mol}$ and a PDI of 1.50 (Table 11). The low PDI value indicates that the polymerization was conducted in a controlled manner.

The surface coverage, A $\left(\mathrm{mg} / \mathrm{m}^{2}\right)$, grafting density, $\sigma$ (chains $\left./ \mathrm{nm}^{2}\right)$ and average distance between grafting sites, $D(\mathrm{~nm})$ of grafted poly (BuMA) brushes were calculated from the $M_{n}$ of the free polymer and the dry film thickness, $h(\mathrm{~nm})$, according to equations 18, 19 and 20 (Table 11). As a result of that the grafting density is 0.29 chains per $\mathrm{nm}^{2}$, poly(PEGMA) brushes might be considered as a semi dilute brush. (509) The gold substrate covered with $24 \pm 0.1 \mathrm{~nm}$ poly (PEGMA) brushes was used as model substrate for RAFT mediated block copolymerization of DMAEMA.

\subsubsection{Investigation of Impact of Monomer / Raft Agent Ratio in Feed on Resulting Thickness and Grafting Density Values of Polymer Brushes Prepared via DTBA Polymerization}

Monomer amount per DTBA in reaction mixtures were varied in order to check and confirm controllability of thickness of resulting polymer brushes by utilizing raft agent precursor (DTBA) both in solution (as free DTBA) and on gold surfaces (as anchor). (Table 10) The ATR-FTIR spectra for poly (MMA) and poly (DMAEMA) brushes synthesized with the ratio of $[\mathrm{M}] /[\mathrm{CTA}] /[\mathrm{I}]=2000 / 4 / 1$ in the presence of free raft agent precursor (DTBA) from gold-DTBA surface for $24 \mathrm{~h}$ [Figure 121 (a), (b)] and with the ratio of $[\mathrm{M}] /[\mathrm{CTA}] /[\mathrm{I}]=10000 / 4 / 1$ in the presence of free raft agent precursor (DTBA) from gold-DTBA surface for $24 \mathrm{~h}$ [Figure 121 (c), (d)] demonstrated characteristic bands at $\sim 1732 \mathrm{~cm}^{-1}$, associated with the carbonyl stretch and strong absorption band 
at $1029 \mathrm{~cm}^{-1}$ resulted from $\mathrm{C}=\mathrm{S}$ stretching vibrations suppressed by absorption bands at $1100 \mathrm{~cm}^{-1}$ and $1264 \mathrm{~cm}^{-1}$ associated with $\mathrm{C}-\mathrm{O}$ stretching of $\mathrm{C}(=\mathrm{O})-\mathrm{O}-\mathrm{C}$ bonds in monomer units and bands at around $1243-1023 \mathrm{~cm}^{-1}$ resulted from vibrations of C-N stretching which belong to PDMAEMA layers. The bands at around 2958, 2922, 2854 $\mathrm{cm}^{-1}$ are considered to be due to aliphatic CH groups. The $2958,2854 \mathrm{~cm}^{-1}$ peaks are due to asymmetric and symmetric stretching vibrations of $\mathrm{CH}_{3}$, respectively, while the $2922 \mathrm{~cm}^{-1}$ peak is assigned to $\mathrm{CH}_{2}$ vibration. The peaks at $1460 \mathrm{~cm}^{-1}$ and $1379 \mathrm{~cm}^{-1}$ are attributed to the $\mathrm{CH}$ bending and rocking respectively. In addition to these bands, there are absorption bands at $703-616 \mathrm{~cm}^{-1}$ attributed to $\mathrm{C}-\mathrm{S}$ stretching. The broad band at $3699-3128 \mathrm{~cm}^{-1}$ which is assigned to $\mathrm{O}-\mathrm{H}$ stretching, results from water vapor which polymer brush coatings absorb due to their hydrophilic natures. Aromatic $\mathrm{C}-\mathrm{H}$ stretching of raft agent precursor DTBA which is supposed to be at around 3100-3000 $\mathrm{cm}^{-1}$ is not clearly observable as a result of being overlapped by both aliphatic $\mathrm{CH}$ stretching and broad band of $\mathrm{O}-\mathrm{H}$ stretching. However, $\mathrm{C}=\mathrm{C}$ aromatic doublets which are expected at around $1480-1420 \mathrm{~cm}^{-1}$, were observed at around $1460 \mathrm{~cm}^{-1}$, being overlapped with $\mathrm{CH}$ bending vibration.

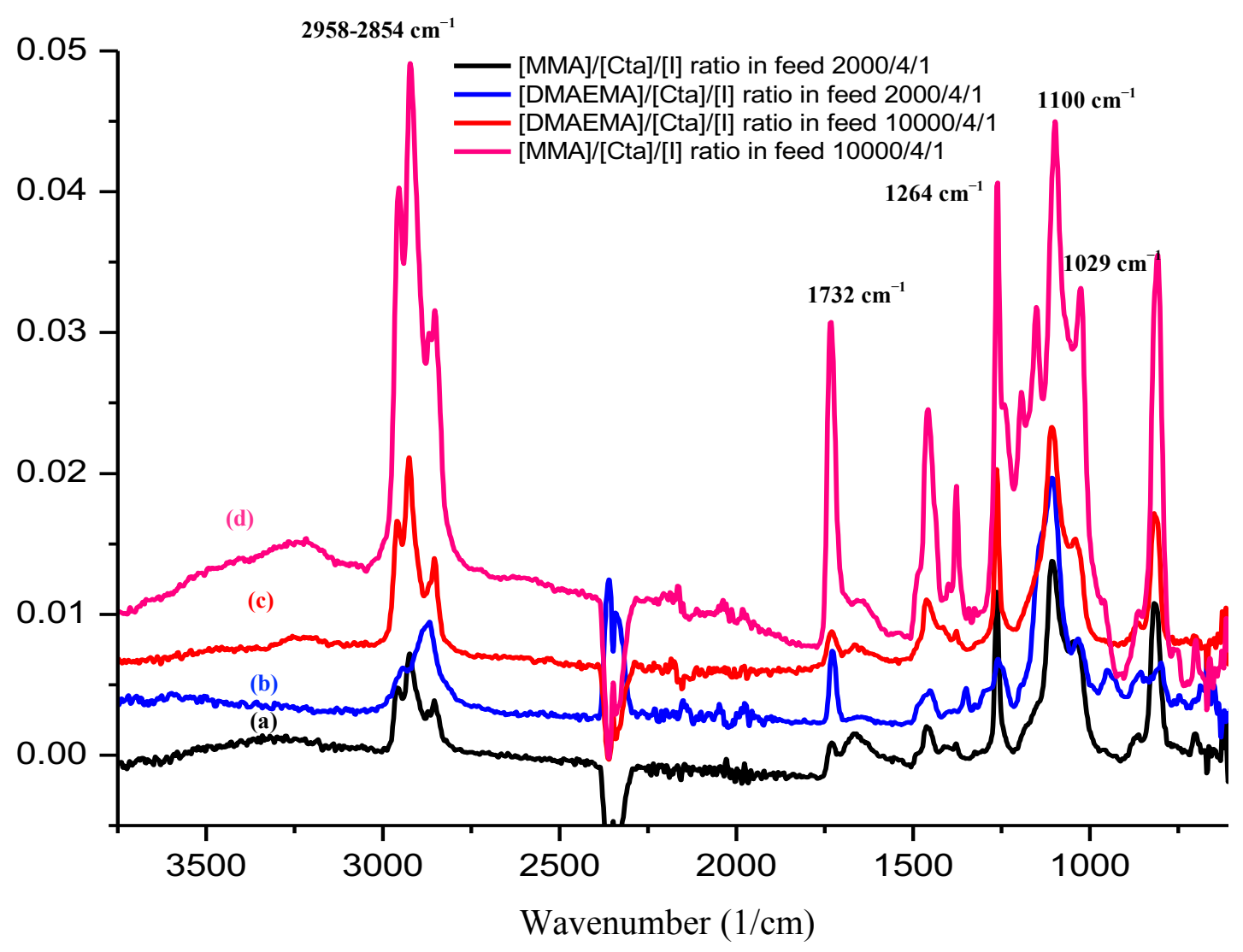

Figure 121 ATR-FTIR spectra of (a) poly (MMA) brush synthesized with the ratio of $[\mathrm{M}] /[\mathrm{DTBA}] /[\mathrm{I}]=2000 / 4 / 1$ in the presence of free raft agent precursor (DTBA) from gold-DTBA surface for $24 \mathrm{~h}$, (b) poly (DMAEMA) brush synthesized with the ratio of $[\mathrm{M}] /[\mathrm{DTBA}] /[\mathrm{I}]=2000 / 4 / 1$ in the presence of free raft agent precursor (DTBA) from gold-DTBA surface for $24 \mathrm{~h}$, (c) poly (DMAEMA) brush synthesized with the ratio of $[\mathrm{M}] /[\mathrm{DTBA}] /[\mathrm{I}]=10000 / 4 / 1$ in the presence of free raft agent precursor (DTBA) from 
gold-DTBA surface for $24 \mathrm{~h}$, (d) poly (MMA) brush synthesized with the ratio of $[\mathrm{M}] /[\mathrm{DTBA}] /[\mathrm{I}]=10000 / 4 / 1$ in the presence of free raft agent precursor (DTBA) from gold-DTBA surface for $24 \mathrm{~h}$.

Increase in water contact angles of the gold surfaces polymerized with the ratio of $[\mathrm{M}]$ :2000 / [CTA]: 4 in feed (PMMA) 71.50 , (PDMAEMA) 60.51 $1^{\circ}$ and the ratio of [M] $: 10000$ / [CTA]: 4 in feed (PMMA) $71.64^{\circ}$ and (PDMAEMA) $63.12^{\circ}$ compared to bare gold $\left(44.35^{\circ}\right)$ are additional confirmations of sucsessful growth of polymer layers. The areas shown in Figure 117 poly (MMA) brush synthesized for $24 \mathrm{~h}$ with the ratio of $[\mathrm{M}] /[\mathrm{DTBA}] /[\mathrm{I}]=2000 / 4 / 1$ in feed in the presence of free raft agent precursor (DTBA) from gold-DTBA surface), Figure 122 (a) (poly (MMA) brush synthesized for $24 \mathrm{~h}$ with the ratio of $[\mathrm{M}] /[\mathrm{DTBA}] /[\mathrm{I}]=10000 / 4 / 1$ in feed in the presence of free raft agent precursor (DTBA) from gold-DTBA surface), Figure 122 (b) (poly (DMAEMA) brush synthesized in the presence of DTBA for $24 \mathrm{~h}$ with the ratio of $[\mathrm{M}] /[\mathrm{DTBA}] /[\mathrm{I}]=$ 2000/4/1 in feed) and Figure 122 (c) (poly (DMAEMA) brush synthesized in the presence of DTBA for $24 \mathrm{~h}$ with the ratio of $[\mathrm{M}] /[\mathrm{DTBA}] /[\mathrm{I}]=10000 / 4 / 1$ in feed) display gaussian distributions which confirm surface uniformity although small aggregates heterogeneously distributed over some areas of samples were observed in related AFM height images. The changes in film morphologies is associated with increases in the RMS roughnesses from $1.3106 \mathrm{~nm}$ (DTBA immobilized gold surfaces) to 2.1821 (Figure 117), 2.3026 [Figure 122 (a)], (with a slight decrease to) 1.0946 [Figure $122(b)$ ] and 2.1875 [Figure 122 (c)] nm respectively.

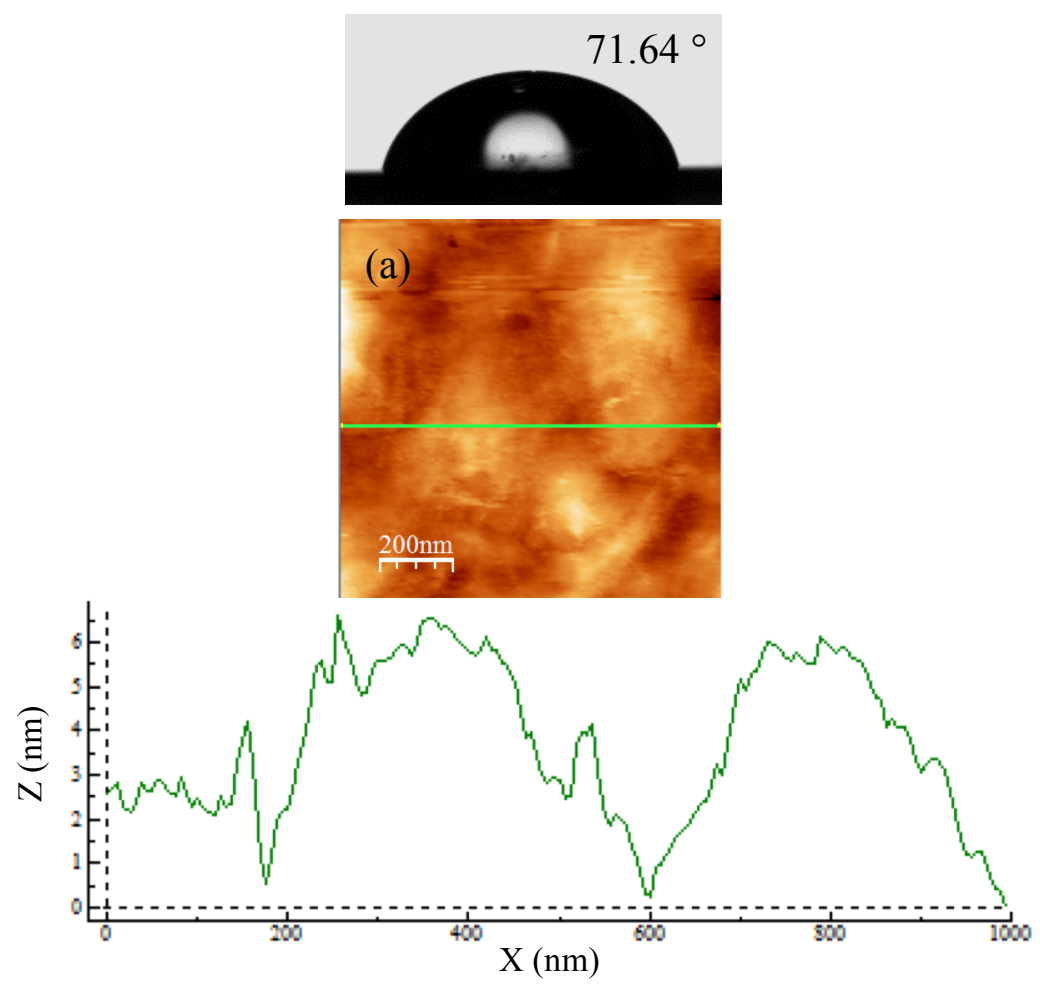



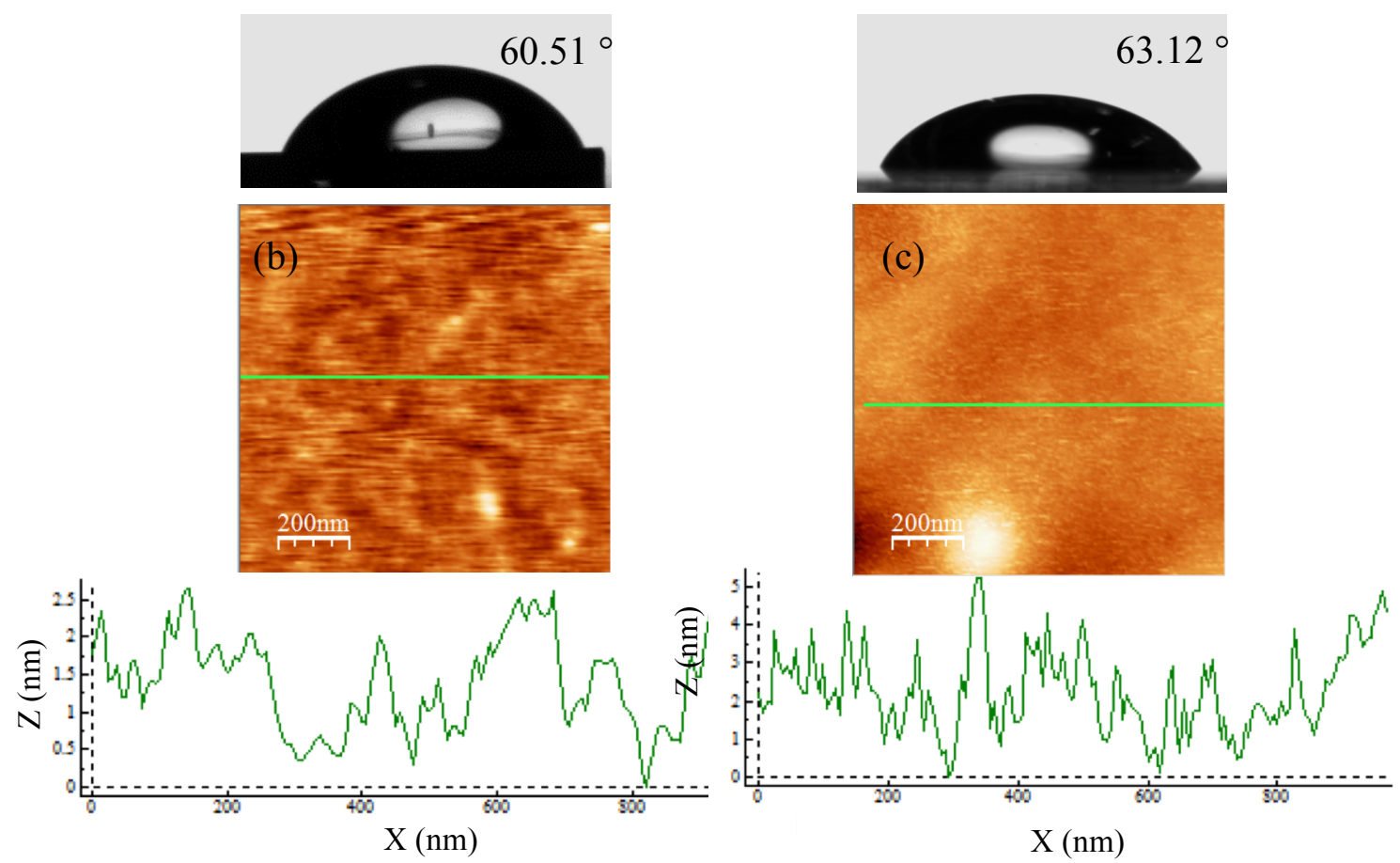

Figure 122 2D AFM topography images $(1 \mu \mathrm{m} \times 1 \mu \mathrm{m})$ in ambient conditions, surface profile analysises and photographs of $1 \mu \mathrm{L}$ water droplets for (a) poly (MMA) brush synthesized in the presence of DTBA for $24 \mathrm{~h}$ with the ratio of $[\mathrm{M}] /[\mathrm{DTBA}] /[\mathrm{I}]=10000 / 4 / 1$ in feed, (b) poly (DMAEMA) brush synthesized in the presence of DTBA for $24 \mathrm{~h}$ with the ratio of $[\mathrm{M}] /[\mathrm{DTBA}] /[\mathrm{I}]=2000 / 4 / 1$ in feed, (c) poly (DMAEMA) brush synthesized in the presence of DTBA for $24 \mathrm{~h}$ with the ratio of $[\mathrm{M}] /[\mathrm{DTBA}] /[\mathrm{I}]=10000 / 4 / 1$ in feed.

The gold surfaces polymerized with the ratio of [MMA]:2000/[DTBA]:4 in feed resulted in poly (MMA) brush layer with a thickness of $24.6 \mathrm{~nm}$ whereas the ratio of [MMA]:10000 / [DTBA]:4 in feed produced a layer with a thickness of $58.7 \mathrm{~nm}$. Similarly, the ratio of [DMAEMA]: 2000 / [DTBA]: 4 in feed resulted in $18 \mathrm{~nm}$ thick poly (DMAEMA) layer whereas the ratio of [DMAEMA]:10000 / [DTBA]: 4 in feed ended up with formation of $43 \mathrm{~nm}$ thick brush layer. (Table 10) This clear increase in thickness of resulting brush with increasing monomer amount per free DTBA in solution is another proof of controllability of thicknesses of polymer brushes synthesized from gold surfaces by utilization of DTBA mediated polymerization technique. The time evolution of number average molecular weights, $M_{n}$ and polydispersities, PDI of the grafted polymer chains on gold, which is assumed to be the same as that of the unbound polymer chains in solution (263) was obtained by GPC analysis. (shown in Table 10) 
Table 10 Summary of impact of monomer [M] /Raft agent precursor [DTBA] / initiator [I] ratio on resulting thickness of polymer brushes

\begin{tabular}{|c|c|c|c|c|c|c|c|}
\hline $\begin{array}{l}{[\mathrm{M}] /[\mathrm{CTA}]} \\
\text { Ratios }\end{array}$ & $\mathbf{M n} / \mathbf{M w}$ & PDI & $\begin{array}{c}\text { Thickness }^{\mathrm{c}} \\
\text { (nm) }\end{array}$ & $\begin{array}{l}\text { Coverage } \\
\left(\mathrm{mg} / \mathrm{m}^{2}\right)\end{array}$ & $\begin{array}{l}\text { Grafting } \\
\text { Density } \\
\text { (chains/ } \\
\mathbf{n m}^{2} \text { ) }\end{array}$ & $\begin{array}{l}\text { Distan } \\
\text { ce } \\
(\mathrm{nm})\end{array}$ & $\begin{array}{l}\text { Static } \\
\text { Contact } \\
\text { Angle }^{d}\end{array}$ \\
\hline $\begin{array}{l}\text { 2000:4:1 } \\
\text { (PMMA) }\end{array}$ & $49806 / 68447$ & 1.37 & 24.6 & 26 & 0.31 & 2 & $71.50^{\circ}$ \\
\hline $\begin{array}{l}1000041^{a} \\
\text { (PMMA) }\end{array}$ & $153900 / 291160$ & 1.89 & 58.7 & 62 & 0.24 & 2.30 & $71.64^{\circ}$ \\
\hline $\begin{array}{l}\text { 2000:4:1 } \\
\text { (PDMAEMA) }\end{array}$ & $40437 / 53843$ & 1.33 & 18 & 24.3 & 0.36 & 1.87 & $60.51^{\circ}$ \\
\hline $\begin{array}{l}\text { 10000:4:1 } \\
\text { (PDMAEMA) }\end{array}$ & $151344 / 88543$ & 1.72 & 43 & 58 & 0.23 & 2.35 & $63.12^{\circ}$ \\
\hline
\end{tabular}

${ }^{a}$ Polymerization of (methyl methacrylate) MMA with dithiobenzoicacid (DTBA) and 2,2'-azobis(isobutyronitrile) (AIBN) in toluene at $60{ }^{\circ} \mathrm{C}$ for $24 \mathrm{~h}$ from gold-DTBA surface, ${ }^{b}$ Polymerization of (2-(dimethylamino) ethyl methacrylate) DMAEMA with DTBA and AIBN in toluene at $60{ }^{\circ} \mathrm{C}$ for $24 \mathrm{~h}$ from gold-DTBA surface, ${ }^{\mathrm{c}}$ Thicknesses were determined by ellipsometry and are an average of five samplings across the sample. Error of thicknesses measured was within $\pm 0.7 \mathrm{~nm},{ }^{d}$ Static contact angles were taken using goniometry and are an average of five samplings across the sample. The standard deviation of contact angles was less than $1^{\circ}$.

GPC trace of free polymers for each sample was monomodal and had narrow molecular weight distribution, which is an indicator of well-defined polymers prepared via DTBA-mediated polymerization without a free CTA in solution. The monomer conversions are $60 \%$ ([MMA]:2000/[DTBA]:4), $69 \%$ ([MMA]:10000/[DTBA]:4), $78.5 \%$ ([DMAEMA]:2000/[DTBA]:4), ([DMAEMA]:10000/[DTBA]:4) 84.1\%; GPC analysises of the free polymers reveal molecular weights of 49806, 153900, 40437, $151344 \mathrm{~g} / \mathrm{mol}$ and PDIs of $1.37,1.89,1.33,1.72$ respectively. The low PDI values are another evidences of the fact that the DTBA mediated polymerizations were conducted in a controlled manner without utilization of free CTAs in solution. As a result of that the grafting density values are varied between $0.23-0.36$ chains per $\mathrm{nm}^{2}$, poly (MMA) brushes might be considered as a brush type between semi-dilute and a high density brush, which is closer to a high density conformation than semi-dilute. (509) 


\subsection{Characterization of Diblock Copolymer Brushes Prepared on Gold}

\subsubsection{Characterization of Diblock Copolymer Brush (PBuMA-b-PDMAEMA) Synthesized on Gold-DTBA-PBuMA Surface in the Presence of Free DTBA via DTBA Mediated Polymerization Technique}

One of the advantages of utilization of DTBA based technique to prepare polymer brushes is the opportunity to produce block copolymer brushes by re-activation of the dormant chains. The preparation of a block copolymer brush is also another confirmation of whether the previous sample has maintained the living characteristics. Thus, the gold substrate covered with $20 \mathrm{~nm}$ poly (BUMA) brushes was used for subsequent formation of diblock copolymer brushes in order to check living nature of the surface bound homopolymer brushes prepared via DTBA mediated polymerization. The formation of second block poly (DMAEMA) brush on the gold substrate covered with poly (BuMA) brush was confirmed by ATR-IR [Figure 123 (c)], AFM and water contact angle measurements (Figure 124).

The ATR-FTIR spectra for the PBuMA-block-PDMAEMA brush demonstrated characteristic band at $\sim 1730 \mathrm{~cm}^{-1}$ associated with the carbonyl stretch, strong band at $1044 \mathrm{~cm}^{-1}$ resulted from $\mathrm{C}=\mathrm{S}$ stretching vibrations suppressed by strong absorption bands at $1094 \mathrm{~cm}^{-1}$ and $1264 \mathrm{~cm}^{-1}$ associated with $\mathrm{C}-\mathrm{O}$ stretching of $\mathrm{C}(=\mathrm{O})-\mathrm{O}-\mathrm{C}$ bonds in monomer units and bands at $1237-1023 \mathrm{~cm}^{-1}$ resulted from vibrations of C-N stretching which belongs to poly (DMAEMA) layer. The bands at around 2958, 2922, $2854 \mathrm{~cm}^{-1}$ are considered to be due to aliphatic $\mathrm{CH}$ groups. The $2958,2854 \mathrm{~cm}^{-1}$ peaks are due to asymmetric and symmetric stretching vibrations of $\mathrm{CH}_{3}$, respectively, while the $2922 \mathrm{~cm}^{-1}$ peak is assigned to $\mathrm{CH}_{2}$ vibration. A slight shift is observed in stretching vibrations of $\mathrm{CH}_{3}$ of homopolymer (PBuMA) $\left(2872 \mathrm{~cm}^{-1}\right)$ after blockcopolymerization with DMAEMA as a result of being overlapped by the characteristic peaks for the $\mathrm{N}\left(\mathrm{CH}_{3}\right)_{2}$ of PDMAEMA segment $\left(2854 \mathrm{~cm}^{-1}\right)$, which generally appears at $\sim 2830$ $\mathrm{cm}^{-1}$. It is clearly observable that a shoulder between $2854-2808 \mathrm{~cm}^{-1}$ attributed to $\mathrm{N}\left(\mathrm{CH}_{3}\right)_{2}$ stretching band of PDMAEMA appears. The peaks at $1466 \mathrm{~cm}^{-1}$ and 1379 $\mathrm{cm}^{-1}$ are attributed to the $\mathrm{CH}$ bending and rocking respectively. In addition to these bands, there are absorption bands at around $668-624 \mathrm{~cm}^{-1}$ attributed to $\mathrm{C}-\mathrm{S}$ stretching. Moreover, appearance of the broad band at $3664-3122 \mathrm{~cm}^{-1}$ which is assigned to O-H stretching, resulting from water vapor which second brush layer (PDMAEMA) absorbs is another confirmation of formation of a second hydrophilic block segment (PBuMAblock-PDMAEMA). The aromatic C-H stretching peak which belongs to DTBA, expected at around $3100-3000 \mathrm{~cm}^{-1}$ is not clearly observable as a result of being suppressed by both aliphatic $\mathrm{C}-\mathrm{H}$ stretching and broad band of $\mathrm{O}-\mathrm{H}$ stretching whereas $\mathrm{C}=\mathrm{C}$ aromatic doublets, which is expected at a value between $1480-1420 \mathrm{~cm}^{-1}$, were observed at $1466 \mathrm{~cm}^{-1}$, being overlapped with $\mathrm{CH}$ bending vibration. 


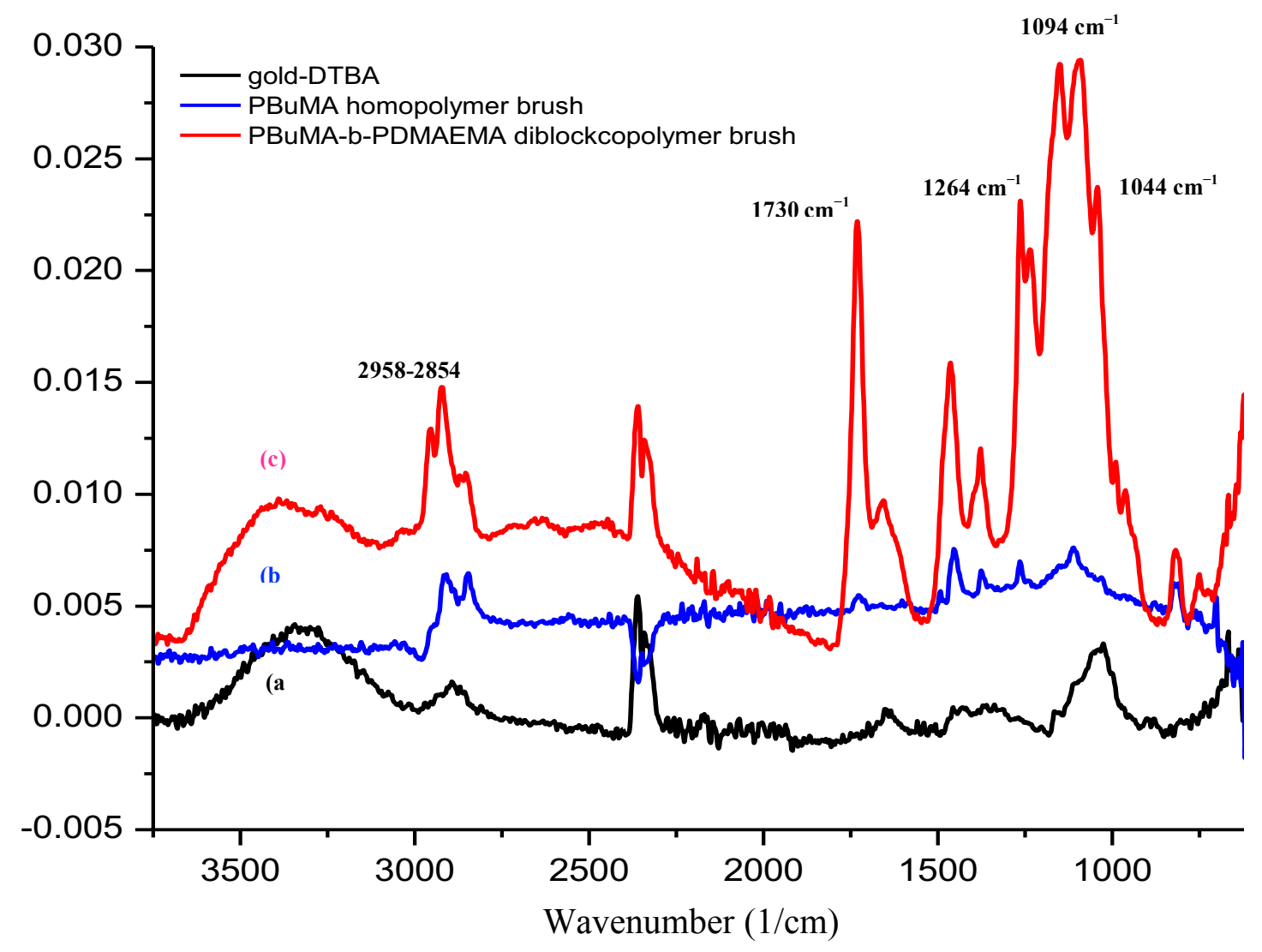

Figure 123 ATR-FTIR spectra of (a) DTBA on gold, (b) poly (BuMA) brush synthesized in the presence of free raft agent precursor (DTBA) from gold-DTBA surface for $24 \mathrm{~h}$, (c) poly(BuMA)-block-poly(DMAEMA) brush synthesized in the presence of free raft agent precursor (DTBA) from gold-DTBA-PBuMA surface for $24 \mathrm{~h}$.

The goniometry demonstrated a decrease in static water contact angle from $83.37^{\circ}$ (PBuMA layer) to $58.62^{\circ}$ (PBuMA-block-PDMAEMA), which is expected due to the more hydrophilic nature of second layer (PDMAEMA). Ellipsometry indicated a thickness increase of $30 \mathrm{~nm}$ from that of the first (PBuMA) homopolymer layer whereas the total thickness of blockcopolymer brush is $50 \mathrm{~nm}$ (Table 11). The area shown in Figure 124 for 24 hours polymerization and the other areas measured on same sample (data not shown) display gaussian distribution which is an indicator of surface homogenitiy. 


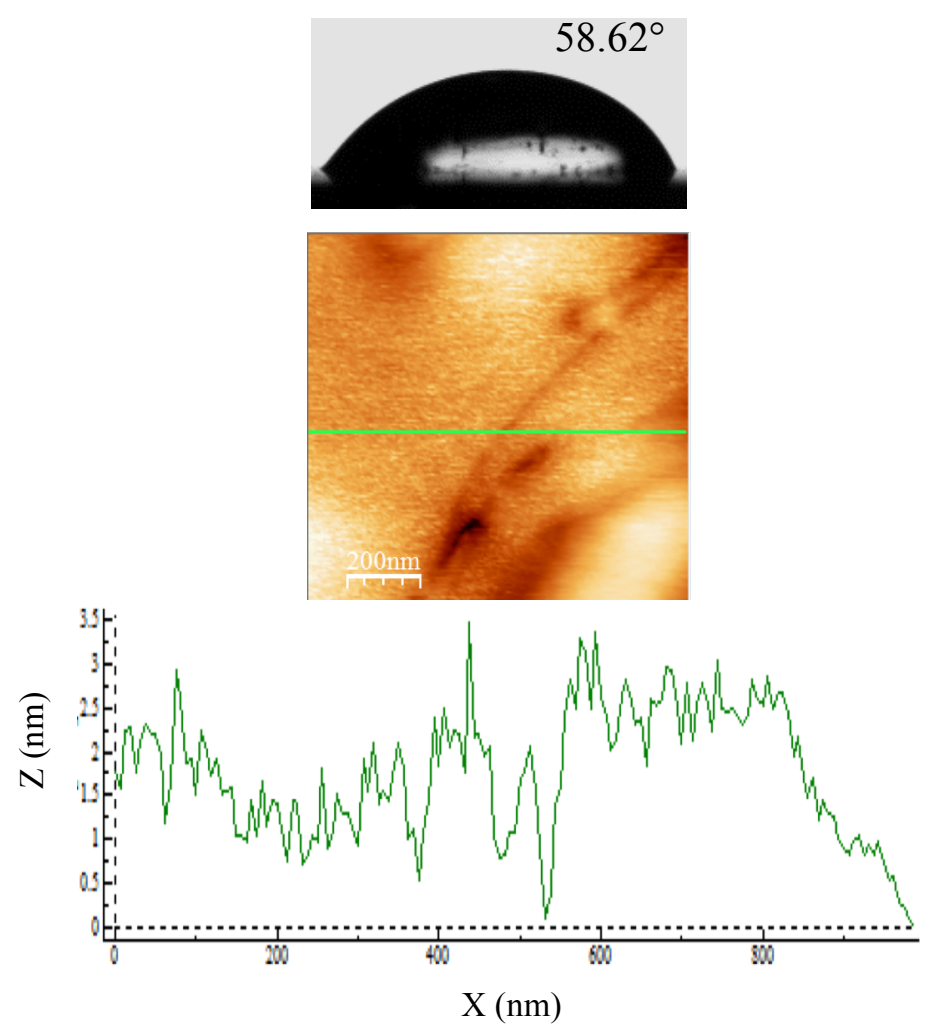

Figure 124 2D AFM topography image $(1 \mu \mathrm{m} \times 1 \mu \mathrm{m})$ in ambient conditions, surface profile analysis and photograph of $1 \mu \mathrm{L}$ water droplets for PBuMA-block-PDMAEMA brush synthesized in the presence of DTBA for $24 \mathrm{~h}$ with the ratio of $[\mathrm{M}] /[\mathrm{DTBA}] /[\mathrm{I}]=2000 / 4 / 1$ in feed.

The RMS roughness and roughness average values increased from 0.8059 to 1.7748 $\mathrm{nm}$ and from 0.6287 to 1.3717 respectively after blockcopolymerization. GPC trace of free polymer was monomodal and had narrow molecular weight distribution, which is a proof of well-defined polymers prepared via DTBA mediated polymerization without utilization of a free CTA in solution. After 24 hours polymerization, the monomer conversion is $44 \%$. GPC analysis of the free polymers reveals a $M_{n}$ of $55579 \mathrm{~g} / \mathrm{mol}$ and a PDI of 1.66 (Table 11). The low PDI value indicates that the polymerization was conducted in a controlled manner.

The surface coverages, A $\left(\mathrm{mg} / \mathrm{m}^{2}\right)$, grafting densities, $\sigma$ (chains $\left./ \mathrm{nm}^{2}\right)$, and average distances between grafting sites, $D(\mathrm{~nm})$, of grafted block poly (DMAEMA) brushes were calculated from the $M_{n}$ of the free polymer and the dry film thickness, $h(\mathrm{~nm})$, according to equations 18, 19 and 20 (Table 11). As a result of that the grafting density is 0.44 chains per $\mathrm{nm}^{2}$, second block poly (DMAEMA) layer might be considered as a brush type between semi-dilute and a high density brush, which is closer to a high density conformation than semi-dilute. (509) 


\subsubsection{Characterization of Diblock Copolymer Brush (PPEGMA-b-PDMAEMA) Synthesized on Gold-DTBA-PPEGMA Surface in the Presence of Free CTA (CDB) via RAFT Mediated Polymerization Technique}

The livingness of homopoly (PEGMA) brushes synthesized in previous step in the presence of free CTA (DTSCPA) from gold-DTBA surface was checked by block extension with DMAEMA. The formation of second segment of blockcopolymer brush (PDMAEMA) on the gold substrate covered with $24 \pm 0.1 \mathrm{~nm}$ poly (PEGMA) brushes was confirmed by ATR-IR [Figure 125 (c)], AFM and water contact-angle measurements (Figure 126). The ATR-FTIR spectra for the PPEGMA-blockPDMAEMA brush demonstrated characteristic band at $1730 \mathrm{~cm}^{-1}$ associated with the carbonyl stretch, strong band at $1044 \mathrm{~cm}^{-1}$ resulted from $\mathrm{C}=\mathrm{S}$ stretching vibrations suppressed by absorption bands at $\sim 1108 \mathrm{~cm}^{-1}, 1264 \mathrm{~cm}^{-1}$ associated with C-O stretching resulted from vibrations of $\mathrm{C}-\mathrm{O}-\mathrm{C}$ bonds in monomer units and bands at $1243-1023 \mathrm{~cm}^{-1}$ resulted from vibrations of C-N stretching which belongs to DMAEMA segment. The bands at around 2951, 2931, $2773 \mathrm{~cm}^{-1}$ are considered to be due to aliphatic $\mathrm{CH}$ groups. The $2951,2773 \mathrm{~cm}^{-1}$ peaks are due to asymmetric and symmetric stretching vibrations of $\mathrm{CH}_{3}$, respectively, while the $2931 \mathrm{~cm}^{-1}$ peak is assigned to $\mathrm{CH}_{2}$ vibration. A huge shift is observed in stretching vibrations of $\mathrm{CH}_{3}$ of homopolymer (PPEGMA) $\left(2854 \mathrm{~cm}^{-1}\right.$ ) after blockcopolymerization with DMAEMA $\left(2773 \mathrm{~cm}^{-1}\right)$ as a result of appearance of the characteristic peak for the $-\mathrm{N}\left(\mathrm{CH}_{3}\right)_{2}$ of PDMAEMA segment $\left(2823 \mathrm{~cm}^{-1}\right)$, which generally appears at $\sim 2830 \mathrm{~cm}^{-1}$. It is clearly observable that an individual peak at $2823 \mathrm{~cm}^{-1}$ attributed to $\mathrm{N}\left(\mathrm{CH}_{3}\right)_{2}$ stretching band of PDMAEMA shows up after block extension. The peaks at $1460 \mathrm{~cm}^{-1}$ and 1389 $\mathrm{cm}^{-1}$ are attributed to the $\mathrm{CH}$ bending and rocking respectively. In addition to these bands, there are bands at around 701-624 $\mathrm{cm}^{-1}$ attributed to C-S stretching. Moreover, a drastic increase in the broad band at $3691-3163 \mathrm{~cm}^{-1}$ which is assigned to $\mathrm{O}-\mathrm{H}$ stretching, resulting from water vapor which hydrophilic first brush layer absorbs (PEGMA) is another confirmation of formation of hydrophilic another layer (PDMAEMA) which leads to absorbtion of more water vapor. The aromatic $\mathrm{C}-\mathrm{H}$ stretching peak which belongs to DTBA, expected at around $3100-3000 \mathrm{~cm}^{-1}$ is not clearly observable as a result of being suppressed by both aliphatic $\mathrm{C}-\mathrm{H}$ stretching and broad band of $\mathrm{O}-\mathrm{H}$ stretching whereas $\mathrm{C}=\mathrm{C}$ aromatic doublets, which is expected at a value between $1480-1420 \mathrm{~cm}^{-1}$, were observed at $1460 \mathrm{~cm}^{-1}$, being overlapped with $\mathrm{CH}$ bending vibration. Static water contact angle increased from $43^{\circ}$ (PPEGMA) to $63.30^{\circ}$ (PPEGMA-block-PDMAEMA), which is another proof for formation of a relatively more hydrophobic second polymer layer. Ellipsometry indicated a thickness increase of $24 \mathrm{~nm}$ from that of the first (PPEGMA) homopolymer layer whereas the total thickness of blockcopolymer brush is $48 \mathrm{~nm}$ (Table 11). The area shown in Figure 126 for 24 hours polymerization displays gaussian distribution which is an indicator of surface homogenitiy although small aggregates heterogeneously distributed over some areas of sample were observed in related AFM height image. The RMS roughness and 
roughness average values increased from 2.1821 to $3.1771 \mathrm{~nm}$ and from 1.6239 to 2.0139 respectively.

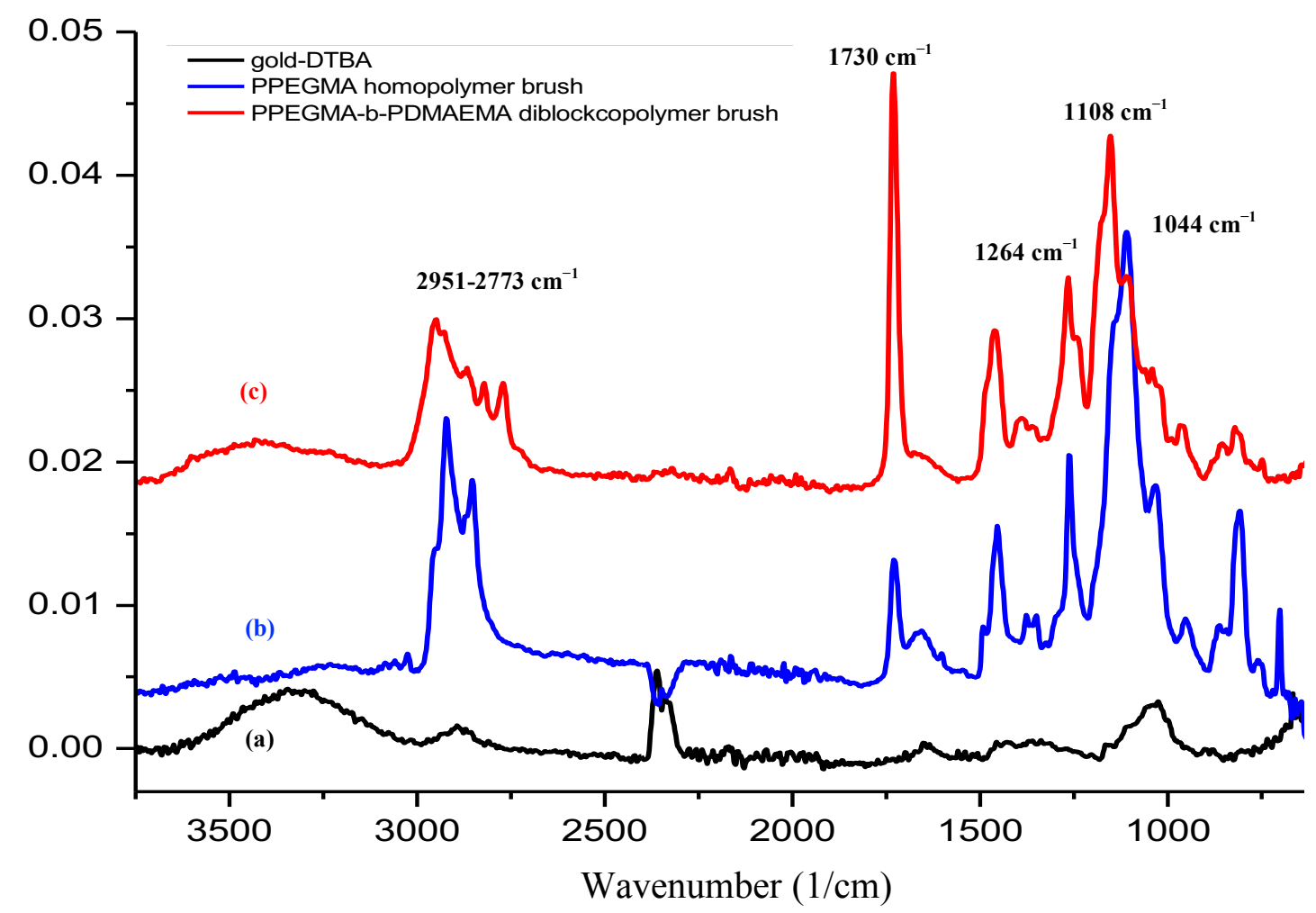

Figure 125 ATR-FTIR spectra of (a) DTBA on gold, (b) poly (PEGMA) brush synthesized in the presence of free raft agent (DTSCPA) from gold-DTBA surface for $24 \mathrm{~h}$, (c) poly (PEGMA)-block-poly (DMAEMA) brush synthesized in the presence of free raft agent (CDB) from gold-DTBA-Poly(PEGMA) surface for $24 \mathrm{~h}$.

GPC trace of free polymer was monomodal and had narrow molecular weight distribution, which are characteristics of well-defined polymers prepared via RAFTmediated polymerization. After 24 hours polymerization, the monomer conversion is $78 \%$. GPC analysis of the free polymers reveals a $M_{n}$ of $42469 \mathrm{~g} / \mathrm{mol}$ and a PDI of 1.31 (Table 11). The low PDI value indicates that the polymerization was conducted in a controlled manner. The surface coverage, A $\left(\mathrm{mg} / \mathrm{m}^{2}\right)$, grafting density, $\sigma$ (chains $/ \mathrm{nm}^{2}$ ), and average distance between grafting sites, $D(\mathrm{~nm})$ of grafted poly (DMAEMA) brushes were calculated from the $M_{n}$ of the free polymer and the dry film thickness, $h$ (nm), according to equations 18,19 and 20 (Table 11). As a result of that the grafting density is 0.46 chains per $\mathrm{nm}^{2}$, second block segment poly (DMAEMA) might be considered as a brush type between semi-dilute and a high density brush, which is closer to a high density than semi-dilute. (509) 


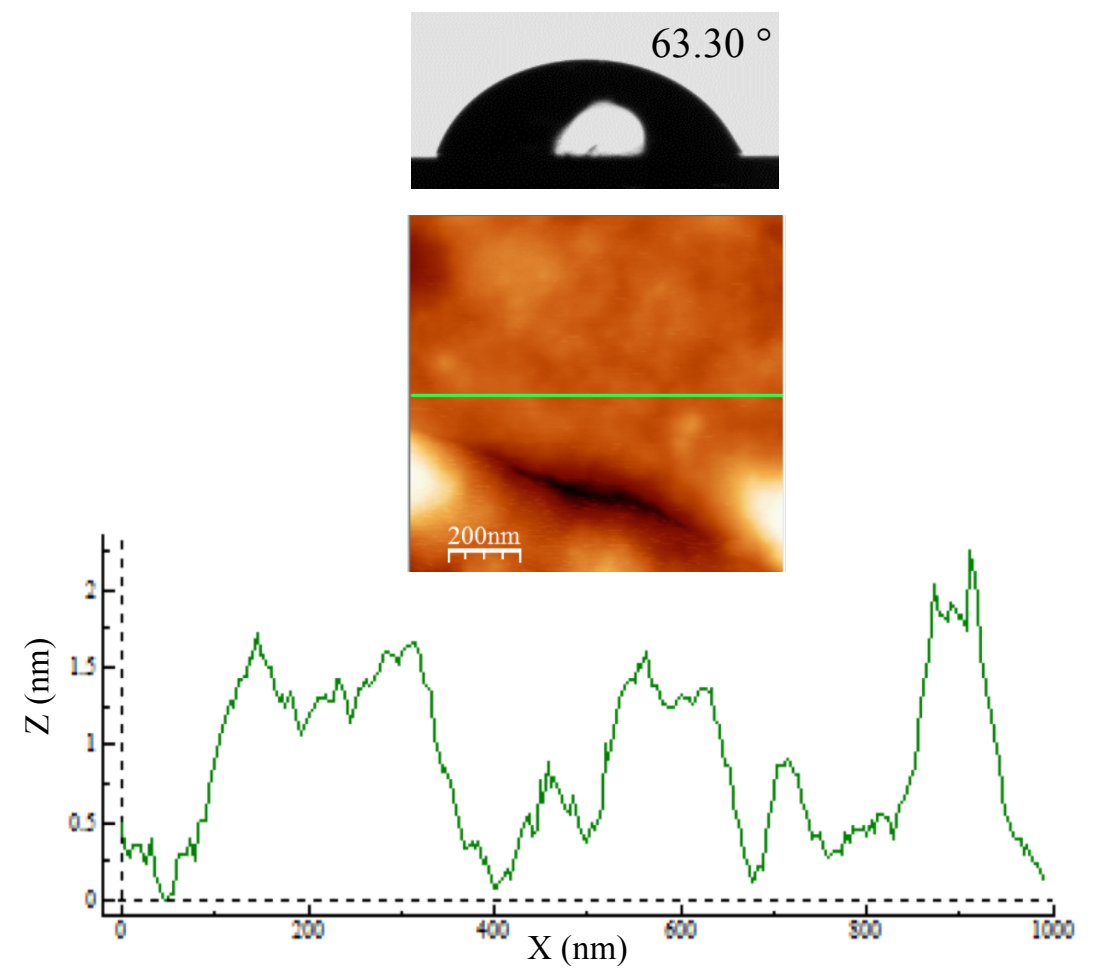

Figure 126 2D AFM topography images $(1 \mu \mathrm{m} \times 1 \mu \mathrm{m})$ in ambient conditions, surface profile analysis and photograph of $1 \mu \mathrm{L}$ water droplet for PPEGMA-block-PDMAEMA brushes synthesized in the presence of free CTA Cumyldithiobenzoate (CDB) for 24 $\mathrm{h}$ with the ratio of $[\mathrm{M}] /[\mathrm{CTA}] /[\mathrm{I}]=2000 / 4 / 1$ in feed.

Table 11 Summary of DTBA and RAFT mediated blockcopolymerizations from goldDTBA surfaces

\begin{tabular}{|l|l|l|l|l|l|l|}
\hline & & & & & \\
\hline
\end{tabular}


${ }^{a}$ Polymerization of (buthyl methacrylate) BuMA with dithiobenzoicacid (DTBA) and $2,2^{\text {' }}$-azobis(isobutyronitrile) (AIBN) in toluene at $60{ }^{\circ} \mathrm{C}$ for $24 \mathrm{~h}$ from gold-DTBA surface, ${ }^{b}$ Copolymerization of (2-(dimethylamino)ethyl methacrylate) DMAEMA with DTBA and AIBN in toluene at $60^{\circ} \mathrm{C}$ for $24 \mathrm{~h}$ from gold-DTBA-PBuMA surface,

${ }^{c}$ Polymerization of (polyethyleneglycolmethyl ether methacrylate) PEGMA with 4Cyano-4-[dodecylsulfanylthiocarbonyl)sulfanyl] pentanoicacid (DSTCSPA) and AIBN in toluene at $60{ }^{\circ} \mathrm{C}$ for $24 \mathrm{~h}$ from gold-DTBA surface, ${ }^{d}$ Copolymerization of (2(dimethylamino)ethyl methacrylate) DMAEMA with Cumyldithiobenzoate (CDB) and AIBN in toluene at $60{ }^{\circ} \mathrm{C}$ for $24 \mathrm{~h}$ from gold-DTBA-PPEGMA surface, ${ }^{\mathrm{e}}$ Thicknesses were determined by ellipsometry and are an average of five samplings across the sample. Error of thicknesses measured was within $\pm 0.7 \mathrm{~nm} .{ }^{f}$ Static contact angles were taken using goniometry and are an average of five samplings across the sample. The standard deviation of contact angles was less than $1^{\circ}$.

\subsection{Summary and Conclusions}

In this work, for the first time to our knowledge, we introduced a novel straightforward method, which is an easier alternative over multistep approaches commonly used for immobilization of controlling agents onto gold surface in order to prepare brushes. This method is based on chemisorption tendency of raft agent precursor dithiobenzoicacid toward gold. The basic difference of the technique that we improved compared to the ones in literature is the fact that it doesn't require any special reaction or extra treatment for immobilization of raft agents onto gold surface. Moreover, it doesn't even require any raft agent neither on gold nor in solution as free CTA. To the best of our knowledge, this is the easiest technique improved in order to construct polymer brushes on gold surfaces.

DTBA mediated polymerization technique has been sucsessfully used on DTBAchemisorbed gold substrate to prepare well defined homopolymer and diblockcopolymer brushes with different thicknesses and wettability properties. The homopolymer and diblockcopolymer brushes prepared demonstrated the linear growth of film thickness at the early stage of polymerization and the surface coverage controlled by the increase in molecular weight of polymer brushes. Block extension of homopolymer brushes obtained at first step by further confirmed "living character" of brushes obtained via raft agent precursor and the fact that postpolymerization is possible based on this "livingness" being maintained. In addition to that, impact of monomer [M] $\backslash$ raft agent precursor [DTBA] ratio in feed on resulting thickness of polymer brushes was investigated. The presence of free DTBA in reaction mixture, which results in free polymer chains in solution, is essential both in order to estimate molecular weights and to be able to control polymerization. The ellipsometric thicknesses and molecular weights were used to estimate the grafting parameters of chains attached to the gold surface. DTBA mediated polymerization technique can be 
utilized to obtain well-defined polymer and diblockcopolymer brushes with targeted molecular weights and thicknesses as high as $58.7 \mathrm{~nm}$.

To best of our knowledge, for the first time, the advantages of chemisorption property of dithiobenzoicacid toward gold and ability to control polymerization was sucsessfully combined with an attempt to prepare polymer and diblockcopolymer brushes on gold in a controlled manner without utilization of any raft agent. This work is the easiest approach in literature leading to a new avenue for preparation of polymer brushes on gold surfaces. 
8 Characterization of Star Polymer Brushes Prepared on Gold via Novel Z-Raft Star Polymerization Approach

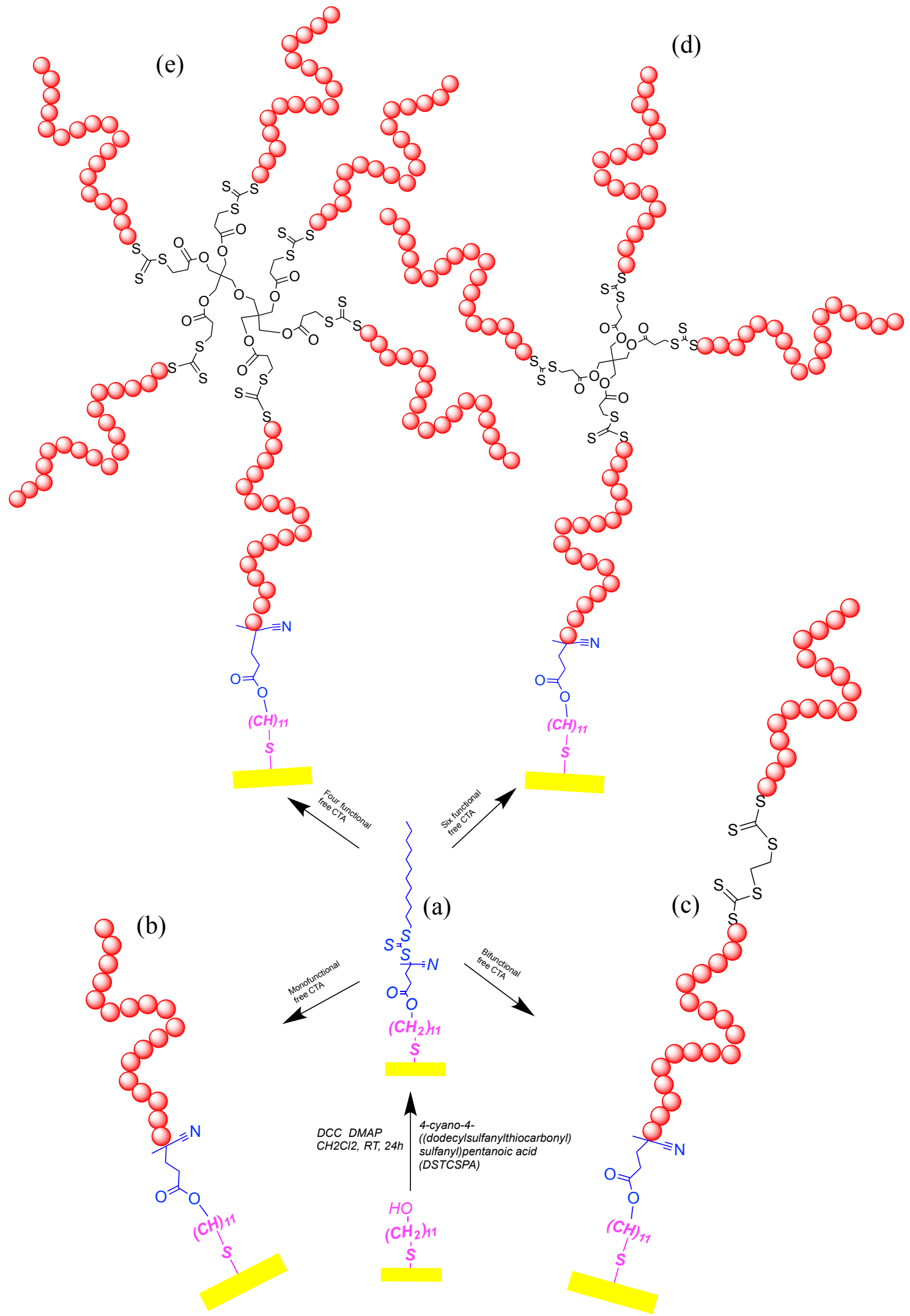


Scheme 4 Schematic diagram illustrating the processes of (a) immobilization of 4Cyano [dodecyl sulfanyl thiocarbonyl) sulfanyl] pentanoicacid (DSTCSPA) and the RAFT-mediated polymerization of PEGMA (b) in the presence of linear free CTA DSTCSPA (4-Cyano[dodecylsulfanylthiocarbonyl)sulfanyl]pentanoicacid) (c) in the presence of bifunctional free CTA EBPEDTTC (ethane-1,2-diyl bis(1phenylethyl)ditrithiocarbonate) (d) in the presence of tetra functional free CTA PETSPETTCP (Penta-erythritol-tetrakis-(3-(S-phenylethyl trithiocarbonate))propanoate) (e) in the presence of hexa functional free CTA DPEHPETTC (Dipentaerythriol-hexakis(3-(S-methoxopropionate-trithiocarbonate))-propanoate) from the gold-MUD-CTA(DSTCSPA) surfaces.

\subsection{Characterization of Self Assembling Monolayer and Raft Agents Immobilized on Gold Surface}

It is known that thiols are highly susceptible to be chemisorped onto gold surface. (19), (225) MUD molecules were immobilized on gold surface by SAM process mentioned in the Experimental section. The resulting MUD thickness was measured by ellipsometry at $1.6 \pm 0.3 \mathrm{~nm}$. The formation of MUD layer on gold was also confirmed by the presence of the peaks at about $2600-2540 \mathrm{~cm}^{-1}$, which are assigned to the aliphatic $\mathrm{CH}_{2}$ stretching vibrations. [Figure 128 (a)] Another evidence for the successful self assembly of MUD on gold is observed as a clear decrease of static contact angle from $61.56^{\circ}$ (bare gold) to $30.53^{\circ}$ (MUD layer on gold). In the second step, CTA was immobilized to $\mathrm{OH}$ functional gold surface via Steiglich esterification reaction. The CTA immobilization was confirmed by the appearance of ester bands at $1734 \mathrm{~cm}^{-1}$ associated with $\mathrm{C}=\mathrm{O}$ stretching vibration and $1259 \mathrm{~cm}^{-1}$ attributed to $\mathrm{C}-\mathrm{O}$ stretching vibrations [Figure 128 (b)]. The $\mathrm{C}=\mathrm{S}$ stretching band at around $1045 \mathrm{~cm}^{-1}$ further confirmed successful covalent coupling of the CTA on the reactive SAM. The thickness of CTA layer was measured by ellipsometry as $1.8 \pm 0.4 \mathrm{~nm}$. Surface morphology and profiles of bare gold, MUD layer and CTA-modified gold substrates are shown in Figure 127 (a), (b), (c) respectively. Root mean square roughness (RMS) and roughness average of the bare gold is 0.6936 and 0.548 respectively whereas that of MUD layer on gold is 0.9021 and 0.6545 . After CTA coupling, these values decreased to 0.3512 and 0.2612 . In addition to that, the sucsessful immobilization of CTA resulted in a change in surface wettability observed by a dramatic increase of static contact angle from $30.53^{\circ}$ (MUD layer on gold) to $73.84^{\circ}$ (CTA layer on gold). 

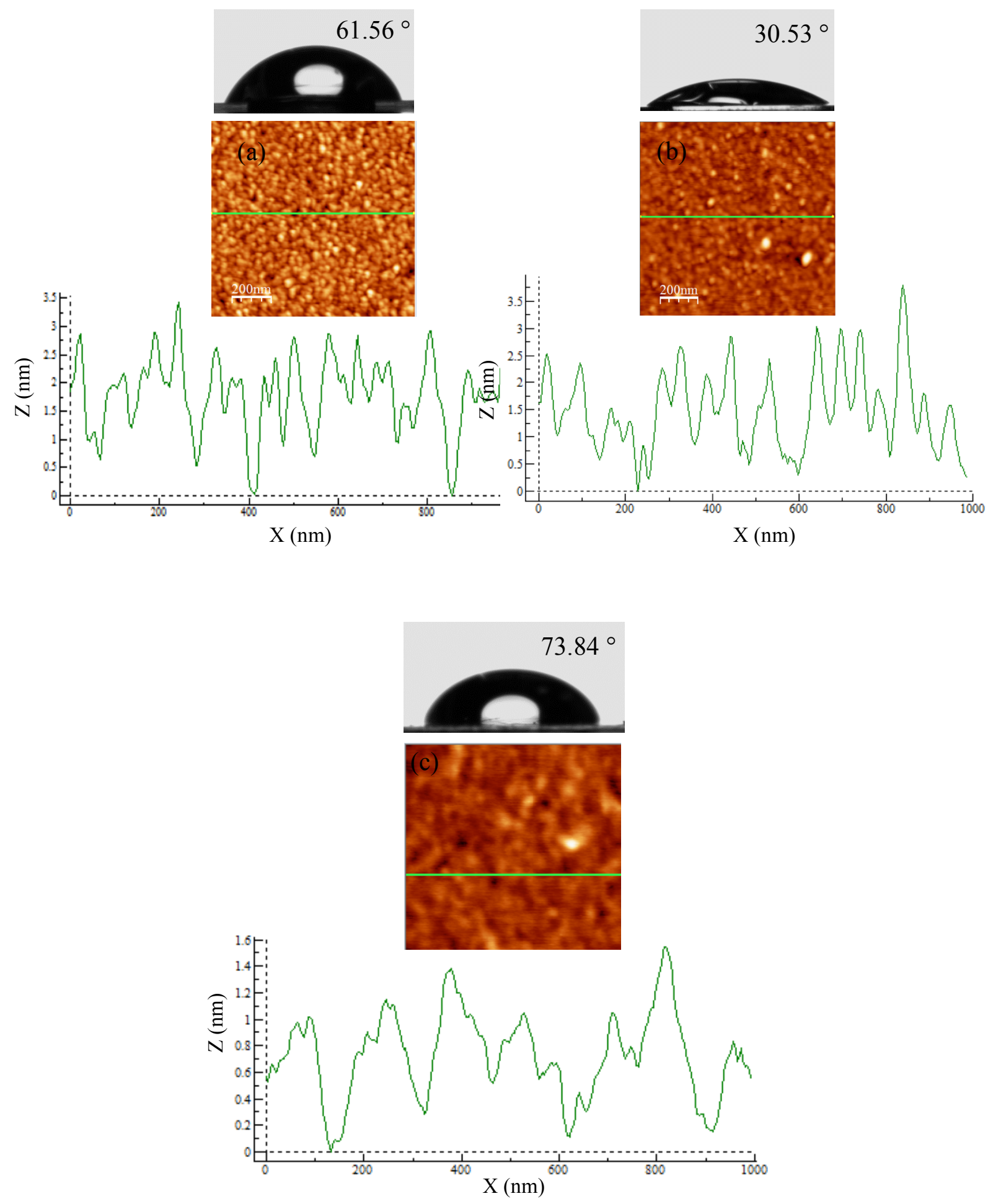

Figure 127 2D AFM images $(1 \mu \mathrm{m} \times 1 \mu \mathrm{m})$ in ambient conditions, surface crosssection analysis and photographs of $1 \mu \mathrm{L}$ water droplets for (a) bare gold (b) goldMUD (c) gold-MUD-linear CTA (DSTCSPA). 


\subsection{Kinetic Investigation of Synthesis of Tetrafunctional Star Poly (PEGMA) Brush from Gold-MUD-CTA Surface}

In order to achieve a high grafting density of the polymer, in the case of homopolymer brush formation, it is important to immobilize CTA on gold surface with a high grafting density. In the case of star polymer formation based on the novel approach including Z-RAFT process combined with $\mathrm{R}$ group approach on surface, it is not required for all of the CTA sites on substrate surface to contribute in the growth of polymer chains to achieve formation of dense layer. Even the CTA sites on substrate have relatively low density, star polymer formation might still result in dense polymer brush layer independent from surface CTA density due to both nature of star polymers and originality of the novel approach developed. This independent nature makes this approach more advantageous over both homopolymer coatings and other star polymer approaches improved against nonspecific protein adsorbtion.

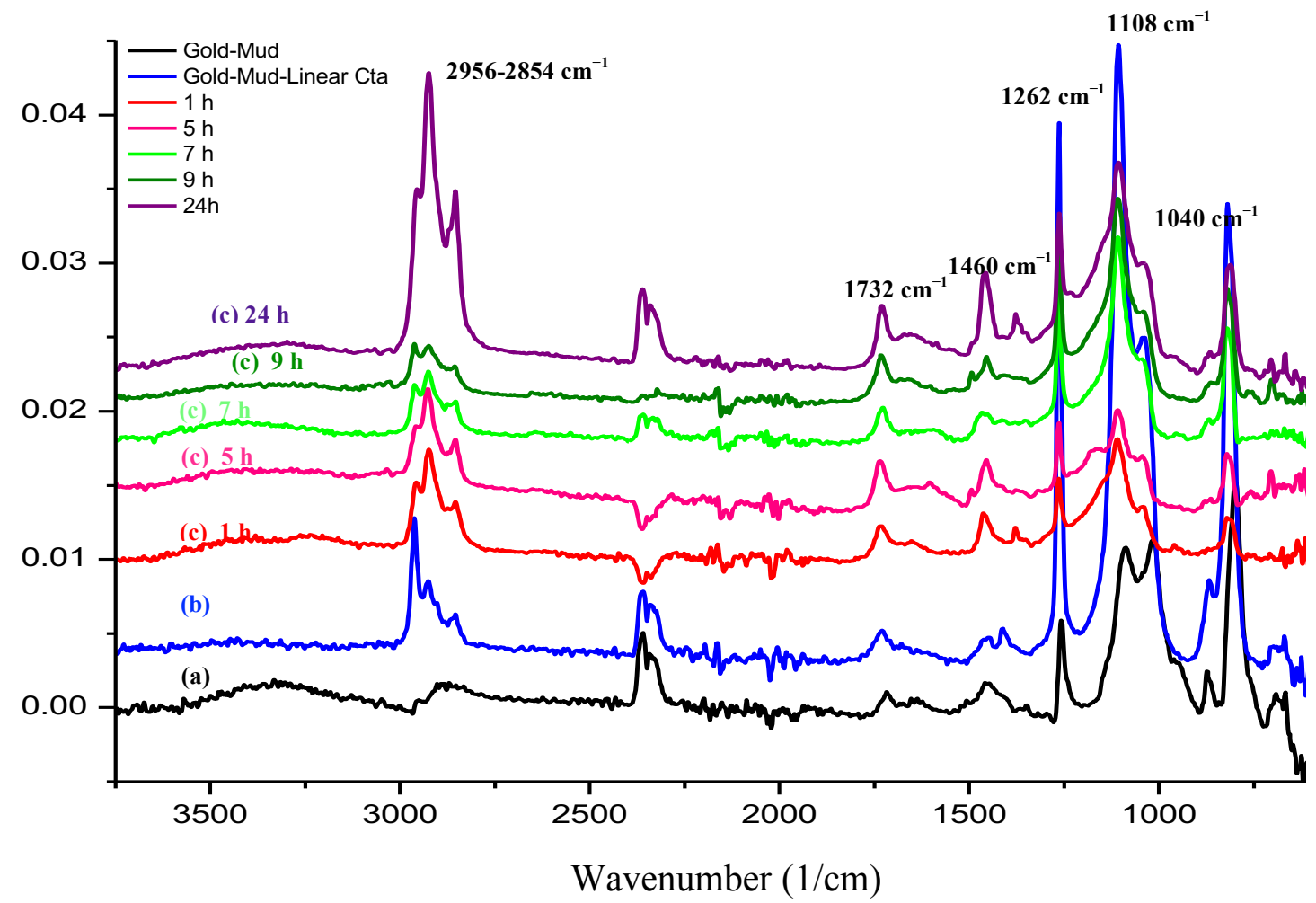

Figure 128 ATR-FTIR spectra of (a) gold-MUD, (b) gold-MUD-CTA (DSTCSPA) and (c) star poly (PEGMA) brush kinetics synthesized for 1, 5, 7, 9, $24 \mathrm{~h}$.

The formation of poly (PEGMA) star brushes was confirmed by ATR-IR [Figure 128 (c)] AFM and water contact angle measurements. (Figure 129) The ATR-FTIR spectra for poly (PEGMA) star brush demonstrated characteristic bands at $\sim 1732 \mathrm{~cm}^{-1}$ associated with the carbonyl stretch, a strong band at $1040 \mathrm{~cm}^{-1}$ resulted from $\mathrm{C}=\mathrm{S}$ stretching vibrations suppressed by absorption bands at $\sim 1108 \mathrm{~cm}^{-1}, 1262 \mathrm{~cm}^{-1}$ associated with $\mathrm{C}-\mathrm{O}$ stretching resulted from vibrations of $\mathrm{C}-\mathrm{O}-\mathrm{C}$ ether bonds in the 
ethylene glycol units. The bands at around 2956, 2924, $2854 \mathrm{~cm}^{-1}$ are considered to be due to aliphatic $\mathrm{CH}$ groups. The $2956,2854 \mathrm{~cm}^{-1}$ peaks are due to asymmetric and symmetric stretching vibrations of $\mathrm{CH}_{3}$, respectively, while the $2924 \mathrm{~cm}^{-1}$ peak is assigned to $\mathrm{CH}_{2}$ vibration. The peaks at $1460 \mathrm{~cm}^{-1}$ and $1379 \mathrm{~cm}^{-1}$ are attributed to the $\mathrm{CH}$ bending and rocking respectively. In addition to these bands, there are bands at $705-620 \mathrm{~cm}^{-1}$ attributed to C-S stretching. The broad band at $3668-3128 \mathrm{~cm}^{-1}$ which is assigned to $\mathrm{O}-\mathrm{H}$ stretching, results from water vapor which PPEGMA star brush coating absorbs due to its hydrophilic nature. Moreover, $\mathrm{C}=\mathrm{C}$ aromatic doublets, which are expected at a value between $1480-1420 \mathrm{~cm}^{-1}$, are observed at $1460 \mathrm{~cm}^{-1}$, being overlapped with $\mathrm{CH}$ bending vibration. The little shoulder visible at around 3128-3018 is a part of the band associated with aromatic $\mathrm{C}-\mathrm{H}$ stretching which is expected to be at around $3100-3000 \mathrm{~cm}^{-1}$. However, it is not clearly observable as a result of being suppressed by broad band of O-H stretching.

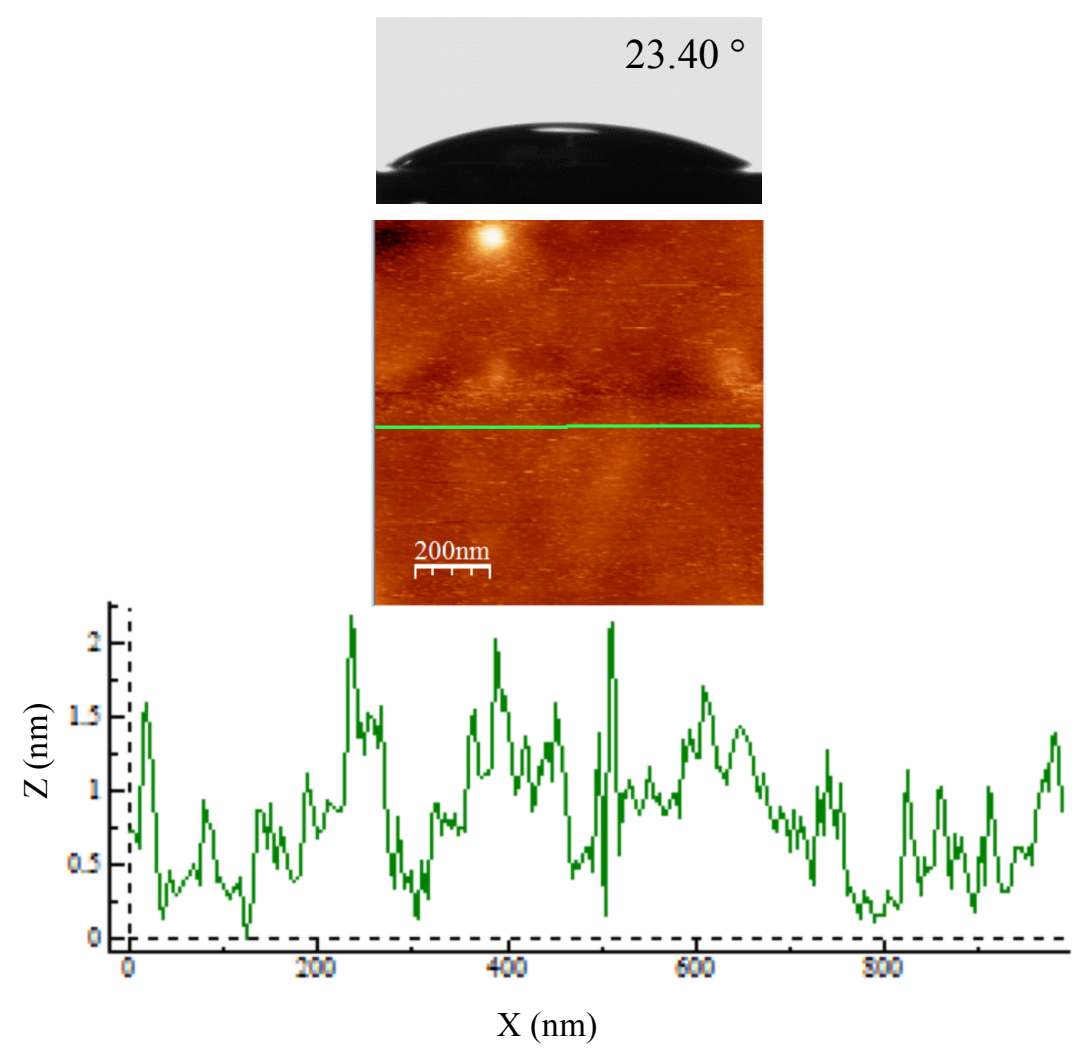

Figure 129 2D AFM image $(1 \mu \mathrm{m} \times 1 \mu \mathrm{m})$ in ambient conditions, surface cross section analysis and photograph of $1 \mu \mathrm{L}$ water droplet for poly (PEGMA) brush synthesized for $24 \mathrm{~h}$ in the presence of tetra functional free CTA (PETSPETTCP).

The time evolution of number-average molecular weight, $M_{n}$ and polydispersity, PDI of the grafted polymer chains on gold, which is assumed to be the same as that of the unbound polymer chains in solution (263), was obtained by GPC analysis (shown in Table 12). Typical GPC traces of free polymers with the increase of polymerization were monomodal and had relatively narrow molecular weight distributions for a star 
polymer, which are characteristics of well-defined polymers prepared via RAFTmediated polymerization. After $24 \mathrm{~h}$, the monomer conversion is $43.5 \%$ and the resulting thickness value is $40 \mathrm{~nm}$. GPC analysis of the free polymers reveals a $M_{n}$ of $82,814 \mathrm{~g} / \mathrm{mol}$ and a PDI of 1.73 (Table 12). Figure 130 (d) presents the evolution of $M_{n}$ of the free star poly (PEGMA) brushes as a function of monomer conversion. The fact that the experimental molecular weights of the free polymers determined by GPC increased linearly with monomer conversion and the PDI values are all below 1.97 indicate that the polymerization was conducted in a controlled manner.

(a)
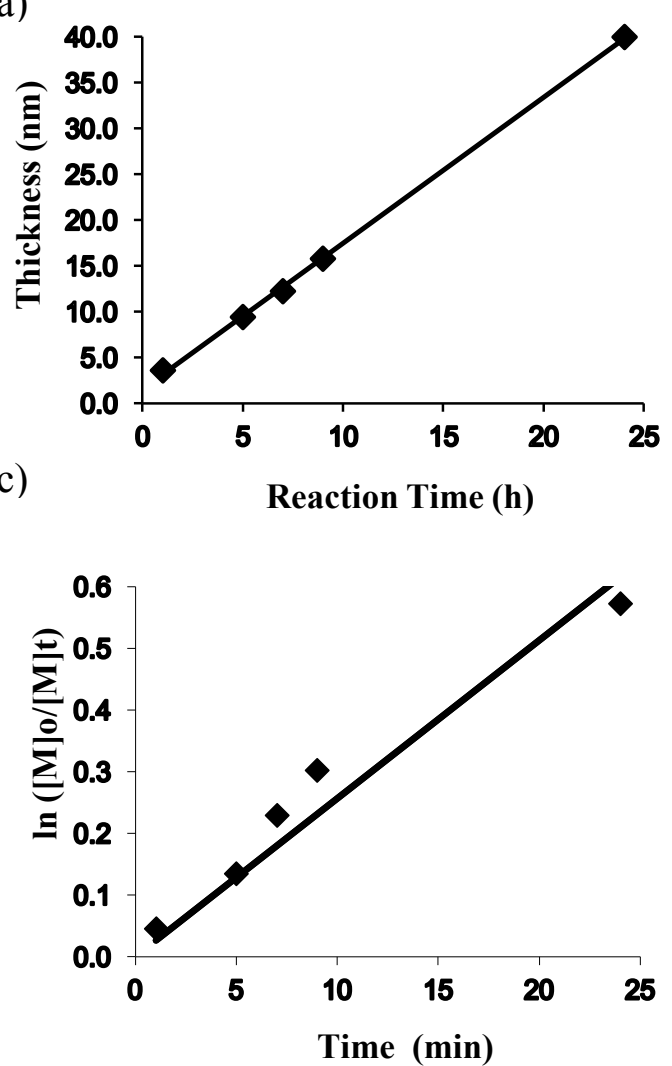

(b)
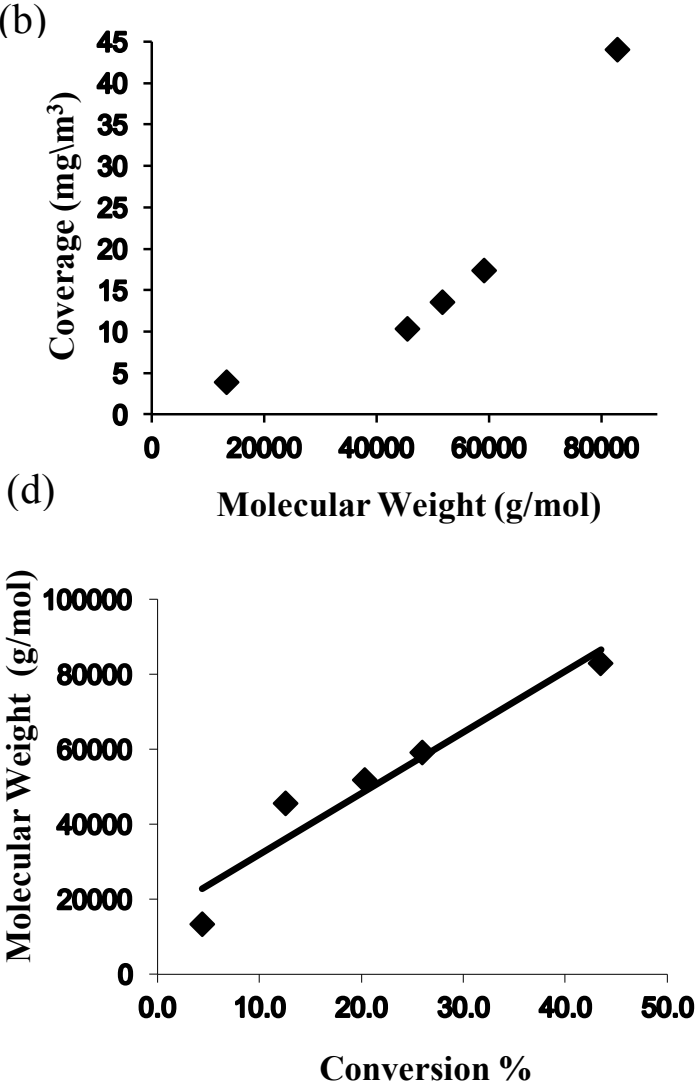

Figure 130 Results of Z-RAFT star polymerization kinetics of PEGMA in Toluen at $60^{\circ} \mathrm{C}$ in the presence of free tetra functional CTA PETSPETTCP (Penta-erythritoltetrakis-(3-(S-phenylethyl trithiocarbonate))-propanoate) a) thickness profiles and kinetics of star poly(PEGMA) brushes, b) the surface coverage plotted against molecular weight of star poly(PEGMA) brushes, c) plot of pseudo-first-order kinetics of star poly(PEGMA) brushes, d) molecular weights of star poly(PEGMA) brushes with inreasing monomer conversion.

Additional evidence of controlled polymerization is also provided by the free star poly (PEGMA) brushes formed from the free star shaped raft agent in solution. Figure 130 (c) shows the linear relationship between $\left.\ln \left[\mathrm{M}_{\mathrm{o}}\right] \backslash \mathrm{M}_{\mathrm{t}}\right]$ and time, where $\left[\mathrm{M}_{\mathrm{o}}\right]$ is the initial monomer concentration and $\left[\mathrm{M}_{\mathrm{t}}\right]$ is the monomer concentration remained in reaction mixture after $t$ hour polymerization time. The result indicates that the concentration of the growing species remains constant and first-order kinetic is 
obtained. The dependence of ellipsometric thickness of poly (PEGMA) star brush grown from the chain transfer agent immobilized surface on polymerization time is shown in Figure 130 (a). It is clearly observable that the star brush thickness increases linearly with polymerization.

Table 12 Summary of polymerization kinetics of star polymer brushes

\begin{tabular}{|c|c|c|c|c|c|c|c|}
\hline $\begin{array}{l}\text { Reaction } \\
\text { Time } \\
\text { (h) }\end{array}$ & $\mathbf{M n} / \mathbf{M w}$ & PDI & $\begin{array}{c}\text { Thickness }^{\text {a }} \\
\text { (nm) }\end{array}$ & $\begin{array}{l}\text { Coverage } \\
(\mathrm{mg} / \\
\left.\mathbf{m}^{2}\right)\end{array}$ & $\begin{array}{l}\text { Grafting } \\
\text { Density } \\
\left(\text { chains } / \mathbf{n m}^{2} \text { ) }\right.\end{array}$ & $\begin{array}{l}\text { Distan } \\
\text { ce } \\
(\mathrm{nm})\end{array}$ & $\begin{array}{l}\text { Static } \\
\text { Contact } \\
\text { Angle }^{b}\end{array}$ \\
\hline 1 & $13341 / 17026$ & 1.27 & 3.6 & 3.89 & 0.17 & 2.7 & $49.22^{\circ}$ \\
\hline 5 & $45465 / 87383$ & 1.92 & 9.4 & 10.15 & 0.13 & 3 & $48.71^{\circ}$ \\
\hline 7 & $51750 / 102350$ & 1.97 & 12.25 & 13.23 & 0.15 & 2.9 & $48.47^{\circ}$ \\
\hline 9 & $59182 / 114350$ & 1.93 & 15.73 & 17 & 0.17 & 2.7 & $42.68^{\circ}$ \\
\hline 24 & $82814 / 143480$ & 1.73 & 40 & 43.2 & 0.31 & 2 & $23.40^{\circ}$ \\
\hline
\end{tabular}

Polymerization of (poly (ethylene glycol) methyl ether methacrylate) PEGMA with tetra functional free CTA PETSPETTCP (Penta-erythritol-tetrakis-(3-(S-phenylethyl trithiocarbonate))-propanoate) and AIBN in toluene at $60{ }^{\circ} \mathrm{C}$ for $24 \mathrm{~h},{ }^{a}$ Thicknesses were determined by ellipsometry and are an average of five samplings across the sample. Error of thicknesses measured was within $\pm 0.5 \mathrm{~nm},{ }^{b}$ Static contact angles were taken using goniometry and are an average of five samplings across the sample. The standard deviation of contact angles was less than $1^{\circ}$.

The surface coverages, A $\left(m g / m^{2}\right)$, grafting densities, $\sigma$ (chains $\left./ \mathrm{nm}^{2}\right)$, and average distances between grafting sites, $D(\mathrm{~nm})$, of grafted star poly (PEGMA) brushes were calculated from the $M_{n}$ of the free polymer and the dry film thickness, $h(\mathrm{~nm})$ according to equations 18, 19, 20 and summarized in Table 12. As a result of that the grafting densities range between 0.13 and 0.31 chains per $\mathrm{nm}^{2}$, star poly(PEGMA) brushes might be considered as a brush type between semi-dilute and a high density brush, which is closer to a high density conformation than semi-dilute. (509)

\subsection{Characterization of Poly (PEGMA) Brushes Synthesized in the Presence of Linear and Multifunctional Raft Agents from Gold-MUD-CTA}

Arm numbers of free CTAs used in solution were increased from one (linear raft agent) to six (hexafunctional star shaped raft agents) in order to investigate impact of functionality of raft agents on resulting thickness of polymer brush synthesized via SIRAFT on gold surfaces. (Scheme 5) 


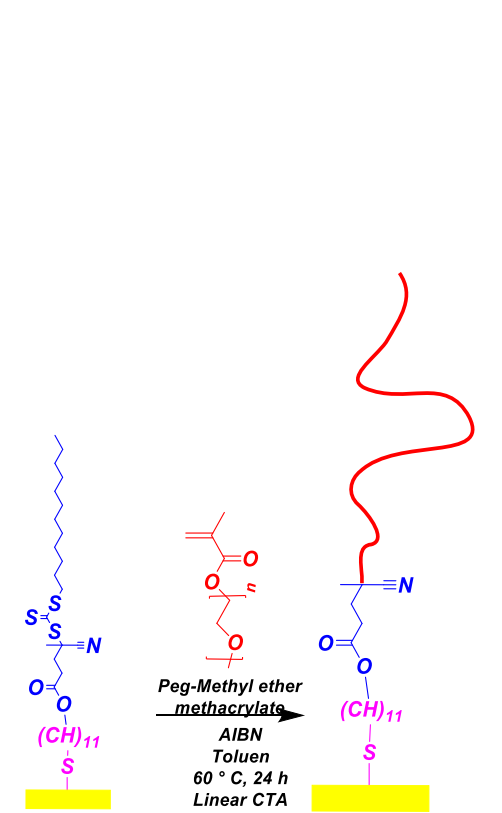

(a)

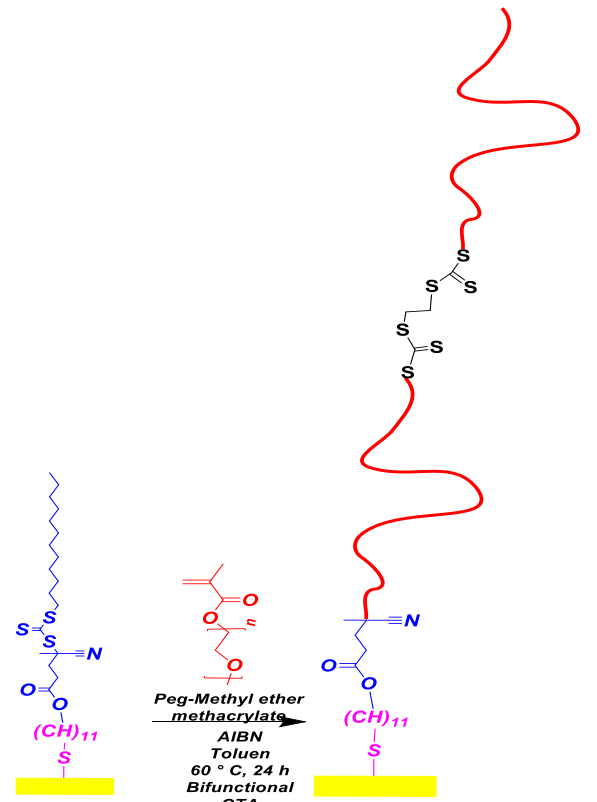

(c)

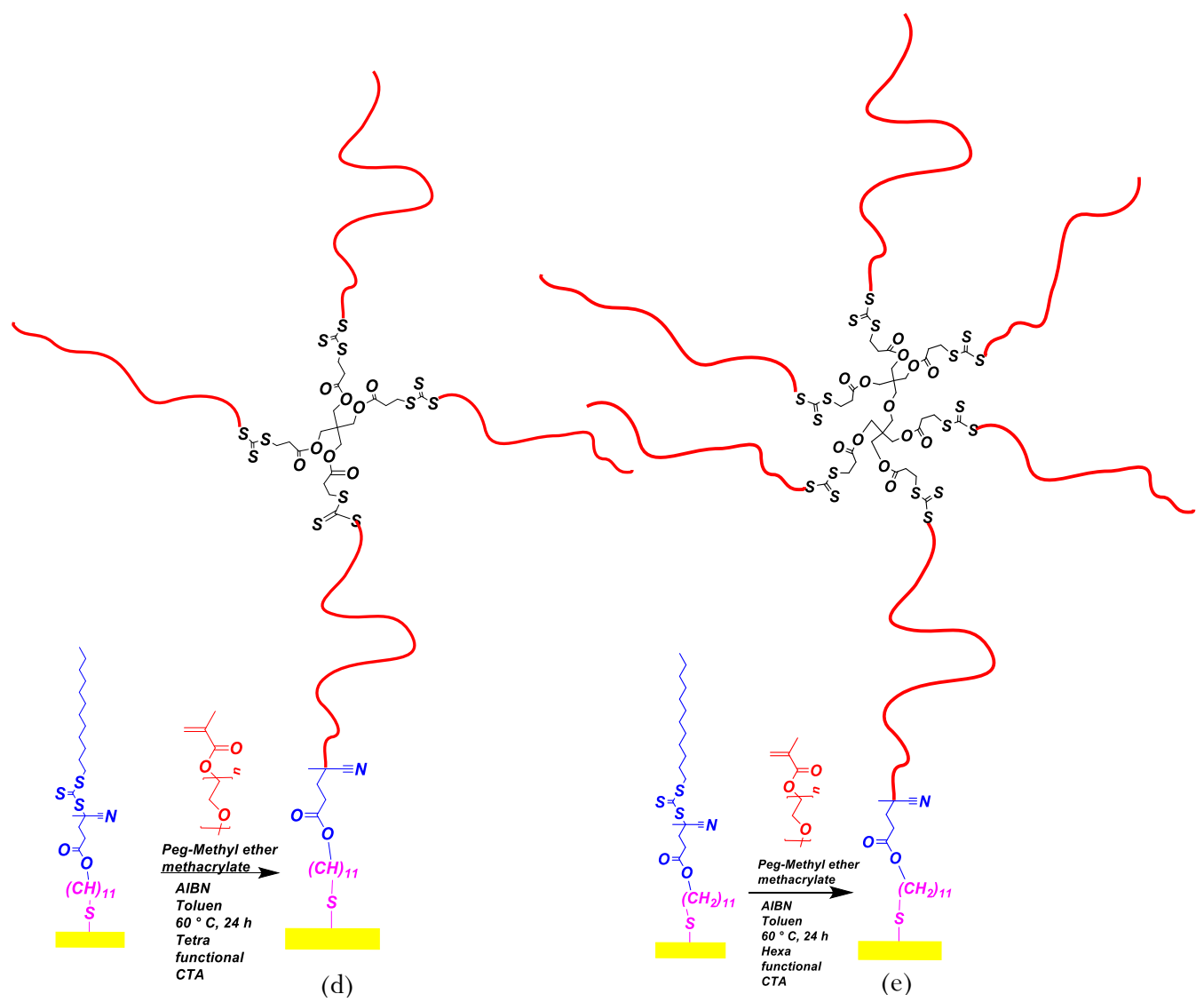

(e)

Scheme 5 Schematic diagram illustrating processes of (a) immobilization of 4Cyano[dodecylsulfanylthiocarbonyl) sulfanyl]pentanoicacid (DSTCSPA) and the RAFT-mediated polymerization of PEGMA (b) in the presence of linear free CTA DSTCSPA (4-Cyano[dodecylsulfanylthiocarbonyl)sulfanyl]pentanoicacid) (c) in the presence of bifunctional free CTA EBPEDTTC (ethane-1,2-diyl bis(1phenylethyl)ditrithiocarbonate) (d) in the presence of tetra functional free CTA PETSPETTCP (Penta-erythritol-tetrakis-(3-(S-phenylethyl trithiocarbonate))- 
propanoate) (e) in the presence of hexa functional free CTA DPEHPETTC (Dipentaerythriol-hexakis(3-(S-methoxopropionate-trithiocarbonate))-propanoate) from the gold-MUD-CTA(DSTCSPA) surfaces.

The growth of poly (PEGMA) brushes from the gold-MUD-linear CTA (DSTCSPA) surface in the presence of monofunctional free CTA (DSTCSPA) [Scheme 5 (a)], bifunctional free CTA (EBPEDTTC) [Scheme 5 (b)], tetra functional free CTA (PETSPETTCP) [Scheme 5 (c)], hexa functional free CTA (DPEHPETTC) [Scheme 5 (d)] was confirmed by ATR-FTIR [Figure 131 (a), (b), (c), (d), (e) ], AFM and water contact angle measurements [Figure 132 (a), (b), (c), (d)] respectively. The ATR-FTIR spectra of star poly (PEGMA) brushes demonstrated characteristic bands at $\sim 1728 \mathrm{~cm}^{-1}$ associated with the carbonyl stretch, a strong band at $1034 \mathrm{~cm}^{-1}$ resulted from $\mathrm{C}=\mathrm{S}$ stretching vibrations suppressed by strong absorption bands at $1104 \mathrm{~cm}^{-1}$ resulted from vibrations of C-O-C ether bonds in the ethylene glycol units. The bands at around $2949,2914,2870 \mathrm{~cm}^{-1}$ are considered to be due to aliphatic $\mathrm{CH}$ groups. The $2949,2870 \mathrm{~cm}^{-1}$ peaks are due to asymmetric and symmetric stretching vibrations of $\mathrm{CH}_{3}$, respectively, while the $2914 \mathrm{~cm}^{-1}$ peak is assigned to $\mathrm{CH}_{2}$ vibration. The peaks at $1455 \mathrm{~cm}^{-1}$ and $1389 \mathrm{~cm}^{-1}$ are attributed to the $\mathrm{CH}$ bending and rocking respectively. In addition to these bands, there is a strong absorption band at $1264 \mathrm{~cm}^{-1}$ associated with $\mathrm{C}-\mathrm{O}$ stretching and bands at $667-616 \mathrm{~cm}^{-1}$ attributed to $\mathrm{C}-\mathrm{S}$ stretching. The broad band at $3676-3049 \mathrm{~cm}^{-1}$ which is assigned to $\mathrm{O}-\mathrm{H}$ stretching, results from water vapor which PPEGMA brush coating absorbs due to its hydrophilic nature. The little shoulder visible at around 3084-3000 is a part of the band associated with aromatic C-H stretching which is expected to be at around $3100-3000 \mathrm{~cm}^{-1}$ but not clearly observable as a result of being suppressed by both aliphatic C-H stretching and broad band of O$\mathrm{H}$ stretching. Also, $\mathrm{C}=\mathrm{C}$ aromatic doublets which are expected at a value between $1480-1420 \mathrm{~cm}^{-1}$, were observed at around $1455 \mathrm{~cm}^{-1}$, being overlapped with $\mathrm{CH}$ bending vibration. Clear decreases in water contact angles from $73.84^{\circ}$ (gold-MUDCTA layer) to $45.42^{\circ}$ [poly(PEGMA) brush synthesized in the presence of free linear CTA (DSTCSPA)], 37 $7^{\circ}$ [poly(PEGMA) brush synthesized in the presence of bifunctional free CTA (EBPEDTTC)], 23.40 ${ }^{\circ}$ poly(PEGMA) brush synthesized in the presence of tetra functional free CTA (PETSPETTCP)], 20.90 [poly(PEGMA) brush synthesized in the presence of hexa functional free CTA (DPEHPETTC)] also confirmed that relatively more hydrophilic polymer layers than CTA immobilized gold surface was obtained. All the areas shown in Figure 132 (a) [poly(PEGMA) brush synthesized in the presence of free linear CTA (DSTCSPA)], Figure 132 (b) [poly(PEGMA) brush synthesized in the presence of bifunctional free CTA (EBPEDTTC)], Figure 132 (c) [poly (PEGMA) brush synthesized in the presence of tetra functional free CTA (PETSPETTCP)], Figure 132 (d) [poly(PEGMA) brush synthesized in the presence of hexa functional free CTA (DPEHPETTC)] display gaussian distribution which is an indicator of surface homogenity. The change in film morphology is associated with a slight increase in the RMS roughness from $0.3512 \mathrm{~nm}$ (gold-MUD-CTA layer) to 0.9202 [Figure 132 (a)], 1.5609 [Figure 132 (b)], 1.059 [

Figure 132 (c)], 0.6317 [Figure 132 (d)] nm (PEGMA brush layers). 


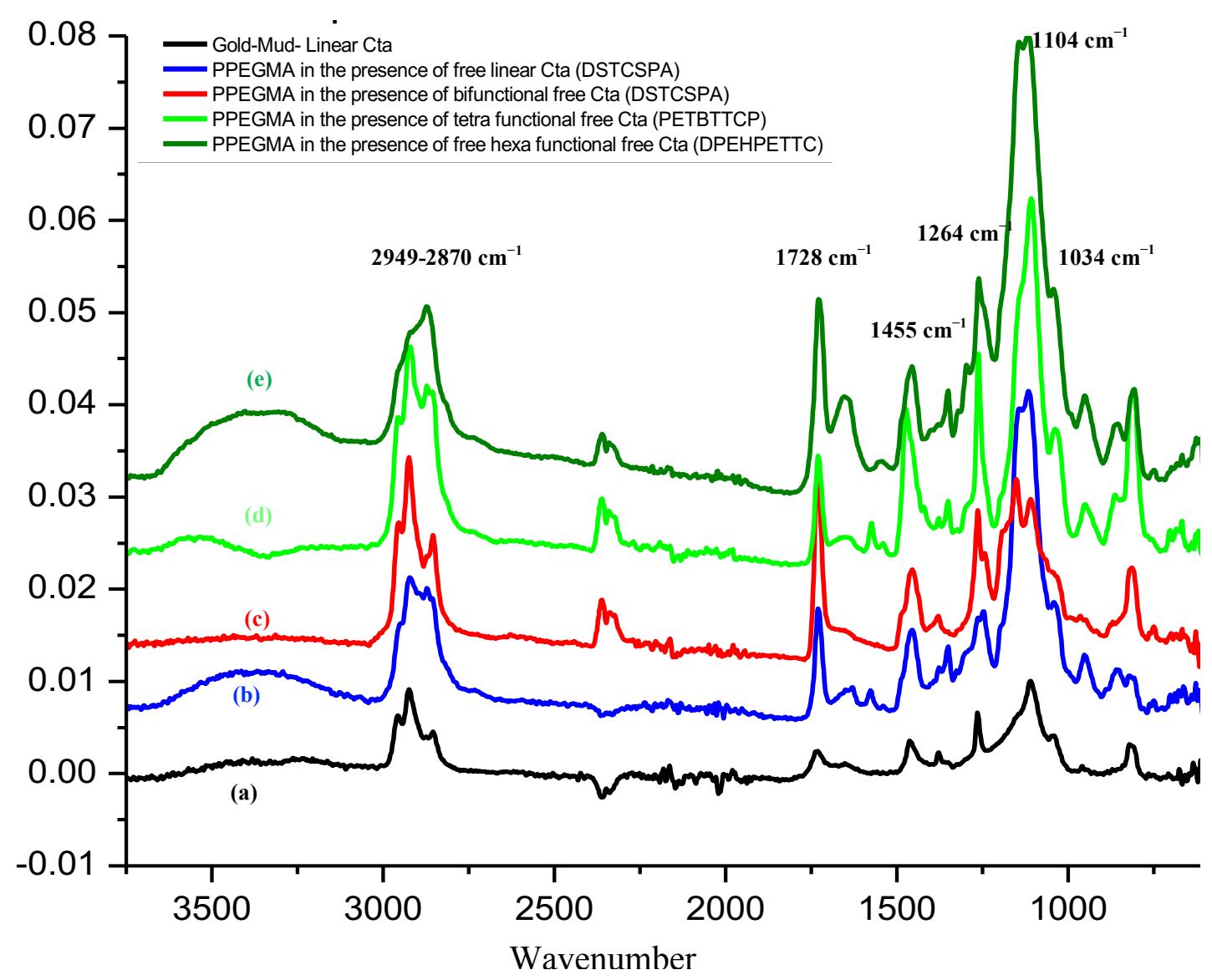

Figure 131 ATR-FTIR spectras of (a) Gold-Mud-Linear CTA (DSTCSPA) and poly (PEGMA) brush synthesized for $24 \mathrm{~h} \mathrm{(b)}$ in the presence of free linear CTA (DSTCSPA) (c) in the presence of bifunctional free CTA (EBPEDTTC) (d) in the presence of tetra functional free CTA (PETSPETTCP) (e) in the presence of hexa functional free CTA (DPEHPETTC).

GPC trace of free polymers were monomodal and had narrow molecular weight distributions, which are characteristics of well-defined polymers prepared via RAFTmediated polymerization. The monomer conversions and resulting thicknesses after 24 $\mathrm{h}$ polymerization are $52.4 \%, 16 \mathrm{~nm}$ (PEGMA synthesized in the presence of linear CTA); $30 \%, 24.3 \mathrm{~nm}$ (PEGMA synthesized in the presence of bifunctional CTA); 43.5 $\%, 40 \mathrm{~nm}$ (PEGMA synthesized in the presence of tetrafunctional CTA); $33 \%, 73 \mathrm{~nm}$ (PEGMA synthesized in the presence of hexafunctional CTA) respectively. (Table 13) GPC analysis of the free polymers reveals a $M_{n}$ of 34050 (PEGMA synthesized in the presence of linear CTA), 50908 (PEGMA synthesized in the presence of bifunctional CTA), 82814 (PEGMA synthesized in the presence of tetrafunctional CTA), 103670 $\mathrm{g} / \mathrm{mol}$ (PEGMA synthesized in the presence of hexafunctional CTA) and a PDI of $1.07,1.77,1.73,1.53$ respectively. (Table 13) The low PDI values indicate that the polymerizations were conducted in a controlled manner. 

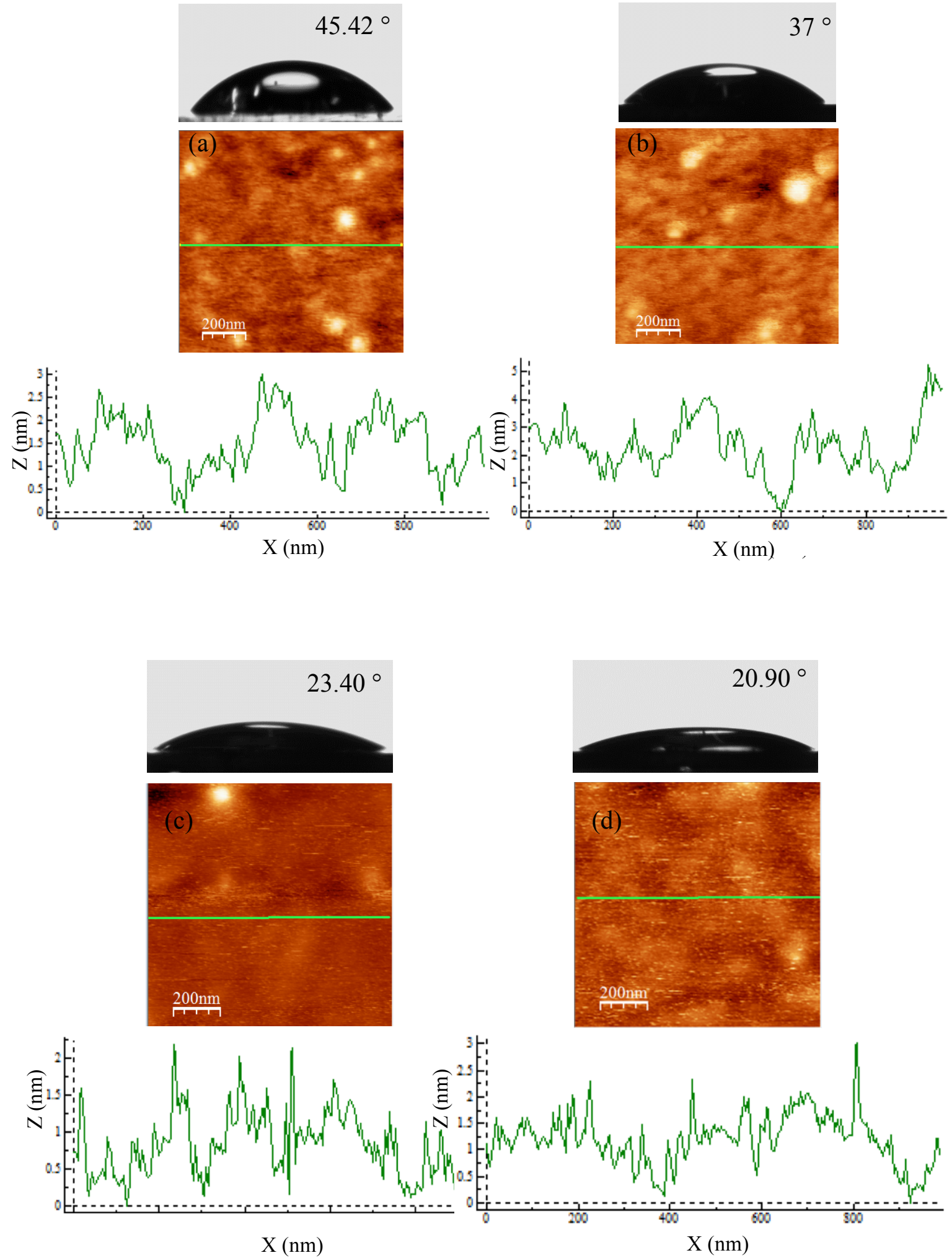

Figure 132 2D AFM images $(1 \mu \mathrm{m} \times 1 \mu \mathrm{m})$ in ambient conditions, surface crosssection analysises, and photographs of $1 \mu \mathrm{L}$ water droplets for poly (PEGMA) brushes synthesized for $24 \mathrm{~h}$ (a) in the presence of free linear CTA (DSTCSPA) (b) in the presence of bifunctional free CTA (EBPEDTTC) (c) in the presence of tetra functional free CTA (PETSPETTCP) (d) in the presence of hexa functional free CTA (DPEHPETTC). 
The surface coverages, A $\left(\mathrm{mg} / \mathrm{m}^{2}\right)$, grafting densities, $\sigma$ (chains $\left./ \mathrm{nm}^{2}\right)$, and average distances between grafting sites, $D(\mathrm{~nm})$, of grafted polymer brushes were calculated from the $M_{n}$ of the free polymer and the dry film thickness, $h(\mathrm{~nm})$, according to equations 18, 19, 20 and summarized in Table 13. As a result of that the grafting densities are between 0.30 and 0.46 chains per $\mathrm{nm}^{2}$, poly (PEGMA) brushes might be considered as a brush type between semi-dilute and a high density brush, which is closer to a high density conformation than semi-dilute. (509)

Table 13 Summary of polymerization of 2, 4,6 Arm star polymer brushes

\begin{tabular}{|l|l|l|l|l|l|l|}
\hline & & & & & & \\
\hline
\end{tabular}

Polymerization of (poly(ethylene glycol) methyl ether methacrylate) PEGMA ${ }^{\text {a }}$ with monofunctional free CTA 4-Cyano [dodecylsulfanylthiocarbonyl) sulfanyl] pentanoicacid (DSTCSPA), ${ }^{b}$ with bifunctional free CTA ethane-1,2-diyl bis(1phenylethyl) ditrithiocarbonate (EBPEDTTC), ${ }^{\mathrm{c}}$ with tetra functional free CTA Pentaerythritol-tetrakis-(3-(S-phenylethyl trithiocarbonate))-propanoate (PETSPETTCP), ${ }^{\mathrm{d}}$ with hexa functional free CTA Dipentaerythriol-hexakis (3-(S-methoxopropionatetrithiocarbonate))-propanoate (DPEHPETTC) and AIBN in toluene at $60{ }^{\circ} \mathrm{C}$ for $24 \mathrm{~h}$ from gold-mud-linear CTA (DSTCSPA) surfaces, ${ }^{\mathrm{e}}$ Thicknesses were determined by Ellipsometry and are an average of five samplings across the sample. Error of thicknesses measured was within $\pm 1 \mathrm{~nm},{ }^{f}$ Static contact angles were taken using goniometry and are an average of five samplings across the sample. The standard deviation of contact angles was less than $1^{\circ}$.

\subsection{Investigation of Protein Resistivity of Star Poly (PEGMA) Brushes Prepared on Gold Surface}

Fouling from solutions of main blood plasma proteins (Human Serum Albumin, Fibrinogen) and Insulin on PEGMA brushes of various thicknesses and architectures was investigated using Spectroscopic Ellipsometry and HPLC-Mass spectrometry. Bare gold, Mercaptoundecanol (MUD) immobilized gold and Dodecanthiol (DDT) 
tethered gold surfaces were chosen as model SAMs in order to check and compare protein resistive properties of surfaces modified with different type of coatings. DDT confined gold surface was expected to lead to high protein fouling as a result of its hydrophobic nature, whereas the one covered with MUD was expected to reduce the fouling.

PEGMA brushes grown from gold surfaces were incubated in a solution of Insulin in Methanol and solutions of HSA and Fbg in PBS $\left(1 \mathrm{mg} \mathrm{mL}^{-1}\right)$ respectively. Two hours later, gold samples were taken out of protein solutions and placed into empty vials. They were rinsed one time with $5 \mathrm{ml}$ Methanol (for I) or Milli Q water (for HSA and Fbg) and dried under a stream of Argon. After one time rinsing step, thickness of proteins foulded on all gold samples were measured via Ellipsometry. Ellipsometric thicknesses of adsorbed proteins confirmed that protein resistive character of surfaces becomes more pronounced with the increasing number of arms of star shaped free CTAs used in solution phase. According to these results, DDT confined gold surfaces has $16.5 \mathrm{~nm}$ thick protein layer while the maximum thickness of protein layer foulded on gold surface coated with PEGMA brush is $2.28 \mathrm{~nm}$. (Table 14) All polymer brush types (homopolymer, bi-, tetra and hexa functional star polymers) displayed similar resistance to proteins. The spectroscopic Ellipsometry results confirmed successfull prevention of protein fouling on PEGMA star brush coatings.

In order to double check the ellipsometric results, the amount of proteins adsorbed on surfaces was investigated via HPLC-Mass measurements as well. Proteins foulded on gold samples were desorbed via two times more rinsing and cleaning steps $(5 \mathrm{ml}$ $\mathrm{MeOH}$ or Water). These vials full with Methanol and Milli-Q water cleaning solutions containing related protein residues, each of which belongs to a different gold sample, were gathered in an individual vial for every protein type used for each gold sample. Each of these vials with full of a protein type which belongs to related gold sample, were kept under the fume hood so that all solvents were evaporated. After evaporation of solvents, each of these protein residues which belong to different substrate types were dissolved with $5 \mathrm{ml}$ of Methanol (for I) or water (for HSA and Fbg).

Insulin solutions were used for quantitative analysis and measured via HPLC-Mass spectrometry in order to compare integral areas of base peaks (retention time at around 5.8-6.6 $\mathrm{min}$ ), which are correlated to the concentration of related proteins. The larger the integral area under the base peak the higher the concentration of protein in solution which correspondes to the amount of protein desorbed from the surface via cleaning, which had been previously foulded on surface during protein adsorbtion studies. According to quantitative analysis of these protein solutions, the amount of protein foulded on gold is the least for all PPEGMA brush types (homopolymer, bi-, tetra and hexa functional star polymers), whereas it is the most for Gold-DDT sample (4.13 E8). There was no peak observed at the drift time of Insulin at HPLC-Mass spectrums of gold surfaces coated with PPEGMA brushes. The lack of signal indicated the presence of the neglicible amount of Insulin residue in cleaning solution $(5 \mathrm{ml})$, which 
corresponded to the amount of desorbed proteins from brush coated gold surfaces. This finding confirmed successfull prevention of protein fouling on PEGMA star brush coatings as a result of the fact that the amount of desorbed proteins should have been equal to the amount of the adsorbed proteins.

Table 14 Summary of Protein Adsorbtion Studies of homo poly (PEGMA), 2, 4 and 6 Armed Star Polymer Brushes

\begin{tabular}{|c|c|c|c|c|}
\hline \multirow[t]{2}{*}{$\begin{array}{l}\text { Polymer brush type } \\
\text { on gold }\end{array}$} & \multirow{2}{*}{$\begin{array}{l}\text { Integral } \\
\text { area of } \\
\text { base peak } \\
\text { of Insulin }{ }^{\text {a }} \\
\qquad \text { I }^{\mathrm{c}}\end{array}$} & \multicolumn{3}{|c|}{$\begin{array}{l}\text { Thickness }(\mathrm{nm}) \text { of adsorbed protein } \\
\text { layer on related gold substrate }{ }^{\mathrm{b}}\end{array}$} \\
\hline & & I & $\mathrm{HSA}^{\mathrm{d}}$ & $\mathrm{Fbg}^{\mathrm{e}}$ \\
\hline Bare Substrate & $9.82 \mathrm{E} 7$ & 7.2 & 6.53 & 12 \\
\hline Gold-DDT (Dodecanthiol) & $4.13 \mathrm{E} 8$ & 10.6 & 10.5 & 16.5 \\
\hline $\begin{array}{l}\text { Gold-MUD } \\
\text { undecanol) }\end{array}$ & $3.0 \quad$ E7 & 5.5 & 5.06 & 11.37 \\
\hline Homo (PEGMA) on gold & - & 2 & 0.95 & 2.28 \\
\hline $\begin{array}{l}2^{*} \text { Arm PEGMA Polymer } \\
\text { brush }\end{array}$ & - & 0.73 & 0.38 & 1.26 \\
\hline $\begin{array}{l}\text { 4* Arm PEGMA STAR } \\
\text { Polymer brush }\end{array}$ & - & 0.23 & 0.28 & 1 \\
\hline $\begin{array}{l}\text { 6* Arm PEGMA STAR } \\
\text { Polymer brush }\end{array}$ & - & 0.1 & 0.1 & 0.83 \\
\hline
\end{tabular}

${ }^{a}$ Quantitative analysis of the proteins foulded on gold substrates covered with different type coatings was investigated via HPLC-Mass spectrometry. Integral area of the base peak in the HPLC-Mass spectrum of related protein which belongs to related substrate was calculated. ${ }^{\mathrm{b}}$ Thickness analysis of the proteins foulded on gold substrates covered with different type coatings via Spectroscopic Ellipsometry. Thickness values are an average of five samplings across the sample. Error of thicknesses measured was within $\pm 0.1 \mathrm{~nm}{ }^{\mathrm{c}}$ Insulin ${ }^{\mathrm{d}}$ Human Serum Albumin ${ }^{\mathrm{e}}$ Fibrinogen.

\subsection{Summary and Conclusions}

In this work, a novel method for formation of star polymer brushes on gold surfaces was introduced. To the best of our knowledge, this is the easiest technique improved 
in order to construct star polymer brushes on gold surfaces. Z-Raft star polymerization has been sucsessfully conducted in the presence of a free star shaped raft agent in solution phase by utilization of surface tethered controlling agents (R group approach) in order to prepare well defined star polymer brushes with different thicknesses on gold. The star polymer brushes demonstrated the surface coverage controlled by the increase in molecular weight of polymer brushes. The presence of star shaped free CTAs in reaction mixture, which results in free star polymer chains in solution, is essential in order to form star polymers on gold surfaces and to estimate molecular weights. The ellipsometric thicknesses and molecular weights were used to estimate the grafting parameters of chains attached to the gold surface.

Z-RAFT star polymerization technique was sucsessfully utilized to obtain welldefined star polymer brushes of up to $73 \mathrm{~nm}$ thick. Moreover, poly (PEGMA) star polymer brushes were exposured to proteins such as HSA, Fibronectin and Insulin with an attempt to check protein resistivity properties of brushes. Star polymer brushes demonstrated excellent resistance to protein fouling. To best of our knowledge, for the first time, the advantages of raft polymerization such as lack of any toxic catalyst and novel approach improved based on the exchange mechanism between star shaped free CTAs and surface tethered monofunctional CTAs were sucsessfully combined in order to construct star polymer brushes as protein resistive coatings. This work is a new avenue for preparation of antifouling coatings which is extremely important for biomedical applications. 


\section{General Conclusion}

In the frame of this thesis work, novel Raft based synthetic routes were developed and optimized to prepare multi-functional polymeric coatings with complex topologies for specific biomedical applications. Traditional approaches used for preparation of polymer brushes and novel strategies developed in this work were compared in Table $\mathbf{1 5}$ in terms of advantages and drawbacks.

Furthermore, a novel Z-Raft based approach was introduced to prepare surface confined star polymer brush which reduces non-specific protein adsorption which is considered to be one of the major causes for the failure of various blood-contacting implantable devices. It was revealed that the beneficial features of Pegma star polymer surfaces, whose generation requires complex procedures, could effectively be retained by using much easier approach, which is a new avenue to prepare anti-fouling coatings that have extreme significance for biomedical applications. In face of the limited number of work that has addressed RAFT polymerization from solid surfaces so far, this thesis exploited the capacity of RAFT for generating novel organic/inorganic composite materials, which cannot easily be produced by alternative methods. In combination with the Z-RAFT star polymerizations, a multitude of new and unexplored surface-confined polymer materials are thus envisaged and strongly encourage to direct further fundamental and applied research effort into this fascinating field, which is still in its infancy.

Table 15 Comparement of Raft based approaches used for synthesis of polymer brushes 


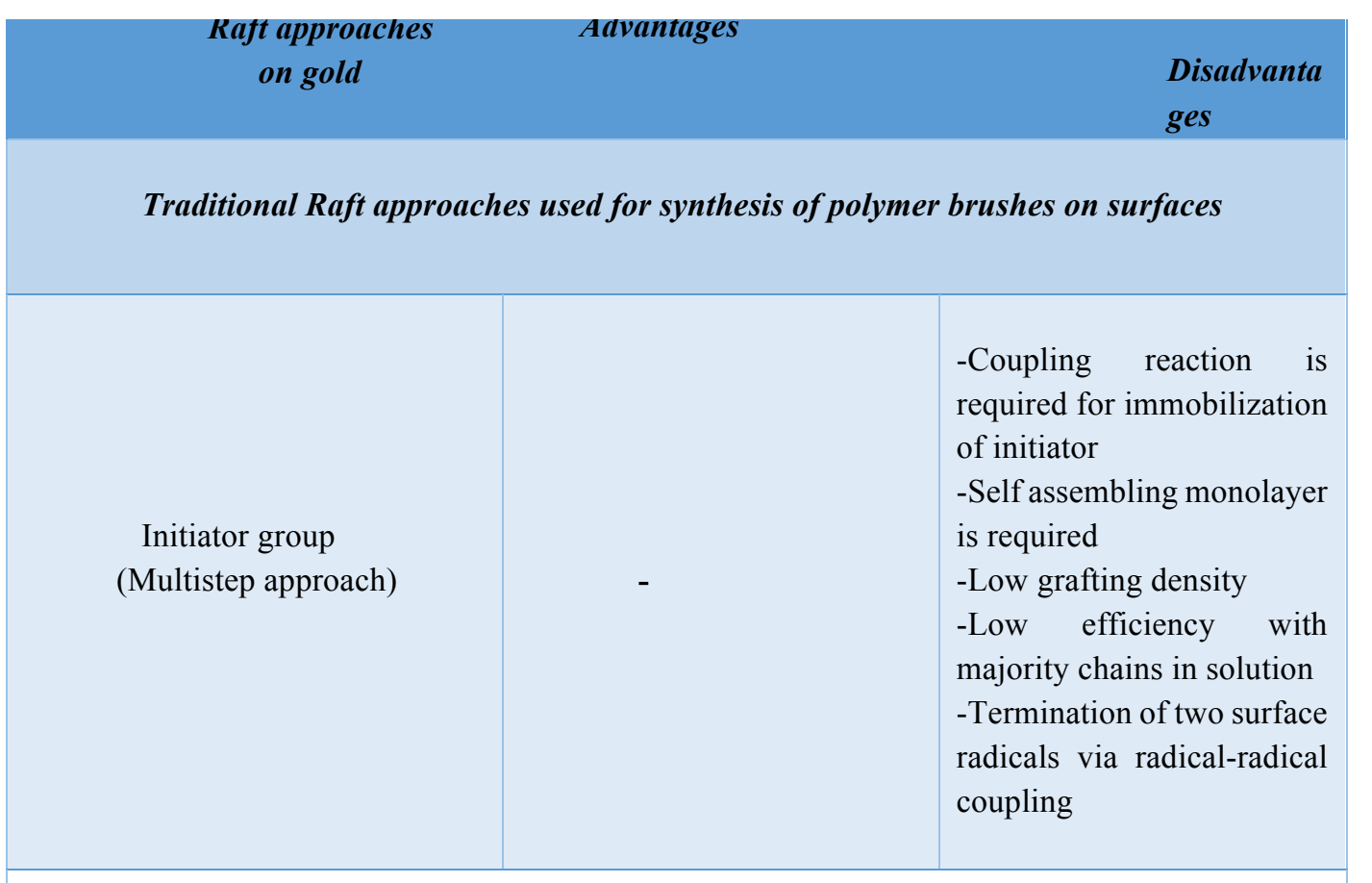

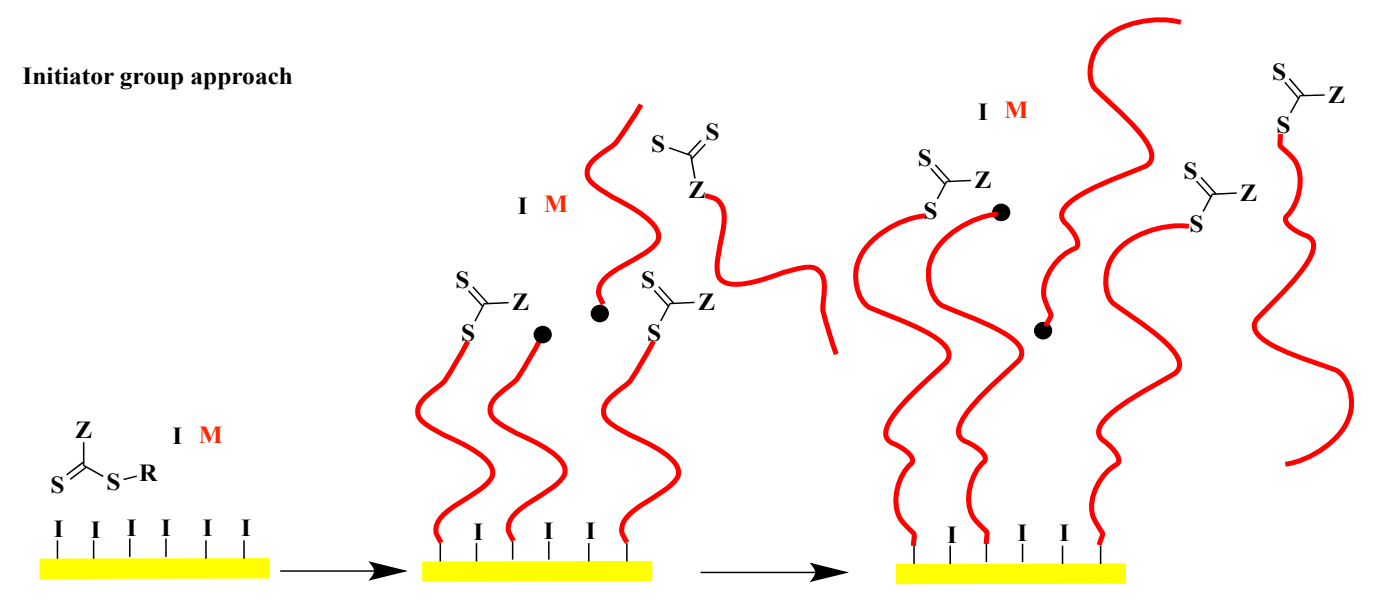

Not applied in this work

Multistep approach)
(Mroup




\section{Summary}

1) Introduce of a commercial raft agent through its $R$ group onto gold surface via traditional multistep approach

2) Investigation of polymerization kinetics

3) Variation of monomers for different applications

4) Variation of architecture (e.g. homo, block)

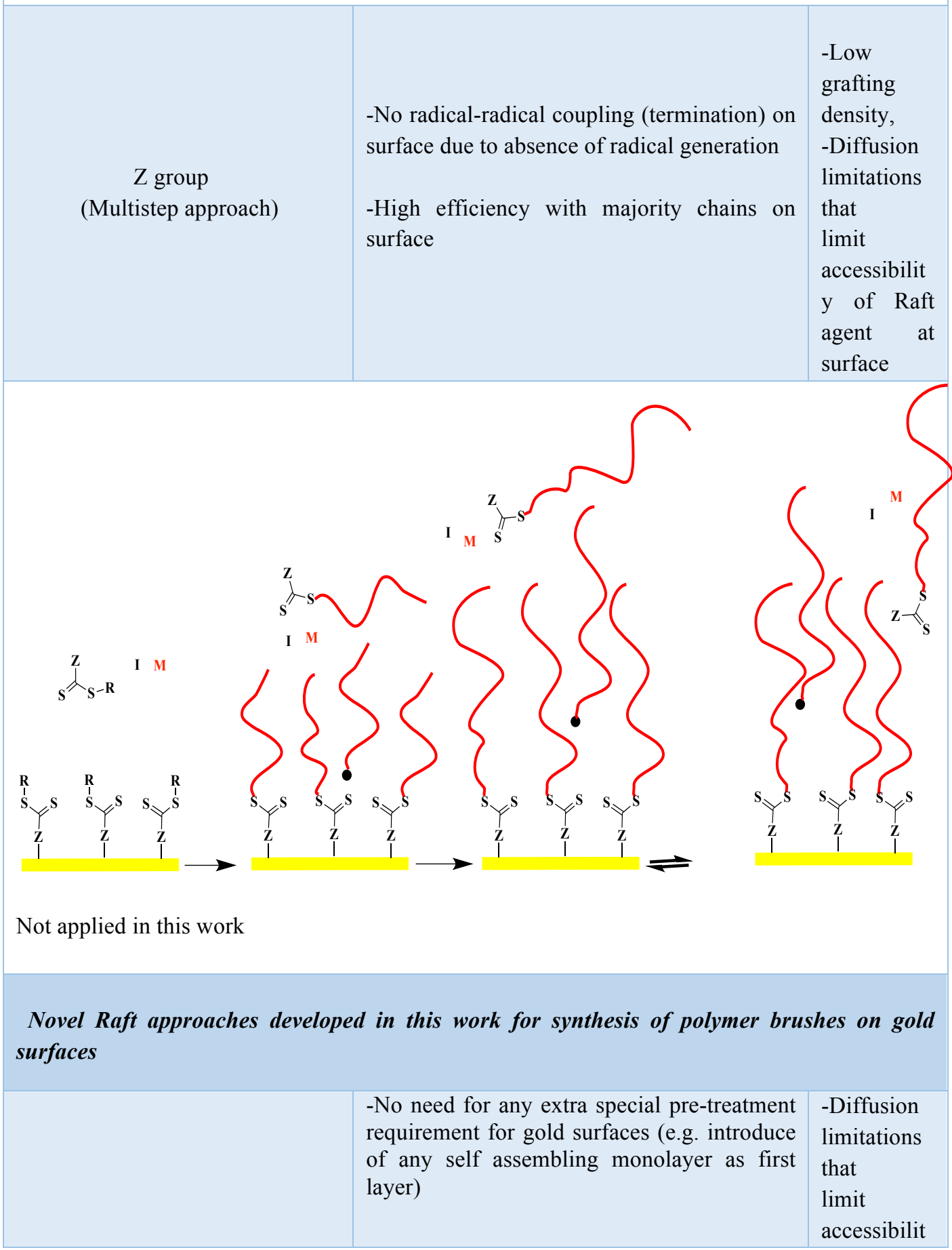


Direct attachment of linear and raft agents via chemisorption (Straight forward approach)
-No obligation of diligent design, synthesis and utilization of terminally functionalized Raft agent, which is mandatory (in traditional approaches) for successful immobilization of chain transfer agents onto surfaces and thus formation of dense polymer brushes

-No need for utilization of any catalysts or chemical reaction in order to couple raft agent with the end functionality of self assembling monolayer on surface

-No need for any specific conditions/ optimization of process conditions to attach raft agents

-The possibility to introduce any desired commercial raft agent onto gold without limitation of "appropriate terminal functionality"

-This versatility of raft agents used as both surface confined CTA and free CTA in reaction mixtures, enables polymerization of various monomer types on gold surfaces and thereby opens up opportunities to alter physicochemical characteristics of gold surface easily and thus enhance breadth of application areas of gold surface.

-No radical-radical termination

-High grafting density achievable (in the range of $0.6-0.85$ )

-High thickness achievable (in the range of $80-166 \mathrm{~nm}$ ) $y$ of Raft agent at

surface

-The requiremen $\mathrm{t}$ for design and synthesis of multifuncti onal Raft agents

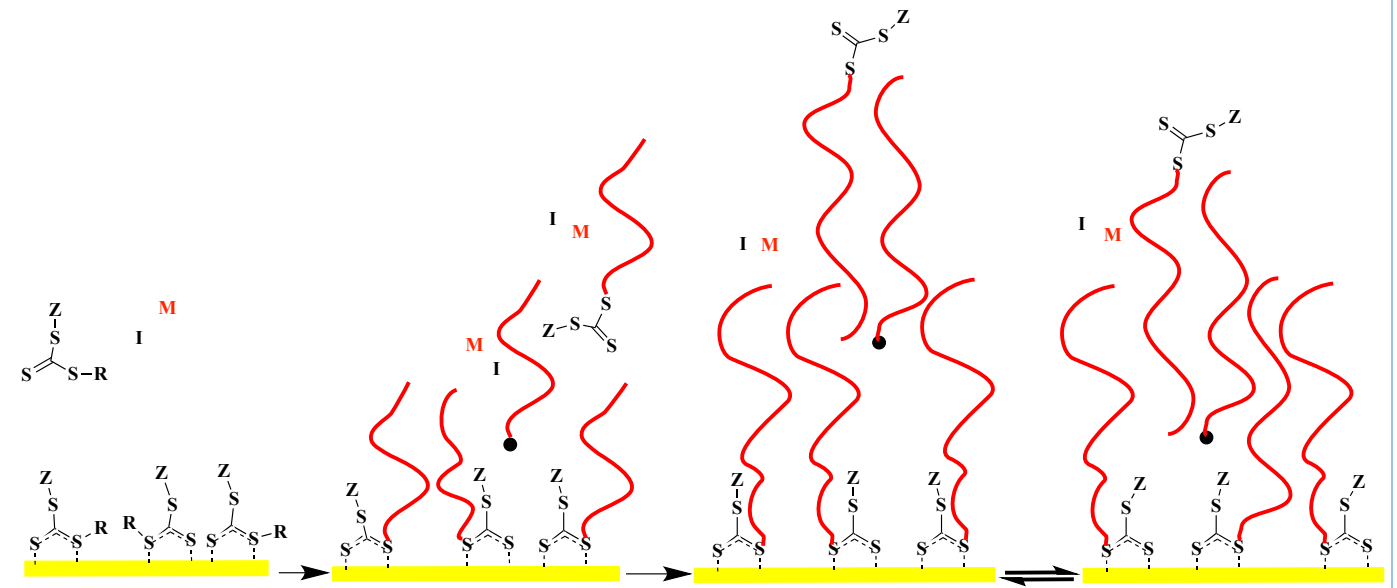




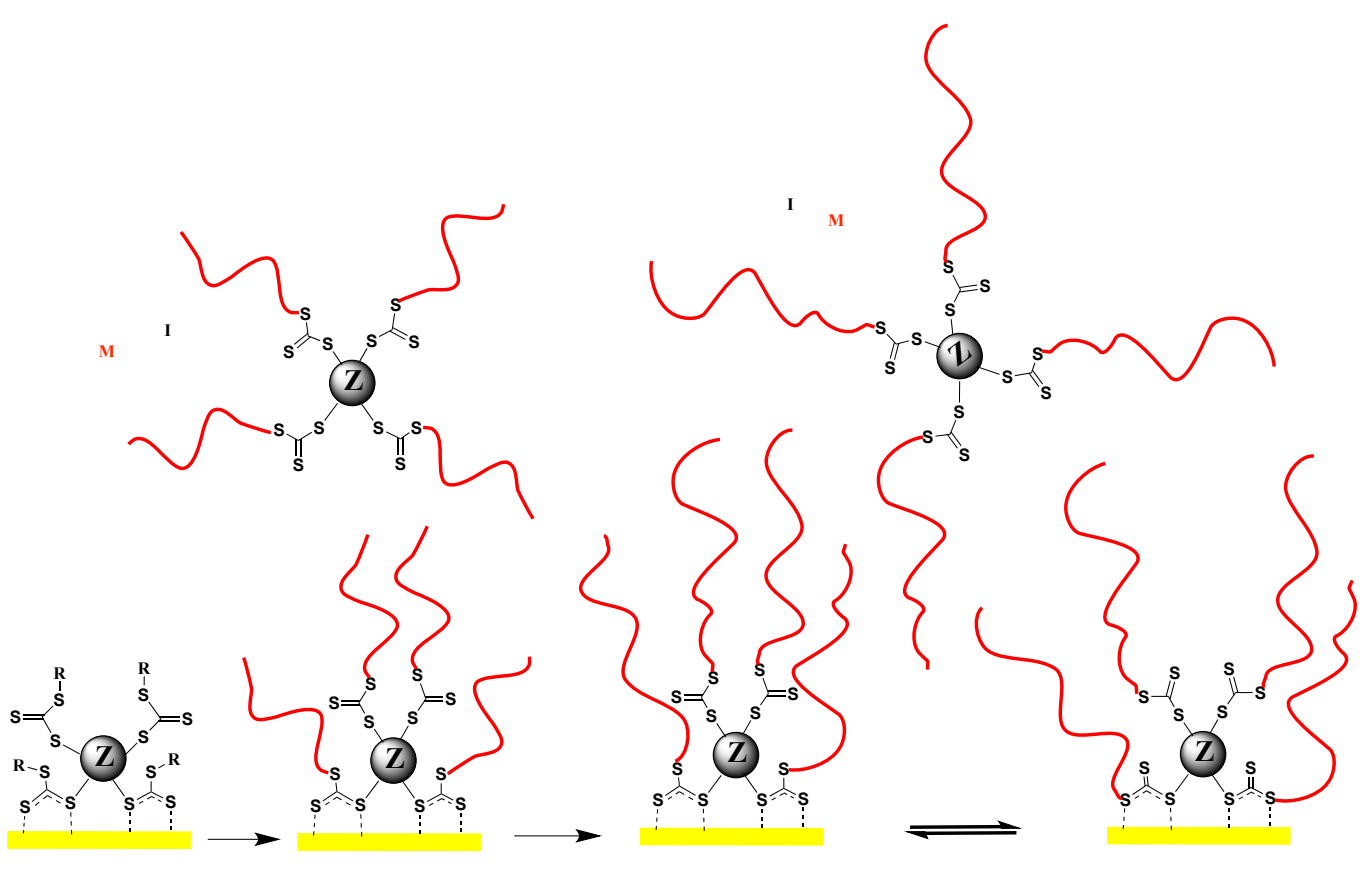

\section{Summary}

A new one step approach was represented for synthesis of polymer brush, which is based on chemisorption of raft agent through its sulfur groups and which is similar-comparable to $\mathrm{Z}$ group approach.This chemisorption is represented as an alternative to conventional $\mathrm{Z}$ group approach, which offers higher grafting density than traditional $\mathrm{Z}$ group approach.

1) Introduce of various linear and multifunctional raft agents onto gold surface

2) Investigation of polymerization kinetics

3) Variation of monomer for different applications

4) Variation of architecture (e.g. homo, block)

Direct attachment of DTBA via chemisorption (Straight forward approach)
- No radical-radical termination

-No need for any extra special pre-treatment requirement for gold surfaces (e.g. introduce of any self assembling monolayer as first layer)

-No obligation of diligent design, synthesis and utilization of terminally functionalized Raft agent, which is mandatory (in traditional approaches) for successful immobilization of chain transfer agents onto surfaces and thus formation of dense polymer brushes

-No need for utilization of any raft agents
-Diffusion limitations that limit accessibilit $y$ of Raft agent at surface 


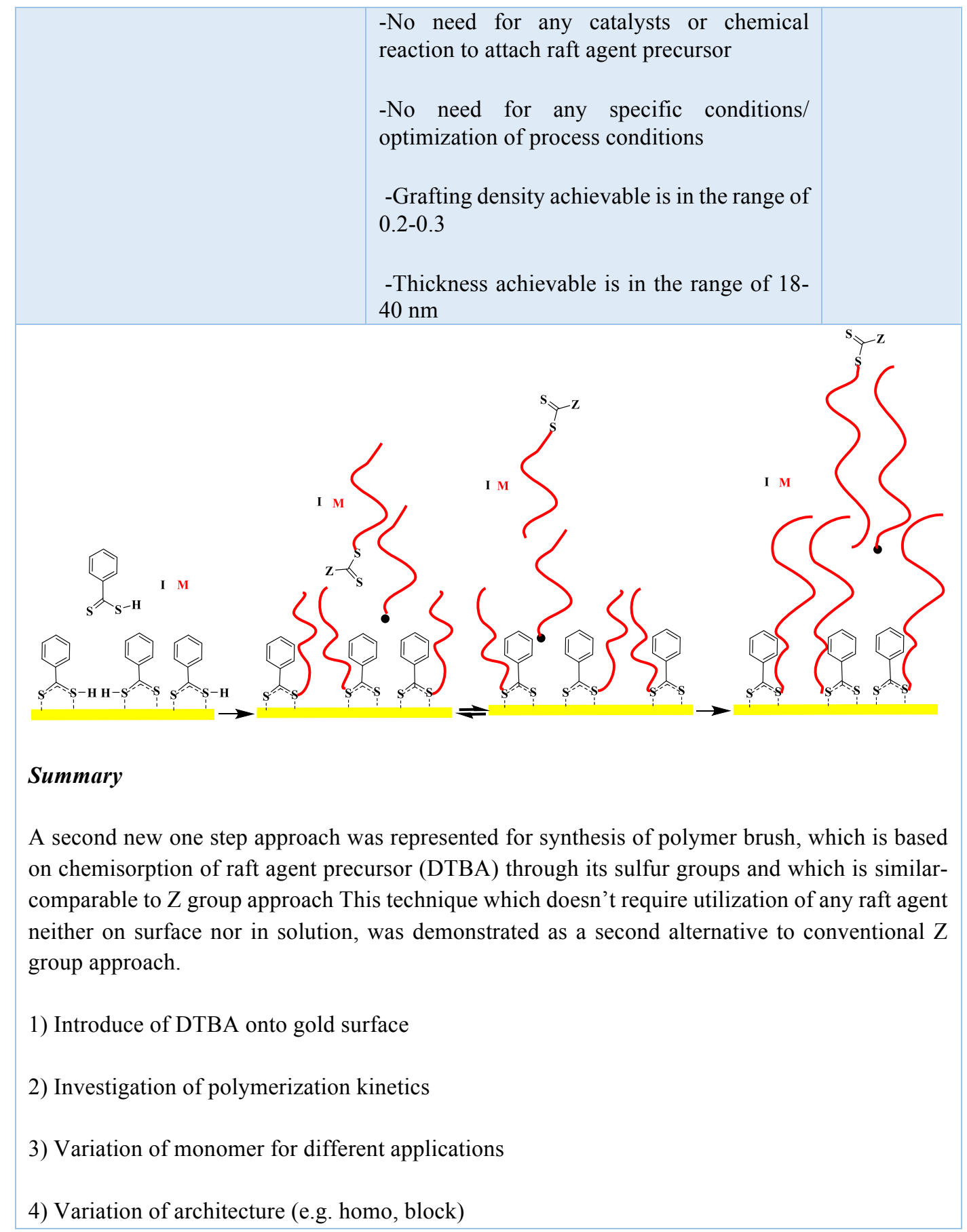




\section{Z-Raft star approach}

(A novel approach which requires less steps in comparision to the traditional procedures used for preparation of star polymers)
-High grafting density achievable (in the range of $0.3-0.5)$

-High thickness achievable (in the range of 40-70 nm)
-A suitable coupling reaction is required for anchoring the linear raft agent on to gold surface

-Self assembling monolayer is required as first layer

-Termination of two surface radicals via radical-radical coupling

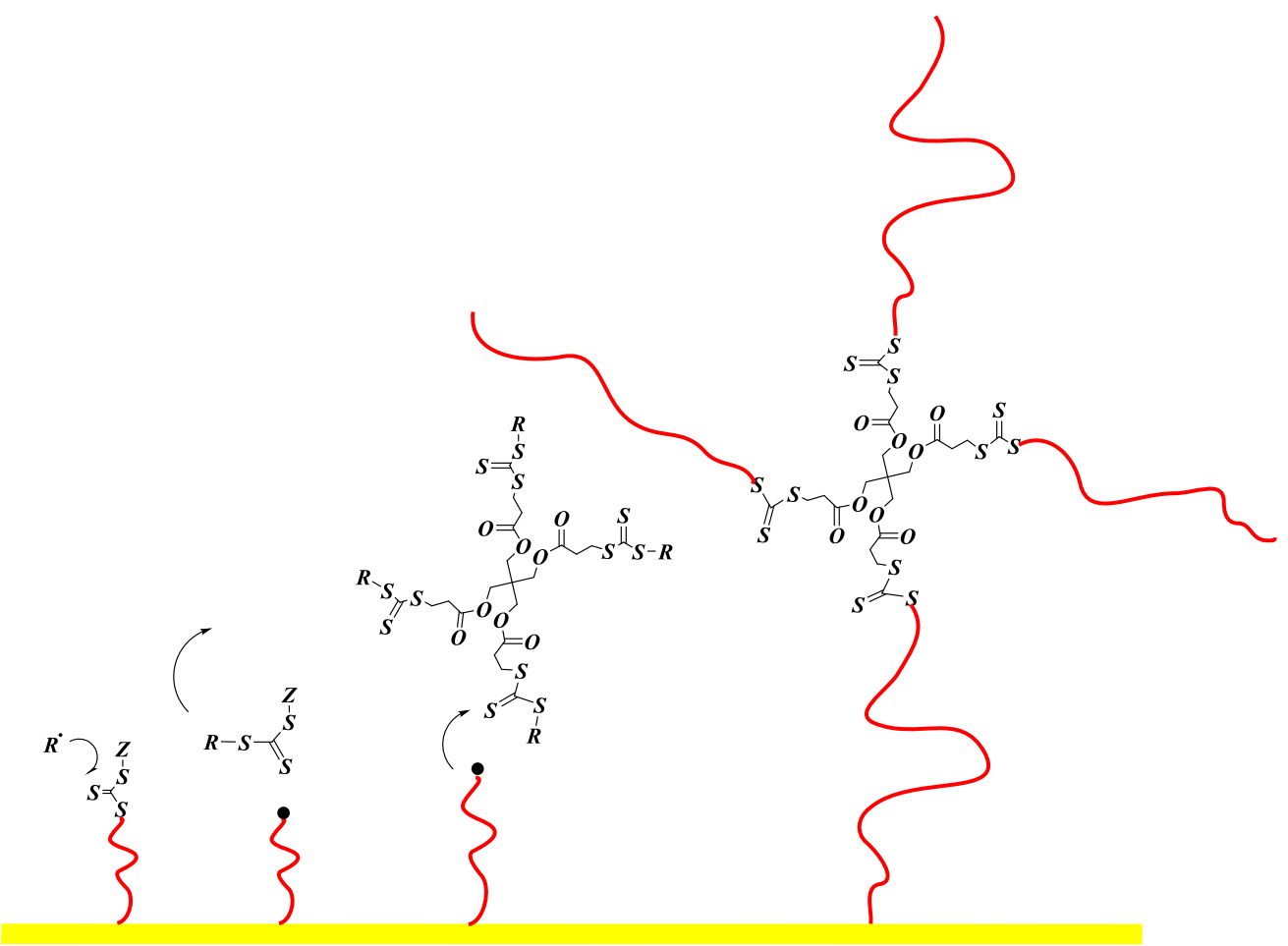

\section{Summary}

1) Introduce of a commercial raft agent through its $R$ group onto gold surface via traditional multistep approach

2) Investigation of star polymerization kinetics (free tetra functional CTA)

3) Variation of free CTAs from linear to hexa functional CTAs 


\section{Experimental Part}

In this part, the experimental setup characterization techniques and evaluation procedures used to analyze the synthesized brushes followed for this thesis are described. Characterization methods of synthesized materials include: gel permeation chromatography (GPC), attenuated total reflectance fourier transform infrared spectroscopy (ATR-FTIR), atomic force microscopy (AFM), contact angle (CA) measurements, high pressure liquid chromatography (HPLC) mass spectrometry and spectroscopic ellipsometry (SE).

\subsection{Chemicals and Equipments}

\subsubsection{Materials}

2,2'-azobis(isobutyronitrile) (AIBN, Akzo Nobel, 98\%) were recrystallized twice from methanol and dried under high vacuum before use. The inhibitors in Butyl methacrylate (BUMA (Mw=142.2 g/mol), 99\%, Sigma Aldrich), 2- (Dimethylamino) ethyl methacrylate (DMAEMA (Mw $=157.21 \mathrm{~g} / \mathrm{mol}), 98 \%$, Sigma Aldrich), Poly(ethyleneglycol)methyl ether methacrylate (PEGMA (Mw=500 g/mol), Sigma Aldrich), Methyl methacrylate (MMA (Mw=100.12 g/mol), 99\%, Sigma Aldrich) were removed by inhibitor remover (Replacement packing for removing hydroquinone and monomethylether hydroquinone, Sigma Aldrich). The purified monomers were then stored under an argon atmosphere at $-20{ }^{\circ} \mathrm{C}$. 4-Cyano-4[dodecylsulfanylthiocarbonyl)sulfanyl]pentanoicacid (DSTCSPA) (SigmaAldrich,97\%), 2-phenyl-2-propylbenzodithioate (CDB) (Sigma-Aldrich,99\%), dichloromethane (anhydrous, Sigma-Aldrich, 99.8\%), 4-(dimethylamino)pyridine (DMAP, Sigma-Aldrich, 99\%), N,N`-Dicyclohexylcarbodiimide (DCC, SigmaAldrich, 99\%), 11-Mercapto-1-undecanol (MUD, 97\%, Sigma Aldrich), Ethanol (Absolute, $96 \%$, VWR Chemicals), Tetrahydrofuran (THF, $99.9 \%$, Sigma Aldrich), Methanol (99.9 \%, VWR Chemicals), Hexan (99 \%, Fluka), Toluen (99.9 \%, Sigma Aldrich), Insulin solution from Bovine Pancreas (10 mg $\backslash \mathrm{ml}$ in $25 \mathrm{mM}$ HEPES, Sigma Aldrich), Fibrinogen from Human Plasma (50-70 \% protein, powder, $100 \mathrm{mg}$, Sigma Aldrich), Albumin from Human Serum (lyophilized powder, $\geq 97 \%$, Sigma Aldrich), Phosphate Buffer Solution (PBS) (pH 7.4, Sigma Aldrich) were used as received. Star shaped raft agents including Ethane-1,2-diylbis(1-phenylethyl) ditrithiocarbonate (EBPEDTTC), Penta - erythritol - tetrakis - (3- (S- phenyl ethyl trithiocarbonate))propanoate (PETSPETTCP), Dipentaerythriol- hexakis (3- (S- methoxo propionate trithio carbonate))-propanoate (DPEHSMPETTC) and Dithiobenzoicacid (DTBA) were synthesized according to literature. (510) Throughout all experiments, ultrapure water (resistivity $18.2 \mathrm{M} \Omega \mathrm{cm}$ at $25{ }^{\circ} \mathrm{C}$, total organic carbon $<5 \mathrm{ppb}$ ) from a Millipore water purification system was used. Gold-coated silicon wafers $(99.999 \% \mathrm{Au}$, layer thickness $1000 \AA$ with a titanium adhesion layer) were provided by Sigma-Aldrich. To remove the organic residues on the surface, the gold substrates were washed with 
"piranha" solution, a mixture of 98 wt \% concentrated sulfuric acid (75 vol \%) and hydrogen peroxide (25 vol \%). Caution: Piranha solution reacts violently with organic materials and should be handled carefully! After rinsing with copious amounts of deionized water, the gold flats were blown dry with purified argon.

\subsubsection{Instruments}

\subsubsection{Gel Permeation Chromatography (GPC)}

\subsection{Working Principle and Data Analysis}

Gel Permeation Chromatography (GPC), also known as Size Exclusion Chromatography (SEC), is used for characterizing molecular weights of polymers (Figure 133). GPC is a chromatographic technique that utilizes columns to separate polymers on the basis of size, in another words, hydrodynamic volume (radius of gyration) of the analytes.

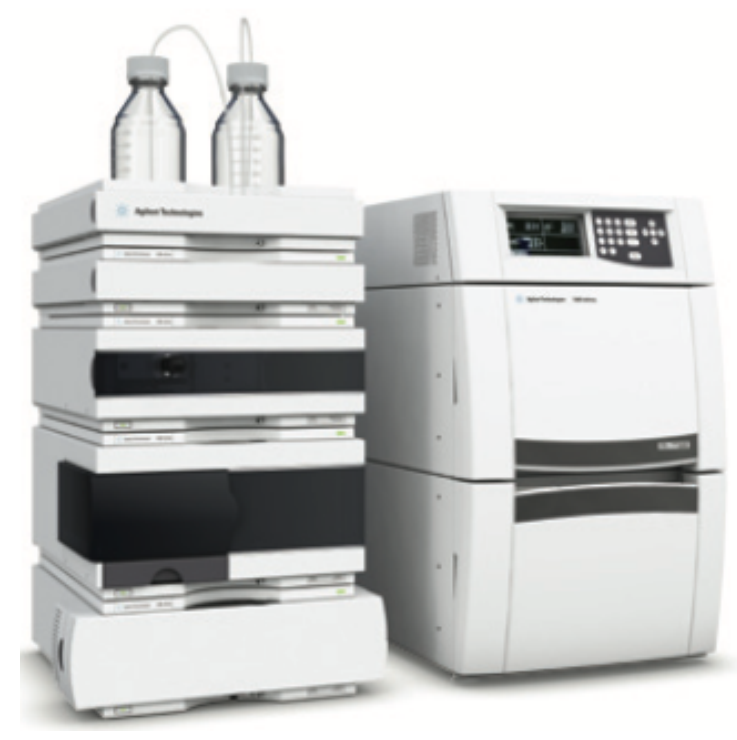

Figure 133 A typical GPC instrument including A. Autosampler, B.Column C.Pump, D.RI Detector, E.UV-vis Detector. (511)

Separation occurs via a column or a set of columns which is composed of porous particles. As the polymer travels through the column, the smaller molecules can penetrate into the pores more easily, therefore spend more time in these pores, increasing their retention time and elute at a relatively later elution volume (Figure 134). Conversely, the molecules that are too large to penetrate into the pores of the column elute first and come out of columns earlier according to BOCOF's law (Big Ones Come Out First). (Figure 135) 

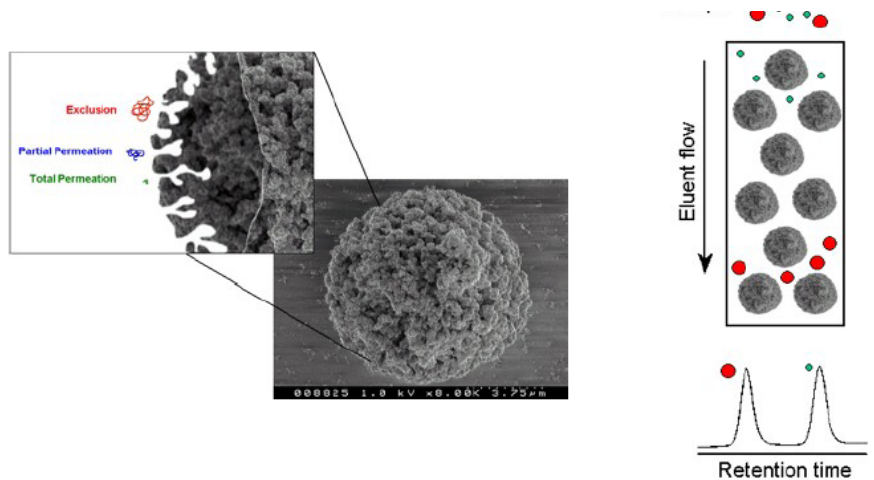

Figure 134 Seperation based upon size in solution. (57)

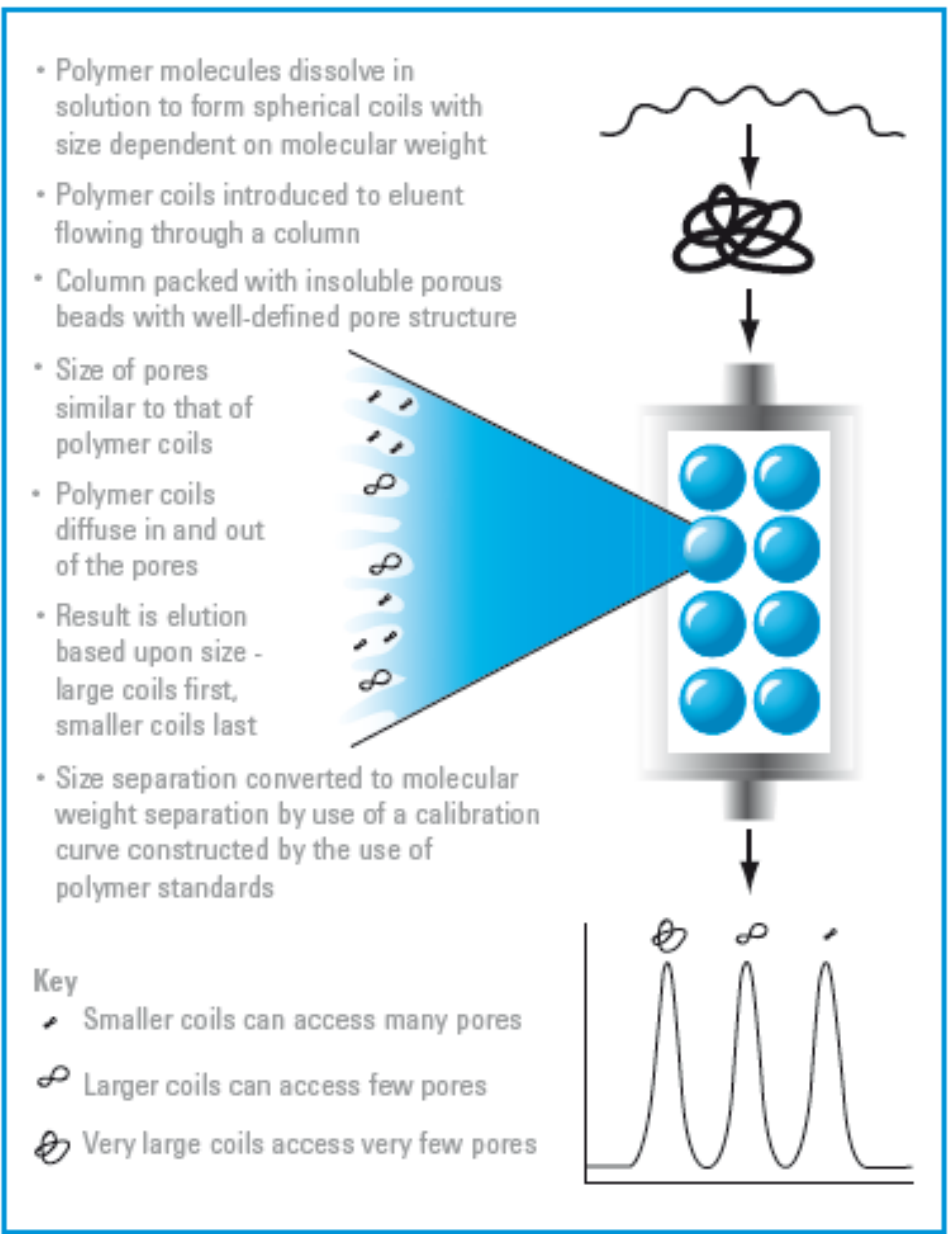

Figure 135 The summary of GPC/SEC mechanism. (512)

Monomers have a single molecular weight, socalled as monodisperse. Polymers have a distribution of chain lengths, and, therefore, molecular weights. Polymers can be characterized by a variety of definitions for molecular weight including the number average molecular weight $\left(M_{n}\right)$, the weight average molecular weight $\left(M_{w}\right)$, the size 
average molecular weight $\left(\mathrm{M}_{\mathrm{z}}\right)$, or the viscosity molecular weight $\left(\mathrm{M}_{\mathrm{v}}\right)$. GPC allows for the determination of PDI as well as $M_{v}$ and based on other data, the $M_{n}, M_{w}$ and $\mathrm{M}_{\mathrm{z}}$ can be determined. When characterizing polymers, it is important to consider the polydispersity index (PDI) as well the molecular weight. The PD is the ratio of the weight average and number average molecular weights and is called as the polydispersity, or sometimes simply, the dispersity of the polymer. Depending on how the polymerization was carried out, this distribution can be narrow, or quite broad. As an example, a polymer obtained via Controlled Radical Polymerization techniques (e.g. RAFT polymer) will have a narrow distribution of molecular weights. (Figure 136). On the other hand, a free radical polymerization may produce a polymer with a very broad distribution of chain lengths and molecular weights. (Figure 137) Controlling the kinetics of the polymerization is extremely important in obtaining a desired molecular weight distribution.

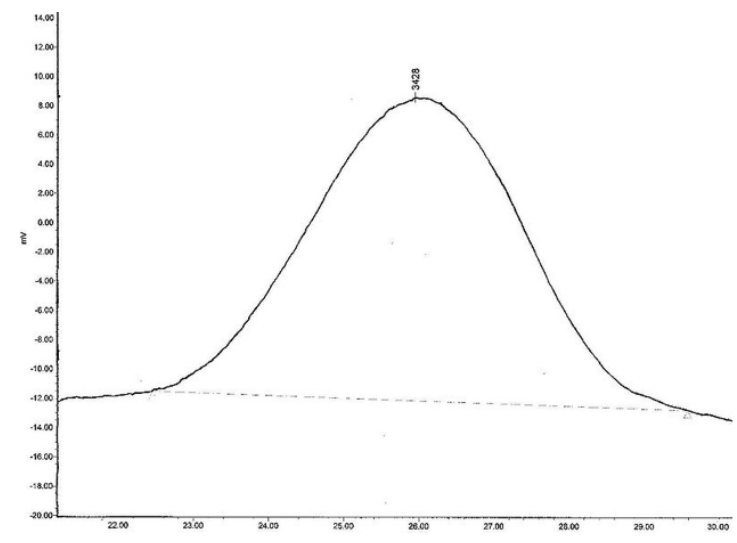

Figure 136 GPC Separation of Anionically Synthesized Polystyrene; $M_{n}=3,000 \mathrm{~g} / \mathrm{mol}$, $\mathrm{PDI}=1.32$. (513)

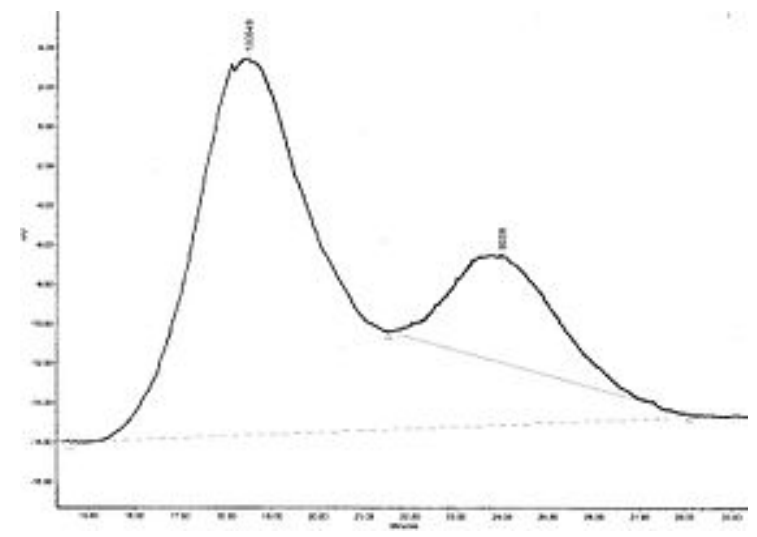

Figure 137 GPC Separation of Free-Radical Synthesized Polystyrene; $M_{n}=24,000$ $\mathrm{g} / \mathrm{mol}, \mathrm{PDI}=4.96$. (513)

Molecular weight average across this distribution is assigned by simply doing statistics. During the analysis the detector tells how much material elutes from the 
column at any given time as a peak. The integral area of that sample peak can be assumed as a histogram which is divided into a number of slices. There is a height, $\left(\mathrm{H}_{\mathrm{i}}\right.$, also represented as concentration, $\left.\mathrm{C}_{\mathrm{i}}\right)$ a retention time, and a molecular weight, $\left(\mathrm{M}_{\mathrm{i}}\right)$, attributed to each slice. That sample peak is converted into a molecular weight and then into a molecular weight distribution by reference to a calibration curve of molecular weight as a function of retentionlelution time (or volume). (Figure 138)

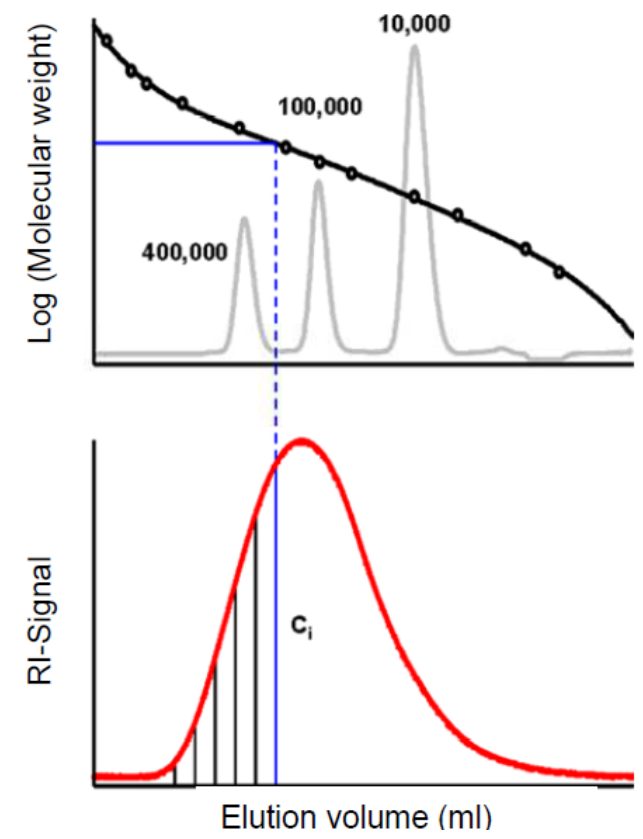

Figure 138 Principle of conventional calibration. (514)

The fact that hydrodynamic volume $\left(\mathrm{V}_{\eta}\right)$ is proportional to the product of the intrinsic viscosity of the polymer in the SEC eluent $([\eta])$ and molecular weight $(M)$ might be used as the universal calibration parameter. (512) The Universal Calibration is based on the fact that the intrinsic viscosity and the molecular weight are related to the size of the molecules in solution (Figure 139) by Hydrodynamic volume $=\mathrm{k} x$ molecular weight $\mathrm{x}$ intrinsic viscosity.

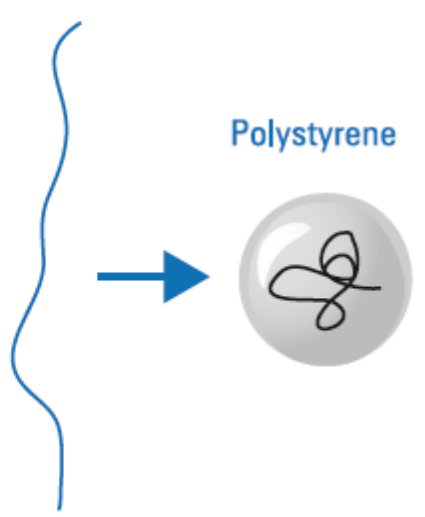

Figure 139 Size of a polymer dissolved in a common solvent. (512) 
The Mark-Houwink-Sakurada equation explains a relation between the intrinsic viscosity $([\eta])$ and molecular weight $(M)$ : (515)

where $[\eta]$ is intrinsic viscosity, $M$ is molecular weight and $\alpha$ and $\mathrm{K}$ are the Mark-Houwink constants. Rearranging this into a log form gives equation 22:

$\log [\eta]=\log \mathrm{K}+\alpha \log \mathrm{M}$

Plotting this relationship gives a straight line with intercept of $\log \mathrm{K}$ and a slope of $\alpha$ (Figure 140). This is a useful relationship because the values of $K$ and $\alpha$ are constant for a given solvent and polymer combination, and because the value of $\alpha$ gives information about the dynamic behavior of the polymer molecules in solution. For most polymers the value of $\alpha$ ranges between 0.5 and 0.8 , with lower values indicating a more compact dense structure (following the 'hard sphere' model), 0.7-0.8 being expected for a random coil polymer in a good solvent, and increasing values indicating a larger, more solvated structure (the limiting value of 2.0 is indicative of the 'rigid rod' model, most of the components of the molecule are far from the centre of gravity, similar to that expected for a spinning rod structure in solution). The Mark-Houwink relationship is thus an excellent way of probing the solution behavior of polymer molecules.

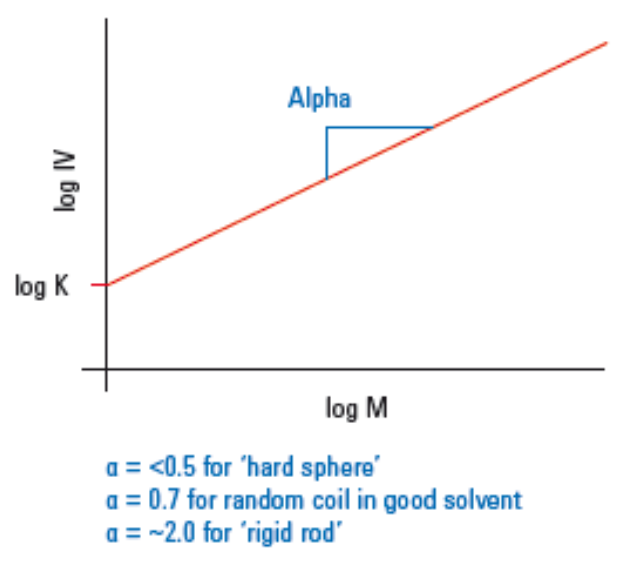

Figure 140 The plot which gives information about the dynamic behavior of the polymer molecules in solution. (512)

Basing on the Mark-Houwink-Sakurada equation, the molecular weight of a polymer can be determined from data on the intrinsic viscosity. Since the intrinsic viscosity of a polymer is directly related to the elution volume of the polymer in size-exclusion chromatography, it is possible to determine the values of $\mathrm{K}$ and $\alpha$ (Mark-Houwink- 
Sakurada constants) by measuring several monodisperse samples of polymer and than graphically using a line of best fit. Once $\mathrm{K}$ and $\alpha$ are known, a plot of $\log$ [ $\eta$ ] $\mathrm{M}$ versus elution volume (or elution time) for a particular solvent, column and instrument provides a universal calibration curve which can be used for any polymer in that solvent. By determining the retention volumes (or times) of monodisperse polymer standards (e.g. solutions of monodispersed polystyrene in THF), a calibration curve can be obtained by plotting the logarithm of the molecular weight versus the retention time or volume.

The GPC sorts the polymer chains by volume and as intrinsic viscosity is related to the volume of the polymer chain, the GPC data is the same for the two different polymers. Therefore, once the calibration curve is obtained, the gel permeation chromatogram of any other polymer can be obtained in the same solvent and the molecular weights (usually $\mathrm{M}_{\mathrm{n}}$ and $\mathrm{M}_{\mathrm{w}}$ ) and the complete molecular weight distribution for the polymer can be determined. Forexample, if GPC calibration curve is known for polystyrene in toluene, polyethylene in toluene can be run in a GPC and molecular weight of polyethylene can be found according to polystyrene calibration curve via the equation 23 below: (516)

$$
\mathrm{K}_{1} M_{1}{ }^{1+\alpha}{ }_{1}=\mathrm{K}_{2} M_{2}{ }^{1+\alpha}{ }_{2}
$$

A typical calibration curve is shown above in Figure 138 and the molecular weight from an unknown sample can be obtained from the calibration curve.

\subsection{Components of GPC}

The system consists of a pump, a type of injector (either manual or automated), the column set, the detector(s), and a kind of data handling device. (Figure 141) For GPC measurements, after the sample is dissolved in an appropriate solvent (generally organic solvents) and filtered, it is injected into the column where the separation of multi component mixture takes place. The fresh eluent is constantly feeded into the column via a pump. A detector or multiple detectors might be used to gain additional information about the polymer sample. The availability of a detector makes the fractionation convenient and accurate. A degasser might be used additionally while using THF with a refractive index detector. The columns are almost always heated to some elevated temperature, even for room temperature soluble applications not only for dissolution purposes but also to increase the resolution of the separation, enhance the permeation process, decrease the viscosity of the solvent (DMF, for example) and reduce backpressure across the column bank. 


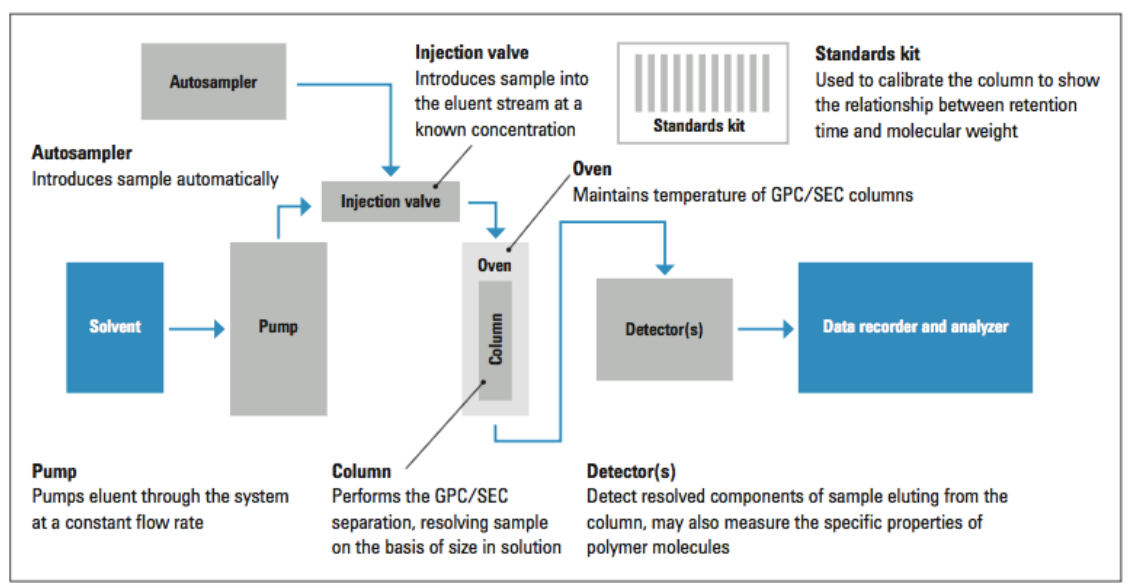

Figure 141 The main components of a GPC/SEC system. (517)

\subsection{Gel (Stationary Phase)}

Gel is used as stationary phase for GPC. The pore size of a gel must be carefully chosen in order to achieve a sucsessful separation. Since the separation is based on the size of the sample in solution, there should be no interaction such as adsorption, partition, etc. with the column packing. For this reason, the gel forming agent should be free of ionizing groups and low affinity for the substances to be separated. PLgel, sephadex, bio-Gel (cross-linked polyacrylamide), agarose gel and styragel are commercial gels used for different separation requirements. (518)

\subsection{Eluent (Mobile Phase)}

The SEC mobile phase (the eluent) is generally chosen as the same solvent which is used to dissolve the polymer. The sample must be dissolved in a suitable solvent due to the fact that the separation is not based on molecular weight, but on the size of the polymer being analyzed in solution. Therefore, the eluent should be a good solvent which permits high detector response from the polymer and should be able to wet the packing surface. are tetrahydrofuran (THF), o-dichlorobenzene and trichlorobenzene are the most common eluents for polymers that dissolve at room temperature GPC. The concentration of the sample in solution depends on the molecular weight. For a polymer of molecular weight $\sim 100,000$, a concentration of $0.10 \%(\mathrm{w} / \mathrm{v})$ is typical.

\subsection{Column Selection}

The columns are packed with a crosslinked gel such as styrene/divinylbenzene copolymer (for organic applications), which is composed of surface pores which act as a molecular filter. These pores can vary from small to quite large. The larger size molecules will not fit into the smaller pores and not be retained. Conversely, the smaller molecules will fit into most of the pores and be retained longer. Molecules that 
are not retained elute through the free volume outside of the particles $\left(\mathrm{V}_{\mathrm{o}}\right)$, while smaller molecules that are completely retained are eluted with volume of solvent held in the pores $\left(\mathrm{V}_{\mathrm{i}}\right)$. (Figure 142) The total volume can be considered by the equation 24 , where $\mathrm{V}_{\mathrm{g}}$ is the volume of the polymer gel and $\mathrm{V}_{\mathrm{t}}$ is the total volume: (513), (519)

$\mathrm{V} t=\mathrm{V} g+\mathrm{V} i+\mathrm{V} o$

Eq 22

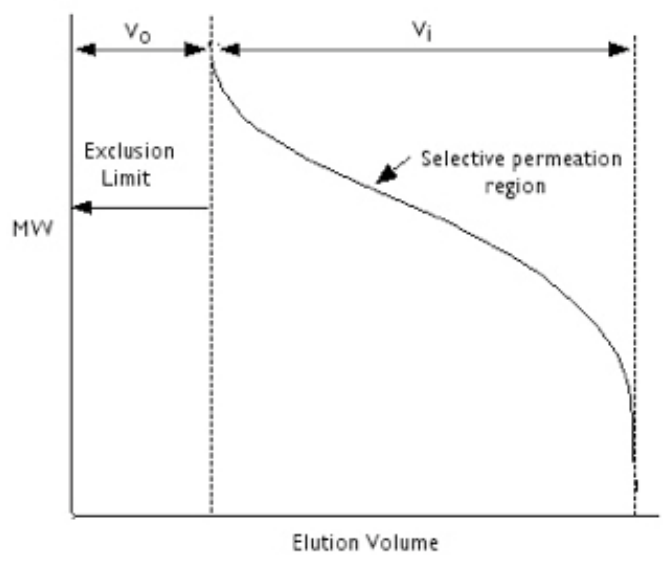

Figure 142 Range of molecular weights that can be separated for each packing material. (513)

Since a limited range of molecular weights can be separated by each type of column, the size and shape of the pores for the packing should be chosen according to the range of molecular weight of analytes to be separated. For polymer separations the pore sizes should be on the order of the polymers being analyzed. If a sample has a broad molecular weight range it may be necessary to use multiple column system to fully resolve the sample. Resolution increases with the incrase of number of columns used. "Linear" columns socalled as "Mixed Bed" columns are the most common types in use. These columns are composed of different pore sizes in order to cover a broader molecular weight range. If the blending of pores is done carefully enough, the column calibration curve may indeed be linear. The drawback of utilization of these mixed bed columns is that resolution over a finite molecular range is not as good as the one getting from individual pore size columns. Multiple-column systems in series are generally preferred in order to ensure that there is enough pore volume which provides successful separation. The following table (Figure 143) lists the molecular weight range of separation for individual pore size columns of styrene / divinylbenzene packings, based on polystyrene chain length exclusion limits (in Angstroms): 


\begin{tabular}{|ll}
\hline Molecular weight range & Pore size \\
\hline $100-1000$ & $50 \mathrm{~A}$ \\
\hline $250-2500$ & $100 \mathrm{~A}$ \\
\hline $1,000-18,000$ & $500 \mathrm{~A}$ \\
\hline $5,000-40,000$ & $10^{3} \mathrm{~A}$ \\
\hline $10,000-200,000$ & $10^{4} \mathrm{~A}$ \\
\hline $50,000-1,000,000$ & $10^{5} \mathrm{~A}$ \\
\hline $200,000->5,000,000$ & $10^{6} \mathrm{~A}$ \\
\hline $500,000-\sim 20,000,000$ & $10^{7} \mathrm{~A}$ \\
\hline$\sim 1,000-10,000,000$ & Mixed Bed - High \\
\hline$\sim 100-100,000$ & Mixed Bed - Low \\
\hline
\end{tabular}

Figure 143 The molecular weight range of separation for individual pore size columns of styrene/divinylbenzene packings. (513)

\subsection{Pump}

The pumps are fluid handling devices. The most important thing in choosing a fluidics module for GPC analysis is the flow precision. Since the calibration of the system is a plot of retention time (or volume) vs. the log of the molecular weight, any minor flow fluctuation might result in a potentially large error in molecular weight. For this reason, It is important to choose the most precise fluid-handling device, whose flow precision under $0.075 \%$ without any flow rate correction. Piston or peristaltic pumps are two types of pumps generally preferred for uniform delivery of relatively small liquid volumes for GPC.

\subsection{Detector Selection}

In GPC, the concentration by weight of polymer in the eluting solvent is monitored continuously by utilising a detector. Detector types can be divided into two main categories. The first is concentration sensitive detectors which includes UV absorption, differential refractometer (DRI) or refractive index (RI) detectors, infrared (IR) absorption and density detectors. Molecular weight sensitive detectors include low angle light scattering detectors (LALLS), multi angle light scattering (MALLS). The most sensitive detector is the differential UV photometer and the most common detector is the differential refractometer (DRI). The GPC analysis depends on the 
number and type of detectors used in the experiment. The dual detector GPC system used for this thesis employs the following two kinds of detectors: RI and UV detector.

\subsection{Refractive Index (RI) Detector}

The RI detector is a concentration sensitive detector that measures the difference in refractive index ( $\mathrm{dRI}$ ) between the eluent in the reference side, and the sample + eluent in the sample side. In some cases, the difference in refractive index for the sample and eluent is very small, resulting in a poor signal. In that case, another eluent should be used in order to dissolve the polymer and provide a significant d RI. The RI detection is based on the fact that a beam of the light is deflected as it passes through a flow cell including the reference solvent (whose refractive index is $n_{o}$ ) in reference part, whereas the solution sample (whose refractive index is $n$ ) in the sample part. (55) The beam is refracted at the liquid-glass interfaces separating the two compartments. (Figure 144) The area under the RI peak is proportional to $d n \mid d c$, the refractive index increment, and $C$, the concentration of the sample (Equation 25, 26). (514)

$$
R I_{\text {Area }} \propto C \frac{d n}{d c}
$$

Where

$$
\frac{d n}{d c}=\frac{n-n_{o}}{C}
$$

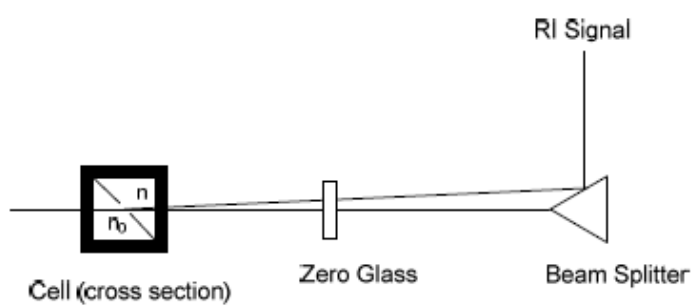

Figure 144 Schematic representation of deflection type refractometer. (57)

During the analysis, the GPC column separates the polymer molecules on the basis of their size (hydrodynamic volume) in solution, and the detector determines the amount of material eluting from the column as a function of retention time. This is converted to molecular weight by the use of a calibration curve -a graph that relates retention time (time of elution from the column) to the molecular weight of the polymer. The calibration curve is itself generated by the elution behavior of a series of 
commercially available polymer standards of known molecular weight, with low polydispersity indexes. During calculations, the integration of the peak for the sample eluting from the column can be assumed as a histogram which consists of a number of small fractions (slices). For each slice (i), the $M_{i}$ represents the molecular weight whereas the number of molecules got from detector response is $\mathrm{N}_{\mathrm{i}}$. (Figure 145) The retention time of each slice is used to determine its molecular weight by reference to the calibration curve. The area or height of the slice allows the percentage of the whole sample in the slice to be determined, and from the percentage and molecular weight data the molecular weight distribution is calculated.

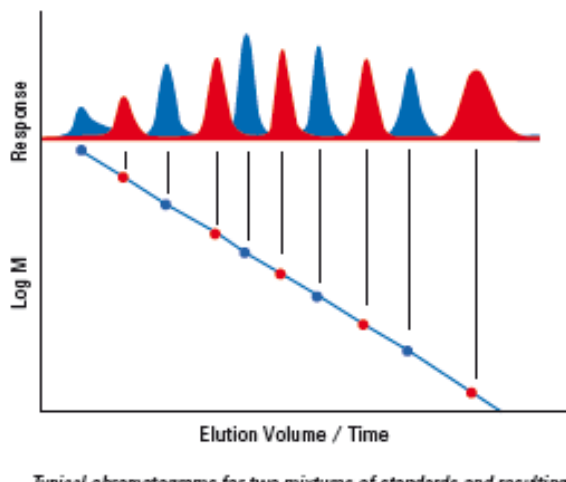

$$
\begin{aligned}
& \text { Typical chromatog } \\
& \text { calibration curve }
\end{aligned}
$$

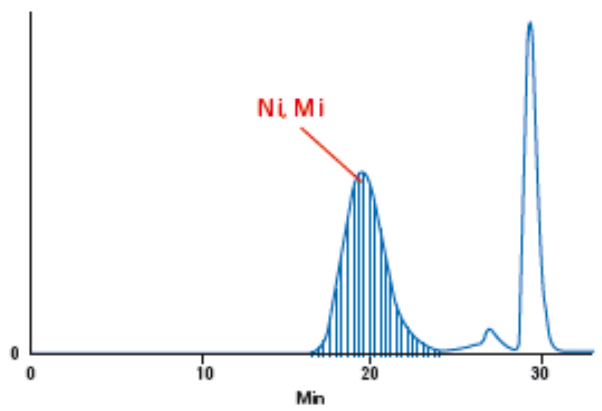

Figure 145 A peak sliced during GPC calculations. (512)

The molecular weight of an unknown sample is calculated by dividing the area below the curve in individual slices and projecting the retention volume of each slice on the calibration curve. Polymers commonly used for calibration are polymethyl methacrylate (in THF, ethyl acetate, acetone or DMF), polystyrene (in THF, toluene or chloroform), polyethylene glycol (in aqueous eluents, DMF or DMSO).

\subsection{Ultraviolet Absorbance (UV) Detector}

Another detector that is used often for GPC is the UV detector. A pump and some form of injection device, such as an autosampler, creates a flow of mobile phase through a flow cell which is situated in the UV detector. Light from the UV detector is passed through the flow cell via fiber-optic cables. Compounds including chromophore which is able to absorb UV, give a response on the detector, which is 
directly proportional to the concentration. The UV detector measures absorbance at the user-specified wavelength. The software records the absorbance data as a chromatogram along with all the other data channels. This is then used to calculate concentration or conjugate data. The UV detector is excellent for styrenic type polymers, (polystyrene, styrene/isoprene, styrene/butadiene, ABS, etc.), epoxies, phenolics, polycarbonates, polyurethanes, and aromatic polyesters. UV detectors have many advantages such as:

* Excellent tool for the compositional analysis of co-polymers or protein conjugates.

* Choose the most appropriate wavelength for sample.

* Unmatched baseline stability and data reproducibility.

\subsection{Equipment Properties and Measurement Conditions}

SEC characterization of all samples was performed with THF (THF; HPLC-grade from Sigma-Aldrich) as the eluent containing of toluene (>99.7 \%, dry, from SigmaAldrich) as internal standard (flow velocity $1.0 \mathrm{~mL} \cdot \mathrm{min}-1$ at $35^{\circ} \mathrm{C}$ ) using an Agilent 1260 Infinity system. It was composed of an autosampler, an isocratic solvent pump, a PSS GRAM (polyester copolymer network) precolumn $(8 \times 50 \mathrm{~mm})$, three PSS GRAM separation columns $\left(8 \times 300 \mathrm{~mm}\right.$, nominal particle size $=10^{-5} \mathrm{~m}$; pore sizes $=$ 30,103 , and $103 \AA$ ) maintained at $45^{\circ} \mathrm{C}$ in a column compartment, an $80 \mathrm{~Hz} \mathrm{UV}$ detector (set to a wavelength of $310 \mathrm{~nm}$ for the RAFT polymers) and a refractive index (RI) detector. The flow rate of the mobile phase was $8 \times 10-4 \mathrm{~L} \mathrm{~min}^{-1}$. The whole setup was calibrated with a total of 12 PSS poly (methyl methacrylate) standards ( $\bar{M} p$ $=0.8-1820 \mathrm{~kg} \mathrm{~mol}-1)$ of low dispersity with toluene as internal standard. All samples were filtered through a $450 \mathrm{~nm}$ PTFE syringe filter prior to injection.

\subsubsection{High Pressure Liquid Chromatography (HPLC) Mass Spectrometry}

\subsection{Definitions $\backslash$ Basic Principle}

Mass spectrometry is a common analytical technique used for production, subsequent separation and identification of charged species. The mass spectrometry technique is based on separation of gas phase ions according to their $\mathrm{m} \backslash \mathrm{z}$ (mass to charge ratio) value. These charged species are produced as gas phase ions under atmospheric pressure conditions by a variety of ionisation methods such as Electrospray Ionisation (EI) and Atmospheric Pressure Chemical Ionisation (APCI). The separation of gas phase ions is achieved within the mass spectrometer using electrical andlor magnetic fields for differentiation of ions. High pressure liquid chromatography (HPLC) is a widely used technique for separation, identification and quantification of all components in a mixture. (Figure 146) In this technique, a pressurized liquid solvent containing the sample mixture is passed through a column filled with a solid adsorbent 
material. The separation is based on the fact that each component in the sample interacts differently with the adsorbent material in the column, leading to different flow rates for each component.

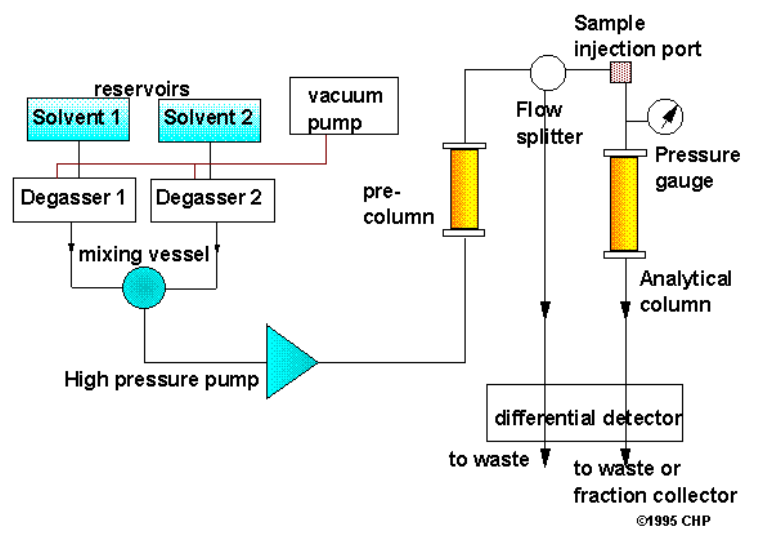

Figure 146 HPLC mass spectrometer used for this work. (520)

In HPLC, operational pressures are significantly higher (50-350 bar) than the traditional (low pressure) liquid chromatography which relies on the force of gravity to pass the mobile phase through the column. Due to HPLC columns which are made with smaller sorbent particles (2-50 micrometer in average particle size), the resolving power of HPLC (the ability to distinguish between compounds) is better than other chromatographic techniques. HPLC instrument typically includes a sampler, pumps, and a detector. (Figure 147) The sampler or autosampler brings the sample mixture into the mobile phase stream whereas the pumps adjust the desired flow and composition of the mobile phase and pass it through the column. The detector generates a signal proportional to the amount of sample component coming out from the column which allows quantitative analysis of the sample components.

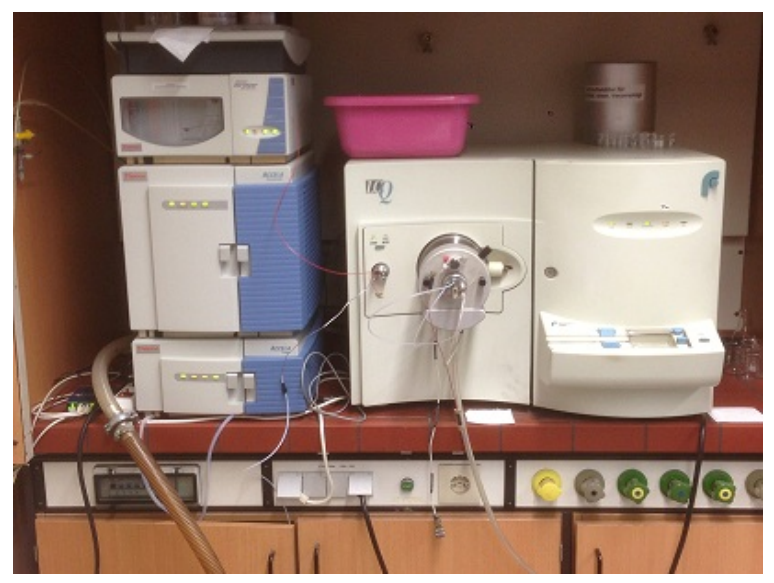

Figure 147 The schematic of an HPLC instrument. (521)

There are various detectors commonly used in HPLC system such as UV/Vis, photodiode array (PDA) or based on mass spectrometry. Most HPLC instruments also 
have a column oven used for adjustment of the temperature the separation is performed at. LCMS (Liquid Chromatography Mass Spectrometry) which combines these two techniques covers a broad range of application areas. The applicability conditions and advantages of both HPLC as a separative technique and MS as a means of detecting analyte species are listed below (522):

* For HPLC analysis the analyte must be soluble in the mobile phase.

* HPLC samples are prepared in a solvent system that has the same or less organic solvent than the mobile phase.

* HPLC can analyse samples over a wide polarity range including ionic samples.

* HPLC has no real upper molecular weight limit and large proteins of many thousands of Daltons may be analysed. Solubility in the mobile phase may preclude the analysis of very large molecules.

* Allows specific compound identification (structural elucidation via spectral interpretation combined with elemental composition from accurate mass analysers is possible).

Very sensitive (fempto-gram amounts have been detected by certain mass analyzer types).

Highly selective (certain analyzer and experiment combinations can lead to highyl selective and sensitive analysis of a wide range of analytes).

\subsection{Working Principle}

LC-MS analysis combines two different techniques including HPLC as a separative technique and MS as a means of detecting analyte species in order to produce and identify charged particles. (Figure 148) The entire process is a multi-step approach which typically includes following steps: Separation of the sample components were accomplished by using a HPLC column. The analytes are differentially partitied between the mobile phase (eluent) and the stationary phase coated onto a support material and packed into the column. The mechanism of retention and therefore separation depends on the mode of chromatography which may include Hydrophobic interaction, Ion Exchange, Ion-pair, Surface Localisation, etc. 


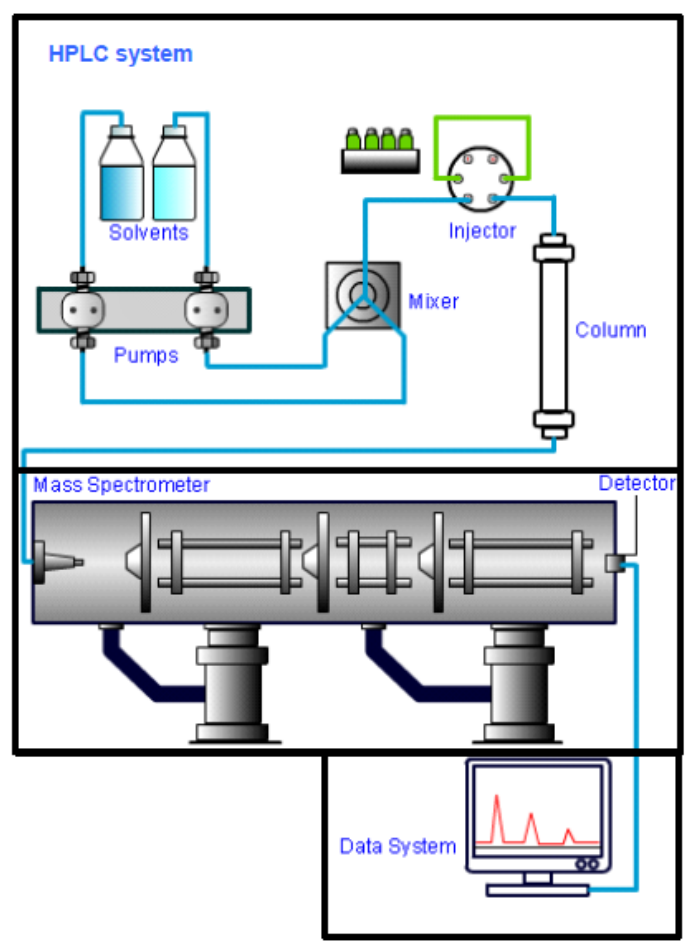

Figure 148 Schematic representation of HPLC-Mass Spectrometer. (522)

The separated sample species are then sprayed into an Atmospheric Pressure Ion Source (API) where they are converted to ions in the gas phase. Then, these ions are separated ions according to their mass to charge ratio in the Mass Analyzer which is used to isolate ions of specific mass to charge ratio or to 'scan' over all ion $\mathrm{m} \backslash \mathrm{z}$ values present. The ions emergent from the mass analyzer are counted by the detector which can also amplify the signal generated from each ion.

\subsection{HPLC Mass Set Up}

The mass spectrometer consists of an atmospheric ionisation chamber, a vacuum system, an analyzer and a detector. All parts are summarized below:

Ion source: It is used to spray the HPLC eluent into the atmospheric region.

Skimmer Cone: A cone with a sampling orifice which is used to reduce the gas load entering the vacuum system of the mass analyser device.

Quadrupole: Device which consists of four parallel equidistant rods, is used to separate ions according to their mass to charge ratio $(\mathrm{mlz})$ via utilization of electric fields. Ions are separated while passing along the central axis of these rods. 
* Collision Cell: Ions coming out of the first mass analyzer are accelerated by using a potential difference and collide with neutral gas molecules such as $\mathrm{H}_{2}$, $\mathrm{N}_{2}$ or Ar leading to analyte fragmentation.

Detector: The separated ions are detected and transformed into a usable signal via the detector. Electron multiplier, Dynode, Photodiode and Multi Channel Plate (MCP) are ion detection systems widely employed in most modern mass spectrometer systems.

Vacuum system: Mass analyzers require high levels of vacuum in order to operate in a predictable and efficient way. The vacuum systems used for most modern LC-MS systems consist of two or more differentially pumped vacuum chambers with a variety of designs.

\subsection{Ionisation}

Ionisation is the production process of ions whereby electrons are either removed or added to atoms or molecules by the use of strong electric fields in the vapour or condensed phase. In LC-MS charge may also be applied to the molecule via association with other charged molecules-for example a proton $\left(\mathrm{H}^{+}\right)$. The ionization process which the sample is ionized or desolvated under atmospheric pressure conditions is called Atmospheric Pressure Ionisation (API). (Figure 149) The most common ionisation methods employed in LC-MS are Electrospray Ionisation (ESI) (ionisation in the condensed phase), Atmospheric Pressure Chemical Ionisation (APCI) (ionisation in the gas phase), Atmospheric Pressure Photo Ionisation (APPI) (ionisation in the gas phase).
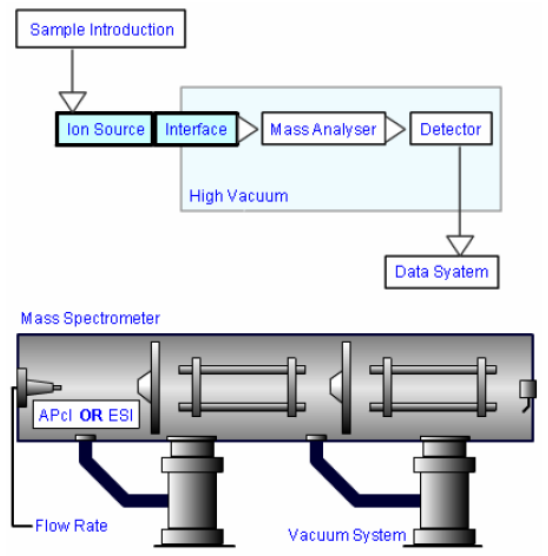

Figure 149 Where the "Flow rate" label denotes the effluent (analyte plus eluent and additivates) coming from the HPLC system. (522) 


\subsection{Atmospheric Pressure Ionisation (API)}

The solvent elimination and ionisation steps are combined and take place in the ion source. There are two main considerations common to all interface types: First step includes desolvation of the analyte molecule. Here, the solvent molecules are removed from the HPLC eluent. In second step, the analyte molecules are converted into charged molecules (ions). Here, ions are formed sothat the analyte (or analyte derivatives) can be transported from the interface into the mass spectrometer where they will be filtered from other masses and subsequently detected. (Figure 150)

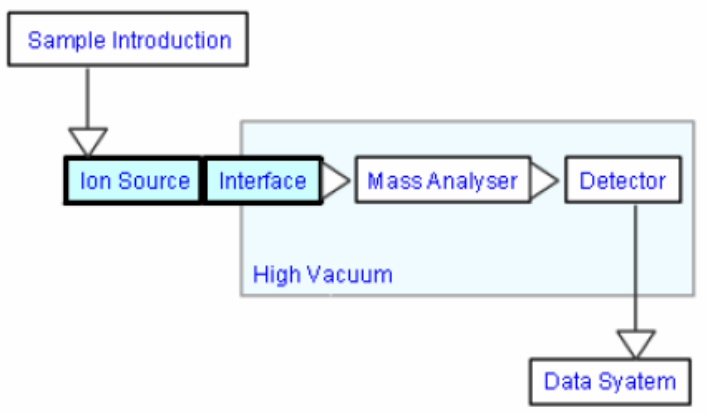

Figure 150 The schematic diagram of ion production process. (522)

The two basic modes of ionisation used in API LC-MS are Electrospray Ionisation (ESI) and Atmospheric Pressure Chemical Ionisation (APCI). In electrospray ionisation analyte ions are pre-formed in the mobile phase prior to entering the API interface whereas ions are formed via charge transfer processes in the gas phase within the API interface in Atmospheric Pressure Chemical Ionisation.

\subsection{Mass Analysers}

Analyte ions or fragments of analyte ions created in the Atmospheric Pressure Ionisation (API) interface or in the regions between the API interface and the high vacuum region of the mass analyser (products of collision-induced dissociation etc.) are separated or filtrated in Mass Analyser. (Figure 151) There are many types of mass analyser which differ in the fundamental way in which they separate species on a massto-charge basis. The most employed ones are Quadrupole, Time of Flight (TOF) and Magnetic Sector Mass Analyzers. 


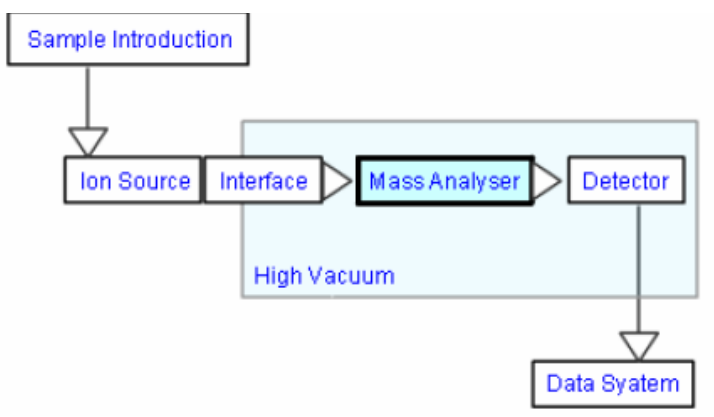

Figure 151 Schematic representation of mass analyzer located in high vacuum region where analyte ions or fragments of analyte ions are separated according to their $\mathrm{m} \backslash \mathrm{z}$ (mass to charge ratio) value. (522)

Quadrupole and Ion Trap Mass analysers:

Electric fields are used to separate ions according to their mass to charge ratio $(\mathrm{mlz})$ in quadrupole mass analyzing devices which consists of four parallel equidistant rods (or poles), as ions pass along the central axis of the quadruple. (Figure 153) Ions are separated by using controlled voltages which are applied to the mass analyser rods which impart an electrostatic field inside the analysing device. $\mathrm{X}$ and $\mathrm{Y}$ determine the position of an ion from the centre of the rods.
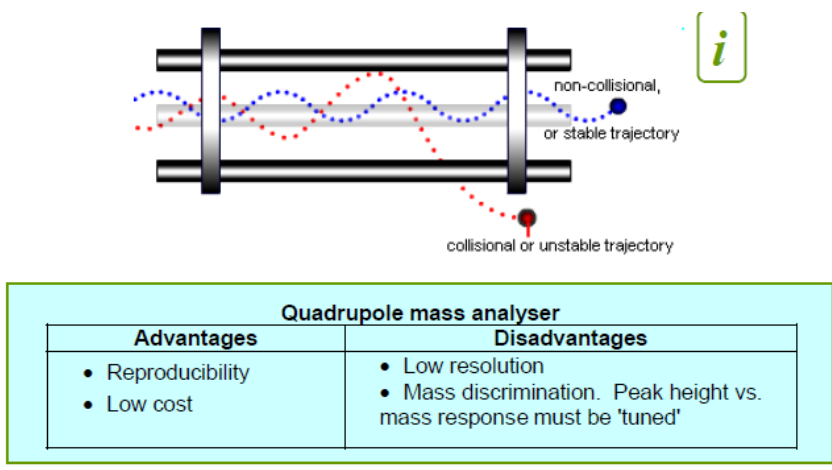

Figure 152 Schematic represantation of non-collisional or collisional trajectory. (522)

If $x$ and $y$ values remain smaller than $r_{o}$, the ion can accomplish to pass through the quadrupole without touching the rods. (Figure 152) This is named as a non-collisional or stable trajectory. If the $\mathrm{x}, \mathrm{y}$ values exceed $\mathrm{r}_{\mathrm{o}}$, ion will collide with a rode and become discharged and subsequently pumped to waste. This is known as an unstable or collisional trajectory. 


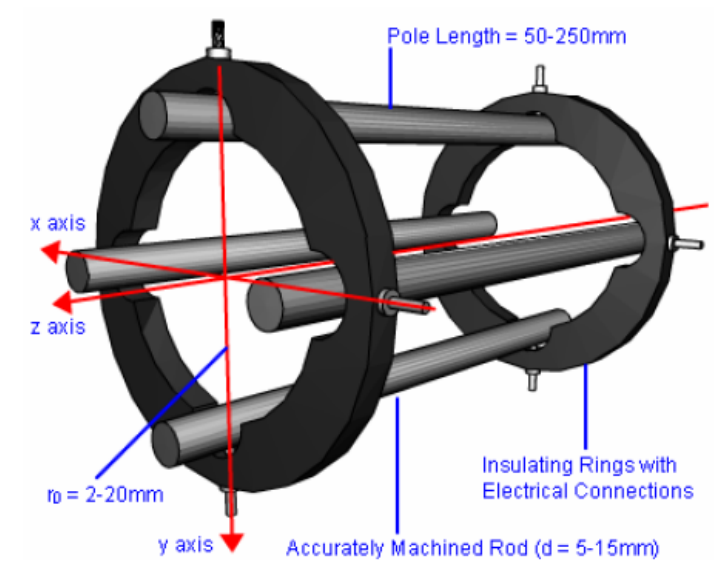

Figure 153 Schematic representation of a quadrupole. (522)

* Time of Flight (TOF) mass analysers utilize differences in flight times of accelerated ions through an extended flight path in order to be able to separate ions.

* Magnetic Sector Mass Analysers use magnetic fields to select ions. Ions are seperated by directing the beam of ions of interest towards the detector via magnetic fields. The analyte and fragment ions are plotted in terms of their mass to charge ratio $(\mathrm{m} \backslash \mathrm{z})$ against the abundance of each mass shown in Figure 154.

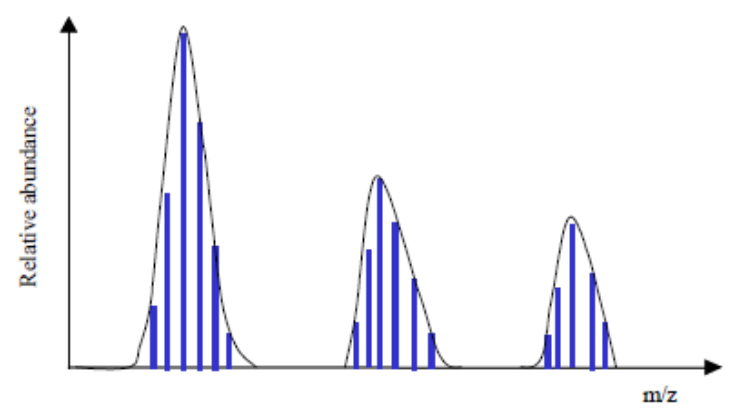

Figure 154 A typical mass spectrum. (522)

\subsection{Detectors}

After the ions are separated and passed the mass analyzer, they are detected and transformed into a usable signal via a detector. The detector generates a signal from incident ions in two ways: it generates secondary electrons, which are further amplified, or induces a current which is generated by moving charges. Ion detector systems divide into two main categories including point detectors and array detectors. In the case of point detectors, ions are not spatially resolved and sequentially bumped into a detector situated at a single point within the spectrometer geometry. In the case 
of Array detectors, ions are spatially resolved and all ions arrive simultaneously and are recorded along a plane consisting of a block of detectors. (Figure 155)

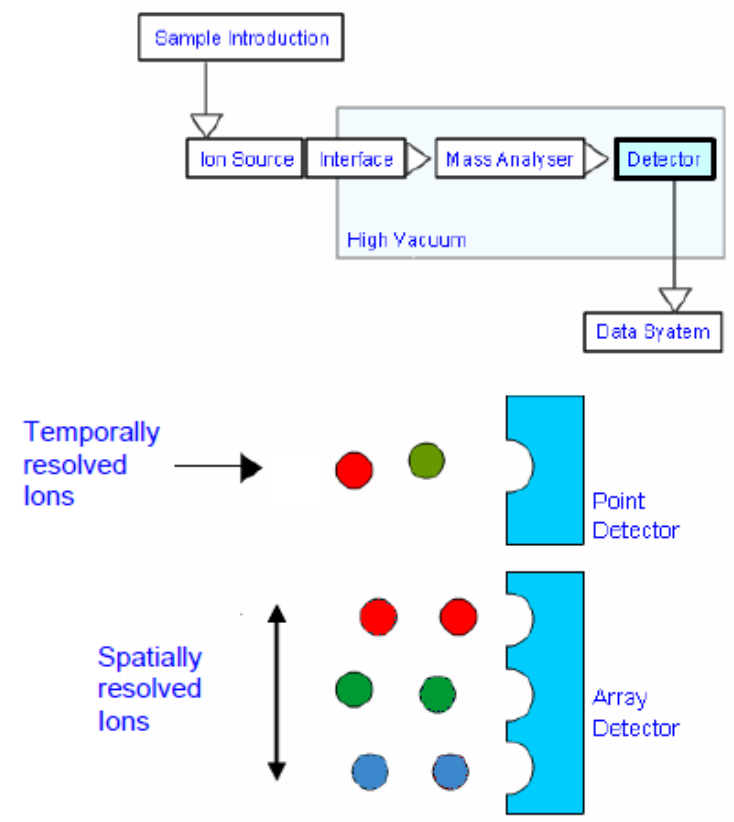

Figure 155 Schematic represantation of point and array detectors. (522)

\subsection{Equipment Properties $\backslash$ Measurement Conditions}

HPLC/ESI-MS used a VG PLATFORM mass spectrometer (Micromass, Manchester, UK) equipped with optional electrospray ionization (ESI) and atmospheric pressure chemical ionization (APCI) facilities and linked to a Jasco HPLC instrument (Jasco, Gross-Umstadt, Germany) with a ternary pump (PU-980/LG-980-02), integrated degassing unit (DG-980-50) and UV detector (UV-975). An RP-C18 column, Nucleosil $100 \mathrm{C} 18(250 \mathrm{~mm} \times 3 \mathrm{~mm}$, particle size $5 \mu \mathrm{m})$, and a precolumn Nucleosil $100 \mathrm{C}(20 \mathrm{~mm} \times 4 \mathrm{~mm}$, particle size $5 \mu \mathrm{m})$ was used for the chromatographic separation.

\subsubsection{Spectroscopic Ellipsometry (SE)}

\subsection{Definition $\backslash$ Basic Principles}

Ellipsometry is an optical technique used for investigating the dielectric properties of thin films. (Figure 156) This technique is based on the fact that the polarization state of light changes when the light beam is reflected from a surface. If the surface is covered by a thin film (or a stack of films), the entire optical system of film \& substrate influences the change in polarization. It is therefore possible to deduce information about the film properties, especially the film thickness. 


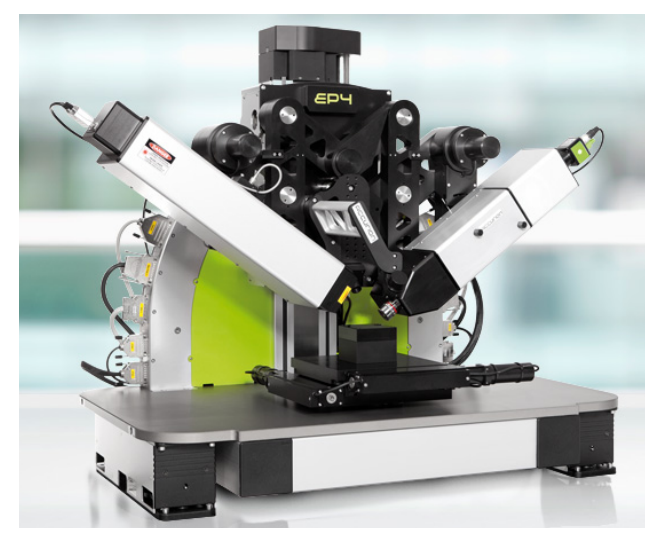

Figure 156 A typical Spectroscopic Ellipsometer. (523)

Ellipsometry measures a change in polarization (light wave`s electric field behavior in space and time) as light reflects from an interface, which is represented as an amplitude ratio $\Psi$, and a phase difference, $\Delta$. (Figure 157) The amount of change in polarization upon reflection depends on the optical properties and thickness of individual layers on the surface, the substrate and the ambient. (524)

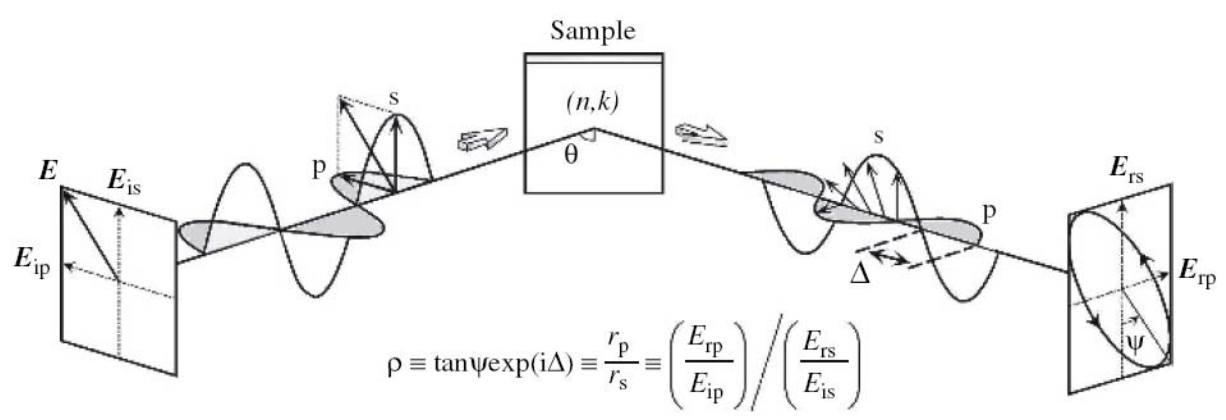

Figure 157 Measurement principle of ellipsometry. (525)

Thus, ellipsometry is primarily used to determine film thickness and optical constants. However, it is also applied to characterize composition, roughness, and other material properties associated with a change in optical response. To correlate the measured changes in light polarization with surface properties, a theoretical optical model is required. With the help of the model it is possible to determine refractive index and thickness of the adsorbed layer by iteration of the ellipsometric angles and $\Delta$, $\Psi$. (Delta) is defined as the change of the phase difference between the p- and scomponents of the polarized light upon reflection, while (Psi) is the angle whose tangent is the ratio of the magnitudes of the total reflection coefficients of the p-and s- component. The total reflection coefficient is the ratio of the amplitudes of the outgoing wave to the incoming wave. Therefore, the results are highly dependent on the assumption made about the surface properties. 


\subsection{Polarization of Light}

Light (electromagnetic wave) is generally described with the strength and direction of the electric field $\mathrm{E}$, which has a stronger interaction with matter than the magnetic field. Monochromatic light (at a point in space E) is resolved into three independent harmonic oscillations along an $\mathrm{x}, \mathrm{y}, \mathrm{z}$ coordinate system. Assuming that the light wave is a plane wave which travels along the $\mathrm{z}$-axis, the $\mathrm{E}$ vector becomes orthogonal to $\mathrm{z}$ and thus it can be described by two harmonic oscillations along $\mathrm{x}$ and $\mathrm{y}$. These oscillations have the same frequency, but generally different amplitude and phase. As a result, the E vector moves along an ellipse at a fixed point in space. The behavior with time of a vector field at a fixed point in space is called polarization. This elliptical state of polarization is the most general state of polarization.

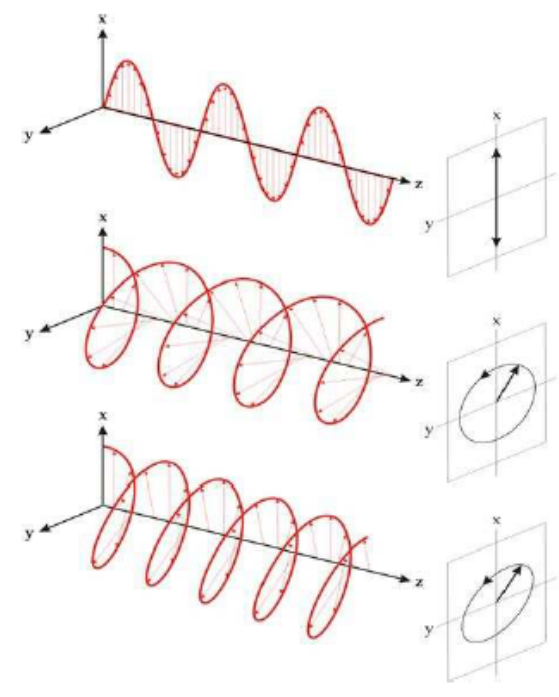

(a)

(b)

(c)

Figure 158 Polarization states of light. (523)

Depending on the magnitude of $\mathrm{x}$ and $\mathrm{y}$ oscillations as well as their difference in phase, three different states of polarization are possible: If the phases are equal, the resulting ellipse degenerates into a straight line (linear polarization) (Figure 158 (a)). If the phase difference is $+/-90^{\circ}$ the ellipse becomes a circle (circular polarization) (Figure 158 (b)). Thus, linear and circular polarization are specialized cases of the general elliptical state. For all other phase differences, a "true" ellipse evolves. (elliptical polarization) (Figure 158 (c))

\subsection{The Proper Coordinate System}

In Ellipsometry, when a beam of light hits a surface under obligue incidence, the angle of incidence (AOI) equals the angle of reflection. The incident and the reflected beam span is called as the plane of incidence. (Figure 159) Light which is polarized 
parallel to this plane is named p-polarized (p-polarised) whereas polarization direction perpendicular is called s-polarized (s-polarised).

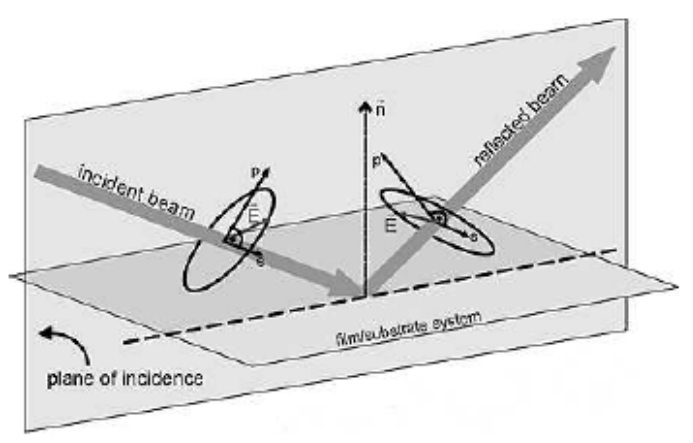

Figure 159 Reflection from the sample (film/substrate system) changes the ellipse of polarization. (523)

The directions of $\mathrm{x}$ and $\mathrm{y}$ oscillations are described as parallel to the plane of incidence (for $\mathrm{x}$ ) and perpendicular (for $\mathrm{y}$ ). These directions are re-named as $\mathrm{p}$, which means parallel (for $\mathrm{x}$ ) and $\mathrm{s}$, which means perpendicular (for $\mathrm{y}$ ) when the plane of incidence is considered. Thus, the electric field $\mathrm{E}$ is split into its $\mathrm{p}$ and $\mathrm{s}$ components.

\subsection{Reflection at Surfaces}

Ellipsometry investigates how p- and s- components change in relation to each other upon reflection. The sample is irradiated with a known polarization of light and the reflected polarization is measured. (526) Thus, the fundamental equation of ellipsometry is the following $(\mathrm{R}(\mathrm{p}, \mathrm{s})$ : reflectivity):

$$
\tan (\psi) \mathrm{e}^{\mathrm{i} \Delta}=\frac{\mathrm{R} p}{\mathrm{R} s}
$$

$\Psi$ represent the rate of amplitude Fresnel coefficients $r_{p}$ and $r_{s}$ for the light waves polarized in the plane $p$ (parallel with plane of incidence) and $s$ (perpendicular on plane of incidence); $\Delta$ is the phase difference between the mentioned plane $p$ and $s$. In spectroscopic ellipsometry, $(\Delta, \Psi)$ are measured by changing the wavelength of light. The sample may be composed of multiple layers of different optical properties. In this case, it would have a complex optical system with multiple reflections at the interfaces of the layers which superimpose to finally form the reflected light wave with an altered state of polarization. In particular, as a result of that the $\mathrm{p}$ and $\mathrm{s}$ components undergo different overall phase shifts and also exhibit different reflective properties, the shape and the size of the ellipse of polarization are changed upon reflection. This change is a measure of the properties of the optical system of the sample. The incident and reflected $\mathrm{E}$ vectors are connected by the reflection matrix $\mathrm{R}$ of the sample which is defined with equation 28 : 


$$
\left[\frac{E p, \text { ou }}{E s, \text { out }}\right]=\left[\begin{array}{l}
R p p \\
R s p
\end{array}\right] \chi\left[\frac{E p, \text { in }}{E s, \text { in }}\right]
$$

The concept of ellipsometry is to measure the change of the polarization state of the light wave to obtain information about the sample (the matrix R).

\subsection{Ellipsometry Set up $\backslash$ Components}

The basic components of an ellipsometer are: a light source, some optical components to modify the polarization and a detector. (Figure 160) Light is emitted by a light source and linearly polarized by a polarizer. It passes through an optional compensator which is so called as retarder (quarter wave plate) and reaches the sample. After reflection, the light passes a compensator (optional) and an analyzer, which is another polarizer and falls into the detector.

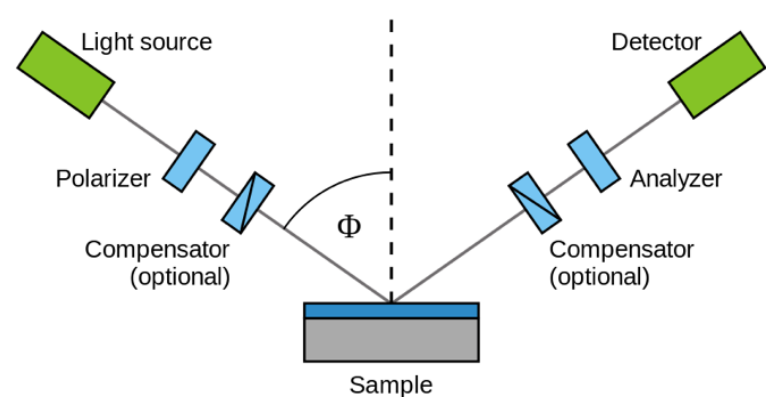

Figure 160 Schematic setup of an ellipsometry experiment. (527)

The main optical components used in ellipsometer are polarizers and retarders. A polarizer is a component that produces light in a special state of polarization by suppressing one component of the incident unpolarized light, thus allowing only the other component to pass. If the incident beam is already polarized, the transmitted intensity will depend on the amplitude of the component of $\mathrm{E}$ along the axis of the polarizer. In this case the polarizer is called an analyser since it measures the ratio of the $\mathrm{p}$ and s components. Optical retarders are used to shift the phase of one component of the incident light. A typical retarder is a "quarterwave plate" which has a "fast" and a "slow" axis leading to a phase shift of $90^{\circ}$ in the components of $\mathrm{E}$ along these axes. Depending on the orientation of the quarter-wave plate it transforms the ellipse of polarization. Forexample, linearly polarized light is transformed into circularly polarized light when the orientation of the quarter-wave plate is set to $45^{\circ}$ in respect to the linear polarization axis. If the $\mathrm{s}$ and $\mathrm{p}$ amplitudes are equal at the input, a rotatable combination of a linear polarizer $\mathrm{P}$ and a quarter-wave compensator $\mathrm{C}$ (PC) acts as a variable polarization filter that can generate any desired elliptical state of polarization at the output. 


\subsection{Single Wavelength Ellipsometry}

Single-wavelength ellipsometry utilizes a monochromatic light source which is usually a laser in the visible spectral region (e.g. a HeNe laser with a wavelength of $632.8 \mathrm{~nm}$ ). The advantage of this ellipsometry technique is that laser beam can be focused on a small spot size. Since lasers have a higher power than broad band light sources, laser based ellipsometry can be used for imaging. The biggest disadvantage of this technique, the limitation of experimental output. Only one set of $\Psi$ and $\Delta$ values is obtained per measurement.

\subsection{Spectroscopic Ellipsometry}

The term "spectroscopic ellipsometry" means ellipsometric measurements being carried out at a variety of wavelengths of the incident beam. A spectroscopic ellipsometry measurement provides large set of $\Delta \& \Psi$ angles (for every wavelength) as a function of photon energy. Due to the fact that Spectroscopic Ellipsometry (SE) utilizes broad band light sources, which cover a certain spectral range in the visible (VIS) and parts of both the ultraviolet (UV) and the near infrared (NIR) range of the electromagnetic spectrum., it is possible to obtain complex refractive index or the dielectric function tensor in the corresponding spectral region, which allows the characterization of complex multilayer systems even when several physical parameters are unknown. Thick layers, complex materials and multilayer systems usually cannot be characterized by using single wavelength ellipsometry.

Spectroscopic Ellipsometry allows simultaneous determination of several optical parameters (refractive index, extinction coefficient, film thickness, roughness anisotropy, etc.) of complex multilayer coatings. The more unknown parameters are included in an optical model, the more independent sets of $\Delta \& \Psi$ are needed to perform reliable numerical fitting of the wanted parameters. Spectroscopic ellipsometry yields enough information from the sample to make modelling feasible even when several physical parameters of the sample have to be determined. In most of the cases, the optical constants of the materials included in a complex sample are known as a function of photon energy and can be loaded into an optical model from a database. Typical fields of applications of spectroscopic ellipsometry are summarized as (Figure 161) :

- multilayer stacks with more than one unknown layer thickness

- characterization of volume fractions or compositions of binary material layers via effective medium theories

- $\quad$ surface roughness 
- material-void volume fraction, e.g. in a layer of nanoparticles

- alloy compositions

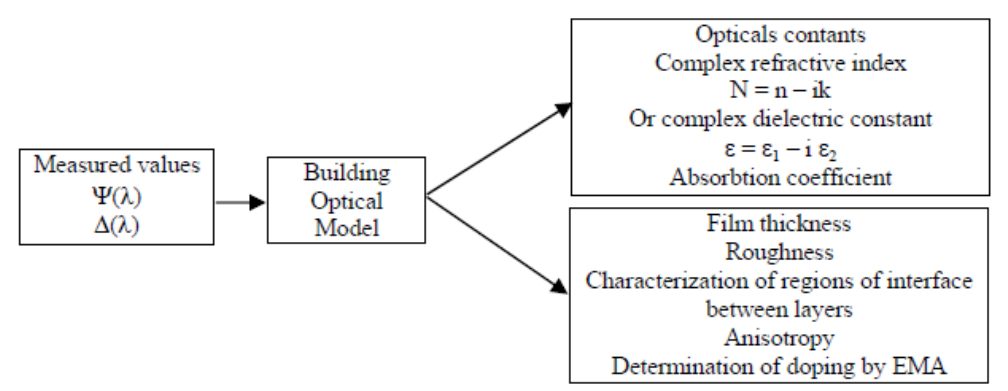

Figure 161 Characterization of physical properties by spectroscopic ellipsometry. (525)

Compared to single wavelength ellipsometry, main advantage of spectroscopic ellipsometry is the fact that it allows calculation of both the optical constants and layer thickness of a thin film layer simultaneously by using dielectric function models (e.g. Tauc-Lorentz model, Cauchy model, or Drude model). These models assume a functional relationship between the material's electric permittivity $\varepsilon$ and the vacuum wavelength $\lambda$ (or equivalent photon energy) of the probing light. Each function model has a certain small number of free parameters whose values are obtained from numerical optimization of the optical model that is chosen for the sample. The relationship $N=\sqrt{\varepsilon}$ then yields the complex refractive index $N$ that is defined as $N=\mathrm{n}+\mathrm{ik}$ with the refractive index $\mathrm{n}$ and the extinction coefficient $\mathrm{k}$.

\subsection{Imaging-Nulling Ellipsometry}

Ellipsometry is a macroanalysis technique, i.e., the sample size cannot be any smaller than a few millimeters. The development of imaging ellipsometry (Figure 162), which combines the power of ellipsometry with microscopy, has overcome this limitation.

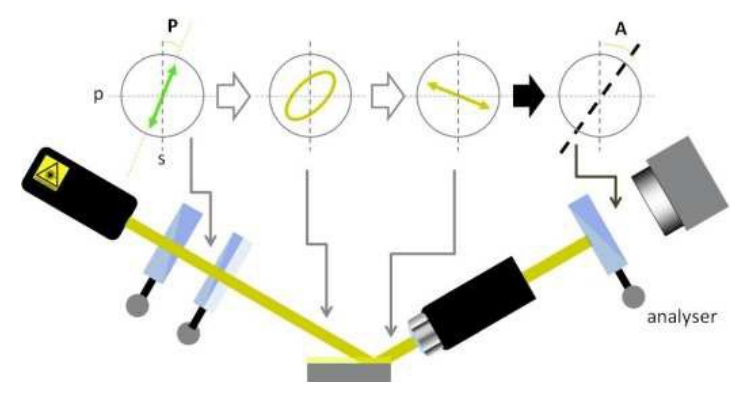

Figure 162 State of pollarisation during nulling ellipsometry. (523) 
Imaging ellipsometry is based on the concept of nulling. When linearly polarized light with an axis pointing anywhere but in the s or $\mathrm{p}$ direction is incident on a sample, polarization state of the reflected light will be elliptical. The other way around, when the same elliptical state of polarization hits the surface, it reflects in a linear state of polarization. In general, it can be concluded that using the right PC combination always results in an elliptical polarization which can produce a perfect linearly polarized reflection. This particular state can be detected by using a second polarizer as an analyzer in the reflected beam. A linearly polarized beam can be extinguished by setting the analyzer to a $90^{\circ}$ position with respect to the axis of the linear polarization. This is called "nulling", which is equivalent to finding a minimum in the signal of a photo-detector. In Nulling ellipsometry, the film under investigation is placed onto a reflective substrate. The film and the substrate have different refractive indexes. In order to obtain data about film thickness, the light reflecting off of the substrate must be nulled. Iterative routines can be found that allow one to actually find the right angle settings for $\mathrm{P}, \mathrm{C}$ and $\mathrm{A}$ to fulfill the Null condition. The most commonly used method is "fixed compensator nulling scheme". In this method, the compensator is fixed at a certain angle whereas polarizer and analyzer are rotated. A rotation of polarizer is followed by a rotation of analyzer while keeping polarizer at its minimum signal position which leads to a Null. The light source consists of a monochromatic laser of the desired wave length. (528)

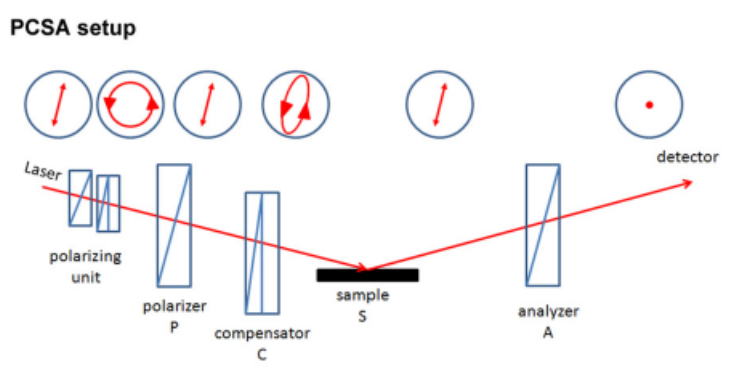

Figure 163 PCSA (Polarizer-Compensator-Sample-Analyzer) setup commonly used in Nulling ellipsometry. (529)

Typically, imaging ellipsometers are fabricated in such a way so that the laser (L) fires a beam of light which immediately passes through a linear polarizer $(\mathrm{P})$. The linearly polarized light then passes through a quarter wave length compensator (C) which transforms the light into elliptically polarized light. (530) This elliptically polarized light then reflects off the sample (S), passes through an analyzer (A). This design is called as a LPCSA configuration. (Figure 163) The orientation of the angles of $\mathrm{P}$ and $\mathrm{C}$ should be chosen in such a way that the elliptically polarized light is completely linearly polarized after it is reflected off the sample. Another approach for fullfilling Nulling conditions is PSCA set up. (Figure 164) 


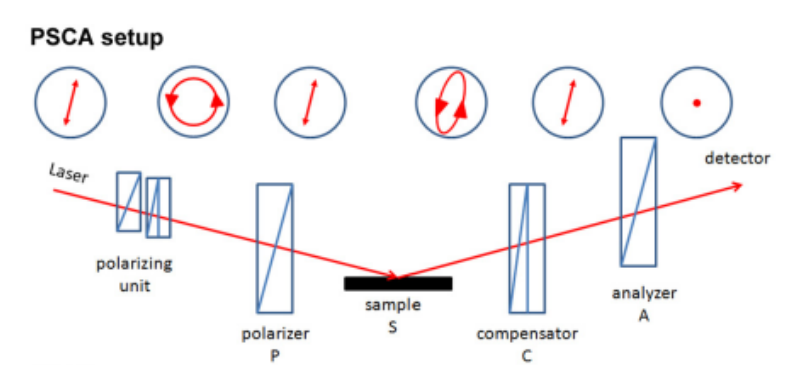

Figure 164 PSCA (Polarizer-Sample-Compensator-Analyzer) setup commonly used in Nulling ellipsometry. (529)

The ellipsometric null condition is obtained when the analyzer is perpendicular with respect to the polarization axis of the reflected light achieving complete destructive interference (the state at which the absolute minimum of light flux is detected at the $\mathrm{CCD}$ camera). The angles of the polarizer $(\mathrm{P})$ and the analyzer $(\mathrm{A})$ are adjusted to match the condition $\mathrm{I}(\mathrm{P}, \mathrm{A})=0$; the intensity at the detector is 0 . The angles of $\mathrm{P}, \mathrm{C}$, and A obtained are used to determine the $\Psi$ and $\Delta$ values of the material with the equations 29 and 30 respectively. (530)

$\psi=\mathrm{A}$

$\Delta=2 P+\pi / 2$

Eq 28

Where $\mathrm{A}$ and $\mathrm{P}$ are the angles of the analyzer and polarizer under null conditions respectively. If a quarter wave plate is used as compensator (C) and fixed in $45^{\circ}, \Psi$ and $\Delta$ are given by the equations 31 and 32 respectively:

$\Psi=|A A| \Delta=2 P P \pm 90^{\circ}$ for $\mathrm{C}=-45^{\circ}$

$\Psi=|A A| \Delta=-2 p p \pm 90^{\circ}$ for $\mathrm{C}=+45^{\circ}$

A model based on Fresnel's equations, is used to fit the film thickness $d$ and the refractive index n. (531) Main advantage of nulling ellipsometry is the fact that one measures angles instead of light flux, thus partly avoiding problems of the stability of the light source or non-linearity of the detectors. When an objective and a spatially resolving detector, e.g. a sensitive CCD camera are added to the Ellipsometer, it is called as Imaging Ellipsometry. The objective images the illuminated area of the sample onto the camera. As a consequence, areas that have different optical properties cause a different signal in the camera image. Especially those areas that are currently fulfilling the condition of the ellipsometric "Null", where the optical parameters are such that the light reflected is extinguished for that particular setting of $\mathrm{P}, \mathrm{C}$ and $\mathrm{A}$, and will appear dark in the image. Where this condition is not met higher light intensities are incident at the detector, producing brighter image regions. Changing the 
settings of $\mathrm{P}, \mathrm{C}$, and $\mathrm{A}$ it is now possible to find the Null for these regions, which will cause the former dark areas to appear bright.

The main advantage of such an imaging ellipsometer is that the signal one gets is not the average over an entire laser beam spot on the sample, but spatially resolved to show the details of the sample. By using the imaging ellipsometer, it is possible both to get immediate qualitative information and to restrict ellipsometric analysis to a particular region of interest within the field-of-view. Due to the fact that the imaging is done at an inclined observation angle, only a limited area of the entire field of view appears to be well-focused when using conventional optics. The Imaging Ellipsometer overcomes this limitation by using a motorized focusing mechanism to collect a series of images with different foci within the region of interest. All of the images are then compiled into a single, in focus image of the sample. Applying proprietary algorithms allows one to map the Nulls for the entire image, if necessary. This yields a two-dimensional map of the ellipsometric data that can be transformed into a thickness map of the sample or another quantity.

\subsection{Data Analysis $\backslash$ Optical Modelling}

Ellipsometry measures the complex reflectance ratio, $\rho$, of a system, which is quantified by the amplitude component $\Psi$ and the phase difference $\Delta$. The polarization state of the light incident upon the sample is decomposed into $s$ component (oscillating perpendicular to the plane of incidence, parallel to the sample surface) and $p$ component (oscillating paralel to the plane of incidence). The amplitudes of the $s$ and $p$ components, after reflection are normalized to their initial values and symbolised with $R_{s s}$ and $R_{p p}$ respectively. The ratio of these complex reflection coefficients $R p p$ and Rss, is the actual value which is measured by Ellipsometer and called as the complex reflectance ratio, $\rho$ (a complex quantity) which is determined by equation 33.

$\rho=\frac{R p p}{R s s}=\frac{|R p p|}{|R s s|} \times \mathrm{e}^{\mathrm{i}(\delta p p-\delta s s)}=\tan (\Psi) \mathrm{e}^{\mathrm{i} \Delta}$

$\Psi$ is an angle whose tangent gives the ratio of amplitude change for the $\mathrm{p}$ and $\mathrm{s}$ components and upon reflection :

$$
\tan (\Psi)=\frac{|R p p|}{|R s s|}
$$

and $\Delta$ denotes the relative phase shift (difference) of the $\mathrm{p}$ and s component upon reflection :

$$
\Delta=\delta \mathrm{pp}-\delta \mathrm{ss}
$$


A maximal difference should have been achieved by adjusting the angle of incidence to a value which is close to the Brewster angle. (532) Since ellipsometry is measuring the ratio (difference) of two values (rather than the absolute value of either), it is very robust, accurate, and reproducible. The result of nulling is a set of angles of $\mathrm{P}, \mathrm{C}$ and A. There are formulas that relate these numbers to the ellipsometric angles $\Delta$ and $\Psi$ and thus to the reflection matrix R. It is symbolically shown with equation 36 :

$\Psi, \Delta=f(P, C, A)$

Eq 34

In general, it is not possible to calculate optical constants directly from the ellipsometric angles due to the fact that the formulas that describe $\mathrm{R}$ as a function of these parameters are complicated and can not be inverted. Therefore, one has to develop a layer model which considers the optical constants (refractive index or dielectric function tensor) and thickness parameters of all individual layers of the sample, which are defined in the right order. (Figure 165) Unknown optical constants and/or thickness parameters are varied by using an iterative procedure (least-squares minimization) and $\Psi, \Delta$ values are calculated by using the Fresnel equations. The calculated $\Psi$ and $\Delta$ values (model) are compared with the measured values (experimental data), which is called as "fitting" procedure. In terms of the fitting, the film thickness in the model should be changed until the resulting values of $\Delta$ and $\Psi$ similar to the measured data. The ones which match the experimental data best provide the optical constants and thickness parameters of the sample. This can be a tedious and complicated task, especially if more than one parameter is to be deduced. The optical modeling can be regarded as the most critical point in ellipsometry.

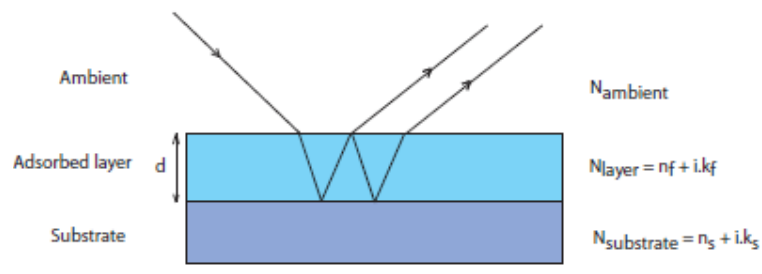

Figure 165 Three phase model for ellipsometric measurement. (57)

Two measurable real quantities are obtained via a single nulling measurement. Thus it is in principle possible to measure e.g. a complex index of refraction or the real index of refraction plus a film thickness or another combination of two real numbers. However, often one needs to measure more than this since multilayer systems are investigated, e.g. two thicknesses plus two refractive indices for a double layer system. To accomplish this task, the number of independently measurable quantities should be increased. This can be achieved by doing spectroscopic ellipsometry, which measures at different wavelengths, where each wavelength introduces a new unknown refractive index (due to dispersion) but provides two new values for $\Delta$ and $\Psi$. Since it requires 
very extensive mathematical apparatus and a lot of numerical calculus, it is a very tricky task to determine all unknowns for a multilayer system.

\subsection{Equipment Properties $\backslash$ Measurement Conditions}

Ellipsometry measurements were taken on an imaging spectroscopic ellipsometer (EP4_SE, Accurion) with EP4_mahl (Accurion) software for modeling. Measurements at five spots per sample were taken with light incident at $55^{\circ}$ angle from the surface normal using wavelengths from 400 to $720 \mathrm{~nm}$. Optical constants for a bare gold substrate, cut from the same wafer as the samples to be characterized, were measured by ellipsometry and used as the baseline for all polymer film samples. Film thickness of the polymer layer on samples, regardless of modification, was obtained using a $\mathrm{n}, \mathrm{k}$ fixed model, allowing the modeling software to fit thickness. Refractive indices were fixed at 1.45 for all respective surface modifications and brushes.

\subsubsection{Attaneuted Total Reflectance Infrared (ATR-IR) Spectroscopy}

\subsection{Definition $\backslash$ Basic Principle}

Infrared (IR) spectroscopy is a non-destructive spectroscopic technique used for functional group characterization. Infrared spectroscopy is divided into three categories: far $\left(4 \sim 400 \mathrm{~cm}^{-1}\right)$, mid $\left(400 \sim 4,000 \mathrm{~cm}^{-1}\right)$, and near-infrared $(4,000 \sim$ $\left.14,000 \mathrm{~cm}^{-1}\right)$. (533) Mid-infrared region is used for measuring organic compounds. The basic concept behind this spectroscopic technique is measurement of the different frequencies at which the sample absorbs and the intensities of these absorptions. The fact that each chemical functional group absorbs radiation at a specific frequency allows identification of the sample's chemical composition by determination of these specific frequencies. When an infrared light interacts with the matter, chemical bonds stretch, contract and bend. As a result, a chemical functional group tends to adsorb infrared radiation in a specific wavenumber range regardless of the structure of the rest of the molecule.

An IR spectrum is produced by absorption of energy due to vibrations of polar covalent bonds. The detected vibrations of the molecules in related sample, caused by IR-light, enable drawing conclusions on its chemical composition. Absorption bands of substances are generated during each analysis and can directly be assigned to a certain molecular bond (e.g. $\mathrm{C}=\mathrm{O}$ ) or to frequencies of functional groups. Fourier Transform Infrared Spectroscopy provides specific information about chemical bonding and molecular structures, making it useful for analyzing organic materials and certain inorganic materials. FTIR is an improved technique to make the IR measurements easier and faster, in which the IR beam is guided through an interferometer. A FTIR spectrum is obtained from performing a mathematical Fourier Transform on the interferogram. The obtained spectra was evaluated by interpreting 
the bands of the FTIR directly and tentatively assigned to chemical compounds, based on published data.

\subsection{ATR-IR Set Up \Working Principle}

ATR (Attenuated Total Reflection)-FTIR is a method for analyzing solid samples using infrared spectroscopy. (Figure 166) This method is based on the measurement of absorption from the reflected beam when an infrared beam is reflected off the sample. Unfortunately, the reflected radiation generally has a very low intensity. In order to overcome this limitation, the infrared beam is bounced through a crystal such as $\mathrm{ZnSe}$ or Ge at an angle such that the beam is totally internally reflected.

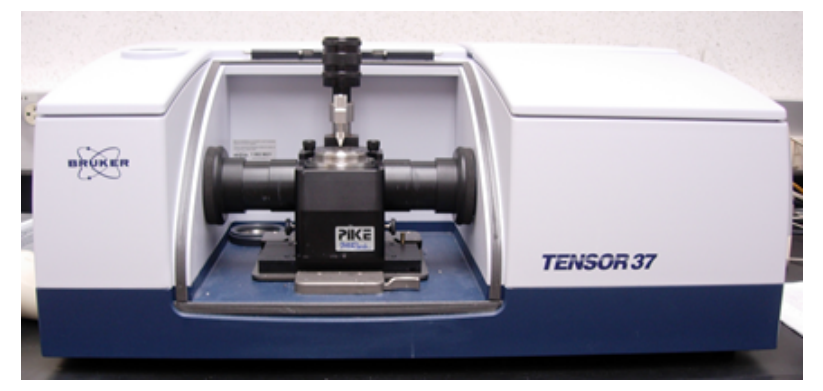

Figure 166: A typical ATR-IR spectrometer. (534)

The solid sample which sits on the crystal, is pressed onto the crystal to ensure contact to the evanescent IR field. (535) IR radiation is focused onto the end of the crystal and than reflects across the length of the crystal. Thus, the evanescent IR radiation penetrates several ten to hundred nanometers from the surface of the crystal into the material and finally will be reflected off the sample. (Figure 167) The ATR-FTIR setup which adds a universal sampling accessory to FTIR provides reproducible qualitative and quantitative analysis of thin films and is a non-destructive method for determination of different coating characteristics such as the chemical composition or the presence of different chemical functions. Therefore, a sample can be analyzed several times. FTIR-spectra of solid, liquid or gaseous samples are generated in a few minutes.

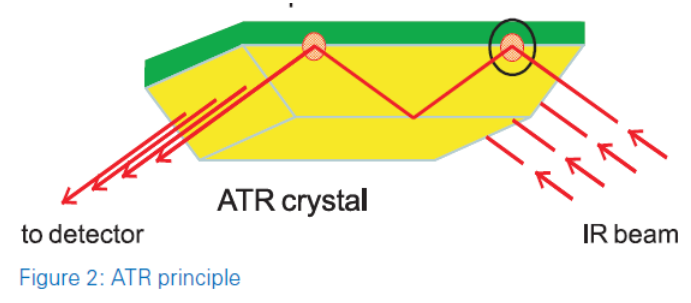

Figure 167 ATR-FTIR setup to measure coating characteristics on the coated substrate. (536) 
A deuteriumtriglycine-sulfate (DTGS) -detector, frequently used in modern FTIR spectroscopes, allows analyzing measurements of spectra within a frequency range from $600-4200 \mathrm{~cm}^{-1}$. However, especially in the wave number area between 1800 and $600 \mathrm{~cm}^{-1}$, the so-called fingerprint region, the bonds can be directly interpreted and ascribed to a particular chemical composition in the sample. The spectra can be analyzed quantitatively (quantities of components in the sample), qualitatively (eductproduct comparison to follow reactions; direct interpretation of spectra, comparison with a reference spectrum)

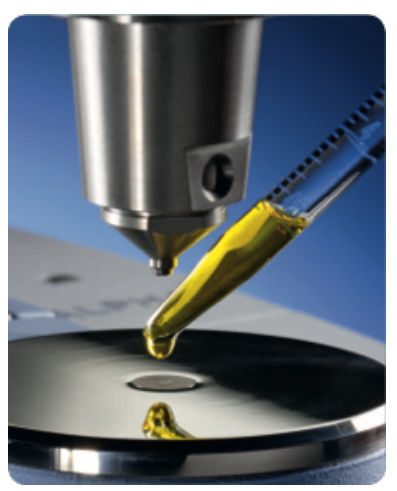

Figure 168 Diamond ATR accessory in Bruker Optics’ ALPHA. (536)

An IR transparent material with a high refractive index is used as the ATR crystal. (Figure 168) The infrared beam enters the ATR crystal at an angle of typically $45^{\circ}$ and is totally reflected at the crystal to the sample interface. The light is not reflected directly on the boundary surface due to its wave like properties. Instead, it is reflected by a virtual layer within the optically less dense sample. (Figure 169) The fraction of the light wave that reaches into the sample is called the evanescent wave. Penetration depth of the evanescent wave depends on the wavelength, the refractive indices of ATR crystal and sample and the angle of the entering light beam.

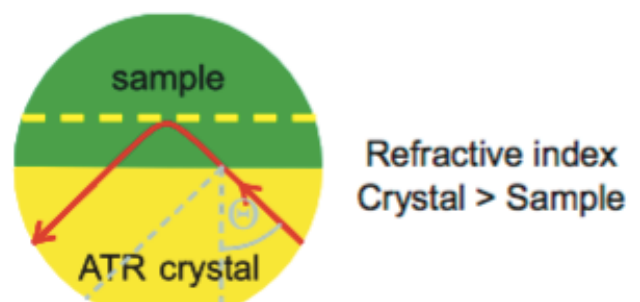

Figure 169 The reflection of light by a virtual layer within the optically less dense sample (Goos-Hänchen effect; dotted yellow line). (536)

The refractive index of the crystal must be considerably higher than that of the sample (Table 16). As the typical refractive indices for ATR crystals are between 2 to 4 and typical values for organic substances (e.g. polymers) range from ca. 1.2 to 1.5, a large majority of IR active samples can be measured. The light beam typically penetrates 
into a few microns (ca. 0.5 - $3 \mu \mathrm{m}$ ). The evanescent wave will be attenuated in spectral regions where the sample absorbs energy. After one or several internal reflections, the IR beam exits the ATR crystal and is directed to the IR-detector. Good sample contact can be obtained by utilization of modern small ATR crystals and robust pressure clamps. Crystal materials generally used for ATR are diamond, zinc selenide ( $\mathrm{ZnSe}$ ) and germanium. Their properties are listed in the following table:

Table 16 ATR crystal types (536)

\begin{tabular}{|l|l|l|l|l|}
\hline Material & $\begin{array}{l}\text { Spectral } \\
\text { region }\left(\mathrm{cm}^{-1}\right)\end{array}$ & $\begin{array}{l}\text { Refractive } \\
\text { index }\end{array}$ & $\begin{array}{l}\text { Depth of } \\
\text { penetration } \\
\text { at } 45^{\circ}, \\
1000 \mathrm{~cm}^{-1} \\
(\mu \mathrm{m})\end{array}$ & $\begin{array}{l}\text { Hard- } \\
\text { ness } \\
(\text { Knoop })\end{array}$ \\
\hline $\mathrm{ZnSe}$ & $20,000-500$ & 2.43 & 1.66 & 130 \\
\hline $\mathrm{ZnS}$ & $22,000-750$ & 2.25 & 1.54 & 355 \\
\hline $\mathrm{Ge}$ & $5,000-600$ & 4.01 & 0.65 & 550 \\
\hline $\mathrm{Si}$ & $10,000-100$ & 3.42 & 0.81 & 11150 \\
\hline Diamond & $45,000-10$ & 2.40 & 1.66 & 9,000 \\
\hline
\end{tabular}

Since diamond is very robust and chemically inert, it is an ideal crystal material for ATR measurements which allows measurement of a wide range of samples. Another advantage of using diamond as crystal is that diamond cannot be scratched, damaged and is completely insoluble. Moreover, as the diamond ATR spectrum shows stronger signals due to the higher penetration depth compared to other crystal types, it is commonly preffered for thin film characterization.

\subsection{Equipment Properties $\backslash$ Measurement Conditions}

The FTIR-ATR spectra of MUD layer, CTA layer, and polymer brush layers were recorded in the wave number range from 4500-600 $\mathrm{cm}^{-1}$ with an Equinox 55 spectrometer (Bruker Optics, Ettlingen, Germany) including a deuteriumtriglycinesulfate-detector and an attached ATR-unit (DuraSamplIR, SensIR Europe, Warrington, England). The unit has a plunger on the top side which is pressed on the sample with constant pressure. FTIR-ATR measurements were conducted with a resolution of $4 \mathrm{~cm}^{-1}$ and a number of 32 scans per five different areas of each sample. OPUS 6.5 software (Bruker Optics, Ettlingen, Germany) was used for spectra evaluation.

\subsubsection{Atomic Force Microscopy (AFM)}

\subsection{Definition $\backslash$ Basic Principles}

The atomic force microscope is one type of scanning probe microscopes (SPM) with a resolution in the nanometer regime, which allows true 3D-profiling of nonconductive surfaces (537) (in comparison to a scanning tunneling microscope, which was invented earlier, but is only capable to probe conductive surfaces). (538) Main 
difference of AFM from other optical or electron microscopes which assemble two dimensional pictures using the reflection amplitude of photons or electrons from the surface is that a cantilever moves within the surface potential of the sample and collects a height profile. Optical or electron microscopy can in principle obtain height information from several pictures. This requires a large $\mathrm{z}$ resolution in order to achieve high resolution. Main drawback of optical microscopy is that it is difficult to achieve high resolution as a result of that the wavelength is not small enough and interference efects arise. In the case of electron microscopy, focusing is troublesome which might lead to errors. (539)

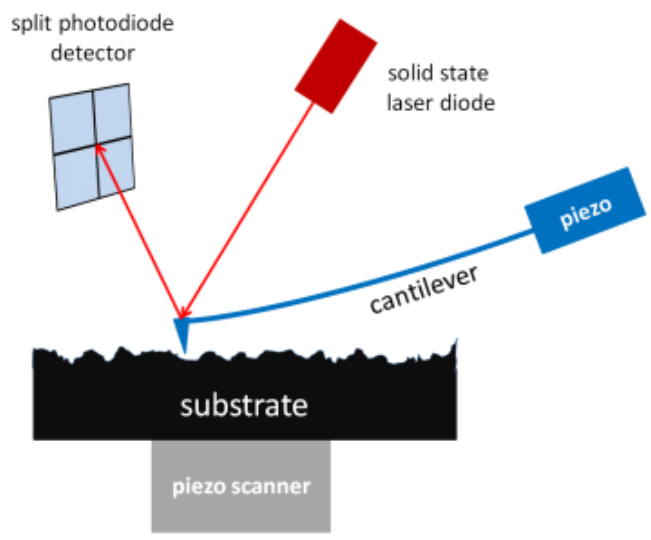

Figure 170 General setup of an atomic force microscope with beam deflection detection. (540)

In contrast, AFM is more advantageous than these techniques in many situations. The main principal is based on a small tip which is scanned across the surface and thus probes attractive and repulsive forces on a flat spring (cantilever) arising from the surface. The widely used beam deflection detection setup of an AFM is shown in Figure 170. Depending on the forces on the cantilever it will bend in z-direction according to Hook's law (not considering lateral forces which will also cause torsion), the bending is detected by a laser beam, deflected from the cantilever onto a split photodiode detector. An electrical feedback loop controls the interaction between a piezo scanner and the photodiode, resulting in a topographical image of the surface.

\subsection{AFM Imaging Modes}

There are various imaging modes available for capturing AFM images. Depending on the nature of the sample and the desired information about the surface, different modes which are operated in different ranges of the surface potential can be utilised for AFM measurements. (541) The bending of the cantilever is dependent on the surface potential which is divided into three sections. (Figure 171) There is no interaction far from the surface, that is, the force to the cantilever is zero. Getting closer to the surface results in that the cantilever is pulled onto the surface by an attractive 
force caused e.g. by Van der Waals forces. There is a minimum in the surface potential while the cantilever is being driven towards the surface. Even closer to the surface, the force becomes repulsive and the tip is rejected from the surface. These repulsive interactions are based on e.g. Van der Waals forces, dipole-dipole interaction and coulomb repulsion.

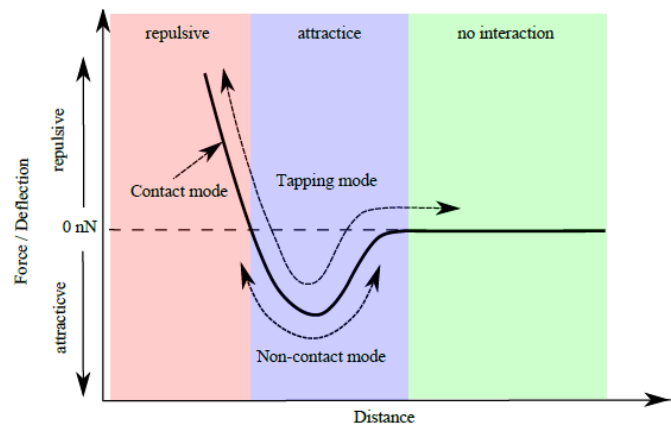

Figure 171 Plot of a surface potential and application area for contact, non-contact and tapping mode. The force-distance curve is divided into three sections. There is no interaction between tip and surface in the region shown with green. The attractive regime is demonstrated with blue. Closer to the surface, the tip is rejected in the repulsive regime demonstrated with pink. (542)

One basic operation type is the contact mode in which, the tip is dragged across the surface far in the repulsive section of the surface potential. It is a static mode operation technique. The feedback loop regulates a constant cantilever deflection, that is, the bend angle is kept constant by the z piezo during the scan, thus the force between tip and sample remains constant. The distance which the scanner moves vertically at each $(\mathrm{x}, \mathrm{y})$ data point is stored by the computer in order to form the topographical image. Main advantages of the contact mode are the highest atomic resolution and high scanning speed. Main drawback is that soft samples may be damaged as a result of that the surface forces in contact mode are very high.

On the contrary of the contact mode, non-contact mode works in the attractive regime. It is a dynamic mode operation technique in which the piezo in the cantilever holder is used to excite the cantilever at its resonance frequency. The detection is an AC signal which characterizes the cantilever motion. The cantilever oscillates with an amplitude of a few nanometer and it does not contact the surface, thus the tip-sample interaction is weak. While scanning the sample, the cantilever`s oscillating amplitude is decreased by van der Waals forces and the feedback loop keeps the oscillation amplitude constant by regulating the distance between tip and surface with the z-piezo, that is, by vertically moving the scanner at each $(\mathrm{x}, \mathrm{y})$ position until a set point amplitude is reached. From this information the topographical image is calculated. The advantage of the noncontact mode technique is that also soft samples can be analyzed and the tip wear is very low. A limiting factor for noncontact mode in air is a contamination layer, present 
on most surfaces under ambient conditions. This layer causes a shielding of the tipsurface interaction and restricts the resolution to a few nanometers.

A third operation mode is the tapping mode so called as AC mode. The cantilever is oscillating similar to the non-contact mode but near its resonance frequency, resulting in amplitudes of $20 \mathrm{~nm}$ to $100 \mathrm{~nm}$. The tip lightly "taps" on the sample surface during scanning, getting in direct contact with the surface for a very small fraction of the oscillation period and is inuenced from attractive as well as repulsive regime. When imaging in air, the typical amplitude of the oscillation allows the tip to contact the surface without getting stuck. By vertical movement of the piezo scanner, a constant oscillation amplitude is maintained. Thus, information about the topography is obtained, as well as information about a phase shift during oscillation due to different mechanical properties of the sample surface. (543) Main advantage of Tapping mode over other operation types is a low sample wear with a good resolution. Compared to contact mode, tapping mode does not apply so much force to the sample since the cantilever only taps on the surface. Compared to non-contact mode, there is no "instability" problem due to noise sensitivity in Tapping mode AFM and is not inuenced by the contamination layer as the tip is able to pass through it onto the sample surface. The tapping mode operation is preffered for this work due to several reasons summarized below:

- A high resolution is essential in order to analyze the surface roughness.

- Reproducible image conditions are important to sucsessfully compare all samples (prepared with different approaches) eachother. Since it is not as susceptible to noise as the non-contact mode, it is easier to work in identical conditions using tapping mode.

-The tapping mode is commonly used for polymeric substrates or other soft materials.

\subsection{AFM Set Up}

An AFM generally consists of three components: the microscope stage, control electronics and a computer software. The whole setup of an AFM is shown in Figure 172. All measurement instruments are located in a hood to decrease noise from the environment such as temperature fluctuations resulting from air conditioning. The microscopy stage which consists of a sample holder and a mechanical positioning system is located on a vibration damping platform in order to increase the resolution. 


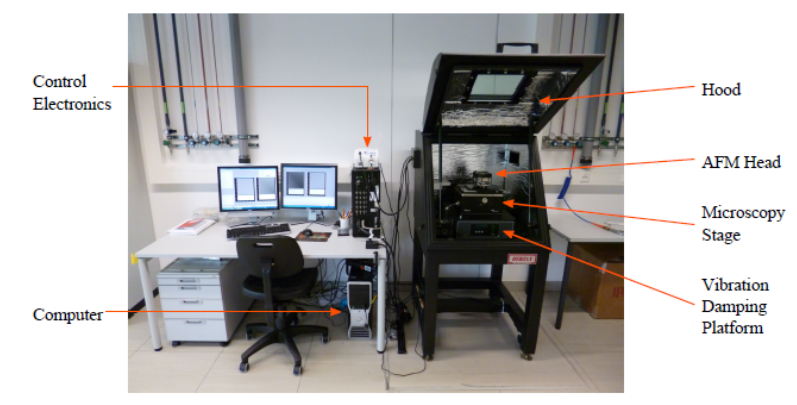

Figure 172 A typical AFM set up. (542)

The AFM head which is responsible for the scanning mechanism sits on top of the microscopy stage. This head contains piezoelectric transducers and force sensors used for controlling and monitoring the AFM tip. The control electronics which are responsible for the fast data processing connects the AFM head and the computer by generating the signals to drive the scanner and digitizing the signals coming from the AFM. These digitized datas are recorded by the computer and processed by the software to be converted into the images. The scanning process is determined by the user in the computer software which sends this information to the control electronics.

\subsection{Working Principle}

The AFM head includes the integral elements used for the height measurement. A super luminescent diode shines on a cantilever including a sharp tip which has a radius of a few nanometers at its bottom. The light is reflected of the back of the cantilever, collimated through a lens system and finally reachs a photo detector via an adjustable photo detector mirror. This cantilever position detection scheme is called optical lever detection. (Figure 173) The photo detector is a photo diode which is divided into four quadrants, each of which generates a voltage proportional to the amount of light shining on it. The sum signal is a measure for the total amount of light collected by the photo detector and shown in equation 37 :

$\mathrm{V}_{\mathrm{Sum}}=\mathrm{V}_{\mathrm{A}}+\mathrm{V}_{\mathrm{B}}+\mathrm{V}_{\mathrm{C}}+$

The deflection voltage is the difference between the voltages generated in the top and bottom half of the photo diode, which is determined with equation 38 :

$\mathrm{V}_{\text {Def }}=\mathrm{V}_{\text {top }}-\mathrm{V}_{\text {bottom }}=\mathrm{V}_{\mathrm{A}}+\mathrm{V}_{\mathrm{B}}-\left(\mathrm{V}_{\mathrm{C}}+\mathrm{V}_{\mathrm{D}}\right)$

Both of them are important parameters for the position measurement of the cantilever. The position of reflected beam is determined by the angle of the cantilever. When the cantilever pushes down on a hard surface, it experiences a repulsive force and bends 
upwards, that is, the spot moves up on the detector resulting in the fact that the top voltage exceeds the bottom voltage. The resulting detection signal will be positive. When the distance between cantilever and surface is increased, the cantilever experiences an attractive force and bends down, that is, the spot moves downwards on the detector resulting in the fact that more light is detected in the bottom half of the photo diode and thus the detection voltage becomes negative.

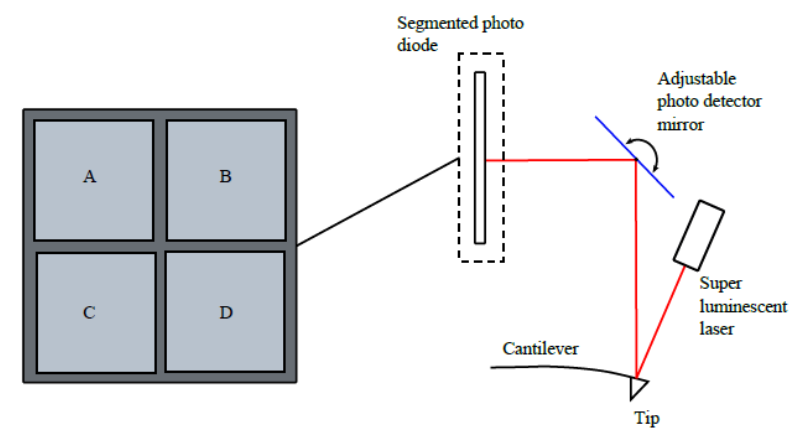

Figure 173 Schematic of the optical cantilever detection and the segmented photo diode. A laser shines onto the cantilever. The light is refected of its back and led to a photo diode via an adjustable photo detector mirror. The photo diode is segmented into four parts which generate the two basic parameters of the AFM setting the photo diode requires only a limited resolution in the micrometers regime. The $\mathrm{x}-\mathrm{y}$ - and z-position of the cantilever is controlled by piezoelectric transducers. They consist of piezoelectric materials which convert electrical potential into mechanical motion. (542)

In general, the detection signal is a function of the cantilever angle. For this reason, there might be changes of the spot position on the photo diode caused by small variations of the cantilever in the range of sub nanometers as a result of the long optical path. In order to increase the position accuracy, linear variable differential transformers are used in each direction. These transformers consist of three coils placed around a cylindrical ferromagnetic core connected to the cantilever and converts any mechanical motion of the ferromagnet into an electrical signal. In this way the AFM has an additional independent system for position control to detect and calibrate thermal drifts. During the movement of the cantilever, the distance between the cantilever tip and the sample is kept constant. The lever bends up and down according to surface structures with various heights.

However, when it comes to extracting height information from the detection signal, it is not applicable due to two main reasons: The obligation for frequent calibration of detection signal due to the fact that it is different for each cantilever and light source alignment. Because of that the detection signal is readjusted several times during the measurement, there is a reproducibility problem. Second reason is that the force between tip and sample and thus the detection signal is nonlinearly dependent on the distance between tip and sample. In addition to that, the bending of the cantilever is 
nonlinear aswell for increasing bend angles. To overcome these limitations, a feedback loop is used to control the height of the cantilever by the $\mathrm{z}$ piezo. The height of the cantilever is adjusted up or down to keep the angle of the lever constant sothat the detection signal is kept constant at a characteristic level. The control voltage of the $\mathrm{z}$ piezo then traces the height structure of the surface.

\subsection{Optimization of Scan Conditions}

The optimization of scanning parameters is very important for AFM data acquisition. Scanning process differs for various surface structures and materials. Also, the optical calibration of the laser spot and tip wear result in varying measurement parameters. A proper optimization of these parameters is crucial in order to achieve reproducible measurements.

\subsection{Cantilever Tuning}

A proper mounting of the tip in the cantilever holder is essential. The tip should be well aligned in the pocket under the spring clip. This newly placed cantilever should be tested with the "auto tune" function of the control software "IgorPro" which sweeps the oscillation frequency of the driving piezo over a set frequency range and records the oscillation amplitude of the detection signal. A power spectral density measurement should be done for determination of the frequency range. For this reason, time dependence of the thermal noise is measured. The resonance frequency of the cantilever is calculated via a Fourier analysis. This calculated frequency should match with the one supplied by the manufacturer. If they don't match, the cantilever might not be mounted correctly. Afterwards, the cantilever is excited around its resonance and the amplitude is measured at the photo detector. The transmission amplitude of the cantilever should be a single, Lorentz-shaped peak in the transmission spectrum. (Figure 174) The phase should have a 180 degree shift at the frequency of maximum transmission amplitude. Multiple or misshaped peaks in the frequency spectrum indicate a problem with the cantilever mounting. The cantilever could be broken or not correctly fixed under the spring clip. A measure for the quality of the cantilever adjustment is the fraction of the resonance frequency over the full width half maximum (FWHM) which should be in between 100 and 150 for good measurements.

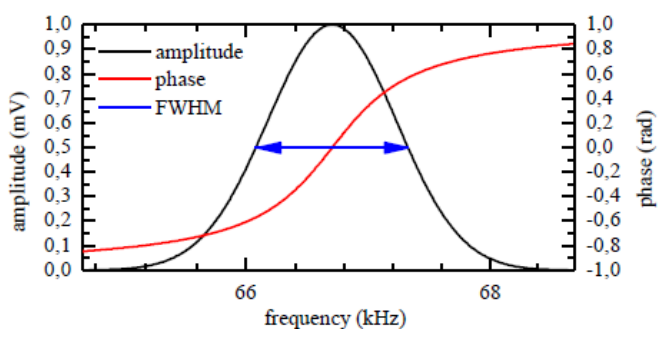


Figure 174 Idealized transmission spectrum of the auto tune process. The oscillation amplitude is depicted in black and shows a Lorentzian peak. The phase is colored red. The phase has a 180 degree shift at the frequency of maximum transmission amplitude. The full width half maximum (FWHM) is measured at half of the peak height plot. The point of maximum amplitude is chosen as operation frequency during the scan. (542)

\subsection{Scan Parameters and Imaging Conditions}

Initial scan parameters which are dependent on the surface structures on the sample have to be set for each image acquisition. Optimization of the scan area is done by setting a width to height ratio in order to scan a rectangular area. Secondly, the resolution is set. The software divides the scan area into scan lines which consist of a number of scan points. The resolution is defined by these scan lines. A high resolution improves the image quality but also increases the measurement time. The other parameter is the scan speed respectively the scan rate. The scan speed is the speed of the cantilever during scanning and the scan rate is the frequency of line scans. Calibration is generally done for the scan speed since it doesn't depend on the image size. It is important to keep scan speed constant in order to accomplish reproducible image conditions since it is responsible for image artifacts. If the scan speed is too high, the tip can not track the surface properly and the resulting image quality is not good. On the other hand, a low scan speed suffers from thermal noise which degrades the image quality. At the beginning of the measurement, it is necessary to obtain an overview of the surface structures and to position the tip nearby the region of interest (ROI).

\subsection{Choice of Cantilever Type}

The choice of the cantilever type is dependent on the AFM mode applied for image acquisition. There are two different types: diving board cantilevers and V legged cantilevers. The most common type is the diving board cantilever which consists of a rectangular leg with the tip at one end whereas the $\mathrm{V}$ legged cantilever has two legs forming a V shape. Main advantages of diving board over $\mathrm{V}$ legged cantilever is that there are more methods calibrating a diving board cantilevers and the calculation of the spring constant is easier. Therefore, diving board cantilevers whose spring constant is smaller are preffered for AC mode imaging. (544) The low spring constant makes the cantilever ideal for observing surface topography with low tip abuse. Additionally, the tip is positioned at the very end of the cantilever, which makes the tip positioning easier. 


\subsection{Equipment Properties $\backslash$ Measurement Conditions}

The morphology of the surfaces mentioned in this thesis was recorded on a MFP3D AFM from Asylum Research an Oxford Instruments company which is controlled by the software program "IgorPro". (545) The image processing is done with the free ware "WSxM 5.0 Develop 8.1" from Nanotec electronica. (546) A rectangular-shaped (Olympus AC240TS-R3) cantilever was used to acquire the images in the tapping mode. The normal spring constant of the cantilever was $2 \mathrm{~N} / \mathrm{m}$ whereas the resonance frequency was $70 \mathrm{kHz}$. The dimensions of the scanned areas were $1.00 \mu \mathrm{m} \times 1.00 \mu \mathrm{m}$. Scans were performed with 256 lines and 256 points per line at a scan rate of $1.00 \mathrm{~Hz}$.

\subsubsection{Contact Angle Meter Instrument}

\subsection{Definition and Basic Principle}

The measurement of solid/liquid/vapor contact angle is one of the most sensitive techniques commonly used for characterization of surfaces. The method is based on surface/interface energy and surface tension. Surface tension (ST) and Surface free energy (SFE) are equivalent physical terms; the first is conventionally used for liquids and the second for solids. Atoms or molecules at an interface which are not surrounded from every side differ in behaviour than molecules in the bulk substance surrounded from everyside. They either lose some of their interaction energy, in the case of the ideal surface/vacuum interface or share some of the interaction energy with the molecules in the surrounding medium. The surface tension is determined by the tendency of liquids to minimize their surface area or the occurrence of capillary phenomena. (547) In a liquid the surface tension results from the fact that a molecule at the surface is in contact with fewer neighboring molecules that it can interact with than in the bulk of the liquid. Remaining at the surface is less attractive for molecules of a pure liquid. This is why liquids attempt to achieve as small as surface area as possible; work is required to increase a surface. In principle the same applies to the SFE of solid phases. However, it is hardly possible to directly measure the amount of work required to increase a surface, as it is difficult to differentiate this work from the work of deformation of the bulk phase The SFE of a solid can be measured indirectly by using its wettability by liquids. This is where the contact angle enters the picture. The measurement of surface free energy by contact angle is based on the equilibrium at the three-phase boundary (Figure 175). When a drop of liquid is placed on a solid surface, it will modify its shape depending on the different surface/interfacial tensions, until an equilibrium is reached. 


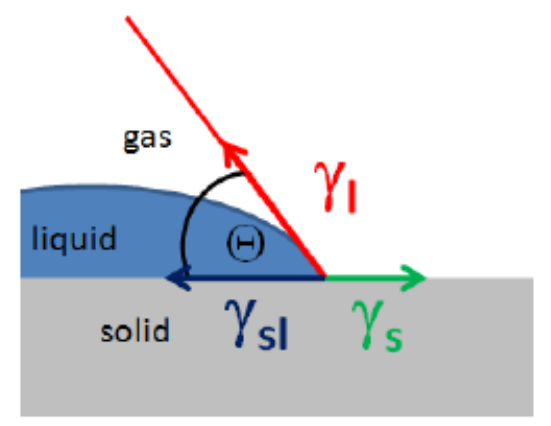

Figure 175 Contact angle and interface tensions at the three phase contact line. (529)

Two different possibilities may occur; either the liquid completely spreads on the solid or a finite contact angle $(\mathrm{CA}, \Theta)$ is established. In the second case a three-phase contact line is formed, at which all three phases (including a solid, a liquid and a gas or non-mixable liquid phase) are in contact. Thomas Young described the three-phase equilibrium by relating the contact angle to the interfacial tensions $\gamma \mathrm{s}, \gamma \mathrm{l}$, and $\gamma \mathrm{sl}$ (Figure 175) which results in the equation 39 or interfacial equilibrium. (548), (549)

$$
\gamma_{\mathrm{s}}-\gamma_{s L}=\gamma_{L} \cos \theta
$$

If $\gamma_{\mathrm{s}}$ is smaller than $\gamma_{\mathrm{sl}}\left(\gamma_{\mathrm{s}}<\gamma_{\mathrm{sl}}\right)$, the interfacial tension of the wetted surface is higher than one of the "dry" solid surface (which is in contact to air). The resulting contact angle will be larger than $90^{\circ}$ which means that the solid surface is considered to be hydrophobic in case of that the liquid is water. In the second case, if $\gamma_{\mathrm{s}}$ is bigger than $\gamma_{\mathrm{sl}}\left(\gamma \mathrm{s}>\gamma_{\mathrm{s} l}\right)$, the wetted surface is energetically more favorable than the "dry" surface. In this case, the resulting contact angle will be smaller than $90^{\circ}$ if the liquid is water and the solid surface is considered to be hydrophilic. The contact angle and the interface tension are important characteristics of a coating since coatings are often exposured to contact of liquids (usually water) in daily use. The most common and easiest method to determine the contact angle is to observe the sessile drop with a camera in front of the drop and a light source behind. And the angle is fitted by a computer using the Laplace equation.

\subsection{Goniometer Set Up}

The contact angle goniometer set up consists of a horizontal movable stage to mount a solid sample, an illumination source, a micrometer pipette to form a liquid drop and a camera to record the drop image. (Figure 176) The measurement is done by taking photographs of the drop with a camera and simply aligning the tangent of the sessile drop profile at the contact point with the surface. A detailed examination of the intersection profile can be achieved by using relatively high magnification. Additionaly, a motor driven syringe can be used to control the rate of liquid addition 
and removal which allows investigation of advancing, receding or dynamic contact angles.

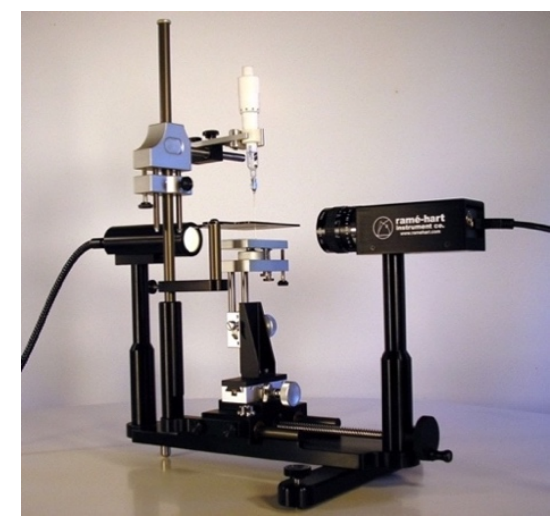

Figure 176 A goniometer set up used for contact angle measurements. (550)

\subsection{Dynamic or Static Measurement}

The contact angle describes the relationship between the surface tension of the liquid and that of the solid as well as the interfacial tension between the phases. From this relationship, the Young contact angle $\theta$ is used to describe the wetting processes with equation 40:

$$
\cos \theta=\sigma_{\mathrm{s}}-\gamma_{\mathrm{sl}} \backslash \sigma_{1}
$$

where $\sigma_{\mathrm{s}}$ and $\sigma_{\mathrm{l}}$ represent the surface tensions of the solid and liquid and $\gamma_{\mathrm{sl}}$ is the interfacial tension. The contact angle can be determined with either a constant volume (static contact angle) or a varying drop volume (dynamic contact angle) which is divided into two categories; advancing (with increasing drop volume) and receding (with decreasing drop volume) contact angle. The contact angle of a liquid is identical at all positions on a theoretical, ideal solid surface due to the lack of chemical, topographical inhomogeneities and lack of any chemical reaction between the components which occurs at the phase contact points. In an ideal system the static contact angle doesn't differ from the dynamic one; in both cases an equilibrium contact angle is formed as is described in the Young equation.

However, in practice, systems therefore contact angle values deviates from the ideal state to a greater or lesser degree as a result of that roughness affects the wettability. The real contact angles could be increased or decreased by the influences such as chemical inhomogenenities on surfaces or soluble components which may diffuse from the solid surface into the solution depending on the nature of the system. Another reason for deviation from the equilibrium value of the Young equation might be differences in energy between neighboring positions which result in occurrence of energy barriers which cause resistance to the wetting process. As a consequence, 
measurement conditions must be evaluated in order to be able to interpret the contact angle and its variations in a reasonable manner.

In advancing angle, the drop is forced to wet a neighboring position by the increase in the deposition volume. As the volume increases the contact angle initially increases, without any change in the contact area. Contact angle increases up to a point. After that point which a limiting contact angle has been achieved, angle no longer changes. Instead the drop borderline moves outward with a constant contact angle. Because the angle is measured at a 'fresh' contact line, the advancing angle method is frequently used. If an inhomogeneous surface is to be determined by static contact angle, then a large number of single drop measurements is required to obtain a reliable mean value. Static measurements are also usually appropriate when inhomogeneities are to be determined instead of being statistically eliminated. A meaningful correlation between position and wettability might be provided by measuring the static contact angles at many sample positions.

\subsection{Selecting Method and Drop Type}

The drop type describes the arrangement of sample and drop in the image. Selection of the type depends on the measuring set up used. There are different drop types used for analysis such as sessile drop, captive bubble, pendant drop. The sessile drop is the standart arrangement for contact angle measurements. (Figure 177) A drop lying on the solid surface forms a characteristic contact angle with the surface at the three-phase contact point.

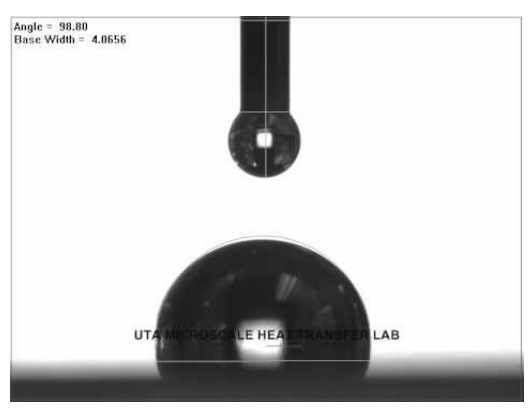

Figure 177 Sessile drop on a plain aluminium surface. (551)

\subsection{Shape Analysis and Baseline}

Drop shape analysis (DSA) is an image analysis method used for determination of the contact angle from the shadow image of a sessile drop and the surface tension or interfacial tension from the shadow image of a pendant drop. An image of the drop, which is located onto a solid sample (sessile drop) or at the tip of a needle (pendant drop), is recorded with the help of a camera and transferred to the drop shape analysis software. Determination of the contact angle is accomplished in two steps. In the first 
step, a contour recognition is carried out based on a gray level analysis of the drop image. An optically determined contour line around the phase boundary in the drop image is formed. In the second step, a geometrical model describing the drop shape is fitted to the contour, that is, this drop contour is described mathematically. The contact angle is obtained from the angle between this drop contour function and the sample surface, which is named as the baseline. (shown in Figure 178) The mathematical description of the baseline depends on its shape: a straight line equation is used for a flat surface whereas a circular function is used for rounded substrates.
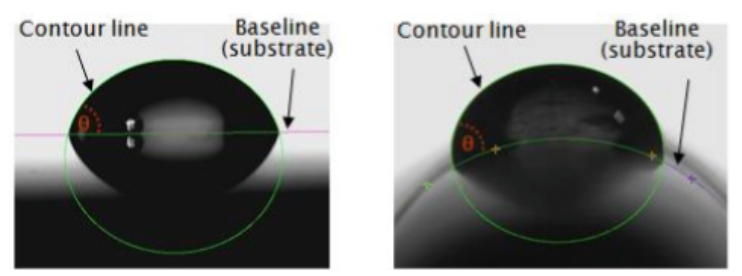

Figure 178 Contour analysis (shown in green) on a flat and a curved sample surface. (552)

\subsection{Models for Contour Analysis (Young-Laplace Method)}

There are several models in the drop shape analysis programs for mathematical description of the drop contour which is a curved line. (552) The suitability of the model to be used for analysis of the contour depends on that the actual contour follows the requirements of the model. For this reason, the programs show both the optically determined and calculated contour lines. The quality of the contour analysis depends on the agreement of these two lines. There are many methods available for contour analyses such as Circle method, Conic section method, Polynomial method and Young-Laplace method. (Table 17) The Young-Laplace fit is suitable for symmetrical drop shapes that are not affected by interferences such as sample tilting or contact with the deposition needle. The Young-Laplace method takes the characteristic drop shape under the influence of gravity into account with a sophisticated iteration method. It is also used for determination of the surface tension from the shape of a pendant drop.

Table 17 Some methods used for determination of the contact angle with different measuring ranges and applications (552)

\begin{tabular}{|c|c|c|c|c|}
\hline & Circle method & $\begin{array}{l}\text { Conic section } \\
\text { method }\end{array}$ & $\begin{array}{l}\text { Polynomial } \\
\text { method }\end{array}$ & Young-Laplace-Fit \\
\hline $\begin{array}{l}\text { Assumed drop } \\
\text { shape }\end{array}$ & Circular arc & Ellipse & $\begin{array}{l}\text { No prior } \\
\text { assumption }\end{array}$ & $\begin{array}{l}\text { Ideal sessile drop } \\
\text { oblated by its own } \\
\text { weight }\end{array}$ \\
\hline \multicolumn{5}{|l|}{$\begin{array}{l}\text { Recommended } \\
\text { measuring range }\end{array}$} \\
\hline & $x$ & & & \\
\hline$\frac{10-100^{\circ}}{100-180^{\circ}}$ & & $x$ & $\frac{x}{x}$ & $\frac{x}{x}$ \\
\hline \multicolumn{5}{|l|}{$\begin{array}{l}\text { Drop weight } \\
\text { (VolumexDensity) }\end{array}$} \\
\hline small & $\mathrm{X}$ & $\mathrm{X}$ & $\mathrm{X}$ & $\mathrm{X}$ \\
\hline large & & $x$ & $x$ & $x$ \\
\hline Very large & & & $x$ & $x$ \\
\hline $\begin{array}{l}\text { Dosing } \\
\text { Static (contour } \\
\text { without needle) }\end{array}$ & $x$ & $x$ & $\mathrm{x}$ & $\mathrm{x}$ \\
\hline $\begin{array}{l}\text { Dynamic (contour } \\
\text { with needle) }\end{array}$ & & $x$ & $\bar{x}$ & \\
\hline $\begin{array}{l}\text { Contour shape } \\
\text { Symmetrical }\end{array}$ & $\bar{x}$ & $x$ & $x$ & $x$ \\
\hline $\begin{array}{l}\text { Slightly } \\
\text { asymmetrical }\end{array}$ & & $\mathrm{x}$ & $\mathrm{x}$ & \\
\hline Highly asymmetrical & & & & \\
\hline
\end{tabular}




\subsection{Equipment Properties $\backslash$ Measurement Conditions}

The static water contact-angle measurements were performed in a sessile drop experiment at room temperature using a Goniometer (OCA 20) equipped with a microliter syringe. Deionized water $(18.2 \mathrm{M} \Omega \mathrm{cm}$ resistivity_ Advantage A10 Millipore system) was used as the wetting liquid. All the reported values are the average of static contact angles at 5 different spots per sample.

\section{Self Assembly Processes and Polymerisations}

\subsection{Self Assembly Process}

\subsubsection{Self Assembly of MUD onto Gold Surface}

Gold-coated silicon substrates were diced into $10 \mathrm{~mm} \times 10 \mathrm{~mm}$ samples, degreased in acetone and ethanol in an ultrasonic bath, and then dried under a stream of Argon. The substrates were cleaned in a piranha solution (H2O2:H2SO4, 1:3 v/v \%) for 10 min and rinsed in deionized water for $10 \mathrm{~min}$. (Caution: Piranha solution reacts violently with organic materials and should be handled carefully!) gold-MUD was prepared at room temperature by overnight immersion of gold-coated silicon substrates in $1 \mathrm{mM}$ ethanolic solution of 11-Mercapto-1-undecanol (MUD). Samples were finally sonicated for $10 \mathrm{~min}$ in ethanol and dried under an Argon stream.

\subsubsection{Self Assembly of Dithiobenzoicacid (DTBA) onto Gold Surface}

Dithiobenzoicacid (DTBA) coated gold substrates were prepared at room temperature by overnight immersion of gold-coated silicon substrates in $1 \mathrm{mM}$ DTBA solution of THF. Samples were finally sonicated for $10 \mathrm{~min}$ in THF and dried under an Argon stream.

\subsubsection{Self Assembly of Raft Agents onto Gold Surface}

The gold substrates with the surface-immobilized CTA was prepared at room temperature by overnight immersion of gold-coated silicon substrates in $1 \mathrm{mM}$ solution of related CTAs. Each of (2_Phenyl_2_Propylbenzodithioate(CDB),4Cyano[dodecylsulfanylthiocarbonyl)sulf anyl] pentanoic acid (DSTCSPA), ethane-1,2-diyl bis(1-phenylethyl) ditrithiocarbonate (EBPEDTTC), Penta-erythritol-tetrakis-(3-(S-phenylethyl trithiocarbonate)- propanoate (tetra functional CTA) (PETSPETTCP), Dipentaerythriol-hexakis(3-(S-methoxopropionate-trithiocarbonate)) propanoate (hexa functional CTA) (DPEHSMPETTC) CTAs (0.01 mmol) was dissolved in $10 \mathrm{~mL}$ of anhydrous THF. Then, gold substrates were placed into these CTA solutions and 
left under argon atmosphere at room temperature for $24 \mathrm{~h}$. After this immobilization step, the gold substrates with the surface-immobilized CTA were repeatedly washed with THF, ethanol, water and dried under a stream of argon. The ellipsometric thicknesses of the CTAs chemisorbed on gold flats were measured at five different spots on each sample and averaged.

\subsubsection{Immobilization of Raft Agent onto Gold-MUD Surface}

CTA (DSTCSPA) (34 mmol), DMAP (20.4 mmol) and DCC (24.48 mmol) were dissolved in $85 \mathrm{~mL}$ of anhydrous dicholorometan and divided into 17 different vials. Then, hydroxyl-terminated gold substrates were placed into these reaction solutions. The reaction solution was left to react at room temperature for $24 \mathrm{~h}$ under argon atmosphere. After the reaction, the gold substrates with the surface-immobilized CTA were repeatedly washed with dicholorometan, ethanol and dried under a stream of argon.

\subsection{Polymerizations}

\subsubsection{Polymerization of BuMA in the Presence of Linear Free CTA from Gold- MUD-CTA}

The RAFT-mediated polymerizations of BuMA (39.2 mmol) was carried out in Toluen (12.42 mL), initiator AIBN (0.0196 mmol), and free CTA (DSTCSPA) (0.0784 mmol) in glass vials. The monomer and solvent were degassed via three pump-freezethaw cycles and were transferred along with six glass vials filled with initiator, Raft agents into an argon-filled glove box (oxygen content below $4 \mathrm{ppm}$ ). The six individual reaction mixtures consisting of same amount of initiator, monomer, Raft agent and solvent were prepared in the box. After the gold substrates with the surfaceimmobilized CTA were placed into these six vials filled with identical reaction mixtures, vials were sealed with Teflon/rubber lids and subsequently inserted into an oil bath, thermostated at a given temperature at $60^{\circ} \mathrm{C}$. The vials were removed after preset time intervals and cooled in an ice bath to quench the reaction. Monomer-topolymer conversions were determined gravimetrically. Therefore, the polymer was precipitated from the reaction mixture (with Methanol), dried to constant weight under vacuum in a heating cabinet. The molecular weight distribution of the polymer was measured by gel permeation chromatography (GPC). For ellipsometric measurements, the gold flats were also removed from the reactor after preset time intervals, washed with THF and water in an ultrasonic bath and dried with Argon stream. The ellipsometric thicknesses of the dry polymer films were measured at five different spots on each sample and averaged. 


\subsubsection{Polymerization of DMAEMA in the Presence of Linear Free CTA from Gold-MUD-CTA}

The RAFT-mediated polymerizations of DMAEMA (39.2 mmol) was carried out in Toluen (12.42 mL), initiator AIBN (0.0196 mmol), and free CTA (CDB) (0.0784 $\mathrm{mmol}$ ) in glass vials. The monomer and solvent were degassed via three pump-freezethaw cycles and were transferred along with six glass vials filled with initiator, Raft agents into an argon-filled glove box (oxygen content below 4 ppm). The six individual reaction mixtures consisting of same amount of initiator, monomer, Raft agent and solvent were prepared in the box. After the gold substrates with the surfaceimmobilized CTA were placed into these six vials filled with identical reaction mixtures, vials were sealed with Teflon/rubber lids and subsequently inserted into an oil bath thermostated at a given temperature at $60^{\circ} \mathrm{C}$. The vials were removed after preset time intervals and cooled in an ice bath to quench the reaction. Monomer-topolymer conversions were determined gravimetrically. Therefore, the polymer was precipitated from the reaction mixture (with Hexan), dried to constant weight under vacuum in a heating cabinet. The molecular weight distribution of the polymer was measured by gel permeation chromatography (GPC). For ellipsometric measurements, the gold flats were also removed from the reactor after preset time intervals, washed with Methanol, THF and water in an ultrasonic bath and dried with Argon stream. The ellipsometric thicknesses of the dry polymer films were measured at five different spots on each sample and averaged.

\subsubsection{Copolymerization of BuMA from Gold-PPEGMA Surface}

The RAFT-mediated copolymerization of BuMA (39.2 mmol) was carried out in Toluen (12.42 mL), initiator AIBN (0.0196 mmol), and free CTA (DSTCSPA) (0.0784 $\mathrm{mmol}$ ) in a glass vial. The monomer and solvent were degassed via three pump-freezethaw cycles and were transferred along with the glass vial filled with initiator and Raft agent into an argon-filled glove box (oxygen content below $4 \mathrm{ppm}$ ). The reaction mixture consisting of initiator, monomer, Raft agent and solvent were prepared in the box. The gold substrate coated with $9.9 \pm 0.1 \mathrm{~nm}$ poly (PEGMA) brushes, which was obtained from previous 5 hours homopolymerization step, was placed into the vial filled with reaction mixture. Afterwards vial was sealed with Teflon/rubber lid and inserted into an oil bath, thermostated at a given temperature at $60^{\circ} \mathrm{C}$. The vial was removed 24 hours later and cooled in an ice bath to quench the reaction. Monomer-topolymer conversion was determined gravimetrically. Therefore, the polymer was precipitated from the reaction mixture (with Methanol), dried to constant weight under vacuum in a heating cabinet. The molecular weight distribution of the polymer was measured by gel permeation chromatography (GPC). For ellipsometric measurements, the gold flat was removed from the vial, washed with THF, water in an ultrasonic bath and dried with Argon stream. The ellipsometric thickness of the dry polymer film was measured at five different spots on the sample and averaged. 


\subsubsection{Copolymerization of PEGMA from Gold-PDMAEMA Surface}

The RAFT-mediated copolymerization of PEGMA (19.6 mmol) was carried out in Toluen (12.42 mL), initiator AIBN (0.0196 mmol) and free CTA (DSTCSPA) (0.0784 $\mathrm{mmol}$ ) in a glass vial. The monomer and solvent were degassed via three pump-freezethaw cycles and were transferred along with the glass vial filled with initiator and Raft agent into an argon-filled glove box (oxygen content below $4 \mathrm{ppm}$ ). The reaction mixture consisting of initiator, monomer, Raft agent and solvent were prepared in the box. The gold substrate coated with $25 \pm 0.1 \mathrm{~nm}$ poly(DMAEMA) brushes, which was obtained from previous 24 hours homopolymerization step, was placed into the vial filled with reaction mixture. Afterwards vial was sealed with Teflon/rubber lid and inserted into an oil bath, thermostated at a given temperature at $60^{\circ} \mathrm{C}$. The vial was removed 24 hours later and cooled in an ice bath to quench the reaction. Monomer-topolymer conversion was determined gravimetrically. Therefore, the polymer was precipitated from the reaction mixture (with Hexan), dried to constant weight under vacuum in a heating cabinet. The molecular weight distribution of the polymer was measured by gel permeation chromatography (GPC). For ellipsometric measurements, the gold flat was removed from the vial, washed with Metanol, water in an ultrasonic bath and dried with Argon stream. The ellipsometric thickness of the dry polymer film was measured at five different spots on the sample and averaged.

\subsubsection{Polymerization of DMAEMA in the Presence of Free CTA (CDB) from Gold-CDB Surface}

The RAFT-mediated polymerization of DMAEMA (41.2 mmol) was carried out in Toluen (13.32 mL), initiator AIBN (0.04 mmol), and free CTA (CDB) $(0.16 \mathrm{mmol})$ in a glass vial. The monomer and solvent were degassed via three pump-freeze-thaw cycles and were transferred along a glass vial filled with initiator and Raft agent into an argon-filled glove box (oxygen content below $4 \mathrm{ppm}$ ). The reaction mixture consisting of initiator, monomer, Raft agent and solvent were prepared in the box. The gold substrate with the surface immobilized CTA (CDB) was placed into the vial filled with reaction mixture. Afterwards vial was sealed with Teflon/rubber lid and inserted into an oil bath, thermostated at a given temperature at $60^{\circ} \mathrm{C}$. The vial was removed 24 hours later and cooled in an ice bath to quench the reaction. Monomer-to-polymer conversion was determined gravimetrically. Therefore, the polymer was precipitated from the reaction mixture (with Hexan), dried to constant weight under vacuum in a heating cabinet. The molecular weightof the polymer was measured by gel permeation chromatography (GPC). For ellipsometric measurements, the gold flat was removed from the vial, washed with Metanol, THF, water in an ultrasonic bath and dried with Argon stream. Ellipsometric thicknesses of the dry polymer film was measured at five different spots on the sample and averaged. 


\subsubsection{Polymerization of BuMA in the Presence of Bifunctional Free CTA (EBPEDTTC) from Gold-EBPEDTTC Surface}

The RAFT-mediated polymerization of BuMA (38.3 mmol) was carried out in Toluen (12 mL), initiator AIBN (0.153 mmol), and free CTA (EBPEDTTC) (0.0766 $\mathrm{mmol}$ ) in glass vials. The monomer and solvent were degassed via three pump-freezethaw cycles and were transferred along with five glass vials filled with initiator and Raft agents into an argon-filled glove box (oxygen content below 4 ppm). The five individual reaction mixtures consisting of same amount of initiator, monomer, Raft agent and solvent were prepared in the box. After the gold substrates with the surfaceimmobilized CTA (EBPEDTTC) were placed into these vials filled with identical reaction mixtures, they were sealed with Teflon/rubber lids and subsequently inserted into an oil bath, thermostated at a given temperature at $60^{\circ} \mathrm{C}$. The vials were removed after preset time intervals and cooled in an ice bath to quench the reaction. Monomerto-polymer conversions were determined gravimetrically. Therefore, the polymer was precipitated from the reaction mixture (with Methanol), dried to constant weight under vacuum in a heating cabinet. The molecular weight of the polymer was measured by gel permeation chromatography (GPC). For ellipsometric measurements, the gold flats were also removed from the reactor after preset time intervals and washed with Methanol, THF, water in an ultrasonic bath and dried with Argon stream. Ellipsometric thicknesses of the dry polymer films were measured at five different spots on each sample and averaged.

\subsubsection{Polymerization of DMAEMA in the Presence of Bifunctional Free CTA (EBPEDTTC) from Gold-EBPEDTTC Surface}

The RAFT-mediated polymerization of DMAEMA (38.3 mmol) was carried out in Toluen $(13.2 \mathrm{~mL})$, initiator AIBN (0.0192 mmol) and free CTA (EBPEDTTC) (0.0766 $\mathrm{mmol}$ ) in a glass vial. The monomer and solvent were degassed via three pump-freezethaw cycles and were transferred along with the glass vial filled with initiator and Raft agent into an argon-filled glove box (oxygen content below 4 ppm). The reaction mixture consisting of initiator, monomer, Raft agent and solvent were prepared in the box. Then, the gold substrate coated with the CTA (EBPEDTTC) was placed into the vial filled with the reaction mixture and the vial was sealed with Teflon/rubber lid. The vial was subsequently inserted into an oil bath, thermostated at a given temperature at $60^{\circ} \mathrm{C}$. 24 hours later, it was removed and cooled in an ice bath to quench the reaction. Monomer to polymer conversion was determined gravimetrically. Therefore, the polymer was precipitated from the reaction mixture (with Hexan), dried to constant weight under vacuum in a heating cabinet. The molecular weight of the polymer was measured by gel permeation chromatography (GPC). For ellipsometric measurements, the gold flat was removed from the vial, washed with Metanol, THF and water in an ultrasonic bath and dried with Argon stream. Ellipsometric thickness of the dry polymer film was measured at five different spots on the sample and averaged 


\subsubsection{Polymerizations of PEGMA in the Presence of Monofunctional Free CTA (DSTCSPA) from Gold-EBPEDTTC，Gold-PETSPETTCP， Gold- DPEHPETTC Surfaces}

The RAFT-mediated polymerizations of PEGMA (18 mmol) was carried out in Toluen $(18 \mathrm{~mL})$, initiator AIBN $(0.018 \mathrm{mmol})$, and free CTA (DSTCSPA) $(0.072$ $\mathrm{mmol})$ in glass vials. The monomer and solvent were degassed via three pump-freezethaw cycles and were transferred along with three glass vials filled with initiator, Raft agent into an argon-filled glove box (oxygen content below $4 \mathrm{ppm}$ ). The three individual reaction mixtures consisting of same amount of initiator, monomer, Raft agent and solvent were prepared in the box. After CTA immobilized gold substrates were placed into these three vials filled with identical reaction mixtures, they were sealed with Teflon/rubber lids and subsequently inserted into an oil bath, thermostated at a given temperature at $60^{\circ} \mathrm{C}$. The vials were removed after 24 hours polymerization and cooled in an ice bath to quench the reaction. Monomer to polymer conversions were determined gravimetrically. Therefore, the polymers were precipitated from the reaction mixtures (with Hexan), dried to constant weight under vacuum in a heating cabinet. The molecular weights of the polymers were measured by gel permeation chromatography (GPC). For ellipsometric measurements, the gold flats were also removed from the reactor, washed with Methanol, THF and water in an ultrasonic bath and dried with Argon stream. Ellipsometric thicknesses of the dry polymer films were measured at five different spots on each sample and averaged.

\subsubsection{Polymerization of PEGMA in the Presence of Bifunctional Free CTA (EBPEDTTC) from Gold-EBPEDTTC Surface}

The RAFT-mediated polymerization of PEGMA ( $18 \mathrm{mmol})$ was carried out in Toluen $(18 \mathrm{~mL})$, initiator AIBN $(0.072 \mathrm{mmol})$ and free CTA (EBPEDTTC) $(0.036 \mathrm{mmol})$ in a glass vial. The monomer and solvent were degassed via three pump-freeze-thaw cycles and were transferred along with the vial filled with initiator and Raft agent into an argon-filled glove box (oxygen content below $4 \mathrm{ppm}$ ). The reaction mixture consisting of initiator, monomer, Raft agent and solvent were prepared in the box. After the gold substrate with the surface-immobilized CTA (EBPEDTTC) was placed into this vial filled with reaction mixture, vial was sealed with Teflon/rubber lid and subsequently inserted into an oil bath, thermostated at a given temperature at $60^{\circ} \mathrm{C}$. The vial was removed 24 hour later and cooled in an ice bath to quench the reaction. Monomer to polymer conversion was determined gravimetrically. Therefore, the polymer was precipitated from the reaction mixture (with Hexan), dried to constant weight under vacuum in a heating cabinet. The molecular weight of the polymer was measured by gel permeation chromatography (GPC). For ellipsometric measurement, the gold flat was also removed from the reactor, washed with Methanol, THF, water 
in an ultrasonic bath and dried with Argon stream. Ellipsometric thickness of the dry polymer film was measured at five different spots on sample and averaged.

\subsubsection{Polymerization of PEGMA in the Presence of Tetrafunctional Free CTA (PETSPETTCP) from Gold-PETSPETTCP and Gold-DPEHPETTC Surface}

The RAFT-mediated polymerization of PEGMA $(18 \mathrm{mmol})$ was carried out in Toluen $(18 \mathrm{~mL})$, initiator AIBN $(0.18 \mathrm{mmol})$ and free CTA (PETSPETTCP) $(0.045 \mathrm{mmol})$ in two glass vials. The monomer and solvent were degassed via three pump-freeze-thaw cycles and were transferred along glass vials filled with initiator, Raft agent into an argon-filled glove box (oxygen content below $4 \mathrm{ppm}$ ). The reaction mixture consisting of same amount of initiator, monomer, Raft agent and solvent were prepared in the box. The gold substrates with the surface-immobilized CTA (PETSPETTCP) and surface immobilized CTA (DPEHSMPETTC) were placed into the vials filled with identical reaction mixtures. After vials were sealed with Teflon/rubber lids, they were inserted into an oil bath, thermostated at a given temperature at $60^{\circ} \mathrm{C}$. The vials were removed 24 hours later and cooled in an ice bath to quench the reaction. Monomer to polymer conversions were determined gravimetrically. Therefore, the polymers were precipitated from the reaction mixtures (with Hexan), dried to constant weight under vacuum in a heating cabinet. The molecular weights of the polymers were measured by gel permeation chromatography (GPC). For ellipsometric measurements, the gold flats were removed from the vial, washed with Metanol, THF and water in an ultrasonic bath and dried with Argon stream. Ellipsometric thicknesses of the dry polymer films were measured at five different spots on each sample and averaged.

\subsubsection{Copolymerization of PEGMA in the Presence of Bifunctional Free CTA (EBPEDTTC) from Gold-EBPEDTTC-PDMAEMA Surface}

RAFT mediated polymerization of PEGMA (18 mmol) was carried out in Toluen (18 $\mathrm{mL})$, initiator AIBN (0.036 mmol), and free bifunctional CTA (EBPEDTTC) (0.018 $\mathrm{mmol}$ ) in a glass vial. The monomer and solvent were degassed via three pump-freezethaw cycles and were transferred along a glass vial filled with initiator and Raft agent into an argon-filled glove box (oxygen content below $4 \mathrm{ppm}$ ). The reaction mixture consisting of initiator, monomer, RAFT-agent and solvent were prepared in the box. The gold substrate coated with $27 \pm 0.1 \mathrm{~nm}$ poly(DMAEMA) brushes, which was obtained from previous homopolymerization step, was placed into the vial filled with reaction mixture. Afterwards vial was sealed with Teflon/rubber lid and inserted into an oil bath, thermostated at a given temperature at $60^{\circ} \mathrm{C}$. The vial was removed 24 hours later and cooled in an ice bath to quench the reaction. Monomer to polymer conversion was determined gravimetrically. Therefore, the polymer was precipitated from the reaction mixture (with Hexan), dried to constant weight under vacuum in a heating cabinet. The molecular weight of the polymer was measured by gel permeation 
chromatography (GPC). For ellipsometric measurement, the gold flat was removed from the vial, washed with Metanol, THF and water in an ultrasonic bath and dried with Argon stream. Ellipsometric thickness of the dry polymer film was measured at five different spots on the sample and averaged.

\subsubsection{Polymerization of MMA in the Presence of Free DTBA from Gold-DTBA Surface}

DTBA mediated polymerizations of MMA (39.2 mmol) were carried out in Toluen $(4 \mathrm{~mL})$, initiator AIBN $(0.0196 \mathrm{mmol})$, and free DTBA $(0.0784 \mathrm{mmol})$ in glass vials. The monomer and solvent were degassed via three pump-freeze-thaw cycles and were transferred along with six glass vials filled with initiator, DTBA into an argon-filled glove box (oxygen content below $4 \mathrm{ppm}$ ). The six individual reaction mixtures consisting of same amount of initiator, monomer, DTBA and solvent were prepared in the box. After the DTBA coated gold substrates were placed into these six vials filled with identical reaction mixtures, vials were sealed with Teflon/rubber lids and subsequently inserted into an oil bath, thermostated at a given temperature at $60^{\circ} \mathrm{C}$. The vials were removed after preset time intervals and cooled in an ice bath to quench the reaction. Monomer to polymer conversions were determined gravimetrically. Therefore, the polymer was precipitated from the reaction mixture (with Methanol), dried to constant weight under vacuum in a heating cabinet. The molecular weight distributions of the polymers were measured by gel permeation chromatography (GPC). For ellipsometric measurements, the gold flats were also removed from the reactors after preset time intervals and washed with THF, water in an ultrasonic bath and dried with Argon stream. The ellipsometric thicknesses of the dry polymer films were measured at five different spots on each sample and averaged.

\subsubsection{Polymerization of BuMA in the Presence of Free DTBA from Gold-DTBA Surface}

DTBA mediated polymerization of BuMA (39.2 mmol) was carried out in Toluen (12.42 mL), initiator AIBN (0.0196 mmol) and free CTA (DSTCSPA) (0.0784 mmol) in a glass vial. The monomer and solvent were degassed via three pump-freeze-thaw cycles and were transferred along the glass vial filled with initiator, DTBA into an argon-filled glove box (oxygen content below $4 \mathrm{ppm}$ ). The reaction mixture consisting of initiator, monomer, DTBA and solvent were prepared in the box. The gold substrate coated with DTBA was placed into the vial filled with reaction mixture. After vial was sealed with Teflon/rubber lid, it was inserted into an oil bath, thermostated at a given temperature at $60^{\circ} \mathrm{C}$. The vial was removed 24 hours later and cooled in an ice bath to quench the reaction. Monomer to polymer conversion was determined gravimetrically. Therefore, the polymer was precipitated from the reaction mixture (with Metanol), dried to constant weight under vacuum in a heating cabinet. The molecular weight distribution of the polymer was measured by gel permeation chromatography (GPC). 
For ellipsometric measurement, the gold flat was removed from the vial and washed with THF, water in an ultrasonic bath and dried with Argon stream. The ellipsometric thickness of the dry polymer film was measured at five different spots on the sample and averaged.

\subsubsection{Polymerization of PEGMA in the Presence of Free CTA (DSTCSPA) from Gold-DTBA Surface}

RAFT mediated polymerization of PEGMA (39.2 mmol) was carried out in Toluen (12.42 mL), initiator AIBN (0.0196 mmol) and free CTA (DSTCSPA) (0.0784 mmol)) in a glass vial. The monomer and solvent were degassed via three pump-freeze-thaw cycles and were transferred along the glass vial filled with initiator and Raft agent into an argon-filled glove box (oxygen content below $4 \mathrm{ppm}$ ). The reaction mixture consisting of initiator, monomer, Raft agent and solvent were prepared in the box. The gold substrate coated with DTBA was placed into the vial filled with reaction mixture. After vial was sealed with Teflon/rubber lid, it was inserted into an oil bath, thermostated at a given temperature at $60^{\circ} \mathrm{C}$. The vial was removed 24 hours later and cooled in an ice bath to quench the reaction. Monomer to polymer conversion was determined gravimetrically. Therefore, the polymer was precipitated from the reaction mixture (with Hexan), dried to constant weight under vacuum in a heating cabinet. The molecular weight distribution of the polymer was measured by gel permeation chromatography (GPC). For ellipsometric measurement, the gold flat was removed from the vial, washed with Metanol, THF and water in an ultrasonic bath and dried with Argon stream. The ellipsometric thickness of the dry polymer film was measured at five different spots on the sample and averaged.

\subsubsection{Copolymerization of DMAEMA in the Presence of Free DTBA from Gold- DTBA-PBuMA Surface}

DTBA mediated blockcopolymerization of DMAEMA (39.2 mmol) was carried out in Toluen $(6.60 \mathrm{~mL})$, initiator AIBN $(0.0196 \mathrm{mmol})$ and free DTBA $(0.0784 \mathrm{mmol})$ in a glass vial. The monomer and solvent were degassed via three pump-freeze-thaw cycles and were transferred along the glass vial filled with initiator and DTBA into an argon-filled glove box (oxygen content below $4 \mathrm{ppm}$ ). The reaction mixture consisting of initiator, monomer, DTBA and solvent were prepared in the box. The gold substrate covered with $20 \pm 0.1 \mathrm{~nm}$ Poly(BUMA) brush, which was obtained from previous homopolymerization step was placed into the vial filled with reaction mixture. After vial was sealed with Teflon/rubber lid, it was inserted into an oil bath, thermostated at a given temperature at $60^{\circ} \mathrm{C}$. The vial was removed 24 hours later and cooled in an ice bath to quench the reaction. Monomer to polymer conversion was determined gravimetrically. Therefore, the polymer was precipitated from the reaction mixture (with Hexan), dried to constant weight under vacuum in a heating cabinet. The molecular weight distribution of the polymer was measured by gel permeation 
chromatography (GPC). For ellipsometric measurement, gold flat was removed from the vial, washed with Metanol, THF, water in an ultrasonic bath and then dried with Argon stream. The ellipsometric thickness of dry polymer film was measured at five different spots on the sample and averaged.

\subsubsection{Copolymerization of DMAEMA in the Presence of Free CTA (CDB) from Gold-DTBA-PPEGMA Surface}

RAFT mediated blockcopolymerization of DMAEMA (39.2 mmol) was carried out in Toluen (13.20 mL), initiator AIBN (0.0196 mmol), and free CTA (CDB) (0.0784 $\mathrm{mmol}$ ) in a glass vial. The monomer and solvent were degassed via three pump-freezethaw cycles and were transferred along with the glass vial filled with initiator and Raft agent into an argon-filled glove box (oxygen content below $4 \mathrm{ppm}$ ). The reaction mixture consisting of initiator, monomer, Raft agent and solvent were prepared in the box. The gold substrate covered with $24 \pm 0.1 \mathrm{~nm}$ poly(PEGMA) brushes, which was obtained from previous homopolymerization step, was placed into the vial filled with the reaction mixture. Afterwards vial was sealed with Teflon/rubber lid and inserted into an oil bath, thermostated at a given temperature at $60^{\circ} \mathrm{C}$. The vial was removed 24 hours later and cooled in an ice bath to quench the reaction. Monomer to polymer conversion was determined gravimetrically. Therefore, polymer was precipitated from the reaction mixture (with Hexan), dried to constant weight under vacuum in a heating cabinet. The molecular weight distribution of the polymer was measured by gel permeation chromatography (GPC). For ellipsometric measurement, the gold flat was removed from the vial, washed with Metanol, THF, water in an ultrasonic bath and dried with Argon stream. The ellipsometric thickness of the dry polymer film was measured at five different spots on the sample and averaged.

\subsubsection{Polymerization of PEGMA in the Presence of Bifunctional Free CTA (EBPEDTTC) from Gold-MUD-CTA Surface}

The RAFT-mediated polymerization of PEGMA ( $18 \mathrm{mmol})$ was carried out in Toluen $(18 \mathrm{~mL})$, initiator AIBN $(0.18 \mathrm{mmol})$, and free CTA (EBPEDTTC) $(0.09 \mathrm{mmol})$ in a glass vial. The monomer and solvent were degassed via three pump-freeze-thaw cycles and were transferred along the glass vial filled with initiator and Raft agent into an argon-filled glove box (oxygen content below $4 \mathrm{ppm}$ ). The reaction mixture consisting of initiator, monomer, Raft agent and solvent were prepared in the box. The gold substrate with the surface-immobilized linear CTA (DSTCSPA) was placed into the vial filled with reaction mixture. After the vial was sealed with Teflon/rubber lid, it was inserted into an oil bath, thermostated at a given temperature at $60^{\circ} \mathrm{C}$. The vial was removed 24 hours later and cooled in an ice bath to quench the reaction. Monomer to polymer conversion was determined gravimetrically. Therefore, polymer was precipitated from the reaction mixture (with Hexan), dried to constant weight under vacuum in a heating cabinet. The molecular weight distribution of the polymer was 
measured by gel permeation chromatography (GPC). For ellipsometric measurement, the gold flat was removed from the vial, washed with Metanol, THF, water in an ultrasonic bath and dried with Argon stream. Ellipsometric thickness of the dry polymer film was measured at five different spots on the sample and averaged.

\subsubsection{Polymerization of PEGMA in the Presence of Tetrafunctional Free CTA (PETSPETTCP) from Gold-MUD-CTA Surface}

The RAFT-mediated polymerization kinetics of PEGMA (18 mmol) was carried out in Toluen $(18 \mathrm{~mL})$, initiator AIBN $(0.18 \mathrm{mmol})$ and free cta (PETSPETTCP) $(0.045$ $\mathrm{mmol}$ ) in glass vials. The monomer and solvent were degassed via three pump-freezethaw cycles and were transferred along with five glass vials filled with initiator and Raft agent into an argon-filled glove box (oxygen content below $4 \mathrm{ppm}$ ). The five reaction mixtures consisting of same amount of initiator, monomer, Raft agent and solvent were prepared in the box. After the gold substrates with the surfaceimmobilized linear CTA (DSTCSPA) were placed into these five vials filled with identical reaction mixtures, they were sealed with Teflon/rubber lids and subsequently inserted into an oil bath, thermostated at a given temperature at $60^{\circ} \mathrm{C}$. The vials were removed after preset time intervals and cooled in an ice bath to quench the reaction. Monomer to polymer conversions were determined gravimetrically. Therefore, polymer was precipitated from the reaction mixture (with Hexan), dried to constant weight under vacuum in a heating cabinet. The molecular weight distribution of the polymer was measured by gel permeation chromatography (GPC). For ellipsometric measurements, the gold flats were also removed from the reactor after preset time intervals, washed with Methanol, THF, water in an ultrasonic bath and dried with Argon stream. Ellipsometric thicknesses of the dry polymer films were measured at five different spots on each sample and averaged.

\subsubsection{Polymerization of PEGMA in the Presence of Hexafunctional Free CTA (DPEHPETTC) from Gold-MUD-CTA Surface}

The RAFT-mediated polymerization of PEGMA (18 mmol) was carried out in Toluen $(18 \mathrm{~mL})$, initiator AIBN $(0.18 \mathrm{mmol})$, and free hexa functional CTA (DPEHPETTC) $(0.03 \mathrm{mmol})$ in a glass vial. The monomer and solvent were degassed via three pumpfreeze-thaw cycles and were transferred along the glass vial filled with initiator and Raft agent into an argon-filled glove box (oxygen content below $4 \mathrm{ppm}$ ). The reaction mixture consisting of initiator, monomer, Raft agent and solvent were prepared in the box. The gold substrate with the surface-immobilized linear CTA (DSTCSPA) was placed into the vial filled with reaction mixture. Afterwards vial was sealed with Teflon/rubber lid and inserted into an oil bath, thermostated at a given temperature at $60^{\circ} \mathrm{C}$. The vial was removed 24 hours later and cooled in an ice bath to quench the reaction. Monomer to polymer conversion was determined gravimetrically. Therefore, polymer was precipitated from the reaction mixture (with Hexan), dried to constant 
weight under vacuum in a heating cabinet. The molecular weight distribution of the polymer was measured by gel permeation chromatography (GPC). For ellipsometric measurements, the gold flat was removed from the vial, washed with Metanol, THF, water respectively in an ultrasonic bath and dried with Argon stream. Ellipsometric thickness of the dry polymer film was measured at five different spots on the sample and averaged

\subsubsection{Protein Adsorbtion Experiments}

Fouling from solutions of main blood plasma proteins (Human Serum Albumin, Fibrinogen) and Insulin on PEGMA brushes with different thicknesses and architectures was investigated using Spectroscopic Ellipsometry and HPLC-Mass spectrometry. Gold surfaces coated with PEGMA brushes were incubated in a solution of Insulin in Methanol and solutions of HSA and Fbg in PBS $\left(1 \mathrm{mg} \mathrm{mL}^{-1}\right)$ respectively. After 2 hours, gold flats were taken out of protein solutions and placed into empty vials. They were rinsed one time with $5 \mathrm{ml}$ Methanol (for I) or MilliQ water (for HSA and Fbg) and dried under a stream of Argon. After thicknesses of proteins foulded on gold surfaces were investigated via Spectroscopic Ellipsometry, gold flats were rinsed with $5 \mathrm{ml}$ Methanol or MilliQ water two times more. These vials of Methanol and MilliQ water used for cleaning steps, which contains related protein residues, each of which belongs to a different gold sample, were gathered in a vial for every protein type used for each gold sample. Each of these vials full of a protein type which belongs to a gold sample, were individually kept under the fume hood so that all solvents were evaporated. After evaporation of solvents, each of these protein residues which belong to different substrate types were dissolved with $5 \mathrm{ml}$ of Methanol or water. Only Insulin solutions were used for quantitative investigation and measured via HPLC-Mass spectroscopy. 


\section{Appendix}

\subsection{List of Abbreviations and Symbols}

a

A

A

$\mathrm{AC}$

AIBN

AFM

$\mathrm{Al}$

API

APCI

APPI

ATRP

ATR-IR

ATR-FTIR

AuNps

BDTB

BPO

BUMA

$\mathrm{C}, \mathrm{C}_{\mathrm{i}}$

$\mathrm{C}$

$\mathrm{C}_{\text {tr }}$

CA

CDB

$\mathrm{CH}_{2} \mathrm{CL}_{2}$

C(L)RP

CTA

CTAP

d

d or D

DBTTC
Size of the monomer

Coverage

Analyzer

Alternating current

2,2'-azobis(isobutyronitrile)

Atomic force microscopy

Albumin

Atmospheric Pressure Ion

Atmospheric Pressure Chemical Ionisation

Atmospheric Pressure Photo Ionisation

Atom transfer radical polymerization

Attenauted Total Reflectance Infrared spectroscopy

Attenuated total reflection Fourier Transform Infrared Spectroscopy

Gold nanoparticles

Benzyldithiobenzoate

Benzoyl peroxide

Butyl methacrylate

Concentration

Quarter wave length compensator

Transfer constant

Contact Angle

2-phenyl-2-propylbenzodithioate

Dichloromethane

Controlled (Living) Radical Polymerization Technique

Chain Transfer Agent

Chain Transfer Agent Precursor

Film thickness

Average distance between the anchor points

Dibenzyl trithiocarbonate 


\begin{tabular}{|c|c|}
\hline DCC & N, N`-Dicyclohexylcarbodiimide \\
\hline $\operatorname{dn} \backslash \mathrm{dc}$ & Refractive index increment \\
\hline DMAEMA & 2-(dimethylamino)ethyl methacrylate \\
\hline DMAP & 4-(dimethylamino)pyridine \\
\hline DMF & Dimethylformamide \\
\hline DMSO & Dimethylsulfoxide \\
\hline \multirow[t]{2}{*}{ DPEHSMPETTC } & Dipentaerythriol-hexakis(3-(S \\
\hline & methoxopropionatetrithiocarbonate))propanoate \\
\hline DRI & Differential refractometer \\
\hline DSA & Drop shape analysis \\
\hline DSTCSPA & 4-Cyano-4-[dodecylsulfanylthiocarbonyl) sulfanyl] pentanoicacid) \\
\hline DTBA & Dithiobenzoicacid \\
\hline DTGS & Deuteriumtriglycinesulfate \\
\hline EBPEDTTC & Ethane-1,2-diylbis(1-phenylethyl) ditrithiocarbonate \\
\hline EI & Electrospray Ionisation \\
\hline Ep, out Es, out & Reflected vectors \\
\hline$E p_{\text {, in }} E s$, in & Incident vectors \\
\hline ESI & Electrospray Ionisation \\
\hline $\mathrm{EtOH}$ & Ethanol \\
\hline f & Initiator effectiveness \\
\hline Fbg & Fibrinogen \\
\hline FRP & Conventional Free Radical Polymerization \\
\hline FTIR & Fourier Transform Infrared Spectroscop \\
\hline FWHM & Full width half maximum \\
\hline g & Number of monomers in a blob \\
\hline GPC & Gel Permation Chromatography \\
\hline $\mathrm{H}$ & Brush height \\
\hline $\mathrm{H}_{\mathrm{i}}$ & Height \\
\hline HDPE & High-density polyethylene \\
\hline HPLC & High pressure liquid chromatography \\
\hline HPLC-Mass & High pressure liquid chromatography-mass spectrometry \\
\hline HSA & Human serum albumin \\
\hline$[\mathrm{I} \cdot]$ & Primary radical concentration \\
\hline
\end{tabular}




\begin{tabular}{|c|c|}
\hline [In] & Initiator concentration \\
\hline In & Insulin \\
\hline IR & Infrared \\
\hline IR-RAS & Grazing incidence infrared reflection absorption spectroscopy \\
\hline i & Each slice \\
\hline $\mathrm{k}$ & Extinction coefficient \\
\hline $\mathrm{K}$ & Mark-Houwink constants \\
\hline $\mathrm{k}_{\text {add }}$ & Addition rate coefficient \\
\hline $\mathrm{k}_{\mathrm{d}}$ & Rate coefficient of the decomposition of initiator \\
\hline $\mathrm{k}_{\mathrm{t}}, \mathrm{k}_{\mathrm{T}}$ & Rate coefficient of termination \\
\hline $\mathrm{k}_{\mathrm{tr}}$ & Rate coefficient of transfer \\
\hline $\mathrm{k}_{\mathrm{p}, \mathrm{k}_{\mathrm{P}}}$ & Rate coefficient of propagation \\
\hline $\mathrm{k}_{\beta}$ & Fragmentation rate coefficient \\
\hline $\mathrm{L}$ & Laser \\
\hline LALLS & Low angle light scattering detectors \\
\hline LCMS & Liquid chromatography mass spectrometry \\
\hline LCST & Lower critical solution temperature \\
\hline LDPE & Low density polyethylene \\
\hline $\mathrm{M}, \mathrm{M}_{\mathrm{i}}$ & Molecular weight \\
\hline [M] & Monomer concentration \\
\hline $\mathrm{M}_{\mathrm{n}}$ & Number average molecular weight \\
\hline $\mathrm{M}_{\mathrm{n}}$ & Number average molecular weight \\
\hline $\mathrm{M}_{\mathrm{v}}$ & Viscosity molecular weight \\
\hline $\mathrm{M}_{\mathrm{z}}$ & Size average molecular weight \\
\hline $\mathrm{M}_{\mathrm{w}}$ & Weight average molecular weight \\
\hline MALLS & Multi angle light scattering \\
\hline $\mathrm{MeOH}$ & Methanol \\
\hline MMA & Methyl methacrylate \\
\hline MUD & 11-Mercapto-1-undecanol \\
\hline MS & Mass spectrometry \\
\hline $\mathrm{mlz}$ & Mass to charge ratio \\
\hline$n_{o}, n$ & Refractive index \\
\hline $\mathrm{N}$ & Complex refractive index \\
\hline
\end{tabular}




\begin{tabular}{|c|c|}
\hline $\mathrm{N}$ & Number of monomer \\
\hline $\mathrm{N}$ & Degree of polymerization \\
\hline $\mathrm{N} / \mathrm{g}$ & Number of correlation blobs per chain \\
\hline $\mathrm{N}_{\mathrm{A}}$ & Avogadro's number \\
\hline $\mathrm{N}_{\mathrm{i}}$ & Number of molecules got from detector response \\
\hline NMP & Nitroxide mediated polymerization \\
\hline p component & $\begin{array}{l}\text { Polarization state of the light (incident upon the sample) which is } \\
\text { oscillating paralel to the plane of incidence }\end{array}$ \\
\hline $\mathrm{P}$ & Linear polarizer \\
\hline $\mathrm{P}_{\mathrm{n}} \cdot \mathrm{P}_{\mathrm{m}} \cdot \mathrm{P}$ & Active polymeric radical (active species) \\
\hline PAA & Poly (acrylic acide) \\
\hline PAM & Poly (acrylamide) \\
\hline PBS & Phosphate Buffer Solution \\
\hline $\mathrm{PC}$ & Combination of a linear polarizer and a quarter-wave compensator \\
\hline PCSA & Polarizer-Compensator-Sample-Analyzer \\
\hline PDA & Photodiode array \\
\hline PDE & Phenyldithioester \\
\hline PDI & Polydispersity index \\
\hline PDMAEMA & Poly (2-(dimethylamino) ethyl methacrylate) \\
\hline PEG & Poly (ethylene glycol) \\
\hline PEGMA & Poly (ethyleneglycol) methyl ether methacrylate \\
\hline PELs & Polyelectrolytes \\
\hline PETSPETTCP & Penta-erythritol-tetrakis-(3-(S-benzyl trithiocarbonate))-propanoate \\
\hline PGMA & Poly (glycidyl methacrylate) \\
\hline PHEMA & Poly (2-hydroxyethyl methacrylate) \\
\hline PMEP & Poly (2-methacryloyloxyethyl phosphate) \\
\hline PNIPAM-co- & Poly (N-isopropylacrylamide)-co-poly (N-vinylimidazole) \\
\hline \multicolumn{2}{|l|}{ PNVI } \\
\hline PSBMA & Poly (sulfobetaine methacrylate) \\
\hline PSCA & Polarizer-Sample-Compensator-Analyzer \\
\hline $\mathrm{P}-\mathrm{X}$ & Dormant species \\
\hline $\mathrm{R} \cdot$ & Generated free radical \\
\hline$[\mathrm{R} \cdot]$ & Radical concentration \\
\hline
\end{tabular}




\begin{tabular}{|c|c|}
\hline RAFT & Reversible addition fragmentation chain transfer \\
\hline $\mathrm{r}_{\mathrm{b}}$ & Brutto polymerization rate \\
\hline$r_{d}$ & Rate of initiation reaction (decomposition of initiator) \\
\hline $\mathrm{R}_{\mathrm{g}}$ & Radius of gyration \\
\hline RI & Refractive index \\
\hline$r_{p}$ & Rate of propagation reaction \\
\hline $\mathrm{Rp}$ & Rate of polymerization \\
\hline $\mathrm{R}_{\mathrm{pp}} \mathrm{R}_{\mathrm{ps}} \quad \mathrm{R}_{\mathrm{sp}} \mathrm{R}_{\mathrm{ss}}$ & Reflection matrix \\
\hline$r_{t}$ & Rate of termination reaction \\
\hline $\mathrm{r}_{\mathrm{tr}}$ & Rate of transfer reaction \\
\hline $\mathrm{R}-\mathrm{M}$ & Active polymeric radical \\
\hline$r_{0}$ & Radius of the equidistant rod \\
\hline ROI & Region of interest \\
\hline ROP & Ring-opening polymerization \\
\hline ROMP & Ring-opening metathesis polymerization \\
\hline S & Sample \\
\hline s component & $\begin{array}{l}\text { Polarization state of the light incident upon the sample which is } \\
\text { oscillating perpendicular to the plane of incidence (parallel to the } \\
\text { sample surface) }\end{array}$ \\
\hline SAM & Self-assembled monolayer \\
\hline $\mathrm{SE}$ & Spectroscopic Ellipsometry \\
\hline SEC & Size exclusion chromatography \\
\hline SERS & Surface enhanced raman spectroscopy \\
\hline SFE & Surface free energy \\
\hline SFRP & Stable free radical polymerization \\
\hline SI-CLRP & Surface initiated controlled-living radical polymerization technique \\
\hline SIP & Surface initiated polymerization \\
\hline SI-PIMP & Surface-initiated photoiniferter-mediated polymerization \\
\hline SI-RAFT & Surface initiated reversible addition fragmentation chain transfer \\
\hline SPM & Scanning probe microscopes \\
\hline SPR & Surface plasmon resonance spectroscopy \\
\hline ST & Surface tension \\
\hline $\mathrm{t}$ & Reaction time \\
\hline
\end{tabular}




\begin{tabular}{|c|c|}
\hline THF & Tetrahydrofuran \\
\hline $\mathrm{T}_{\mathrm{g}}$ & Glass transition temperature \\
\hline TOF & Time of Flight \\
\hline UCST & Upper critical solution temperature \\
\hline UV & Ultraviolet \\
\hline QCM & Quartz crystal microbalance \\
\hline$[\mathrm{X}]$ & Concentration of the transferring agent \\
\hline XPS & X-ray photoelectron spectroscopy \\
\hline $\mathrm{V}_{\mathrm{g}}$ & Volume of the polymer gel \\
\hline $\mathrm{V}_{\mathrm{i}}$ & Volume of solvent held in the pores \\
\hline $\mathrm{V}_{\mathrm{o}}$ & Free volume outside of the particles \\
\hline $\mathrm{V}_{\mathrm{t}}$ & Total volume \\
\hline $\mathrm{V}_{\eta}$ & Hydrodynamic volume \\
\hline $\mathrm{ZnSe}$ & Zinc selenide \\
\hline$\Phi(\mathrm{z})$ & Segment density profile of the surface-attached chains \\
\hline$\sigma$ & Grafting density \\
\hline$v$ & Quality of solvent \\
\hline$\xi$ & Size of a blob \\
\hline$\Sigma$ & Reduced tethered density \\
\hline$\rho$ & $\begin{array}{l}\text { Complex reflectance ratio of a system which is the ratio of complex } \\
\text { reflection coefficients } \mathrm{Rpp} \text { and Rss (The amplitudes of the } \mathrm{s} \text { and } \mathrm{p} \\
\text { components, after reflection are normalized to their initial values and } \\
\text { symbolised with } \mathrm{R}_{\mathrm{ss}} \text { and } \mathrm{R}_{\mathrm{pp}} \text { respectively) }\end{array}$ \\
\hline 0 & Bulk density of the polymer brush \\
\hline [n] & Intrinsic viscosity \\
\hline$\alpha$ & Mark-Houwink constant \\
\hline$\Psi$ & $\begin{array}{l}\text { Rate of amplitude Fresnel coefficients } \mathrm{rp} \text { and } \mathrm{rs} \text { for the light waves } \\
\text { polarized in the plane } \mathrm{p} \text { (parallel with plane of incidence) and } \mathrm{s} \\
\text { (perpendicular on plane of incidence) }\end{array}$ \\
\hline$\Delta$ & Phase difference between the mentioned plane $\mathrm{p}$ and $\mathrm{s}$ \\
\hline$\varepsilon$ & Material's electric permittivity \\
\hline & Vacuum wavelength (or equivalent photon energy) of the probing light \\
\hline
\end{tabular}




$\begin{array}{ll}\gamma \mathrm{s}, \gamma \mathrm{l}, \text { and } \gamma \mathrm{sl} & \text { Interfacial tensions } \\ \sigma_{\mathrm{s}} \text { and } \sigma_{1} & \text { Surface tensions of the solid and liquid } \\ \theta & \text { Young contact angle }\end{array}$

\subsection{List of Figures}

Figure 1 Different approaches to functionalize specific surfaces with small molecules

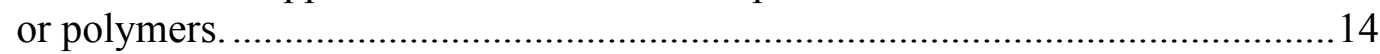

Figure 2 Conventional Free Radical Polymerization. (45) .........................................16

Figure 3 Reaction equilibrium in a $C R P(50)$.........................................................19

Figure 4 Molecular weight distributions resulting from a FRP (black) and a CRP (red)

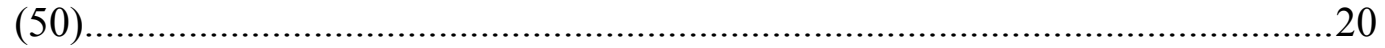

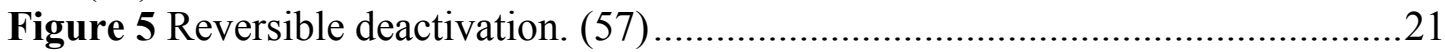

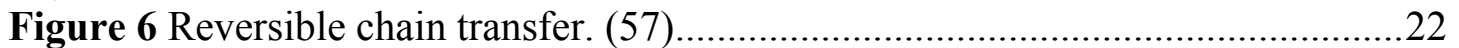

Figure 7 Typical molecular weight and PDI vs. conversion for a living radical

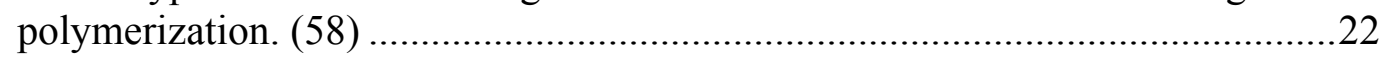

Figure 8 ATRP polymerization mechanism. (57) ...................................................2. 23

Figure 9 Raft polymerization schematic. The number of chains of each type shown here is not in proportion to that expected for a well-designed experiment. On average, all living chains grow simultaneously and have equal chain length because equilibration of the dormant and active chain ends is rapid with respect to propagation. A raft agent is represented as ' $\mathrm{ZC}(=\mathrm{S}) \mathrm{S}$ '. (45).

Figure 10 Typical molecular weight distrubions for a conventional and a RAFT polymerization of styrene under identical experimental conditions. (45) ............24

Figure 11 General structure of a chain transfer agent...........................................25

Figure 12 Examples of the different classes of thiocarbonylthio RAFT agents. (50)25

Figure 13 Overall reaction in RAFT polymerization. (34) .......................................26

Figure 14 Mechanism for reversible addition-fragmentation chain transfer (RAFT). (45)

Figure 15 Mechanism of RAFT polymerization. (80) ............................................27

Figure 16 Comparison of the developments of the molecular mass of the polymers with consumed monomer in the FRP-case (black) and the CRP-case (red). (57) ........28

Figure 17 Schematic of general RAFT polymerization. (50) ...................................28

Figure 18 Structure of different classes of reagents currently used as RAFT agents.

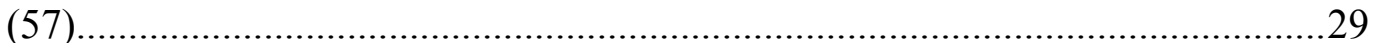

Figure 19 Structure of some R groups and dithiobenzoates. (57) ............................30

Figure 20 Canonical form of (a) xanthates and (b) dithiocarbamates. (57) .................31

Figure 21 Guideline for selection of RAFT agents. (45) ......................................... 31

Figure 22 Schematic of (a) cross-termination between propagating and intermediate radicals of the pre-equilibrium, (b) the core-equilibrium, and (c) self-termination reaction between intermediate radicals of the core-equilibrium. (45) ..................33

Figure 23 Schematic illustration of copolymers types. (57) ....................................33

Figure 24 Schematic illustration of linear diblock copolymerization via RAFT. (57)

Figure 25 Self-assembled monolayers are formed by simply immersing a substrate into a solution of the surface-active compound. The driving force for the spontaneous 
formation of the 2D assembly includes chemical bond formation of molecules with the surface and intermolecular interactions. (19) ...............................................36

Figure 26 Adduct of ammonia and boron trifluoride. The arrow $\rightarrow$ indicates that both electrons in the bond originate from the amine moiety. (137)

Figure 27 Examples of a) a bidentate ligand - Ethane-1,2-diamine has two lone pairs of electrons shown in green b) a tridentate ligand. 1,4,7-triazaheptane (also known as diethylenetriamine) has three lone pairs of electrons shown in green. (139)...41

Figure 28: Surface active organosulfur compounds that form monolayers on gold. (19)

Figure 29 Schematic diagram of an ideal, single-crystalline SAM of alkanethiolates supported on a gold surface with a (111) texture. (19) .......................................4

Figure 30 Chemical reaction of thiolate bond formation. (19) ................................45

Figure 31 Schematic representation of the organisation of gold and sulphur atoms. . 45

Figure 32 Illustration of the organization of the thiol self-assembly in solution. (212)

Figure 33 Chemisorption Configuration of the BDTB (Left) and of the DBTTC

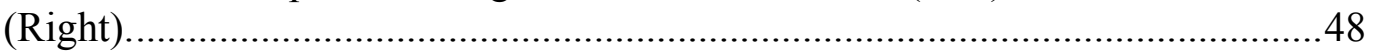

Figure 34 Structures of phenyldithioester derivatives used in SERS based study......48

Figure 35 Some monomers illustrating the concept of carbon backbone..................49

Figure 36 The structure of high density polyethylene. (230).....................................50

Figure 37 The structure of low density polyethylene. (230) ...................................50

Figure 38 Various architectures of polymers. (230) ...............................................51

Figure 39 Schematic illustration of brush types with respect to geometry of substrate (a) Planar brushes (b) Cylindrical brushes (c) Spherical brushes. (234) ..............52

Figure 40 Two hundred chains of a polymer brush (chain length $\mathrm{N}=100$ ) under good

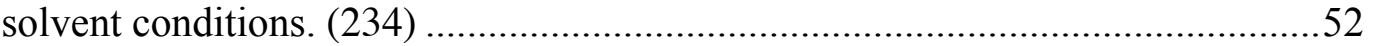

Figure 41 Schematic illustration of the Alexander de Gennes model for the theoretic description of polymer brushes. The chain segments with the "blobs" (indicated by the circles) behave as random ("Gaussian") coils. (D represents the average distance between anchor points) (234) (a) Average end to end distance of a nongrafted polymer chain in a given solvent (b) If the brush height $(\mathrm{H})$ of grafted chain is not higher than the end-to-end distance $(\mathrm{R})$ of the identical non-grafted polymer chain in the same solvent, resulting conformation is a mushroom (c) If tethering points are high enough, the macromolecular chains stretch away from the surface so that in the given solvent the brush height $(\mathrm{H})$ is larger than the end-toend distance of the identical non-grafted chains dissolved in the same solvent. If the distance between grafting points (D) is smaller than the chain end-to-end distance, it leads to formation of a real brush conformation. (25), (237) .............53

Figure 42 (a) "mushroom", (b) "pancake" and (c) "brush" used for the different possible conformations of surface-attached polymers. (234) ...............................55

Figure 43 Classification of polymer brushes with respect to grafting density............57

Figure 44 Synthetic strategies for the preparation of polymer brushes: (a) physisorption of diblock copolymers via preferential adsorption of the red blocks to the surface (grafting to approach); (b) chemisorption via reaction of appropriately endfunctionalized polymers with complementary functional groups at the substrate surface (grafting to approach); (c) polymer brushes grown via surface-initiated polymerization techniques (grafting from approach). (33) ................................58

Figure 45 Schematic description of the physisorption of a block copolymer. (25) ....58

Figure 46 Preparation of polymer brushes on surfaces via SI-CRP through (a) grafting to (b) grafting from approaches. (244) .59 
Figure 47 Schematic description of the "grafting to" process of a polymer chain. (244)

Figure 48 Schematic representation of the "grafting from" process of a polymer chain. (249).

Figure 49 Anchoring types of a Raft agent to a surface via $\mathrm{Z}$ and $\mathrm{R}$ group approach.

Figure 50 Structures of Raft agents used in this study.

Figure 51 SI-RAFT Polymerization: (A) Bimolecular Process as reported by Baum and Brittain (263) for the Preparation of PMMA Brushes from Azo-Functionalized Silicon Wafers; (B) R-Group Approach To Grow PBA Brushes from Dithiobenzoate Modified Silica Nanoparticles as Described by Li and Benicewicz; (264) (C) Z-Group Approach for the Grafting of PMA Brushes from Silica Particles Supported Trithiocarbonate Derivative. (265).

Figure 52 Overview of different polymer brush architectures that can be prepared via surface-initiated controlled radical polymerization. (A) block copolymer brushes; (B) random copolymer brushes; (C) cross-linked polymer brushes; (D) free-standing polymer brushes; (E) hyperbranched polymer brushes; (F) highly branched polymer brushes; $(\mathrm{G})$ Y-shaped binary mixed polymer brushes; $(\mathrm{H})$ standard binary mixed brushes; (I) molecular weight gradient polymer brushes; (J) grafting density gradient polymer brushes; $(\mathrm{K}, \mathrm{L})$ chemical composition gradient polymer brushes. (33)....

Figure 53 Schematic represantation of bottle brush formation by polymerization of HEMA and LA. (25)

Figure 54 Basic mechanism describing the in situ reduction and immobilization of a RAFT-prepared (co)polymer on a gold surface. (301).

Figure 55 (a) Schematic Illustration of RAFT-Based DNA Detection and (b) the Chemical Reaction of RAFT Polymerization Grafting on DNA-Immobilized Surfaces. (302).

Figure 56 (A) DNA-polymer conjugates grafted on the surface using RAFT polymerization; (B) Chemical steps in preparation of CTA-coupled ssDNA molecules. (305) 70

Figure 57 General scheme for the preparation of protein and cell-resistant PPEGMEMA brushes from the electrodeposited RAFT agent. (306) .................71

Figure 58: Stepwise fabrication process for creating streptavidin-immobilized poly(OEGA) brushes. (307)

Figure 59: Schematic diagram of (a) preparation of thiol terminated polymer (PPgMAMPC-SH), (b,c) immobilization of Biotin- $\mathrm{N}_{3}$ and $\mathrm{PNA}_{3} \mathrm{~N}_{3}$ on PPgMAMPC-modified gold surface, and (d,e) specific binding between

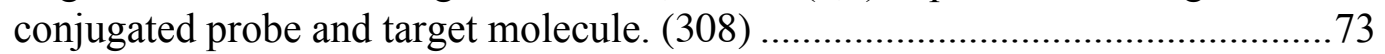

Figure 60 Some examples for applications of polymer brushes. (309) ......................73

Figure 61 Schematic representation of physical and chemical stimuli in solution, and surfaces \& interfaces state. (311)

Figure 62 Solvent responsiveness of a binary mixed homopolymer brush: solvent $\mathrm{B}$ is a nonselective solvent, whereas solvents $\mathrm{A}$ and $\mathrm{C}$ are selective for the red and blue segments, respectively. (33)

Figure 63 Solvent-responsive behavior of binary mixed homopolymer brushes. (41)

Figure 64 Structural changes in a diblock copolymer brush upon variations in solvent quality; solvent B is a good solvent for both blocks, while solvent A is a good solvent for the blue block but a nonsolvent for the red block. (33). 
Figure 65 Planar polyelectrolyte (PEL) brush with ionizable groups (a) swells when the groups dissociate a polar solvent (b); the dissociation is $\mathrm{pH}$ dependent for weak polyelectrolytes (c); addition of salt leads to the shrinkage of the PEL brush. (41)

Figure 66 Mixed two-component brush: lateral phase segregation of chains in nonselective solvent (a), and combined lateral and layered segregation in a selective solvent (b, c). (41)....

Figure 67 Mixed weak polyelectrolyte brush forms a neutral complex in the isoelectric point (a); in acidic or basic solutions positively or negatively charged polymers, respectively, are stretched while a counterpart chains are collapsed and segregated to the grafting surface $(b, c)$. (41)...

Figure 68 The block-copolymer brush constituted of more soluble A blocks and less soluble B blocks. The brushes grafted by the A-blocks (a) form B-polymer micelles shielded by the A-polymers (a, right). In good solvent for the A-polymers Bmicelles are located at the top of the brush (a, left). The brushes grafted by the Bpolymers (b) form dumbbell-like micelles (b, right). In good solvent for the Apolymers the A-blocks are stretched and the B-blocks form micelles segregated to

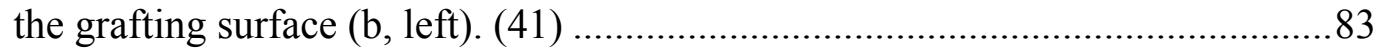

Figure 69 Poly (acrylic acid) brushes from a silica substrate. (353)..........................84

Figure 70 Poly-(N,N'-(dimethylamino)ethyl methacrylate) from silica substrate. (354)

Figure 71 Approaches used to prepare star-shaped polymers. (50) ...........................93

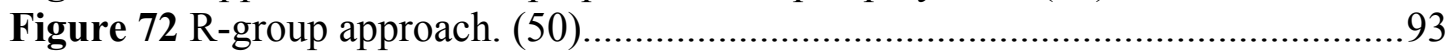

Figure 73 Z-group approach. (50) .....................................................................94

Figure 74 Schematic depiction of the formation of polymer brushes via (a) "grafting to" (b) "grafting from" technique with $\mathrm{R}_{\mathrm{g}}$ as the radius of gyration of a random coiled polymer in a good solvent, $L$ as the layer thickness, $d$ the average lateral distance between grafting points, $\mathbf{M}$ an arbitrary monomer, and $\mathbf{I}$ an initiation site able to induce a surface initiated polymerization (c) Initiator group approach on

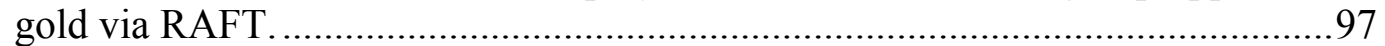

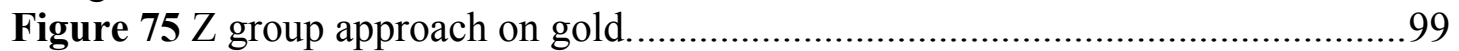

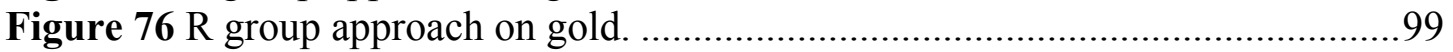

Figure 77 Novel straightforward strategies developed for preparation of various brush architectures on gold planar surfaces............................................................ 102

Figure 78 Chemical structure of the "free" chain transfer agents used in this work.104 Figure 79 Chemical structure of the "free" chain transfer agents used in this work.105 Figure 80 Polymer brush formation on gold via novel approach based on chemisorption of (a) linear and (b) multifunctional raft agent.

Figure 81 Chemical structure of the "free" chain transfer agent precursor and chain transfer agents used in this work.

Figure 82 Polymer brush formation on gold via novel approach based on chemisorption of DTBA. 108

Figure 83 Chemical structures of the "free" chain transfer agents used in this work.

Figure 84 The mechanism of novel Z-Raft approach leading to surface-confined star polymers.

Figure 85 2D AFM topography images $(1 \mu \mathrm{m} \times 1 \mu \mathrm{m})$ in ambient conditions, surface profile analysis and photographs of $1 \mu \mathrm{L}$ water droplets for (a) Bare gold, (b) MUD layer, (c) CTA layer. 115 
Figure 86 ATR-FTIR spectra of (a) MUD layer, (b) CTA layer, (c) poly (BuMA) brush kinetics synthesized for $1,3,5,7,24 \mathrm{~h}$.

Figure 87 2D AFM topography image $(1 \mu \mathrm{m} \times 1 \mu \mathrm{m})$ in ambient conditions, surface profile analysis and photograph of $1 \mu \mathrm{L}$ water droplet for poly (BuMA) brushes synthesized for $24 \mathrm{~h}$ with the ratio of $[\mathrm{M}] /[\mathrm{CTA}] /[\mathrm{I}]=2000 / 4 / 1$ in feed. 118

Figure 88 Results of Raft mediated polymerizations of BuMA in Toluen at $60{ }^{\circ} \mathrm{C}$ with the ratio of $[\text { Monomer }]_{\mathrm{o}} \backslash[\text { freeCTA }]_{\mathrm{o}} \backslash[\mathrm{AIBN}]_{\mathrm{o}}=2000 \backslash 4 \backslash 1$ in feed a) thickness profiles of poly (BuMA) brushes , b) the surface coverage plotted against molecular weight of poly (BuMA) brushes, c) plot of pseudo-first-order kinetics of poly (BuMA) brushes, d) molecular weights of poly (BuMA) brushes with inreasing monomer conversion.

Figure 89 ATR-FTIR spectra of (a) CTA layer, (b),(c),(d),(e),(f) poly (DMAEMA) brush kinetics synthesized for $1,3,5,7,24 \mathrm{~h}$ respectively.

Figure 90 2D AFM topography image $(1 \mu \mathrm{m} \times 1 \mu \mathrm{m})$ in ambient conditions, surface profile analysis and photograph of $1 \mu \mathrm{L}$ water droplet for poly (DMAEMA) brushes synthesized for $24 \mathrm{~h}$ with the ratio of $[\mathrm{M}] /[\mathrm{CTA}] /[\mathrm{I}]=2000 / 4 / 1$ in feed.

122

Figure 91 Results of Raft mediated polymerizations of DMAEMA in Toluen at $60{ }^{\circ} \mathrm{C}$ with the ratio of [Monomer $]_{\mathrm{o}} \backslash[\text { freeCTA }]_{\mathrm{o}} \backslash[\mathrm{AIBN}]_{\mathrm{o}}=2000 \backslash 4 \backslash 1$ in feed a) thickness profile of poly (DMAEMA) brushes, b) the surface coverage plotted against molecular weight of poly (DMAEMA) brushes, c) plot of pseudo-first-order kinetics of poly (DMAEMA) brushes, d) molecular weights of poly (DMAEMA) brushes with inreasing monomer conversion.

Figure 92 2D AFM topography image $(1 \mu \mathrm{m} \times 1 \mu \mathrm{m})$ in ambient conditions, surface profile analysis and photograph of $1 \mu \mathrm{L}$ water droplet for poly(PEGMA) brushes synthesized for $5 \mathrm{~h}$ with the ratio of $[\mathrm{M}] /[\mathrm{CTA}] /[\mathrm{I}]=2000 / 4 / 1$.

Figure 93 ATR-FTIR spectra of (a) PPEGMA brush synthesized for $5 \mathrm{~h}$ and (b) PPEGMA-block-PBuMA brush synthesized for $24 \mathrm{~h}$ 126

Figure 94 2D AFM topography image $(1 \mu \mathrm{m} \times 1 \mu \mathrm{m})$ in ambient conditions, surface profile analysis and photograph of $1 \mu \mathrm{L}$ water droplet for PPEGMA-block-PBuMA brushes synthesized for $24 \mathrm{~h}$ with the ratio of $[\mathrm{M}] /[\mathrm{CTA}] /[\mathrm{I}]=2000 / 4 / 1$ in feed.

Figure 95 ATR-FTIR spectra of (a) PDMAEMA brush synthesized for $24 \mathrm{~h}$ and (b) PDMAEMA-block-PPEGMA brush synthesized for $24 \mathrm{~h}$.

Figure 96 2D AFM topography image $(1 \mu \mathrm{m} \times 1 \mu \mathrm{m})$ in ambient conditions, surface profile analysis and photograph of $1 \mu \mathrm{L}$ water droplet for PDMAEMA-blockPPEGMA brush synthesized for $24 \mathrm{~h}$ with the ratio of $[\mathrm{M}] /[\mathrm{CTA}] /[\mathrm{I}]=2000 / 4 / 1$ in feed.

Figure 97 2D AFM topography images $(1 \mu \mathrm{m} \times 1 \mu \mathrm{m})$ in ambient conditions, surface profile analysis and photographs of $1 \mu \mathrm{L}$ water droplets for (a) poly (BuMA) brushes synthesized for $24 \mathrm{~h}$ with the ratio of $[\mathrm{M}] /[\mathrm{CTA}] /[\mathrm{I}]=6000 / 4 / 1$ in feed, (b) poly (BuMA) brushes synthesized for $24 \mathrm{~h}$ with the ratio of $[\mathrm{M}] /[\mathrm{CTA}] /[\mathrm{I}]=10000 / 4 / 1$ in feed, (c) poly (DMAEMA) brushes synthesized for $24 \mathrm{~h}$ with the ratio of $[\mathrm{M}] /[\mathrm{CTA}] /[\mathrm{I}]=10000 / 4 / 1$ in feed.

Figure 98 ATR-FTIR spectra of (a) poly (BuMA) brush synthesized for $24 \mathrm{~h}$ with the ratio of $[\mathrm{M}] /[\mathrm{CTA}] /[\mathrm{I}]=2000 / 4 / 1$ in feed, (b) poly (DMAEMA) brush synthesized for $24 \mathrm{~h}$ with the ratio of $[\mathrm{M}] /[\mathrm{CTA}] /[\mathrm{I}]=2000 / 4 / 1$ in feed (c) poly (BuMA) brush synthesized for $24 \mathrm{~h}$ with the ratio of $[\mathrm{M}] /[\mathrm{CTA}] /[\mathrm{I}]=6000 / 4 / 1$ in feed, (d) poly (DMAEMA) brush synthesized for $24 \mathrm{~h}$ with the ratio of $[\mathrm{M}] /[\mathrm{CTA}] /[\mathrm{I}]=$ 
$10000 / 4 / 1$ in feed and (e) poly (BuMA) brush synthesized for $24 \mathrm{~h}$ with the ratio

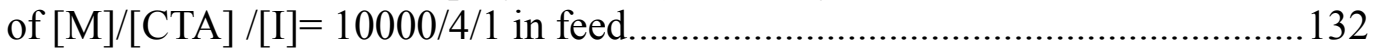

Figure 99 ATR-FTIR spectra of (a) gold-CDB (2_Phenyl_2_Propylbenzodithioate),

(b) gold-EBPEDTTC (ethane-1,2-diyl bis(1-phenylethyl) ditrithiocarbonate) (c) gold-PETSPETTCP

(Penta-erythritol-tetrakis-(3-(S-phenylethyl trithiocarbonate))-propanoate (tetra functional)) (d) gold-DPEHSMPETTC Dipentaerythriol-hexakis(3-(S-methoxopropionate-trithiocarbonate))-

propanoate(hexa functional).

Figure $1002 \mathrm{D}$ AFM topography images $(1 \mu \mathrm{m} \times 1 \mu \mathrm{m})$ in ambient conditions, surface profile analysis, and photographs of $1 \mu \mathrm{L}$ water droplets for (a) gold-CDB (2_Phenyl_2_Propylbenzodithioate), (b) gold-EBPEDTTC (ethane-1,2-diyl bis(1phenylethyl) ditrithiocarbonate) (c) gold-PETSPETTCP (Penta-erythritoltetrakis-(3-(S-phenylethyl trithiocarbonate))-propanoate (tetra functional)), (d) gold-DPEHSMPETTC Dipentaerythriol-hexakis(3-(S-methoxopropionatetrithiocarbonate))-propanoat (hexa functional). .............................................140

Figure 101 ATR-FTIR spectra of (a) gold-EBPEDTTC and (b),(c),(d),(e),(f) poly (BuMA) brush kinetics synthesized in the presence of free CTA (EBPEDTTC) from gold-EBPEDTTC surface for 1,3,7,12, $24 \mathrm{~h}$ respectively.

Figure 102 2D AFM topography image $(1 \mu \mathrm{m} \times 1 \mu \mathrm{m})$ in ambient conditions, surface profile analysis, and photograph of $1 \mu \mathrm{L}$ water droplet for poly (BuMA) synthesized in the presence of bifunctional free CTA (EBPEDTTC) and AIBN in toluene at $60{ }^{\circ} \mathrm{C}$ for $24 \mathrm{~h}$ from gold-EBPEDTTC surface.

142

Figure 103 Results of RAFT mediated polymerizations of BuMA from goldEBPEDTTC surface in the presence of bifunctional free CTA ethane-1,2-diyl bis(1-phenylethyl) ditrithiocarbonate (EBPEDTTC) in Toluen at $60^{\circ} \mathrm{C}$ a) thickness profile of poly (BuMA) brushes, b) the surface coverage plotted against molecular weight of poly (BuMA) brushes, c) plot of pseudo-first-order kinetics of poly (BuMA) brushes, d) molecular weights of poly (BuMA) brushes with inreasing monomer conversion. 145

Figure 104 ATR-FTIR spectra of (a) gold-CDB and (b) poly (DMAEMA) brush synthesized in the presence of mono functional free CTA (CDB) from gold-CDB surface for $24 \mathrm{~h}$ with 2000:4:1 ratio of [DMAEMA] / [CTA] / [I] in feed. ....... 146

Figure 105 2D AFM topography image $(1 \mu \mathrm{m} \times 1 \mu \mathrm{m})$ in ambient conditions, surface profile analysis and photograph of $1 \mu \mathrm{L}$ water droplet for poly (DMAEMA) synthesized in the presence of linear free CTA (CDB) and AIBN in toluene at 60 ${ }^{\circ} \mathrm{C}$ for $24 \mathrm{~h}$ from gold-CDB surface.

Figure $1062 \mathrm{D}$ AFM topography images $(1 \mu \mathrm{m} \times 1 \mu \mathrm{m})$ in ambient conditions, surface profile analysis and photograph of $1 \mu \mathrm{L}$ water droplet for poly (DMAEMA) brushes synthesized in the presence of bifunctional free CTA (EBPEDTTC) and AIBN in toluene at $60{ }^{\circ} \mathrm{C}$ for $24 \mathrm{~h}$ from gold-EBPEDTTC surface...................149

Figure 107 ATR-FTIR spectra of (a) gold-EBPEDTTC, (b) poly (PEGMA) brush synthesized in the presence of bifunctional free CTA (EBPEDTTC) from goldEBPEDTTC for $24 \mathrm{~h}$ and (c) poly (PEGMA) brush synthesized in the presence of mono functional free CTA (DSTCSPA) from gold-EBPEDTTC for $24 \mathrm{~h} . . . . . . . .150$

Figure $1082 \mathrm{D}$ AFM topography images $(1 \mu \mathrm{m} \times 1 \mu \mathrm{m})$ in ambient conditions, surface profile analysis and photographs of $1 \mu \mathrm{L}$ water droplets for (a) poly (PEGMA) synthesized in the presence of bifunctional free CTA (EBPEDTTC) and AIBN in toluene at $60{ }^{\circ} \mathrm{C}$ for $24 \mathrm{~h}$ from gold-EBPEDTTC surface (b) poly (PEGMA) synthesized in the presence of linear free CTA (DSTCSPA) and AIBN in toluene at $60{ }^{\circ} \mathrm{C}$ for $24 \mathrm{~h}$ from gold-EBPEDTTC surface. 151 
Figure 109 ATR-FTIR spectra of (a) gold-PETSPETTCP, (b) poly (PEGMA) brush synthesized in the presence of monofunctional free CTA (DSTCSPA) from goldPETSPETTCP surface for $24 \mathrm{~h}$, (c) poly (PEGMA) brush synthesized in the presence of tetra functional free CTA (PETSPETTCP) from gold-PETSPETTCP surface for $24 \mathrm{~h}$.

Figure $1102 D$ AFM topography images $(1 \mu \mathrm{m} \times 1 \mu \mathrm{m})$ in ambient conditions, surface profile analysis and photographs of $1 \mu \mathrm{L}$ water droplets for (a) poly (PEGMA) brushes synthesized in the presence of tetra functional free CTA (PETSPETTCP) and AIBN in toluene at $60{ }^{\circ} \mathrm{C}$ for $24 \mathrm{~h}$ from gold-PETSPETTCP surface, (b) poly (PEGMA) brushes synthesized in the presence of linear free CTA (DSTCSPA) and AIBN in toluene at $60{ }^{\circ} \mathrm{C}$ for $24 \mathrm{~h}$ from gold-PETSPETTCP surface. ..............154

Figure 111 ATR-FTIR spectra of (a) gold-DPEHPETTC (b) poly (PEGMA) brush synthesized in the presence of monofunctional free CTA (DSTCSPA) from goldDPEHPETTC surface for $24 \mathrm{~h}$ and (c) poly (PEGMA) brush synthesized in the presence of tetra functional free CTA (PETSPETTCP) from gold-DPEHPETTC surface for $24 \mathrm{~h}$.

Figure 112 2D AFM topography images $(1 \mu \mathrm{m} \times 1 \mu \mathrm{m})$ in ambient conditions, surface profile analysis and photographs of $1 \mu \mathrm{L}$ water droplets for (a) poly (PEGMA) brushes synthesized in the presence of tetra functional free CTA (PETSPETTCP) and AIBN in toluene at $60{ }^{\circ} \mathrm{C}$ for $24 \mathrm{~h}$ from gold-DPEHPETTC surface, (b) poly (PEGMA) brushes synthesized in the presence of linear free CTA (DSTCSPA) and AIBN in toluene at $60{ }^{\circ} \mathrm{C}$ for $24 \mathrm{~h}$ from gold-DPEHPETTC surface. ................156

Figure 113 ATR-FTIR spectra of (a) gold-EBPEDTTC, (b) poly (DMAEMA) brush synthesized in the presence of bifunctional free CTA (EBPEDTTC) from goldEBPEDTTC surface for $24 \mathrm{~h}$ and (c) poly (DMAEMA)-block-poly (PEGMA) brush synthesized in the presence of bifunctional free CTA (EBPEDTTC) from gold-EBPEDTTC-poly (DMAEMA) surface.

Figure $1142 \mathrm{D}$ AFM topography image $(1 \mu \mathrm{m} \times 1 \mu \mathrm{m})$ in ambient conditions, surface profile analysis and photograph of $1 \mu \mathrm{L}$ water droplet for poly (PEGMA) brush synthesized in the presence of bifunctional free CTA (EBPEDTTC) and AIBN in toluene at $60{ }^{\circ} \mathrm{C}$ for $24 \mathrm{~h}$ from gold-EBPEDTTC-PDMAEMA surface (PDMAEMA-b-poly (poly (ethylene glycol) methyl ether methacrylate) (PPEGMA)).

Figure 115 2D AFM topography images $(1 \mu \mathrm{m} \times 1 \mu \mathrm{m})$ in ambient conditions, surface profile analysises and photographs of $1 \mu \mathrm{L}$ water droplets for (a) bare gold, (b) DTBA on gold.

Figure 116 ATR-FTIR spectra of (a) DTBA on gold and (b) poly (MMA) brush kinetics conducted in the presence of free raft agent precursor (DTBA) for 1,3,5,7, $24 \mathrm{~h}$.

Figure $1172 \mathrm{D}$ AFM topography image $(1 \mu \mathrm{m} \times 1 \mu \mathrm{m})$ in ambient conditions, surface profile analysis and photograph of $1 \mu \mathrm{L}$ water droplets for poly (MMA) brush synthesized in the presence of DTBA for $24 \mathrm{~h}$ with the ratio of $[\mathrm{M}] /[\mathrm{DTBA}] /[\mathrm{I}]=2000 / 4 / 1$ in feed.

Figure 118 Results of DTBA mediated polymerizations of MMA in Toluen at $60{ }^{\circ} \mathrm{C}$, $[\text { Monomer }]_{\mathrm{o}} \backslash[\text { free DTBA }]_{\mathrm{o}} \backslash[\mathrm{AIBN}]_{\mathrm{o}}=2000 \backslash 4 \backslash 1$ in the presence of free raft agent precursor dithiobenzoicacid (DTBA) a) thickness profiles and kinetics of poly (MMA) brushes, b) the surface coverage plotted against molecular weight of poly (MMA) brushes, c) plot of pseudo-first-order kinetics of poly (MMA) brushes, d) molecular weights of poly (MMA) brushes with increasing monomer conversion. 
Figure 119 2D AFM topography image $(1 \mu \mathrm{m} \times 1 \mu \mathrm{m})$ in ambient conditions, surface profile analysis and photograph of $1 \mu \mathrm{L}$ water droplet for poly (BuMA) brush synthesized in the presence of DTBA for $24 \mathrm{~h}$ with the ratio of $[\mathrm{M}] /[\mathrm{DTBA}] /[\mathrm{I}]=2000 / 4 / 1$ in feed. 171

Figure $1202 D$ AFM topography images $(1 \mu \mathrm{m} \times 1 \mu \mathrm{m})$ in ambient conditions, surface profile analysis and photograph of $1 \mu \mathrm{L}$ water droplet for poly (PEGMA) brush synthesized in the presence of free CTA (DSTCSPA) for $24 \mathrm{~h}$ with the ratio of $[\mathrm{M}] /[\mathrm{CTA}] /[\mathrm{I}]=2000 / 4 / 1$ in feed. 173

Figure 121 ATR-FTIR spectra of (a) poly (MMA) brush synthesized with the ratio of $[\mathrm{M}] /[\mathrm{DTBA}] /[\mathrm{I}]=2000 / 4 / 1$ in the presence of free raft agent precursor (DTBA) from gold-DTBA surface for $24 \mathrm{~h}$, (b) poly (DMAEMA) brush synthesized with the ratio of $[\mathrm{M}] /[\mathrm{DTBA}] /[\mathrm{I}]=2000 / 4 / 1$ in the presence of free raft agent precursor (DTBA) from gold-DTBA surface for $24 \mathrm{~h}$, (c) poly (DMAEMA) brush synthesized with the ratio of $[\mathrm{M}] /[\mathrm{DTBA}] /[\mathrm{I}]=10000 / 4 / 1$ in the presence of free raft agent precursor (DTBA) from gold-DTBA surface for $24 \mathrm{~h}$, (d) poly (MMA) brush synthesized with the ratio of $[\mathrm{M}] /[\mathrm{DTBA}] /[\mathrm{I}]=10000 / 4 / 1$ in the presence of free raft agent precursor (DTBA) from gold-DTBA surface for $24 \mathrm{~h}$. 174

Figure 122 2D AFM topography images $(1 \mu \mathrm{m} \times 1 \mu \mathrm{m})$ in ambient conditions, surface profile analysises and photographs of $1 \mu \mathrm{L}$ water droplets for (a) poly (MMA) brush synthesized in the presence of DTBA for $24 \mathrm{~h}$ with the ratio of $[\mathrm{M}] /[\mathrm{DTBA}] /[\mathrm{I}]=10000 / 4 / 1$ in feed, (b) poly (DMAEMA) brush synthesized in the presence of DTBA for $24 \mathrm{~h}$ with the ratio of $[\mathrm{M}] /[\mathrm{DTBA}] /[\mathrm{I}]=2000 / 4 / 1$ in feed, (c) poly (DMAEMA) brush synthesized in the presence of DTBA for $24 \mathrm{~h}$ with the ratio of $[\mathrm{M}] /[\mathrm{DTBA}] /[\mathrm{I}]=10000 / 4 / 1$ in feed. 176

Figure 123 ATR-FTIR spectra of (a) DTBA on gold, (b) poly (BuMA) brush synthesized in the presence of free raft agent precursor (DTBA) from gold-DTBA surface for $24 \mathrm{~h}$, (c) poly(BuMA)-block-poly(DMAEMA) brush synthesized in the presence of free raft agent precursor (DTBA) from gold-DTBA-PBuMA surface for $24 \mathrm{~h}$ 179

Figure $1242 \mathrm{D}$ AFM topography image $(1 \mu \mathrm{m} \times 1 \mu \mathrm{m})$ in ambient conditions, surface profile analysis and photograph of $1 \mu \mathrm{L}$ water droplets for PBuMA-blockPDMAEMA brush synthesized in the presence of DTBA for $24 \mathrm{~h}$ with the ratio of $[\mathrm{M}] /[\mathrm{DTBA}] /[\mathrm{I}]=2000 / 4 / 1$ in feed. 180

Figure 125 ATR-FTIR spectra of (a) DTBA on gold, (b) poly (PEGMA) brush synthesized in the presence of free raft agent (DTSCPA) from gold-DTBA surface for $24 \mathrm{~h}$, (c) poly (PEGMA)-block-poly (DMAEMA) brush synthesized in the presence of free raft agent (CDB) from gold-DTBA-Poly(PEGMA) surface for 24 h. 182

Figure 126 2D AFM topography images $(1 \mu \mathrm{m} \times 1 \mu \mathrm{m})$ in ambient conditions, surface profile analysis and photograph of $1 \mu \mathrm{L}$ water droplet for PPEGMA-blockPDMAEMA brushes synthesized in the presence of free CTA Cumyldithiobenzoate (CDB) for $24 \mathrm{~h}$ with the ratio of $[\mathrm{M}] /[\mathrm{CTA}] /[\mathrm{I}]=2000 / 4 / 1$ in feed. 183

Figure 127 2D AFM images $(1 \mu \mathrm{m} \times 1 \mu \mathrm{m})$ in ambient conditions, surface cross-section analysis and photographs of $1 \mu \mathrm{L}$ water droplets for (a) bare gold (b) gold-MUD (c) gold-MUD-linear CTA (DSTCSPA). 188

Figure 128 ATR-FTIR spectra of (a) gold-MUD, (b) gold-MUD-CTA (DSTCSPA) and (c) star poly (PEGMA) brush kinetics synthesized for 1, 5, 7, 9, $24 \mathrm{~h}$. 189

Figure 129 2D AFM image $(1 \mu \mathrm{m} \times 1 \mu \mathrm{m})$ in ambient conditions, surface cross section analysis and photograph of $1 \mu \mathrm{L}$ water droplet for poly (PEGMA) brush 
synthesized for $24 \mathrm{~h}$ in the presence of tetra functional free CTA (PETSPETTCP).

190

Figure 130 Results of Z-RAFT star polymerization kinetics of PEGMA in Toluen at $60^{\circ} \mathrm{C}$ in the presence of free tetra functional CTA PETSPETTCP (Penta-erythritoltetrakis-(3-(S-phenylethyl trithiocarbonate))-propanoate) a) thickness profiles and kinetics of star poly(PEGMA) brushes, b) the surface coverage plotted against molecular weight of star poly(PEGMA) brushes, c) plot of pseudo-first-order kinetics of star poly(PEGMA) brushes, d) molecular weights of star poly(PEGMA) brushes with inreasing monomer conversion.

Figure 131 ATR-FTIR spectras of (a) Gold-Mud-Linear CTA (DSTCSPA) and poly (PEGMA) brush synthesized for $24 \mathrm{~h} \mathrm{(b)} \mathrm{in} \mathrm{the} \mathrm{presence} \mathrm{of} \mathrm{free} \mathrm{linear} \mathrm{CTA}$ (DSTCSPA) (c) in the presence of bifunctional free CTA (EBPEDTTC) (d) in the presence of tetra functional free CTA (PETSPETTCP) (e) in the presence of hexa functional free CTA (DPEHPETTC). 195

Figure 132 2D AFM images $(1 \mu \mathrm{m} \times 1 \mu \mathrm{m})$ in ambient conditions, surface cross-section analysises, and photographs of $1 \mu \mathrm{L}$ water droplets for poly (PEGMA) brushes synthesized for $24 \mathrm{~h}$ (a) in the presence of free linear CTA (DSTCSPA) (b) in the presence of bifunctional free CTA (EBPEDTTC) (c) in the presence of tetra functional free CTA (PETSPETTCP) (d) in the presence of hexa functional free CTA (DPEHPETTC). 196

Figure 133 A typical GPC instrument including A. Autosampler, B.Column C.Pump, D.RI Detector, E.UV-vis Detector. (511)......................................................2209

Figure 134 Seperation based upon size in solution. (57) .......................................210

Figure 135 The summary of GPC/SEC mechanism. (512) ...................................210

Figure 136 GPC Separation of Anionically Synthesized Polystyrene; $M_{n}=3,000$ g/mol, $\mathrm{PDI}=1.32$. (513)

Figure 137 GPC Separation of Free-Radical Synthesized Polystyrene; $M_{n}=24,000$ $\mathrm{g} / \mathrm{mol}, \mathrm{PDI}=4.96$. (513)

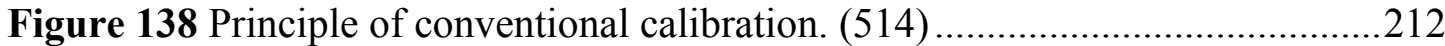

Figure 139 Size of a polymer dissolved in a common solvent. (512).....................212

Figure 140 The plot which gives information about the dynamic behavior of the

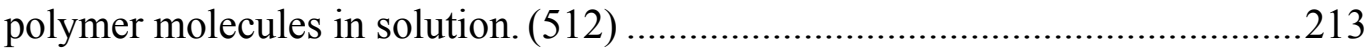

Figure 141 The main components of a GPC/SEC system. (517)...........................215

Figure 142 Range of molecular weights that can be separated for each packing material. (513)

Figure 143 The molecular weight range of separation for individual pore size columns

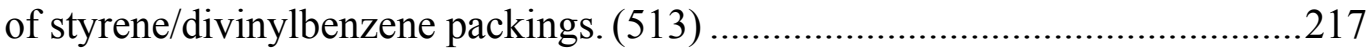

Figure 144 Schematic representation of deflection type refractometer. (57) ...........218

Figure 145 A peak sliced during GPC calculations. (512) ....................................219

Figure 146 HPLC mass spectrometer used for this work. (520)...........................221

Figure 147 The schematic of an HPLC instrument. (521) ...................................221

Figure 148 Schematic representation of HPLC-Mass Spectrometer. (522)..............223

Figure 149 Where the "Flow rate" label denotes the effluent (analyte plus eluent and additivates) coming from the HPLC system. (522) ............................................224

Figure 150 The schematic diagram of ion production process. (522) .....................225

Figure 151 Schematic representation of mass analyzer located in high vacuum region where analyte ions or fragments of analyte ions are separated according to their $\mathrm{m} \backslash \mathrm{z}$ (mass to charge ratio) value. (522)

Figure 152 Schematic represantation of non-collisional or collisional trajectory. (522) 
Figure 153 Schematic representation of a quadrupole. (522) ..............................227

Figure 154 A typical mass spectrum. (522) .......................................................227

Figure 155 Schematic represantation of point and array detectors. (522) ...............228

Figure 156 A typical Spectroscopic Ellipsometer. (523) .......................................229

Figure 157 Measurement principle of ellipsometry. (525) ....................................229

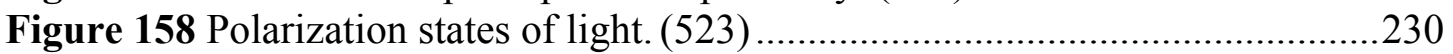

Figure 159 Reflection from the sample (film/substrate system) changes the ellipse of polarization. (523)

Figure 160 Schematic setup of an ellipsometry experiment. (527) .......................232

Figure 161 Characterization of physical properties by spectroscopic ellipsometry.

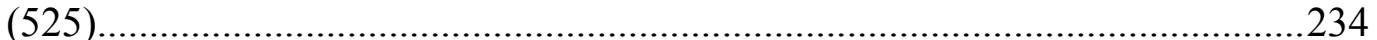

Figure 162 State of pollarisation during nulling ellipsometry. (523) .....................234

Figure 163 PCSA (Polarizer-Compensator-Sample-Analyzer) setup commonly used in Nulling ellipsometry. (529) .235

Figure 164 PSCA (Polarizer-Sample-Compensator-Analyzer) setup commonly used

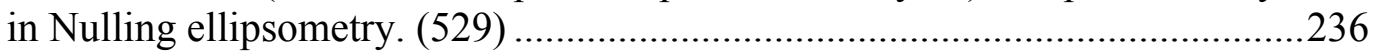

Figure 165 Three phase model for ellipsometric measurement. (57) ......................238

Figure 166: A typical ATR-IR spectrometer. (534) ….......................................240

Figure 167 ATR-FTIR setup to measure coating characteristics on the coated substrate.

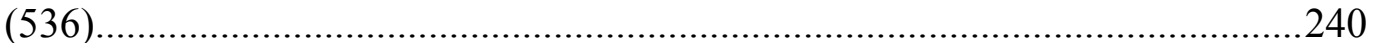

Figure 168 Diamond ATR accessory in Bruker Optics' ALPHA. (536)..................241

Figure 169 The reflection of light by a virtual layer within the optically less dense sample (Goos-Hänchen effect; dotted yellow line). (536) ................................241

Figure 170 General setup of an atomic force microscope with beam deflection detection. (540). 243

Figure 171 Plot of a surface potential and application area for contact, non-contact and tapping mode. The force-distance curve is divided into three sections. There is no interaction between tip and surface in the region shown with green. The attractive regime is demonstrated with blue. Closer to the surface, the tip is rejected in the repulsive regime demonstrated with pink. (542) ..............................................24

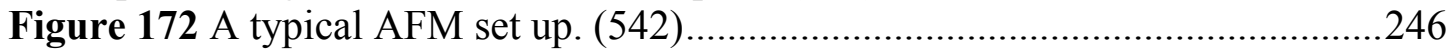

Figure 173 Schematic of the optical cantilever detection and the segmented photo diode. A laser shines onto the cantilever. The light is refected of its back and led to a photo diode via an adjustable photo detector mirror. The photo diode is segmented into four parts which generate the two basic parameters of the AFM setting the photo diode requires only a limited resolution in the micrometers regime. The $\mathrm{x}-, \mathrm{y}-$ and $\mathrm{z}$-position of the cantilever is controlled by piezoelectric transducers. They consist of piezoelectric materials which convert electrical potential into mechanical motion. (542)

Figure 174 Idealized transmission spectrum of the auto tune process. The oscillation amplitude is depicted in black and shows a Lorentzian peak. The phase is colored red. The phase has a 180 degree shift at the frequency of maximum transmission amplitude. The full width half maximum (FWHM) is measured at half of the peak height plot. The point of maximum amplitude is chosen as operation frequency during the scan. (542)

Figure 175 Contact angle and interface tensions at the three phase contact line. (529)

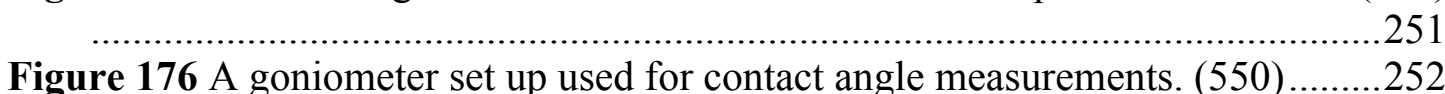

Figure 177 Sessile drop on a plain aluminium surface. (551) ..............................253 
Figure 178 Contour analysis (shown in green) on a flat and a curved sample surface. (552).

\subsection{List of Tables}

Table 1 Classification of Chemical Bonds

Table 2 Overview of initiators, iniferters, and Raft agents that have been used to grow polymer brushes from gold surfaces

Table 3 Summary of polymerization kinetics of poly (BuMA) brushes.................119

Table 4 Summary of polymerization kinetics of poly (DMAEMA) Brushes .......123

Table 5 Summary of diblockcopolymer brushes....................................................129

Table 6 Summary of impact of monomer [M] / raft agent [CTA] ratio in feed on resulting thickness of polymer brushes ........................................................133

Table 7 Summary of polymerization kinetics of poly (BuMA) brushes from gold-

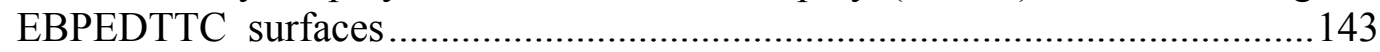

Table 8 Summary of polymerizations and diblockcopolymerization from gold-CTA surfaces

Table 9 Summary of polymerization kinetics of poly (MMA) brushes from goldDTBA surfaces

Table 10 Summary of impact of monomer [M] /Raft agent precursor [DTBA] / initiator [I] ratio on resulting thickness of polymer brushes

Table 11 Summary of DTBA and RAFT mediated blockcopolymerizations from goldDTBA surfaces

Table 12 Summary of polymerization kinetics of star polymer brushes. 192

Table 13 Summary of polymerization of 2, 4, 6 Arm star polymer brushes. 197

Table 14 Summary of Protein Adsorbtion Studies of homo poly (PEGMA), 2, 4 and 6 Armed Star Polymer Brushes

Table 15 Comparement of Raft based approaches used for synthesis of polymer brushes 201

Table 16 ATR crystal types (536). 242

Table 17 Some methods used for determination of the contact angle with different measuring ranges and applications (552) .254

\subsection{Bibliography}

1. Ramakrishna, S, et al. 9, 2001, Composites Science and Technology, Vol. 61, pp. 1189-1224.

2. Ratner, B D, et al. An introduction to biomaterials in Medicine. s.l. : Academic Press, 1996.

3. Mizutani, A, et al. 2008, Biomaterials, Vol. 29, pp. 2073-2081.

4. Balazs, A C, et al. 1997, MRS Bull., Vol. 22, pp. 16-21.

5. Motornov, M, et al. 2007, ACS Nano, Vol. 2, pp. 41-52.

6. Ma, $\boldsymbol{H} \boldsymbol{W}$, et al. 4, 2004, Advanced Materials, Vol. 16, pp. 338-341.

7. Kaholek, M, et al. 2006, Chem. Mater., Vol. 18, p. 3660. 
8. Costa, A C, Composto, R J and Vlcek, P. 2003, Macromolecules, Vol. 36, p. 3254.

9. Raviv, U, et al. 2003, Nature, Vol. 425, p. 163.

10. Ohno, K, et al. 2006, Macromolecules, Vol. 39, p. 1245.

11. Almeida, E. 3, 2001, Ind. Eng. Chem Res., Vol. 40, pp. 15-20.

12. Cartier, M Ed. Handbook of Surface Treatment and Coatings. New York: John Wiley \& Sons, 2003.

13. Swaraj, P. Surface Coatings Science and Technology. New York : John Wiley \& Sons, 1995.

14. Freund, L B and Suresh, S. Thin Films: Stress, Defect Formation and Surface Evolution. Cambridge : Cambridge University Press, 2003.

15. Provder, $\boldsymbol{T}$ and Urban, $\boldsymbol{M} \boldsymbol{W}$. Film Formation in Coatings. Mechanism, Properties, and Morphology. Washington : ACS Symp. Series 790, ACS, 2001.

16. Pascault, J P, et al. Thermosetting Polymers. New York : Marcel Dekker, Inc., 2002.

17. Port, $\boldsymbol{A} \boldsymbol{B}$ and Cameron, $\boldsymbol{C}$. Film Formation. [book auth.] A $R$ Marrion. The Chemistry and Physics of Coatings. 2nd Ed. Cambridge : RSC, 2004.

18. Bigelow , W C, Pickett, D L and Zisman , W. 1946, A. J. Colloid Interface Sci., Vol. 1, pp. 513-538.

19. Ulman, A. 1996, Chem. Rev., Vol. 96, p. 1533.

20. Nuzzo, R G and Allara, D L . 1983, J. Am. Chem. Soc., Vol. 105, p. 4481.

21. Sagiv, J. 1980, J. Am. Chem. Soc., Vol. 102, p. 92.

22. Wasserman, S R, Tao, Y T and Whitesides, J M. 1989, Langmuir, Vol. 5, p. 1074.

23. Walczak, M W, et al. 1991, J. Am. Chem. Soc., Vol. 113, p. 2370.

24. Porter, M D , Bright, T B and Allara, D L. 109, 1987, J. Am. Chem. Soc., p. 3559.

25. Advincula , $\boldsymbol{R} \boldsymbol{C}$, et al. Polymer Brushes: Synthesis, Characterization, Applications. NewYork : Wiley-VCH, 2004.

26. Zhao, B and Brittain, W J. 2000, Prog. Polym. Sci., Vol. 25, pp. 677-710.

27. Boyes, S G, et al. 2004, J. Surf. Sci., Vol. 570, pp. 1-12.

28. Fries, $\boldsymbol{K}$, et al. 47, 2008, Chem. Commun., pp. 6288-6290.

29. Zhou, F and Huck, W T S. 33, 2006, Phys. Chem. Chem. Phys., Vol. 8, pp. $3815-$ 3823 .

30. Boulatov, $\boldsymbol{R}$, [ed.]. Polymer Mechanochemistry. 2015.

31. Advincula , $\boldsymbol{R} \boldsymbol{C}$, et al. Polymer Brushes: Synthesis, Characterization, Applications. NewYork: Wiley-VCH, 2004. 
32. Zhou, J H, et al. 2006, Chem. Comm., Vol. 46, pp. 4820-4822.

33. Barbey, R, et al. 11, 2009, Chem Rev., Vol. 109, pp. 5437-5427.

34. Choi, I S and Langer, R. 2001, Macromolecules, Vol. 34, pp. 5361-5363.

35. Juang, A, et al. 5, 2001, Langmuir, Vol. 17, pp. 1321-1323.

36. Hawker, C J, Bosman, A W and Harth , E. 2001, Chem. Rev., Vol. 101, pp. 3661 3688 .

37. Teare, D O H, et al. 2005, Langmuir, Vol. 21, pp. 11425-11430.

38. Bhat, $\boldsymbol{R} \boldsymbol{R}$, et al. 2006, Adv Polym Sci, Vol. 198, pp. 51-124.

39. Glinel, K, et al. 2009, Bioconjugate Chem., Vol. 20, pp. 71-77.

40. Feng, W, et al. 1, 2007, Advances in Polymer Technology, Vol. 26, pp. 1-13.

41. Minko, S. 4, 2006, Polymer Reviews, Vol. 46, pp. 397-420.

42. Moad, G and Solomon, D H. 1995, Polymer International, Vol. 42, p. 346.

43. Gilbert, R G. Polymer International. London : Academic Press, 1995. p. 352. Vol. 41.

44. Akzo Nobel. Initiators for High Polymers. s.l. : BTB Communication , 2006 . pp. 130.

45. Moad, G, Rizzardo, E and Thang, S H. 2005, Aus.J.Chem, Vol. 58, p. 379.

46. Buback, M, et al. 2007, Macromol. Chem. Phys., Vol. 208, pp. 772-783.

47. Wang, J S and Matyjaszewski, K. 1995, Macromolecules, Vol. 28, p. 7901.

48. Boschmann, D. Sternpolymere mittels RAFT-Polymerisation . Physical Chemistry, Chemistry. Göttingen : s.n., 2008. PhD Thesis.

49. Boyer, C, et al. 2005, Macromolecules, Vol. 38, pp. 10353-10362.

50. Förster, N. Nano-Carrier Synthesis via Z-RAFT Star Polymerisation. Physical Chemistry. Goettingen : s.n., 2012. PhD thesis.

51. Matyjaszewski, K, [ed.]. Controlled/Living Radical Polymerization: Progress in ATRP, NMP, and RAFT. Washington : ACS Symp. Series, 2000. Vol. 768.

52. Webster, O W. 1991, Science, Vol. 251, p. 887.

53. Cowie, J M G. Polymers Chemistn' and Physics of Modern Materials. London : Blackie Academic and Professional, 1991.

54. Otsu, T and Yoshida, M. 1982, Makromol.Chem.Rapid Commun., Vol. 3, p. 4391.

55. Otsu, T and Yoshida, M. 1982, Makromol.Chem.Rapid Commun., Vol. 3, p. 127.

56. Turner, S R and Blevins, R W. 1990, Macromolecules, Vol. 23, p. 1856.

57. Hojjati, B. Synthesis and Characterization of TiO2 nanocomposites via reversible addition fragmentation chain transfer polymerization. 2010. PhD Thesis. 
58. Moad, G, et al. 2000, Polym. Int., Vol. 49, p. 993.

59. ISO 13220:1999. Particle Size Analysis - Laser Diffraction Methods . s.l. : ISO G., In press.

60. Pyun, J and Matyjaszewski, K. 2001, Chem. Mater., Vol. 13, p. 3436.

61. Odian, G. Principles of polymerization. New York: John Wiley Sons, Inc, 1981. p. Chapter 3.

62. Hu, B , et al. 2006, Polymer, Vol. 47, p. 7653.

63. Chiefari, J, et al. 2003, Macromolecules, Vol. 36, pp. 2273-2283.

64. Barner Kowollik, C, et al. 2003, Polym. Sci., Part A: Polym. Chem., Vol. 41, p. 365.

65. Barner-Kowollik, C, [ed.]. Handbook of RAFT Polymerization. s.l. : Wiley-VCH, 2008.

66. Macromolecules. Chiefari, J, et al. 1998, Vol. 31, p. 5559.

67. Mayadunne, R, et al. 1999, Macromolecules, Vol. 32, p. 6977.

68. Boyer, C, et al. 2009, Chem. Rev., Vol. 109, p. 5402.

69. Yosikawa, S and Tsubokawa, N. 1996, Polym. J., Vol. 28, p. 317.

70. Hojjati, B and Charpentier, P A. 2008, Polym.Sci. part A: Polym Chem, Vol. 46, p. 3926.

71. Cacioli, P, et al. 1986, J Macromol. Sci.: Chem.A, Vol. 23.

72. Meijs , G E , Rizzardo, E and Thang, S H . 1988, Macromolecules, Vol. 21, p. 3122.

73. Meijs G, E, Rizzardo, E and Thang, S H. 1990, Polym. Bull. (Berlin), Vol. 24, p. 501.

74. Meijs, G E, Rizzardo, E and Thang, S H. 1992, Polym. Prepi., Vol. 33, p. 893.

75. Meijs G, E, et al. 1992, Macromol. Chem. Phvs., Vol. 193, p. 369.

76. Mitsukami, Y, et al. 2001, Macromolecules, Vol. 34, p. 2248.

77. Stange, T G, Mathew, $R$ and Evans, D F. 1992, Langmuir, Vol. 8, p. 920.

78. Barner Kowolik, C. 2001, Macromolecules, Vol. 34, p. 7849.

79. Chong, Y K, et al. 1999, Macromolecules, Vol. 32, p. 2071.

80. Vana, P, Davis, T P and Barner-Kowolik, C. 2002, Macromolecular Theory and Simulations, Vol. 11, p. 823.

81. Moad, G, Rizzardo, E and Thang, S H. 2006, Aust. J. Chem., Vol. 56, pp. 669692. 
82. Moad, G, Rizzardo, E and Thang, S H. 2005, Aust. J. Chem., Vol. 58, pp. 379410.

83. Moad, G, Rizzardo, E and Thang, S H. 2008, Polymer, Vol. 49, p. 1079.

84. Lee, $\boldsymbol{T} \boldsymbol{W}$, et al. 2001, Advanced Materials, Vol. 13, p. 211.

85. Chong, Y K, Krstina, J and Le, T P T. 2003, Macromolecules, Vol. 36, p. 2256.

86. Hawthorne, D G, et al. 1999, Macromolecules, Vol. 32, p. 5457.

87. Barner-Kowollik, C, et al. 2001, J. Polym. Sci.: Part A., Vol. 39, pp. 1353-1365.

88. Barner-Kowollik, C, et al. 2001, Macromolecules, Vol. 34, pp. 7849-7857.

89. Monteiro, M J and De Brouwer, H. 2001, Macromolecules, Vol. 34, pp. 349-352.

90. Kwak, Y, et al. 2002, Macromolecules, Vol. 35, pp. 3026-3029.

91. Mc Leary, J B , et al. 2004, Macromolecules, Vol. 37, pp. 2383-2394.

92. Barner-Kowollik, C, et al. 2006, Polym.sci.Part A: Polym.chem, Vol. 44, p. 5809.

93. Drache, M, et al. 2005, Polymer, Vol. 46, p. 8483.

94. Arita, T, et al. 2004, e-Polymers, Vol. 003, pp. 1-14.

95. Kwak, Y, Goto, A and Fukuda, T . 2004, Macromolecules, Vol. 37, pp. 12191225.

96. Arita, T, et al. 2005, Macromol. Mater. Eng., Vol. 290, p. 283.

97. Barner-Kowollik, C, Vana, P and Davis , T P. Handbook of Radical Polymerization. [ed.] M Krzysztof and P D Thomas . Newyork : John Wiley and Sons, Inc., 2002.

98. Toy, A A , et al. 2004, Macromolecules, Vol. 37, pp. 744-751.

99. Bozovic-Vukic, J, et al. 2007, Macromolecules, Vol. 40, p. 7132.

100. Zhou, J H, et al. 2006, Chem. Comm., Vol. 46, pp. 4820-4822.

101. Hua, F J and Yang, Y L. 2001, Polymer, Vol. 42, p. 1361.

102. Suna, X, et al. 2005, Polymer, Vol. 46, p. 5251.

103. Guang-Qiang, C, et al. 2000, Macromolecules, Vol. 33, p. 232.

104. Cianga, L, et al. 2003, Designed Monomers and Polymers, Vol. 6, p. 145.

105. Rizzardo, E, et al. 2001, Macromol Symp, Vol. 174, p. 209.

106. Tsujii, Y, et al. 2001, Macromolecules, Vol. 34, p. 8872.

107. Discher, D E and Eisenberg, A . 2002, Science, Vol. 297, p. 967.

108. Mecke, A, Dittrich, C and Meier, W. 2006, Soft Matter, Vol. 2, p. 751.

109. Wikipedia. [Online] http://en.wikipedia.org/wiki/Micelle .

110. Riess, G. 2003, Prog. Polym.Sci., Vol. 28, p. 1107. 
111. Müller, T J J and and Bunz, U H F. Functional Organic Materials. s.l. : WileyCVH, 2007.

112. Ulman, A. Organic Thin Films and Surfaces: Directions for the Nienties. Boston : Academic Press. p. 1995. Vol. Volume 20.

113. Smits, E C P , et al. 2008, Nature, Vol. 455, p. 956.

114. Nanotechnology. [Online] http://www.nanotech-now.com/nanotechnologyglossary-S-U.htm..

115. Gracias, D H, et al. 2000, Science, Vol. 289, pp. 1170-1172.

116. Grzybowski, B A and and Whitesides, G M. 2002, Science, Vol. 295, pp. 24182421.

117. Schreiber, F. 5-8, 2000, Progress in Surface Science, Vol. 65, pp. 151-256.

118. Ishida, T, et al. 8, 1998, Langmuir, Vol. 14, pp. 2092-2096.

119. Nuzzo, R G, Dubois, L H and and Allara, D L. 2, 1990, Journal of the American Chemical Society, Vol. 112, pp. 558-569.

120. Bain, C D , et al. 1, 1989, Journal of the American Chemical Society, Vol. 111, pp. 321-335.

121. Kajikawa, K, et al. 8B, 1997, Japanese Journal of Applied Physics Part 2-Letters, Vol. 36, pp. 1116-L1119.

122. Ginnai, T M, Oxley, D P and and Pritchard, R G. 1, 1980, Thin Solid Films, Vol. 68, pp. 241-256.

123. Vincett, P S and and Roberts, G G. 1, 1980, Thin Solid Films, Vol. 68, pp. 135171.

124. Rohwerder, M and and Stratmann, M. 7, 1999, Mrs Bulletin, Vol. 24, pp. 4347.

125. Itoh, M, et al. 2, 1994, Journal of the Electrochemical Society, Vol. 141, pp. 352358.

126. Laibinis, $\boldsymbol{P} \boldsymbol{E}$ and and Whitesides, G M . 6, 1992, Journal of the American Chemical Society, Vol. 114, pp. 1990-1995.

127. Aramaki K Shimizu K Sakakibara M. Aramaki, K, et al. 6, 1993, Journal of the Electrochemical Society, Vol. 140, pp. 1561-1567.

128. Laibinis, $\boldsymbol{P} \boldsymbol{E}$ and and Jennings, $\boldsymbol{G}$ K. 1996, Abstracts of Papers of the American Chemical Society, Vol. 212, pp. 20-COLL.

129. Zurcher, S, et al. 4, 2006, Journal of the American Chemical Society, Vol. 128, pp. 1064-1065. 
130. Volmeruebing, M, Reynders, B and and Stratmann, M. 1, 1991, Werkstoffe Und Korrosion-Materials and Corrosion, Vol. 42, pp. 19-34.

131. Nozawa, K, Nishihara, H and and Aramaki, K. 9, 1997, Corrosion Science, Vol. 39, pp. 1625-1639.

132. [Online] https://en.wikipedia.org/wiki/Chemical_bond.

133. The Covalent Bond in H 2. Rioux, F. 5, 2001, The Chemical Educator, Vol. 6 , pp. 288-290.

134. Compendium of Chemical Terminology . 2nd ed. . s.l. : IUPAC, 1997. Online corrected version: (2006-).

135. Compendium of Chemical Terminology. 2nd ed. . s.l. : IUPAC, 1997. Online corrected version: (2006-).

136. Compendium of Chemical Terminology. 2nd ed. . s.l. : IUPAC, 1997. Online corrected version: (2006-).

137. [Online] https://en.wikipedia.org/wiki/Dipolar_bond.

138. [Online] https://en.wikipedia.org/wiki/Chelation .

139. [Online] http://www.chemicool.com/definition/ligand.html.

140./Online]http://chemwiki.ucdavis.edu/Core/Inorganic_Chemistry/Organometalli

c_Chemistry/Ligand_Binding_in_Coordination_Complexes/CC5.CChelation.

141. [Online] https://en.wikipedia.org/wiki/Coordination_complex.

142. Cotton, Frank Albert, Geoffrey, Wilkinson and Carlos, A Murillo. Advanced Inorganic Chemistry. s.l. : ISBN 978-0-471-19957-1, 1999. p. 1355.

143. Miessler, Gary L and Donald, Arthur Tarr . Inorganic Chemistry. s.l. : ISBN 978-0-13-841891-5, 1999. p. 642.

144. [Online] https://en.wikipedia.org/wiki/Transition_metal_thiolate_complex.

145. [Online] http://www.sigmaaldrich.com/technical-documents/articles/materialmatters/self-assembled-monolayers. html .

146. Dubois, L H and Nuzzo, R G. 43, 1992, Ann. Phys. Chem., p. 437.

147. Bain, C D and Whitesides, G M. 1, 1989, Adv. Mater., p. 506.

148. Supramolecular architecture. Folkers, J P, et al. [ed.] T Bein. Washington, DC : American Chemical Society, 1992. ACS Symposium Series. Vol. 499, pp. 10-23.

149. Lee, T R, et al. 1991, Pure Appl. Chem., Vol. 63, p. 821.

150. Whitesides, G M and Ferguson, G S. 1, 1988, Chemtracts-Org. Chem., p. 171.

151. J. Am. Chem. Soc. Sellers, H, et al. 1993, Vol. 115, p. 9389.

152. Troughton, $\boldsymbol{E} \boldsymbol{B}$, et al. 1988, Langmuir, Vol. 4, p. 365. 
153. Katz, E, Itzhak, N and Willner, I. 1992, J. Electroanal. Chem., Vol. 336, p. 357.

154. Sabatani, E, et al. 1993, Langmuir, Vol. 9, p. 2974.

155. Bryant, M A , Joa, , S L and Pemberton, J E. 1992, Langmuir, Vol. 9, p. 753.

156. Hill, W and Wehling, B. 1993, J. Phys. Chem., Vol. 97, p. 9451.

157. Li , T T T, Liu, H Y and Weaver, M J. 1984, J. Am. Chem. Soc., Vol. 106, p. 1233.

158. Cooper, J M, Greenough, K R and McNeil, C J . 1993, J. Electroanal. Chem., Vol. 347, p. 267.

159. Uvdal, K, Bodo, P and Liedberg, B . 1992, J. Colloid Interf. Sci., Vol. 149, p. 162.

160. Ihs, A, Uvdal, K and Liedberg, B . 1993, Langmuir, Vol. 9, p. 733.

161. Arndt, Th, Schupp, H and Schepp, W. 1989, Thin Solid Films, Vol. 178, p. 319. 162. Mielczarski, J A and Yoon, R H. 1991, Langmuir, Vol. 7, p. 101.

163. Edwards, TR G, et al. 1989, J. Chem. Soc., Chem. Commun., p. 1041.

164. Arduengo, A J, et al. 1990, J. Am. Chem. Soc., Vol. 112, p. 6153.

165. Xue, G, et al. 1991, J. Electroanal. Chem., Vol. 310, p. 139.

166. Bharathi, S, Yegnaraman, V and Rao, G P. 1993, Langmuir, Vol. 9, p. 1614.

167. Samanat, M G, Broen, C A and Gordon, J G. 1992, Langmuir, Vol. 8, p. 1615.

168. Ulman, A. 1989, J. Mater. Educ., Vol. 11, p. 205.

169. Laibinis, P E, et al. 1991, J. Am. Chem. Soc., Vol. 113, p. 7152.

170. Walczak, M W, et al. 1991, J. Am. Chem. Soc., Vol. 113, p. 2370.

171. Laibinis, P E and Whitesides, G M. 1992, J. Am. Chem. Soc., Vol. 112, p. 1990.

172. Ihs, A and Liedberg, B . 1994, Langmuir, Vol. 10, p. 734.

173. Walczak, M W, et al. 1991, J. Am. Chem. Soc., Vol. 113, p. 2370.

174. Ihs, A and Liedberg, B. 1994, Langmuir, Vol. 10, p. 734.

175. Laibinis, P E and Whitesides, G M. 1992, J. Am. Chem. Soc., Vol. 114, p. 9022.

176. Shimazu, K, et al. 1994, Bull. Chem. Soc. Jpn., Vol. 67, p. 863.

177. Demoz, A and Harrison, D J. 1993, Langmuir, Vol. 9, p. 1046.

178. Muskal, N, et al. 1995, J. Am. Chem. Soc., Vol. 117, p. 1147.

179. Stratmann, M. 1990, Adv. Mater., Vol. 2, p. 191.

180. Volmer, M, Stratmann, M and Viefhaus, H. 1990, Surf. Interface Anal., Vol. 16, p. 278.

181. Liu, Q and Xu, Z. 1995, Langmuir, Vol. 11, p. 4617. 
182. Brust, M, et al. 1994, J. Chem. Soc., Chem. Commun., p. 801.

183. Sheen, C W, et al. 1992, J. Am. Chem. Soc., Vol. 114, p. 1514.

184. Gu, Y, et al. 1995, Langmuir, Vol. 11, p. 1849.

185. Biebuyck, H A , Bain, C D and Whitesides, G M. 1994, Langmuir, Vol. 10, p. 1825.

186. Mohri, $N$, et al. 1995, Langmuir.

187. Textor, M, et al. 7, 2000, Langmuir, Vol. 16, pp. 3257-3271.

188. Hofer, R. Surface Modification for Optical Biosensor Applications. Natural Sciences. Zurich : ETH Zurich, 2000. PhD Thesis.

189. Smith, R K, Lewis, P A and Weiss, P S . 2004, Progress in Surface Science, Vol. 75, p. 1.

190. Love, J C, et al. 2005, Chem. Rev., Vol. 105, p. 1103.

191. Somorjai , G A. Chemistry in Two Dimensions - Surfaces. New York : Cornell University Press, 1982.

192. Rodriguez, J A , et al. 2003, J. Am. Chem. Soc., Vol. 125, p. 276.

193. Roy, P R, et al. 21, 2004, Electroanalysis, Vol. 16, pp. 1743-1822.

194. Porter, M D, et al. 1987, J. Am. Chem. Soc., Vol. 109, p. 3559.

195. Nuzzo, R G, Fusco, F A and Allara, D L . 1987, J. Am. Chem. Soc., Vol. 109, p. 2358 .

196. Bain, C D , Biebuyck, H A and Whitesides, G M. 1989, Langmuir, Vol. 5, p. 723.

197. Nuzzo, R G, Zegarski, B R and Dubois, L H. 1987, J. Am. Chem. Soc., Vol. 109, p. 733.

198. Li, Y, et al. 1992, J. Am. Chem. Soc., Vol. 114, p. 2428.

199. Widrig, C A , Chung, C and Porter, M D. 1991, J. Electroanal. Chem., Vol. 310, p. 335 .

200. Bryant, M A and Pemberton, J E. 1991, J. Am. Chem. Soc., Vol. 113, p. 3630.

201. Bryant, M A and Pemberton, J E. 1991, J. Am. Chem. Soc., Vol. 113, p. 8284.

202. Dubois, L H and Nuzzo, R G. 1992, Ann. Phys. Chem., Vol. 43, p. 437.

203. Bain, C D and Whitesides, G M. 1989, J. Am. Chem. Soc., Vol. 111, p. 7164.

204. Bain, C D and Whitesides, G M. 1989, Angew. Chem. Int. Ed., Vol. 28, p. 506.

205. Bain, C D , Evall, J and Whitesides, G M. 1989, J. Am. Chem. Soc., p. 7155.

206. Sai , P T and and Raychaudhuri, A K. 2007, J. Phys. D: Appl. Phys., Vol. 40, p. 3182. 
207. Ron, H, et al. 1998, J. Phys. Chem B, Vol. 102, p. 9861.

208. Mekhalif, Z, et al. 2003, Langmuir, Vol. 19, p. 637.

209. Wang, Y, Hush, N S and and Reimers, J R . 2007, J. Am. Chem. Soc., Vol. 129, p. 14532.

210. Nelles, G, et al. 1998, Langmuir, Vol. 14, p. 808.

211. Grönbeck, H, Curioni, A and and Andreoni, W. 2000, J. Am. Chem. Soc., Vol. 122, p. 3839 .

212. [Online] http://www.ifm.liu.se/applphys/sensor/self.html-24/01/2010 .

213. Yamada, R, Sakai, H and Uosaki, K . 1999, Chem. Lett., p. 667.

214. Ishida, T, et al. 1997, Langmuir, Vol. 13, p. 4638.

215. Kawasaki, M, et al. 2000, Langmuir, Vol. 16, p. 1719.

216. Ulman, A. Thin Films-Self-Assembled Monolayers of Thiols. Boston : Academic Press, 1998.

217. Heister, $\boldsymbol{K}$, et al. 1999, Langmuir, Vol. 15, pp. 5440-5443.

218. Porter M D. Zhong, C J, Brush, R C and Anderegg, J. 1999, Langmuir, Vol. 15, pp. 518-525.

219. Dishner, M H, Hemminger, J C and Feher, F J. 1996, Langmuir, Vol. 12, pp. 6176-6178.

220. Tao, Y T, et al. 1998, Langmuir, Vol. 14, pp. 145-150.

221. Colorado, $\boldsymbol{R} \boldsymbol{J r}$, Villazana, $\boldsymbol{R} \boldsymbol{J}$ and Lee , T R. 1998, Langmuir, Vol. 14, pp. 6337-6340.

222. His, A, Uvdal, K and Liedberg, B . 1993, Langmuir, Vol. 9, pp. 733-739.

223. Mielczarski, J A and Yoon, R H. 1991, Langmuir, Vol. 7, pp. 101-108.

224. Zhao, Y, et al. 2005, J. Am. Chem. Soc., Vol. 127, pp. 7328-7329.

225. Duwez, A S , et al. 2006, Macromolecules, Vol. 39, pp. 2729-2731.

226. Blakey, I, et al. 2, 2010, Langmuir, Vol. 26, pp. 692-701.

227. Sanchez-Cortes, $\boldsymbol{S}$, et al. 2001, Langmuir, Vol. 17, pp. 1157-1162.

228. Sanchez-Cortes, S and Garcia-Ramos, J V . 2001, Surf. Sci., Vol. 473, pp. 133142.

229. Park, J S, et al. 2005, Langmuir, Vol. 21, pp. 2902-2911.

230. [Online] http://www.cmu.edu/gelfand/k12-teachers/polymers/polymerarchitecture.

231. Boschmann, D and Vana, P . 2007, Macromolecules, Vol. 40, pp. 2683-2693.

232. Huang, X, et al. 2011, Macromolecules, Vol. 44, pp. 8351-8360. 
233. Chen, Y. 2012, Macromolecules, Vol. 45, pp. 2619-2631.

234. [Online] https://www.aps.org/units/dpoly/resources/upload/zhulina-talk.pdf.

235. Alexander, S. 1977, J. Physique, Vol. 38, p. 977.

236. De Gennes, P G. 1980, Macromolecules, Vol. 13, p. 1069.

237. Jones, $R \boldsymbol{A} L$ and Richards, $R \boldsymbol{W}$. Polymer at surfaces and interfaces. s.l. : Cambridge University Press. p. 1999.

238. Minko, S. 2006, Journal of Macromolecular Science, Part C: Polymer Reviews, Vol. 46, pp. 397-420.

239. Edmondson, S, Osborne, V L and Huck, W T S. 2004, Chem. Soc. Rev., Vol. 33, p. 14.

240. Zengin, A and Caykara, T J. 2012, Polym. Sci., Part A: Polym. Chem, Vol. 50, pp. 4443-4450.

241. Choi, J, et al. 2012, J.Polym. Sci, Part A: Polym. Chem, Vol. 50, pp. 4010-4018. 242. Xie, S, Zhang, S and Wang, F. 2004, J Appl Polym Sci, Vol. 94, p. 1018.

243. Minko, S, et al. 2003, J. Am. Chem. Soc., Vol. 125, p. 3896.

244. [Online] http://de.slideshare.net/anihilator01/polymer-brushes .

245. Yu, K, et al. 2004, Surface Science, Vol. 572, p. 490.

246. Minko, S, et al. 2002, Langmuir, Vol. 18, p. 289.

247. Luzinov, I, et al. 2000, Macromolecules, Vol. 33, p. 1043.

248. Pyun , J , Kowalewski , T and Matyjaszewski, K . 2003, Macromol. Rapid. Commun, Vol. 24, p. 1043.

249. [Online] http://de.slideshare.net/anihilator01/polymer-brushes .

250. Sakellariou, G, et al. 2006, J. Poly Sci. part A: poly chem., Vol. 44, p. 769.

251. Yoshikawa, S, et al. 1995, J. Poly Sci. part A: poly chem., Vol. 33, p. 2251.

252. Husemann, M, et al. 1999, Angew. Chem. Inter. Ed., Vol. 111, p. 688.

253. Voccia, S, et al. 2004, Langmuir, Vol. 20, p. 10670.

254. Yavuz, M S, et al. 2009, Langmuir, Vol. 25, p. 13120.

255. Liu, X, Guo , S and Mirkin, C A. 2003, Angew. Chem. Inter. Ed., Vol. 42, p. 4785.

256. Farhan, T and Huck, W T S. 2004, European polymer journal, Vol. 40, p. 1599.

257. Fristrup, C J, Jankova, K and Hvilsted, S. 2009, Soft Matter, Vol. 5, p. 4623.

258. Husemann, M, et al. 1999, Angew chem Int Ed Engl., Vol. 38, p. 647.

259. Brinks, M K, et al. 2007, Angew. Chem. Int. Ed, Vol. 46, p. 5231.

260. Raula, J, et al. 2003, Langmuir, Vol. 19, p. 3499. 
261. Perrier, S and Takolpuckdee, P J. 2005, Polym. Sci., Part A: Polym. Chem., Vol. 43, p. 5347.

262. Hernandez-Guerrero, M, et al. 2005, Eur. Polym. J., Vol. 41, p. 2264.

263. Baum, M and Brittain, W J. 2002, Macromolecules, Vol. 35, p. 610.

264. Li , C Z and Benicewicz, B C. 2005, Macromolecules, Vol. 38, p. 5929.

265. Zhao, Y and Perrier, S. 2007, Macromolecules, Vol. 40, p. 9116.

266. Chen, YW, et al. 2006, Polym. Sci., Part A: Polym. Chem., Vol. 44, p. 3071.

267. Rowe-Konopacki, M D and Boyes, S G. 2007, Macromolecules, Vol. 40, p. 879.

268. Yuan, K, et al. 2007, Mater. Lett., Vol. 61, p. 2033.

269. Li, D L , et al. 2008, J. Polym. Sci., Part A: Polym. Chem., Vol. 46, p. 970.

270. Li, L, Kang, E and Neoh, K. 2008, Appl. Surf. Sci., Vol. 254, p. 2600.

271. Hojjati, B, Sui, $R$ H and Charpentier, P A. 2007, Polymer, Vol. 48, p. 5850.

272. Roy, D, Guthrie, J T and Perrier, S. 2005, Macromolecules, Vol. 38, p. 10363.

273. Peng, Q, et al. 2006, Macromolecules, Vol. 39, p. 5577.

274. Zhao, Y L and Perrier, S. 2006, Macromolecules, Vol. 39, p. 8603.

275. Wang, G J, et al. 2007, Polymer, Vol. 48, p. 728.

276. Stenzel, M H, Zhang, L and Huck, W T S. 2006, Macromol. Rapid Commun., Vol. 27, p. 1121.

277. Takolpuckdee, P, Mars, C A and Perrier, S. 2005, Org. Lett. , Vol. 7 , p. 3449.

278. Perrier, S and Takolpuckdee, P. 2005, Macromolecules, Vol. 38, p. 6770.

279. Jones, D M , Brown, A A and and Huck, W T S . 2002, Langmuir , Vol. 18 , p. 1265.

280. Ma, H, et al. 2006, Adv. Funct. Mater., Vol. 16, p. 640.

281. Otsu, T, Ogawa, T and Yamamoto, T. 1986, Macromolecules, Vol. 19, p. 2087.

282. Nakayama, Y and Matsuda, T. 1999, Langmuir, Vol. 15, p. 5560.

283. Husseman, M, et al. 1999, Macromolecules, Vol. 32, p. 1424.

284. Matyjaszewski, K, et al. 1999, Macromolecules, Vol. 32, p. 8716.

285. Tomlinson, M R, Efimenko, K and Genzer, J. 2006, Macromolecules, Vol. 39, p. 9049 .

286. Shah, $\boldsymbol{R} \boldsymbol{R}$, et al. 2000, Macromolecules, Vol. 33, p. 597.

287. Kim, J B, Bruening, M L and Baker, G L . 2000, J. Am. Chem. Soc., Vol. 122, p. 7616 .

288. Nuss, $\boldsymbol{S}$, et al. 2001, Angew. Chem. Int. Ed., Vol. 40, p. 4016.

289. Kim , D J , et al. 2005, Macromol. Chem. Phys., Vol. 206, p. 1941. 
290. Kang, S M, et al. 2006, Nanotechnology, Vol. 17, p. 4719.

291. Li, D X, et al. 2007, AdV. Funct. Mater., Vol. 17, p. 3134.

292. Li, D X, et al. 2007, Chem. Mater., Vol. 19, p. 412.

293. Li , D X, et al. 2007, Chem.sEur. J., Vol. 13, p. 2224.

294. Duan, H W, et al. 2004, Angew. Chem., Int. Ed., Vol. 43, p. 5639.

295. Duan, $\boldsymbol{H} \boldsymbol{W}$, et al. 2005, Angew. Chem., Int. Ed., Vol. 44, p. 1717.

296. Duan, $\boldsymbol{H} \boldsymbol{W}$, et al. 2005, Langmuir, Vol. 21, p. 11495.

297. Mandal, T K, Fleming, M S and Walt, D R . 2002, Nano Lett., Vol. 2, p. 3.

298. Ohno, K, et al. 2002, Macromolecules, Vol. 35, p. 8989.

299. Ohno, K, et al. 2003, Angew. Chem., Int. Ed., Vol. 42, p. 2751.

300. Roth, P J and Theato, P. 2008, Chem. Mater., Vol. 20, p. 1614.

301. Sumerlin, B S, et al. 19, 2003, Langmuir, pp. 5559-5562.

302. He, P, et al. 2008, Anal. Chem., Vol. 80, pp. 3633-3639.

303. Lou , X, et al. 2005, Anal. Chem., Vol. 77, pp. 4698-4705.

304. Lou, X and He, L. 2006, Langmuir, Vol. 22, pp. 2640-2646.

305. He, P and and He, L. 2009, Biomacromolecules, Vol. 10, pp. 1804-1809.

306. Tria, M. C. R., et al. 2010, Biomacromolecules, Vol. 11, pp. 3422-3431.

307. Zengin, A and Caykara, T. 2012, J Polym Sci Part A: Polym Chem, Vol. 50, pp. 4443-4450.

308. Wiarachai, O, et al. 2016, Langmuir, Vol. 32, p. 1184-1194.

309. [Online] https://www.aps.org/units/dpoly/resources/upload/zhulina-talk.pdf.

310. Lee, H L , et al. 2010, Progress in Polymer Science, Vol. 35, p. 24.

311. Liu, F and Urban, M W. 2010, Progress in Polymer Science, Vol. 35, p. 3.

312. Sun, TL , et al. 2004, Angew Chem Int Ed, Vol. 43, p. 357.

313. Li , L, Li , J and and Lukehart, C M. 2008, Sens. and Act. B: Chemi., Vol. 130, p. 783.

314. Lue, S J, Hsu, J J and Wei, T C . 2008, J. Membr. Sci., Vol. 321, p. 146.

315. Moran, M T, et al. 2007, J Biomed Mater Research, Vol. 81A, p. 870.

316. Chen, J K, et al. 2008, Macromolecules, Vol. 41, p. 8729.

317. Bumbu, G G, et al. 2004, Macromol. Chem. Phys., Vol. 205, p. 1713.

318. Bumbu, G G, et al. 2007, Langmuir, Vol. 23, p. 2203.

319. Aoki, H, Kitamura, M and Ito, S . 2008, Macromolecules, Vol. 41, p. 285.

320. Zhao, B, Haasch, $\boldsymbol{R}$ T and MacLaren, S J. 2004, J. Am. Chem. Soc., Vol. 126, p. 6124 . 
321. Santer, S and Ruhe, J. 2004, Polymer, Vol. 45, p. 8279.

322. Santer, S, et al. 2006, AdV. Mater., Vol. 18, p. 2359.

323. Li, D J, Sheng, X and Zhao, B. 2005, J Am. Chem. Soc., Vol. 127, p. 6248.

324. Granville, A M , et al. 2004, Macromolecules, Vol. 37, p. 2790.

325. Rowe, M D , Hammer, B A G and Boyes, S G. 2008, Macromolecules, Vol. 41, p. 4147.

326. Xu, C, et al. 2006, Macromolecules, Vol. 39, p. 3359.

327. Gao, X, et al. 2008, Langmuir, Vol. 24, p. 8303.

328. Yin, Y, et al. 2007, Macromolecules, Vol. 40, p. 5161.

329. Prokhorova, S A , et al. 2003, Nanotechnology, Vol. 14, p. 1098.

330. Xu, C, et al. 2005, Langmuir, Vol. 21, p. 11136.

331. Boyes, S G, et al. 2002, Macromolecules, Vol. 35, p. 4960.

332. Huang, WX, et al. 2003, Nanotechnology, Vol. 14, p. 1075.

333. Kumar , N A . Molecular Dynamics Simulations of Polyelectrolyte Brushes. Potsdam : s.n., 2006. PhD Thesis.

334. Ruhe, J, et al. 2004, AdV. Polym. Sci., Vol. 165, p. 79.

335. Pincus, P. 1991, Macromolecules, Vol. 24, pp. 2912 - 2919.

336. Israels, R, Leermakers, F A M and Fleer, G J . 1994, Macromolecules, Vol. 27, pp. 3087-3093.

337. Guo, X and Ballauff, M. 2001, Phys. Rev. E, Vol. 64, p. 051406.

338. Minko, S, et al. 2002, Phys. Rev. Lett., Vol. 88, p. 035502.

339. Milner, S T, Witten, T A and Cates, M E. 1989, Macromolecules, Vol. 22, pp. 853-861.

340. Kent, M S , et al. 1996, Macromolecules, Vol. 29, pp. 2843-2849.

341. Goedel, W A, et al. 1999, Macromolecules, Vol. 32, pp. 7599-7609.

342. Minko, S, et al. 2003, Macromolecules, Vol. 36, pp. $7268-7279$.

343. Shusharina, N P and Linse, P . 2001, Eur. Phys. J. E, Vol. 6, pp. 147-155.

344. Houbenov, N, Minko, S and Stamm, M. 2003, Macromolecules, Vol. 36, pp. 5897-5901.

345. Biesheuvel, P M and Stuart, M A C. 2004, Langmuir, Vol. 20, pp. 2785-2791.

346. Zhulina, E B , Singh, C and Balazs, A C. 1996, Macromolecules, Vol. 29, pp. 6338-6348.

347. Zhulina, E B , Singh, C and Balazs, A C. 1996, Macromolecules, Vol. 29, pp. $8254-8259$. 
348. Zhao, B, et al. 2000, J. Am. Chem. Soc., Vol. 122, pp. $2407-2408$.

349. Zhao, B , et al. 2000, Macromolecules, Vol. 33, pp. 8821-8827.

350. Zhao, B and Brittain, W J. 2000, Macromolecules, Vol. 33, pp. 8813-8820.

351. Boyes, S G , et al. 2003, Macromolecules, Vol. 36, pp. 9539-9548.

352. Treat, N D, et al. 2006, Macromolecules, Vol. 39, p. 26.

353. Wu, T, et al. 2007, Macromolecules, Vol. 40, p. 87565.

354. Sanjuan, S, et al. 2007, Langmuir, Vol. 23, p. 5769.

355. Geoghegan, M, et al. 2006, Soft Matter, Vol. 2, p. 1076.

356. Kurosawa, S, et al. 2004, Biosens. Bioelectron., Vol. 20, p. 1165.

357. Liu, G M and Zhang, G Z . 2008, J. Phys. Chem. B, Vol. 112, p. 10137.

358. Li , D, et al. 2008, Macromolecules, Vol. 41, p. 7254.

359. Zhou, F , et al. 2006, J. Am. Chem. Soc., Vol. 128, p. 5326.

360. Zhou, F and Huck, W T S. 2005, Chem. Commun., p. 5999.

361. Abu-Lail, N I, et al. 2006, Sens. Actuators, B, Vol. 114, p. 371.

362. Sanjuan, S and Tran, Y. 2008, Macromolecules, Vol. 41, p. 8721.

363. Ayres, N, Cyrus, C D and Brittain, W J. 2007, Langmuir, Vol. 23, p. 3744.

364. Gong, P, et al. 2007, Macromolecules, Vol. 40, p. 8765.

365. Ayres, N, Boyes, S G and Brittain, W J. 2007, Langmuir, Vol. 23, p. 182.

366. Langmuir. Choi, E Y, et al. 2007, Vol. 23, p. 10389.

367. Zhou, F, et al. 2008, Nano Lett., Vol. 8, p. 725.

368. Spruijt, E, Choi, E Y and Huck, W T S. 2008, Langmuir, Vol. 24, p. 11253.

369. Azzaroni, O, et al. 2005, Macromolecules, Vol. 38, p. 10192.

370. Moya, S, et al. 2005, Angew. Chem., Int. Ed., Vol. 44, p. 4578.

371. Azzaroni, O, Brown, A A and Huck, W T S . 2007, AdV. Mater., Vol. 151, p. 19.

372. Shimada, $N$ and and Maruyama, A. Thermoresponsive Polymers with Functional Groups Selected for Pharmaceutical and Biomedical Applications. 2013. pp. 235-241. Vol. Chapter 14.

373. Hoffman, A S. 1995, Artificial Organs, Vol. 19, pp. 458-467.

374. Ward, M A and Georgiou, K T . 3, 2013, Polymers, Vol. 3, pp. 1215-1242.

375. Nagase, K, et al. 2008, Langmuir, Vol. 24, p. 511.

376. Nagase, K, et al. 2008, Biomacromolecules, Vol. 9, p. 1340.

377. Idota , N, et al. 2006, Langmuir, Vol. 22, p. 425.

378. Nagase, K, et al. 2007, Langmuir, Vol. 23, p. 9409. 
379. Matsuda, T and Ohya, S. 2005, Langmuir, Vol. 21, p. 9660.

380. Xu, F J, et al. 2006, Biomaterials, Vol. 27, p. 1236.

381. Li , D J, Dunlap, J R and Zhao, B. 2008, Langmuir, Vol. 24, p. 5911.

382. Lokuge, I, Wang, X and Bohn , P W. 2007, Langmuir, Vol. 23, p. 305.

383. Schild, H G. 1992, Prog. Polym. Sci., Vol. 17, p. 163.

384. Mark, J E. Polymer Data Handbook. s.l. : Oxford University Press, Inc., 1999.

385. He, Q, et al. 2007, Langmuir, Vol. 23, p. 3981.

386. Wei, Q S , Ji, J and Shen, J C . 2008, Macromol. Rapid Commun., Vol. 29, p. 645.

387. Jhon, Y K, et al. 2006, Macromol. Rapid Commun., Vol. 27, p. 697.

388. Rahane, S B , et al. 2008, II.AdV. Funct. Mater., Vol. 18, p. 1232.

389. Balamurugan, S , et al. 2003, Langmuir, Vol. 19, p. 2545.

390. Li , J, Chen, X R and Chang, Y C. 2005, Langmuir, Vol. 21, p. 9562.

391. Wu, T, et al. 2008, Chem. Mater., Vol. 20, p. 101.

392. Benetti, E M, Zapotoczny, S and Vancso, G J. 2007, AdV. Mater., Vol. 19, p. 268.

393. Yim, H, et al. 2004, Macromolecules, Vol. 37, p. 1994.

394. Yim, H, et al. 2005, Phys ReV E Stat Nonlinear, Soft Matter Phys., Vol. 72, p. 051801 .

395. Yim, H, et al. 2004, Polym. Sci., Part B: Polym. Phys., Vol. 42, p. 3302.

396. Yim, H, et al. 2006, Macromolecules, Vol. 39, p. 3420.

397. Plunkett, K N, et al. 2006, Langmuir, Vol. 22, p. 4259.

398. Li, D J, et al. 2006, Langmuir, Vol. 22, p. 3344.

399. Jonas, A M , et al. 2008, Nano Lett., Vol. 8, p. 3819.

400. Azzaroni, O, Brown, A A and Huck, W T S . 2006, Angew. Chem., Int. Ed., Vol. 45, p. 1770.

401. Schulz, D N, et al. 1986, Polymer, Vol. 27, p. 1734.

402. Jonas, A M, et al. 2007, Macromolecules, Vol. 40, p. 4403.

403. Xia , F, et al. 2006, AdV. Mater., Vol. 18, p. 432.

404. Kizhakkedathu, J N, et al. 2004, Polymer, Vol. 45, p. 7471.

405. Wang, X, et al. 2007, Macromol. Rapid Commun., Vol. 28, p. 828.

406. Andruzzi, L, et al. 2005, Langmuir, Vol. 21, p. 2495.

407. Chang, Y, et al. 2008, Langmuir, Vol. 24, p. 5453.

408. Ladd, J, et al. 2008, Biomacromolecules, Vol. 9, p. 1357. 
409. Ista, L K and and Lopez, G P. 1998, Journal of Industrial Microbiology \& Biotechnology, Vol. 20, p. 121.

410. Kong, B, et al. 2009, Biomaterials, Vol. 30, p. 5514.

411. Lutolf, M P and Hubbell, J A . 2005, Nat. Biotechnol., Vol. 23, p. 47.

412. Stevens, M M and George, J H. 2005, Science, Vol. 310, p. 1135.

413. Balachandra, A M, Baker, G L and Bruening, M L J. 2003, Membr. Sci., Vol. 227, pp. 1-14.

414. Sun, L, Baker, G L and Bruening, M L. 2005, Macromolecules, Vol. 38, p. 2307.

415. Wang, H J, et al. 2006, J., Am. Chem. Soc., Vol. 128, p. 15954.

416. Singh, N, et al. 2008, J. Membr. Sci., Vol. 311, p. 225.

417. Zhu, L P, et al. 2008, J. Membr. Sci., Vol. 320, p. 407.

418. Holmberg, S, et al. 2002, J. Polym. Sci. Part A: Polym. Chem., Vol. 40, p. 591.

419. Chen, Y W, et al. 2007, Polymer, Vol. 48, p. 7604.

420. Lee, S B , et al. 2004, Biomacromolecules, Vol. 5, p. 877.

421. Murata, H, et al. 2007, Biomaterials, Vol. 28, p. 4870.

422. Ramstedt, M, et al. 2007, Langmuir, Vol. 23, p. 3314.

423. Cheng, G, et al. 2007, Biomaterials, Vol. 28, p. 4192.

424. Cringus-Fundeanu, I, et al. 2007, Langmuir, Vol. 23, p. 5120.

425. Zhang, F, et al. 2008, Biomaterials, Vol. 29, p. 4751.

426. Fundeanu, I, et al. 2008, Colloids Surf., B, Vol. 64, p. 297.

427. Ignatova, M, et al. 2006, Langmuir, Vol. 22, p. 255.

428. Zhang, F, et al. 2007, Ind. Eng. Chem. Res., Vol. 46, p. 9077.

429. Yao, F, et al. 2008, J. Membr. Sci., Vol. 319, p. 149.

430. Hawker, C J, et al. 1997, J. Am. Chem. Soc., Vol. 119, pp. 9903-9904.

431. Douglas, J F , Roovers, J and Freed, K F. 1990, Macromolecules, Vol. 23, pp. 4168-4180.

432. Acad. Sci. Stockmayer, W H, Fixmann, M and Ann, N Y. 1953, Vol. 57, pp. 334352.

433. Burchard, W. 1999, Advances in Polymer Science., Vol. 143, pp. 113-194.

434. Mendrek, B, et al. 2010, European Polymer Journal, Vol. 46, pp. 2341-2351.

435. Kowalczuk-Bleja, A, et al. 2005, Polymer, Vol. 46, pp. 8555-8564.

436. Burchard, W. 1999, Advances in Polymer Science, Vol. 143, pp. 113-194. 
437. Stenzel, M H and Davis, T P. 2002, J. Polym. Sci.:Part A., Vol. 40, pp. 44984512.

438. Boschmann, D, et al. 2008, Polymer, Vol. 49, pp. 5199-5208.

439. Boschmann, D, et al. 2, 2008, Polymer Preprints., Vol. 49, pp. 189-190.

440. Barner-Kowollik, C, et al. 2003, J. Polym. Sci.: Part A., Vol. 41, pp. 365-375.

441. Stenzel-Rosenbaum, M A , et al. 2001, J. Polym. Sci.: Part A., Vol. 39, pp. 27772783.

442. Boschmann, D and Vana, P. 2005, Polymer Bulletin., Vol. 53, pp. 231-242.

443. Prime, K L and and Whitesides, G M . 1993, J. Am. Chem. Soc., Vol. 115, p. 10714.

444. Mrabet, B, et al. 2009, Surface science, Vol. 603, p. 2422.

445. Li, L, et al. 2005, Phys. Chem. B, Vol. 109, p. 2934.

446. Jeon , S I , et al. 1991, J. Colloid Interface Sci., Vol. 142, p. 149.

447. Feng, W, Brash, J L and and Zhu, S. 2006, Biomaterials, Vol. 6, p. 847.

448. Sofia , S J, Premnath, V and and Merrill, E W. 1998, Macromolecules, Vol. 31, p. 5059.

449. Brash , J L and Horbett, T A. Proteins at Interfaces: Physicochemical and Biochemical Studies. Division of Colloid and Surface Chemistry. Washington : American Chemical Society, 1987. Meeting.

450. Brash , $\boldsymbol{J}$ L and Horbett , $\boldsymbol{T}$ A. Proteins at Interfaces II: Fundamentals and Applications. Division of Colloid and Surface Chemistry . Washington : American Chemical Society, 1995. Meeting.

451. Andrade, J D and Hladly, V. 1986, Advances in Polymer Science, Vol. 79, p. 1. 452. Kondo, A and Fukuda, H. 1998, Journal of Colloid and Interface Science, Vol. 198, p. 34.

453. Petrella, A, et al. 2005, J Phys Chem B, Vol. 109, p. 1554.

454. Kocher, M, et al. 1998, Appl. Phys. Lett., Vol. 72, p. 650.

455. Ohno, K, et al. 2006, Macromolecules, Vol. 39, p. 1245.

456. Ma, H W, et al. 2004, Advanced Materials, Vol. 16, p. 338.

457. Milner, S T. 1991, Science, Vol. 251, pp. 905-909.

458. Park, J W and Thomas, E L. 2002, J. Am. Chem. Soc., Vol. 124, pp. 514-515.

459. Cullen, S P , et al. 2007, Langmuir, Vol. 24, pp. 913- 920.

460. Brown, A A , et al. 2005, Eur. Polym. J., Vol. 41, pp. 1757-1765. 
461. Senaratne, W, Andruzzi, L and Ober, C K. 2005, Biomacromolecules, Vol. 6, pp. 2427-2448.

462. Skaff, H and Emrick, T. 2004, Angew. Chem., Int. Ed., Vol. 43, pp. 5383-5386.

463. Pirri, G, et al. 2006, Anal. Chem., Vol. 78, pp. 3118-3124.

464. Hong, C, You, Y and Pan, C. 2005, Chem. Mater., Vol. 17, pp. 2247-2254.

465. Balastre, M, et al. 2002, Macromolecules, Vol. 35, pp. 9480-9486.

466. Pyun, J and Matyjaszewski, K. 2001, Chem. Mater., Vol. 13, p. 3436.

467. Zhou, F and Huck, W T S. 2006, Phys. Chem. Chem. Phys., Vol. 8, p. 3815.

468. Barner, L, et al. 2004, J. Polym. Sci., Part A: Polym. Chem., Vol. 42, pp. 50675076.

469. Yoshikawa, C, et al. 2005, Macromolecules, Vol. 38, pp. 4604-4610.

470. Yu, W H, Kang, E T and Neoh, K G. 2004, Ind. Eng. Chem. Res. , Vol. 43, pp. 5194-5202.

471. Zhai, G, et al. 2004, Ind. Eng. Chem. Res., Vol. 43, pp. 1673-1680.

472. Yu, W H, Kang, E T and Neoh, K G. 2005, Langmuir, Vol. 21, pp. 450-456.

473. Hong, C, You, Y and Pan, C J. 2006, Polym. Sci., Part A: Polym. Chem., Vol. 44, pp. 2419-2427.

474. Hong, C, You, Y and Pan , C. 2006, Polymer, Vol. 47, pp. 4300-4309.

475. Xu, G, et al. 2006, Polymer, Vol. 47, pp. 5909-5918.

476. Li , C, et al. 2006, Macromolecules, Vol. 39, pp. 3175-3183.

477. Beija , M , Marty, J D and Destarac, M. 2011, Prog. Polym. Sci., Vol. 36, p. 845.

478. Wypych, F and Satyanarayana, K G. 2005, J Colloid Interface Sci, Vol. 285, p. 532.

479. McEuen, P L, et al. 1999, Microelectronic Engineering, Vol. 47, p. 417.

480. Katz, D A. 2002, Polymer, Vol. 4, p. 13.

481. Wager, C M , Haddleton, D M and Bon, S A F. 2004, Eur. Polym. J., Vol. 40, pp. 641-645.

482. Zengin, A and Caykara, T J . 2012, Polym. Sci., Part A: Polym. Chem., Vol. 50, pp. 4443-4450.

483. Lowe, A B , et al. 2002, J. Am. Chem. Soc., Vol. 124, pp. 11562-115623.

484. Sumerlin, B S, et al. 2003, Langmuir, Vol. 19, pp. 5559-5562.

485. Zhu, M Q, et al. 2004, J. Am. Chem. Soc., Vol. 126, pp. 2656-2657.

486. Shan, J, et al. 2003, Macromolecules, Vol. 36, pp. 4526-4533. 
487. Llauro, M F , et al. 2004, J. Polym. Sci., Part A, Vol. 42, pp. 5439-5462.

488. Loiseau, J, et al. 2003, Macromolecules, Vol. 36, pp. 3066-34077.

489. Lima, V, et al. 2005, J. Polym. Sci., Part A, Vol. 43, pp. 959-973.

490. Vana, P . 2007, Macromol. Symp., Vol. 248, pp. 71-81.

491. Peppas, N A and Langer, R . 1994, Science, Vol. 263, pp. 1715-1720.

492. Ratner, B D. 2000, J. Biomater. Sci-Polym. E, Vol. 11, pp. 1107-1119.

493. Xue, L and Greisler, H P J. 2003, Vasc. Surg., Vol. 37, pp. 472-480.

494. Frost, M C and Meyerhoff, M E . 2002, Curr. Opin. Chem. Biol., Vol. 6, pp. 633-641.

495. Frost, M C, Batchelor, M M and Lee, Y. 2003, Zhang, H.; Kang, Y.; Wilson, B.; Oh, G. S.; Gifford, R.; Rudich, S. M.; Meyerhoff, M. E., Microchem. J., Vol. 74, pp. 277-288.

496. Wisniewski, N and Reichert, M. 2000, Colloids Surf., B, Vol. 18, p. 197.

497. Benesch, J, et al. 2001, J. Biomater. Sci. Polym. Ed., Vol. 12, pp. 581-597.

498. Riedel, T, et al. 2013, Langmuir, Vol. 29, pp. 3388-3397.

499. Rodriguez-Emmenegger, C, et al. 2009, Langmuir, Vol. 25, pp. 6328-6333.

500. Gollander , $\boldsymbol{C} \boldsymbol{G}$, et al. Poly (ethyleneglycol) Chemistry Biotechnical and Biomedical Applications. [ed.] J. M. Harris. Newyork : s.n., 1992. p. 221.

501. Schwendel, D, et al. 2001, Langmuir, Vol. 17, p. 5717.

502. Zhu, B, et al. 2001, Journal of Biomedical Materials Research, Vol. 56, p. 406.

503. Tziampazis, E, Kohn, J and Moghe , P V. 2000, Biomaterials, Vol. 21, p. 511.

504. Feldman, K, et al. 1999, Journal of the American Chemical Society, Vol. 121, p. 10134.

505. Irvine, D J, et al. 1998, J. Biomed. Mater. Res, Vol. 40, p. 498.

506. Groll, J, et al. 2005, Langmuir, Vol. 21, p. 1991.

507. Hoffmann, J, et al. 2006, J. Biomater. Sci. Polym. Ed., Vol. 17, p. 985.

508. Zengin, A , Yildirim, E and Caykara, T. 2013, J. Polym. Sci., Part A: Polym. Chem., Vol. 51, pp. 954-962.

509. Tsujii , Y, et al. 2006, Adv. Polym. Sci., Vol. 197, p. 145.

510. Mitsukami, Y, et al. 2001, Macromolecules, Vol. 34, pp. 2248-2256.

511. Agilent GPC/SEC Solutions-Comprehensively Better Polymer Analysis. [Online] https://www.agilent.com/cs/.../5990-8844EN.pdf.

512. A guide to multi-detector gel permeation chromatography. [Online] https://www.agilent.com/cs/library/primers/public/5990-7196EN.pdf. 
513. [Online] https://en.wikipedia.org/wiki/Gel_permeation_chromatography .

514. [Online] http://www.viscotek.com/theory.htm. .

515. Hiemenz, C P and and Lodge, P T. Polymer Chemistry . 2nd Edition. s.l. : Boca Raton: CRC P, 2007. pp. 338-339. Vol. 336.

516. California Polytechnic State University. [Online] http://chemweb.calpoly.edu/djones/chem444/GPC.pdf.

517. [Online] https://www.agilent.com/cs/library/primers/Public/59906969EN\%20GPC\%20SEC\%20Chrom\%20Guide.pdf.

518. Helmut, D. Gel Chromatography, Gel Filtration, Gel Permeation, Molecular Sieves. s.l. : Springer Verlag. p. 1969.

519. Skoog , D A. Principles of Instrumental Analysis. 6th Edition. Belmont : Thompson Brooks/Cole, 2006. Vol. Chapter 28.

520. [Online] https://www.org.chemie.uni-goettingen.de/analytik/.

521. [Online] http://www.chemicool.com/img1/graphics/hplc-sch.gif .

522. Mass Spectrometry Fundamental LC-MS Introduction. [Online] http://www.ecs.umass.edu/eve/background/methods/chemical/Openlit/Chromacade my\%20LCMS\%20Intro.pdf.

523. Acurrion. [Online] http://www.accurion.com/imagingellipsometry/technology/principles-of-imaging-ellipsometry.pdf .

524. Benesch, J. Null Ellipsometry and Protein Adsorption to Model Biomaterials. Linköpings : s.n., 2001. PhD Thesis.

525. Fujiwara, H. Spectroscopic Ellipsometry Principles and Applications. s.l. : Wiley, 2009.

526. Azzam, R M A and Bashara, N M. Ellipsometry and Polarized Light. Amsterdam : Elsevier Science B. V., 1987.

527. Wikipedia. [Online] https://en.wikipedia.org/wiki/Ellipsometry .

528. Tompkins, G H and Irene, E A. Handbook of Ellipsometry . Newyork: William Andrew, Inc, 2005. p. 13.

529. Kessler, D . Inorganic-Organic Hybrid Polymers: Solution-Processible Coating Materials for Defined Surface Functionalization. Mainz : s.n., 2009. PhD Thesis.

530. Tompkins, $\boldsymbol{G} \boldsymbol{H}$ and Irene, E A. Handbook of Ellipsometry. Newyork: William Andrew Inc, 2005. p. 329.

531. Tompkins, H and Haber, E A . Handbook of Ellipsometry. New York: William Andrew Publishing, 2005. 
532. Butt, $\boldsymbol{H} \boldsymbol{J}$, Graf , $\boldsymbol{K} \boldsymbol{H}$ and and Michael, K. Measurement of Adsorption Isotherms , Physics and Chemistry of Interfaces. s.l. : Weinheim: Wiley-VCH, 2006. pp. 206-09.

533. [Online] http://www.nuance.northwestern.edu/keckii/ftir1.asp.

534. [Online] http://www.kutztown.edu/Images/Chemistry/Bruker_Tensor.png .

535. Kendall , D S. Infrared Spectroscopy of Coatings, Coatings Technology, Fundamentals, Testing, and Processing Techniques. [ed.] A A. Boca Raton : CRC Press, 2007.

536. Bruker. A Versatile Tool for FT-IR Spectroscopy, Attenuated Total Reflection (ATR). . Application Note AN 79.

537. Binnig, G, Quate, C F and Gerber, C. 1986, Phys. Rev.Lett., Vol. 56, p. 930.

538. Binnig, G, et al. 1982, Phys. Rev. Lett., Vol. 50, p. 120.

539. Sterr, $\boldsymbol{F}$. Optimization of Josephson Junction Nanofabrication for Superconducting Quantum Circuits. Mainz : s.n., 2013. PhD Thesis.

540. Kessler, D. Inorganic-Organic Hybrid Polymers: Solution-Processible Coating Materials for Defined Surface Functionalization. Mainz : s.n., 2009. PhD Thesis.

541. Hinterdorfer, P and Dufrene, Y F. 2006, Nature Methods, Vol. 3, p. 5.

542. Muller, D M. Atomic Force Microscopy on Nano Scale Josephson Contacts. Muenchen : s.n., 2013.

543. Giessibl, F. 2003, Rev. Modern Phys., Vol. 75, p. 949.

544. Eaton, P and and West, P. Atomic force microscopy . s.l. : Oxford Univ. Press, 2010.

545. WaveMetrics . s.l. : Igor Pro, 1989.

546. Horcas, I, et al. 2007, Rev. Sci. Instrum., Vol. 78, p. 013705.

547. Holly , F J. Physiochemical Aspects of Polymer Surfaces. New York : Plenum Press, 1983.

548. Padday, J F. 3-4, 1968, Journal of Colloid and Interface Science, Vol. 28, p. 557. 549. Befay, P and and Petre, G. 1969, Surface Colloid Science, Vol. 3, pp. 27-80. 550. [Online] http://www.ramehart.com/images/standard_goniometer.jpg . 551. [Online] 1. http://images.google.de/imgres?imgurl=https\%3A\%2F\%2Fi.ytimg.com\%2Fvi\% FXNH-

NnLODdg\%2Fhqdefault.jpg\&imgrefurl=https\%3A\%2F\%2Fwww.youtube.com\%2 Fwatch\%3Fv\%3DXNH-NnLODdg\& $h=360 \& w=480 \&$ tbnid $=c-$ 
_XR5_K74fV5M\%3A\&docid=5QdZ9i3BI6SzIM\&ei=OF5QV6rZIsaPgAbY65uYCg $\&$ tbm $=$ isch \& client $=$ safari\&iact $=r c \&$ uact $=3 \&$ dur $=714 \&$ page $=1 \&$ start $=0 \&$ ndsp $=30$ \&ved=0ahUKEwiq1cqN4YnNAhXGB8AKHdj1BqMQMwgzKAswCw\&bih=744\&bi $w=1199$.

552. Kruss. [Online] Technical Note-TN311e-Practical Contact Angle Measurement. http://www.kruss.de . 


\subsection{Acknowledgements}

The best part of my PhD studies in Germany is not only writing this thesis but also the fact that I had the opportunity to meet a lot of interesting people from different scientific, cultural and personal backgrounds, who have been around me during my $\mathrm{PhD}$ and supported me in achieving my goals by whatever means they could. This work would not have been possible without the help and support of many people. It is my pleasure to express my gratitude to all the people who supported me during my $\mathrm{PhD}$.

I would like to extend my heartfelt gratitude to my advisor, Prof. Philipp Vana, not only for his supervision, insight, advice, guidance throughout all stages of this research but also for his humanistic, kind style of management and the trust he gave me in fulfilling this interesting and interdisciplinary thesis. He provided me enthusiastic support and encouragement in various ways. I am very proud to have had the opportunity to work with him.

Many thanks go to Prof. Marcus Muller and Prof. Jorg Enderlein, members of the Advisory committee, for their important guidance and suggestions. I would like to express my sincere gratitude to Dr. Iwan Schaap and Dr. Mitja Platen of the third institute of Physics department, University of Goettingen for AFM support; Prof. JeanChristophe Baret, Dr. Quentin Brosseua and Dr. Renaud Dufour of Self Organization and Dynamics department of the Max Planck Institute for Goniometer support and mask design; Dr. Christian Rolling of Acurrion for Spectroscopic Ellipsometer; Prof. Andrea Polle and Dr. Rodica Pena of the department of Forest Botany and Tree Physiology for ATR spectrometer.

In addition, I would like to thank to DFG (German Research Foundation) and SFB 937 (Collective behavior of soft and biological matter) for the financial support.

I am also very grateful to fellow colleagues and friends for their continuous support, and advices.

Words fail me to express my appreciation to my mother Nuran Catli and my aunt Nuray Altiok, whose dedications, loves, compassions and emotional supports have always been with me during not only my $\mathrm{PhD}$ studies but also my entire life.

Finally, I would like to thank to my best friend Berna Turgut and my cousin Zehra Altiok for their lifelong friendships and moral support. 


\subsection{Curriculum Vitae}

\section{CANDAN CATLI}

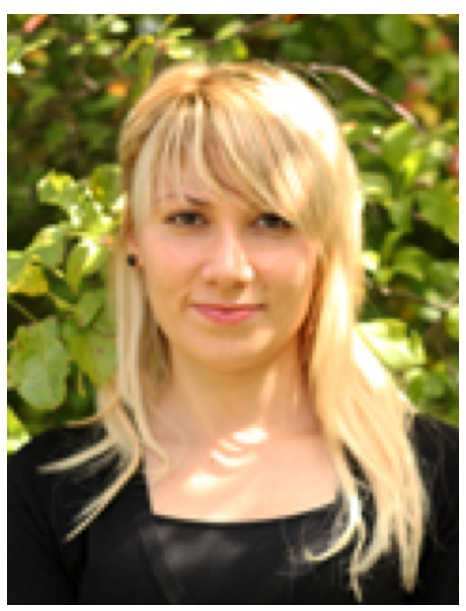

\section{SCIENTIFIC INTERESTS}

Smart polymeric thin films, protein resistive coatings, controlled radical polymerization techniques (CRP), reversible addition fragmentation chain transfer technique (RAFT), surface initiated polymerizations from metal and metaloxide surfaces, polymer brushes, star polymers.

\section{EDUCATION}

\section{1-2016: Doctoral studies}

Department of Physical Chemistry, George August University of Goettingen, Germany Doctoral Thesis: Synthesis and characterization of polymer brushes with complex topologies on gold surfaces via Surface Initiated Reversible Addition Fragmentation Chain Transfer Technique (SI-RAFT)

2008-2010: Graduate studies

Department of Polymer Science and Technology, Istanbul Technical University, Turkey

Master Thesis: Novel synthetic approaches for multi-functional ATRP initiators

2003-2008: B.Sc.

Department of Chemical Engineering, Istanbul University, Turkey

Diploma Thesis: Feasibility study of establishment of high density polyethylene production company 


\section{PUBLICATIONS}

Catli, C., Vana, P., Synthesis of Surface Initiated Diblock Copolymer Brushes from Gold Flat Substrates Utilizing the Raft Polymerization Technique; in preparation

Catli, C., Vana, P., Raft Mediated Synthesis of Diblock Copolymer Brushes on Gold Flat Surfaces via Direct Attachment of Raft Agents; in preparation

Catli, C., Vana, P., The Easiest Route for Polymer Brush Fabrication on Gold Utilizing Raft Agent Precursor Dithiobenzoicacid; in preparation

Catli, C. and Vana, P., Gold Surfaces with Protein Repellence Properties via Z-Raft Star Polymerizations; in preparation

Catli, C. and Vana, P., A Novel Three Thiol Anchored Raft Agent for Surface Initiated Raft Polymerizations from Gold Flat Surfaces; in preparation

Catli, C., Vana, P., Fabrication of Patterned Polymer Brushes on Titanium Surfaces via RAFT Polymerization Technique; in preparation

\section{INTERNSHIPS -TEACHING}

2012-Present: Tutor in Vacuum Technic Laboratory, Department of Physical Chemistry, George August University of Göttingen

2011-Present: Researcher at the project of the SFB 937, Deutsche Forschungsgemeinschaft (DFG)

2009-2010: Tutor in Polymer Characterization Laboratory, Istanbul Technical University

2008-2010: Researcher at the project of the Scientific and Technological Research Council of Turkey (TUBITAK)

2005-2007: Tutor in General Chemistry Laboratory, Istanbul University

2005: Internship at the AK-KIM Chemical Company, Yalova, Turkey (6 weeks)

2004: Internship at the AK-SA Acrylic Fiber Company, Yalova, Turkey (6 weeks) 


\section{PROJECTS}

2011-Present: "Synthesis and Characterization of Polymer Brushes with Complex Topologies on Gold Surfaces via Reversible Addition Fragmentation Chain Transfer Technique" (DFG)

2011-Present: “ Polymer Brushes in Motion ”, SFB 937 (DFG)

2008-2010: "Baroplastic Elastomers: A Novel Processing for Plastic Processing and Recycling", The Scientific and Technological Research Council of Turkey (TUBITAK)

2008-2010: "Novel Synthetic Approaches for Multifunctional ATRP Initiators” (Master) 2007: "Feasibility Study of Establishment of High Density Polyethylene Production Company" (Bachelor)

\section{SYMPOSIUMS}

11-13 November 2015: SFB 937 Autumn School, Hohegeis, Germany

16-17 October 2014: Niedersächsisches Katalyse Symposium, George August University, Göttingen, Germany (Poster Presantation)

18-19 October 2012: Niedersächsisches Katalyse Symposium, George August University, Göttingen, Germany (Poster Presantation)

19 July 2012: SFB 937 Summer Symposium, Campus MPI Fassberg, Göttingen, Germany (Poster Presantation)

14-15 February 2012: SFB 937 Winter Symposium, Eisenach, Germany (Oral Presantation)

10-15 July 2011: IP'11-International Symposium on Ionic Polymerizations, Akron, Ohio, USA

18-19 April 2009: Novel Polymeric Materials and Technology Symposium, Çanakkale Onsekiz Mart University, Canakkale, Turkey (Oral Presentation)

9-13 June 2008: 4.Nanoscience and Nanotechnology Congress, Istanbul Technical University, Istanbul, Turkey (Poster Presantation) 